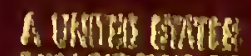

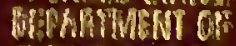

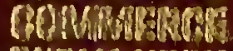

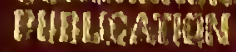
(i)

MHE WWGML HULCAWON 39

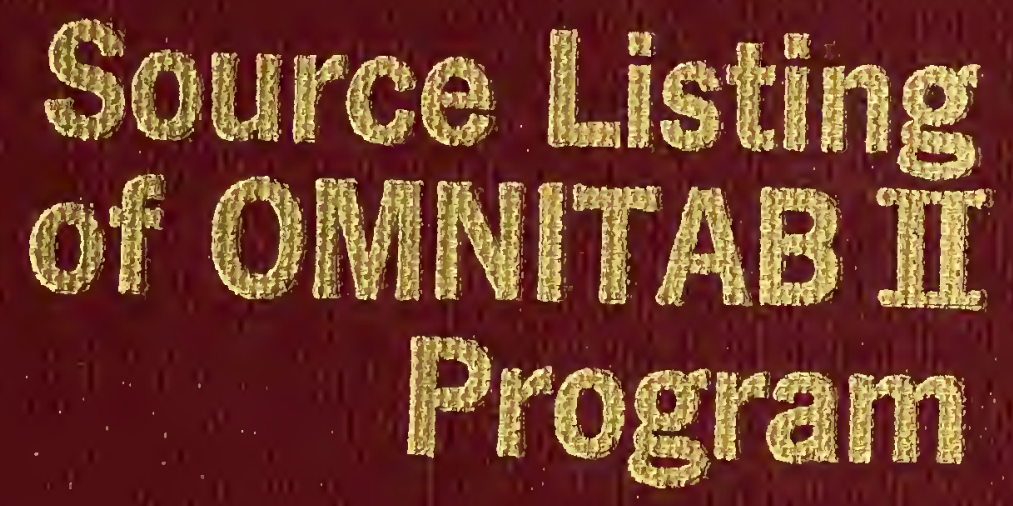

HBM

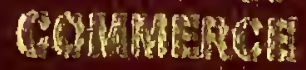

Natong: Puroger

Buncharde 





\title{
Source Listing of OMNITAB II Program
}

\author{
Sally T. Peavy, Ruth N. Varner, and David Hogben \\ Statistical Engineering Laboratory \\ Applied Mathematics Division \\ Institute for Basic Standards \\ National Bureau of Standards \\ Washington, D.C. 20234
}

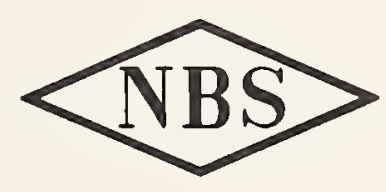

U., ,National Bureau of Standards Special Publication 339

Nat. Bur. Stand. (U.S.), Spec. Publ. 339, 371 pages (Dec. 1970)

CODEN: XNBSA

Issued December 1970 
NATIONAL BUREAU OF STANDARDS

APR 51979
LO32\%
$0 \mathrm{C} 100$
$\cup 57$
No.339
1970
Copor

Library of Congress Catalog Card Number: 79-609406 


\section{Contents}

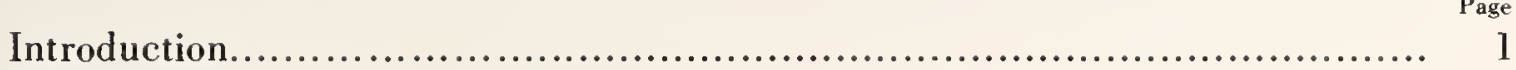

Description................................................................... 1

Programming Techniques............................................................. 2

OMNITAB II Operating System Routines....................................... 3

References................................................................... 4

Table of Contents for Subprogram Listings...................................... 5

Listing of Subprograms........................................................... 7

Appendix-OMNIT Flow Chart................................................ 346 



\title{
Sally T. Peavy, Ruth N. Varner, and David Hogben
}

\begin{abstract}
OMNITAB II is a general-purpose interpretive computing system designed to allow a nonprogrammer to use a high-speed computer easily, accurately and effectively. The system permits the user to perform arithmetic operations including complex arithmetic, trigonometric calculations, miscellaneous function calculations, statistical analysis, Bessel function calculations, and operations on matrices and arrays.
\end{abstract}

The OMNITAB II system contains 177 subprograms written in the ANSI FORTRAN language. Every effort has been made to make the system transportable. This publication contains a complete listing of all these subprograms. The listing is preceded by a brief introduction which describes the programming techniques used; the use of system library functions; and the subprograms used to control the flow of operations in the OMNITAB system.

Key words: Accuracy: algorithms; ANSI FORTRAN; documentation; error checking; machine independent: OMNITAB II operating system subprograms; OMNITAB II source listing; programming techniques; transportable; user-oriented computing system.

\section{Introduction}

The OMNITAB II computing system enables the nonprogrammer to use a large digital computer to perform data, statistical, and numerical analysis without having any prior knowledge of computers or computer languages. The user writes simple English instructions to obtain accurate results easily and effectively. The instructions reference reliable, varied, and sophisticated algorithms for analysis and manipulation. The tedious task of formatting data for input and output is alleviated by OMNITAB II through free field input, readable printing, and automatic printouts. Formatting capability exists, if more flexibility is desired. The system permits the user to perform: arithmetic operations including complex arithmetic, trigonometric calculations, data manipulations, miscellaneous function calculations, statistical analysis, numerical analysis, Bessel function calculations, operations on matrices and arrays, and thermodynamic calculations. Although OMNITAB has been specifically designed for nonprogrammers, many experienced programmers find uses for OMNITAB.

OMNITAB was developed at the National Bureau of Standards under the guidance of Joseph Hilsenrath who contributed the basic ideas and philosophy, see Hilsenrath et al. [1966]. The program was written initially in a mixture of symbolic machine language and FORTRAN. With the advent of third generation computers and the success of OMNITAB, it became necessary to rewrite the program and to make it as machine independent as possible. Walter J. Gilbert undertook this task in 1966 and initiated a number of changes and new features. Since 1968, the Statistical Engineering Laboratory, Applied Mathematics Division, has been responsible for the development, maintenance, and management of OMNITAB. NBS OMNITAB II Version 5.0 (Hogben et al. [1970]) is the result of this effort.

\section{Description}

This publication is one of four which constitute the documentation for OMNITAB II. A user's guide is given in Hogben et al. [1970]. A systems programmer's guide is contained in Peavy et al. [1970]. Problems and results for assessing the success of implementing OMNITAB II are given in Varner et al. [1970]. No attempt is made to make this publication independent of the other three. In particular, the reader should consult Hogben et al. [1970] for a general understanding of OMNITAB II and for an explanation of terms used in conjunction with OMNITAB II.

The OMNITAB II software system contains 177 subprograms written in American National Standard (ANSI) FORTRAN language. This publication contains a listing of all of these subprograms in section 7. The subprograms are listed alphabetically by the subprogram name; except for the main program which appears first. Each subprogram has a unique identification in columns 73-75. Sequential line numbers appear in columns 76-79. This provides a means of easily updating the subprogram when improvements are made. Each subprogram has been tidied using the program of Murphy [1966].

A table of contents giving the subprogram name, subprogram identification, and page number is given in section 6 on pages 5 and 6 . Along with the identification, symbols are used to indicate the main function of each subprogram. The symbol denotes the main purpose and not necessarily the sole function of the subprogram. For example, OMNIT is an executive subprogram, but it also executes the command NOTE.

The material contained herein is a complete listing of the OMNITAB II program with some exceptions. Subprograms which compute the square root, sin, cos, tan, input and output and other subprograms which are normally part of the operating system library are not included; see Peavy et al. [1970]. 
Each subprogram has been tested as thoroughly as possible. A few errors which escaped detection in the preliminary testing were subsequently detected in extensive use and corrected. Despite considerable effort to make OMNITAB II error free, a few errors are to be expected in such a large system. We believe that any errors that remain are few in number. Any reader who finds errors or has any comments is encouraged to write to us so that improvements can be passed on to other users.

\section{Programming Techniques}

The subprograms which comprise the OMNITAB II system were the efforts of many individuals working independently with considerable latitude. Thus, the programming techniques used in the subprograms are varied. However, each subprogram had to satisfy the following criteria.

(a) Programming language. All subprograms are written in the ANSI FORTRAN language. Thus, the routines which scan the OMNITAB II instructions, manipulate and pack characters, and involve input-output are lengthy and to a limited extent inefficient. These routines could be written in machine language for a particular computer installation if efficiency were sufficiently important. However, more attention has been devoted to developing a system which is transportable and easily used. Since OMNITAB II is designed primarily for the analysis of small-to-moderate amounts of data, these considerations are far more important than machine efficiency up to a certain point.

Every effort was made to produce machine independent subprograms in order to make the entire system transportable. Where it was necessary to supply alphanumeric information, a maximum of three characters per machine word was permitted. The only exception is in the case where user defined formats are packed to a full capacity of the machine word. Formats containing information for printout were restricted to 120 characters per line. Variable names were used in all references to input-output devices. These special features are discussed further in Peavy et al. [1970]. All FORTRAN compilers do not accept every ANSI FORTRAN statement and efforts were made to avoid the use of such statements.

(b) Algorithms. The policy is to use the most reliable and accurate algorithms. In some instances it was not feasible to adhere to this policy completely. Sometimes, a good algorithm was accepted with the intention of improving it at a later date. This is particularly true for the commands with comprehensive automatic printing. Again, efficiency was subordinated to accuracy when necessary. Most calculations are performed using single precision floating-point arithmetic. In a few instances, where more accuracy was deemed desirable, double precision floating-point arithmetic was used. Double precision arithmetic was used primarily to obtain full single precision accuracy.

(c) Error checking. Every subprogram which executes a command is required to do extensive error checking. Besides checking for correct number and type of arguments and previous errors, the subprograms have to perform unique checks for the particular command that is to be executed. Specific messages are printed and execution of further OMNITAB instructions may be affected depending upon the severity of the error. However, this does not prevent OMNITAB from scanning the remaining instructions for errors unless the instruction is stored. See Hogben et al. [1970] and Peavy et al. [1970] for further details.

(d) Elementary functions. The original OMNITAB had its own programs to compute the elementary functions (SIN, LOGE, etc.) which were of known accuracy. In order to make OMNITAB II as transportable as possible, this procedure was not followed and the system library functions of the particular computer system are used. The accuracy of system library subroutines varies from one computer to another. Some are quite accurate; others are surprisingly inaccurate as was explained excellently by Cody [1970].

Many system library functions terminate execution if an arithmetic fault is encountered; for example, in computing the square root of a negative number. This confuses and hinders the nonprogrammer since the messages upon termination are cryptic and often the fault is not significant. Efforts have been made to eliminate this nuisance in OMNITAB II. The subprograms are not permitted to use any of the library functions directly. Instead, they reference other function routines which check the values of the arguments to be used by the system library functions. See Peavy et al. [1970] for a list of these function routines. Only if the values are arithmetically legitimate and within certain bounds will the system library functions be used. Otherwise, a very explicit diagnostic message will be printed. The result is set equal to zero and execution is continued.

(e) Input-output routines. Input-output conversion routines were also part of the old OMNITAB, but again these had to be dropped in favor of using operating system routines to make OMNITAB more transportable. There is one important exception. The subprogram RFORMT does the necessary output conversion for many of the basic printing commands. RFORMT does its own conversion to enable the printing of numbers in a more readable form. The conversion is as accurate as or more accurate than the standard operating system output conversion routines. See Hogben [1970] for further details.

(f) Division by zero. Computer operating systems handle division by zero in many different ways. In some instances a run may be terminated, in other cases it may be ignored, and sometimes just 
a message is printed that division by zero was attempted. The OMNITAB II commands which specifically call for division, such as DIVIDE and ADIVIDE, provide a check on zero before division is attempted. If the divisor is zero, the result is set equal to zero, a message is printed, and execution is continued.

In some commands division by zero can occur in nonobvious places depending upon the algorithm which is used, as in the FIT command. When it is known that a divisor can equal zero, a check on the divisor was provided. However, a check on the divisor is not made in every instance that division is used. It is possible that an error termination could occur, but it is not likely.

(g) Comment statements. Comments in the form of FORTRAN comment statements are dispersed in most subprograms. Some of the comments are very detailed and complete while others are rather limited. The use of comments was at the discretion of the programmer and no standard style or format was required. Due to recent changes in OMNITAB II, some of the comments may not be pertinent. No effort has been made to edit the comments.

(h) Stand alone subprograms. Some of the subprograms may be used separately outside the context of the OMNITAB II system. More often, the programmer took advantage of the OMNITAB II system when writing a particular subprogram. A list of all the stand alone programs is given below. Some use the OMNITAB II elementary function routines and would have to be modified accordingly. These exceptions are shown in parentheses. A number of other subprograms, not listed here, such as RFORMT could be easily modified to make them stand alone.
ACCDIG
(FLOG10)
computes the accuracy of one number compared to another
BEZERO
(FDSQRT)
computes zeros of Bessel function of order zero
BEZONE
computes zeros of Bessel func- tion of order one
BJORCK least squares quadratic fit for CORRELATION
CBEI (FDSIN, FDCOS, FDSQRT, FDEXP)
computes Bessel functions of complex argument, with and without scale factors
DETRNK computes the determinant and
FREQCY (FLOG10) rank of a matrix
HDIAG (FSQRT)
computes eigenvalues and eigen- vectors of a matrix
INTRP provides n-point Lagrangian in- terpolation
QRion of a column of numbers
computes the frequency distribu-
MXTXP
(calls SORTSM below) per- forms matrix multiplication $\mathrm{X}^{\prime} \mathrm{X}$ or $\mathrm{XX}^{\prime}$
PVTRI determines if matrix is upper or lower triangular
RANKO
RANKX
(equivalent to RANKX below)
RCSUM computes row and column sums of a matrix
RNJBK
SKSYMV
SORTSM
SPINV
SYMV
machine independent pseudo ran- dom number generator
tests for skew symmetry of a matrix
sorts products of matrix multipli- cation and sum
matrix inversion with minimum round-off error accumulation test for matrix symmetry

\section{OMNITAB II Operating System Routines}

The main program of the OMNITAB II system is small and contains one call to the OMNIT subprogram. The OMNIT and XECUTE subprograms are the two major routines in the OMNITAB II system. A detailed flow chart of OMNIT, prepared using CAL-COMP [1968], is given in the appendix. A capsule summary of the chief function of each of the 34 executive subprograms is given below. In addition, there are four block data subprograms which are BLOCK, LBCONS, LOOKTB and PHYSIC. The reader can consult Peavy et al. [1970] for further details.

Main Program
AARGS

ADRESS

AERR

ASTER

CHKCOL

CKIND

ERROR

EXPAND

INFERR

INPUT

LOCATE

LOOKUP

\section{calls OMNIT}

converts arguments of an instruction

computes memory location of first row of worksheet column tallys and prints arithmetic faults

checks proper use of asterisks checks for valid column numbers and computes memory locations

checks mode of instruction arguments

prints fatal errors

sets up IARGS, ARGS and

KIND for use of arguments by subprograms

prints informative diagnostics reads all cards

locates stored instructions assigns values to $\mathrm{L} 1$ and $\mathrm{L} 2$ which are used by XECUTE 
MTXCHK

NNAME

NONBLA

OMCONV

OMNIT

OUTPUT

PACK

PREPAK

READQ

RNDOWN

SETQ

SETUP

STMT

STORE

TAPOP

VARCON

VECTOR

XECUTE

XOMNIT

XPND

XSTOP determines if matrix fits in worksheet and computes memory location

converts command name to a unique number

looks for next non-blank character in scanning card

converts input card images to standard code

main subprogram which controls flow of operations

outputs card images to scratch unit

packs or unpacks alphanumeric characters

controls packing/unpacking of characters for OMNITAB II system

converts and stores data

prints location of error in stored instructions

converts and stores data

initializes system at the very beginning

assembles and checks an instruction number

stores numbered instructions

sets up arguments for tape operation commands

checks for legitimate variables within asterisk

vectorizes a constant

calls appropriate subprogram to execute an instruction

initializing for OMNITAB command

sets up IARGS, ARGS and KIND for stored instructions

terminates use of OMNITAB and returns control to operating system

OMNITAB II is a large system and its success is due in no small part to the fact that many specialists have contributed to its development. We especially thank J. Hilsenrath for his continued interest and advice. Walter J. Gilbert laid the foundation for OMNITAB II by developing the scan routine, many of the basic subroutines, and by adding several new features. Joseph M. Cameron formulated the set of instructions for matrix and array operations in addition to providing guidance, particularly in the development of the least squares curve fitting instructions. The following individuals, besides the authors, not only programmed many of the subprograms, but checked them out as well: Robert C. McClenon, Carla G. Messina, Bradley A. Peavy, and Philip J. Walsh. M. Stuart Scott did a fine job of developing CORRELATION. Irene A. Stegun and Ruth Zucker provided the subroutine ERRINT which is used for the commands ERROR and CERF; see Stegun and Zucker [1970]. Roy H. Wampler and William J. Hall provided the subroutine BJORCK for use with the subprogram CORREL. We thank John Mandel for providing an approximation to percentage points of the studentized range, which is used in the subprogram ONEWAY. Shirley G. Bremer gave valuable assistance in general maintenance and housekeeping. We thank Bruce W. Ramsay and Robert J. Arms of the Computer Services Division for their administrative and technical support. The staff of the Statistical Engineering Laboratory, under Joan R. Rosenblatt, made invaluable contributions and suggestions arising out of their varied experiences in statistical consulting. Carla G. Messina merits additional thanks for preparing section 7 for computerized phototypesetting. Last, but not least, special thanks are given to all the users who made valuable comments and stimulated modifications and additions to the system.

\section{References}

American Standard Fortran (1966). American National Standards Institute, New York.

CAL-COMP (1968). Flowgen/F - A Fortran Flowchart Generator. California Computer Products, Inc.

Cody, W. J. (1970). Software for the elementary functions. Presented at Mathematical Software Symposium, Purdue University, April 1-3.

Hilsenrath, J., Ziegler, G. G., Messina, C. G., Walsh, P. J., and Herbold, R. J. (1966). OMNITAB: A Computer Program For Statistical And Numerical Analysis. Nat. Bur. Stand. (U.S.), Handb. 101. Superintendent of Documents, U.S. Government Printing Officc, Washington, D.C. 20402. Reissued January 1968 with corrections.

Hogben, David (1970). Readable printing of single precision numbers. To be submitted to J. Res. Nat. Bur. Stand.

Hogben, David, Peavy, S. T., and Varner, R. N. (1970). OMNITAB II User's Reference Manual. Nat. Bur. Stand. (U.S.), Tech. Note 552, Superintendent of Documents, U.S. Government Printing Office, Washington, D.C. 20402.

Murphy, H. M., Jr. (1966). Tidy, a computer code for renumbering and editing Fortran source programs. Air Force Systems Command, Kirkland Air Force Base, New Mexico.

Peavy, S. T., Varner, R. N., and Bremer, S. G. (1970). A Systems Programmer's Guide For Implementing OMNITAB II. Nat. Bur. Stand. (U.S.), Tech. Note 550, Superintendent of Documents, U.S. Government Printing Office, Washington, D.C. 20402.

Stegun, Irene A., and Zucker, Ruth (1970). Automatic computing for special functions. J. Res. Nat. Bur. Stand. (U.S.), 74B (Math. Sci.), No. 3, 211-225, July-Sept.

Varner, R. N., and Peavy, S. T. (1970). Test Problems And Results For OMNITAB II. Nat. Bur. Stand. (U.S.), Tech. Note 551, Superintendent of Documents, U.S. Government Printing Office, Washington, D.C. 20402. 


\section{Table of Contents for Subprogram Listings}

The following symbols are used to indicate the main function of each subprogram. The number of subprograms in each category is given in parentheses on the extreme right.

E OMNITAB II executive system control subprogram

Function routine as defined in 3 (d)

I executes instruction(s) (and performs calculations)

C performs computations for subprogram(s) which execute instruction(s)

\begin{tabular}{|c|c|c|c|c|c|c|c|}
\hline \multirow{2}{*}{$\frac{\text { Subprogram name }}{\text { Main Program }}$} & \multicolumn{2}{|c|}{ Identification } & \multirow{3}{*}{$\begin{array}{r}\text { Page } \\
7 \\
8\end{array}$} & \multirow{3}{*}{$\begin{array}{c}\text { Subprogram name } \\
\text { EXCHNG } \\
\text { EXPAND }\end{array}$} & \multicolumn{2}{|c|}{ Identification } & \multirow{3}{*}{$\begin{array}{r}\text { Page } \\
77 \\
78\end{array}$} \\
\hline & OMS & $\mathrm{E}$ & & & EXC & $I$ & \\
\hline AARGS & AAR & $\mathrm{E}$ & & & EXD & $\mathrm{E}$ & \\
\hline ABRIDG & ABR & I & 10 & EXPCON & EXN & $\bar{I}$ & 80 \\
\hline ACCDIG & $\mathrm{ACC}$ & $\mathrm{C}$ & 11 & EXTREM & EXT & I & 82 \\
\hline ADRESS & ADR & $\mathrm{E}$ & 11 & FCOS & $\mathrm{FCO}$ & $\mathrm{F}$ & 83 \\
\hline AERR & AER & $\mathrm{E}$ & 12 & FDCOS & FDC & $\mathrm{F}$ & 83 \\
\hline ALLSUB & ALL & I & 14 & FDEXP & FDE & $\mathrm{F}$ & 83 \\
\hline APRINT & APR & I & 17 & FDLOG & FDL & $\mathrm{F}$ & 84 \\
\hline ARITH & ARI & I & 20 & FDPCON & FDP & $\mathrm{F}$ & 84 \\
\hline ARYVEC & ARY & I & 22 & FDSIN & FDS & $\mathrm{F}$ & 84 \\
\hline ASTER & AST & $\mathrm{E}$ & 25 & FDSQRT & FDQ & $\mathrm{F}$ & 85 \\
\hline BEGIN & BEG & I & 27 & FEXP & FEX & $\mathrm{F}$ & 85 \\
\hline BEJN & $\mathrm{BEJ}$ & $\mathrm{C}$ & 28 & FEXP2 & $\mathrm{FX} 2$ & $\mathrm{~F}$ & 85 \\
\hline BESSEL & BES & $\mathrm{I}$ & 30 & FIXFLO & FIX & $\mathrm{I}$ & 86 \\
\hline BEZERO & $\mathrm{BEZ}$ & $\mathrm{C}$ & 36 & FLIP & FLI & I & 87 \\
\hline BEZONE & $\mathrm{BEO}$ & $\mathrm{C}$ & 37 & FLOG & FLE & $\mathrm{F}$ & 88 \\
\hline BINTJO & BIN & $\mathrm{C}$ & 38 & FLOG10 & FLT & $\mathrm{F}$ & 88 \\
\hline BJORCK & BJO & $\mathrm{C}$ & 39 & FNEC & FNE & I & 89 \\
\hline BLOCK & BLO & $\mathrm{B}$ & 41 & FNEIC & FNC & $\mathrm{I}$ & 90 \\
\hline CBEI & CBI & $\mathrm{C}$ & 42 & FNKC & FKC & $\bar{I}$ & 91 \\
\hline CBEK & CBK & $\mathrm{C}$ & 44 & FOURIA & FOU & $\mathrm{C}$ & 92 \\
\hline CHANGE & CHA & I & 46 & FPPT & FPP & C & 93 \\
\hline CHKCOL & CHK & $\mathrm{E}$ & 46 & FPROB & FPR & I & 94 \\
\hline CKIND & CKI & $\overline{\mathrm{E}}$ & 47 & FRDIST & FRD & I & 95 \\
\hline CMPARA & CMP & $\mathrm{C}$ & 47 & FREQCY & FRE & C & 96 \\
\hline CMSEPA & CMS & I & 48 & FSIN & FSI & $\mathrm{F}$ & 97 \\
\hline COALES & COA & $\mathrm{I}$ & 51 & FSQRT & FSQ & $\mathrm{F}$ & 97 \\
\hline COMELL & COM & $\mathrm{C}$ & 54 & FTANH & FTA & $\mathrm{F}$ & 97 \\
\hline COMPLX & $\mathrm{COX}$ & I & 55 & FUNC'T & FUN & I & 98 \\
\hline CORREL & COR & I & 57 & GENER & GEN & I & 102 \\
\hline CSPINV & CSP & $\mathrm{C}$ & 62 & GQUAD & QUA & I & 103 \\
\hline DBEJ & DBE & $\mathrm{C}$ & 64 & HDIAG & HDI & $\mathrm{C}$ & 104 \\
\hline DEFINE & DEF & I & 66 & HEADS & HEA & C & 108 \\
\hline DETRNK & DET & $\mathrm{C}$ & 67 & HISTGM & HIS & I & 110 \\
\hline DHRND & DHR & C & 68 & IFS & IFS & I & 111 \\
\hline DIMENS & DIM & I & 69 & INFERR & INF & $\mathrm{E}$ & 113 \\
\hline DUMMYA & DMA & I & 70 & INPUT & INP & $\mathrm{E}$ & 116 \\
\hline DUMMYB & DMB & $\mathrm{I}$ & 70 & INTERP & INT & I & 117 \\
\hline DUMMYC & DMC & I & 70 & INTRP & INR & $\mathrm{C}$ & 119 \\
\hline DUMMYD & DMD & $\mathrm{I}$ & 70 & INVCHK & INK & $\mathrm{C}$ & 121 \\
\hline DUMMYE & DME & I & 70 & INVCOR & INC & C & 123 \\
\hline DUMMYF & DMF & $\mathrm{I}$ & 70 & INVERT & INV & I & 125 \\
\hline ERASE & ERA & I & 71 & ITERAT & ITE & $\mathrm{I}$ & 127 \\
\hline ERRINT & ERT & $\mathrm{C}$ & 72 & LBCONS & LBC & B & 132 \\
\hline ERROR & ERR & $\mathrm{E}$ & 74 & LIST & LIS & I & 133 \\
\hline
\end{tabular}


6. Table of Contents for Subprogram Listings (continued)

\begin{tabular}{|c|c|c|c|c|c|c|c|}
\hline Subprogram name & \multicolumn{2}{|c|}{ Identification } & Page & Subprogram name & \multicolumn{2}{|c|}{ Identification } & Page \\
\hline LOCATE & LOC & $\mathrm{E}$ & 133 & PUNCH & PUN & I & 255 \\
\hline LOOKTB & LOT & $\mathrm{B}$ & 134 & PVTRI & PVT & $\mathrm{C}$ & 256 \\
\hline LOOKUP & LOU & $\mathrm{E}$ & 14.3 & RANKO & RKO & $\mathrm{C}$ & 257 \\
\hline MATRIX & MAT & I & 145 & RANKS & RAS & I & 258 \\
\hline MDAMAD & MDA & I & 149 & RANKX & RAX & $\mathrm{C}$ & 259 \\
\hline MEIGEN & MEI & $\mathrm{I}$ & 151 & RCSUM & RCS & $\mathrm{C}$ & 260 \\
\hline MISC2 & MIS & I & 153 & READQ & REQ & $\mathrm{E}$ & 261 \\
\hline MIST & MST & $\mathrm{C}$ & 156 & READX & REX & I & 262 \\
\hline MKRON & MKR & $\mathrm{I}$ & 158 & REPINC & REP & I & 264 \\
\hline MMULT & MMU & I & 160 & RESET & RES & $\mathrm{I}$ & 268 \\
\hline MOP & MOP & $\mathrm{I}$ & 162 & RFORMT & RFO & $\mathrm{C}$ & 269 \\
\hline MOVE & MOV & I & 164 & RNDOWN & RND & $\mathrm{E}$ & 273 \\
\hline MPROP & MPR & I & 165 & RNJBK & RNJ & $\vec{C}$ & 273 \\
\hline MRAISE & MRA & $\mathrm{I}$ & 173 & RPRINT & $\mathrm{RPR}$ & $\mathrm{C}$ & 274 \\
\hline MSCROW & MSC & $\mathrm{I}$ & 176 & SELECT & SEL & I & 279 \\
\hline MTRIAN & MTR & I & 178 & SET & SET & I & 282 \\
\hline MTXCHK & MCK & $\mathrm{E}$ & 181 & SETQ & STQ & $\mathrm{E}$ & 283 \\
\hline MXTX & MXT & $\mathrm{I}$ & 182 & SETUP & STP & $\mathrm{E}$ & 284 \\
\hline MXTXP & MXP & $\mathrm{C}$ & 184 & SKSYMV & SKS & $\mathrm{C}$ & 285 \\
\hline NNAME & NNA & $\mathrm{E}$ & 185 & SORDER & SOD & I & 286 \\
\hline NONBLA & NON & $\mathrm{E}$ & 186 & SORTSM & SOM & $\mathrm{C}$ & 288 \\
\hline NOTEPR & NOT & $\mathrm{I}$ & 186 & SPACE & SPA & I & 289 \\
\hline OANOVA & $\mathrm{OAN}$ & $\mathrm{C}$ & 187 & SPINV & SPI & $\mathrm{C}$ & 290 \\
\hline OCOEFF & $\mathrm{OCO}$ & $\mathrm{C}$ & 189 & STATIS & STA & I & 292 \\
\hline OCOVAR & $\mathrm{OCV}$ & $\mathrm{C}$ & 191 & STMT & STM & $\mathrm{E}$ & 300 \\
\hline OMCONV & OMC & $\mathrm{E}$ & 192 & STORE & STO & $\mathrm{E}$ & 301 \\
\hline OMNIT & OMN & $\mathrm{E}$ & 193 & STORMT & STT & $\mathrm{C}$ & 302 \\
\hline ONEWAY & ONE & $\mathrm{I}$ & 199 & STRUVE & STR & $\mathrm{C}$ & 303 \\
\hline OPONE & $\mathrm{OPO}$ & C & 206 & SYMV & SYM & $\mathrm{C}$ & 303 \\
\hline ORTHO & ORT & I & 208 & TAPOP & TAP & $\mathrm{E}$ & 304 \\
\hline ORTHRV & ORV & C & 225 & TAPOP2 & TP2 & I & 305 \\
\hline ORTPLT & ORP & $\mathrm{C}$ & 227 & THERMO & THE & I & 307 \\
\hline OUTPUT & OUT & $\mathrm{E}$ & 230 & ТРСТРТ & TPC & C & 313 \\
\hline PACK & PAC & $\mathrm{E}$ & 231 & TRANSF & TRA & C & 314 \\
\hline PAGE & PAG & $\bar{I}$ & 232 & TWOWAY & TWO & I & 316 \\
\hline PDMOTE & PDM & I & 233 & VARCON & VAR & $\mathrm{E}$ & 332 \\
\hline PHYCON & $\mathrm{PHY}$ & I & 235 & VECTOR & VEC & $\mathrm{E}$ & 332 \\
\hline PHYSIC & $\mathrm{PHC}$ & $\mathrm{B}$ & 236 & XECUTE & XEC & $\mathrm{E}$ & 333 \\
\hline PLOT & PLO & I & 238 & XFORMT & $\mathrm{XFO}$ & I & 341 \\
\hline PREPAK & PRE & $\mathrm{E}$ & 245 & XHEAD & $\mathrm{XHE}$ & I & 341 \\
\hline PRINTX & PRI & I & 248 & XOMNIT & XOM & $\mathrm{E}$ & 342 \\
\hline PROB & PRB & $\mathrm{C}$ & 250 & XPND & XPN & $\mathrm{E}$ & 344 \\
\hline PROCHK & PRK & $\mathrm{C}$ & 252 & XSTOP & XST & $\mathrm{E}$ & 345 \\
\hline PROROW & PRO & I & 253 & & & & \\
\hline
\end{tabular}




\section{Listing of Subprograms}

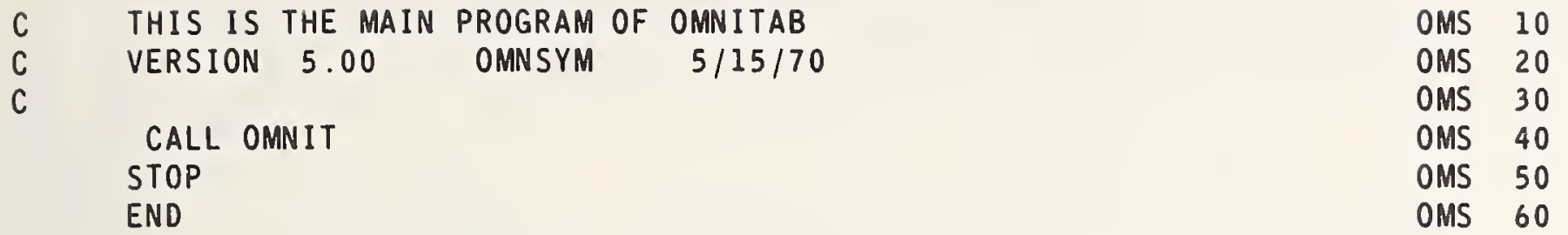


THIS SUBROUTINE ASSEMBLES A FLOATING POINT NUMBER FROM A STRING O

C DIGITS ETC. M INITIALLY POINTS AT THE FIRST NUMBER. IT IS LEFT POINTING AT THE FIRST CHARACTER AFTER THE NUMBER.

AAR 40

AAR 50

C VALUE RETURNED IN ARG

KARG $=1=$ FLOATING POINT, $=0=$ INTEGER, $-1=$ ERROR.

C

$A R G=K A R D(M)$

$S I G N=1$.

JEXP $=0$

I $X S=1$

IEXP $=0$

$K A R G=0$

C

LOOK BACK FOR MINUS SIGN AND/OR DECIMAL POINT

C

$K=K A R D(M-1)$

IF (K.NE.37) GO TO 10

$K A R G=1$

IEXP $=-1$

$K=K A R D(M-2)$

10 IF (K.EQ.38) SIGN=-1.

$20 \quad M=M+1$

$K=K A R D(M)$

IF (K.GE.10) GO TO 30

IEXP $=$ IEXP-KARG

$A R G=10 . * A R G+F L O A T(K)$

GO TO 20

30 IF (K.NE.37) GO TO 50

C

C DECIMAL POINT FOUND

C

IF (KARG.EQ.0) GO TO 40

CALL ERROR (3)

$K A R G=-1$

RETURN

$40 \quad K A R G=1$

GO TO 20

C CHECK FOR EXPONENT E X, E+X, E-X, $+X,-X$

C
50

$M=M+1$

$K=K A R D(M)$

IF (K.NE.44) IF (K-10) $70,65,65$

$60 \quad M=M+1$

$K=K A R D(M)$

IF $(K-10) \quad 70,90,90$

65 IF (K.NE.38) IF (K-39) $90,60,90$

IXS $=-1$

GO TO 60

$70 \quad K A R G=K A R G+1$

80 JEXP $=10^{*}$ JEXP $+K$

AAR $\quad 70$

AAR 80

AAR 90

AAR 100

AAR 110

AAR 120

AAR 130

AAR 140

AAR 150

AAR 160

AAR 170

AAR 180

AAR 190

AAR 200

AAR 210

AAR 220

AAR 230

AAR 240

AAR 250

AAR 260

AAR 270

AAR 280

AAR 290

AAR 300

AAR 310

AAR 320

AAR 330

AAR 340

AAR 350

AAR 360

AAR 370

AAR 380

AAR 390

AAR 400

AAR 410

AAR 420

AAR 430

AAR 440

AAR 450

AAR 460

AAR 470

AAR 480

AAR 490

AAR 500

AAR 505

AAR 510

AAR 520

AAR 530

AAR 535

AAR 540

AAR 550

AAR 560

AAR 570 
$M=M+1$

$K=K A R D(M)$

IF (K.LT.10) GO TO 80

C

C

C

90 IF (KARG.NE.0) GO TO 110

$100 \quad A R G=S I G N * A R G$

RETURN

$110 \quad K A R G=1$

IEXP $=I X S * J E X P+I E X P$

C

C

C

THE FOLLOWING CODING YIELDS MORE ACCURATE RESULTS THAN THE OBVIOUS ARG $=$ ARG * 10.* IEXP

$$
J E X P=I A B S(I E X P)
$$

IF (JEXP.GT.IFIX(XALOG)) GO TO 140

IF (IEXP) $120,100,130$

$120 \quad A R G=A R G / F E X P 2(10.0, F L O A T(J E X P))$

GO TO 100

$130 \quad A R G=A R G * F E X P 2(10.0, F L O A T(J E X P))$

GO TO 100

140 CALL ERROR (102)

$A R G=0$.

GO TO 100

END
AAR 580

AAR 590

AAR 600

AAR 610

AAR 620

AAR 630

AAR 640

AAR 650

AAR 660

AAR 670

AAR 680

AAR 690

AAR 700

AAR 710

AAR 720

AAR 730

AAR 740

AAR 750

AAR 760

AAR 770

AAR 780

AAR 790

AAR 800

AAR 810

AAR 820

AAR 830 
THE COMMAND ABRIDGE MAY BE USE IN THE FOLLOWING WAYS

ABRIDGE ROW,, OF COL ++,++, (USE RPRINT UNLESS IOSWT HAS BEEN SET BY FIXED OR FLOATING)

ABRIDGE / / ROW, , OF COL ++, ++ (USE SPECIFIED FORMAT)

$A B R \quad 20$

ABRIDGE WITH FLOATING PT. ARGS USES RPRINT

COMMON /BLOCKE/ NAME (4),L1,L2, ISRFLG

ABR 100

COMMON /BLOCRC/ NRC,RC(12600)

ABR 110

DIMENSION ARGS $(100)$

ABR 120

EQUIVALENCE (ARGS(1), RC (12501))

ABR 130

COMMON /FMAT/ IFMTX (6), IOSWT, IFMTS (6), LHEAD (96)

ABR 140

COMMON /HEADER/ NOCARD $(80), \operatorname{ITLE}(60,6)$, LNCNT, IPRINT, NPAGE, IPUNCH

$A B R \quad 150$

COMMON /BLOCKD/ IARGS (100), KIND(100), ARGTAB (100), NRMAX, NROW, NCOL, NABR 170

IARGS, VWXYZ (8), NERROR

COMMON /KFMT/ KFMT (100)

IF (NARGS.NE.0) GO TO 15

ABR 180

CALL ERROR (205)

\section{RETURN}

15 IF (L2.EQ.1) IF (IOSWT) $70,70,80$

CALL PREPAK (4, IND, L2, IND, KFMT)

IF (IND.NE.0) GO TO 90

$I P=1$

IF (NARGS.LE.1) GO TO 10

$20 \quad L L=I A R G S(1)$

$\operatorname{IARGS}(1)=1$

IF (LL.LE.O.OR.LL.GT.NROW) GO TO 10

ABR 190

CALL CHKCOL (I)

IF (I.NE.0) GO TO 10

IF (NERROR.NE.0) RETURN

DO $30 \quad I=2$, NARGS

ABR 200

$J=I A R G S(I)+L L$

$30 \quad \operatorname{ARGS}(I)=R C(J-I)$

IF (NPAGE.EQ.0) CALL PAGE (0)

GO TO $(40,50)$, IP

ABR 210

ABR 220

ABR 225

ABR 230

ABR 240

ABR 250

ABR 260

ABR 270

ABR 280

ABR 290

ABR 300

ABR 310

ABR 320

ABR 330

ABR 340

ABR 350

ABR 355

ABR 360

WRITE (IPRINT, KFMT) (ARGS (I), I=2, NARGS)

$A B R 370$

GO TO 60

$A B R \quad 380$

WRITE (IPRINT, IFMTX) (ARGS (I), I=2, NARGS)

$A B R 390$

60 RETURN

70 IF (NPAGE.EQ.0) CALL PAGE (0)

CALL RPRINT

$A B R \quad 400$

ABR 410

ABR 420

RETURN

ABR 430

ABR 470

ABR 480

ABR 490

$A B R 500$

$A B R 510$

CALL ERROR (222)

GO TO 70

ABR 520 
SUBROUTINE ACCDIG (AX, X, AD,N)

$\begin{array}{llll}C & \text { VERSION } 5.00 & \text { ACCDIG } & 5 / 15 / 70 \\ C & \text { RETURNS NUMBER OF ACCURATE DIGITS, AD }\end{array}$

ACC 10

WRITTEN BY DAVID HOGBEN, SEL, NBS. 10/29/69.

DATA ADMAX /8.0/

DIMENSION $A X(1), X(1), A D(1)$

DO $100 \quad I=1, N$

$D I F F=A X(I)-X(I)$

IF (DIFF) $20,10,20$

ACC 20

C

10

$A D(I)=A D M A X$

GO TO 100

$20 A D(I)=-F L O G 10(A B S(D I F F))+F L O G 10(A B S(X(I)))$

$A D(I)=A M I N I(A D M A X, A D(I))$

$A D(I)=A M A X I(-A D M A X, A D(I))$

100 CONTINUE

RETURN

END

ACC 30

ACC 40

ACC 50

ACC 60

ACC 70

ACC 80

ACC 90

ACC 100

ACC 110

ACC 120

ACC 130

ACC 140

ACC 150

ACC 160

ACC 170

$\begin{array}{lll}\text { SUBROUTINE ADRESS (I,J) ADR } 10 & \text { AD }\end{array}$

$\begin{array}{lllll}\text { VERSION } 5.00 \quad \text { ADRESS } & 5 / 15 / 70 & \text { ADR } & 20\end{array}$

COMMON /BLOCRC/ NRC,RC(12600) $\quad$ ADR 30

COMMON /BLOCKD/ IARGS (100), KIND(100), ARGTAB (100), NRMAX,NROW,NCOL, NADR 40

IARGS, VWXYZ (8), NERROR

ADR 50

DIMENSION ARGS $(100)$

ADR 60

EQUIVALENCE (ARGS(1), RC (12501))

ADR 70

C

CALCULATE ADDRESS OF ARGUMENT( I ). IF ARGUMENT( I ) IS A

ADR 80

FLOATING POINT NUMBER, $J=-(I+N R C)$. IF ILLEGAL COLUMN NUMBERADR 100

$\mathrm{J}=0$. IF OK, $\mathrm{J}=$ ADDRESS

ADR 110

C

C

IF (KIND(I).EQ.O) GO TO 10

ADR 120

$J=-(I+N R C)$

GO TO 30

ADR 130

ADR 150

IF (IARGS (I).GE.I.AND.IARGS (I).LE.NCOL) GO TO 20

ADR 160

10

$J=0$

ADR 170

GO TO 30

ADR 180

$J=N R O W^{*}($ I ARGS $(I)-1)+1$

$\begin{array}{ll}20 & \text { J }=\text { NROW* } \\ 30 & \text { RETURN }\end{array}$

END

ADR 190

ADR 200

ADR 210

ADR 220 
WHEN ARITHMETIC TROUBLES DEVELOP, THIS ROUTINE TALLIES THEM AND THAER 70

PRINTS THE RESULTS WHEN THE COMMAND IS DONE.

AER 80

ARIHMETIC MESSAGES MUST HAVE THE FOLLOWING TYPE OF FORMAT:

AER 90

1) THE FIRST TWO CHARACTERS MUST BE **

AER 110

2) MESSAGE PLUS OTHER INFO MUST NOT BE LONGER THAN 84 CHARACTERSAER 120

3) IF MESSAGE IS LESS THAN 84 CHAR, ADD NX AT END OF FORMAT AER 130

IF MORE THAN 10 ARITHMETIC ERROR MESSAGES ARE NEEDED THEN

AER 140

DIMENSION OF MESS(10) MUST BE CHANGED AND KMESS MUST BE SET =

AER 150

TO DIMENSION SIZE OF MESS.

AER 160

DIMENSION MESS (10)

AER 170

DATA KMESS/10/

IF (I) $160,30,10$

ALSO COMPUTED GO TO MUST BE CHANGED. SEE NOTEAER 180

DATA COMING IN

AER 190

AER 200

AER 210

DUMP RESULTS, END OF COMMAND

AER 240

AER 250

AER 260

AER 270

C

IF (LLIST.LT.2.OR.LLIST.EQ.4) GO TO 160

AER 280

AER 290

AER 300

DO $150 \mathrm{~J}=1$, KMESS

AER 310

IF (MESS(J).EQ.0) GO TO 150

AER 320

WRITE (ISCRAT, 250)

AER 330

WRITE (ISCRAT, 180) $\operatorname{MESS(J)}$

AER 340

AER 350

AER 360

C

THIS COMPUTED GO TO MUST BE CHANGED IF MORE THAT 10 ARITHMETIC ERRORS ARE ADDED

AER 370

AER 380

AER 390

GO TO $(40,50,60,70,80,90,100,110,120,130)$, J

40 WRITE (ISCRAT, 101)

GO TO 140

AER 400

AER 410

AER 420

AER 430

WR ITE (ISCRAT, 102)

GO TO 140

AER 440

WR ITE (ISCRAT, 103)

GO TO 140

AER 450

AER 460

WRITE (ISCRAT, 104) MESS (J)

GO TO 140

AER 470

AER 480

WRITE (ISCRAT, 105) MESS (J)

GO TO 140

AER 490

AER 500

WRITE (ISCRAT, 106) MESS(J)

GO TO 140

100 WRITE (ISCRAT, 107) MESS(J)

GO TO 140

AER 505

AER 510

AER 515

AER 517

WRITE (ISCRAT, 108) MESS (J)

AER 520

GO TO 140

AER 525

WRITE (ISCRAT, 109) MESS (J)

AER 530

GO TO 140

AER 535

WRITE (ISCRAT, 240) J

AER 540 
140 IF (LEVEL.NE.0) CALL RNDOWN

AER 550

WRITE (ISCRAT, 250)

AER 560

$\operatorname{MESS}(\mathrm{J})=0$

AER 570

150 CONTINUE

AER 580

IF (LEVEL.NE.0) GO TO 20

AER 590

I SWERR $=0$

NERR $=0$

AER 600

GO TO 20

AER 610

AER 620

C

C INITIALIZATION SECTION

AER 630

AER 640

C

160 DO $170 \mathrm{~J}=1$, KMESS

AER 650

$170 \operatorname{MESS}(\mathrm{J})=0$

I SWERR $=0$

AER 660

NERR $=0$

AER 670

GO TO 20

AER 680

AER 690

AER 700

AER 710

C

180 FORMAT (5IH** ARITHMETIC FAULT IN ABOVE COMMAND, ZERO RETURNED, I 4, AER 720 $16 H$ TIMES, 23X)

101 FORMAT (42H** NEGATIVE ARGUMENT TO SQRT, LOG OR RAISE, 42X)

AER 730

102

FORMAT (43H** EVALUATION OF EXPONENT PRODUCES OVERFLOW, $41 \mathrm{X}$ )

AER 740

103

FORMAT (45H** ARGUMENT OUT OF BOUNDS TO INVERSE FUNCTION, 39X)

AER 750

104

FORMAT ( $51 \mathrm{H}^{* *}$ ARGUMENT TOO LARGE FOR SIN OR COS, ZERO. RETURNED, I 4 , AER 770

16H TIMES, 23X)

AER 780

105 FORMAT (61H**BESSEL ARGUMENTS SCALED TO AVOID OVER/UNDER FLOW.RE AER 790 ITURNED , I4,6H TIMES, 13X)

240 FORMAT (16H** ERROR MESSAGE, I 2,66X)

AER 800

AER 810

250

FORMAT $(84 \mathrm{X})$

AER 820

106

FORMAT ( $33 H^{* *}$ DIVISION BY ZERO, RESULT SET $=0, I 4,6 \mathrm{H}$ TIMES, $41 X$ )

AER 830

107 FORMAT (44H** TRIG FUNCTIONS NOT DEFINED, RESULTS SET=0, I 4, 6H TIMEAER 840 1S, $30 X)$

AER 850

108 FORMAT $\left(66 \mathrm{H}^{* *}\right.$ ONE OF THE VALUES COMPARED IS ZERO, ABSOLUTE TOLERANCAER 860 IE WAS USED, I $4,6 \mathrm{H}$ TIMES, 8X)

AER 870

109 FORMAT $\left(71 H^{*} \times\right.$ FOR ELLIPTICAL INTEGRALS IS $=1.0$ OR GREATER. RESULTAER 880 1 IS SET TO $0.0 \ldots$, I $4,6 H$ TIMES, 3X)

END

AER 890

AER 900 
COMMON /BLOCKD/ IARGS(100), KIND(100), ARGTAB $(100)$, NRMAX, NROW, NCOL, NALL 40

IARGS, VWXYZ (8), NERROR

ALL $\quad 50$

DIMENSION ARGS $(100)$

EQUIVALENCE (ARGS (1), RC (12501))

ALL $\quad 60$

COMMON /BLOCKE/ NAME (4),L1,L2, ISRFLG

ALL $\quad 70$

COMMON /SCRAT/ NS, NS2, A(13500)

ALL 80

DIMENSION SCRA(1)

EQUIVALENCE (SCRA, A)

EQUIVALENCE (L11,LL1), (L22,LL2)

PROGRAMMED BY PHILIP J. WALSH (NBS 453.40) MAY, 1967

ALL 90

ALL 100

ALL 110

ALL 120

ALL 130

ALL 140

ALL 150

COMMAND IS OF THE FORM XXXX OF ORDER ++ OF COL ++, STORE IN ++ ALL 160

$X X X X$ MAY BE (A) NLSUB FOR NORMALIZED LAGUERRE POLYNOMIALS

(B) LSUB FOR LAGUERRE POLYNOMIALS

(C) HSUB FOR HERMITE POLYNOMIALS

(D) USUB FOR CHEBYSHEV POLYNOMIALS

(E) PSUB FOR LEGENDRE POLYNOMIALS

(F) TSUB FOR CHEBYSHEV POLYNOMIALS

ALL 170

ALL 180

ALL 190

ALL 200

ALL 210

ALL 220

SEE RECURSIVE FORMULAE FOR THESE POLYNOMIALS FURTHER IN CODE

ALL 230

EACH OF THE COMMANDS REQUIRE THREE ARGUMENTS

IF (NARGS.EQ.3) GO TO 10

CALL ERROR (10)

GO TO 210

10 IF (KIND (1)+KIND(3).EQ.0) GO TO 30

20 CALL ERROR (3)

ALL 240

ALL 250

ALL 260

ALL 270

ALL 280

GO TO 210

ALL 290

ALL 300

C CHECK THAT $X$ IS WITHIN WORKSHEET AND GET ADDRESS OF ARGUMENT COLUMALL 310

30 CALL ADRESS $(2, L 11)$

IF (LII) $20,40,50$

40 CALL ERROR (11)

GO TO 210

$50 \operatorname{IARGS}(4)=\operatorname{IARGS}(1)+\operatorname{IARGS}(3)-1$

$\operatorname{KIND}(4)=0$

CALL ADRESS $(4, L 22)$

IF (L22.LE.0) GO TO 40

CALL ADRESS $(3, L 22)$

IF (NRMAX.NE.0) GO TO 60

CALL ERROR (9)

GO TO 210

60 IF (NERROR.NE.0) GO TO 210

I JK=LL 1

$\mathrm{I} J=\mathrm{LL} 2$

DO $110 \quad I=1$, NRMAX

$\operatorname{SCRA}(1)=\mathrm{RC}($ I JK)

GO TO $(70,70,80,80,90,90)$, L2

$70 \quad \mathrm{RC}(\mathrm{IJ})=1 .-\mathrm{SCRA}(1)$

GO TO 100

$80 \quad \mathrm{RC}(\mathrm{IJ})=2 .{ }^{*} \mathrm{SCRA}(1)$

GO TO 100

$90 \quad \mathrm{RC}(\mathrm{IJ})=\operatorname{SCRA}(1)$

$100 \quad \mathrm{IJK}=\mathrm{I} \mathrm{JK}+1$

$110 \quad I J=I J+1$

IF (IARGS (1).EQ.1) GO TO 210

$N=I$ ARGS $(1)-1$

DO $200 \mathrm{~J}=1$, NRMAX

ALL 320

ALL 330

ALL 340

ALL 350

ALL 360

ALL 370

ALL 380

ALL 390

ALL 400

ALL 410

ALL 420

ALL 430

ALL 440

ALL 450

ALL 460

ALL 470

ALL 480

ALL 490

ALL 500

ALL 510

ALL 520

ALL 530

ALL 540

ALL 550

ALL 560

ALL 570

ALL 580

ALL 590 
$I J K=L L I+J$

ALL 600

$I J=L L 2+J$

ALL 610

$\operatorname{SCRA}(1)=1.0$

$\operatorname{SCRA}(2)=R C(I J K-1)$

ALL 620

$\operatorname{SCRA}(3)=R C(I J-1)$

ALL 630

$\operatorname{SCRA}(4)=1.0$

$\operatorname{SCRA}(5)=2.0$

DO $190 \quad I=1, N$

$\operatorname{IARGS}(4)=\operatorname{IARGS}(3)+I$

CALL ADRESS $(4, L L 22)$

GO TO $(120,130,140,150,160,170)$, L2$$
\text { L2 }=1 \text { NLSUB }
$$

NORMALIZED LAGUERRE POLYNOMIALS

ALL 640

ALL 650

ALL 660

ALL 670

ALL 680

ALL 690

ALL 700

ALL 710

RECURSION FORMULA $L(N+1)=(1 .+2 \cdot * N-X) * L(N)-N * * 2 * L(N-1)$

ALL 720

$L(0)=1$.

$L(1)=-X+1$

$L(2)=X * * 2-4 \cdot 0 * X+2$.

$L(3)=-X * 3+9.0 * X * * 2-18.0 * X+6$.

ALL 730

ALL 740

ALL 750

ALL 760

$L(N)=\operatorname{EXP}(X) *(D N / D X N(X * * N * \operatorname{EXP}(-X)))$

ALL 770

ALL 780

ALL 790

ALL 800

$\operatorname{SCRA}(4)=1$

$\operatorname{SCRA}(6)=1 \cdot 0+2 \cdot 0 * \operatorname{SCRA}(4)$

$\operatorname{SCRA}(7)=\operatorname{SCRA}(4) * \operatorname{SCRA}(4)$

$\operatorname{SCRA}(8)=(\operatorname{SCRA}(6)-\operatorname{SCRA}(2)) * \operatorname{SCRA}(3)-\operatorname{SCRA}(7) * \operatorname{SCRA}(1)$

GO TO 180

$C$
$C$
$C$
$C$
$C$
$C$
$C$
$C$
$C$
$C$
$C$
$C$
$C$
$C$
$C$

L2 $=2$

LSUB

LAGUERRE POLYNOMIALS

ALL 810

ALL 820

ALL 830

ALL 840

ALL 850

RECURSION FORMULA $L(N+1)=\left(\left(\left(2 *^{*} N+1\right)-X\right) * L(N)-N * L(N-1)\right) /$ $(\mathrm{N}+1)$

ALL 860

ALL 870

$L(0)=1$.

$L(1)=-X+1$.

$L(2)=.5(X X * 2-4 . * X+2)$

$L(3)=(-X * * 3+9 . * x * 2-18 * x+6) /$.6 .

ALL 880

ALI. 890

ALL 900

ALL 910

ALL 920

* See abramowitz, m. and stegun, i.A., handboOK of mathematical all 930 FUNCTIONS, NATIONAL BUREAU OF STANDARDS APPLIED MATHEMATICSALL 940 SERIES 55, SUPERINTENDENT OF DOCUMENTS, U.S. GOVERNMENT PRINTING OFFICE, WASHINGTON, D.C. 20402

ALL 950

ALL 960

ALL 970

* SEE HILSENRATH, ZIEGLER, MESSINA, WALSH, HERBOLD, , OMNITAB, NBS HANDBOOK 101 (MARCH 4, 1966) - FOR FORMULAE USED

$130 \quad \operatorname{SCRA}(4)=1$

$\operatorname{SCRA}(6)=\operatorname{SCRA}(4)+1.0$

$\operatorname{SCRA}(7)=\operatorname{SCRA}(4)+\operatorname{SCRA}(6)$

$\operatorname{SCRA}(8)=((\operatorname{SCRA}(7)-\operatorname{SCRA}(2)) * \operatorname{SCRA}(3)-\operatorname{SCRA}(4) * \operatorname{SCRA}(1)) / \operatorname{SCRA}(6)$

GO TO 180

C L2 $\quad=3$ HSUB HERMITE POLYNOMIALS

C RECURSION FORMULA $\mathrm{H}(\mathrm{N}+1)=2.0 * \mathrm{X} * \mathrm{H}(\mathrm{N})-2.0 * \mathrm{~N} * \mathrm{H}(\mathrm{N}-1)$

ALL 980

ALL 990

ALL 1000

ALLI010

ALL1020

ALL1030

ALL 1040

ALL1050

ALL1060

ALL1070

$H(0)=1$.

$H(1)=2.0 * X$

$H(2)=4.0 * X * 2-2$.

$H(3)=8.0 * X * 3-12 . * X$

ALL1080

ALL1090

ALL1 100

ALL 1110

ALL1120

$\operatorname{SCRA}(8)=2.0 *(\operatorname{SCRA}(2) * \operatorname{SCRA}(3)-\operatorname{SCRA}(4) * \operatorname{SCRA}(1))$

ALLI1 130

$\operatorname{SCRA}(4)=\operatorname{SCRA}(4)+1.0$

ALLI1 140

GO TO 180

C $L 2=4$ USUB CHEBYSHEV POLYNOMIALS

ALLI150

ALL 1160

RECURSION FORMULA $U(N)=2.0 * X * U(N-1)-U(N-2)$

ALL1170

ALLI180 


$$
\begin{array}{ll}
C & U(0)=1 . \\
C & U(1)=2.0 * X \\
C & U(2)=4.0 * X * 2-1.0 \\
C & U(3)=8.0 * X * 3-4.0 * X
\end{array}
$$

ALL 1300

ALL 1310

ALL 1320

ALL1330

ALL 1340

ALL1350

ALL 1360

ALL 1370

ALL 1380

ALL 1390

ALL 1400

ALL 1410

RECURSION FORMULA

ALL 1420

ALL 1430

$T(0)=1$.

$T(1)=X$

$T(2)=2 \cdot * x * * 2-1$.

$T(3)=4 . * X * 3-3 . * X$

ALL 1440

ALL 1450

ALL 1460

ALL 1470

ALL1480

$\operatorname{SCRA}(8)=2.0 * \operatorname{SCRA}(2) * \operatorname{SCRA}(3)-\operatorname{SCRA}(1)$

ALL 1490

CONTINUE

$L J M N=L L 22+J$

$R C(L J M N-1)=S C R A(8)$

ALL 1500

$\operatorname{SCRA}(1)=\operatorname{SCRA}(3)$

ALL 1510

$\operatorname{SCRA}(3)=\operatorname{SCRA}(8)$

ALL 1520

CONTINUE

ALL 1530

190

CONT INUE

ALL1540

200

RETURN

ALL 1550

END

ALL 1560

ALL 1570 

COMMON /BLOCKD/ IARGS(100),KIND(100), ARGTAB (100), NRMAX, NROW,NCOL,NAPR 40 IARGS, VWXYZ ( 8), NERROR

APR 50

DIMENSION ARGS $(100)$

APR 60

EQUIVALENCE (ARGS(1),RC(12501))

APR 70

COMMON /BLOCKE/ NAME (4),L1,L2, ISRFLG

APR 80

COMMON /ABCDEF/ L (48)

APR 90

COMMON /HEADER/ NOCARD $(80), \operatorname{ITLE}(60,6), \operatorname{LNCNT}$, IPRINT, NPAGE, IPUNCH

APR 100

COMMON /FMAT/ IFMTX (6), IOSWT, IFMTS (6), LHEAD (96)

COMMON /SCRAT/ NS, NS2, A(13500)

COMMON /KFMT/ KFMT (100)

DIMENSION IFRV (3)

DATA IFRV (1), IFRV (2), IFRV (3)/3H1X, ,3HI5, 3H2X, /

C $\quad$ LI $=4$ APRINT

C LI $=7$ MPRINT

MPRINT PRINTS ROW/COL TITLE, APRINT DOES NOT.

ALL READABLE IF POSSIBLE, OTHERWISE ALL FLOATING.

WRITTTEN BY DAVID HOGBEN, SEL, NBS. $8 / 18 / 69$.

$* * * * *$

6 FORMAT STATEMENTS FOLLOW WHICH MAY NEED MODIFY IF CHANGES MADE $* * * * *$

IF (NARGS.EQ.4) GO TO 30

APR 110

APR 120

APR 130

APR 140

APR 150

APR 160

APR 170

APR 180

APR 190

APR 200

APR 210

APR 220

APR 230

APR 240

CALL ERROR (205)

$\begin{array}{ll}10 & \text { CALL ERR } \\ 20 & \text { RETURN }\end{array}$

APR 250

$30 \quad \mathrm{~J}=1$

$30 \quad \begin{aligned} & \mathrm{J} \\ & \mathrm{I}=\mathrm{I}\end{aligned}$

APR 260

CALL CKIND (I)

IF (I.NE.0) GO TO 10

APR 270

APR 280

APR 290

$K=I$ ARGS (I)

CALL MTXCHK (J)

IF (J.NE.O) GO TO 10

IF (NERROR.NE.0) RETURN

C CHECK TO SEE IF NPAGE=0. IF YES, BEGIN A NEW PAGE. CALL PAGE (0)

IF (NPAGE.EQ.0) CALL PAGE (0)

C IF $L 2=1$ IOSWT $=0$ USE READABLE FORMAT

IF (L2.EQ.1.AND.IOSWT.EQ.0) GO TO 190

C IF $L 2=1$ IOSWT $=1$ USE FIXED OR FLOATING

IF (L2.EQ.1.AND.IOSWT.EQ.1) GO TO 60

APR 300

APR 310

APR 320

APR 330

APR 340

APR 342

APR 344

APR 350

APR 360

APR 370

C IF (L2.NE.1) USE SPECIFIED FORMAT
C FORMAT SHOULD SPECIFY FORMAT FOR ONLY ONE ROW

CALL PREPAK (4, IND,L2, IND, KFMT)

IF (IND.NE.0) GO TO 50

$I A=I$ ARGS ( 3 )

$J 1=I A R G S(1)$

$\mathrm{J} 2=\mathrm{J} 1+(\operatorname{IARGS}(4)-1) *$ NROW

DO $40 \quad I=1$, IA

WRITE (IPRINT, KFMT) (RC (J), J=J $1, J 2, N R O W)$

$\mathrm{J} l=\mathrm{J} l+1$

$\mathrm{J} 2=\mathrm{J} 2+1$

APR 380

APR 390

APR 400

APR 410

APR 420

APR 440

APR 450

APR 460

APR 470

APR 480

APR 490

APR 500

CONT INUE

RETURN

C NO FORMAT IS FOUND SO USE READABLE FORMAT

50 CALL ERROR (222)

GO TO 190

C FIXED OR FLOATING FORMAT USED

60 DO $70 \quad \mathrm{I}=1,100$

$70 \quad \operatorname{KFMT}(\mathrm{I})=\mathrm{L}(45)$

APR 510

APR 520

APR 530

APR 540

APR 550

APR 560

APR 570

APR 590 
IF (LI.EQ.7) GO TO 90

DO $80 \quad I=1,6$

APR 600

$\operatorname{KFMT}(\mathrm{I})=\operatorname{IFMTX}(\mathrm{I})$

GO TO 110

$90 \operatorname{KFMT}(1)=\operatorname{IFMTX}(1)$

$\operatorname{KFMT}(2)=\operatorname{IFRV}(1)$

$\operatorname{KFMT}(3)=\operatorname{IFRV}(2)$

$\operatorname{KFMT}(4)=\operatorname{IFRV}(3)$

DO $100 \quad I=2,6$

APR 610

APR 620

APR 630

APR 640

APR 650

APR 660

APR 670

APR 680

$100 \operatorname{KFMT}(I+3)=\operatorname{IFMTX}(\mathrm{I})$

APR 690

$110 K A=I$ ARGS (1)

$L L=\operatorname{IARGS}(3)$

APR 700

$I B B=I A R G S(4)$

APR 710

$I B B P=8$

IF (LI.EQ.7) IBBP $=7$

APR 720

APR 730

I IA=IARGS (2)

120 IF (IBB.GT.IBBP) GO TO 130

APR 740

$I B=I B B$

$\mathrm{IBB}=0$

GO TO 140

$130 \quad I B B=I B B-I B B P$

$I B=I B B P$

$140 \mathrm{~KB}=(\mathrm{IB}-1) * N R O W+K A$

$K B P=K B+N R O W$

I $2 A=I 1 A+I B-1$

IF (LI.EQ.4) GO TO 150

APR 750

APR 760

APR 770

APR 780

APR 790

APR 800

APR 810

APR 820

APR 830

APR 840

WRITE (IPRINT, 280) L(28),L(25),L(33),L(37),L(13),L(25),L(22), (JJ, JAPR 860

$I J=I I A, I 2 A)$

$M R V=K$

150 DO $180 \quad M=1, L L$

IF (LI.EQ.4) GO TO 160

WRITE (IPRINT, KFMT) MRV, (RC (K), K=KA, KB, NROW)

APR 870

APR 880

APR 890

APR 900

$M R V=M R V+1$

GO TO 170

160 WRITE (IPRINT, KFMT) (RC (K), K=KA, KB, NROW)

$170 \quad K A=K A+1$

$K B=K B+1$

180 CONTINUE

IF (IBB.EQ.0) RETURN

WRITE (IPRINT, 330)

C PRINT NEXT SET OF COLUMNS

APR 910

APR 920

APR 930

APR 940

APR 950

APR 960

APR 970

APR 980

APR 990

APR 1000

$K A=K B P$

I I $A=I 2 A+1$

GO TO 120

C THE NEXT CARD MUST BE CHANGED IF WIDTH OF COLUMN CHANGED

APR 1010

APR 1020

APR 1030

APR 1040

THE CARD AFTER IT MUST BE CHANGED IF NUMBER OF COLUMNS CHANGES

APR 1050

2 CALLS TO RFORMT LATER NEED TO BE CHANGED IF NO. OF SD NOT 8.

APR 1060 NWMX IS DETERMINED BY 120/8-2 WHERE 120 IS THE NUMBER OF

APR 1070

APR 1080

APR 1100

APR 1110

APR 1120

APR 1130

APR 1140

APR 1150

APR 1160

APR 1170

APR 1180

DO $210 \quad I=1, K C$

APR 1190 
$A(K 1)=R C(K 2)$

APR 1200

$\mathrm{Kl}=\mathrm{K} 1+\mathrm{l}$

APR 1210

$\mathrm{K} 2=\mathrm{K} 2+1$

APR 1220

$K 2=K 2+N R O W-K R$

$K S I Z E=K R * K C$

CALL RFORMT (A (1),KSIZE, 8, NWIDTH,NDECS, NWMX $+1, A(1), A(1), 0,0)$

APR 1230

APR 1240

MINIMUM OF TWO BLANK SPACES ON LEFT

APR 1250

$N B L A N K=N W M X+2-N W I D T H$

APR 1260

APR 1270

I $1=1$

I 1 A =I ARGS ( 2)

APR 1280

$K 1=N S T A R T-1$

APR 1290

APR 1300

LOOP ON BLOCKS

APR 1310

$\mathrm{I} 2=\mathrm{I} I+\mathrm{MCOL}-\mathrm{I}$

I $2=$ MINO $(I 2, K C)$

APR 1320

APR 1330

$I 2 A=I$ ARGS $(2)+I 2-1$

APR 1340

$\mathrm{K} 2=\mathrm{K} 1+(\mathrm{I} 2-\mathrm{I} 1) * \mathrm{NROW}$

APR 1350

$\mathrm{K} 4=\mathrm{K} 2$

APR 1360

IF (LI.EQ.4) GO TO 230

APR 1370

WRITE (IPRINT, 280) L (28), L (25),L (33),L (37), L (13),L (25),L (22), (JJ, JAPRI380

$I J=I 1 A, I 2 A$ )

C LOOP ON ROWS

APR 1390

APR 1400

$230 \quad 00270 \mathrm{JJ}=1, K R$

APR 1410

$\mathrm{Kl}=\mathrm{KI}+\mathrm{I}$

$\mathrm{K} 2=\mathrm{K} 2+1$

APR 1420

$J J J=K S T A R T+J J$

IF (NWIDTH.LE.NWMX.OR.L1.NE.7) GO TO 240

C WRITE FLOATING IF MPRINT (L $1=7)$ AND NWIDTH GT NWMAX

APR 1430

APR 1440

WRITE (IPRINT, 300$)$ JJJ, $(R C(K 3), K 3=K 1, K 2$, NROW)

GO TO 270

$240 \quad L L=1$

$K=K 1$

C LOOP ON COLUMNS

DO 250 I I $=$ I I, I 2

CALL RFORMT ( $A, 1,8, N W I D T H, N D E C S, 0, R C(K), A(L L), N B L A N K, 0)$

APR 1450

APR 1460

APR 1470

APR 1480

APR 1490

APR 1500

APR 1510

APR 1520

$K=K+N R O W$

APR 1530

APR 1540

$L L=L L+N W M X+2$

$N L=L L-1$

IF (LI.EQ.7) GO TO 260

APR 1550

A.PR 1560

APR 1570

WRITE (IPRINT, 310 ) (A (LL), LL=2, NL)

APR 1580

GO TO 270

APR 1590

APR 1600

APR 1610

APR 1620

APR 1630

APR 1640

APR 1650

APR 1660

APR 1670

APR 1680

APR 1690

APR 1700

C

GO TO 220

APR 1710

FORMAT $(1 X, 7 A 1,7(6 X, 15,4 X))$

FORMAT $(1 X, 15,2 X, 112 A 1)$

FORMAT $(1 X, 15,2 X, 1 P 7 E 15.6)$

APR 1720

FORMAT $(1 X, 119 A 1)$

$\begin{array}{ll}320 & \text { FORMAT (IH) } \\ 330 & \text { FORMAT (IX) }\end{array}$

APR 1730

APR 1740

APR 1750

END 
SUBROUTINE ARITH

C VERSION 5.00 ARITH $5 / 15 / 70$
COMMON/BLOCRC/NRC,RC $(12600)$

ARI 10

ARI 20

COMMON /BLOCKD/ IARGS (100), KIND (100), ARGTAB $(100)$, NRMAX, NROW, NCOL, NARI 40

IARGS, VWXYZ (8), NERROR

DIMENSION ARGS $(100)$

ARI 50

EQUIVALENCE (ARGS (1), RC (12501))

ARI 60

COMMON /BLOCKE/ NAME (4),L1,L2, ISRFLG

ARI 70

D IMENS ION II (5), KK(5)

ARI 80

EQUIVALENCE (I I (1), I I), (I I (2), I 2) , ( I I (3), I 3) ,

ARI 90

1 ( I I (4), I 4), (I I (5), I 5)

ARI 100

C

THIS SUBROUTINE PERFORMS ADD, SUB, MULT, DIV AND RAISE FOR

ARI 105

THREE, FOUR AND FIVE ARGUMENTS

$\mathrm{L} 2=1 \quad \mathrm{ADD}$

$\bar{L} 2=2$ SUBTRACT

ARI 110

ARI 120

ARI 130

ARI 140

$\mathrm{L} 2=3 \quad$ MULTIPLY

ARI 150

$L 2=4 \quad$ DIVIDE

ARI 160

$\mathrm{L} 2=5 \quad$ RAISE

$L 2=6 \quad$ ACCURATE DIGITS

AR I 170

ARI 180

ARI 190

ARI 200

IF (NARGS.LT.3.OR.NARGS.GT.5) CALL ERROR(10)

IF (NARGS.EQ.4) CALL ERROR (29)

IF (KIND (NARGS) . NE.0) CALL ERROR (20)

IF (L2.NE.6) GO TO 5

IF (NARGS.EQ.3) GO TO 5

CALL ERROR (212)

NARGS $=3$

5 DO $30 \quad I=1$, NARGS

$K K(I)=1$

CALL ADRESS (I, II (I))

ARI 210

ARI 215

ARI 220

ARI 222

ARI 224

ARI 226

ARI 228

ARI 230

ARI 240

IF (I I (I) ) $20,10,30$

ARI 250

10 CALL ERROR (11)

ARI 260

ARI 270

RETURN

ARI 280

$20 \quad \mathrm{KK}(\mathrm{I})=0$

I I $(I)=-I I(I)$

30 CONTINUE

IF (NRMAX.LE.0) CALL ERROR (9)

IF (NERROR.NE.0) RETURN

$J J=I I$ (NARGS) +NRMAX-I

IF (NARGS.NE.3) GO TO 120

DO $110 \quad \mathrm{I}=\mathrm{I} 3, \mathrm{JJ}$

GO TO $(50,60,70,80,90,95), \mathrm{L} 2$

$50 \quad R C(I)=R C$ ( I I) $+R C$ (I 2)

GO TO 100

ARI 290

ARI 300

ARI 310

ARI 320

ARI 330

ARI 380

ARI 390

ARI 400

ARI 410

ARI 420

ARI 430

$R C(I)=R C$ ( I 2$)-R C$ ( I I)

GO TO 100

ARI 440

ARI 450

ARI 460

$\mathrm{RC}(\mathrm{I})=\mathrm{RC}(\mathrm{I} 1) * \mathrm{RC}(\mathrm{I} 2)$

ARI 470

GO TO 100

80 IF (RC (I2).NE.0.0) GO TO 85

ARI 480

$R C(I)=0.0$

ARI 490

CALL ERROR (106)

ARI 500

GO TO 100

ARI 510

$85 \quad \mathrm{RC}(\mathrm{I})=\mathrm{RC}$ ( I I) / RC ( I 2 )

ARI 520

GO TO 100

ARI 530

$90 \quad R C(I)=F E X P 2(R C(I 1), R C(I 2))$

ARI 540

GO TO 100

ARI 545

CALL ACCDIG(RC (I 1), RC (I2), RC (1), 1)

ARI 547

$100 \quad I I=I I+K K(1)$

ARI 550 


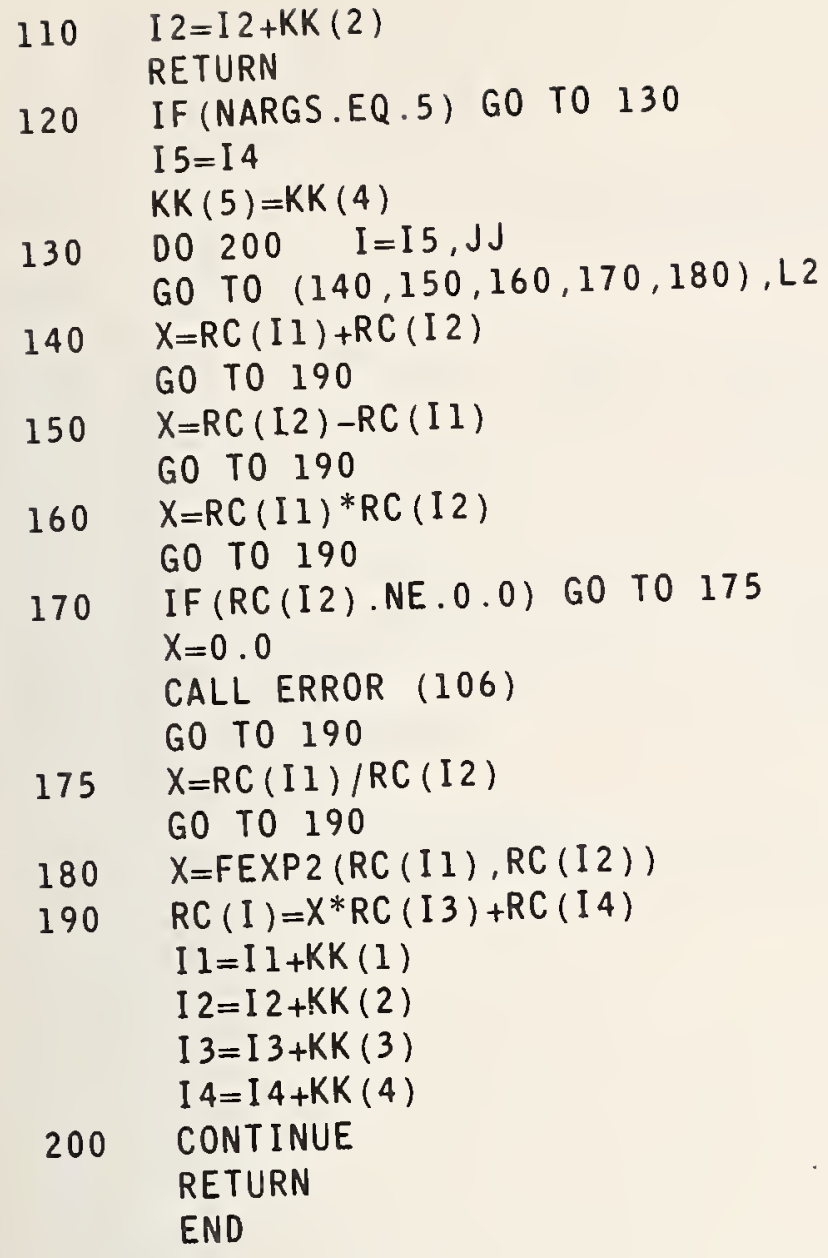


SUBROUTINE ARYVEC

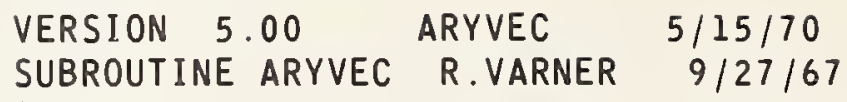


IROWSV $=I$ ARGS $(6)$

ARY 620

GO TO 60

ARY 630

$\begin{array}{ll}\text { ARY } & 640 \\ \text { ARY } & 650\end{array}$

50

$\operatorname{IARGS}(12)=\operatorname{IARGS}(4)$

$\operatorname{IARGS}(11)=1$

ARY 660

$\operatorname{IARGS}(9)=\operatorname{IARGS}(6)$

ARY 660

$J=3$

ARY 670
ARY 680

ARY 690

GO TO 90

$70 \quad \mathrm{~J}=3$

$\operatorname{IARGS}(12)=1$

$\operatorname{IARGS}(11)=\operatorname{IARGS}(3)$

ARY 700

ARY 710

$\operatorname{IARGS}(7)=\operatorname{IARGS}(4)$

ARY 720

ARY 730

IF (NARGS.EQ.6) GO TO 80

ARY 740

$\operatorname{IARGS}(9)=\operatorname{IARGS}(6)$

ARY 750

GO TO 90

$80 \quad \operatorname{IARGS}(9)=1$

$90 \operatorname{IARGS}(6)=\operatorname{IARGS}(5)$

$\operatorname{IARGS}(5)=1$

CALL MTXCHK (J)

IF (J-1) $120,100,110$

100 CALL ERROR (3)

$$
\text { RETURN }
$$

ARY 760

ARY 770

ARY 780

ARY 790

ARY 800

ARY 810

ARY 820

ARY 830

ARY 840

ARY 850

ARY 860

ARY 870

ARY 880

ARY 890

ARY 900

ARY 910

ARY 920

ARY 930

ARY 940

ARY 950

ARY 960

ARY 970

ARY 980

ARY 990

ARY 1000

ARY 1010

ARY 1020

ARY 1030

ARY 1040

ARY 1050

ARY 1060

ARY 1070

ARY 1080

ARY 1090

ARY 1100

ARY 1110

ARY 1120

ARY 1130

ARY 1140

ARY 1150

ARY 1160

ARY 1170

ARY 1180

ARY 1190

180

$I A=I A+I A D I$

ARY 1200 
$A(I C)=S U M$

ARY 1210

I $C=I C+1$

ARY 1220

GO TO $(190,200), L 2$

ARY 1230

190

$I A P=I A P+1$

GO TO 210

ARY 1240

$I B P=I B P+N R O W$

ARY 1250

200

CONTINUE

ARY 1260

ARY 1270

ARY 1280

ARY 1290

STORE RESULTS IN WORKSHEET

ARY 1300

I $S=1$

DO $220 \quad I=1$, IP

ARY 1310

$R C(I C S)=A(I S)$

ARY 1320

ARY 1330

I $S=I S+I$

ARY 1340

$I C S=I C S+I A D 2$

ARY 1350

220 CONTINUE

RETURN

ARY 1360

ARY 1370

END

ARY 1380 
SUBROUTINE ASTER

COMMON /BLOCKA/ MODE, M,KARD (83), KARG, ARG, ARG2, NEWCD(80), KRDEND AST 30

DIMENSION NAM(2)

AST 40

AST 50

ASTERISKS HAVE BEEN FOUND, LOOK FOR A SPECIAL FORM OF ARGUMENT. AST 60

FORMS CAN BE..

AST 70

AST 80

AST 90

*PHYSCON* A PHYSICAL CONSTANT NAME, FL.PT.

AST 100

**VARCON** A -VARIABLE- CONSTANT TO BE USED AS AN INTEGER (TRUN)AST 110

*VARCON* A -VARIABLE- CONSTANT TO BE USED AS A FL.PT. NUMBER AST 120

**ROW, COLUMN** A WORKSHEET ENTRY TO BE TRUNCATED AND USED AS INTAST 130

*ROW, COLUMN* A WORKSHEET ENTRY TO BE USED AS FLOATING POINT AST 140

AST 150

NONBLA IS A FUNCTION WHICH RETURNS THE NEXT NON-BLANK CHARACTER AST 160

IN THE CARD AND ALSO POINTS M AT IT

AST 170

KARG $=1$, SINGLE $*$ KARG $=0$, DOUBLE $*$.

AST 180

AST 190

$L=K A R G$

$K=N O N B L A(M)$

IF (K.NE.40) GO TO 20

AST 200

AST 210

AST 220

AST 230

AST 240

A LONG LINE OF ASTERISKS FOUND, SKIP OVER THEM AND IGNORE AST 250

$K A R G=7$

$10 \quad M=M+1$

IF $(K A R D(M)-40) 100,10,100$

20 IF (K.GE.36) GO TO 60

IF (K.GE.10) GO TO 40

AST 260

AST 270

AST 280

AST 290

AST 300

AST 310

AST 320

NUMBER IS FIRST NON-BLANK CHARACTER, SET $N=$ COMMA

AST 330

AST 340

$\mathrm{N}=43$

30 CALL AARGS

IF (KARG.NE.0) GO TO 60

IF (NONBLA (M) .EQ.N) IF $(N-40) \quad 35,37,35$

GO TO 60

35 IF (NONBLA(M+1).GE.10) GO TO 60

C

C SET $N=$ ASTERISK

C

\section{$\mathrm{N}=40$}

$\mathrm{T}=\mathrm{ARG}$

GO TO 30

$37 \quad A R G 2=A R G$

$A R G=T$

$K A R G=5$

GO TO 90

AST 350

AST 360

AST 370

AST 375

AST 380

AST 390

AST 400

AST 410

AST 420

AST 430

AST 440

AST 450

AST 460

AST 470

AST 480

AST 490

C

\section{LETTER FOUND FIRST}

AST 500

C

C

40 CALL NNAME (NAM(1))

AST 510

AST 520

CALL PHYCON (NAM(1))

AST 530

IF (ARG.EQ.0.) GO TO 50

AST 540

AST 550

C

PHYSICAL CONSTANT FOUND, SET KARG $=1$ 
IF (L) $60,60,80$

AST 600

C

NAME NOT IN PHYSICAL CONSTANT LIST, TRY VARIABLE LIST

AST 610

AST 620

AST 630

50 CALL VARCON (NAM(1))

AST 640

IF (ARG.NE.0.) GO TO 70

AST 650

AST 660

$60 \quad K A R G=1$

RETURN

AST 670

$70 \quad K A R G=3$

AST 680

IF (NONBLA(M).NE.40) GO TO 60

AST 690

$90 \quad M=M+1$

AST 700

AST 710

CHECK THAT THE NUMBER OF ASTERISKS AT THE END OF THE EXPRESSION

AST 720

IS THE SAME AS AT THE BEGINNING. $L=0$ MEANS 2, $L=1$ MEANS 1

AST 730

AST 740

IF (L.NE.0) IF (KARD (M) -40) $95,60,95$

AST 745

IF (KARD (M).NE.40.OR.KARD (M+1).EQ.40) GO TO 60

AST 750

$95 \quad M=M+1$

$K A R G=K A R G+L$

AST 760

100 RETURN

AST 770

AST 780

AST 800 
$\begin{array}{llrr}\text { C VERSION } 5.00 & \text { BEGIN } & 5 / 15 / 70 \\ & \text { COMMON/BLOCKA/ MODE, M,KARD (83), KARG, }\end{array}$

BEG 20

COMMON /BLOCKA/ MODE, M,KARD (83), KARG, ARG, ARG2,NEWCD (80), KRDEND

BEG 30

COMMON /BLOCKE/ NAME (4),L1,L2, ISRFLG

BEG 40

COMMON /BLOCKB/ NSTMT, NSTMTX, NSTMTH, NCOM, LCOM, IOVFL, COM(2000) BEG 50

COMMON /BLOCRC/ NRC, RC (12600)

BEG 60

COMMON /BLOCKD/ IARGS(100), KIND(100), ARGTAB (100), NRMAX, NROW, NCOL, NBEG 70

IARGS, VWXYZ (8), NERROR

BEG 80

DIMENSION ARGS(100)

BEG 90

EQUIVALENCE (ARGS(1),RC(12501))

BEG 100

BEG 110

THIS SUBROUTINE CONTAINS THE CODING FOR BEGIN AND SCAN

BEG 120

IF (L2.EQ.1) GO TO 20

BEG 140

BEG 150

BEG 160

SCAN (CARD UP TO AND INCLUDING CARD COLUMN ++ )

BEG 170

IF (NARGS.GT.1) CALL ERROR (221)

BEG 180

IF (NARGS.GE.1.AND.KIND(1).EQ.0.AND.IARGS (1).GE.6.AND.IARGS (1).LE.BEG 190 180) GO TO 10

$K=205$

GO TO 30

BEG 200

BEG 210

KRDEND=IARGS ( 1 )

BEG 220

GO TO 40

BEG 230

BEG 240

BEGIN STORING INSTRUCTIONS AT NUMBER ++

BEG 250

IF NO NUMBER IS GIVEN, I IS ASSUMED.

BEG 260

BEG 270

BEG 280

IF (MODE.EQ.1) GO TO 50

BEG 290

$\mathrm{K}=5$

BEG 300

CALL ERROR (K)

BEG 310

RETURN

BEG 320

IF (NARGS-1) $70,90,60$

BEG 330

$K=10$

BEG 340

GO TO 30

BEG 350

$\begin{array}{ll}70 & \text { NSTMT }=0 \\ 80 & \text { MODE }=3\end{array}$

BEG 360

BEG 370

GO TO 40

BEG 380

IF (KIND(1).EQ.0) GO TO 100

BEG 390

$K=20$

BEG 400

GO TO 30

BEG 410

IF (IARGS(1).GT.0.AND.IARGS(1).LT.1000) GO T0 110

BEG 420

$K=7$

BEG 430

GO TO 30

BEG 440

NSTMT $=10^{*}(\operatorname{IARGS}(1)-1)$

BEG 450

GO TO 80

BEG 460

END

BEG 470 


\begin{tabular}{|c|c|c|c|}
\hline & SUBROUTINE BEJN (IST, R,Z) & BEJ & 10 \\
\hline C & VERSION $5.00 \quad$ BEJN & BEJ & 20 \\
\hline C & IF IST $=0$ ENTRY IS FOR BEJN & BEJ & 30 \\
\hline \multirow[t]{7}{*}{ C } & IF IST $=1$ ENTRY IS FOR BEIN & BEJ & 40 \\
\hline & DIMENSION R(I) & BEJ & 50 \\
\hline & DOUBLE PRECISION $X, R, Z, A, B, C, D, E, F, G, P, Q, Y$ & BEJ & 60 \\
\hline & $Y=1 . D 0$ & BEJ & 70 \\
\hline & IF (IST.NE.0) $Y=-1 . D 0$ & BEJ & 80 \\
\hline & $X=Z$ & BEJ & 90 \\
\hline & $0010 \quad N=1,100$ & BEJ & 100 \\
\hline \multirow[t]{7}{*}{10} & $R(N)=0.0$ & BEJ & 110 \\
\hline & $L A=0$ & BEJ & 120 \\
\hline & IF $(X . L E .60$.$) GO TO 30$ & BEJ & 130 \\
\hline & $L A=1$ & BEJ & 140 \\
\hline & IF $(X . L E .100$.$) GO TO 20$ & BEJ & 150 \\
\hline & CALL ERROR (225) & BEJ & 160 \\
\hline & GO TO 130 & BEJ & 170 \\
\hline 20 & $X=X / 2 . D 0$ & BEJ & 180 \\
\hline \multirow[t]{9}{*}{30} & $A=X / 2 . D 0$ & BEJ & 190 \\
\hline & IF $(X . G T .15$.$) GO TO 100$ & BEJ & 200 \\
\hline & $B=1 . D 0$ & BEJ & 210 \\
\hline & $C=1 . D O$ & BEJ & 220 \\
\hline & $D 040 \quad N=1,100$ & BEJ & 230 \\
\hline & $J=N$ & BEJ & 240 \\
\hline & $B=B * A / C$ & BEJ & 250 \\
\hline & $C=C+1 \cdot D 0$ & BEJ & 260 \\
\hline & IF $(B . L T . .5 D-30)$ GO T0 50 & BEJ & 270 \\
\hline 40 & CONTINUE & BEJ & 280 \\
\hline \multirow[t]{13}{*}{50} & $D=B * A / C$ & BEJ & 290 \\
\hline & $A=A * 2$ & BEJ & 300 \\
\hline & $K=X+6 . D 0$ & BEJ & 310 \\
\hline & $E=K$ & BEJ & 320 \\
\hline & $\mathrm{F}=\mathrm{K}+\mathrm{J}$ & BEJ & 330 \\
\hline & $G=F+1 . D 0$ & BEJ & 340 \\
\hline & $P=1 . D 0$ & BEJ & 350 \\
\hline & $Q=1 . D 0$ & BEJ & 360 \\
\hline & DO $60 \mathrm{~N}=1, \mathrm{~K}$ & BEJ & 370 \\
\hline & $P=1 . D O-P^{*} A /(E * F) * Y$ & BEJ & 380 \\
\hline & $Q=1 . D O-Q^{*} A /\left(E^{*} G\right) * Y$ & BEJ & 390 \\
\hline & $E=E-1 . D 0$ & BEJ & 400 \\
\hline & $F=F-1 . D 0$ & BEJ & 410 \\
\hline \multirow[t]{3}{*}{60} & $G=G-1 . D 0$ & BEJ & 420 \\
\hline & $R(J+1)=B * P$ & BEJ & 430 \\
\hline & $R(J+2)=D * Q$ & BEJ & 440 \\
\hline \multirow[t]{3}{*}{70} & $D 080 \quad N=1, J$ & BEJ & 450 \\
\hline & $K=J-N+1$ & BEJ & 460 \\
\hline & $A=K$ & BEJ & 470 \\
\hline \multirow[t]{8}{*}{80} & $R(K)=2 . D 0 * A * R(K+1) / X-R(K+2) * Y$ & BEJ & 480 \\
\hline & IF $(L A . E Q .0)$ GO TO 130 & BEJ & 490 \\
\hline & $L A=L A-1$ & BEJ & 500 \\
\hline & $A=R(1) * R(100)$ & BEJ & 510 \\
\hline & $B=.000$ & BEJ & 520 \\
\hline & DO $90 \quad N=1.99$ & BEJ & 530 \\
\hline & $K=100-N$ & BEJ & 540 \\
\hline & $A=A+R(N+1) * R(K)$ & BEJ & 550 \\
\hline \multirow[t]{4}{*}{90} & $B=B+R(N) * R(K)$ & BEJ & 560 \\
\hline & $J=98$ & BEJ & 570 \\
\hline & $R(100)=A$ & BEJ & 580 \\
\hline & $R(99)=B$ & BEJ & 590 \\
\hline
\end{tabular}


$X=Z$

GO TO 70

$100 \quad K=1.5^{*} X$

$B=1.00$

$\mathrm{C}=\mathrm{K}$

DO $110 \mathrm{~N}=1, \mathrm{~K}$

$B=A * B / C$

$110 \quad C=C-1.00$

$P=2$. D -9

IF (LA.EQ.1) $P=5 . D-20$

$\mathrm{C}=\mathrm{K}+1$

DO $120 \mathrm{~N}=1,30$

$J=K+N$

$B=B * A / C$

$C=C+1 . D 0$

IF (B.LT.P) GO TO 50

IF (J.EQ.98) GO TO 50

120 CONTINUE

GO TO 50

130 RETURN

END
BEJ 600

BEJ 610

BEJ 620

BEJ 630

BEJ 640

BEJ 650

BEJ 660

BEJ 670

BEJ 680

BEJ 690

BEJ 700

BEJ 710

BEJ 720

BEJ 730

BEJ 740

BEJ 750

BEJ 760

BEJ 770

BEJ 780

BEJ 790

BEJ 800 
SUBROUTINE BESSEL

$\begin{array}{llll}C & \text { VERSION } 5.00 & \text { BESSEL } 5 / 15 / 70 & B E S \\ 20\end{array}$

COMMON /BLOCKD/ IARGS $(100)$, KIND (100), ARGTAB $(100)$, NRMAX, NROW, NCOL, NBES 30

IARGS, VWXYZ ( 8 ), NERROR

COMMON /BLOCRC/ NRC, RC (12600)

BES 40

EQUIVALENCE (ARGS(1), RC (12501))

BES 50

DIMENSION ARGS(100)

BES 60

COMMON /SCRAT/ NS, NS2,A(13500)

BES 70

DOUBLE PRECISION DBEJ , X, Y, E,P , Q, S, T, BINTJO , COMELL , Z, DXEX, XEX

BES 80

DOUBLE PRECISION FDCOS, FDEXP

DOUBLE PRECISION AA $(1000), B(1000), W(100)$

EQUIVALENCE $(A(1), A A),(A(2001), B),(A(4001), W)$

BES 90

BES 100

COMMON /BLOCKE/ NAME (4),L1,L2, ISRFLG

BES 110

DIMENSION R(1), IA(1), KI (1)

BES 120

EQUIVALENCE (R, RC), (IA, I ARGS), (KI, KIND), (NR, NRMAX)

BES 130

COMMON /ABEKI/ $X, Y, P, Q, S, T$

BES 140

DOUBLE PRECISION DSNCOS, DXEXP

BES 150

COMMON /CONSLB/ XTRIG, XEXP

COMMON /DCONLB/ DSNCOS, DXEXP

BES 160

BES 170

BES 180

C

C

WJG PATCH )())()()())()()()()()()()()()()()()(

$X E X=X E X P-3.0$

DXEX $=D X E X P-4.000$

IF (NARGS.GE.2) GO TO 10

CALL ERROR (10)

RETURN

C

END PATCH $)(1)()()()()()()())()()()()()$

C

10 IF (L2.GT.28) GO T0 250

$\mathrm{N}=0$

$\mathrm{L}=\mathrm{L} 2 / 2$

$\mathrm{L}=2 * \mathrm{~L}$

IF (L.EQ.L2) $N=1$

IF (L2.GT.12) GO TO 110

IF (NARGS.GT.2) CALL ERROR (10)

CALL ADRESS (NARGS,J)

IF (J.LE.O) CALL ERROR (11)

$L T=1$

IF (KI (1).EQ.1) GO TO 20

BES 190

BES 200

BES 210

BES 220

BES 225

BES 227

BES 230

BES 240

BES 250

BES 260

BES 270

BES 280

BES 290

BES 300

BES 310

BES 320

BES 330

BES 340

BES 350

BES 360

BES 370

BES 380

BES 390

CALL ADRESS ( $1, J A)$

IF (JA.LE.0) CALL ERROR (11)

$L T=2$

$20 \quad M=1$

IF (NERROR.NE.O) RETURN

IF (L2.GT.2) $\quad M=5$

IF (L2.GT.4) $\quad M=3$

IF (L2.GT.6) $\quad M=7$

IF (L2.GT.8) $\quad M=3$

IF (L2.GT.10) $\quad M=7$

$\mathrm{L}=0$

IF (L2.GT.4) $L=1$

IF (L2.GT.8) $\quad \mathrm{L}=2$

IF (LT.EQ.1) GO TO 70

DO $50 \quad I=1, N R$

$X=R(J A)$

$J A=J A+1$

BES 400

BES 410

BES 420

BES 430

BES 440

BES 450

BES 460

BES 470

BES 480

BES 490

BES 500

BES 510

BES 520

BES 530

BES 540

BES 550

BES 560

$Y=1 . D 0$

BES 570 
IF (L.EQ.0) GO TO 33

BES 580

IF (L.EQ.2) GO TO 30

BES 590

IF (DABS (X). LT.XEX) GO TO 33

BES 600

IF 1 XI IS GREATER THAN XEXP AND LESS THAN DXEXP THE RESULTS BES 610

WILL BE SCALED BY EXP (X) OR EXP $(-X)$.

BES 620

IF IXI IS GREATER THAN DXEXP THE SUBROUTINE DBEJ DOES THE SCALINGBES 630 AND A MESSAGE IS PRINTED.

BES 640

THIS APPLIES TO THE FOLLOWING COMMANDS BIZERO, BIONE, BKZERO, BES 650

BKONE

CALL ERROR (105)

IF $(D A B S(X) . G T . D X E X) \quad$ GO TO 33

BES 660

$Y=F D E X P(X)$

IF (M.EQ.3) $Y=1.00 / Y$

33 IF (M.EQ.5.OR.M.EQ.7) IF (X) 35,35,40

GO TO 40

BES 670

BES 680

BES 690

BES 700

BES 705

BES 710

$\mathrm{R}(\mathrm{J})=0$.

CALL ERROR ( 101$)$

GO TO 50

C $\quad \mathrm{L} 2=1, M=1, N=0, L T=2, L=0 \quad$ :BJZERO OF ++ STORE IN ++

$L 2=2, M=1, N=1, L T=2, L=0 \quad$ :BJONE OF ++ STORE IN ++

$L 2=3, M=5, N=0, L T=2, L=0 \quad: B Y Z E R O O F++$ STORE IN ++

$L 2=4, M=5, N=1, L T=2, L=0$ :BYONE OF ++ STORE IN ++

$\mathrm{L} 2=5, M=3, N=0, L T=2, L=1 \quad$ :BIZERO OF ++ STORE IN ++

$\mathrm{L} 2=6, \mathrm{M}=3, \mathrm{~N}=1, \mathrm{LT}=2, \mathrm{~L}=1 \quad$ :BIONE OF ++ STORE IN ++

$\mathrm{L} 2=7, M=7, N=0, L T=2, L=1 \quad$ :BKZERO OF ++ SOTRE IN ++

$\mathrm{L} 2=8, M=7, N=1, \mathrm{LT}=2, \mathrm{~L}=1$ :BKONE OF ++ STORE IN ++

$L 2=9, M=3, N=0, L T=2, L=2$ :EXJZERO OF ++ STORE IN ++

$L 2=10, M=3, N=1, L T=2, L=2$ :EXIONE OF ++ STORE IN ++

BES 720

BES 730

BES 740

BES 750

BES 760

BES 770

BES 780

BES 790

BES 800

BES 810

BES 820

BES 830

BES 840

$L 2=11, M=7, N=0, L T=2, L=2$ :EXKZERO OF ++ STORE IN ++

BES 850

$L 2=12, M=7, N=1, L T=2, L=2$ :EXKONE OF ++ STORE IN ++

BES 860

$R(J)=Y^{*} \operatorname{DBEJ}(X, N, M)$

BES 870

$\mathrm{J}=\mathrm{J}+\mathrm{l}$

BES 880

RETURN

BES 890

$X=A R G S(1)$

$Y=1 . D 0$

IF (L.EQ.0) GO TO 90

BES 900

BES 910

BES 920

IF (L.EQ.2) GO TO 80

IF (DABS $(X)$.LT.XEX) GO TO 90

C SEE COMMENTS ABOVE ON BOUNDS OF $X$ BEFORE DBEJ IS CALLEO CALL ERROR (105)

80 IF (DABS $(X)$.GT.DXEX) GO TO 90

$Y=F D E X P(X)$

IF (M.EQ.3) $Y=1.00 / Y$

C $\quad L 2=1, M=1, N=0, L T=1, L=0 \quad$ BJZERO OF ** STORE IN ++

BES 930

BES 940

BES 950

BES 960

BES 970

BES 980

BES 990

BES 1000

$L 2=2, M=1, N=1 \quad L T=1, L=0 \quad$ BJONE OF ** STORE IN ++

BES 1010

$\mathrm{L} 2=3, \mathrm{M}=5, \mathrm{~N}=0, \mathrm{LT}=1, \mathrm{~L}=0 \quad:$ BYZERO OF ** STORE IN ++

BES 1020

$\mathrm{L} 2=4, \mathrm{M}=5, \mathrm{~N}=1, \mathrm{LT}=1, \mathrm{~L}=0$ :BYONE OF $* *$ STORE IN ++

BES 1030

$L 2=5, M=3, N=0, L T=1, L=1 \quad: B I Z E R O$ OF ** STORE IN ++

BES 1040

$\mathrm{L} 2=6, \mathrm{M}=3, \mathrm{~N}=1, \mathrm{LT}=1, \mathrm{~L}=1$ :BIONE OF ** SOTRE IN ++

BES 1050

$\mathrm{L} 2=6, \mathrm{M}=3, \mathrm{~N}=1, \mathrm{LT}=1, \mathrm{~L}=1 \quad$ :BIONE OF ** STORE IN ++

BES 1060

$\mathrm{L} 2=7, M=7, N=0, L T=1, L=1 \quad: B K Z E R O$ OF $* *$ SOTRE IN ++

BES 1070

$L 2=8, M=7, N=1, L T=1, L=1$ :BKONE OF ** STORE IN ++

BES 1080

$L 2=9, M=3, N=0, L T=1, L=2$ :EXIZERO OF ** STORE IN ++

BES 1090

$L 2=10, M=3, N=1, L T=1, L=2$ :EXIONE OF ** STORE IN ++

BES 1100

$L 2=11, M=7, N=0, L T=1, L=2$ :EXKZERO OF ** STORE IN ++

BES 1110

$L 2=12, M=7, N=1, L T=1, L=2$ :EXKONE OF ** STORE IN ++

BES 1120

$X=Y * \operatorname{DBEJ}(X, N, M)$

BES 1130

DO $100 \quad I=1, N R$

BES 1140

$R(J)=X$

BES 1150 
$M=1$

BES 1200

IF (L2.GT. 14) $M=2$

BES 1210

IF (L2.GT.16) $M=1$

BES 1220

IF (L2.GT. 18) $\quad M=2$

BES 1230

$L=0$

BES 1240

IF (L2.GT.16) $\mathrm{L}=1$

BES 1250

$Y=.78539816339700$

BES 1260

$L V=0$

BES 1270

$J X=0$

BES 1280

BES 1290

CALL ADRESS (NARGS,J2)

BES 1300

IF (J2.LE.0) CALL ERROR (11)

BES 1310

CALL ADRESS (NARGS-1,Jl)

BES 1320

IF (JI.LE.0) CALL ERROR (11)

BES 1330

IF (KI (1).EQ.1) GO TO 130

BES 1340

CALL ADRESS $(1, J A)$

IF (JA.LE.0) CALL ERROR (11)

BES 1350

$\mathrm{LT}=1$

$130 \quad K=0$

$K A=0$

IF $(L T+L V . E Q .0) \quad$ GO TO 200

BES 1360

BES 1370

BES 1380

BES 1390

BES 1400

IF (LV.EQ.0) GO TO 230

IF (LT.EQ.O) GO TO 240

140 IF (NERROR.NE.0) RETURN

DO $190 \quad I=1, N R M A X$

BES 1410

BES 1420

BES 1430

IF (KA.EQ.0) $X=R$ (JA)

BES 1440

$J A=J A+1$

$E=1 . D 0$

IF (JX.NE.O) $Y=R(J B)$

BES 1450

BES 1460

BES 1470

$\mathrm{JB}=\mathrm{JB}+1$

C $\quad L 2=15, M=2, N=0, L=0$

C $\quad L 2=16, M=2, N=1, L=0$

C $\quad L 2=19, M=2, N=0, L=1$

C $\quad L 2=20, M=2, N=1, L=1$

C $\quad L 2=23, M=2, N=0, L=0$

C $\quad L 2=24,7=2, N=1, L=0$

C $\quad L 2=27, M=2, N=0, L=1$

C $\quad L 2=28, M=2, N=1, L=1$

IF (M.EQ.2) CALL CBEK

C $\quad L 2=13, M=1, N=0, L=0$

C $\quad L 2=14, M=1, N=1, L=0$

C $\quad L 2=18, M=1, N=1, L=1$

C $\quad L 2=21, M=1, N=0, L=0$

C $\quad L 2=22, M=18 \mathrm{~N}=1, L=0$

C $\quad L 2=25, M=1, N=0, L=1$

C $\quad L 2=26, M=1, N=1, L=1$

IF (M.EQ.1) CALL CBEI

BES 1480

BES 1490

:KBKZERO OF \$\$ PUT REAL IN ++ IMAGINARY ++ BES1500

:KBKONE OF \$\$ PUT REAL IN ++ IMAGINARY ++ BES1510

:KEXKZR OF \$\$ PUT REAL IN ++ IMAGINARY ++ BES1520

:KEXKONE OF \$\$ PUT REAL IN ++ IMAGINARY ++ BES1530

:CKZERO OF \$\$ OHI \$ UT REAL ++ IMAG ++

BES 1540

:CKONE OF \$\$PHI \$ PUT REAL ++ IMAG ++ BES1550

:CEKZERO OF \$\$PHI \$ PUT REAL ++ IMAG ++ BES1560

:CEKONE OF \$\$ PHI \$\$PUT REAL ++ IMAG ++ BES1570

BES 1580

:KBIZERO OF \$\$ PUT REAL IN ++ IMAGINARY ++ BES 1590

:KBIONE OF \$\$ PUT REAL IN ++ IMAGINARY ++ BESI600

:KEXIONE OF \$\$ PUT REAL IN ++ IMAGINARY ++ BES1610

:CIZERO OF \$\$ PHI \$\$ PUT REAL ++ IMAG ++ BES1620

:CIONE OF \$ PHI \$ PUT REAL ++ IMAG ++ BES1630

:CEIZERO OF \$ HI \$ UT REAL ++ IMAG ++ BES1640

:CEIONE OF \$\$ PHI \$ PUT REAL ++ IMAG ++ BES1650

$Z=X * F D C O S(Y)$

BES 1660

BES1670

IF (L.EQ.1) GO TO 150

BES1680

IF (DABS (Z).LT.XEX) GO TO 160

BES 1690

CALL ERROR (105)

$150 \quad E=F D E X P(Z)$

BES 1700

BES 1710

IF (M.EQ.1) $E=1 . D 0 / E$

BES 1720

160 IF (N.EQ.0) GO TO 170

C STORE INTO WORK SHEET RESULTS OF COMMANDS KBIONE, KBKONE 
C KEXIONE, KEXKONE, CIONE, CEIONE, CEKONE

BES 1750

$R(J I)=E * S$

$R(J 2)=E^{*} T$

BES 1760

GO TO 180

BES 1770

C STORE INTO WORK SHEET RESULTS OF COMMANDS KBIZERO, KBKZERO,

BES 1780

BES 1790

C KEXIZER, KEXKZER, CIZERO, CEIZERO, CEKZERO

$170 \quad \mathrm{R}(\mathrm{J} l)=E * P$

$R(J 2)=E^{*} Q$

BES 1800

BES 1810

BES 1820

$180 \quad \mathrm{Jl}=\mathrm{Jl}+1$

BES 1830

BES 1840

$\mathrm{J} 2=\mathrm{J} 2+1$

RETURN

200 IF (JX.EQ.0) GO TO 240

BES 1850

BES 1860

$Y=\operatorname{ARGS}(2)$

BES 1870

$X=A R G S(1)$

BES 1880

$K A=1$

BES 1890

$\mathrm{JX}=0$

BES 1900

GO TO 140

BES 1910

210 IF (NARGS.GT.4) CALL ERROR (10)

BES 1920

$J X=1$

$L V=0$

BES 1930

IF (KI (2).EQ.1) GO T0 220

BES 1940

CALL ADRESS $(2, J B)$

BES 1950

IF (JB.LE.0) CALL ERROR (11)

BES 1960

$L V=1$

$220 \quad M=1$

IF (L2.GT.22) $M=2$

IF (L2.GT.24) $M=1$

IF (L2.GT.26) $M=2$

$L=0$

IF (L2.GT.24) $L=1$

GO TO 120

BES 1970

BES 1980

BES 1990

BES2000

BES 2010

BES2020

BES2030

BES2040

BES2050

230 IF (JX.EQ.0) GO TO 140

$Y=A R G S(2)$

$J X=0$

BES2060

GO TO 140

BES2070

BES2080

$240 \quad K A=1$

$X=A R G S(1)$

GO TO 140

250 IF (L2.FT.32) GO T0 350

260 IF (NARGS.GT.2) CALL ERROR (10)

CALL ADRESS (NARGS,J)

IF (J.LE.0) CALL ERROR (11)

$L T=0$

IF (KI(1).EQ.1) GO TO 270

CALL ADRESS $(1, J A)$

IF (JA.LE.0) CALL ERROR (11)

$L T=1$

270 IF (NERROR.NE.0) RETURN

IF (LT.EQ.0) $X=A R G S(1)$

IF (L2.GT.37) GO TO 310

IF (L2.EQ.32) GO TO 310

IF (L2.GT.29) GO T0 290

DO $280 \mathrm{~N}=1$, NR

IF (LT.EQ.1) $\quad X=R(J A)$

$\mathrm{JA}=\mathrm{J} A+1$

$L 2=29$

BES2090

BES 2100

BES 2110

BES 2120

BES2 130

BES 2140

BES2 150

BES 2160

BES2 170

BES2180

BES2190

BES2200

BES 2210

BES2220

BES2230

BES2240

BES2250

BES2260

BES2270

BES2280

BES2290

$R(J)=B I N T J O(X, W, Z)$

BES2300

BES 2310

$\mathrm{J}=\mathrm{J}+1$

BES2320

RETURN

BES2330 
IF (L2.EQ.31) $K=2$

DO $300 \mathrm{~N}=1, \mathrm{NR}$

BES 2360

IF (LT.EQ. I) $\quad X=R$ (JA)

BES 2370

$\mathrm{JA}=\mathrm{J} A+1$

C $\quad L 2=30, K=1$

:ELLIPTICAL FIRST OF \$\$ STORE IN ++

BES2380

C $\quad L 2=31, K=2$

:ELLIPTICAL SECOND OF \$\$ STORE IN ++

BES2390

$R(J)=\operatorname{COMELL}(X, K)$

BES2400

$300 \quad \mathrm{~J}=\mathrm{J}+1$

BES2410

RETURN

BES2420

BES2430

310 IF (LT.EQ.1) CALL ERROR (20)

BES2440

$Z=X$

BES 2450

$K=N R$

BES 2460

IF (K.LE.100) GO T0 330

BES 2470

$K=100$

$\mathrm{J} A=\mathrm{J}+100$

BES 2480

DO $320 \quad I=K, N R$

BES2490

$R(J A)=0.0$

$320 \quad \mathrm{JA}=\mathrm{J} A+1$

C L2 $=32$ :BESJN $X=* *$ STORE IN ++

330 IF (L2.EQ.32) CALL BEJN $(0, W, Z)$

C L2 $=38 \quad: B E S I N \quad X=* *$ STORE IN ++

IF (L2.EQ.38) CALL BEJN $(1, W, Z)$

IF (L2.EQ.39) GO TO 460

DO $340 \mathrm{~N}=1, \mathrm{~K}$

BES2500

BES 2510

BES 2520

BES 2530

BES2540

BES 2550

BES2560

BES2570

$R(J)=W(N)$

$340 \quad \mathrm{~J}=\mathrm{J}+1$

RETURN

350 IF (L2.GT.34) GO T0 390

$L=N R$

IF (NR.GT.1000) $L=1000$

IF (NARGS.GT.2) CALL ERROR (10)

CALL ADRESS (NARGS,J)

IF (J.LE.0) CALL ERROR (11)

IF (KI (1).EQ.1) CALL ERROR (20)

BES 2580

BES2590

BES2600

BES2610

BES2620

BES 2630

BES2640

BES2650

BES 2660

BES2670

BES 2680

CALL ADRESS ( $1, J A)$

IF (JA.LE.0) CALL ERROR (II)

IF (NERROR.NE.O) RETURN

IF (L2.EQ.33) GO TO 360

C L2 $=34$ :ZEROS BJZERO STORE IN ++ AND ++

BES 2690

BES 2700

BES 2710

BES2720

CALL BEZONE (AA, B, I, L)

GO TO 370

C $\quad L 2=33$

:ZEROS BJONE STORE IN ++ AND ++

BES 2730

BES 2740

BES 2750

BES2760

BES 2770

BES 2780

DO $380 \quad N=1, L$

$R(J A)=A A(N)$

BES 2790

$R(J)=B(N)$

BES 2800

$J A=J A+1$

BES 2810

$J=J+1$

BES 2820

BES2830

RETURN

BES 2840

BES 2850

IF (NARGS.GT.2) CALL ERROR (10)

BES2860

CALL ADRESS (NARGS,J)

BES2870

IF (J.LE.O) CALL ERROR (11)

BES 2880

$L T=0$

BES2890

IF (KI(1).EQ.1) GO TO 400

BES2900

CALL ADRESS $(1, J A)$

BES2910

IF (JA.LE.0) CALL ERROR (11)

BES2920 
400 IF (LT.EQ.0) $X=A R G S(1)$

IF (NERROR.NE.0) RETURN

BES2940

$\mathrm{K}=0$

BES 2950

IF (L2.EQ.36) $\mathrm{K}=1$

BES2960

DO $420 \mathrm{~N}=1, \mathrm{NR}$

BES 2970

IF (LT.NE.0) $X=R(J A)$

$\mathrm{JA}=\mathrm{JA}+1$

C $\quad \mathrm{L} 2=35, \mathrm{~K}=0$

:STRUVE ZERO OF \$\$ STORE IN ++

BES2980

$L 2=36, K=1$

:STRUVE ONE OF $\$ \$$ STORE IN ++

BES2990

CALL STRUVE $(X, Y, Z, W)$

BES 3000

IF (K.EQ.O) GO TO 410

BES 3010

BES 3020

BES3030

C STORE RESULTS FOR STRUVE ONE

BES 3040

$R(J)=Z$

GO TO 420

C STORE RESULTS OF STRUVE ZERO

BES 3050

BES 3060

$410 \quad R(J)=Y$

$420 \mathrm{~J}=\mathrm{J}+1$

RETURN

430 IF (L2.GT.37) GO T0 260

IF (NARGS.GT.3) CALL ERROR (10)

CALL ADRESS (NARGS,J)

IF (J.LE.0) CALL ERROR (11)

IF (KI(1).EQ.1) CALL ERROR (20)

CALL ADRESS $(1, J A)$

IF (JA.LE.0) CALL ERROR (11)

$J B=I A(2)$

IF (KI (2).NE.0) CALL ERROR (3)

IF (NERROR.NE.0) RETURN

$K=I A(2)$

$L N R=N R M A X$

IF (LNR.GT.1000) LNR $=1000$

DO $440 \quad N=1$, LNR

BES 3070

BES3080

BES 3090

BES 3100

BES 3110

BES 3120

BES 3130

BES 3140

BES 3150

BES 3160

BES3 170

BES 3180

BES3 190

BES3200

BES 3210

BES3220

BES3230

$A A(N)=R(J A)$

$440 \quad J A=J A+1$

C $L 2=37$ :HARMONIC OF $++\ldots$ STORE IN ++

CALL FOURIA (AA, B (1),B(2), K,L)

BES 3240

BES 3250

BES 3260

BES3270

BES 3280

DO $450 \mathrm{~N}=1, \mathrm{JB}$

BES 3290

$R(J)=B(N)$

BES 3300

$\mathrm{J}=\mathrm{J}+\mathrm{l}$

RETURN

BES 3310

BES 3320

BES 3330

IF (X.LT.XEXP) GO TO 470

BES 3340

CALL ERROR (225)

RETURN

C $\quad L 2=39$

:BESKN $X=* *$ STORE IN ++

BES 3350

BES 3360

BES 3370

BES 3380

$A A(2)=\operatorname{DBEJ}(X, 1,7)$

BES 3390

$R(J)=A A(1)$

$R(J+1)=A A(2)$

BES 3400

$\mathrm{J}=\mathrm{J}+2$

DO $480 \quad I=3, K$

BES 3410

$Z=I-2$

BES 3420

$A A(I)=A A(I-2)+2 *{ }^{*} A A(I-1) / X$

BES 3430

$R(J)=A A(I)$

BES 3440

BES 3450

IF (AA(I).GT.3.E37) GO TO $490 \quad$ BES3460

$480 \mathrm{~J}=\mathrm{J}+1$

RETURN

BES 3470

BES 3480

BES 3490

BES 3500

DO $500 \mathrm{JA}=1, K$

BES 3510

$\mathrm{J}=\mathrm{J}+\mathrm{I}$

RETURN

BES 3520

END

BES 3530 
SUBROUTINE BEZERO ( $A, B, M, L)$

C VERSION 5.00 BEZERO $5 / 15 / 70$

DOUBLE PRECISION $A(1), B(1), X, Y, A A, A B, A C, F D S Q R T$

$\mathrm{KB}=1$

$N=M$

$10 \quad J=4 * N-1$

IF (J.GT.44) GO TO 130

GO TO $(20,30,40,50,60,70,80,90,100,110,120), N$

$20 \quad X=2.40482557700$

$Y=.519147497300$

GO TO 140

$30 \quad X=5.5200781103 D 0$

$Y=-.340264806500$

GO TO 140

$40 \quad X=8.6537279129 D 0$

$Y=.271452299900$

GO TO 140

$50 \quad X=11.791534439100$

$Y=-.2324598314 D 0$

GO TO 140

$60 \quad X=14.930917708600$

$Y=.206546433100$

GO TO 140

$70 \quad X=18.071063967900$

$Y=-.18772880300$

GO TO 140

$80 \quad X=21.211636629900$

$Y=.1732658942 D 0$

GO TO 140

$90 \quad X=24.3524715308 D 0$

$Y=-.161701550700$

GO TO 140

$100 \quad X=27.493479132 D 0$

$Y=.152181213800$

GO TO 140

$110 \quad X=30.634606468400$

$Y=-.144165977700$

GO TO 140

$120 \quad X=33.775820213600$

$Y=.137296943400$

GO TO 140

$130 \quad X=J$

$X=X * 3.141592653600$

BEZ 10

BEZ 20

BEZ 30

BEZ 40

BEZ 50

BEZ 60

BEZ 70

BEZ 80

BEZ 90

BEZ 100

BEZ 110

BEZ 120

BEZ 130

BEZ 140

BEZ 150

BEZ 160

BEZ 170

BEZ 180

BEZ 190

BEZ 200

BEZ 210

BEZ 220

BEZ 230

BEZ 240

BEZ 250

BEZ 260

BEZ 270

BEZ 280

BEZ 290

BEZ 300

BEZ 310

BEZ 320

BEZ 330

BEZ 340

BEZ 350

BEZ 360

BEZ 370

BEZ 380

BEZ 390

BEZ 400

BEZ 410

BEZ 420

BEZ 430

$A A=1 . D 0 / X * * 2$

BEZ 440

$A B=1 . D 0+2 . D 0^{*} A A^{*}(1 . D 0-A A *(31 . D 0-A A *(3779 . D 0-A A * 6277237 . D 0 / 7 . D 0) /$ $15 . D 0)(3 . D 0)$

$\mathrm{J}=\mathrm{N} / 2$

$\mathrm{J}=2 * \mathrm{~J}$

$A C=1 . D 0$

IF (J.EQ.N) $A C=-1 . D 0$

$Y=A C * 1.595769122 D 0 *(1 . D 0-A A * 2 * 56 . D 0 / 3 . D 0) / F D S Q R T(X)$

BEZ 450

BEZ 460

BEZ 470

BEZ 480

BEZ 490

BEZ 500

$X=X * A B / 4 . D 0$

$140 \quad A(K B)=X$

$B(K B)=Y$

$\mathrm{N}=\mathrm{N}+\mathrm{l}$

$K B=K B+1$

IF (KB.LE.L) GO TO 10

RETURN

END

BEZ 510

BEZ 520

BEZ 530

BEZ 540

BEZ 550

BEZ 560

BEZ 570

BEZ 580

BEZ 590 
SUBROUTINE BEZONE (A,B, M,L)

C VERSION 5.00 BEZONE $5 / 15 / 70$

DOUBLE PRECISION A(l),B(l),R, S, T, X,Y, FDSQRT

BEO 10

$\mathrm{KB}=1$

$\mathrm{N}=\mathrm{M}$

$10 \mathrm{~J}=4 * \mathrm{~N}+1$

IF (J.GT.46) GO TO 130

GO TO $(20,30,40,50,60,70,80,90,100,110,120)$, N

$20 \quad X=3.8317059702 D 0$

$Y=-.402759395700$

GO TO 140

$30 \quad X=7.015586669800$

$Y=.300115752500$

GO TO 140

$40 \quad X=10.173468135100$

$Y=-.249704877100$

GO TO 140

$50 \quad X=13.323691936300$

$Y=.2183594072 D 0$

GO TO 140

$60 \quad X=16.470630050900$

$Y=-.196465371500$

GO TO 140

$70 \quad X=19.615858510500$

$Y=.18006337500$

GO TO 140

$80 \quad X=22.7600843806 D 0$

$Y=-.167184600500$

GO TO 140

$90 \quad X=25.903672087600$

$Y=.156724986300$

GO TO 140

$100 \quad X=29.046828534900$

$Y=-.148011110000$

GO TO 140

$110 \quad X=32.189679911000$

$Y=.140605798200$

GO TO 140

$120 \quad X=35.332307550100$

$Y=-.134211240300$

GO TO 140

$130 \quad X=J$

$X=X * 3.141592653600$

$\mathrm{R}=1 . \mathrm{DO} / \mathrm{X} * 2$

$S=1 . D 0-6 . D O * R *(1 . D 0-R *(1 . D 0-R *(157.2 D 0-130080.6 D 0 * R / 7 . D 0)))$

$\mathrm{J}=\mathrm{N} / 2$

$\mathrm{J}=2 * \mathrm{~J}$

$T=1 . D 0$

IF (J.NE.N) T=-1.D0

$Y=T * 1.595769122 D 0 *(1 . D 0+R * 2 * 24 . D 0 *(1 . D 0-81.6 D 0 * R)) / F D S Q R T(X)$

$X=S^{*} X / 4 . D 0$

$140 \quad A(K B)=X$

$B(K B)=Y$

$\mathrm{N}=\mathrm{N}+1$

$K B=K B+1$

IF (KB.LE.L) GO TO 10

RETURN

END

BEO 20

BEO 30

BEO 40

BEO 50

BEO 60

BEO 70

BEO 80

BEO 90

BEO 100

BEO 110

BEO 120

BEO 130

BEO 140

BEO 150

BEO 160

BEO 170

BEO 180

BEO 190

BEO 200

BEO 210

BEO 220

BEO 230

BEO 240

BEO 250

BEO 260

BEO 270

BEO 280

BEO 290

BEO 300

BEO 310

BEO 320

BEO 330

BEO 340

BEO 350

BEO 360

BEO 370

BEO 380

BEO 390

BEO 400

BEO 410

BEO 420

BEO 430

BEO 440

BEO 450

BEO 460

BEO 470

BEO 480

BEO 490

BEO 500

BEO 510

BEO 520

BEO 530

BEO 540

BEO 550

BEO 560

BEO 570

BEO 580 


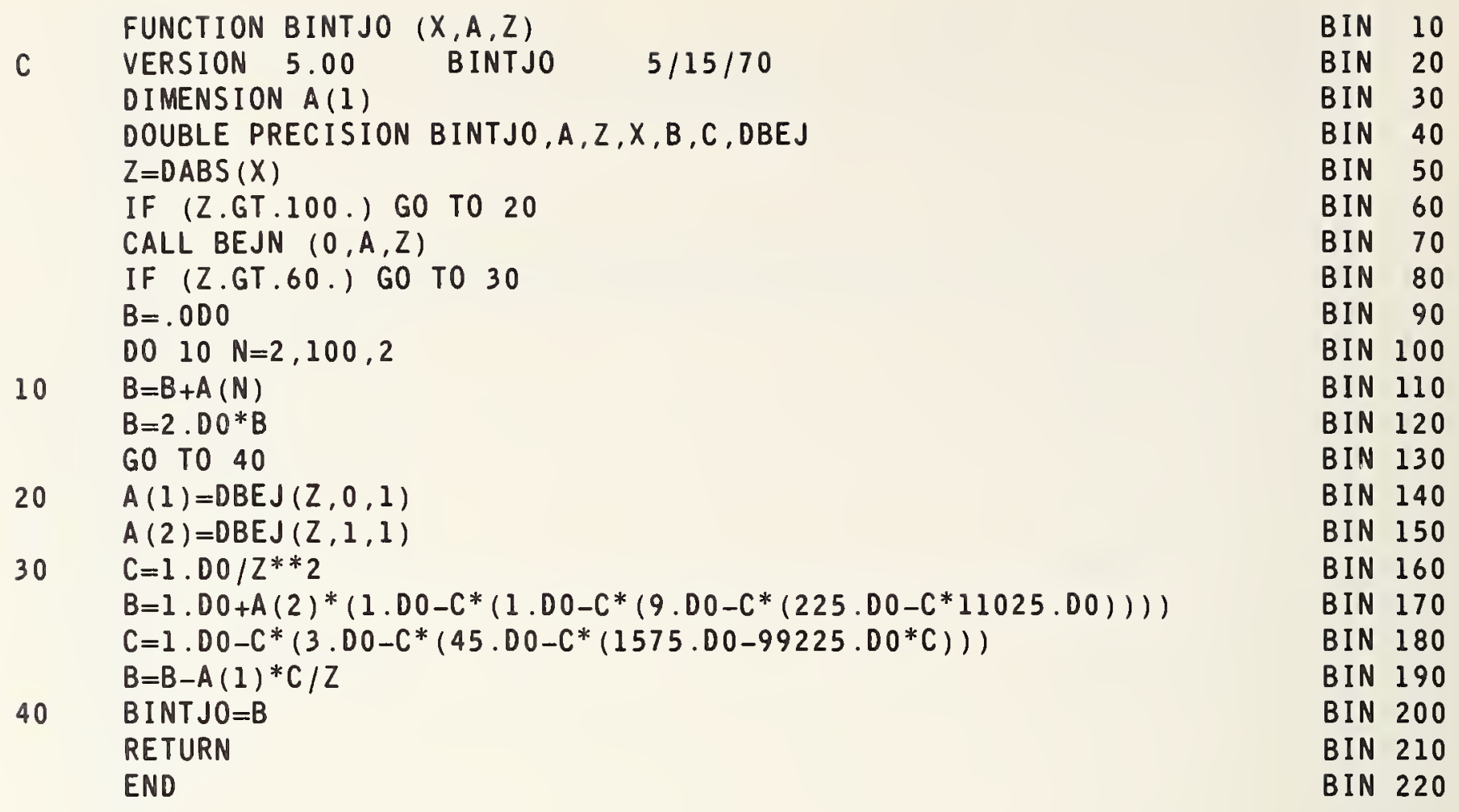


SUBROUTINE BJORCK ( $X, B, N P, A, F)$

BJO 40

THIS SUBROUTINE WAS ADAPTED BY ROY H. WAMPLER AND M. STUART SCOTT,BJO 50

NATIONAL BUREAU OF STANDARDS, WASHINGTON, D. C., JULY 1969, FROM BJO 60

A SUBROUTINE CALLED 'BJORCK' WHICH WAS WRITTEN BY WILLIAM J. HALL, BJO 70

NATIONAL BUREAU OF STANDARDS, BOULDER, COL. THIS ROUTINE USES THEBJO 80

MODIFIED GRAM-SCHMIDT ALGORITHM GIVEN BY AKE BJORCK IN 'SOLVING BJO 90

LINEAR LEAST SQUARES PROBLEMS BY GRAM-SCHMIDT ORTHOGONALIZATION' BJO 100

'BIT' VOL. 7 (1967), PAGES 1-21.

BJO 110

DOUBLE PRECISION C,D,R,Y,FDSQRT

BJO 115

DIMENSION $X(1), B(1), A(3,1)$

DIMENSION $C(3,3), D(3), R(3), Y(4)$

BJO 120

C

C INITIALIZE A AND FORM SUM OF SQUARES OF THE B VECTOR

BJO 130

$\mathrm{Y}(4)=0 . \mathrm{DO}$

BJO 140

DO $10 \quad I=1, N P$

BJO 150

$A(1, I)=1.0$

$A(2, I)=X(I)$

$A(3, I)=X(I) * X(I)$

BJO 160

BJO 170

BJO 180

BJO 190

$Y(4)=Y(4)+B(I) * B(I)$

BJO 200

10

$N F=3$

BJO 210

$D(1)=0 . D 0$

BJO 220

$Y(1)=0 . D 0$

BJO 230

DO $20 \quad I=1, N P$

BJO 240

$D(1)=A(1, I) * A(1, I)+D(1)$

BJO 250

$Y(1)=A(1, I) * B(I)+Y(1)$

BJO 260

$Y(1)=Y(1) / D(1)$

BJO 270

$I R=0$

BJO 280

DO $60 \mathrm{~K}=2$, NF

BJO 290

DO $40 \mathrm{~J}=\mathrm{K}, \mathrm{NF}$

BJO 300

$I R=I R+1$

BJO 310

$R(I R)=0 . D 0$

BJO 320

DO $30 \quad I=1, N P$

BJO 330

$R(I R)=A(K-I, I) * A(J, I)+R(I R)$

BJO 340

$R(I R)=R(I R) / D(K-1)$

BJO 350

DO $40 \quad I=1$, NP

BJO 360

BJO 370

$A(J, I)=A(J, I)-A(K-I, I) * R(I R)$

BJO 380

$D(K)=0 . D O$

BJO 390

$Y(K)=0 . D 0$

BJO 400

DO $50 \quad I=1, N P$.

BJO 410

$B(I)=B(I)-A(K-1, I) * Y(K-1)$

BJO 420

$Y(K)=A(K, I) * B(I)+Y(K)$

BJO 430

$D(K)=A(K, I) * A(K, I)+D(K)$

BJO 440

$Y(K)=Y(K) / D(K)$

BJO 450

IRS $=-N F$

BJO 460

DO $90 \mathrm{~K}=1$, NF

BJO 470

$I R S=I R S+N F-K+I$

BJO 480

$I R=I R S$

BJO 490

DO $90 \mathrm{JJ}=1, K$

BJO 500

$J=K-J J+1$

BJO 510

$C(K, J)=Y(J)$

BJO 520

IF $(J J-1) \quad 90,90,70$

BJO 530

DO $80 \quad I=2, J J$

BJO 540

$C(K, J)=C(K, J)-C(K, K-I+2) * R(I R)$

BJO 550

$I R=I R-1$

BJO 560

BJO 570

$I R=I R-N F+K$

BJO 580 
$F=Y(3) * Y(3) * F L O A T(N P-3) /(Y(4)-Y(1) * Y(1)-Y(2) * Y(2)-Y(3) * Y(3))$ RETURN 
C BLOCK DATA BLOCK

C VERSION 5.00 BLOCK $5 / 15 / 70$

BLOCK DATA

COMMON / ABCDEF / L ( 48 )

COMMON / HEADER/NOCARD $(80), \operatorname{ITLE}(60,6)$, LNCNT , IPR INT , NPAGE , I PUNCH

COMMON/FMAT / I FMTX ( 6), IOSWT, IFMTS ( 6), LHEAD (96)

C BLOCK

ABCDEF

$$
\begin{aligned}
& L(1)=1 H 0 L(2)=1 H 1 L(3)=1 H 2 L(4)=1 H 3 L(5)=1 H_{4} \\
& L(6)=1 H 5 L(7)=1 H_{6} L(8)=1 H_{7} \quad L(9)=1 H 8 \quad L(10)=1 H 9 \\
& L(11)=1 H A \quad L(12)=1 H B \quad L(13)=1 H C \quad L(1 J)=1 H D \quad L(15)=1 H E \\
& L(16)=1 H F \quad L(17)=1 H G \quad L(18)=1 H H \quad L(19)=1 H I \quad L(20)=1 H J \\
& L(21)=1 \mathrm{HK} \quad L(22)=1 \mathrm{HL} \quad L(23)=1 \mathrm{HM} \quad L(24)=1 \mathrm{HN} \quad L(25)=1 \mathrm{HO} \\
& L(26)=1 H P \quad L(27)=1 H Q \quad L(28), 1 H R \quad L(29)=1 H S \quad L(30)=1 H T \\
& L(31)=1 H U \quad L(32)=1 H V \quad L(33)=1 H W \quad L(34)=1 H X \quad L(35)=1 H Y \\
& L(36)=1 \mathrm{HZ} \quad L(37)=1 \mathrm{H} / \mathrm{L}(38)=1 \mathrm{H} . \quad L(39)=1 \mathrm{H}-\quad L(40)=1 \mathrm{H}_{+} \\
& L(41)=1 H^{*} \quad L(42)=2 H(L(43)=1 H) \quad L(44)=1 H, \quad L(45)=1 H \\
& L(46)=1 H=L(47)=1 H \$(48)=1 H \text {, }
\end{aligned}
$$

BLO 10

BLO 20

BLO 30

BLO 40

BLO 50

BLO 60

BLO 70

BLO 80

BLO 90

BLO 100

BLO 110

BLO 120

BLO 130

BLO 140

BLO 150

BLO 160

BLO 170

BLO 180

DATA $L(1), L(2), L(3), L(4), L(5), L(6), L(7), L(8), L(9), L(10) /$

BLO 190

$1 \mathrm{IHO}, 1 \mathrm{H1}, 1 \mathrm{H} 2,1 \mathrm{H} 3,1 \mathrm{H} 4,1 \mathrm{H} 5,1 \mathrm{H} 6,1 \mathrm{H} 7,1 \mathrm{H} 8,1 \mathrm{H} 9 /$

BLO 200

DATA L(11),L(12),L(13),L(14),L(15),L(16),L(17),L(18),L(19),L(20)/BL0 210

$1 \mathrm{HHA}, 1 \mathrm{HB}, 1 \mathrm{HC}, 1 \mathrm{HD}, 1 \mathrm{HE}, 2 \mathrm{HF}, 1 \mathrm{HG}, 1 \mathrm{HH}, 1 \mathrm{HI}, 1 \mathrm{HJ} /$

BLO 220

DATA L(21),L(22),L(23),L(24),L(25),L(26),L(27),L(28),L(29),L(30)/BL0 230

1 IHK, IHL, IHM, IHN, 1HO, 1HP, 1HQ, 1HR, 1HS, 1HT/

BLO 240

DATA L(31),L(32),L(33),L(34),L(35),L(36),L(37),L(38),L(39),L (40)/BL0 250

$1 \mathrm{IHU}, 1 \mathrm{HV}, 1 \mathrm{HW}, 1 \mathrm{HX}, 1 \mathrm{HY}, 1 \mathrm{HZ}, 1 \mathrm{H} /, 1 \mathrm{H}, 1 \mathrm{H}-, 1 \mathrm{H}+1$

BLO 260

DATA L (41), L (42), L(13),L (44),L(45),L (46),L(47),L(48)/

$11 H^{*}, 1 \mathrm{H}(, 1 \mathrm{H}), 1 \mathrm{H}, 1 \mathrm{1H}, 1 \mathrm{H}=, 1 \mathrm{H}, 1 \mathrm{H}, 1$

C NOCARD CONTAINS THE MESSAGE WRITTEN SAVED FROM THE OMNITAB CARD

BLO 270

BLO 280

DATA NOCARD ( 1), NOCARD ( 2), NOCARD (3), NOCARD (4),NOCARD ( 5), NOCARD (6), BLO 300 INOCARD ( 7) ,NOCARD ( 8) ,NOCARD (9), NOCARD (10), NOCARD (11), NOCARD (12), BLO 310 2NOCARD (13), NOCARD (14), NOCARD ( 15) ,NOCARD (16), NOCARD (17), NOCARD (18), BLO 320 3 NOCARD (19), NOCARD (20), NOCARD (21), NOCARD (22), NOCARD (23), NOCARD (24), BLO 330 $4 \operatorname{NOCARD}(25), \operatorname{NOCARD}(26), \operatorname{NOCARD}(27), \operatorname{NOCARD}(28), \operatorname{NOCARD}(29), \operatorname{NOCARD}(30), \mathrm{BLO} 340$ 5NOCARD (31), NOCARD (32), NOCARD (33), NOCARD (34), NOCARD (35), NOCARD (36), BLO 350 6NOCARD (37), NOCARD (38), NOCARD (39) , NOCARD (40)/

BLO 360

$7 \mathrm{H}, 1 \mathrm{H}, 1 \mathrm{H}, 1 \mathrm{H}, 1 \mathrm{H}, 1 \mathrm{H}, 1 \mathrm{H}, 1 \mathrm{H}, 1 \mathrm{H}, 1 \mathrm{H}, 1 \mathrm{H}, 1 \mathrm{H}, 1 \mathrm{H}, 1 \mathrm{H}, 1 \mathrm{H}, 1 \mathrm{H}$, $81 \mathrm{H}, 1 \mathrm{H}, 1 \mathrm{H}, 1 \mathrm{H}, 1 \mathrm{H}, 1 \mathrm{H}, 1 \mathrm{H}, 1 \mathrm{H}, 1 \mathrm{H}, 1 \mathrm{H}, 1 \mathrm{H}, 1 \mathrm{H}, 1 \mathrm{H}, 1 \mathrm{H}, 1 \mathrm{H}, 1 \mathrm{H}$, 9 IH, IH, IH, IH , IHO, IHM, IHN, IHI/

DATA NOCARD (41), NOCARD (42), NOCARD (43), NOCARD (44), NOCARD (45),

1 NOCARD (46), NOCARD (47), NOCARD (48), NOCARD (49), NOCARD (50),

2 NOCARD ( 51) , NOCARD (52), NOCARD (53), NOCARD (54), NOCARD (55),

$3 \operatorname{NOCARD}(56), \operatorname{NOCARD}(57), \operatorname{NOCARD}(58), \operatorname{NOCARD}(59), \operatorname{NOCARD}(60)$,

4 NOCARD (61), NOCARD (62), NOCARD (63), NOCARD (64), NOCARD (65),

$5 \operatorname{NOCARD}(66), \operatorname{NOCARD}(67), \operatorname{NOCARD}(68), \operatorname{NOCARD}(69), \operatorname{NOCARD}(70)$,

$6 \operatorname{NOCARD}(71), \operatorname{NOCARD}(72), \operatorname{NOCARD}(73), \operatorname{NOCARD}(74), \operatorname{NOCARD}(75)$,

BLO 370

BLO 380

BLO 390

BLO 400

BLO 410

BLO 420

BLO 430

BLO 440

BLO 450

BLO 460

BLO 470

BLO 480

BLO 490

BLO 500

BLO 510

BLO 520

BLO 530

BLO 540 
C COMPUTES IO (Z) AND II (Z) FOR COMPLEX ARGUMENT $R^{*} E(I S)=Z$ COMMON /ABEKI/ $R, S, A, B, C, D$

DOUBLE PRECISION $A, B, C, D, R, S$

CBI 40

DOUBLE PRECISION $E, F, G, H, P, Q, T, X, Y, Z, V, U, W, A A$

CBI 50

DOUBLE PRECISION FDSIN, FDCOS, FDEXP, FDSQRT

CBI 60

$E=F D C O S(S)$

$F=F D S I N(S)$

IF (R.GT.15.5) GO TO 30

CBI 70

CBI 80

CBI 90

$P=1 . D 0-2 . D 0 * F * * 2$

CBI 100

$A A=P$

CBI 110

$Q=2 . D 0 * E * F$

$W=Q$

CBI 120

CBI 130

$A=1 . D 0$

CBI 140

$\mathrm{B}=0 . \mathrm{DO}$

CBI 150

$C=1 . D 0$

CBI 160

$\mathrm{U}=0 . \mathrm{DO}$

CBI 170

$\mathrm{G}=1 . \mathrm{DO}$

CBI 180

$T=2$. $D 0$

CBI 190

$X=(R / 2 . D O) * * 2$

CBI 200

$\mathrm{V}=\mathrm{X}$

$Y=X$

DO $10 \mathrm{~N}=1,60$

$Z=1 . D 0 / G^{* *} 2$

$H=1 . D 0 /(G * T)$

$A=A+X * Z * P$

$B=B+X * Z * Q$

$\mathrm{C}=\mathrm{C}+\mathrm{V}^{*} \mathrm{H}^{*} \mathrm{P}$

$U=U+V^{*} H^{*} Q$

$X=X * Y * Z$

IF $(X . L T \ldots 5 D-10)$ GO TO 20

CBI 210

CBI 220

CBI 230

CBI 240

CBI 250

CBI 260

CBI 270

CBI 280

CBI 290

CBI 300

CBI 310

$V=V * Y * H$

CBI 320

$Z=P$

$P=Z^{*} A A-Q * W$

$Q=Q^{*} A A+Z^{*} W$

CBI 330

CBI 340

CBI 350

$G=G+1 . D 0$

CBI 360

$10 \quad T=T+1 . D 0$

CBI 370

CBI 380

$D=R^{*}\left(C * F+U^{*} E\right) / 2 . D 0$

CBI 390

$C=R^{*}\left(C^{*} E-U^{*} F\right) / 2 . D 0$

CBI 400

GO TO 60

$30 \quad Z=F D E X P(R * E) / F D S Q R T(6.283185307 D 0 * R)$

CBI 410

$X=S / 2 . D O-R^{*} F$

$Y=Z^{*} F D C O S(X)$

CBI 420

CBI 430

CBI 440

$Z=Z^{*} \operatorname{FDSIN}(X)$

$W=-1 . D 0$

$G=1 . D 0$

$H=3 . D O$

$P=E$

$Q=F$

$T=1$. D 0

CBI 450

CBI 460

CBI 470

CBI 480

CBI 490

CBI 500

$\mathrm{U}=0 . \mathrm{DO}$

CBI 510

$V=1 . D 0$

CBI 520

CBI 530

$X=0 . D O$

CBI 540

$A=1 . D 0$

CBI 550

$B=1 . D 0 /\left(8 \cdot D 0^{*} R\right)$

$C=B$

CBI 560

$D=B$

CBI 570

CBI 580

DO $40 \mathrm{~N}=1,20$

CBI 590 


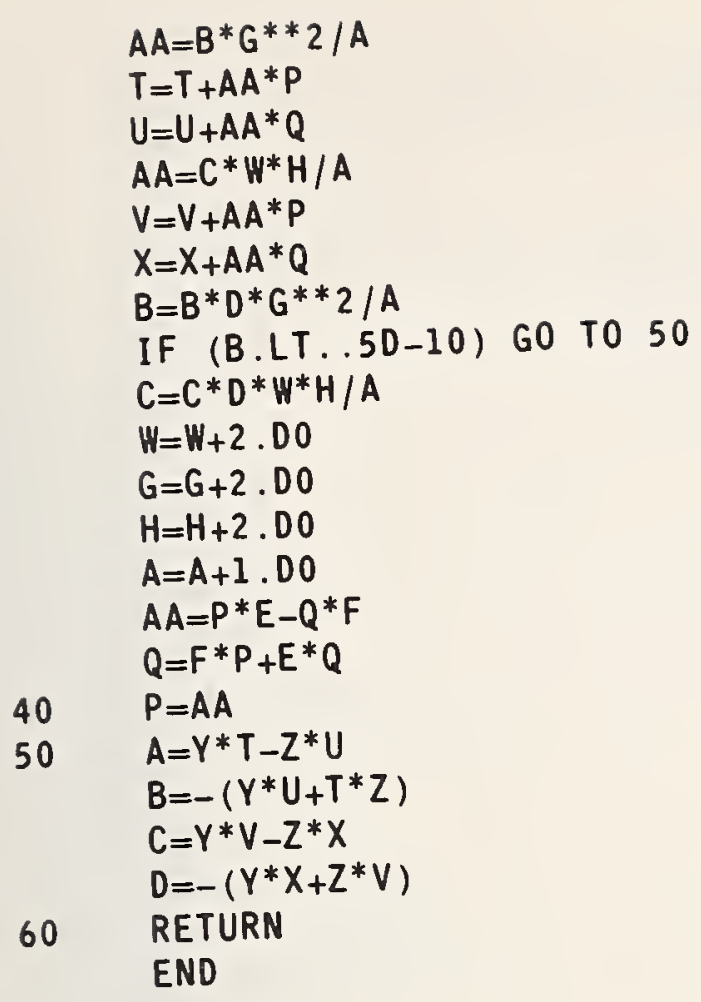


SUBROUTINE CBEK

C VERSION 5.00 CBEK 5/15/70

C COMPUTES $K O(Z)$ AND $K I(Z)$ FOR COMPLEX ARGUMENT $Z=R^{*} E$ (IS)

CBK 10 COMMON /ABEKI/R, S A B,C,D

DOUBLE PRECISION $A, B, C, D, R, S$

CBK 20

CBK 30

CBK 40

DOUBLE PRECISION $E, F, G, H, P, Q, T, U, V, W, X, Y, Z, A A(40), A B(40), A C, A D, A E$ DOUBLE PRECISION FDLOG, FDCOS, FDEXP, FDSIN, FDSQRT

CBK 50

COMMON /SCRAT/ NS, NS2, SCRAT (13500)

CBK 60

EQUIVALENCE (SCRAT $(1700), A A),(\operatorname{SCRAT}(1800), A B)$

CBK 70

CBK 80

IF (R.LE.0.0) GO TO 80

CBK 90

$E=F D C O S(S)$

CBK 100

$F=F D S I N(S)$

CBK 110

IF (R.GT.8.) GO TO 40

CBK 120

$P=1 . D 0-2 . D O * F * * 2$

CBK 130

$Q=2 . D 0 * E * F$

CBK 140

$W=P$

$\mathrm{Z}=\mathrm{Q}$

$X=(R / 2 . D 0) * * 2$

$Y=X$

$\mathrm{V}=\mathrm{X}$

$G=E *(4 \cdot D O * E * * 2-3 \cdot D 0)$

$H=F *(3 \cdot D 0-4 \cdot D O * F * * 2)$

CBK 150

CBK 160

CBK 170

CBK 180

CBK 190

CBK 200

CBK 210

$T=F D L O G(R / 2 . D 0)+.5772156649 D 0$

CBK 220

$A=-T$

$B=-S$

$C=E^{*}(T-0.5 D 0)-S^{*} F$

$U=F *(T-0.5 D 0)+S * E$

$A C=1 . D 0$

$A D=2 . D 0$

$A A(1)=1 . D 0$

$A B(1)=1.25 D 0$

DO $10 \mathrm{~N}=2,40$

$A E=N$

$A A(N)=A A(N-1)+1 . D 0 / A E$

$10 \quad A B(N)=A A(N)+1 . D 0 /(2 . D O *(A E+1 . D 0))$

CBK 230

CBK 240

CBK 250

CBK 260

CBK 270

CBK 280

CBK 290

CBK 300

CBK 310

CBK 320

CBK 330

CBK 340

CBK 350

DO $20 \mathrm{~N}=1,40$

CBK 360

$A E=T-A A(N)$

$D=P * A E-S * Q$

CBK 370

$A E=Q * A E+S * P$

CBK 380

$A=A-D * X / A C * * 2$

CBK 390

$B=B-A E * X / A C * * 2$

CBK 400

$A E=T-A B(N)$

$D=G * A E-H * S$

$A E=H * A E+G * S$

$C=C+D * Y /(A C * A D)$

CBK 410

CBK 420

CBK 430

CBK 440

$U=U+A E^{*} Y /(A C * A D)$

CBK 450

$X=X * V / A C * * 2$

CBK 460

IF $(X . L T \ldots 5 D-10)$ GO TO 30

CBK 470

$Y=Y * V /(A C * A D)$

CBK 480

CBK 490

$A C=A C+1 . D 0$

CBK 500

$A D=A D+1 . D 0$

CBK 510

$A E=P$

CBK 520

$P=A E * W-Q * Z$

CBK 530

$Q=Q * W+A E * Z$

CBK 540

$A E=G$

CBK 550

$G=A E * W-H * Z$

CBK 560

$\begin{array}{ll}20 & H=H^{*} W+A E * Z \\ 30 & C=E / R+R * C / 2 . D 0\end{array}$

CBK 570

CBK 580

$D=-F / R+R^{*} U / 2 . D 0$

CBK 590 
GO TO 70

$40 \quad U=F D E X P(-R * E) * F D S Q R T(1.570796326800 / R)$

$V=R^{*} F+S / 2 . D 0$

$\mathrm{Y}=\mathrm{U}^{*} \mathrm{FDCOS}(\mathrm{V})$

$Z=U^{*} F D S I N(V)$

$W=-1 . D 0$

$G=1 . D 0$

$\mathrm{H}=3 . \mathrm{DO}$

$\mathrm{P}=\mathrm{E}$

$Q=F$

$T=1 . D 0$

$\mathrm{U}=0 . \mathrm{DO}$

$V=1 . D 0$

$X=0 . D 0$

$A=1 . D 0$

$B=1 . D 0 /(8 . D 0 * R)$

$C=B$

$D=B$

$A C=-1 . D 0$

DO $50 \mathrm{~N}=1,12$

$A D=A C * B * G * * 2 / A$

$A E=A C^{*} C^{*} W^{*} H / A$

$T=T+A D * P$

$U=U-A D * Q$

$V=V+A E * P$

$X=X-A E^{*} Q$

$A D=B$

$B=B * D * G * * 2 / A$

IF (B.GT.AD) GO TO 60

IF (B.LT..5D-10) GO TO 60

$C=C * D * W * H / A$

$W=W+2 . D 0$

$\mathrm{H}=\mathrm{H}+2 . \mathrm{DO}$

$G=G+2 . D 0$

$A=A+1 . D 0$

$A C=-1 . D 0 * A C$

$A D=P$

$P=A D * E-Q * F$

$50 \quad Q=Q * E+A D * F$

$60 \quad A=Y^{*} T+U^{*} Z$

$B=Y * U-T * Z$

$C=Y * V+X * Z$

$D=Y * X-V * Z$

70 RETURN

$80 \quad A=0 . D 0$

$B=-.78539816339700$

$C=0 . D 0$

$D=0 . D O$

CALL ERROR (101)

RETURN

END
CBK 600

CBK 610

CBK 620

CBK 630

CBK 640

CBK 650

CBK 660

CBK 670

CBK 680

CBK 690

CBK 700

CBK 710

CBK 720

CBK 730

CBK 740

CBK 750

CBK 760

CBK 770

CBK 780

CBK 790

CBK 800

CBK 810

CBK 820

CBK 830

CBK 840

CBK 850

CBK 860

CBK 870

CBK 880

CBK 890

CBK 900

CBK 910

CBK 920

CBK 930

CBK 940

CBK 950

CBK 960

CBK 970

CBK 980

CBK 990

CBK 1000

CBK 1010

CBK 1020

CBK 1030

CBK 1040

CBK 1050

CBK 1060

CBK 1070

CBK 1080

CBK 1090

CBK 1100 
CHANGE SIGNS OF COLS,,,++++++ ETC.

CHA 40

C

COMMON /BLOCRC / NRC, RC (12600)

CHA 50

COMMON /BLOCKD/ IARGS(100), KIND(100), ARGTAB (100), NRMAX, NROW, NCOL, NCHA 70 IARGS, VWXYZ (8), NERROR

CHA 80

DIMENSION ARGS $(100)$

CHA 90

EQUIVALENCE (ARGS(1), RC(12501))

CHA 100

IF (NARGS) $50,50,10$

10 DO $30 \mathrm{I}=1$, NARGS

CHA 110

CALL ADRESS $(I, J)$

CHA 120

IF (J) $40,60,20$

20 IF (NERROR.NE.0) RETURN

CHA 130

CHA 140

DO $30 \mathrm{~N}=1$, NRMAX

CHA 150

$J \mathrm{~J}=\mathrm{J}+\mathbf{N}-1$

CHA 160

$30 \quad R C(\mathrm{~J})=-R C(\mathrm{~J})$

CHA 170

GO TO 70

CHA 180

40 CALL ERROR (3)

CHA 190

CHA 200

GO TO 70

CHA 210

50 CALL ERROR (10)

CHA 220

GO TO 70

CHA 230

60 CALL ERROR (11)

CHA 240

70 RETURN

CHA 250

END

CHA 260

SUBROUTINE CHKCOL (J)

C VERSION $5.00 \quad$ CHKCOL $5 / 15 / 70$

CHK 10

COMMON /BLOCRC/ NRC,RC(12600)

CHK 20

COMMON /BLOCKD/ I ARGS(100), KIND(100), ARGTAB (100), NRMAX, NROW, NCOL, NCHK 40

IARGS, VWXYZ (8), NERROR

DIMENSION ARGS $(100)$

EQUIVALENCE (ARGS(1), RC(12501))

CHK 50

CHK 60

C

C

THIS ROUTINE CHECKS THAT ALL ,NARGS, ARGUMENTS ARE LEGAL

CHK 70

COLUMN NUMBERS AND CONVERTS THEM IN IARGS TO THEIR BEGINNING ADDRESSES .

IF (NARGS.GT.O) GO TO 20

$10 \mathrm{~J}=1$

GO TO 40

20 DO $30 \quad I=1$, NARGS

CALL ADRESS (I,IARGS(I))

IF (IARGS (I).LE.0) GO TO 10

30 CONTINUE

$\mathrm{J}=0$

40 RETURN

CHK 80

CHK 90

CHK 100

CHK 110

CHK 120

CHK 130

CHK 140

CHK 150

CHK 160

CHK 170

CHK 180

CHK 190

CHK 200

END

CHK 210 


\begin{tabular}{|c|c|}
\hline SUBROUTINE CKIND (J) & CKI \\
\hline VERSION $\quad 5.00$ & CKI \\
\hline CKIND & CKI \\
\hline $5 / 22 / 67$ & CKI \\
\hline THE FIRST J VALUES OF KIND ARE CHECKED & CKI \\
\hline IF ALL ARE $=0$ THEN $\mathrm{J}=0$ & CKI \\
\hline IF $A L L$ ARE $=1$ THEN $J=1$ & CKI \\
\hline IF SOME ARE O AND SOME I & CKI \\
\hline COMMON /BLOCRC/ NRC, RC (12600) & CKI \\
\hline COMMON /BLOCKD/ IARGS $(100)$, KIND $(100)$, ARGTAB $(100)$, NRMAX, NROW, NCOL & NCKI 100 \\
\hline IARGS, VWXYZ ( 8 ), NERROR & CKI 110 \\
\hline DIMENSION ARGS $(100)$ & CKI 120 \\
\hline EQUIVALENCE (ARGS(1), RC (12501)) & CKI 130 \\
\hline $\mathrm{JA}=\mathrm{J}$ & CKI 140 \\
\hline$J=0$ & CKI 150 \\
\hline DO $10 \mathrm{I}=1, \mathrm{JA}$ & CKI 160 \\
\hline IF (KIND(I).NE.0) GO TO 20 & CKI 170 \\
\hline CONT INUE & CKI 180 \\
\hline RETURN & CKI 190 \\
\hline$J=1$ & CKI 200 \\
\hline DO $30 \quad I=1, J A$ & CKI 210 \\
\hline IF (KIND(I).NE.I) GO T0 40 & CKI 220 \\
\hline CONTINUE & CKI 230 \\
\hline RETURN & CKI 240 \\
\hline$J=2$ & CKI 250 \\
\hline RETURN & CKI 260 \\
\hline END & CKI 270 \\
\hline
\end{tabular}

SUBROUTINE CMPARA $\left(X 1, X_{2}, X_{3}, Y_{1}, Y_{2}, Y 3, X, Y\right) \quad$ CMP 10 $\begin{array}{lllll}\text { VERSION } 5.00 & \text { CMPARA } & 5 / 15 / 70 & \text { CMP } & 20\end{array}$ PROGRAM CMPARA WRITTEN BY MRS. CARLA MESSINA NBS-NSRDS JUNE 68 CMP 30 CMPARA IS USED BY THE INSTRUCTION MAXMIN IN PROGRAM CMSEPA $A=\left(\left(Y_{2}-Y_{3}\right) *\left(X_{2}-X_{1}\right)-\left(Y_{2}-Y_{1}\right)^{*}\left(X_{2}-X_{3}\right)\right) /\left(\left(X_{2}-X_{1}\right) *\left(X_{2} * * 2-X_{3} * * 2\right)-\left(X_{2}-X_{3}\right) C M P \quad 50\right.$ $1 *(X 2 * * 2-X 1 * * 2)) \quad$ CMP 60 $B=\left((Y 2-Y 1)-A^{*}\left(X_{2} * * 2-X 1 * * 2\right)\right) /\left(X_{2}-X_{1}\right) \quad$ CMP 70 $C=-A * X 3 * * 2-B * X 3+Y 3 \quad$ CMP 80 $X=-B /\left(2{ }^{*} A\right)$ $Y=A * X * * 2+B * X+C$ RETURN END

CMP 90

CMP 100

CMP 110

CMP 120 


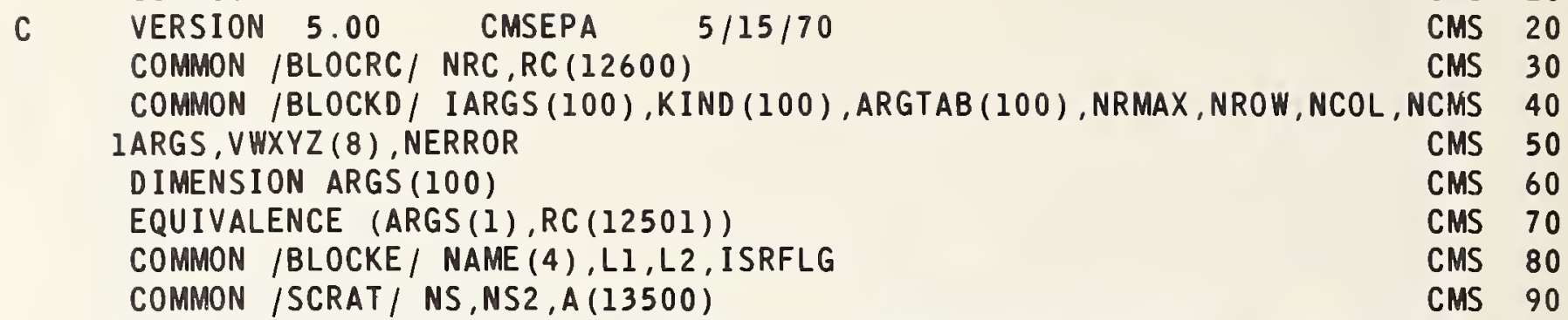

C L2 2 S SEPARATE FROM COL ++ EVERY, , ROW START WITH ROW , STORE INCMS 100

C L2 $=3$ INSERT IN COL ++ FROM COL ++ AT EVERY ,, ROW STARTING AS , CMS 120

C STORE IN COL ++ CMS 130

C L2=4 MAXMIN X IN ++ Y IN ++, XMAX IN ++ YMAX IN ++, XMIN ++ YMIN CMS 140

C PROGRAM CMSEPA WRITTEN BY MRS. CARLA MESSINA NBS-NSRDS JUNE 196 CMS 150 GO TO $(10,10,30,310)$, L2

$10 \quad$ NARGS $=$ NARGS +1

$J=N A R G S$

DO $20 \quad I=2$, NARGS

$\operatorname{IARGS}(\mathrm{J})=\operatorname{IARGS}(\mathrm{J}-1)$

$\operatorname{KIND}(\mathrm{J})=\operatorname{KIND}(\mathrm{J}-1)$

CMS 160

$\mathrm{J}=\mathrm{J}-1$

30 CALL CKIND (J)

IF (J) $40,70,40$

CMS 170

CMS 180

CMS 190

CMS 200

CMS 210

CMS 220

$40 \quad K=3$

CMS 230

CMS 240

50 CALL ERROR (K)

CMS 250

RETURN

CMS 260

70 IF (NARGS-5) $80,100,80$

CMS 270

CMS 280

$K=10$

CMS 290

IF (L2-2) $90,90,50$

CMS 300

90 NARGS=NARGS-1

GO TO 50

$100 \quad M=I A R G S$ (3)

$N=I$ ARGS (4)

DO $120 \quad I=3,4$

IF (IARGS (I)) $130,130,110$

CMS 310

CMS 320

CMS 330

CMS 340

CMS 350

110 IF (IARGS (I)-NROW) $120,120,130$

CMS 360

CMS 370

$120 \operatorname{IARGS}(\mathrm{I})=\operatorname{IARGS}(\mathrm{I})$

CMS 380

CALL CHKCOL (J)

IF (J) $40,140,40$

$130 \quad K=16$

GO TO 50

140 IF (NERROR.NE.0) GO TO 60

IF (NRMAX) $150,150,160$

$150 \quad K=9$

GO TO 50

160 DO $170 \quad I=1$, NARGS

$170 \operatorname{IARGS}(I)=\operatorname{IARGS}(I)-1$

$L=I A R G S(5)$

IF (L2-2) $180,180,210$

C SEPARATE

CMS 390

CMS 400

CMS 410

CMS 420

CMS 430

CMS 440

CMS 450

CMS 460

CMS 470

CMS 480

CMS 490

CMS 500

CMS 510

180 DO $190 \quad I=1$, NRMAX

$J=I \operatorname{ARGS}(1)+I$

CMS 520

CMS 530

$190 \quad A(I)=R C(J)$

DO $200 \mathrm{~K}=\mathbb{N}$, NFMAX, $M$

CMS 540

$L=L+1$

CMS 550

CMS 560

$200 \quad R C(L)=A(K)$

GO TO 60

CMS 570

C INSERT

CMS 580

CMS 590

$210 \quad M=M-1$

CMS 600 
$K A=0$

CMS 640

$I=I \operatorname{ARGS}(1)$

DO $240 \mathrm{~K}=1, \mathrm{~N}$

CMS 650

$\mathrm{I}=\mathrm{I}+\mathrm{I}$

$K A=K A+1$

CMS 660

CMS 670

$240 \quad A(K A)=R C(I)$

CMS 680

$N N=K A+I A R G S(1)$

CMS 690

$M M=I A R G S$ (2)

CMS 700

DO $260 \mathrm{~K}=\mathrm{N}$, NRMAX, $M$

CMS 710

$K A=K A+1$

CMS 720

$M M=M M+1$

CMS 730

$A(K A)=R C(M M)$

CMS 740

DO $250 \quad L L=1, M$

CMS 750

$K A=K A+1$

CMS 760

$N N=N N+1$

$250 \quad A(K A)=R C(N N)$

260 CONTINUE

$I=(N R M A X-N) / M+I$

CMS 770

CMS 780

CMS 790

CMS 800

CMS 810

IF (I+NRMAX-NROW) $270,270,280$

CMS 820

$270 \quad$ NRMAX $=$ NRMAX $+I$

GO TO 290

CMS 830

CMS 840

CMS 850

CMS 860

CALL ERROR (219)

290 DO $300 \mathrm{~K}=1$, NRMAX

$\mathrm{L}=\mathrm{L}+1$

$300 \quad R C(L)=A(K)$

GO TO 60

310 IF (NARGS-6) $80,320,80$

320 CALL CHKCOL (J)

IF (J) $40,330,40$

330 IF (NERROR.NE.0) GO TO 60

$K A=0$

I $U P=-1$

IF (NRMAX) $150,150,340$

340 IF (NRMAX-2) $350,350,390$

350 IF (KA) $370,370,360$

360 CALL ERROR (220)

370 IF (IUP) $380,60,60$

$380 \quad K=219$

GO TO 50

390 DO $400 \mathrm{~K}=1$, NRMAX

$I=I \operatorname{ARGS}(1)+K-1$

$J=I \operatorname{ARGS}(2)+K-1$

$A(K)=R C(I)$

$\mathrm{K} 2=\mathrm{K}+\mathrm{NRMAX}$

$400 \quad A(K 2)=R C(J)$

$I I=I$ ARGS $(3)-1$

$J I=I$ ARGS $(4)-1$

$K I=I A R G S(5)-1$

$L I=I \operatorname{ARGS}(6)-1$

IF (NRMAX-4) $410,520,520$

CMS 870

CMS 880

CMS 890

CMS 900

CMS 910

CMS 920

CMS 930

CMS 940

CMS 950

CMS 960

CMS 970

CMS 980

CMS 990

CMS 1000

CMS 1010

CMS 1020

CMS 1030

CMS 1040

CMS 1050

CMS 1060

CMS 1070

CMS 1080

CMS 1090

CMS 1100

CMS 1110

CMS 1120

CMS 1130

CMS 1140

$K 2=N R M A X+1$

IF $(A(K 2)-A(K 2+1)) \quad 420,380,430$

CMS 1150

CMS 1160

IF $(A(K 2+1)-A(K 2+2)) 380,380,440$

CMS 1170

430 IF $(A(K 2+1)-A(K 2+2)) 450,380,380$
440 IUP $=I U P+1$

CMS 1180 
$480 \quad K A=1$

CMS 1240

GO TO 350

CMS1250

490 CALL CMPARA ( $A(1), A(2), A(3), A(K 2), A(K 2+1), A(K 2+2), X 1, Y 1)$

CMS 1260 IF (IUP) $500,500,510$

$500 \quad R C(K 1+1)=X 1$

CMS 1270

$R C(L 1+1)=Y 1$

CMS 1280

GO TO 60

CMS 1290

$\mathrm{RC}(I 1+1)=\mathrm{XI}$

CMS 1300

510

$R C(L l+1)=Y 1$

CMS 1310

GO TO 60

CMS 1320

$I=N R M A X-2$

CMS 1330

520

DO $730 \mathrm{~K}=1$, I

CMS 1340

IEQUAL $=1$

$\mathrm{K} 2=\mathrm{K}+\mathrm{NRMAX}$

If $(A(K 2)-A(K 2+1)) 530,610,540$

530 If $(A(K 2+1)-A(K 2+2)) 730,730,550$

540 IF $(A(K 2+1)-A(K 2+2)) \quad 560,730,730$

550 IUP $=1$

$$
\text { GO TO } 570
$$

$560 \quad$ IUP $=0$

570 IF $(A(K)-A(K+1)) \quad 580,600,580$

580 IF $(A(K)-A(K+2)) \quad 590,600,590$

590 IF $(A(K+1)-A(K+2)) \quad 680,600,680$

$600 \quad K A=K A+1$

GO TO 730

610 IF (K-1) $730,730,620$

620 IEQUAL $=2$

IF $(A(K-1)-A(K)) \quad 630,600,630$

630 IF $(A(K-1)-A(K+1)) \quad 640,600,640$

640 If $(A(K-1)-A(K+2)) \quad 650,600,650$

650 If $(A(K 2-1)-A(K 2)) \quad 660,730,670$

660 IF $(A(K 2+1)-A(K 2+2)) 730,730,550$

CMS 1350

CMS 1360

CMS 1370

CMS 1380

CMS1390

CMS 1400

CMS1410

CMS 1420

CMS 1430

CMS 1440

CMS 1450

CMS 1460

CMS 1470

CMS 1480

CMS 1490

CMS 1500

CMS 1510

CMS 1520

CMS 1530

CMS 1540

CMS 1550

IF $(A(K 2+1)-A(K 2+2)) \quad 560,730,730$

CMS 1560

CMS 1570

CALL CMPARA ( $A(K), A(K+1), A(K+2), A(K 2), A(K 2+1), A(K 2+2), X 1, Y 1)$ IF (IEQUAL-2) $700,690,690$

690 CALL CMPARA ( $A(K-1), A(K), A(K+1), A(K 2-1), A(K 2), A(K 2+1), X 2, Y 2)$

CMS 1590 $X 1=0.5^{*}(X 1+X 2)$

$Y 1=0.5^{*}\left(Y_{1}+Y_{2}\right)$

700 IF (IUP) $710,710,720$

CMS 1600

CMS 1610

CMS1620

CMS 1630

$I U P=0$

CMS 1640

$K 1=K 1+1$

CMS 1650

$L 1=L I+1$

CMS 1660

$R C(K I)=X I$

CMS 1670

$R C(L 1)=Y 1$

CMS 1680

GO TO 730

CMS 1690

$11=11+1$

CMS 1700

$=\sqrt{ } 1+1$

CMS 1710

$R C(I I)=X I$

CMS 1720

$R C(J I)=Y I$

CMS 1730

CONTINUE

CMS 1740

GO TO 350

CMS 1750 
SUBROUTINE COALES

C VERSION 5.00 COALES 5/15/70

ACOALESCE AND AAVERAGE COMMANDS

WRITTEN BY R. MCCLENON, NSRDS-NBS, NOV. 1969

COA 10

$L I=A C O A L E S C E \quad L 2=$ AAVERAGE

COA 20

COA 30

COMMAND FORM IS --

COA 40

ACOALESCE MATRIX STARTING IN $\mathrm{R}_{++} \mathrm{C}_{++}$

COA 50

COA 60

ACOALESCE ON FIRST COL OF ARRAY IN , ++ $R=, C=$, START STORING COA 80

$\begin{array}{ll}\text { IN }, .++ & \text { COA } 90\end{array}$

AAVERAGE ON FIRST COL OF ARRAY IN , ++ $R=, C=$, , START STORING COA 100

IN , . ++ OR

ACOALESCE ON ** IN FIRST COL OF , $++R=,, C=$, START STORING

COA 110

COA 120

IN , . ++

AAVERAGE ON ** IN FIRST COL OF, , ++ $R=,, C=$, START STORING

COA 130

COA 140

IN , , ++

COMMON /BLOCKE/ NAME (4),L1,L2, ISRFLG

COA 150

COA 160

COA 170

COMMON /BLOCKD/ IARGS (100), KIND(100), ARGTAB (100), NRMAX, NROW, NCOL, NCOA 180

IARGS, VWXYZ (8), NERROR

COMMON /SCRAT/ NS,NS2,A(13500)

COA 190

COMMON /BLOCRC/ NRC,RC(12600)

COA 200

DIMENSION ARGS(100)

COA 210

EQUIVALENCE (ARGS (1), RC (12501))

COA 220

$\mathrm{L} 2=\mathrm{L} 2-8$

IF (NARGS-6) $410,10,20$

$10 \quad K L=1$

$I=6$

CALL CKIND (I)

IF (I-I) $60,420,420$

COA 230

COA 240

COA 250

COA 260

COA 270

COA 280

$\mathrm{KL}=2$

DO $30 \mathrm{~J}=2,7$

IF (KIND(J)) $420,30,420$

COA 290

COA 300

COA 310

COA 320

CONTINUE

IF (NARGS-8) $40,410,410$

COA 330

COA 340

IF (KIND(1)) 420,420,50

COA 350

$\begin{array}{ll}50 & Y=A R G S(1) \\ 60 & K L 5=K L+5\end{array}$

DO $70 \mathrm{~J}=\mathrm{KL}, \mathrm{KL} 5$

IF (IARGS (J)) $430,430,70$

70 CONTINUE

LROW=I ARGS $(K L+2)$

COA 360

COA 370

COA 380

COA 390

COA 400

COA 410

$L C O L=I A R G S(K L+3)$

COA 420

KROW=IARGS (KL)

$K C O L=I$ ARGS $(K L+1)$

IF (KROW+LROW-NROW- 1 ) $80,80,440$

COA 430

COA 440

COA 450

80 IF (KCOL+LCOL-NCOL-1) $90,90,440$

COA 460

MROW=I ARGS $(K L+4)$

$M C O L=I A R G S(K L+5)$

IF (MROW+LROW-NROW-1) $100,100,440$

COA 470

COA 480

COA 490

100 IF (MCOL+LCOL-NCOL-1) 110,110,440

COA 500

IF (NERROR) $400,120,400$

$120 \mathrm{KRR}=\mathrm{KROW}+\mathrm{LROW}-1$

$K C C=K C O L+L C O L-1$

$M R R=M R O W+L R O W-1$

COA 510

COA 520

COA 530

$M C C=M C O L+L C O L-1$

COA 540

COA 550

$\mathrm{N}=0$

COA 560

IF (KL-1) 230,230,130

COA 570

130 DO $140 \mathrm{~J}=2$, LCOL

COA 580

$140 \quad A(J)=0.0$

COA 590 


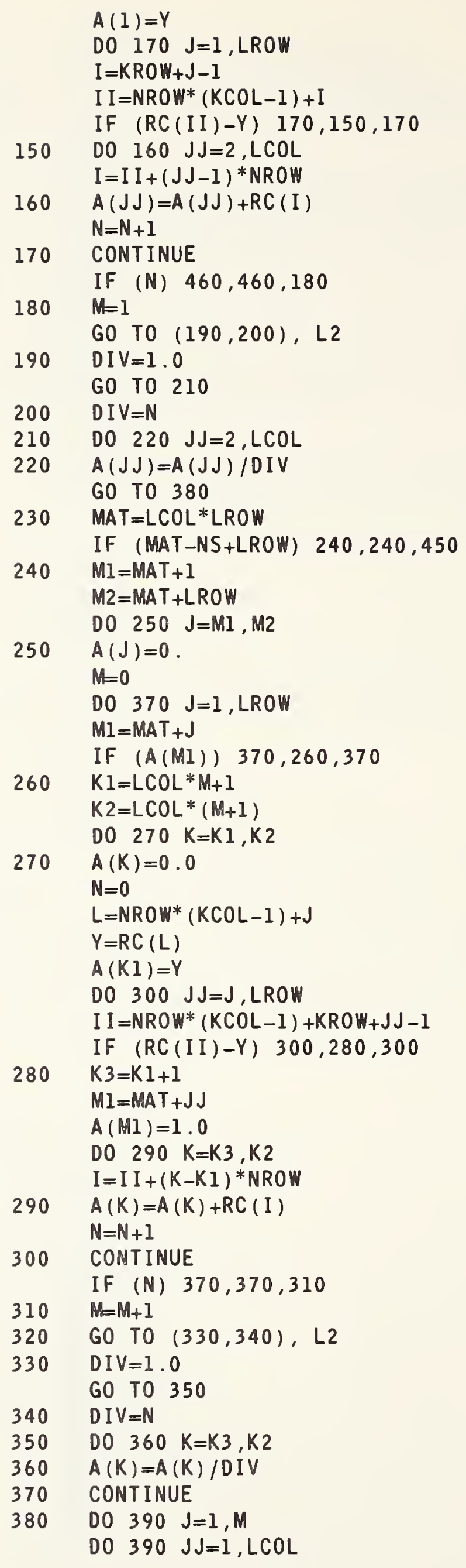




$$
I=L \operatorname{COL}^{*}(J-1)+J J
$$

$I I=N R O W^{*}(M C O L-2+J J)+J+M R O W-1$

$R C$ (II) $=A$ (I)

390 CONTINUE

400 RETURN

COA1210

410

CALL ERROR (10)

COA1220

GO TO 400

COA1230

COA1240

COA 1250

CALL ERROR (20)

GO TO 400

COA 1260

430

CALL ERROR (11)

COA1270

GO TO 400

COA 1280

440 CALL ERROR (17)

COA1290

GO TO 400

COA 1300

COA 1310

COA 1320

COA 1330

COAl 340

COA 1350

COA1360

COA 1370 
FUNCTION COMELL $(Z, I)$

C VERSION 5.00 COMELL $5 / 15 / 70$

C COMPLETE ELLIPTIC INTEGRALS - FIRST AND SECOND KIND

COM 10

DOUBLE PRECISION $Z, X, A, B, C, D, E, P, Q$

DOUBLE PRECISION FDLOG, FDSQRT, COMELL

COM 20

COM 30

COM 40

$X=Z$

IF (DABS(Z).LT.1.DO) GO TO 10

IF (DABS (Z).EQ.1.DO.AND.I.EQ.2) GO T0 10

COM 50

COM 60

COM 70

CALL ERROR (109)

COM 80

$Z=0.0 D O$

COM 83

RETURN

COM 85

COM 90

$10 \quad A=X$

$B=F D S Q R T(1 . D O-A)$

IF $(X . G T \ldots 996 D 0)$ GO TO 50

COM 100

COM 110

$B=(1 . D 0-B) /(1 . D 0+B)$

COM 120

$A=B * 2$

$B=1 . D 0+B$

COM 130

$\operatorname{COM} 140$

$C=1 . D 0$

COM 150

$D=C$

$E=2 . D 0$

IF (I.EQ.1) GO TO 20

COM 160

COM 170

COM 180

$B=1 . D 0 / B$

COM 190

$D=-1 . D 0$

$20 \quad P=A$

DO $30 \quad N=1,90$

$C=C+P *(D / E) * * 2$

$P=P * A *(D / E) * * 2$

IF (P.LT..ID-9) GO TO 40

COM 200

COM 210

COM 220

COM 230

$\operatorname{COM} 240$

COM 250

$D=D+2 . D 0$

$30 \quad E=E+2 . D 0$

$40 \quad A=B * C * 1.570796326 D 0$

GO TO 70

$50 \quad A=F D L O G(4.0 D 0 / B)$

$Q=B * * 2$

IF (I.GT.1) GO TO 60

$B=.2500^{*}(A-1 . D 0)$

$C=.140625 D 0^{*}(A-1.66666666600)$

$D=9.765625 D-2 *(A-1.233333333 D 0)$

$E=1255 . D 0^{*}(A-1.27904761904 D 0) / 16384 . D 0$

COM 260

COM 270

COM 280

COM 290

COM 300

$\operatorname{COM} 310$

$\operatorname{COM} 320$

COM 330

$\operatorname{COM} 340$

COM 350

COM 360

$\operatorname{COM} 370$

$A=A+Q^{*}\left(B+Q^{*}\left(C+Q^{*}(D+Q * E)\right)\right)$

$\operatorname{COM} 380$

GO TO 70

COM 390

COM 400

COM 410

COM 420

$\operatorname{COM} 430$

$\operatorname{COM} 440$

COM 450

$\operatorname{COM} 460$

COM 470

C

$$
\text { COMELL }=A
$$

RETURN

COM 490 
SUBROUTINE COMPLX

$\operatorname{cox} 10$

VERSION $5.00 \quad$ COMPLX $5 / 15 / 70$

$\operatorname{cox} 20$

C

COMMON /BLOCRC/ NRC, RC (12600)

$\operatorname{cox} 30$

COMMON /BLOCKD/ IARGS $(100)$, KIND(100), ARGTAB $(100), N R M A X, N R O W, N C O L, N C O X 50$

IARGS, VWXYZ (8), NERROR

DIMENSION ARGS $(100)$

EQUIVALENCE (ARGS(1), RC(12501))

$\operatorname{cox} 60$

$\operatorname{cox} 70$

COMMON /SCRAT/ NS, NS2,A(13500)

COMMON /BLOCKE/ NAME (4),L1,L2, ISRFLG

$\operatorname{cox} 80$

COMMON /CONSTS/ PI ,E, HALFPI, DEG, RAD, XALOG

$\operatorname{cox} 90$

$\operatorname{cox} 100$

$\operatorname{cox} 110$

$\operatorname{cox} 120$

DIMENSION KK $(6)$

$\operatorname{cox} 130$

EQUIVALENCE (I 1, IARGS (1)), (I2,IARGS (2)), (I3, IARGS (3)), (I 4, IARGSCOX 140

$1(4)),($ I $5, \operatorname{IARGS}(5)),(16, \operatorname{IARGS}(6))$

$\operatorname{cox} 150$

DOUBLE PRECISION D(5), X, Y,FDCOS,FDSIN, FDSQRT

$\operatorname{cox} 160$

$\mathrm{C}$

WRITTEN BY DAVID HOGBEN, SEL, NBS. $4 / 24 / 70$.

$\operatorname{cox} 170$

CADD (E), (E), TO (E), (E) AND PUT IN COLUMNS (C), (C)

$\operatorname{cox} 180$

CSUBTRACT (E), (E), FROM (E), (E) AND PUT IN COLUMNS (C), (C)

$\operatorname{cox} 190$

CMULTIPLY

$(E),(E)$

E) $B Y$

(E), (E) AND PUT IN COLUMNS (C), (C)

$\operatorname{cox} 200$

CDIVIDE (E), (E) BY (E), (E) AND PUT IN COLUMNS (C), (C)

$\operatorname{cox} 210$

CPOLAR OF X IN (E) Y IN (E) PUT R IN COL (C) THETA IN COL (C) COX 240

FIRST ARGUMENT OF EACH PAIR IS REAL, SECOND IS IMAGINARY

$\operatorname{cox} 250$

$\operatorname{cox} 260$

$\operatorname{cox} 270$

VALUES OF L2 ARE ***

$\operatorname{cox} 280$

$1=C A D D, \quad 2=C S U B, \quad 3=C M U L T, \quad 4=C D I V, \quad 5=C R E C T A N \quad 6=C P O L A R$

$\operatorname{cox} 290$

NARGS $=6$ FOR ADD, SUB, MULT, DIV

$\operatorname{cox} 300$

$\operatorname{cox} 310$

NARGS $=4$ FOR CRECTANGULAR AND CPOLAR

$\operatorname{cox} 320$

$\operatorname{cox} 330$

ERROR CHECKING

10 IF (NARGS.NE.6.AND.L2.LT.5) CALL ERROR (10)

$\operatorname{cox} 340$

$\operatorname{cox} 350$

IF (NARGS.NE.4.AND.L2.GT.4) CALL ERROR (10)

IF (KIND (NARGS).NE.0.AND.KIND (NARGS-1).NE.0) CALL ERROR (20)

$\operatorname{cox} 360$

$\operatorname{cox} 370$

IF (NRMAX.EQ.0) CALL ERROR (9)

DO $30 \quad I=1$, NARGS

$K K(I)=1$

CALL ADRESS (I,IARGS(I))

IF (IARGS (I)) $20,40,30$

$20 \quad K K(I)=0$

$\operatorname{IARGS}(I)=-I \operatorname{ARGS}(I)$

30 CONTINUE

GO TO 50

$\operatorname{cox} 380$

$\operatorname{cox} 390$

$\operatorname{cox} 400$

$\operatorname{cox} 410$

$\operatorname{cox} 420$

$\operatorname{cox} 430$

$\operatorname{cox} 440$

$\operatorname{cox} 450$

$\operatorname{cox} 460$

$\operatorname{cox} 470$

$\operatorname{cox} 480$

$\operatorname{cox} 490$

$\operatorname{cox} 500$

Cox 510

COX 520

COX 530

COX 540

$\operatorname{cox} 550$

COX 560

COX 570

COX 580

$70 \quad X=D(1)+D(3)$

$Y=D(2)+D(4)$

COX 590 
GO TO 120

C CDIVIDE

$\operatorname{cox} 670$

C ZERO RETURNED IF DIVISION BY ZERO, DIAGNOSTIC GIVEN. ERROR(104).

$\operatorname{cox} 690$

$\operatorname{cox} 700$ $D(5)=D(3) * * 2+D(4) * * 2$

IF (D(5).GT.0.) GO TO 110

$\operatorname{cox} 710$

CALL ERROR (106)

$X=0.0 D 0$

$Y=0.0 D 0$

GO TO 120

$110 \quad X=(D(1) * D(3)+D(2) * D(4)) / D(5)$

$Y=(D(3) * D(2)-D(1) * D(4)) / D(5)$

$120 R C(I 5)=F D P C O N(X)$

$\mathrm{RC}(I)=\mathrm{FDPCON}(\mathrm{Y})$

$I I=I I+K K(I)$

$\mathrm{I} 2=\mathrm{I} 2+\mathrm{KK}(2)$

$13=I 3+K K(3)$

$I 4=I 4+K K(4)$

130 I $5=15+K K(5)$

RETURN

C CRECTANGULAR AND CPOLAR

$140 \quad M M=L 2-4$

DO $260 \quad I=I 4, \mathrm{~J} J$

$D(1)=R C(I 1)$

$D(2)=R C(I 2)$

GO TO $(150,190)$, MM

C CRECTANGULAR - R, THETA TO $X, Y$

150 IF (RC(II)) $180,160,180$

$160 \quad X=0.000$

$170 \quad Y=0.000$

GO TO 250

$180 \quad X=D(1) * F D C O S(D(2))$

$Y=D(1) * \operatorname{FDSIN}(D(2))$

GO TO 250

C CPOLAR $X, Y$ TO R, THETA

$\operatorname{cox} 720$

$\operatorname{cox} 730$

$\operatorname{cox} 740$

$\operatorname{cox} 750$

$\operatorname{cox} 760$

$\operatorname{cox} 770$

$\operatorname{cox} 780$

$\operatorname{cox} 790$

COX 800

$\operatorname{cox} 810$

$\operatorname{cox} 820$

$\operatorname{cox} 830$

$\operatorname{cox} 840$

$\operatorname{cox} 850$

$\operatorname{cox} 860$

$\operatorname{cox} 870$

$\operatorname{cox} 880$

$\operatorname{cox} 890$

$\operatorname{cox} 900$

$\operatorname{cox} 910$

$\operatorname{cox} 920$

$\operatorname{cox} 930$

$\operatorname{cox} 940$

$\operatorname{cox} 950$

$\operatorname{cox} 960$

$\operatorname{cox} 970$

$\operatorname{cox} 980$

$\operatorname{cox} 990$

$\operatorname{cox} 1000$

Cox 1010

IF (RC (I2)) $220,200,220$

200 IF (RC(II)) $210,160,210$

$\operatorname{cox} 1020$

$\begin{array}{ll}C & Y=0, X \text { NE } 0 \\ 210 & X=D A B S(D(1))\end{array}$

$\operatorname{cox} 1030$

COX 1040

GO TO 170

Cox 1050

220 IF (RC(II)) $240,230,240$

C $X=0, Y$ NE 0

$\operatorname{cox} 1060$

$\operatorname{cox} 1070$

$230 X=D A B S(D(2))$

$\operatorname{cox} 1080$

IF $X=0.0$, THEN THETA=HALFPI*SIGN $(Y)$

$Y=S I G N($ HALFPI, RC (I2))

GO TO 250

$240 \quad X=F D S Q R T(D(1) * * 2+D(2) * * 2)$

$Y=D A T A N 2(D(2), D(1))$

$250 \operatorname{RC}(\mathrm{I} 3)=\operatorname{FDPCON}(\mathrm{X})$

$R C(I)=F D P C O N(Y)$

$I I=I I+K K(I)$

$12=I 2+K K(2)$

$260 \quad I 3=I 3+K K(3)$

RETURN

Cox 1090

COXI100

$\operatorname{cox} 1110$

COX 1120

$\operatorname{cox} 1130$

$\operatorname{cox} 1140$

$\operatorname{cox} 1150$

$\operatorname{cox} 1160$

COX 1170

Cox 1180

Cox 1190

END 
SUBROUTINE CORREL
VERSION 5.00

C

5.00 CORREL

$5 / 15 / 70$

COR 10

COR 20

IARGS, VWXYZ ( 8) , NERROR

COMMON /BLOCKE/ NAME (4),L1,L2, ISRFLG

COMMON /BLOCRC/ NRC, RC (12600)

DIMENSION ARGS $(100)$

COR 40

COR 50

COR 60

COR 70

EQUIVALENCE (ARGS (1), RC (12501))

COMMON /HEADER/ NOCARD $(80), \operatorname{ITLE}(60,6), \operatorname{LNCNT}$, IPRINT, NPAGE, IPUNCH

COR 80

COMMON /SCRAT/ NS, NS2, A (13500)

DIMENSION ERR (3), AVG $(100), \operatorname{SD}(100), T(100)$

EQUIVALENCE (A(13301), AVG(1)), (A(13401),SD(1),T(1))

$L 2=L 2-10$

IF (L2.EQ.1.OR.NARGS.NE.IARGS(1) +1) GO TO 10

COR 90

COR 100

COR 110

COR 120

COR 122

COR 124

CALL ERROR (233)

RETURN

$10 \quad M V A R=99$

IF (NARGS.LT.3) CALL ERROR (10)

NVAR=IARGS $(1)$

IF (NVAR.LT.2.OR.NVAR.GT.MVAR) CALL ERROR (3)

$K E E P=(N A R G S-N V A R+1) / 2$

IF (KEEP.LT.1.OR.KEEP.GT.3.OR.MOD(NARGS-NVAR 2) .EQ.0) CALL ERROR

COR 126

COR 128

COR 130

COR 140

COR 150

COR 160

COR 170

110)

GO TO $(40,30,20)$, KEEP

COR 180

COR 190

COR 200

COR 210

IF (K2.LT.1.OR.K2.GT.NROW) CALL ERROR (16)

I ARGS (NVAR +4$)=1$

COR 220

COR 230

COR 240

IF (KI.LT.1.OR.KI.GT.NROW) CALL ERROR (16)

I ARGS (NVAR +2$)=1$

COR 250

COR 260

COR 270

CALL CHKCOL (J)

IF (J.EQ.0) GO TO 50

COR 280

CALL ERROR (11)

RETURN

50 GO TO $(80,70,60)$, KEEP

60 IARGS $($ NVAR +4$)=$ I ARGS $($ NVAR +5$)+K 2-1$

$70 \quad$ IARGS $(N V A R+2)=I A R G S(N V A R+3)+K I-1$

80 LOTTE $=$ NVAR*NVAR

KURT $=2 *$ LOTTE

C LOT IS SPACE IN ARRAY A RESERVED FOR RANKED DATA

LOT $=$ MAXO (NRMAX* (NVAR+1), $3 *$ LOTTE $+8 *$ NVAR $+8,4 *$ LOTTE)

IF (NRMAX.LT.3) CALL ERROR (9)

IF (NRMAX*NVAR.GT.NRC) CALL ERROR (15)

IF (MAXO(LOT+LOTTE $+100, N R M A X * 4+3 * L O T T E)$.GT.NS) CALL ERROR (23)

IF (NERROR.NE.0) RETURN

COR 290

COR 300

COR 310

COR 320

COR 330

COR 340

COR 350

COR 360

COR 370

COR 380

COR 390

COR 400

COR 410

NVA $=$ NVAR-1

IF (L2.EQ.2) GO TO 130

C RANKS OF OBSERVATIONS

IND $=$ NVAR * NRMAX +1

DO $90 \mathrm{I}=1$, NVAR

$K I=(I-1) * N R M A X+1$

$K 2=I$ ARGS $(I+1)$

90 CALL RANKX (NRMAX,RC (K2),A(IND), A(K1), T(I))

C SPEARMAN RANK CORRELATION COEFFICIENT

$\mathrm{F}=(($ NRMAX-1) *NRMAX* $($ NRMAX +1$)) / 6$

$I 1=$ LOT + LOTTE

$A(I 1)=1$.

DO $120 \mathrm{~J}=1$, NVA

IND $=(\mathrm{J}-1) * N V A R+L O T$

COR 420

COR 430

COR 440

COR 450

COR 460

COR 470

COR 480

COR 490

COR 500

COR 510

COR 520

COR 530

COR 540

COR 550 
$\mathrm{IJ}=\mathrm{J}+1$

DO $120 \mathrm{I}=\mathrm{IJ}, \mathrm{NVAR}$

COR 580

I $l=I N D+I$

COR 590

$\mathrm{I} 2=(\mathrm{I}-1) * N V A R+\mathrm{J}+\mathrm{LOT}$

COR 600

$\mathrm{Kl}=2 . * T(\mathrm{I})+0.4$

COR 610

$K 2=2 . * T(J)+0.4$

COR 620

IF (IFIX (F)-K1.GT.0.AND. IFIX (F)-K2.GT.0) GO TO 100

COR 630

COR 640

$A(I 1)=0$.

GO TO 120

$100 \quad D=0$.

DO $110 \mathrm{~K}=1$, NRMAX

COR 650

COR 660

COR 670

COR 680

$\mathrm{K} I=(\mathrm{I}-\mathrm{I}) * \mathrm{NRMAX}+\mathrm{K}$

COR 690

$\mathrm{K} 2=(\mathrm{J}-1) * \mathrm{NRMAX}+\mathrm{K}$

COR 700

$110 \quad \mathrm{D}=\mathrm{D}+(\mathrm{A}(\mathrm{K} 1)-\mathrm{A}(\mathrm{K} 2)) *(\mathrm{~A}(\mathrm{~K} 1)-\mathrm{A}(\mathrm{K} 2))$

$A(I 1)=(F-D-T(I)-T(J)) /(F S Q R T(F-2 . * T(I)) * F S Q R T(F-2 . * T(J)))$

COR 710

IF $(A B S(A(I 1))$.GT.1.) $A(I 1)=A I N T(A(I 1))$

$120 \quad A(I 2)=A(I 1)$

C MEANS OF OBSERVATIONS

130 DO $150 \quad I=1, N V A R$

$\operatorname{AVG}(\mathrm{I})=0$.

SUMPOS $=0$.

SUMNEG $=0$.

DO $140 \mathrm{~J}=1$, NRMAX

$I 1=I$ ARGS $(I+1)-1+J$

SUMPOS $=S U M P O S+A M A X 1(R C($ I 1$), 0$.

140 SUMNEG $=$ SUMNEG+AMAXI (-RC (I I ), 0.)

$150 \quad$ AVG (I) $=($ SUMPOS-SUMNEG) /FLOAT (NRMAX)

C STANDARD DEVIATIONS

DO $170 \quad I=1$, NVAR

$\mathrm{SD}(\mathrm{I})=0$.

DO $160 \mathrm{~J}=1$, NRMAX

$I I=I$ ARGS $(I+1)-1+J$

$160 \mathrm{SD}(\mathrm{I})=\mathrm{SD}(\mathrm{I})+(\mathrm{RC}(\mathrm{I} I)-\mathrm{AVG}(\mathrm{I})) *(\mathrm{RC}(\mathrm{I} 1)-\mathrm{AVG}(\mathrm{I}))$

170 SD (I) $=$ FSQRT (SD (I))

C SIMPLE CORRELATION COEFFICIENT

$A($ LOTTE $)=1$.

$Z=F L O A T$ (NRMAX-2)

DO $200 \mathrm{~J}=1$, NVA

$I N D=(J-1) * N V A R$

$I \mathrm{I}=\mathrm{IND}+\mathrm{J}$

$A(I 1)=1$.

$\mathrm{I} J=\mathrm{J}+1$

DO $200 \quad I=I J$, NVAR

COR 720

COR 730

COR 740

COR 750

COR 760

COR 770

COR 780

COR 790

COR 800

COR 810

COR 820

COR 830

COR 840

COR 850

COR 860

COR 870

COR 880

COR 890

COR 900

COR 910

COR 920

COR 930

COR 940

COR 950

COR 960

COR 970

COR 980

COR 990

COR 1000

COR 1010

I $I=I N D+I$

COR 1020

I $2=(I-1) * N V A R+J$

CORI030

IF (SD(I).GT.0.AND.SD(J).GT.0.) GO TO 180

COR 1040

$A(I 1)=0$.

GO TO 200

COR 1050

SUMPOS $=0$.

COR 1060

SUMNEG $=0$.

COR 1070

DO $190 \mathrm{~K}=1$, NRMAX

COR 1080

$\mathrm{K} I=\operatorname{IARGS}(\mathrm{I}+1)-1+\mathrm{K}$

COR 1090

$\mathrm{K} 2=\operatorname{IARGS}(\mathrm{J}+1)-1+\mathrm{K}$

COR 1100

$C P=(R C(K 1)-A V G(I)) *(R C(K 2)-A V G(J))$

COR1110

SUMPOS=SUMPOS+AMAXI $(C P, 0$.

COR 1120

COR 1130

SUMNEG $=$ SUMNEG+AMAX $1(-C P, 0$.

COR 1140 
IF $(A B S(A(I 1)) . G T .1) \quad A.(I I)=A I N T(A(I I))$

$200 \quad A(I 2)=A(I 1)$

IF (NVAR.LE.2) GO TO 210

C PARTIAL CORRELATION COEFFICIENT

CALL INVCOR (A (1), NVAR, NVAR, A(LOTTE+1), NVAR+2, A(1), 1, ERR, IND)

IF (IND.EQ.0) GO TO 220

WR ITE (IPRINT, 480)

$210 \quad K E E P=M I N O(K E E P, 2)$

$A(L O T T E+1)=0$.

IF (L2.EQ.1) GO TO 300

GO TO 420

220

DO $230 \mathrm{~J}=2$, NVAR

$K 1=2 *(J-1)$

$\mathrm{K} 2=\mathrm{LOTTE}+(\mathrm{J}-1) *$ NVAR

DO $230 I=1$, NVAR

$\mathrm{I} I=\mathrm{K} 2+\mathrm{I}$

$12=I 1+K I$

$230 \quad A(I 1)=A(I 2)$

DO $240 \mathrm{~J}=1$, NVA

IND $=L O T T E+(J-1) * N V A R$

$\mathrm{K} 1=\mathrm{INO}+\mathrm{J}$

$I J=J+1$

DO $240 \quad I=I J$, NVAR

$\mathrm{K} 2=\mathrm{LOTTE}+(\mathrm{I}-1) * \mathrm{NVAR}+\mathrm{I}$

I $1=I N D+I$

I $2=L O T T E+(I-I) * N V A R+J$

$A(I 1)=-A(I 1) /(F S Q R T(A(K 1)) * F S Q R T(A(K 2)))$

IF (ABS (A(II)).GT.1.) $A(I I)=A I N T(A(I 1))$

$240 \quad A(I 2)=A(I 1)$

DO $250 \mathrm{I}=1$, NVAR

I $1=L O T T E+(I-1) * N V A R+I$

$250 \quad A(I 1)=1$.

IF (L2.EQ.2) GO TO 420

IF (NRMAX.LE.NVAR) GO TO 300

C SIGNIFICANCE LEVEL OF PARTIAL CORRELATION COEFFICIENT $Z=F L O A T$ (NRMAX-NVAR)

DO $290 \mathrm{~J}=1$, NVAR

$I J=(J-1) *$ NVAR + LOTTE

IND $=I J+K U R T$

DO $290 I=J$, NVAR

$I I=I N D+I$

I $2=(I-I) * N V A R+J+3 *$ LOTTE

$K I=I J+I$

IF $(A(K 1)) 270,260,270$

$260 \quad A(I I)=1$.

GO TO 290

270 IF (ABS $(A(K I)) . L T .1) \quad G$.0 TO 280

$A(I 1)=0$.

GO TO 290

$280 \quad F=A(K 1) * A(K 1)$

$F=Z * F /(1,-F)$

CALL PROB (I.,Z,F,A(II))

$290 \quad A(I 2)=A$ (II)

$300 \quad Z=F L O A T$ (NRMAX-2)

DO $340 \mathrm{~J}=1$, NVAR

$I J=(J-1) * N V A R$

IND $=I J+K U R T$

DO $340 \mathrm{I}=\mathrm{J}$, NVAR

$I I=I N D+I$
COR 1150

COR 1160

COR 1170

COR 1180

COR 1190

COR 1200

COR 1210

COR 1220

COR 1230

COR 1240

COR 1250

COR 1260

COR 1270

COR 1280

COR 1290

COR 1300

COR 1310

COR 1320

COR 1330

COR 1340

COR 1350

COR 1360

COR 1370

COR 1380

COR 1390

COR 1400

COR 1410

COR 1420

COR 1430

COR 1440

COR 1450

COR 1460

COR 1470

COR 1480

COR 1490

COR 1500

COR 1510

COR 1520

COR 1530

COR 1540

COR 1550

COR 1560

COR 1570

COR 1580

COR 1590

COR 1600

COR 1610

COR 1620

COR 1630

COR 1640

COR 1650

COR 1660

COR 1670

COR 1680

COR 1690

COR 1700

COR 1710

COR 1720

COR 1730 
$K I=I J+I$

$A(I 1)=1$.

COR 1770

GO TO 340

COR 1780

320 IF $(A B S(A(K 1)) . L T .1$.$) GO TO 330$

COR 1790

$A(I I)=0$.

GO TO 340

$330 \quad F=A(K 1) * A(K I)$

$F=Z * F /(1 .-F)$

CALL PROB $(1, Z, F, A(I 1))$

$340 \quad A(12)=A(I 1)$

C FIRST PRINTING STAGE (SCC,PCC,SIGNIFICANCE LEVELS, SRCC)

NLA $=1$

CALL PAGE (4)

WR ITE (IPRINT, 490) NVAR, NRMAX

CALL MIST (NVAR, A (1), 1,NLA, 1)

CALL MIST (NVAR, A (KURT+1), 1, NLA, 2)

IF (A (LOTTE+1).LE.0.) GO TO 350

COR 1800

COR1810

COR 1820

COR 1830

COR 1840

COR 1850

COR 1860

COR 1870

COR 1880

COR 1890

CALL MIST (NVAR, A (LOTTE+1), 1, NLA, 3)

COR 1900

$F=A B S(A M A X 1(\operatorname{ERR}(1), \operatorname{ERR}(2), \operatorname{ERR}(3)))$

C FOLLOWING STATEMENT WILL BE RESTORED LATER

COR 1910

COR 1920

COR 1930

WRITE (IPRINT, 45) F

COR 1940

C

45 FORMAT (/IH, 72HERROR BOUND FOR INVERSION OF MATRIX OF SIMPLE IRELATION COEFFICIENTS $=$, E14.4)

$N L A=N L A+2$

IF (NRMAX.LE.NVAR) GO TO 350

CALL MIST (NVAR, A $(3 * L O T T E+1), 1, N L A, 4)$

350 CALL MIST (NVAR, A $(L O T+1), 1, N L A, 5)$

IF (NRMAX.GT.3) GO TO 360

WRITE (IPRINT, 500) NRMAX

GO TO 420

$360 \quad Z=F L O A T$ (NRMAX -3 )

IND $=3 *$ LOTTE +NRMAX + 1

DO $400 \mathrm{~J}=1$, NVAR

COR 1950

COR 1960

$11=I \operatorname{ARGS}(\mathrm{J}+1)$

$I J=(J-1) * N V A R$

COR 1970

COR 1990

COR 2000

COR2010

COR2020

COR2030

COR2040

COR2050

COR2060

COR 2070

COR2080

COR2090

DO $400 \quad I=1$, NVAR

COR 2100

COR2110

IF (I.NE.J) GO TO 370

COR2120

$12=K U R T+I J+J$

COR 2130

$A(12)=1$.

GO TO 400

COR2140

COR2150

COR2160

COR 2170

COR2180

COR2190

COR2200

COR2210

COR2220

COR2230

COR2240

COR2250

COR2260

COR2270

COR2280

COR2290

COR2300

COR2310

COR2320 
$A\left(3^{*}\right.$ LOTTE $)=99$.

COR2330

$A\left(5^{*}\right.$ LOTTE $)=95$.

COR2340

DO $410 \mathrm{~J}=1$, NVA

COR2350

IND $=(J-1)$ *NVAR

COR2360

$K l=I N D+J+K U R T$

COR2370

$K 2=K 1+K U R T$

COR2380

$A(K 1)=99$.

COR2390

$A(K 2)=95$.

COR2 400

$\mathrm{I} J=\mathrm{J}+\mathrm{I}$

COR2410

DO $410 \quad I=I J$, NVAR

COR2420

INDEX OF SCC

COR2430

$I I=I N D+I$

COR2440

INDICES OF UPPER, LOWER SCC CONFIDENCE LIMITS (99 PER CENT LEVEL) COR2450 $K 1=(I-1) * N V A R+J+K U R T$

$K 2=K 1+K U R T$

COR2460

COR2470

$Z=.5 * F L O G((1 .+A(I 1)) /(1 .-A(I 1)))$

COR2480

$A(K 1)=A M I N 1(F T A N H(Z+H L 1), 1$.

COR2490

$A(K 2)=A M A X 1$ (FTANH $(Z-H L 1),-1$.)

COR 2500

C INDICES OF UPPER, LOWER SCC CONFIDENCE LIMITS (95 PER CENT LEVEL) COR2510

$K 1=I 1+K U R T$

COR2520

$K 2=K 1+K U R T$

COR2530

$A(K 1)=A M I N 1(F T A N H(Z+H L 2), 1$.

COR 2540

$410 \quad A(K 2)=A M A X 1$ (FTANH $(Z-H L 2),-1$.

COR2550

CALL MIST (NVAR, A (KURT +1), $0, N L A, 7$ )

COR2560

GO TO $(470,450,430)$, KEEP

420

STORE SIMPLE AND PARTIAL CORRELATION COEFFICIENTS IN WORKSHEET

COR2570

COR2580

COR2590

I I=MINO (NVAR, NCOL-(IARGS (NVAR+5) -1) /NROW)

I2=MINO (NVAR, NROW-(IARGS (NVAR+4) -I ARGS $(N V A R+5)))$

COR2600

IF (I1.LT.NVAR.OR.I2.LT.NVAR) CALL ERROR (213)

COR2610

DO $440 \mathrm{~J}=1, \mathrm{I} I$

DO $440 \quad I=1, I 2$

COR2620

COR2630

$K I=(J-1) * N V A R+I+L O T T E$

COR2640

$K 2=I A R G S(N V A R+4)-1+(J-1) * N R O W+I$

COR2650

COR2660

COR2670

COR2680

COR2690

COR2700

COR2710

COR2720

COR2730

COR2740

COR2750

COR2760

$\begin{array}{ll}460 & R C(K 2)=A(K 1) \\ 470 & \text { RETURN }\end{array}$

C

480 FORMAT (IH ,32X,54HMATRIX IS SINGULAR. NO PARTIAL CORRELATIONS COCOR2770
IMPUTED.)

490 FORMAT (/30X,24HCORRELATION ANALYSIS FOR, I3,15H VARIABLES WITH,I5,COR2790 113H OBSERVATIONS)

COR2800

500 FORMAT (1H, 18X,83HNONLINEARITY TEST AND APPROXIMATION OF CONFIDENCOR2810 ICE INTERVALS NOT DEFINED FOR NRMAX $=, 12$ )

END 


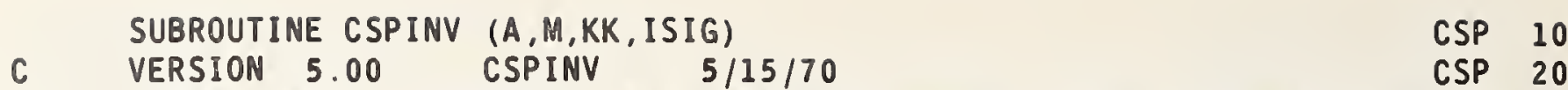

C 7058MI MATRIX INVERSION WITH MINIMUM ROUNDOFF ERROR ACCUMULATION.CSP 30

COMMON / CONLB2 / ER, IS I GD

CSP 35

DATA ONE/1:0/.ZERO/0.0/

CSP 40

DIMENSION A(1)

CSP 50

IS I $G=0$

CSP 60

$\mathrm{N}=\mathrm{M}$

CSP 70

$N N=K K$

CSP 80

$N 2=N+N$

CSP 90

DO $30 \mathrm{~J}=1, \mathrm{~N}$

CSP 100

NJCOL $=(N+J-1) * N N$

CSP 110

DO $30 \quad I=1, N$

CSP 120

$K I N J=N J C O L+I$

CSP 130

IF (I-J) $10,20,10$

CSP 140

$10 \quad A(K I N J)=Z E R O$

CSP 150

GO TO 30

CSP 160

$20 \quad A(K I N J)=$ ONE

CSP 170

30 CONTINUE

CSP 180

C DETERMINE MAXIMUM ABS OF VARIABLE BEING ELIMINATED. THIS BECOMES

CSP 190

$L=0$

$40 \quad L=L+1$

$L C O L=N N * L-N N$

CSP 200

$K L L=L C O L+L$

IF (L-N) $50,100,200$

CSP 210

CSP 220

CSP 230

CSP 240

C FIND THE LARGEST ELEMENT IN THE LTH COLUMN.

CSP 250

$50 \quad \mathrm{~J} l=\mathrm{L}$

$C=A B S(A(K L L))$

CSP 260

$L 1=L+1$

CSP 270

DO $70 \quad I=L 1, N$

CSP 280

$K I L=L C O L+I$

CSP 290

$X=A B S(A(K I L))$

CSP 300

IF (C-X) $60,70,70$

CSP 310

CSP 320

C RECORD THE NUMBER OF THE ROW HAVING THE GREATER ELEMENT.

CSP 330

$60 \quad \mathrm{~J} I=I$

C C BECOMES THE GREATER.

CSP 340

$\mathrm{C}=\mathrm{X}$

CSP 350

CSP 360

70 CONTINUE

CSP 370

C INTERCHANGE ROW JI WITH ROW L. JI IS THE ROW WITH THE LARGEST ELEMCSP 380

C TEST TO SEE IF INTERCHANGING IS NECESSARY.

IF (JI-L) $80,100,80$

$80 \quad$ DO $90 \mathrm{~J}=\mathrm{L}, \mathrm{N} 2$

$J C O L=N N * J-N N$

CSP 390

CSP 400

CSP 410

$K J I J=J C O L+J I$

CSP 420

$H O L D=A(K J I J)$

CSP 430

$\mathrm{KLJ}=\mathrm{JCOL}+\mathrm{L}$

CSP 440

$A(K J I J)=A(K L J)$

CSP 450

$A(K L J)=H O L D$

CSP 460

90 CONTINUE

CSP 470

C IF THE LARGEST ABSOLUTE ELEMENT IN A COLUMN IS MACHINE ZERO WE

CSP 480

C HAVE A SINGULAR MATRIX

CSP 490

$100 \sim$ IF (ABS(A (KLL)) -ER) $110,110,120$

CSP 495

110 IS I G=4

CSP 500

CSP 510

GO TO 200

CSP 520

C ZERO ALL THE ELEMENTS IN THE LTH COLUMN BUT THE PIVOTAL ELEMENT. CSP 530

$120 \quad L 1=1$

$L 2=L-1$

IF (L2) $130,130,150$

CSP 540

CSP 550

CSP 560

130

IF (L-N) $140,170,140$

CSP 570 


$$
\mathrm{L} 2=\mathrm{N}
$$

150 D0 $160 \quad I=L 1, L 2$

$K I L=L C O L+I$

DO $160 \mathrm{~J}=\mathrm{L}, \mathrm{N} 2$

CSP 620

$J C O L=N N * J-N N$

CSP 630

$K I J=J C O L+I$

CSP 640

$K L J=J C O L+L$

CSP 650

CSP 660

$A(K I J)=A(K I J)+Z * A(K L J)$

CSP 670

IF (N-L2) $40,40,130$

CSP 680

CSP 690

C DIVIDE BY DIAGONAL ELEMENTS.

CSP 700

$K K K=N N^{*} I-N N+I$

CSP 710

$Z Z=A(K K K)$

DO $180 \mathrm{~J}=1, \mathrm{~N} 2$

$K K I=N N * J-N N+I$

CSP 720

CSP 730

CSP 740

CSP 750

$180 \quad A(K K I)=A(K K I) / Z Z$

C RETURN AFTER PUTTING A INVERSE INTO B

CSP 760

DO $190 \mathrm{~J}=1, N$

$J C O L=N N * J-N N$

CSP 770

$N J C O L=N N * N+J C O L$

CSP 780

DO $190 \quad I=1, N$

$\mathrm{KIJ}=\mathrm{JCOL}+\mathrm{I}$

CSP 790

CSP 800

CSP 810

$K I N J=N J C O L+I$

CSP 820

CSP 830

$190 \quad A(K I J)=A(K I N J)$

CSP 840

END

CSP 850 


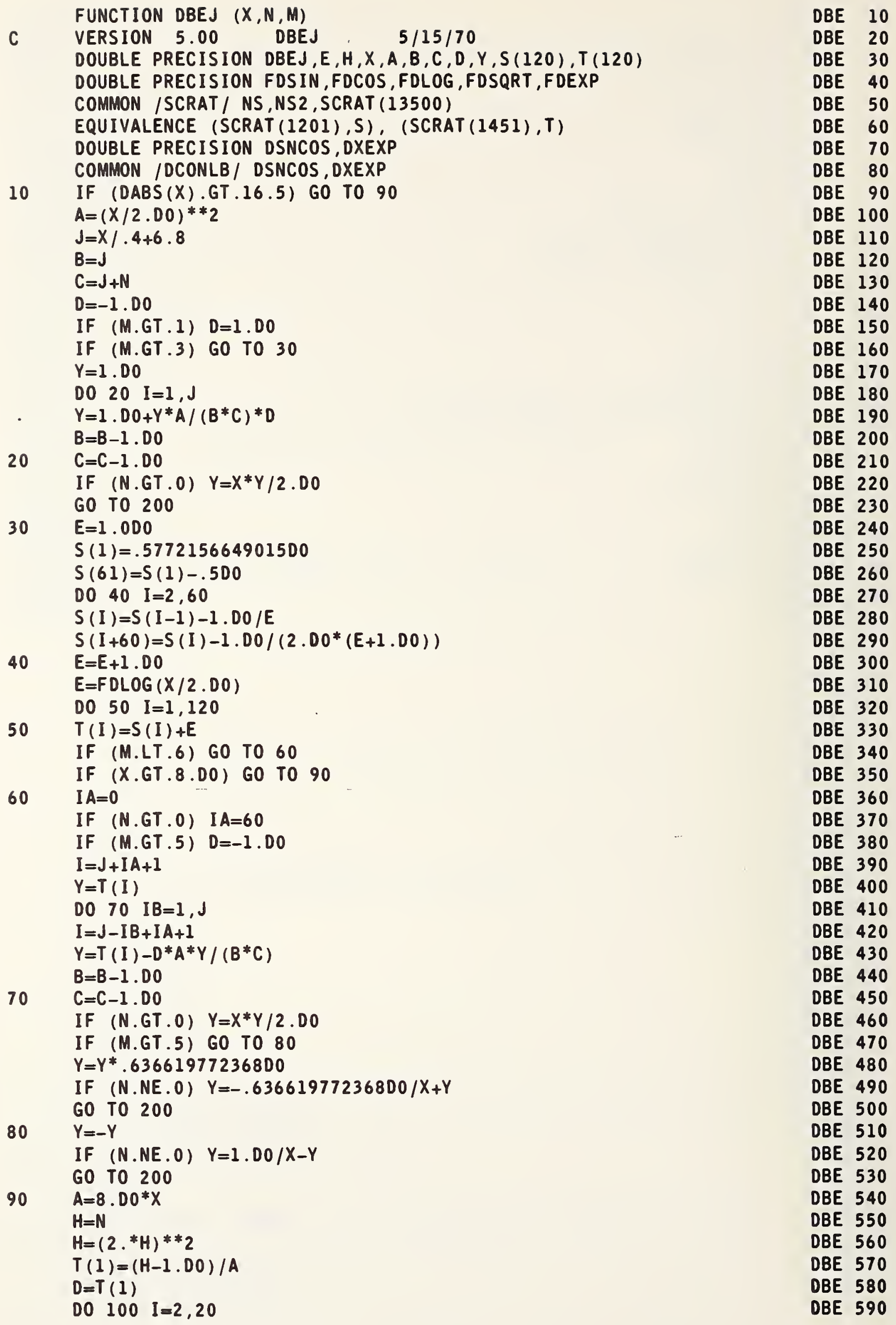




$$
\begin{aligned}
& K=I \\
& B=I \\
& C=(2 * I-1)^{* * 2}
\end{aligned}
$$

$T(I)=(H-C) /(A * B)$

$E=D$

$D=T(I) * D$

$E=D A B S(D / E)$

IF (DABS (D).LT . .5D-10) GO TO 110

IF (E.GT..91DO) GO TO 110

$100 \quad T(I+2)=0.000$

$110 \quad A=-1 . D 0$

IF (M.LE.1) GO TO 150

IF (M.LE.3) GO TO 120

IF (M.LE.5) GO TO 150

$A=1.00$

$120 \quad Y=1.00$

DO $130 \quad I=1, K$

$J=K-I+1$

$130 \quad Y=1.00+A * Y * T(J)$

$A=1.00$

IF (X.LT.DXEXP) A=FDEXP (X)

IF (M.LE.5) GO TO 140

$Y=Y /\left(A^{*} F D S Q R T\left(.63661977236800^{*} X\right)\right)$

GO TO 200

$140 \quad Y=Y * A / F D S Q R T\left(6.28318530700^{*} X\right)$

GO TO 200

$150 \quad Y=F D S Q R T(3.141592653600 * X)$

$J=K / 2$

$K=2 * J$

$\mathrm{J}=\mathrm{J}-1$

$A=1.00$

$\mathrm{H}=\mathrm{A}$

DO $160 \quad I=1, J$

$I A=K-2 * I+1$

$A=1 . D 0-A^{*} T(I A) * T(I A+1)$

$160 \quad H=1 . D 0-H^{*} T(I A) * T(I A-1)$

$A=(1 . D 0-T(1) * T(2) * A) / Y$

$H=T(1)^{*} H / Y$

$B=F D S I N(X)$

$C=F D C O S(X)$

$D=A-H$

$E=A+H$

IF (M.GT.2) GO TO 180

IF (N.EQ.0) GO TO 170

$Y=E * B-D * C$

GO TO 200

$170 \quad Y=D * B+E * C$

GO TO 200

180 IF (N.EQ.O) GO TO 190

$Y=-D * B-E * C$

GO TO 200

$190 Y \quad Y=E * B-D * C$

GO TO 200

200 DBEJ $=Y$

RETURN

END
DBE 600

DBE 610

DBE 620

DBE 630

DBE 640

DBE 650

DBE 660

DBE 670

DBE 680

DBE 690

DBE 700

DBE 710

DBE 720

DBE 730

DBE 740

DBE 750

DBE 760

DBE 770

DBE 780

DBE 790

DBE 800

DBE 810

DBE 820

DBE 830

DBE 840

DBE 850

DBE 860

DBE 870

DBE 880

DBE 890

DBE 900

DBE 910

DBE 920

DBE 930

DBE 940

DBE 950

DBE 960

DBE 970

DBE 980

DBE 990

DBE 1000

DBE 1010

DBE 1020

DBE 1030

DBE 1040

DBE 1050

DBE 1060

DBE1070

DBE 1080

DBE1090

DBE 1100

DBE 1110

DBE 1120

DBE 1130

DBE 1140

DBE 1150 
SUBROUTINE DEFINE

C VERSION 5.00

DEFINE

$5 / 15 / 70$

DEF 10

COMMON /BLOCRC/ NRC,RC (12600)

DEF 20

COMMON /BLOCKD/ IARGS (100), KIND (100), ARGTAB (100), NRMAX, NROW, NCOL, NDEF 40

IARGS, VWXYZ (8), NERROR

DIMENSION ARGS $(100)$

EQUIVALENCE (ARGS(1), RC(12501))

DEF 50

DEF 60

C

C DEFINE \$\$ INTO COLUMN ++

DEF 70

DEF 80

DEFINE \$\$ INTO ROW ++, COL ++.

DEF 85

C DEFINE ROW,++ COL ++ INTO ROW,++ COL ++ .

DEF 90

DEF 100

DEFINE ROW ++, COL ++ INTO COL ++.

DEF 110

IF (NARGS.NE.2) GO TO 5

DEF 120

$J=1$

DEF 122

IF (KIND ( 1 ).EQ.0) CALL ADRESS $(1, J)$

DEF 124

CALL ADRESS $(2, I)$

DEF 125

IF (I) $120,130,2$

DEF 126

DEF 127

2 IF (J) $120,130,60$

DEF 128

IF (NARGS.NE .3) IF (NARGS-4) $115,40,115$

DEF 129

IF (KIND(1).EQ.0) GO TO 40

DEF 130

DEF 140

$I=N A R G S$

GO TO 90

20 IF (NERROR.EQ.0) RC (L)=ARGS (1)

30 RETURN

$40 \quad I=2$

GO TO 90

$50 \quad \operatorname{ARGS}(I)=R C(L)$

IF (NARGS.EQ.4) GO TO 10

CALL ADRESS $(3, I)$

IF (I) $120,130,60$

60 IF (NERROR.NE.0) GO TO 30

IF (NRMAX.EQ.0) GO TO 70

IF (KIND (1).EQ.0.AND.NARGS.EQ.2) GO TO 140

DEF 150

DEF 160

DEF 170

DEF 180

DEF 190

DEF 200

DEF 210

DEF 220

DEF 230

DEF 240

DEF 250

DEF 255

CALL VECTOR (ARGS(I), I)

DEF 260

GO TO 30

$70 \quad I=9$

80 CALL ERROR (I)

GO TO 30

DEF 270

DEF 280

DEF 290

DEF 300

DEF 310

DEF 320

C CHECK AND CALCULATE WORKSHEET ENTRY LOCATION INTO L

DEF 330

DEF 340

90 CALL ADRESS $(I, L)$

DEF 350

IF (L) $120,130,100$

DEF 360

100 IF (KIND(I-1).EQ.0.AND.IARGS(I-1).GT.0.AND.IARGS(I-1).LE.NROW) GO 1 TO 110

$I=16$

GO TO 80

DEF 370

DEF 380

DEF 390

$110 \quad \operatorname{L}=\operatorname{L}+1 \mathrm{ARGS}(\mathrm{I}-1)-1$

IF $(\mathrm{I}-2) 50,50,20$

$115 \quad I=10$

GO TO 80

$120 \quad I=20$

GO TO 80 .

$130 \quad I=11$

GO TO 80

140 DO $150 \quad I J=1$, NRMAX

$R C(I)=R C(J)$

$I=I+I$

$150 \quad J=J+1$

DEF 400

DEF 410

DEF 420

DEF 430

DEF 440

DEF 450

DEF 460

DEF 470

DEF 480

DEF 490

DEF 500

DEF 510

RETURN

DEF 520

END 
SUBROUTINE DETRNK (A, NROW, $N, D E T, R A N K$ )

DET 10

C VERSION 5.00 DETRNK 5/15/70

DET 20

DET 30

EVALUATES THE DETERMINAUT AND RANK OF A

DET 40

A LOCATION OF MATRIX

NROW THE DIMENSION OF A IN DIMENSION STATEMENT

DET 50

N SIZE OF A

DET - THE VALUE OR DETERMIMANT OF A

DET 60

DET 70

DET 80

RANK-RANK OF A

ORIGANAL VALUES OF A ARE DESTROYED

DET 90

DET 100

DET 110

DIMENSION A (NROW, NROW)

$N K=N$

$\mathrm{NN}=\mathrm{N}-1$

DET 120

DET 130

DET 140

$\mathrm{VAL}=1.0$

DET 150

DO $60 \quad I=1$, NN

$\mathrm{I} I=\mathrm{I}+1$

$\mathrm{I} B=\mathrm{I}$

DO $10 \mathrm{~J}=I \mathrm{I}, \mathrm{N}$

IF $(A B S(A(I B, I)) \cdot G E \cdot A B S(A(J, I)))$ GO TO 10

$I B=J$

10 CONTINUE

IF (ABS (A (IB, I )) .GE.1.E-7) GO TO 20

DET 160

DET 170

DET 180

DET 190

DET 200

DET 210

DET 220

DET 230

$N K=N K-1$

GO TO 40

DET 240

DET 250

IF (IB.EQ.I) GO TO 40

DET 260

DO $30 \mathrm{~J}=\mathrm{I}, \mathrm{N}$.

DET 270

$T=A(I, J)$

DET 280

$A(I, J)=A(I B, J)$

$30 \quad A(I B, J)=T$

$V A L=-V A L$

40 DO $50 \mathrm{~J}=I I, N$

$X=A(J, I) / A(I, I)$

DET 290

DET 300

DET 310

DET 320

DET 330

DO $50 \quad K=I, N$

DET 340

DET 350

DET 360

DET 370

DET 380

DET 390

DET 400

DET 410

DET 420

DET 430

DET 440

DET 450

DET 460 
SUBROUTINE DHRND $(X, N, X T)$
C VERSION 5.00 DHRND

C SUBROUTINE TO ROUND $X$ TO $N$ SD AND STORE IN XT

C WRITTEN BY DAVID HOGBEN, SEL, NBS. 4/09/70. DOUBLE PRECISION $Z$

$$
\text { IF (X) } 20,10,20
$$

10

$$
X T=0.0
$$$$
\text { RETURN }
$$

20

$$
\text { IF (N.LT.1) } N=1
$$$$
\text { IF (N.GT.8) } N=8
$$$$
Y=A B S(X)
$$$$
M=F L O G 10(Y)
$$$$
\text { IF (Y.LT.1.0) } M=M-1
$$$$
Z=Y
$$$$
Z=Z * 10 \cdot D 0 * *(8-M)
$$$$
\text { IF }(Z . L T .1 .00+9) \text { GO TO } 30
$$$$
M=M+1
$$$$
Z=Z / 10.000
$$$$
\text { GO TO } 40
$$

30 IF $(2 . G E .1 .0 D+8)$ GO TO 40

$$
M=M-1
$$

$$
Z=10.000 * Z
$$

$40 \quad \times 1=2$

$$
\text { LLI }=X 1
$$

$X 2=Z-D B L E(X 1)$

$$
L L 2=X 2
$$

$L L=L L 1+L L 2+5$

$$
L L 1=L L /(10 * *(9-N))
$$$$
L L 2=L L 1 * 10 * *(9-N)
$$

$L L 2=L L-L L 2$

IF (N.EQ.8) GO TO 70

IF (LL2/10-5*10**(7-N)) $70,50,60$

$50 \quad L L 2=M O D(L L 1,2)$

IF (LL2) $70,70,60$

$60 \quad L L I=L L I+1$

$70 \quad X T=F L O A T$ (LLI)

IF (M.EQ.N-1) GO TO 80

C

$$
\begin{aligned}
& Z=X T \\
& Z=Z * 10.000 * *(M-N+1) \\
& X T=F D P C O N(Z) \\
& X T=S I G N(X T, X) \\
& \text { RETURN } \\
& \text { END }
\end{aligned}
$$$$
80 X T=S I G N(X T, X)
$$

DHR 10

DHR 20

DHR 30

DHR 40

DHR 50

DHR 60

DHR 70

DHR 80

DHR 90

DHR 100

DHR 110

DHR 120

DHR 130

DHR 140

DHR 150

DHR 160

DHR 170

DHR 180

DHR 190

DHR 200

DHR 210

DHR 220

DHR 230

DHR 240

DHR 250

DHR 260

DHR 270

DHR 280

DHR 290

DHR 300

DHR 310

DHR 320

DHR 330

DHR 340

DHR 350

DHR 360

DHR 370

DHR 380

DHR 390

DHR 400

DHR 410

DHR 420

DHR 430

DHR 440 
SUBROUTINE DIMENS

C VERSION 5.00 DIMENS
COMMON /BLOCRC/NRC,RC $(12600)$

DIM 10

DIM 20

DIM 30

COMMON /BLOCKD/ IARGS(100), KIND(100), ARGTAB (100), NRMAX, NROW, NCOL, NDIM 40 IARGS, VWXYZ (8), NERROR

DIMENSION ARGS $(100)$

DIM 50

EQUIVALENCE (ARGS(1), RC (12501))

IF (NARGS.EQ.2) IF (KIND(1)+KIND (2)) 25,27,25

$K=10$

10 CALL ERROR (K)

20 RETURN

$25 \quad K=20$

DIM 60

DIM 70

DIM 75

DIM 80

DIM 90

DIM 100

GO TO 10

DIM 110

DIM 120

27 IF (IARGS(1).GT.0.AND.IARGS(2).GT.0.AND.IARGS (1)*IARGS (2).LE.NRC) DIM 130 IGO TO 30

$\mathrm{K}=3$

DIM 140

GO TO 10

DIM 150

$30 \quad$ NROW=IARGS (1)

DIM 160

DIM 170

NRMAX =MINO (NROW, NRMAX)

DIM 180

GO TO 20

DIM 190

END

DIM 200

DIM 210 
C VERSION 5.00 DUMAYA $5 / 15 / 70$
PRINT 10

DMA 10

DMA 20

DMA 30

10 FORMAT (55H * OMNITAB COMMAND DUMMYA IS NOT AVAILABLE AT THIS TIME)DMA 40 RETURN

DMA 50

END

DMA 60

SUBROUTINE DUMAYB

C VERSION 5.00 DUMMYB $5 / 125 / 70$

DMB 10

PRINT 10

DMB 20

10 FORMAT(55H * OMNITAB COMMAND DUMMYB IS NOT AVAILABLE AT THIS TIME)DMB 40 RETURN

END

DMB 50

DMB 60

SUBROUTINE DUMAYC

C VERSION 5.00 DUMMYC

$5 / 15 / 70$

DMC 10

PRINT 10

DMC 20

10 FORPAT (55H* OMNITAB COMMAND DUMMYC IS NOT AVAILABLE AT THIS TIME) DMC 40

RETURN

DMC 50

END

DMC 60

SUBROUTINE DUMMYD

C VERSION 5.00 DUMMYD $5 / 15 / 70$

DMD 10

PRINT 10

DMD 20

10 FORMAT (55H * OMNITAB COMMAND DUMMYD IS NOT AVAILABLE AT THIS TIME)DMD 40 RETURN

DMD 30

END

DMD 50

DMD 60

SUBROUTINE DUMMYE

C VERSION 5.00 DUMMYE 5/15/70

DME 10

PRINT 10

DME 20

DME 30

10 FORMAT (55H * OMNITAB COMMAND DUMMYE IS NOT AVAILABLE AT THIS TIME) DME 40 RETURN

DME 50

END

DME 60

SUBROUTINE DUMMYF

C VERSION 5.00 DUAMMF $5 / 15 / 70$

DMF 10

PRINT 10

DMF 20

10 FORMAT (55H * OMNITAB COMMAND DUMMYF IS NOT AVAILABLE AT THIS TIME)DMF 40

RETURN

DMF 50

END

DMF 60 
C ERASE COL (C), (C), (C), ETC.

ERA 20

C IF NO COLS SPECIFIED ALL OF WORKSHEET IS ERASED

ERA 30

ERA 40

C

COMMON /BLOCRC/ NRC, RC(12600)

ERA 45

ERA 50

ERA 60

COMMON /BLOCKD/ I ARGS(100), KIND(100), ARGTAB (100), NRMAX, NROW, NCOL, NERA 70

IARGS, VWXYZ (8), NERROR

ERA 80

DIMENSION ARGS(100)

ERA 90

EQUIVALENCE (ARGS (1), RC (12501))

ERA 100

IF (NARGS.EQ.0) GO TO 40

ERA 110

CALL CHKCOL (I)

ERA 120

IF (I.EQ.0) GO TO 20

ERA 130

$I=20$

ERA 140

CALL ERROR (I)

10 RETURN

20 IF (NERROR.NE.0.OR.NRMAX.EQ.0) GO TO 10

ERA 150

ERA 160

DO $30 \mathrm{I}=1$, NIARGS

30 CALL VECTOR (0.,IARGS (I))

ERA 170

ERA 180

GO TO 10

ERA 190

ERA 200

ERA 210

CLEAR ALL OF DIMENSIONED WORKSHEET.

ERA 220

ERA 230

ERA 240

IF (NERROR.NE.O) GO TO 10

ERA 250

NRMAX $=$ NROW*NCOL

CALL VECTOR $(0,1)$

ERA 260

NRMAX $=0$

ERA 270

GO TO 10

ERA 280

END

ERA 290 
SUBROUTINE ERRINT (X,ERF,ERFC)

ERT 10

$\begin{array}{llll}C & \text { VERSION } 5.00 \quad \text { ERRINT } 5 / 15 / 70 & \text { ERT } 20\end{array}$

DOUBLE PRECISION AN, BN, CONS, C1, DN, ERF, ERFC, F, FN, FNM1 , FNM2, FOUR, GN, ERT 25

1 GNM1 , GNM2, ONE , P , PREV , RNBC , SCF , SUM, TN , TOLER , TRRTPI , TWO , ULCF , ULPS , WNERT 30

$2, X, Y, Y S Q, F D E X P$

ERT 40

COMMON / DCONL2 / TRRTPI, NBC, NBM

DATA ONE, THO ,FOUR, ULPS, CONS/1.DO,2.DO,4.DO,1.D0,.83D0/

ERT 90

RNBC $=$ NBC

ERT 100

TOLER $=$ TWO** $(-N B M)$

ERT 120

IF $(X) 20,10,20$

ERT 130

10

$E R F=0$

ERT 140

$E R F C=O N E$

ERT 150

RETURN

ERT 160

$20 \quad Y=A B S(X)$

$Y S Q=Y * * 2$

ERT 170

IF (Y-ULPS) $50,50,30$

ERT 180

ERT 190

$30 \mathrm{Cl}=\mathrm{TWO} *$ * ( (RNBC-ONE)/TWO)

ERT 200

ULCF $=$ CONS ${ }^{*} \mathrm{Cl}$

$S C F=T W O *(C 1 * * 2-R N B C)$

IF $(Y-U L C F) 110,110,40$

ERT 210

ERT 220

ERT 230

ERT 240

$40 \quad E R F=O N E$

$E R F C=0$

GO TO 80

$50 \quad S U M=0$

DN=ONE

$T N=O N E$

$P=T W O * Y S Q$

ERT 250

ERT 260

ERT 270

ERT 280

ERT 290

ERT 300

$D N=D N+T W O$

$T N=P * T N / D N$

ERT 310

ERT 320

$S U M=T N+S U M$

ERT 330

IF (TN-TOLER) $70,60,60$

ERT 340

ERT 350

$70 \quad E R F=(S U M+O N E) * T R R T P I * Y * F D E X P(-Y S Q)$

$E R F C=O N E-E R F$

ERT 360

ERT 370

ERT 380

80 IF (X) $90,100,100$

$90 \quad E R F=-E R F$

ERT 390

ERFC $=$ TWO-ERFC

100 RETURN

$110 \quad \mathrm{FNM} 2=0$

GNM2 $=$ ONE

FNMI $=$ THO $* Y$

GNMI $=T W O * Y S Q+O N E$

ERT 400

ERT 410

ERT 420

ERT 430

ERT 440

PREV $=$ FNMI $/$ GNMI

ERT 450

WN $=$ ONE

ERT 460

$B N=G N M I+F O U R$

$120 \quad A N=-W N *(W N+O N E)$

$F N=B N * F N M 1+A N * F N M 2$

ERT 470

ERT 480

ERT 490

ERT 500

$G N=B N * G N M 1+A N * G N M 2$

ERT 510

$F=F N / G N$

ERT 520

IF (ABS (ONE-(F /PREV))-TOLER) $170,170,130$

ERT 530

130 IF (PREV-F) $140,140,160$

ERT 540

ERT 550

ERT 560

$F N=F N / S C F$

ERT 570

$\mathrm{GN}=\mathrm{GN} / \mathrm{SCF}$

$F N M 1=F N M 1 / S C F$

ERT 580

GNMI $=$ GNMI $/ S C F$

ERT 590

$F N M 2=F N M 1$

ERT 600

GNM2 = GNM1

ERT 610

$F N M 1=F N$

ERT 620

$G N M I=G N$

ERT 630 
$P R E V=F$

ERT 660

GO TO 120

ERT 670

$F=P R E V$

160

ERFC $=F^{*} F D E X P(-Y S Q) * T R R T P I / T W O$

ERT 680

$E R F=O N E-E R F C$

GO TO 80

ERT 690

ERT 700

END

ERT 710

ERT 720 
SUBROUTINE ERROR(I)

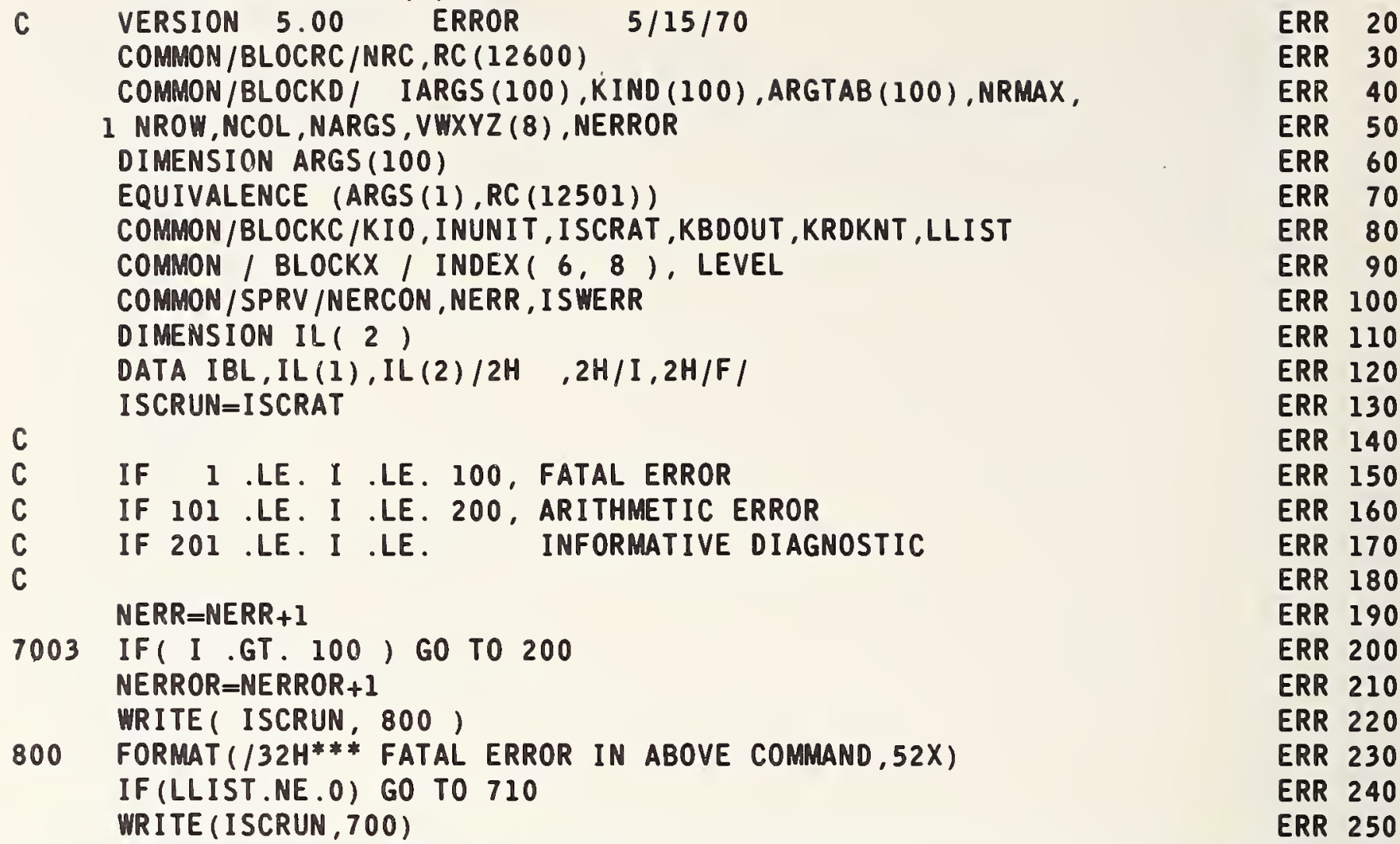

700 FORMAT (66H*** COMMAND WAS NOT LISTED BECAUSE NO LIST OR LIST 0 WASERR 260 1 IN EFFECT, 18X)

ERR 270

710 LLIST $=3$

ERR 280

GO TO $(801,802,803,804,805,806,807,808,809,810,811,812,813,814,815$ ERR 290

$1,816,817,818,819,820,821,822,823,824,825,826,827,828,829,830)$, I ERR 300

801 WRITE (ISCRUN, 1)

1 FORMAT (29H*** NAME NOT FOUND IN LIBRARY, 55X)

ERR 310

GO TO 900

ERR 320

802 WRITE (ISCRUN , 2)

2 FORMAT $\left(28 \mathrm{H}^{* * *}\right.$ ILLEGAL STATEMENT NUMBER, 56X)

ERR 330

GO TO 900

ERR 340

ERR 350

ERR 360

803 HRITE (ISCRUN , 3)

3 FORMAT $\left(28 \mathrm{H}^{* * *}\right.$ ILLEGAL ARGUMENT ON CARD , 56X)

ERR 370

GO TO 900

ERR 380

ERR 390

804 GO TO 900

ERR 400

805 WRITE (I SCRUN, 5)

5 FORMAT (38H*** COMMAND NOT ALLOWED IN REPEAT MODE, 46X)

ERR 410

GO TO 900

ERR 420

806 WRITE (ISCRUN , 6)

ERR 430

ERR 440

6

IN AND FINISH CARDS, 10X)

GO TO 900

807 WRITE (ISCRUN, 7)

7 FORMAT $\left(23 H^{* * *}\right.$ ILLEGAL *STATEMENT* ,61X)

GO TO 900

808 WRITE (ISCRUN , 8 )

8 FORMAT (34H*** PHYSICAL CONSTANT NOT IN TABLE, 50X)

GO TO 900

809 WRITE (ISCRUN, 9)

9 FORMAT $\left(13 H^{* * *}\right.$ NRMAX $\left.=0,71 X\right)$

GO TO 900

810 WRITE (ISCRUN, 10) NARGS

10 FORMAT $\left(3 H^{* * *}\right.$, I $4,34 \mathrm{H}$ IS AN ILLEGAL NUMBER OF ARGUMENTS, 43X) GO TO 900

ERR 450

ERR 460

ERR 470

ERR 480

ERR 490

ERR 500

ERR 510

ERR 520

ERR 530

ERR 540

ERR 550

ERR 560

ERR 570

ERR 580

ERR 590 
811 WRITE (ISCRUN, 11)

11 FORMAT $\left(40 \mathrm{H}^{* * *}\right.$ COLUMN NUMBER TOO BIG OR LESS THAN $\left.1,44 X\right)$ GO TO 900

ERR 610

ERR 620

812 HRITE (ISCRUN , 12)

12 FORMAT (33H*** COMMAND STORAGE AREA OVERFLOW,5IX)

ERR 630 GO TO 900

813 WRITE (ISCRUN , 13)

13 FORMAT $\left(30 \mathrm{H}^{* * *}\right.$ STATEMENT NUMBER NOT FOUND, 54X)

GO TO 900

814 WRITE (I SCRUN , 14)

14 FORMAT (35H*** ILLEGAL OR NO FORMAT DESIGNATOR, 49X)

ERR 640

ERR 650

ERR 660

ERR 670

ERR 680

ERR 690 GO TO 900

815 WR I TE (I SCRUN , 15)

15 FORMAT ( $34 H^{* * *}$ DIMENSIONED AREA EXCEEDS LIMIT, 50X)

ERR 700

ERR 710

ERR 720 GO TO 900

ERR 730

ERR 740

ERR 750

816 WRITE (ISCRUN, 16)

16 FORMAT (27H*** ILLEGAL SIZE ROW NUMBER, 57X)

GO TO 900

ERR 760

ERR 770

ERR 780

817 WRITE (ISCRUN, 17)

17 FORMAT (39H*** DEFINED MATRIX OVERFLOWS WORKSHEET, 45X)

ERR 790 GO TO 900

818 WRITE (ISCRUN, 18 )

18 FORMAT $\left(36 H^{* * *}\right.$ INTEGER ARGUMENT LESS THAN $\left.-8191,48 \mathrm{X}\right)$

ERR 800

ERR 810

ERR 820 GO TO 900

ERR 830

ERR 840

819 WRITE (ISCRUN, 19)

ERR 850 GO TO 900

820 WR ITE (ISCRUN , 20)

20 FORMAT (29H*** IMPROPER TYPE OF ARGUMENT, 55X)

GO TO 900

821 WRITE (ISCRUN, 21)

21 FORMAT (26H*** COMMAND MUST BE STORED,58X)

GO TO 900

ERR 860

ERR 870

ERR 880

ERR 890

ERR 900

ERR 910

ERR 920

ERR 930

822 WRITE (ISCRUN, 22)

22 FORMAT ( 3 IH*** MATRIX IS (NEARLY) SINGULAR, 53X)

ERR 940 GO TO 900

ERR 950

ERR 960

823 WRITE (ISCRUN , 23)

23 FORMAT $\left(28 H^{* * *}\right.$ INSUFF ICIENT SCRATCH AREA, 56X)

ERR 970 GO TO 900

ERR 980

$\begin{array}{lll}824 & \text { WRITE (ISCRUN, 24) } & \text { ERR } 990 \\ 24 & \text { FORMAT (49H*** DEGREE IS LARGER THAN NO. OF NON-ZERO WEIGHTS, 35X) } & \text { ERR1000 }\end{array}$ GO TO 900

825 WRITE (ISCRUN, 25)

ERR 1010

ERR 1020

ERR 1030

FORMAT (35H**NEGATIVE WEIGHTS MAY NOT BE USED, 49X)

ERR 1040 GO TO 900

ERR 1050

826 WRITE (ISCRUN, 26)

26 FORMAT (51H***NUMBER OF COLUMNS IS GREATER THAN NUMBER OF ROHS, 33X) ERR 1060
GO TO 900
ERR 1070

827 WRITE (ISCRUN, 27)

ERR 1080

27 FORMAT (19H***FORMAT NOT FOUND,65X) $\quad$ ERR1090 GO TO 900

ERRI100

C**** THE FOLLOWING CARDS ARE NEEDED ONLY FOR TAPE OPERATIONS ERR 1110

828 WRITE (ISCRUN , 28) ERR 1120

28 FORMAT (47H* INCORRECT TAPE UNIT. COMMAND IS NOT EXECUTED , 37X) ERR 1130 GO TO 900

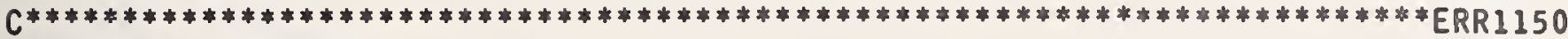

829 NSB $=$ NARGS +1

ERR 1160

WRITE (ISCRUN, 29) NSB

ERR 1170

29 FORMAT (31H* NUMBER OF ARGUMENTS SHOULD BE, I2,51X)

ERR 1180 
830 WRITE (ISCRUN, 30 )

30 FORMAT (48H* AN INCREMENT COMMAND CAN NOT INCREMENT ITSELF. ,36X)

900 IF ( LEVEL .NE. O ) CALL RNDOWN

C FORCE OUT OF REPEAT MODE IF FATAL ERROR

ERR 1190

ERR 1200

ERR 1220

IF ( I .LE. 100$)$ LEVEL $=0$

ERR 1230

WRITE ( ISCRUN, 901 )

ERR 1240

901 FORMAT (84X)

ERR 1250

ERR1260

RETURN

ERR 1270

200 IF (NERR.LE.NERCON.OR.LLIST.NE.3) GO TO 201

ERR 1280

IF (ISWERR . NE.0) RETURN

ERR 1290

IS WERR $=1$

ERR 1300

WRI TE (I SCRUN, 9999 ) NERCON

ERR 1310

9999 FORMAT (/1H*, I5,62H INFORMATIVE AND ARITHMETIC DIAGNOSTICS HAVE BEEERRI320

IN ENCOUNTERED. , 16X/

ERR 1330

284H* ANY SUCH ADDITIONAL DIAGNOSTICS FOR THIS COMMAND OR REPEAT MERRI340 $30 D E$ ARE DISREGARDED. )

RETURN

201 IF (I.GT.200) GO TO 400

ERR 1350

ERR1360

ERR 1370

C

C

C

ARITHMETIC TROUBLES, SET FLAGS

ERR 1380

ERR 1390

ERR 1400

ERR1410

CALL AERR (I-100)

ERR1420

RETURN

ERR1430

INFORMATIVE DIAGNOSTIC

ERR 1440

ERR1450

ERR1460

IF（MOD（LLIST, 2) .EQ. O ) GO TO 250

ERR 1470

IF (LLIST.EQ.0) GO TO 250

ERR1475

CALL INFERR (I)

ERR 1480

GO TO 900

ERR 1490

END

ERR 1500 
SUBROUTINE EXCHNG

C VERSION 5.00 EXCHNG 5/15/70

C

C

C

EXCHANGE COL ++ WITH ++, COL ++ WITH ++, ETC.

COMMON /BLOCRC/ NRC, RC (12600)

COMMON /BLOCKD/ IARGS(100), KIND(100), ARGTAB(100), NRMAX, NROW, NCOL, NEXC 70

IARGS, VWXYZ (8), NERROR

DIMENSION ARGS(100)

EQUIVALENCE (ARGS(1), RC(12501))

IF (NARGS) $70,70,10$

10 IF (NARGS.NE. (NARGS/2)*2) GO TO 70

DO $50 I=1$, NARGS , 2

CALL ADRESS $(I, J)$

IF (J) $60,80,20$

20

CALL ADRESS $(I+1, K)$

IF (K) $60,80,30$

30

IF (NERROR.NE.0) RETURN

DO $40 \mathrm{~N}=1$, NRMAX

$J J=J+N-1$

$\mathrm{KK}=\mathrm{K}+\mathrm{N}-1$

WORK=RC (JJ)

$R C(J J)=R C(K K)$

$R C(K K)=$ WORK

40 CONTINUE

50 CONTINUE

GO TO 90

60 CALL ERROR (3)

GO TO 90

70 CALL ERROR (10)

GO TO 90

80 CALL ERROR (11)

90 RETURN

END
EXC 10

EXC 20

EXC 30

EXC 40

EXC 50

EXC 80

EXC 90

EXC 100

EXC 110

EXC 120

EXC 130

EXC 140

EXC 150

EXC 160

EXC 170

EXC 180

EXC 190

EXC 200

EXC 210

EXC 220

EXC 230

EXC 240

EXC 250

EXC 260

EXC 270

EXC 280

EXC 290

EXC 300

EXC 310

EXC 320

EXC 330

EXC 340 
SUBROUTINE EXPAND ( $J$, WHERE)

C VERSION 5.00 EXPAND 5/15/70

EXD 10

COMMON /BLOCRC/ NRC, RC (12600)

EXD 20

COMMON /BLOCKD/ I ARGS (100), KIND (100), ARGTAB (100), NRMAX, NROW, NCOL, NEXD 40

IARGS, VHXYZ (8), NERROR

DIMENSION ARGS $(100)$

EQUIVALENCE (ARGS(1), RC (12501))

COMMON /BLOCKE/ NAME (4),L1,L2, ISRFLG

EXD 50

EXD 60

DIMENSION WHERE ( 1 )

EXD 70

EXD 80

$\mathrm{C}$

THIS ROUTINE EXPANDS STORED COMMANDS FROM WHERE TO A USABLE

EXD 90

C FORM IN ARGS, IARGS AND KIND.

EXD 100

EXD 110

EXD 120

EXD 130

$I I=0$

EXD 140

$I=0$

EXD 150

$J J J=J$

EXD 160

CONVERT ONLY FIRST ARGUMENT IF COMMAND IS INCREMENT OR RESTORE

EXD 170

IF (LI.NE. 14) GO TO 10

IF (L2.GE.6.AND.L2.LE.8) JJJ=2

EXD 180

EXD 190

$10 \quad I I=I I+I$

EXD 200

$20 \quad I=I+I$

IF (I.GE.JJJ) GO TO 80

EXD 210

$T=$ WHERE (I)

EXD 220

IF (T) $50,40,30$

EXD 230

EXD 240

$30 \quad \operatorname{KIND}(I I)=0$

EXD 250

$\operatorname{IARGS}($ II $)=T-8192$.

EXD 260

GO TO 10

EXD 270

$40 \quad \operatorname{KIND}(I I)=1$

$I=I+1$

ARGS (II) = WHERE (I)

EXD 280

EXD 290

GO TO 10

50 IF (T.EQ.(-1)) GO TO 100

CALL XPND (WHERE (I),K,ARGS (II), KND)

IF (K.GE.O) GO TO 90

$60 \quad K=-K$

70 CALL ERROR (K)

80 RETURN

$90 \quad K I N D(I I)=K N D$

IF (KND.EQ.0) IARGS (II)=ARGS (II)

$I=I+K$

GO TO 10

EXD 300

EXD 310

EXD 320

EXD 330

EXD 340

EXD 350

EXD 360

EXD 370

EXD 380

EXD 390

EXD 400

EXD 410

C

C

IF STORED VALUE $=-1$, THEN ARGS (INTEGER) ARE TO BE EXPANDED FROM

PREVIOUS ARG TO FOLLOWING WITH A MAXIMUM TOTAL OF 100

EXD 420

C

C

$100 \quad I=I+1$

C PICK UP NEXT ARG

I U $=$ WHERE (I)

IF (KIND(II-I).NE.O.OR.I.GE.J) GO TO 190

IF (IU) $170,190,110$

110 IU $=I U-8192$

$120 \mathrm{~K}=I U-I$ ARGS $(I I-I)$

NARGS $=$ NARGS $+I$ ABS $(K)-1$

IF (NARGS.GT.100) GO TO 200

IF (K) $130,20,140$

130 INC $=-1$

$\mathrm{K}=-\mathrm{K}$

EXD 430

EXD 440

EXD 450

EXD 460

EXD 470

EXD 480

EXD 490

EXD 500

EXD 510

EXD 520

EXD 530

EXD 540

EXD 550

EXD 560

EXD 570

GO TO 150

EXD 580

$140 \quad$ INC $=1$

EXD 590 


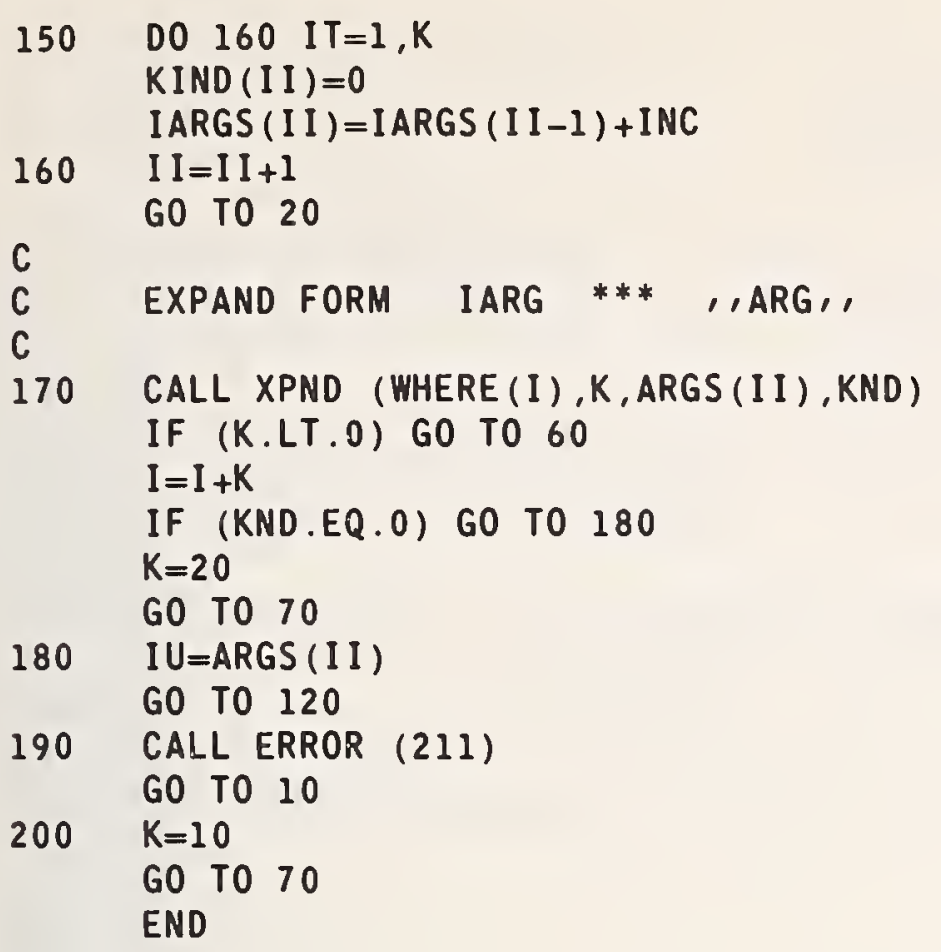




\section{COMMANDS}

L2=1: MVECDIAG

MVECDIAG MATRIX IN $R, C$ SIZE $N$, MVECDIAG MATRIX IN $R, C$ SIZE $N$,

L2=2: MVECMAT MVECMA

\section{MATRIX IN $R, C$ SIZE N,}

PUT ROW BY ROW

AS A VECTOR IN C

MVECMAT MATRIX IN $R$, C SIZE $N$, M PUT ROW BY ROW AS A VECTOR IN $R, C$

EXN 30

EXN 40

EXN 50

EXN 60

PUT DIAGONAL IN C

EXN 70

EXN 80

EXN 90

EXN 100

\section{L2=3: MMATVEC} MMATVEC MMATVEC

VECTOR C PUT

AS ROW

ROW X ROW MAT

MATRIX IN R, C SIZE N, ME

EXN 110

EXN 120

EXN 130

COMMON /BLOCRC/ NRC, RC (12600)

SIZE N X M

EXN 135

EXN 140

COMMON /BLOCKD/ IARGS (100), KIND (100), ARGTAB (100), NRMAX, NROW, NCOL, NEXN 170

IARGS, VWXYZ (8), NERROR

DIMENSION ARGS $(100)$

EQUIVALENCE (ARGS(1), RC(12501))

COMMON /SCRAT/ NS,NS2,A(13500)

COMMON /BLOCKE/ NAME (4),L1,L2, ISRFLG

IF (NARGS.EQ.5.OR.NARGS.EQ.6) GO TO 10

CALL ERROR (10)

RETURN

$J=N A R G S$

$K R R=0$

CALL CKIND (J)

IF $(J . E Q .0)$ IF $(L 2-2) \quad 15,65,125$

EXN 180

EXN 190

EXN 200

EXN 210

EXN 220

EXN 230

EXN 240

EXN 250

EXN 260

EXN 270

EXN 280

CALL ERROR (3)

EXN 285

RETURN

C VEC DIAG ****

EXN 290

EXN 300

$15 \operatorname{IARGS}(7)=\operatorname{MINO}(\operatorname{IARGS}(3), \operatorname{IARGS}(4))$

EXN 310

EXN 320

$\operatorname{IARGS}(8)=1$

EXN 330

IF (NARGS.EQ.6) GO TO 20

EXN 340

$\operatorname{IARGS}(6)=\operatorname{IARGS}(5)$

EXN 350

$\operatorname{IARGS}(5)=1$

EXN 360

$M K K R=226$

EXN 370

IF (IARGS (5)+IARGS (7)-1.LE. NROW) GO TO 30

EXN 380

$\operatorname{IARGS}(7)=$ NROW-IARGS $(5)+1$

EXN 390

KRR $=$ MKKR

EXN 400

C ERROR 226: COLUMN NOT LONG ENOUGH TO STORE ALL ELEMENTS. ONLY NROWEXN 410

C WILL BE STORE

EXN 420

$30 \quad J=2$

CALL MTXCHK (J)

IF (J.NE.O) CALL ERROR (17)

EXN 430

IF (NERROR.NE.O) RETURN

EXN 440

IF (KRR.NE.O) CALL ERROR (KRR)

EXN 450

GO TO $(40,80,140), L 2$

EXN 460

I $A=I$ IARGS (1)

IB $=\operatorname{IARGS}(7)$

DO $50 \quad I=1, I B$

EXN 470

$A(I)=R C$ (IA)

EXN 480

40

$50 \quad$ I $A=I A+N R O W+1$

EXN 490

EXN 500

EXN 510

EXN 520

EXN 530

DO $60 \mathrm{I}=1$, IB

EXN 540

$R C(I A)=A$ (I)

EXN 550

$I A=I A+I$ 
70 MKKR $=226$

EXN 640

$$
\text { GO TO } 20
$$

EXN 650

$80 \operatorname{IB}=\operatorname{IARGS}(7)$

EXN 690

IA=I ARGS (1)

EXN 700

$N=I$ ARGS (3)

EXN 710

$M=I$ ARGS (4)

EXN 720

$I C=1$

EXN 730

DO $100 \quad I=1, N$

EXN 740

I $A A=I A$

EXN 750

DO $90 \mathrm{~J}=1, M$

EXN 760

$A(I C)=R C$ (IAA)

EXN 770

IF (IC.EQ.IB) GO TO 110

EXN 780

IC $=I C+1$

EXN 790

$90 \quad I A A=I A A+N R O W$

EXN 800

EXN 810

$100 \quad I A=I A+1$

EXN 820

110 I $A=I$ ARGS (5)

DO $120 \quad I=1, I B$

EXN 830

$R C(I A)=A(I)$

EXN 840

EXN 850

$120 \quad I A=I A+1$

RETURN

C 125 TAKE A COLUMN AND RESTORE IT TO A MATRIX OR ARRAY.

EXN 860

EXN 870

EXN 880

$125 \operatorname{IARGS}(8)=I$ ARGS (NARGS)

EXN 890

$\operatorname{IARGS}(7)=\operatorname{IARGS}($ NARGS-1)

EXN 900

$\operatorname{IARGS}(6)=\operatorname{IARGS}($ NARGS-2)

EXN 910

$\operatorname{IARGS}(5)=$ IARGS (NARGS-3)

EXN 920

IF (NARGS.EQ.6) GO TO 130

EXN 930

$\operatorname{IARGS}(2)=\operatorname{IARGS}(1)$

EXN 940

$\operatorname{IARGS}(1)=1$

$130 \operatorname{IARGS}(3)=\operatorname{IARGS}(7) * \operatorname{IARGS}(8)$

EXN 950

$\operatorname{IARGS}(4)=1$

IF (IARGS(1)+IARGS(3)-1.LE.NROW) GO TO 30

EXN 960

EXN 970

EXN 980

$\operatorname{IARGS}(3)=$ NROW-IARGS $(1)+1$

EXN 990

$\begin{array}{ll}\text { C KRR }=227 & \text { EXN } 1000 \\ \text { C } 227 \text { ERROR : NOT ENOUGH ELEMENTS IN COL TO RESTORE MATRIX OR ARRAY. EXN1010 }\end{array}$

$\begin{array}{ll}\text { C KRR }=227 & \text { EXN } 1000 \\ \text { C } & 227 \text { ERROR : NOT ENOUGH ELEMENTS IN COL TO RESTORE MATRIX OR ARRAY. EXN1010 }\end{array}$

C ELEMENTS AVAILABLE WILL BE USED.

GO TO 30

EXN1020

EXN1030

$I A=I A R G S(1)$

EXN1040

IB=I ARGS (3)

EXN1050

DO $150 \quad I=1, I B$

EXN1060

$A(I)=R C$ (IA)

EXN1070

$I A=I A+1$

I $A=I$ ARGS (5)

EXN1080

$N=I$ ARGS $(7)$

EXN1090

$M=I$ ARGS (8)

EXN1100

IC $=1$

DO $170 I=1, N$

EXN1110

EXN1120

$I A A=I A$

EXN1130

DO $160 \mathrm{~J}=1, M$

EXN1140

$R C(I A A)=A(I C)$

EXN1150

IF (IC.EQ.IB) RETURN

EXN1160

$I C=I C+1$

EXN1170

160 IAA $=I A A+N R O W$

EXN1180 
SUBROUTINE EXTREM

C VERSION 5.00 EXTREM 5/15/70

EXT 10

COMMON /BLOCRC/ NRC, RC(12600)

EXT 20

COMMON /BLOCKD/ IARGS(100),KIND(100), ARGTAB (100), NRMAX, NROW, NCOL, NEXT 40

IARGS, VWXYZ (8), NERROR

EXT 50

DIMENSION ARGS(100)

EXT 60

EQUIVALENCE (ARGS (1), RC (12501))

EXT 70

COMMON /BLOCKE/ NAME (4),L1,L2, ISRFLG

EXT 80

C

$\mathrm{L} 2=4,5 \mathrm{MAX} \quad \mathrm{L} 2=6,7 \mathrm{MIN}$

EXT 90

C

C

C MAX OF ++ TO ++
C MAX OF ++ TO,++ CORRESP ENTRY OF $++\mathrm{TO}++,++\mathrm{TO}++$, ETC.

EXT 100

MAX OF ++ TO,++ CORRESP ENTRY OF ++ TO,++++ TO,++ ETC.
LIKEWISE FOR MIN.

EXT 110

EXT 120

C LIKEWISE FOR MIN

EXT 130

IF (NARGS.GT.O.AND.MOD(NARGS, 2).EQ.0) GO TO 30

EXT 140

EXT 150

$I=10$

EXT 160

CALL ERROR (I)

EXT 170

10

RETURN

EXT 180

20

CALL CHKCOL (I)

EXT 190

IF (I.EQ.0) GO TO 40

EXT 200

$I=20$

EXT 210

GO TO 10

40 IF (NERROR.NE.0) GO TO 20

$J=0$

IF (NRMAX-1) $50,110,60$

$50 \quad I=9$

GO TO 10

$60 \mathrm{~J}=\operatorname{IARGS}(1)$

$\mathrm{K}=\mathrm{J}+1$

$L=K+N R M A X-2$

IF (L2.GT.5) GO T0 80

C

C FIND MAXIMUM

C

DO $70 \quad I=K, L$

IF $(R C(J) \cdot L T \cdot R C(I)) \quad J=I$

70 CONTINUE

GO TO 100

EXT 220

EXT 230

EXT 240

EXT 250

EXT 260

EXT 270

EXT 280

EXT 290

EXT 300

EXT 310

EXT 320

EXT 330

EXT 340

EXT 350

EXT 360

EXT 370

EXT 380

EXT 390

EXT 400

C

C FIND MINIMUM

EXT 410

C

80 DO $90 \quad I=K, L$

IF (RC(J).GT.RC(I)) $\quad J=I$

EXT 420

EXT 430

EXT 440

90 CONTINUE

EXT 450

$\mathrm{J}=\mathrm{J}-\mathrm{I}$ ARGS (1)

EXT 460

DO $120 I=1, N A R G S, 2$

EXT 470

$K=I A R G S(I)+J$

EXT 480

CALL VECTOR (RC $(K), \operatorname{IARGS}(I+1)$ )

EXT 490

GO TO 20

EXT 500

END

EXT 510 
THIS FUNCTION IS TO TRAP IF ARGUMENT IS GREATER THAN $3.3 E 7$ IN

10 RETURN

20 CALL ERROR (104)

$\mathrm{FCOS}=0$.

GO TO 10

$\mathrm{FCO} 40$

FCO 50

FCO 60

FCO 70

FCO 80

FCO 90

FCO 100

FCO 110

FCO 120

END

FCO 130

FCO 140

DOUBLE PRECISION FUNCTION FDCOS $(X)$

FDC 10

VERSION 5.00 FDCOS $5 / 15 / 70$

FDC 20

FDC 30

THIS FUNCTION IS TO TRAP IF ARGUMENT IS GREATER THAN 3.5016 IN FDC 40 ABSOLUTE VALUE BEFORE SYSTEM DOES. RESULT SET $=0$ WITH DIAGNOSTIC. FDC 50

FDC 60

FDC 70

DOUBLE PRECISION DSNCOS, DXEXP

FDC 80

COMMON /DCONLB/ DSNCOS,DXEXP

FDC 90

DOUBLE PRECISION $X, D C O S$

IF (DABS $(X)$.GT.DSNCOS) GO TO 20

FDC 100

$F D C O S=D C O S(X)$

FDC 110

RETURN

FDC 120

CALL ERROR (104)

FDC 130

FDCOS $=0 . D 0$

FDC 140

GO TO 10

FDC 150

END

FDC 160

DOUBLE PRECISION FUNCTION FDEXP $(X)$

C VERSION 5.00 FDEXP $5 / 15 / 70$

FDE 10

FDE 20

THIS FUNCTION IS TO TRAP IF ARGUMENT IS GREATER THAN 704.DO

FDE 30

C

BEFORE SYSTEM DOES. RESULT IS SET $=0.0$ AND DIAGNOSTIC IS PRINTED.

FDE 40

C

C

DOUBLE PRECISION DSNCOS,DXEXP

FDE 60

COMMON /DCONLB/ DSNCOS,DXEXP

FDE 70

DOUBLE PRECISION $X, D E X P$

FDE 80

IF (X.GT.DXEXP) GO TO 20

FDE 90

FDEXP $=\operatorname{DEXP}(X)$

FDE 100

RETURN

FDE 110

10

CALL ERROR (102)

FDE 120

20

FDEXP $=0.0 D 0$

FDE 130

GO TO 10

FDE 140

END

FDE 150

FDE 160 
DOUBLE PRECISION FUNCTION FDLOG $(X)$

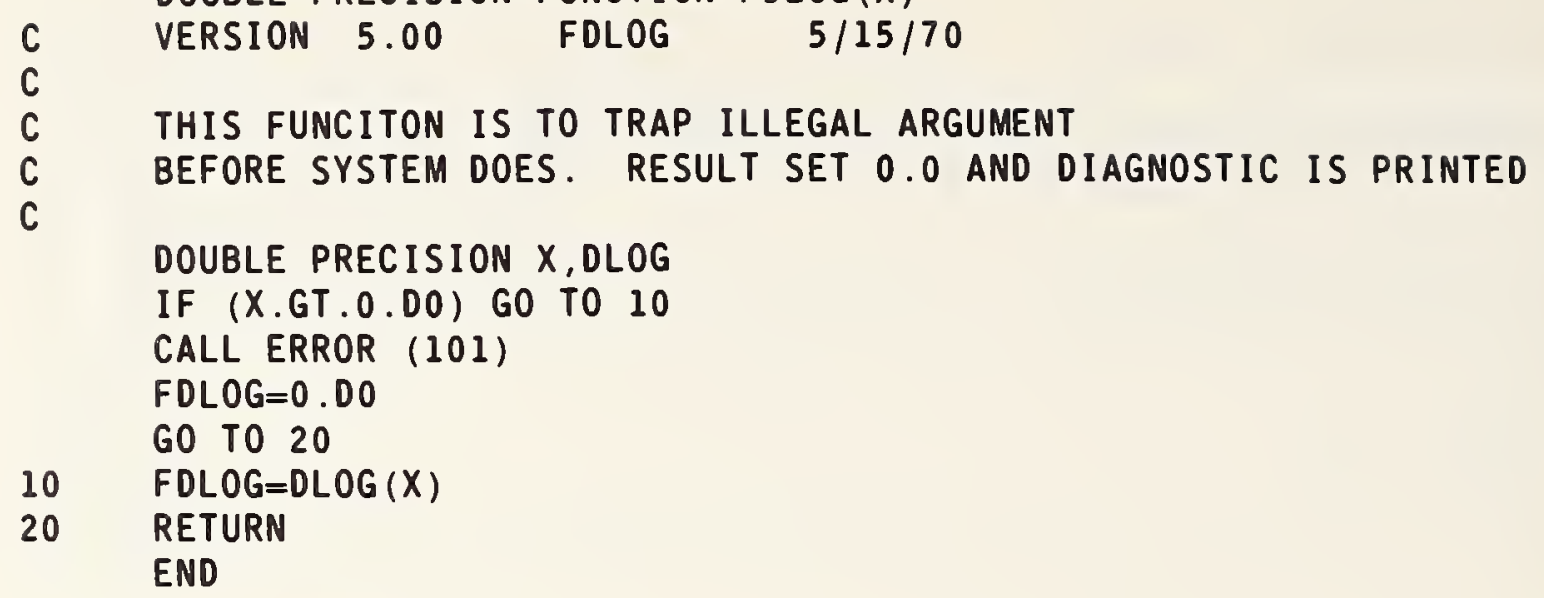

FDL $\quad 10$

FDL 20

FDL $\quad 30$

FDL 40

FDL 50

FDL 60

FDL 70

FDL 80

FDL 90

FDL 100

FDL 110

FDL 120

FDL 130

FDL 140

FUNCTION FDPCON (X)

C VERSION 5.00 FDPCON $5 / 15 / 70$

C WRITTEN BY DAVID HOGBEN, SEL, NBS. $8 / 16 / 69$.

DOUBLE PRECISION $X, D$

$Y=X$

$D=Y$

FDPCON $=X+(X-D)$

RETURN

END

FDP 10

FDP 20

FDP 40

FDP 50

FDP 60

FDP 70

FDP 80

FDP 90

FDP 100

DOUBLE PRECISION FUNCTION FDSIN $(X)$

VERSION 5.00 FDSIN $5 / 15 / 70$

THIS FUNCITON IS TO TRAP IF ARGUMENT IS GREATER THAN 3.5016 IN

FDS 10

$C$
$C$
$C$
$C$
$C$

ABSOLUTE VALUE BEFORE SYSTEM DOES. RESULT SET $=0.0$

FDS 20

FDS 30

FDS 40

FDS 50

FDS 60

DOUBLE PRECISION DSNCOS,DXEXP

FDS 70

COMMON /DCONLB/ DSNCOS,DXEXP

FDS 80

DOUBLE PRECISION X,DSIN

FDS 90

IF (DABS $(X)$.GT.DSNCOS) GO TO 20

FDSIN $=\operatorname{DSIN}(X)$

10 RETURN

20 CALL ERROR (104)

FDSIN $=0.00$

FDS 100

FDS 110

FDS 120

FDS 130

FDS 140

GO TO 10

END

FDS 150

FDS 160 
THIS FUNCTION TRAPS IF ARGUMENT IS NEGATIVE BEFORE SYSTEM DOES. FDQ 40 RESULT SET $=0.0$ AND DIAGNOSTIC IS PRINTED

FDQ 50

DOUBLE PRECISION $X$, DSQRT

FDQ 60

IF (X.LT.O.DO) GO TO 10

FDQ 70

FDSQRT $=$ DSQRT $(X)$

RETURN

FDQ 80

FDQ 90

FDQ 100

CALL ERROR (101)

FDSQRT $=0$. . DO

FDQ 110

RETURN

FDQ 120

END

FDQ 130

FDQ 140

FUNCTION FEXP $(X) \quad$ FEX 10

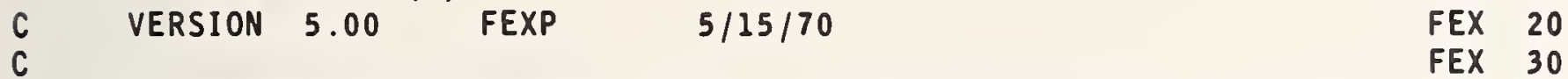

C THIS FUNCTION IS TO TRAP IF ARGUMENT IS GREATER THAN 88.0 BEFORE FEX 40

C SYSTEM DOES. RESULT SET $=0.0 . \quad$ FEX 50

C $\quad$ FEX 60

COMMON /CONSLB/ XTRIG, XEXP $\quad$ FEX 70

IF (X.GT.XEXP) GO TO $20 \quad$ FEX 80

$F E X P=\operatorname{EXP}(X) \quad$ FEX 90

$\begin{array}{lll}10 & \text { RETURN } & \text { FEX } \\ 20 & \text { CALL ERROR (102) } & \text { FEX } 110\end{array}$

$F E X P=0 . \quad$ FEX 120

$\begin{array}{lll}\text { GO TO } 10 & \text { FEX } 130\end{array}$

END

FEX 140

FUNCTION FEXP2 (B,E) $\quad$ FX2 10

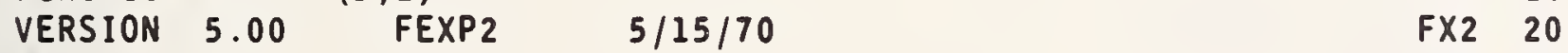

DATA IEXP/60/ $\quad$ FX2 30

$\begin{array}{llll}\text { FX2 } & 40\end{array}$

THIS FUNCTION IS INCLUDED TO CATCH EXPONENTIATION ERRORS BEFORE FX2 50

THE SYSTEM DOES

FX2 60

FX2 70

$\begin{array}{lll}I E=E & F X 2 & 80\end{array}$

IF (E.EQ.FLOAT(IE).AND.IE.LT.IEXP) GO TO $20 \quad$ FX2 90

FEXP2 $=F E X P(E * F L O G(B)) \quad F X 2100$

$\begin{array}{ll}\text { RETURN } & \text { FX2 } 110\end{array}$

$\begin{array}{ll}10 & \text { RETURN } \\ 20 & \text { FEXP2 }=B * * \text { IE }\end{array}$

GO TO 10

FX2 120

FX2 130

END

FX2 140 
SUBROUTINE FIXFLO

C VERSION 5.00

COMMON /ABCDEF/ $L(48)$

FIXFLO $5 / 15 / 70$

FIX 10

COMMON /BLOCRC/ NRC, RC (12600)

FIX 20

FIX 30

FIX 40

COMMON /BLOCKD/ IARGS (100), KIND (100), ARGTAB (100), NRMAX, NROW, NCOL, NFIX 50

IARGS, VWXYZ ( 8 ), NERROR

FIX 60

DIMENSION ARGS(100)

FIX 70

EQUIVALENCE (ARGS(1),RC (12501))

FIX 80

COMMON /BLOCKE/ NAME (4),L1,L2, ISRFLG

FIX 90

COMMON / CONLB2 /ER, IS IGD

FIX 95

COMMON /FMAT / IFMTX (6), IOSWT, IFMTS (6), LHEAD (96)

FIX 100

DIMENSION IB (3)

DATA IB (1), IB (2), IB (3)/2H8F, 2H8E, 2HIP/

FIX 110

FIX 120

$L 2=3$ FOR FIXED, L2 $=4$ FOR FLOAT

FIX 130

$L 2=12$ FOR FLEXIBLE

FIX 140

FIX 150

IF (L2.NE.12) GO TO 5

FIX 160

I OSWT $=0$

RETURN

5 IF (L2.NE.4.OR.NARGS.NE.0) GO TO 8

FIX 170

FIX 180

$I=6$

GO TO 50

8 IF (NARGS.EQ.1) IF (KIND(1)) $30,40,30$

$I=10$

FIX 190

FIX 192

FIX 194

FIX 196

FIX 198

FIX 200

FIX 210

FIX 220

FIX 230

FIX 240

FIX 250

FIX 260

FIX 270

FIX 280

FIX 290

FIX 300

FIX 310

FIX 320

FIX 330

FIX 340

FIX 350

FIX 360

FIX 370

FIX 380

FIX 390

RETURN

FIX 400 
SUBROUTINE FLIP

FLI 10

VERSION 5.00

FLIP

$5 / 15 / 70$

FLI 20

COMMON /BLOCRC/ NRC,RC(12600)

FLI 30

COMMON /BLOCKD/ IARGS(100), KIND(100), ARGTAB (100), NRMAX, NROW, NCOL, NFLI 40

IARGS, VWXYZ (8), NERROR

FLI 50

DIMENSION ARGS $(100)$

FLI 60

EQUIVALENCE (ARGS(1), RC(12501))

FLI 70

EQUIVALENCE (I,IARGS $(100)),(\mathrm{J}, \operatorname{IARGS}(99)),(\mathrm{K}, \operatorname{IARGS}(98)),(\mathrm{KK}, \mathrm{IARGFLI} 80$ 1S(97)), (M,IARGS(96)), (MM,IARGS(95)),(MMM,IARGS(94)),(N,IARGS(9FLI 90

C $23)),($ NN , IARGS (92)), (A,ARGS(1))

FLI 100

C FLIP COL ++ INTO COL ++, ++ INTO ++, ETC.

C IF NARGS $=0$, FLIP THE ENTIRE ARRAY (WORKSHEET).

FLI 110

FLI 120

IF (NARGS.EQ.0) GO TO 40

FLI 130

C

IF (MOD (NARGS, 2).EQ.0) GO TO 30

$I=10$

10 CALL ERROR (I)

20 RETURN

30 CALL CHKCOL (I)

IF (I.EQ.0) GO TO 40

FLI 140

$I=20$

FLI 150

GO TO 10

40 IF (NERROR.NE.0) GO TO 20

FLI 160

FLI 170

FLI 180

FLI 190

FLI 200

FLI 210

FLI 220

FLI 230

IF (NRMAX-1) 50,20,60

FLI 240

$I=9$

FLI 250

50

GO TO 10

FLI 260

$K K=N R M A X-1$

FLI 270

60

$K=K K / 2$

IF (NARGS.EQ.0) GO TO 90

FLI 280

FLI 290

DO $80 \mathrm{I}=1$, NARGS , 2

FLI 300

$M=I$ ARGS $(I)$

$N=I$ ARGS $(I+I)$

FLI 310

FLI 320

$M M=M+K K$

FLI 330

$N N=N+K K$

FLI 340

$M M M=M+K$

FLI 350

DO $70 \mathrm{~J}=M, M M M$

FLI 360

$A=R C(J)$

$R C(N)=R C$ (MM)

FLI 370

FLI 380

$R C(N N)=A$

$\mathrm{N}=\mathrm{N}+1$

$M M=M M-1$

$\begin{array}{ll}70 & \text { NN }=\text { NN }-1 \\ 80 & \text { CONTINUE }\end{array}$

GO TO 20

FLI 390

FLI 400

FLI 410

FLI 420

FLI 430

FLI 440

FLI 450

FLI 460

FLIP ENTIRE ARRAY

FLI 470

FLI 480

FLI 490

$N=1$

DO $110 \quad I=1, N C O L$

FLI 500

$M=N$

FLI 510

$M M=M+K K$

FLI 520

DO $100 \mathrm{~J}=1, \mathrm{~K}$

FLI 530

$A=R C(M)$

FLI 540

$R C(M)=R C(M M)$

FLI 550

$R C(M M)=A$

FLI 560

$M=M+1$

FLI 570

$\begin{array}{ll}100 & M M=M M-1 \\ 110 & N=N+N R O W\end{array}$

FLI 580

FLI 590

GO TO 20

FLI 600

END 
FUNCTION FLOG (X)

VERSION 5.00

FLOG

LIBRARY FUNCTION FOR NATURAL. LOG.

IF $X$ IS ZERO OR NEG., RESULT IS $=0$ AND DIAGNOSTIC IS PRINTED. IF $(X . G T .0$.$) GO TO 10$

CALL ERROR (101)

$F L O G=0$.

GO TO 20

$10 \quad F L O G=A L O G(X)$

20 RETURN

END
FLE 10

FLE 20

FLE 23

FLE 25

FLE 27

FLE 30

FLE 40

FLE 50

FLE 60

FLE 70

FLE 80

FLE 90

FUNCTION FLOGIO (X)

C VERSION 5.00

FLOG10

$5 / 15 / 70$

FLT 10

FLT 20

FLT 30

THIS FUNCTION CHECKS TO SEE IF $X$ IS ZERO OR NEGATIVE BEFORE USINGFLT 40 LIBRARY ALOG1O. INFORMATIVE DIAGNOSTIC IS PRINTED AND O RETURNED.FLT 50

IF (X.GT.0.0) GO TO 20

FLT 60

CALL ERROR (101)

FLT 70

$F L O G 10=0.0$

FLT 80

10 RETURN

FLT 90

$20 \quad F L O G 10=A L O G 10(X)$

GO TO 10

END

FLT 100

FLT 110

FLT 120

FLT 130 


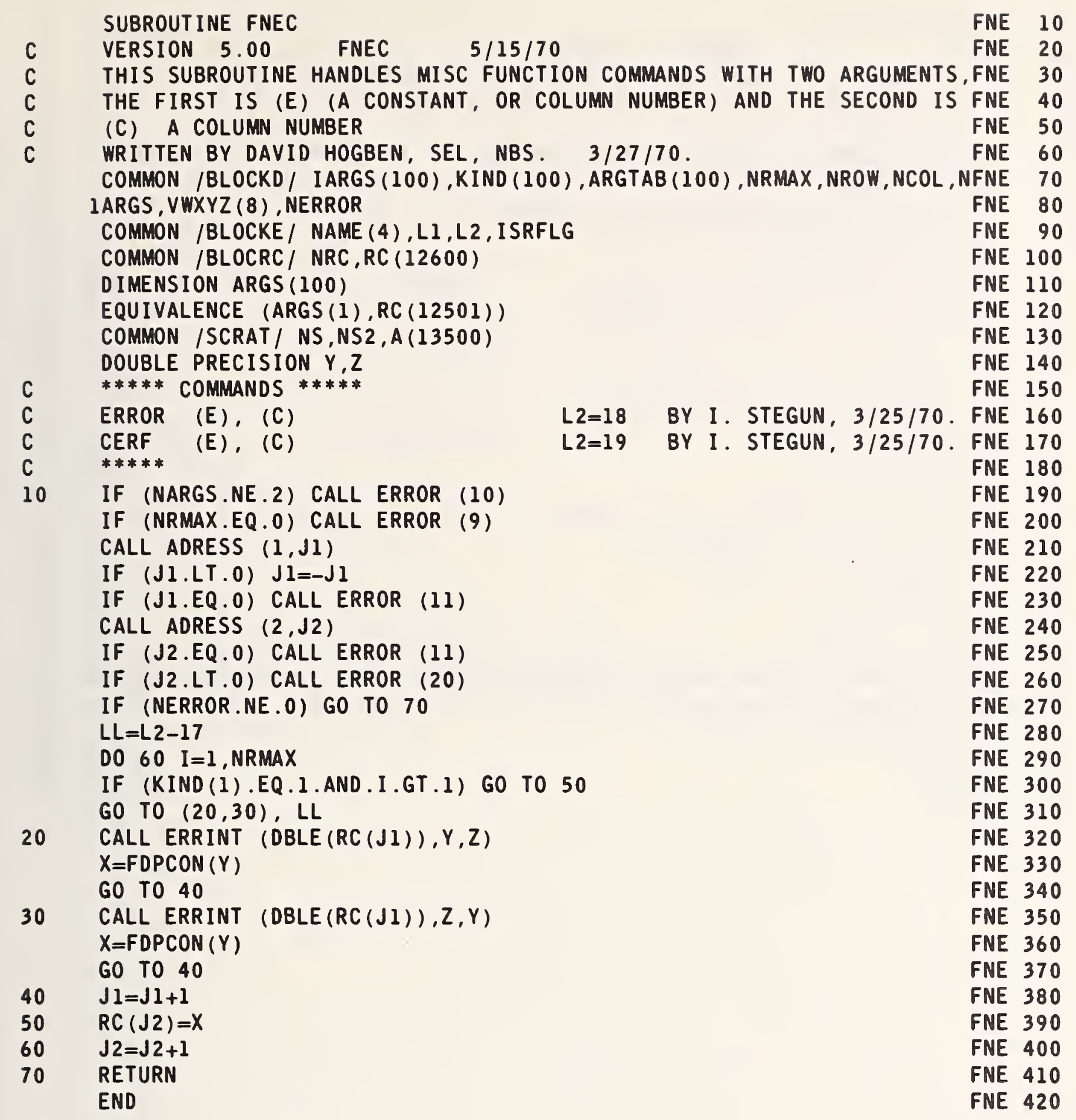




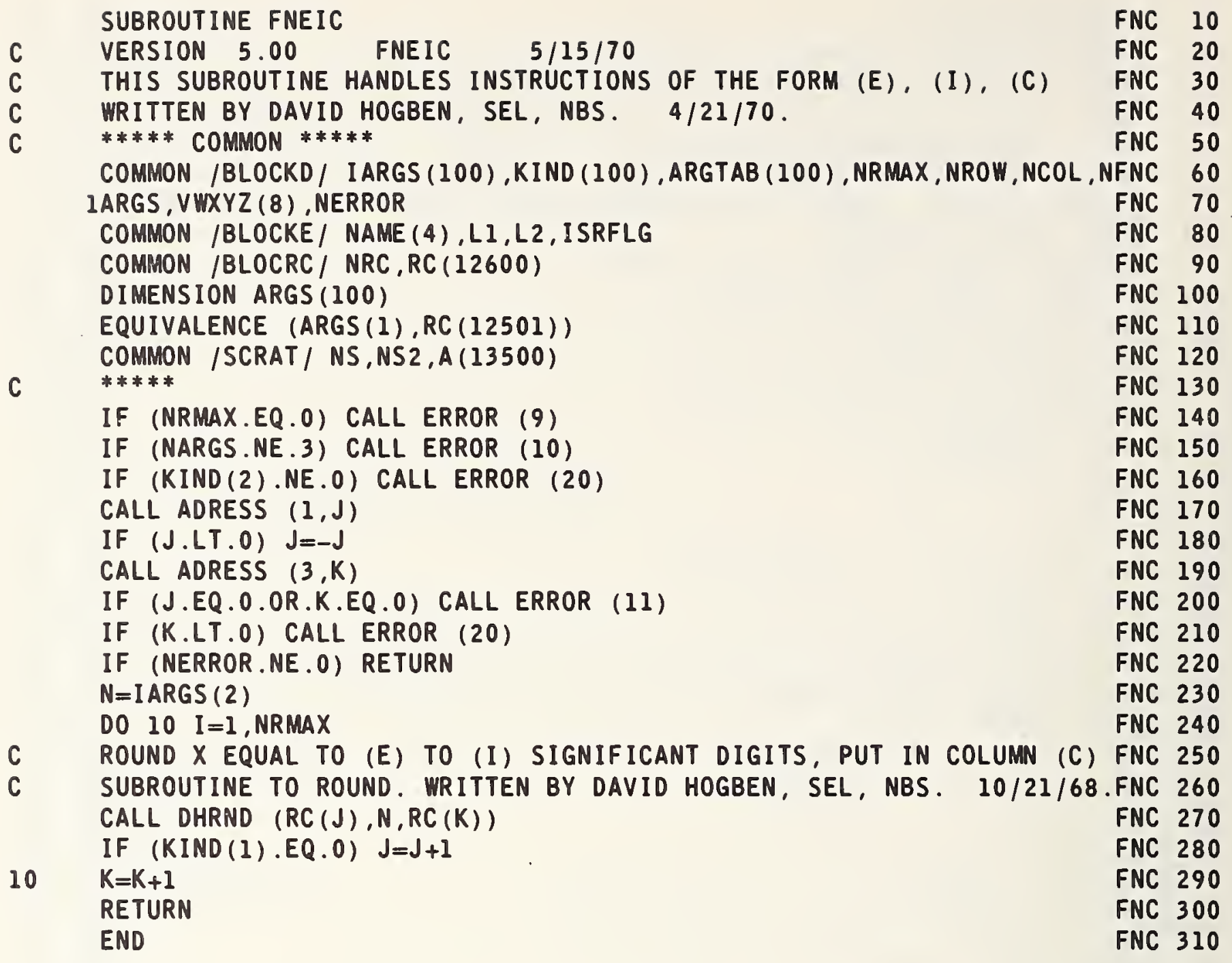


SUBROUTINE FNKC

C VERSION 5.00

FNKC

$5 / 15 / 70$

SUBROUTINE TREATS INSTRUCTIONS OF THE FORM (K), (C)

WRITTEN BY DAVID HOGBEN, SEL, NBS.

***** COMMON *****

COMMON /BLOCKD/ IARGS(100), KIND(100), ARGTAB (100), NRMAX, NROW, NCOL, NFKC 60 IARGS, VWXYZ (8), NERROR

COMMON /BLOCKE/ NAME (4),L1,L2, ISRFLG

COMMON /BLOCRC/ NRC,RC(12600)

DIMENSION ARGS(100)

EQUIVALENCE (ARGS (1), RC(12501))

C

COMMON /SCRAT/ NS,NS2,A(13500)

IF (L1.NE.24.OR.L2.NE.15) RETURN

CALL ERROR (235)

IF (NRMAX.EQ.0) CALL ERROR (9)

IF (NARGS.NE.2) CALL ERROR (10)

CALL ADRESS $(2, J)$

IF (J.EQ.0) CALL ERROR (11)

IF (J.LT.0) CALL ERROR (20)

IF (NERROR.NE.0) RETURN

NST=ARGS ( 1 )

IF (NST.LE.0) NST $=8192.0 * A R G S(1)+0.1$

IF (KIND (1).EQ.0) NST=IARGS (1)

NST $=$ MOD (NST , 8192)

DO $10 \quad I=1$, NRMAX

C RNJBK SHOULD BE REPLACED BY MORE RELIABLE AND EFFICIENT GENERATOR CALL RNJBK (RC (J),NST, NST)

$10 \mathrm{~J}=\mathrm{J}+1$

RETURN

END
FKC 10

FKC 20

FKC 30

FKC 40

FKC 50

FKC 70

FKC 80

FKC 90

FKC 100

FKC 110

FKC 120

FKC 130

FKC 140

FKC 150

FKC 160

FKC 170

FKC 180

FKC 190

FKC 200

FKC 210

FKC 220

FKC 230

FKC 240

FKC 250

FKC 260

FKC 270

FKC 280

FKC 290

FKC 300

FKC 310 
SUBROUTINE FOURIA ( $Y, A, R, N, K A A)$

C VERSION 5.00 FOURIA $5 / 15 / 70$

DOUBLE PRECISION $Y(1), R(1), A, A A, A B, A C, B A, B B, A D$

DOUBLE PRECISION FDCOS, FDSIN

$M=N / 2$

$K=2 * M$

$L=0$

IF (N.EQ.K) GO TO 10

$L=1$

$10 \quad A B=N$

$A A=6.2831853071700 / A B$

$A=0.0$

$R(M)=.0$

$A C=1$.

DO $20 \quad I=1, N$

$A=A+Y(I)$

$R(M)=R(M)+A C * Y(I)$

$20 \quad A C=-1 . * A C$

$A=A / A B$

$R(M)=R(M) / A B$

$J=M+L-1$

$K A=M+1$

DO $40 \mathrm{~K}=1, \mathrm{~J}$

$B A=Y(1)$

$B B=0.0$

$A C=K$

$A C=A C * A A$

DO $30 \quad I=2, N$

$A D=I-1$

$A D=A D * A C$

$B A=B A+Y(I) * F D C O S(A D)$

$30 \quad B B=B B+Y(I) * F D S I N(A D)$

$R(K)=2$. *BA / AB

$R(K A)=2 .{ }^{*} B B / A B$

$40 \quad K A=K A+1$

IF (L.EQ. I) GO TO 50

$R(K A)=0$.

50 RETURN

END
FOU 10

FOU 20

FOU 30

FOU 40

FOU 50

FOU 60

FOU 70

FOU 80

FOU 90

FOU 100

FOU 110

FOU 120

FOU 130

FOU 140

FOU 150

FOU 160

FOU 170

FOU 180

FOU 190

FOU 200

FOU 210

FOU 220

FOU 230

FOU 240

FOU 250

FOU 260

FOU 270

FOU 280

FOU 290

FOU 300

FOU 310

FOU 320

FOU 330

FOU 340

FOU 350

FOU 360

FOU 370

FOU 380

FOU 390 


\begin{tabular}{|c|c|c|c|}
\hline \multirow{4}{*}{ C } & SUBROUTINE FPPT $(V 11, V 12, P 10, X A)$ & FPP & 10 \\
\hline & VERSION $5.0 \quad$ FPPT $5 / 15 / 70$ & FPP & 20 \\
\hline & $V 1=V_{11}$ & FPP & 30 \\
\hline & $V 2=V 12$ & FPP & 40 \\
\hline \multirow{4}{*}{ C } & $P 0=P 10$ & FPP & 50 \\
\hline & CALLS PROB AND THEN USES ISOLATE METHOD FOR SOLVING ITERATIVELY & FPP & 60 \\
\hline & DIMENSION $X(5), P(5)$ & FPP & 70 \\
\hline & IF $\left(V_{1-1}-5\right) 10,10,20$ & FPP & 80 \\
\hline C & USE STUDENT'S T & FPP & 90 \\
\hline C & ONLY GOOD FOR PO=0.05 & FPP & 100 \\
\hline \multirow[t]{3}{*}{10} & CALL TPCTPT $(\mathrm{V} 2, \mathrm{XA})$ & FPP & 110 \\
\hline & $X A=X A * 2.0$ & FPP & 120 \\
\hline & GO TO 70 & FPP & 130 \\
\hline 20 & IF $\left(V_{2}-1.5\right) \quad 30,30,40$ & FPP & 140 \\
\hline $\mathrm{C}$ & ONLY GOOD FOR PO $=0.05$ & FPP & 150 \\
\hline C & SHOULD USE STUDENT:S T & FPP & 160 \\
\hline \multirow[t]{2}{*}{30} & $X A=225.0$ & FPP & 170 \\
\hline & GO TO 70 & FPP & 180 \\
\hline C & TUKEY APPROXIMATION TO NORMAL PERCENT POINT & FPP & 190 \\
\hline 40 & $Y P=-4.91 *(P 0 * * .14-(1 .-P 0) * * .14)$ & FPP & 200 \\
\hline \multirow[t]{5}{*}{ C } & AMS 55 APPROXIMATION 26.5 .22 & FPP & 210 \\
\hline & $H=2: 0 /(1.0 /(V 1-1.0)+1.0 /(V 2-1.0))$ & FPP & 220 \\
\hline & $X L M B D A=(Y P * * 2-3.0) / 6.0$ & FPP & 230 \\
\hline & $H=Y P * F S Q R T(H+X L M B D A) / H$ & FPP & 240 \\
\hline & IF $\left(V_{1}-V_{2}\right) 50,60,50$ & FPP & 250 \\
\hline 50 & $W=W-(1.0 /(V 1-1.0)-1.0 /(V 2-1.0)) *(X L M B D A+0.833333-0.666667 / H)$ & FPP & 260 \\
\hline C & AMS 55 APPROXIMATION 26.6 .16 & FPP & 270 \\
\hline 60 & $X A=F E X P(2 . * W)$ & FPP & 280 \\
\hline \multirow[t]{6}{*}{70} & $X M I N=0.5 * X A$ & FPP & 290 \\
\hline & $X M A X=2.0 * X A$ & FPP & 300 \\
\hline & CALL PROB (V1, V2, XMAX,Q) & FPP & 310 \\
\hline & IF (Q.LE.PO) GO TO 80 & FPP & 320 \\
\hline & $X A=1.9999 * X M A X$ & FPP & 330 \\
\hline & GO TO 70 & FPP & 340 \\
\hline \multirow[t]{4}{*}{80} & CALL PROB $(V 1, V 2, X M I N, Q)$ & FPP & 350 \\
\hline & IF (PO.LE.Q) GO TO 90 & FPP & 360 \\
\hline & $X A=0.5001 * X M I N$ & FPP & 370 \\
\hline & GO TO 70 & FPP & 380 \\
\hline \multirow[t]{3}{*}{90} & $X_{0}=X A$ & FPP & 390 \\
\hline & DO $140 \quad I=1,5$ & FPP & 400 \\
\hline & $X(I)=X M I N+F L O A T(I-1) *(X M A X-X M I N) / 4$. & FPP & 410 \\
\hline 100 & CALL PROB (VI, V2,X(I),P(I)) & FPP & 420 \\
\hline 110 & IF (P0-P(I)) $140,130,120$ & FPP & 430 \\
\hline \multirow[t]{3}{*}{120} & $X M A X=X(I)$ & FPP & 440 \\
\hline & $X M I N=X(I-1)$ & FPP & 450 \\
\hline & GO TO 150 & FPP & 460 \\
\hline \multirow[t]{2}{*}{130} & $X A=X(I)$ & FPP & 470 \\
\hline & GO TO 160 & FPP & 480 \\
\hline 140 & CONTINUE & FPP & 490 \\
\hline & $X A=(X M I N+X M A X) / 2$ & FPP & 500 \\
\hline & EXIT IF EITHER TOLERANCE IS SATISFIED * ABSOLUTE 5E-6, REL. 5E-7 & FPP & 510 \\
\hline & IF $(A B S(X 0-X A) . G T .5 . E-6 . A N D . A B S(X 0-X A) / X A . G T .5 . E-7)$ G0 TO 90 & FPP & 520 \\
\hline \multirow[t]{2}{*}{160} & RETURN & FPP & 530 \\
\hline & END & FPP & 540 \\
\hline
\end{tabular}


SUBROUTINE FPROB

$c$
$c$
$c$
$c$

VERSION 5.00

FPROB

$5 / 15 / 70$

$10 / 13 / 67$

FPR 10

WRITTEN BY S PEAVY

COMMAND IS AS FOLLOWING

$\$$, STORE Q IN COL ++

FPR 20

FPR 30

FPR 40

FPROBABILITY VI \$ V $2 \$ ， F$

COMMON /BLOCRC/ NRC, RC (12600)

FPR 50

COMMON /BLOCKD/ IARGS (100), KIND(100), ARGTAB (100), NRMAX, NROW, NCOL, NFPR 70

IARGS, VWXYZ (8), NERROR

DIMENSION ARGS(100)

FPR 80

EQUIVALENCE (ARGS(1), RC(12501))

FPR 90

IF (NARGS.NE.4) CALL ERROR (10)

FPR 100

IF (KIND(NARGS). NE.O) CALL ERROR (3)

FPR 110

$I I=1$

I $2=1$

I $3=1$

CALL ADRESS (1,IARGS(1))

10 I $I=2$

IF (IARGS (1)) $10,20,30$

FPR 120

FPR 130

FPR 140

$V I=A R G S(1)$

GO TO 40

20 CALL ERROR (11)

30 L=IARGS ( I)

40 CALL ADRESS (2,IARGS (2))

IF (IARGS (2)) $50,60,70$

50 I $2=2$

V $2=A R G S(2)$

GO TO 80

60 CALL ERROR (11)

$70 \quad M=I$ ARGS (2)

80 CALL ADRESS (3, IARGS (3))

IF (IARGS(3)) $90,100,110$

$90 \quad I 3=2$

$F=A R G S(3)$

GO TO 120

100 CALL ERROR (11)

$110 \quad N=I$ ARGS (3)

120 CALL ADRESS (NARGS,K)

IF (K.LE.0) CALL ERROR (11)

IF $(I 1+I 2+I 3 . N E .6)$ GO TO 140

FPR 150

FPR 160

FPR 170

FPR 180

FPR 190

FPR 200

FPR 210

FPR 220

FPR 230

FPR 240

FPR 250

FPR 260

FPR 270

FPR 280

FPR 290

FPR 300

FPR 310

FPR 320

FPR 330

FPR 340

FPR 350

FPR 360

FPR 370

FPR 380

FPR 400

CALL PROB (V1, V2,F,Q)

DO $130 \quad I=1$, NRMAX

FPR 410

FPR 420

$R C(K)=Q$

$130 \quad K=K+1$

RETURN

140 DO $210 I=1$, NRMAX

GO TO $(150,160)$, II

FPR 430

FPR 440

FPR 450

FPR 460

FPR 470

FPR 480

FPR 490

$L=L+1$

FPR 500

160 GO TO $(170,180)$, I

FPR 510

$V 2=R C(M)$

FPR 520

FPR 530

FPR 540

$\mathrm{F}=\mathrm{RC}(\boldsymbol{N})$

FPR 550

$\mathrm{N}=\mathrm{N}+\mathrm{l}$

200 CALL PROB (V1, V2, $F, R C(K))$

$210 \quad K=K+1$

FPR 560

FPR 570

RETURN

FPR 580

END

FPR 590 
SUBROUTINE FRDIST

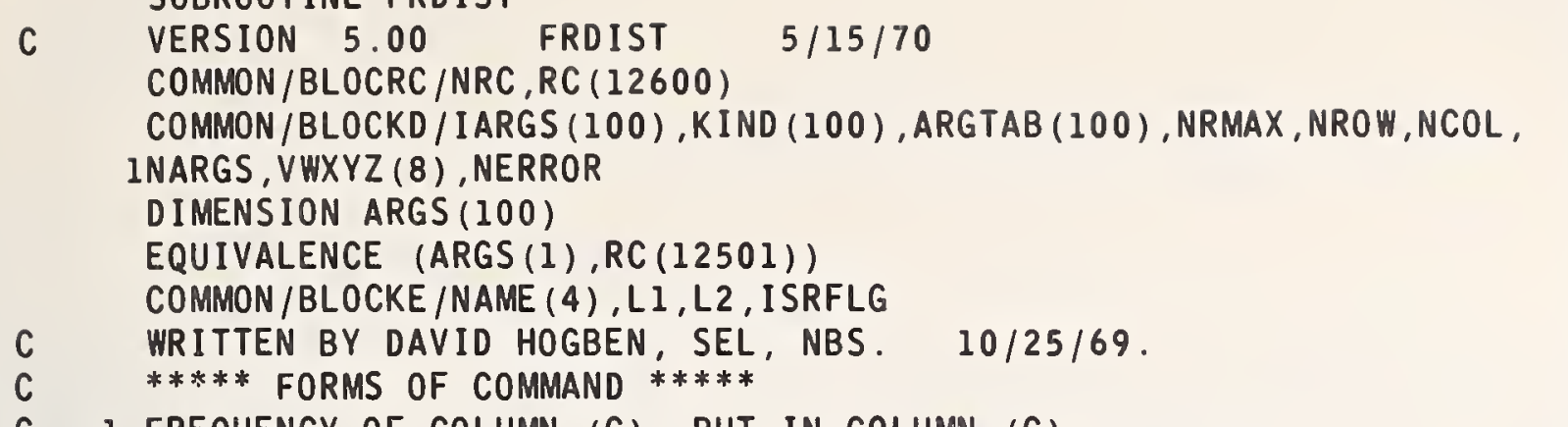

1 FREQUENCY OF COLUMN (C), PUT IN COLUMN (C)

2 FREQUENCY OF COL (C), USE (I) CELLS, PUT IN COLUMN (C)

3 FREQUENCY OF COL (C), USE (I) CELLS, OF LENGTH (A), PUT IN COL (C)FRD 130

4 FREQUENCY OF COL (C), CELLS (I), LENGTH (A), START (A), IN COL (C)FRD 140

5 FREQUENCY OF COL (C), LOWER (C), UPPER IN (C), FREQ IN (C)

FRD 150

7 FREQUENCY OF COL (C), CELLS (I), LENGTH (A), PUT IN (C), (C), (C) FRD 160

8 FREQUENCY OF (C), CELLS (I), LENGTH (A), START (A), IN (C), (C), (C) FRD 170 RESETS NRMAX TO NO. OF CELLS

FRD 180

$* * * * *$

10 IF (NARGS.GT.1 .AND. NARGS.LT.8) GO TO 100

CALL ERROR (10)

RETURN

20 CALL ERROR (3)

RETURN

30 CALL ERROR (11)

RETURN

100 CALL ADRESS $(1, J 1)$

IF (J1) $20,30,110$

FRD 190

FRD 200

FRD 210

FRD 220

FRD 230

FRD 240

FRD 250

FRD 260

FRD 270

FRD 280

110 CALL ADRESS (NARGS,J2)

FRD 290

IF (J2) $20,30,120$

FRD 300

120 NST $=1$

LIMIT $=0$

C FORM (1)

IF (NARGS.EQ.2) GO TO 901

FRD 310

FRD 320

FRD 330

FRD 340

$200 \quad K N=\operatorname{IARGS}(2)$

C FORM (3)

IF (NARGS.EQ.3) GO TO 902

FRD 350

FRD 360

IF (KIND(3).EQ.0) GO TO 500

FRD 370

CELL $=$ ARGS (3)

C FORM (3)

IF (NARGS.EQ.4) GO TO 903

400 IF (KIND(4).EQ.0) GO TO 500

$N S T=0$

STRT $=$ ARGS (4)

C FORM (4)

IF (NARGS.EQ.5) GO TO 904

FRD 380

FRD 390

FRD 400

FRD 410

FRD 420

FRD 430

FRD 440

FRD 450

FRD 460

C FORMS (5), (6), (7), (8)

FRD 470

500 CALL ADRESS (NARGS-2,J3)

IF (J3) $20,30,510$

510 CALL ADRESS (NARGS-1,J4)

IF (J4) $20,30,520$

520 LIMIT $=1$

$\mathrm{J} J=N A R G S-3$

GO TO $(901,902,903,904)$, JJ

FRD 480

FRD 490

FRD 500

FRD 510

FRD 520

FRD 530

FRD 540

$901 \quad K N=0$

FRD 550

FRD 560

FRD 570

FRD 580

FRD 590 
SUBROUTINE FREQCY ( $X, F, N, K, C, N S T A R T$, START, LIMITS , XL, XU)

FRE 10
VERSION 5.00
FREQCY
$5 / 15 / 70$

FRE 20

SUBROUTINE TO CONSTRUCT FREQUENCY DISTRIBUTION IN VECTOR F FOR

FRE 30

VECTOR OF OBSERVATIONS $X$ OF LENGTH N USING K CELLS OF LENGTH C. FRE 40

IF $C=0.0$, THEN $C$ IS DETERMINED BY SUBROUTINE. IF BOTH $K$ AND $C=0.0$, FRE 50

THEN BOTH K AND C ARE DETERMINED BY THE SUBROUTINE.

FRE 60

IF NSTART=1, START DETERMINED. IF NSTART=0, START IS GIVEN.

FRE 70

IF LIMITS=1, LOHER CELL BOUNDARIES ARE PUT IN XL AND UPPER IN XU. FRE 80

WRITTEN BY DAVID HOGBEN, SEL, NBS. 10/25/69.

DIMENSION $X(1), F(1), X L(1), X U(1)$

FRE 90

100 IF (K.GT.0) GO TO 200

FRE 100

$K=1.5+3.3^{*}$ FLOG10(FLOAT $\left.(N)\right)$

$K=\operatorname{MAXO}(K, 5)$

$200 \quad X M I N=X(1)$

$X M A X=X(1)$

DO $250 \quad I=1, N$

IF $(X(I) . L T . X M I N) \quad X M I N=X(I)$

FRE 110

FRE 120

FRE 130

FRE 140

FRE 150

FRE 160

IF $(X(I) . G T . X M A X) \quad X M A X=X(I)$

FRE 170

FRE 180

CONTINUE

IF (C) $300,260,300$

260 RANGE $=X M A X-X M I N$

C=RANGE / FLOAT $(K-1)$

300 IF (NSTART.EQ.0) GO TO 500

START $=X M I N-0.5 * \mathrm{C}$

500 DO $510 \quad I=1, K$

$510 \quad F(I)=0.0$

DO $520 \quad I=1, N$

$J=(X(I)-S T A R T) / C+1.0$

FRE 190

FRE 200

FRE 210

FRE 220

FRE 230

FRE 240

FRE 250

FRE 260

FRE 270

FRE 280

$520 \quad F(J)=F(J)+1.0$

IF (LIMITS.EQ.0) RETURN

FRE 290

FRE 300

$600 \quad X L(1)=S T A R T$

$\mathrm{XU}(1)=\mathrm{XL}(1)+\mathrm{C}$

DO $610 \quad I=2, K$

FRE 310

FRE 320

$X L(I)=X L(I-I)+C$

$610 \quad X U(I)=X L(I)+C$

RETURN

END

FRE 330

FRE 340

FRE 350

FRE 360

FRE 370 
FUNCTION FSIN (X)

VERSION 5.00

FSIN

$5 / 15 / 70$

THIS FUNCTION IS TO TRAP IF ARGUMENT IS GREATER THAN $3.3 E 7$ IN ABSOLUTE VALUE BEFORE SYSTEM DOES. RESULT SET $=0.0$

COMMON /CONSLB / XTRIG, XEXP

IF (ABS $(X)$.GT.XTRIG) GO TO 20

$F S I N=S I N(X)$

10 RETURN

20 CALL ERROR (104)

FSIN $=0$.

GO TO 10

END
FSI $\quad .10$

FSI 20

FSI 30

FSI 40

FSI 50

FSI 60

FSI 70

FSI 80

FSI 90

FSI 100

FSI 110

FSI 120

FSI 130

FSI 140

FUNCTION FSQRT $(X)$

C VERSION 5.00 FSQRT 5/15/70

FSQ 10

FSQ 20

FSQRT CHECKS $X$ FOR NEGATIVE VALUES.

FSQ 23

FSQ 25

FSQ 30

FSQ 40

FSQ 50

FSQ 60

FSQ 70

FSQ 80

GO TO 10

FSQ 90

END

FTA 10

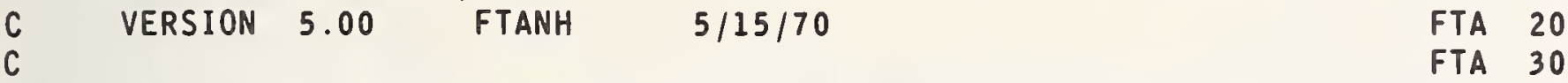

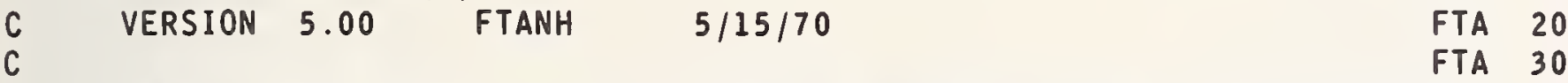

C SINCE TANH FUNCTION USES EXP FUNCTION FTANH CHECKS TO SEE IF THEFTA 40

C ABSOLUTE VALUE OF $2 * X$ IS GREATER THAN XEXP (OR 88.3). IF THISFTA 50

C IS THE CASE, AND ERROR MESSAGE IS PRINTED AND FTANH $=0$. FTA 60

FTA 70

COMMON / CONSLB / XTRIG, XEXP

IF $\left(A B S\left(2{ }^{*} X\right)\right.$. LE.XEXP) GO TO 20

FTA 80

FTA 90

FTA 100

FTANH $=0.0$

FTA 110

FTA 120

FTANH $=$ TANH $(X)$

GO TO 10

FTA 130

END

FTA 140 
SUBROUTINE FUNCT

C VERSION 5.00 FUNCT $5 / 15 / 70$

FUN 10

COMMON /BLOCRC/NRC, RC (12600)

FUN 20

COMMON /BLOCKD/IARGS(100),KIND (100), ARGTAB (100), NRMAX, NROW,NCOL,

1 NARGS, VWXYZ(8), NERROR

DIMENSION ARGS(100)

EQUIVALENCE (ARGS(1), RC (12501))

COMMON /BLOCKE/ NAME (4),L1,L2, ISRFLG

COMMON /CONSTS/ PI, E, HALFPI ,DEG, RAD, XALOG

COMMON /SCRAT/ NS, NS2, A (13500)

DIMENSION II (4), KK (4)

EQUIVALENCE (I I (1), I I), (II (2), I 2), (I I (3), I 3), (I I (4), I 4)

FUN 30

FUN 40

FUN 50

FUN 60

FUN 70

FUN 80

FUN 90

FUN 100

FUN 110

FUN 120

FUN 130

THIS SUBROUTINE HANDLES ALL TWO, THREE, AND FOUR ARGUMENT FUNCTIONFUN 140

IF THE FIRST ARGUMENT IS A CONSTANT, THE FUNCION IS EVALUATED

FUN 150

ONLY ONCE.

\begin{tabular}{|c|c|c|c|c|c|}
\hline$L 2=1$ & SIN & $L 2=2$ & $\cos$ & $L 2=3$ & TAN \\
\hline$L 2=4$ & COT & $L 2=5$ & ARCSIN & $L 2=6$ & ARCCOS \\
\hline$L 2=7$ & ARCTAN & $L 2=8$ & ARCCOT & $L 2=9$ & SIND \\
\hline$L 2=10$ & COSD & $L 2=11$ & TAND & $L 2=12$ & COTD \\
\hline$L 2=13$ & ASIND & $L 2=14$ & ACOSD & $L 2=15$ & ATAND \\
\hline$L 2=16$ & ACOTD & $L 2=17$ & SQRT & $L 2=18$ & EXPONENT \\
\hline$L 2=19$ & NEGEXP & $L 2=20$ & LOGE & $L 2=21$ & LOGTEN \\
\hline$L 2=22$ & ANT I LO & $L 2=23$ & SINH & $L 2=24$ & $\mathrm{COSH}$ \\
\hline$L 2=25$ & TANH & $L 2=26$ & COTH & $L 2=27$ & ASINH \\
\hline$L 2=28$ & $A C O S H$ & $L 2=29$ & ATANH & $L 2=30$ & ACOTH \\
\hline$L 2=31$ & ABSOLU & $L 2=32$ & INTEGE & $L 2=33$ & FRACT I \\
\hline$L 2=34$ & SQUARE & & & & \\
\hline
\end{tabular}

FUN 160

FUN 170

FUN 180

FUN 190

FUN 200

FUN 210

FUN 220

FUN 230

FUN 240

FUN 250

FUN 260

FUN 270

FUN 280

FUN 290

FUN 300

IF (NARGS.LT.2.OR.NARGS.GT.4) CALL ERROR (10)

IF (NARGS.EQ.3) CALL ERROR (29)

FUN 310

FUN 315

DO $30 I=1$, NARGS

$K K(I)=1$

CALL ADRESS (I,II(I))

IF (II (I)) $20,10,30$

10 CALL ERROR (20)

GO TO 30

$20 \quad K K(I)=0$

II (I) $=-I I$ (I)

30 CONTINUE

IF (KK (NARGS) .EQ.0) CALL ERROR (11)

IF (NRMAX.LE .0) CALL ERROR (9)

IF (NERROR . NE . O) RETURN

$N R=N R M A X$

IF (NARGS.EQ.4) GO TO 36

KK $(4)=1$

$I 4=I 2$

IF (NARGS.EQ.3) $\quad 14=13$

$36 \quad I L=I 4$

$I L L=1$

IF (KK (1).EQ.1) GO TO 40

IF (NARGS.EQ.2) NR=1

40 DO $1010 I=1, N R$

GO TO $(50,1005)$, ILL

$50 \quad X=R C(I I)$

GO TO $(110,120,130,140,150,160,170,180,190,200$,

$1210,220,230,240,250,260,270,280,290,300$,

$2310,320,330,340,350,360,370,380,390,400$,

FUN 320

FUN 330

FUN 340

FUN 350

FUN 360

FUN 370

FUN 380

FUN 390

FUN 400

FUN 410

FUN 420

FUN 430

FUN 440

FUN 450

FUN 460

FUN 470

FUN 480

FUN 490

FUN 495

FUN 500

FUN 520

FUN 530

FUN 535

FUN 540

FUN 550

FUN 560

FUN 570 


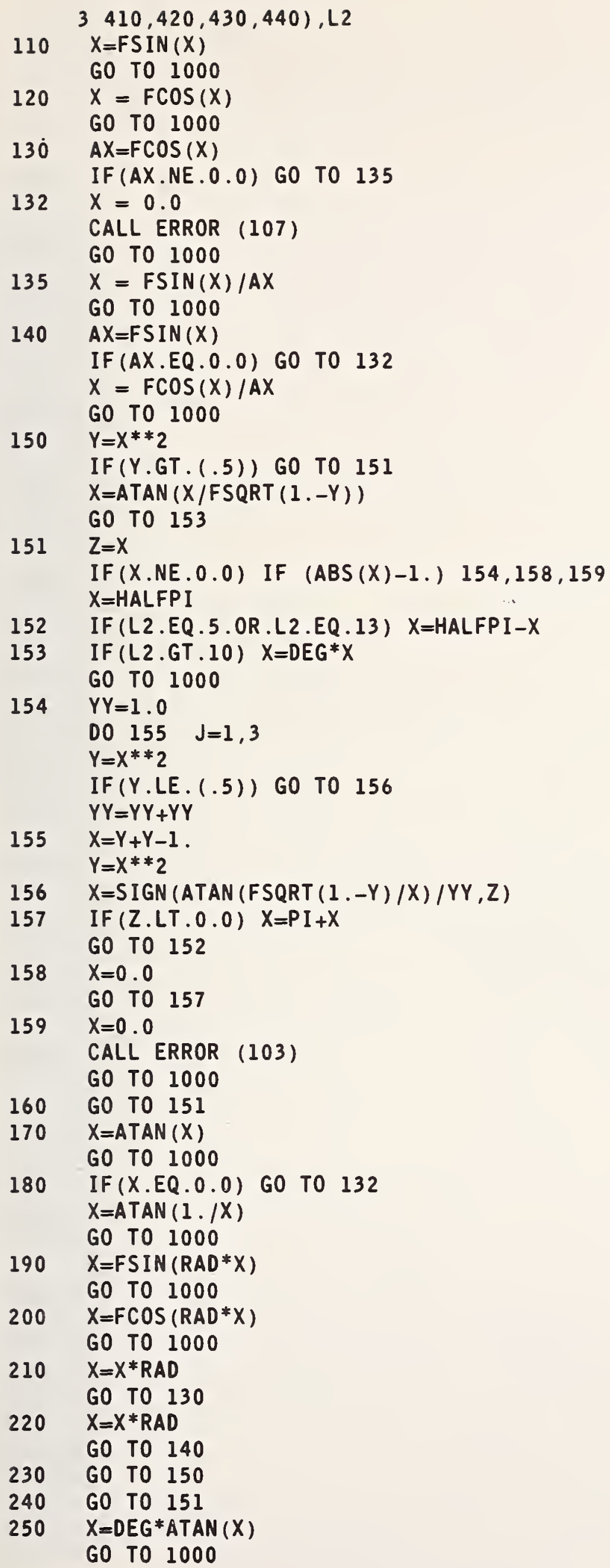

FUN 750

FUN 760

FUN 770

FUN 780

FUN 790

FUN 800

FUN 810

FUN 820

FUN 830

FUN 840

FUN 850

FUN 860

FUN 870

FUN 880

FUN 890

FUN 900

FUN 910

FUN 920

FUN 930

FUN 940

FUN 950

FUN 960

FUN 970

FUN 980

FUN 990

FUN 1000

FUN 1010

FUN 1020

FUN 1030

FUN 1040

FUN 1050

FUN 1060

FUN 1070

FUN 1080

FUN 1090

FUN 1100

FUN 1110

FUN 1120

FUN1130

FUN1 140

FUN1150

FUN1160 


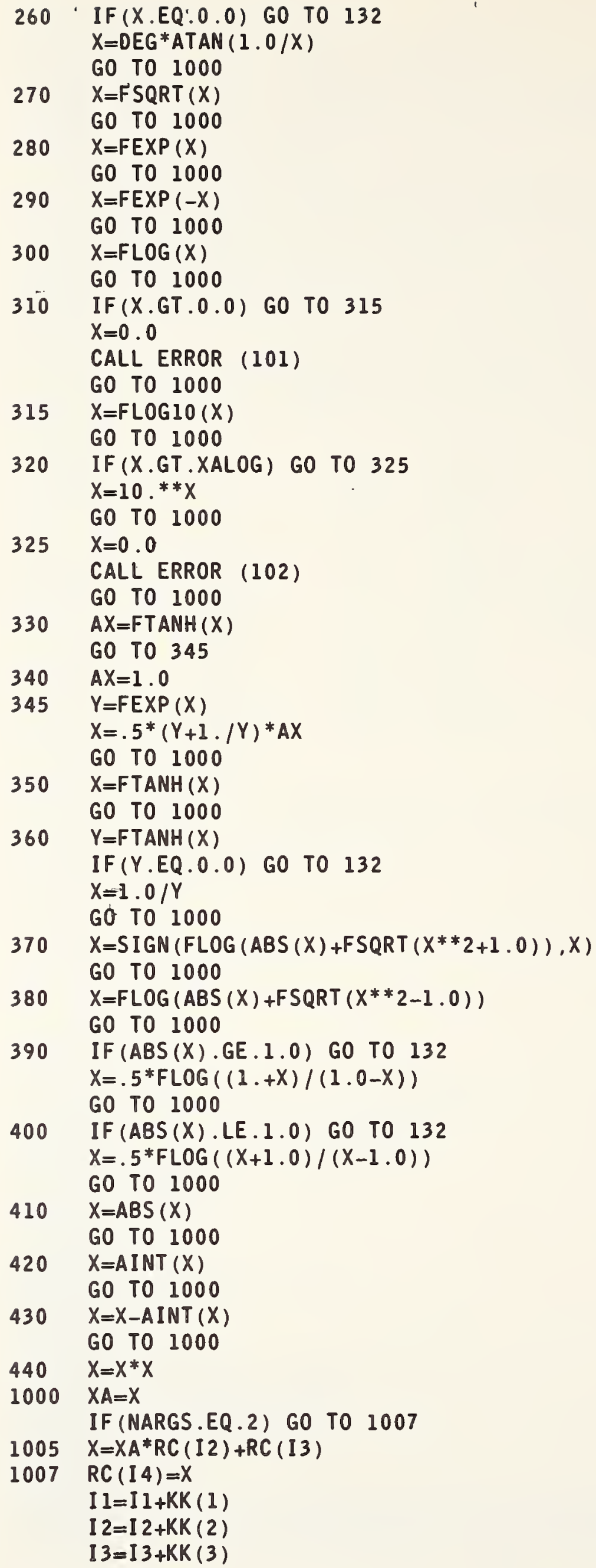


$I 4=I 4+K K(4)$

IF (I.EQ.1.AND .NARGS .NE . 2.AND.KIND(1).EQ.1) ILL=2

FUN 1720

FUN 1722

FUN1725

FUN 1730

IF (KIND(1).EQ.1.AND.NR.EQ.1) CALL VECTOR (RC(IL),IL) RETURN

END

FUN 1740

FUN1750 
COMMON /BLOCKD/ IARGS(100), KIND(100), ARGTAB (100), NRMAX, NROW, NCOL, NGEN 40

IARGS , VWXYZ (8), NERROR

GEN 50

DIMENSION ARGS(100)

GEN 60

EQUIVALENCE (ARGS(1), RC (12501))

GEN 70

C DELETE BLOCKE

GEN 80

GENERATE

NARGS MUST BE .GE. 4 AND EVEN

IF (NARGS.GE.4.AND.MOD(NARGS,2).EQ.0) GO TO 10

GEN 90

GEN 100

CALL ERROR (10)

GO TO 90

C GET STORAGE COLUMN ADDRESS

GEN 110

GEN 120

GEN 130

GEN 140

10 CALL ADRESS (NARGS,J)

IF (J.GT.0) GO TO 20

GEN 150

CALL ERROR (3)

GO TO 90

20 IF (NERROR.NE.0) GO TO 90

C CONVERT INTEGERS TO FLOATING POINT

DO $30 \quad I=2$, NARGS

IF (KIND (I-1).EQ.0) ARGS (I-1)=IARGS (I-1)

30 CONTINUE

$R C(J)=A R G S(I)$

NDROW $=J+N R O W-1$

DO $70 I=4, N A R G S, 2$

IF (ARGS $(I-3) \cdot G T$.ARGS $(I-1)) \quad \operatorname{ARGS}(I-2)=S I G N(\operatorname{ARGS}(I-2),-1$.)

$S=S I G N(1 ., \operatorname{ARGS}(I-2))$

$\operatorname{ENDER}=\operatorname{ARGS}(I-1)-.01 * \operatorname{ARGS}(I-2)$

$40 \quad \mathrm{~J}=\mathrm{J}+1$

$R C(J)=R C(J-1)+A R G S(I-2)$

IF ( $S^{*}(R C(J)$-ENDER) $50,60,60$

C NOT DONE

50 IF (J.LT.NDROW) GO TO 40

C EXCEEDED COLUMN LENGTH

CALL ERROR (201)

GO TO 80

C PASSES GENERATE UPPER BOUND, SET IN UPPER BOUND

GEN 160

GEN 170

GEN 180

GEN 190

GEN 200

GEN 210

GEN 220

GEN 230

GEN 240

GEN 250

GEN 260

GEN 270

GEN 280

GEN 290

GEN 300

GEN 310

GEN 320

GEN 330

GEN 340

GEN 350

GEN 360

GEN 370

GEN 380

GEN 390

GEN 400

CONTINUE

NRMAX = MAXO (NRMAX, J-NDROW+NROW)

RETURN

END

GEN 410

GEN 420

GEN 430 
C VERSION 5.00 GQUAD 5/15/70

C WRITTEN BY DAVID HOGBEN SEL, NBS. $8 / 18 / 69$.

QUA 10

COMMON /BLOCKD/ IARGS (100), KIND(100), ARGTAB (100), NRMAX, NROW, NCOL, NQUA 40

IARGS, VWXYZ (8), NERROR

COMMON /BLOCRC/ NRC, RC(12600)

DIMENSION ARGS $(100)$

EQUIVALENCE (ARGS(1), RC (12501))

C DOUBLE PRECISION USED TO AVOID NOISE IN $8 T H$ DIGIT.

C SLIGHT NOISE MAY BE LEFT DUE TO CONVERSION FROM DP TO SP

DOUBLE PRECISION C, B, BPA, BMA, DELGQ, STOREI, STORE2

QUA 50

QUA 60

QUA 70

QUA 80

QUA 100

QUA 110

QUA 120

C TAKEN FROM SYMBOLIC LISTING PAGE 251 (YELLOW COVER) JULY,1965

QUA 130

C A GOES TO C BECAUSE A DIMENSIONED
10 IF (NARGS.EQ.5) GO TO 20

CALL ERROR (10)

RETURN

C N MUST BE AN EXACT MULTIPLE OF 4 AND LESS THAN NROW

$20 \quad N G Q=I N T(A R G S(1)) * K I N D(1)+\operatorname{IARGS}(1) *(1-K I N D(1))$

IF (MOD(NGQ,4).EQ.0.AND.NGQ.GT.0) GO TO 50

30 CALL ERROR (3)

RETURN

40 CALL ERROR (10)

RETURN

50 IF (NGQ.GT.NROW) GO TO 30

C RESET NRMAX IF NECESSARY

NRMAX $=$ MAXO (NGQ , NRMAX)

CALL ADRESS (4,JPGQ)

IF (JPGQ) $30,40,60$

$60 \quad J P G Q=J P G Q-1$

CALL ADRESS $(5, J W G Q)$

QUA 140

QUA 150

QUA 160

QUA 170

QUA 180

QUA 190

QUA 200

QUA 210

QUA 220

QUA 230

QUA 240

QUA 250

QUA 260

QUA 270

QUA 280

QUA 290

QUA 300

QUA 310

IF (JWGQ) $30,40,70$

$70 \quad J W G Q=J W G Q-1$

IF (NERROR.NE.O) RETURN

C=ARGS (2)*FLOAT (KIND (2)) +FLOAT (IARGS (2)*(1-KIND (2)))

B=ARGS (3)*FLOAT (KIND (3)) +FLOAT (IARGS (3)*(1-KIND (3)))

$D E L G Q=N G Q$

$D E L G Q=4 . D O *(B-C) / D E L G Q$

DO $80 \quad I=1, N G Q, 4$

$\mathrm{B}=\mathrm{C}+\mathrm{DELGQ}$

$B P A=(B+C) / 2 . D 0$

$B M A=(B-C) / 2 . D 0$

$\mathrm{K} I=I+J P G Q$

$\mathrm{K} 2=\mathrm{I}+\mathrm{JWGQ}$

STORE $1=-.86113631159405300 * B M A$

STORE2 $=-.33998104358485600 * B M A$

$R C(K 1)=F D P C O N(S T O R E I+B P A)$

$R C(K 1+1)=F D P C O N(S T O R E 2+B P A)$

$R C(K 1+2)=$ FDPCON (BPA-STORE2)

$R C(K 1+3)=$ FDPCON $(B P A-S T O R E 1)$

$R C(K 2)=F D P C O N(.347854845137454 D 0 * B M A)$

$R C(K 2+1)=F D P C O N(.652145154862546 D 0 * B M A)$

$R C(K 2+2)=R C(K 2+1)$

$R C(K 2+3)=R C(K 2)$

80

$\mathrm{C}=\mathrm{B}$

RETURN

QUA 320

QUA 330

QUA 340

QUA 350

QUA 360

QUA 370

QUA 380

QUA 390

QUA 400

QUA 410

QUA 420

QUA 430

QUA 440

QUA 450

QUA 460

QUA 470

QUA 480

QUA 490

QUA 500

QUA 510

QUA 520

QUA 530

QUA 540

QUA 550

QUA 560

END

QUA 570 
SUBROUTINE HDIAG ( $A, N, I E G E N, U, C O L, V E C T O R, N R O W, H)$

HDI 10

VERSION 5.00 HDIAG $5 / 15 / 70$

DIMENSION A(NROW, 1), H(54,54), U $(54,54), X(54), I Q(54), \operatorname{COL}(1)$, VEHDI 30

ICTOR (NROW, 1), IEGEN (2)

HDI 40

MIHDI3, FORTRAN II DIAGONALIZATION OF A REAL SYMMETRIC MATRIX BY

THE JACOBI METHOD.

MAY 19,1959

CALLING SEQUENCE FOR DIAGONALIZATION

CALL HDIAG ( $H, N$, IEGEN, U, NR)

WHERE H IS THE ARRAY TO BE DIAGONALIZED.

$N$ IS THE ORDER OF THE MATRIX, H.

HDI 50

HDI 60

HDI 70

HDI 80

HDI 90

HDI 100

IEGEN MUST BE SET UNEQUAL TO ZERO IF ONLY EIGENVALUES ARE

HDI 110

TO BE COMPUTED.

HDI 120

HDI 130

HDI 140

IEGEN MUST BE SET EQUAL TO ZERO IF EIGENVALUES AND EIGENVECTORS

HDI 150

ARE TO BE COMPUTED.

U IS THE UNITARY MATRIX USED FOR FORMATION OF THE EIGENVECTORS.

HDI 160

HDI 170

HDI 180

HDI 190

NR IS THE NUMBER OF ROTATIONS.

HDI 200

A DIMENSION STATEMENT MUST BE INSERTED IN THE SUBROUTINE.

HDI 210

DIMENSION $H(\mathbb{N}, \boldsymbol{N}), U(\mathbb{N}, \boldsymbol{N}), X(\boldsymbol{N}), I Q(N)$

HDI 220

HDI 230

HDI 240

HDI 250

THE SUBROUTINE OPERATES ONLY ON THE ELEMENTS OF H THAT ARE TO THE HDI 260 RIGHT OF THE MAIN DIAGONAL. THUS, ONLY A TRIANGULAR

HDI 270 SECTION NEED BE STORED IN THE ARRAY H.

HDI 280

HDI 290

HDI 300

DO $10 \quad I=1, N$

HDI 310

DO $10 \mathrm{~J}=1, \mathrm{~N}$

HDI 320

$H(I, J)=A(I, J)$

HDI 330

CONTINUE

HDI 340

IF (IEGEN (1)) $60,20,60$

HDI 350

DO $50 \quad I=1, N$

HDI 360

DO $50 \mathrm{~J}=1, \mathrm{~N}$

HDI 370

IF (I-J) $40,30,40$

HDI 380

$30 \quad U(I, J)=1.0$

GO TO 50

HDI 390

HDI 400

$U(I, J)=0$.

HDI 410

CONTINUE

HDI 420

HDI 430

$N R=0$

IF (N-1) $440,440,70$

HDI 440

HDI 450

HDI 460

SCAN FOR LARGEST OFF DIAGONAL ELEMENT IN EACH ROW

HDI 470

$X$ (I) CONTAINS LARGEST ELEMENT IN ITH ROW

IQ(I) HOLDS SECOND SUBSCRIPT DEFINING POSITION OF ELEMENT

HDI 480

HDI 490

HDI 500

NMI $1=N-1$

DO $90 \mathrm{I}=1, \mathrm{NMI} I$

HDI 510

$X(I)=0$.

$I P L I=I+1$

HDI 520

HDI 530

DO $90 \mathrm{~J}=$ IPLI,N

IF $(X(I)-A B S(H(I, J))) 80,80,90$

HDI 540

HDI 550

HDI 560

$X(I)=A B S(H(I, J))$

IQ (I ) $=\mathrm{J}$

90 CONTINUE 
C

C

C

C

C

100 DO $130 \quad I=1$, NMI 1

IF (I-1) $120,120,110$

110 IF (XMAX-X(I)) $120,130,130$

$120 \quad X$ MAX $=X$ (I)

IPIV $=I$

$J P I V=I Q(I)$

130 CONTINUE

C

C

IS MAX. $X$ (I) EQUAL TO ZERO, IF LESS THAN HDTEST, REVISE HDTEST

IF (XMAX) $440,440,140$

140 IF (HDTEST) $160,160,150$

150 IF (XMAX-HDTEST) $160,160,190$

$160 \quad \operatorname{HDIMIN}=A B S(H(1,1))$

DO $180 \quad I=2, N$

IF (HDIMIN-ABS(H(I,I))) $180,180,170$

$170 \quad \operatorname{HDIMIN}=A B S(H(I, I))$

180 CONTINUE

C

HDTEST $=$ HD IMIN*RAP

C

C RETURN IF MAX.H(I,J)LESS THAN $(2 * *-27)$ ABSF $(H(K, K)-M I N)$

IF (HDTEST-XMAX) $190,440,440$

$190 \quad N R=N R+1$

C

C

COMPUTE TANGENT, SINE AND COSINE,H(I,I),H(J,J)
HDI 600

HDI 610

HDI 620

HDI 630

HDI 640

HDI 650

HDI 660

HDI 670

HDI 680

HDI 690

HDI 700

HDI 710

HDI 720

HDI 730

HDI 740

HDI 750

HDI 760

HDI 770

HDI 780

HDI 790

HDI 800

HDI 810

HDI 820

HDI 830

HDI 840

HDI 850

HDI 860

HDI 870

HDI 880

HDI 890

HDI 900

HDI 910

HDI 920

TANG $=S I G N(2.0,(H$ (IPIV, IPIV) $-H(J P I V, J P I V))) * H(I P I V, J P I V) /(A B S(H$ (IPIHDI 930

IV, IPIV) -H (JPIV, JPIV)) +FSQRT ( (H (IPIV, IPIV)-H (JPIV, JPIV) )** $2+4.0 * H($ IHDI 940 2PIV, JPIV) **2))

COSINE $=1 . / F S Q R T(1.0+$ TANG**2)

HDI 950

HDI 960

HDI 970

HDI 980

$H I I=H(I P I V, I P I V)$

VHDI 990

1))

HDI 1000

$H(J P I V, J P I V)=C O S I N E * * 2 *(H(J P I V, J P I V)-T A N G *(2 . * H(I P I V, J P I V)-T A N G * H I H D I 1010$

1I))

HDI 1020

HDI 1030

HDI 1040

HDI 1050

HDI 1060

HDI 1070

HDI 1080

HDI 1090

HDI 1100

HDI 1110

HDI 1120

HDI 1130

HDI 1140

HDI 1150

HDI 1160

HDI 1170

HDI 1180 
DO $280 \quad I=1, N M I 1$

IF (I-IPIV) $230,280,220$

220 IF (I-JPIV) $230,280,230$

230 IF (IQ (I)-IPIV) $240,250,240$

240 IF (IQ(I)-JPIV) $280,250,280$

HDI 1200

HDI 1210

HDI 1220

HDI 1230

HDI 1240

HDI1250

$K=I Q(I)$

$H T E M P=H(I, K)$

HDI 1260

$H(I, K)=0$.

HDI 1270

$I P L 1=I+1$

HDI 1280

$X(I)=0$.

C

C

SEARCH IN DEPLETED ROW FOR NEW MAXIMUM

HDI 1290

HDI 1300

HDI 1310

HDI 1320

DO $270 \mathrm{~J}=I \mathrm{PLI}, \mathrm{N}$

IF $(X(I)-A B S(H(I, J))) 260,260,270$

HDI 1330

HDI 1340

HDI 1350

$260 X(I)=A B S(H(I, J))$

$I Q(I)=J$

270 CONTINUE

$H(\mathbb{I}, K)=H T E M P$

280 CONTINUE

HDI 1360

HDI 1370

HDI 1380

HDI 1390

HDI 1400

$X(I P I V)=0$

$X($ JPIV $)=0$.

HDI 1410

HDI 1420

HDI 1430

C

C CHANGE THE OTHER ELEMENTS OF $H$

HDI 1440

DO $410 \quad I=1, N$

C

IF (I-IPIV) $290,410,330$

$290 \quad H T E M P=H(I, I P I V)$

$H(I, I P I V)=C O S I N E * H T E M P+S I N E * H(I, J P I V)$

HDI 1450

HDI 1460

HDI 1470

HDI 1480

HDI 1490

HDI 1500

IF $(X(I)-A B S(H(I, I P I V))) 300,310,310$

HDI 1510

HDI 1520

HDI 1530

HDI 1540

HDI 1550

HDI 1560

HDI 1570

HDI 1580

HDI 1590

HDI 1600

HDI 1610

HDI 1620

HDI 1630

HDI 1640

HDI 1650

HDI1660

HDI 1670

HDI 1680

HDI 1690

HDI 1700

HDI 1710

HD 11720

HDI1730

HDI 1740

HDI 1750

HDI 1760

HDI 1770 
IQ (JPIV) $=$
$410 \quad$ CONTINUE

C

C TEST FOR COMPUTATION OF EIGENVECTORS

C

420

IF (IEGEN (1)) $100,420,100$

DO $430 \quad I=1, N$

HTEMP $=U(I, I P I V)$

$U(I, I P I V)=C O S I N E * H T E M P+S I N E * U(I, J P I V)$

$430 U(I, J P I V)=-S I N E * H T E M P+\operatorname{COSINE*U}(I, J P I V)$

GO TO 100

440 IF (IEGEN (2)-2) $450,470,450$

450 DO $460 \quad I=1, N$

$\operatorname{COL}(I)=H(I, I)$

460 CONTINUE

IF (IEGEN (2).NE.3) GO TO 490

470 DO $480 \quad J=1, N$

DO $480 \quad I=1, N$

$\operatorname{VECTOR}(I, J)=U(I, J)$

480 CONTINUE

490 RETURN

END
HDI 1780

HDI 1790

HDI 1800

HDI 1810

HDI 1820

HDI 1830

HDI 1840

HDI 1850

HDI 1860

HDI 1870

HDI 1880

HDI 1890

HDI 1900

HDI 1910

HDI 1920

HDI 1930

HDI 1940

HDI 1950

HDI 1960

HDI 1970

HDI 1980

HDI 1990 
SUBROUTINE HEADS (LOC,NOO,IN, IO)

HEA 10

VERSION 5.00 HEADS $5 / 15 / 70$

HEA 20

REWRITEN BY S PEAVY

$8 / 8 / 69$

HEA 30

THIS SUBROUTINE INSERTS HEADINGS (IF AVAILABE) OVER THE COLUMNS

HEA 40

WHEN NO FORMAT IS SPECIFIED

LOC LOCATION WHERE COL NUMBERS ARE

HEA 50

HEA 60

HEA 70

NOO NO OF COLUMN HEADINGS TO LOOK FOR. NOO LESS THAN OR $=8$.

HEA 80

IN IF IN $=0$ NEW HEADINGS

IF IN $=1$ PRINT OUT HEADINGS FROM RREVIOUS PAGE

HEA 90

HEA 100

HEA 110

HEA 120

IF A HEADING EXISTS THE 12 CHARACTER HEADING WILL BE PRINTED.

HEA 130

OTHERWISE THE HEADING COLUMN $X X X X$ IS TO BE USED WHERE $X X X X$ IS THE HEA 140 NUMBER CONVERTED FOR DECIMAL PRINTOUT. THE HEADINGS ARE PRINTED

HEA 150

OVER THE DATA WHICH IS IF FORMAT IPBE15.6

HEA 160

IO $=0$ PRINT HEADINGS

IO NOT $=0$ DO NOT PRINT HEADINGS

HEA 170

HEA 180

HEA 190

COMMON /ABCDEF/ $L(48)$

HEA 200

COMMON /HEADER/ NOCARD $(80), \operatorname{ITLE}(60,6)$, LNCNT , IPRINT , NPAGE, IPUNCH

HEA 210

COMMON /FMAT / IFMTX (6), IOSWT, IFMTS (6), LHEAD (96)

DIMENSION LOC (1)

DIMENSION ICOLHD (7)

HEA 220

HEA 230

HEA 240

DATA ICOLHD (1) , ICO

$1 \mathrm{COLHD}(7) / 1 \mathrm{HC}, 1 \mathrm{HO}, 1 \mathrm{HL}, 1 \mathrm{HU}, 1 \mathrm{HM}, 1 \mathrm{HN}, 1 \mathrm{H} /$

HEA 250

NO $=$ NOO

IF (NO.GT.8) $\quad \mathrm{NO}=8$

IF (IN.NE.0) GO TO 80

$I R=1$

DO $70 \quad I=1$, NO

CALL PREPAK (5, IND, I ,LOC (I) , LHEAD (IR))

IF (IND.NE.0) GO TO 10

$I R=I R+12$

GO TO 70

HEA 270

HEA 280

HEA 290

HEA 300

HEA 310

HEA 320

HEA 330

HEA 340

HEA 350

HEA 360

DO 20 IS $=1,7$

HEA 370

LHEAD (IR) = I COLHD (IS)

$20 \quad I R=I R+1$

$K=L O C$ (I)

$K C=1000$

$K D=0$

DO 60 IS $=1,4$

$K A=K / K C$

IF (KA.NE.O) GO TO 30

HEA 380

HEA 390

HEA 400

HEA 410

HEA 420

HEA 430

HEA 440

HEA 450

IF (KD.NE.0) GO TO 40

HEA 460

LHEAD (IR) $=L(45)$

GO TO 50

$30 \quad K D=1$

$40 \quad K A P=K A+1$

$\operatorname{LHEAD}(I R)=L(K A P)$

$50 \quad I R=I R+1$

$K=K-K A * K C$

HEA 470

HEA 480

HEA 490

HEA 500

HEA 510

HEA 520

HEA 530

$K C=K C / 10$

HEA 540

$\operatorname{LHEAD}(I R)=L$ (45)

HEA 550

$I R=I R+I$

HEA 560

CONT INUE

HEA 570

IF (IO.NE.O) RETURN

HEA 580

IS $=N N^{*} 12$

HEA 590 
WRITE (IPRINT, 90) (LHEAD(I), I=1, IS)

HEA 600

RETURN

HEA 610

HEA 620

90 FORMAT $(8(3 X, 12 A 1))$

HEA 630

END

HEA 640 
SUBROUTINE HISTGM

C VERSION 5.00 HISTGM $5 / 15 / 70$

C WRITTEN BY DAVID HOGBEN, SEL, NBS. $10 / 24 / 69$.

COMMON / ABCDEF / L (48)

COMMON/BLOCRC / NRC, RC (12600)

COMMON/BLOCKD/IARGS(100), KIND(100),ARGTAB (100), NRMAX,

1 NROW, NCOL, NARGS, VWXYZ (8), NERROR

DIMENSION ARGS $(100)$

EQUIVALENCE ( ARGS(1), RC(12501))

COMMON / BLOCKE / NAME (4),L1,L2, I SRFLG

COMMON/HEADER/NOCARD $(80), \operatorname{ITLE}(60,6)$, LNCNT , IPRINT , NPAGE , IPUNCH

COMMON / SCRAT /NS, NS2, A (13500)

HIS 10

HIS 20

HISTOGRAM FOR MIDPOINTS IN COLUMN (C), FREQUENCIES IN COLUMN (C)

NHISTOGRAM (C), (C) L2=2 DOES NOT CALL NEW PAGE OR PRINT BLANK LINE BETWEEN CELLS OR HEADING.

\section{$L 2=L 2-7$}

C

C

10 IF (NARGS.EQ.2) GO TO 20

CALL ERROR (10)

RETURN

20 CALL ADRESS $(1, J 1)$

IF (J I) $30,40,50$

30 CALL ERROR (3)

RETURN

40 CALL ERROR (11)

RETURN

50 CALL ADRESS $(2, \mathrm{~J} 2)$

IF (J2) $30,40,60$

60 IF (NRMAX.GT.0) GO TO 70

CALL ERROR (9)

RETURN

HIS 30

HIS 40

HIS 50

HIS 60

HIS 70

HIS 80

HIS 90

HIS 100

HIS 110

HIS 120

HIS 130

HIS 140

HIS 150

HIS 160

HIS 170

HIS 180

HIS 190

HIS 200

HIS 210

HIS 220

HIS 230

HIS 240

HIS 250

HIS 260

HIS 270

HIS 280

HIS 290

HIS 300

HIS 310

70 IF (NERROR.NE.0) RETURN

80 FORMAT (// 25X,35HHISTOGRAM FOR FREQUENCIES IN COLUMN, I5, 22H, MID-HIS 320
IPOINTS IN COLUMN, I5//3X,10HMID-POINTS,7X,9HFREQUENCY /) HIS 330

85 FORMAT (1X,14A1,2X, I5,3X, 95AI)

HIS 340

90 FORMAT (25X, 95Al)

100 CALL RFORMT (RC (J1), NRMAX, 8,NW1,NDEC $1,13, A(1), A(1), 0,0)$

HIS 350

NBLANK $=15-\mathrm{NWI}$

IF (L2.EQ.2) GO TO 110

HIS 360

HIS 370

CALL PAGE (4)

WRITE (IPRINT, 80) IARGS (1), IARGS (2)

HIS 380

HIS 390

$110 \quad L O C 1=J 1$

$\mathrm{LOC} 2=\mathrm{J} 2$

DO $200 \quad I=1$, NRMAX

CALL RFORMT (A (1), 1,8,NW1,NDEC1, $0, R C(L O C 1), A(1), N B L A N K, 1)$

HIS 400

HIS 410

HIS 420

HIS 430

LFREQ $=R C(L O C 2)+0.001$

IF (LFREQ.GT.0) GO TO 140

WRITE (IPRINT, 85) (A (I 1),I $1=2,15)$, LFREQ

GO TO 150

140 I2END = MINO (LFREQ,95)

WRITE (IPRINT , 85) (A (I 1), I $1=2,15)$, LFREQ, ( $(40), I 2=1$, I 2END)

IF (LFREQ.LE.95) GO TO 150

I3END $=$ LFREQ-95

WRITE (IPRINT, 90) (L (40), I3=1, I3END)

$150 \quad \mathrm{LOCl}=\mathrm{LOCl}+1$

$200 \quad \mathrm{LOC} 2=\mathrm{LOC} 2+1$

RETURN

END

HIS 440

HIS 450

HIS 460

HIS 470

HIS 480

HIS 490

HIS 500

HIS 510

HIS 520

HIS 530

HIS 540

HIS 550

HIS 560

HIS 570 
SUBROUTINE IFS

C VERSION 5.00

IFS

IFS 10

COMMON /BLOCRC/ NRC, RC(12600)

IFS 20

COMMON /BLOCKD/ IARGS (100), KIND(100), ARGTAB (100), NRMAX, NROW, NCOL, NIFS 40

IARGS, VWXYZ (8), NERROR

DIMENSION ARGS(100)

EQUIVALENCE (ARGS (1), RC (12501))

COMMON /BLOCKE/ NAME (4),L1,L2, I SRFLG

COMMON /BLOCKX/ INDEX $(6,8)$, LEVEL

DIMENSION II (3), K(3), NNN (7)

EQUIVALENCE (I I, I I (1)), (I 2, I I (2)), ( I 3, I I (3))

LOGICAL TWOARG

IFS 50

IFS 60

IFS 70

IFS 80

IFS 90

IFS 100

IFS 110

IFS 120

IFS 130

THIS COMMAND MAY APPEAR ONLY AS A STORED COMMAND.

IFS 140

IFS 150

IFS 160

IFLT, IFEQ，IFGT，IFGE，IFNE, IFLE CORRESPOND TO L2=9, 14

IFS 170

COMPARE $\quad L 2=15$

IFS 175

IFS 180

COMMANDS MAY HAVE 2 OR 3 ARGUMENTS (ONLY IFEQ AND IFNE MAY HAVE 3) IFS 190

ANY ARGUMENT MAY BE OF ANY TYPE, COLUMN NUMBER OR CONSTANT.

COMPARE MUST HAVE 3 ARGUMENTS

IFS 200

IFS 205

IFS 210

IN COMPARE THE TEST IF FOR RELATIVE ERROR AND GOES

IFS 220

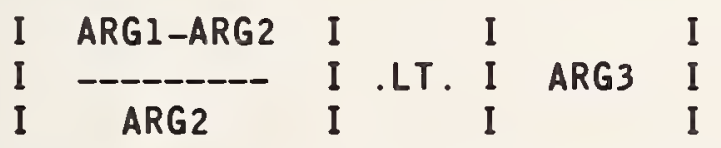

IFS 230

IFS 240

IFS 250

IFS 260

IFS 270

IFS 272

IFS 273

IFS 278

IFS 280

IF IFEQ AND IFNE CONTAIN A THRID ARGUMENT (TOLERANCE) ABSOLUTE IFS 282

ERROR WILL BE COMPUTED

ABS (ARG1-ARG2) .LT. ABS (ARG3)

A GIVEN TOLERANCE IS IGNORED ON IFLT, IFLE, IFGT, IFGE

IFS 284

IFS 286

IFS 290

IFS 300

IFS 310

IFLT 8.32 LT EVERY ENTRY OF COL 34, CONDITION IS TRUE

IFS 320

IFEQ 2. EQ. 5. CONDITION TRUE (USEFUL WHEN INCREMENTING ARGS.) IFS 330

IFS 340

IF CONDITION IS FALSE, NO ACTION IS TAKEN.

IFS 350

IF CONDITION IS TRUE, THERE ARE THO POSSIBILITIES..

IFS 360

1. IF THE TEST COMMAND IS THE LAST ONE IN THE REPEAT LOOP

IFS 370

CURRENTLY BEING EXECUTED, THE LOOP IS TERMINATED (DROPPED IFS 380

BACK TO THE NEXT OUTER LEVEL IF MORE THAN ONE LEVEL DEEP). IFS 390

2. IF THE TEST COMMAND IS NOT THE LAST ONE, ALL THAT HAPPENS IS IFS 400

THAT THE REST OF THE LOOP IS NOT PERFORMED. THAT IS, IF THE IFS 410

LOOP COUNTER HAS NOT REACHED ITS UPPER LIMIT, IT IS ADVANCED IFS 420

ONE AND THE LOOP IS BEGUN FROM THE TOP AGAIN.

IFS 430

IFS 440

IF (LEVEL.GT.0) GO TO 10

IFS 450

CALL ERROR (21)

IFS 460

GO TO 120

IFS 470

IF (NARGS.EQ.2) IF (L2-15) $40,150,40$

IFS 480

IF (NARGS.EQ.3) GO TO 30

IFS 490

CALL ERROR (10)

IFS 500

GO TO 120

IFS 510 
30 IF (L2.EQ.10.0R.L2.EQ.13.0R.L2.EQ.15) G0 T0 40

IFS 540

CALL ERROR (212)

IFS 550

NARGS $=2$

IFS 560

40 DO $60 \quad I=1, N A R G S$

IFS 570

CALL ADRESS (I, I I (I))

IFS 580

IF (II (I) ) $50,20,60$

IFS 590

$50 \quad$ I I (I) $=-$ I I (I)

IFS 600

$60 \quad K(I)=1-K I N D$ (I)

IFS 610

IF (NRMAX.NE .0.OR.KIND(1) +KIND (2).EQ.2) IF (NERROR) $120,65,120$ IFS 615

CALL ERROR (9)

GO TO 120

$65 \quad \operatorname{NNN}(4)=0$

$\operatorname{NNN}(5)=0$

$\operatorname{NNN}(6)=0$

TWOARG=NARGS .EQ 2

IFS 630

IFS 640

IFS 650

IFS 660

DO $110 \quad I=1$, NRMAX

IFS 670

IF (TWO ARG) IF (RC(II)-RC(I2)) 75,77,80

IFS 680

IFS 685

C CHECK EQ,NE WITHIN BOUNDS

IFS 690

$T=A B S(R C$ (I 1$)-R C($ I 2$))$

IF (L2.NE.15) GO T0 66

IF (RC(II).NE.0.0.AND.RC(I2).NE.0.0) GO TO 63

CALL ERROR (108)

GO TO 66

$63 T=A B S(T / R C(I 2))$

66 IF (T-ABS (RC (I3)) )70,80,80

$70 \quad N N N(5)=N N N(5)+1$

GO TO 90

C CHECK IFS WITHOUT BOUNDS

$75 \quad$ NNN $(4)=N N N(4)+1$

GO TO 100

$77 \quad \operatorname{NNN}(5)=\operatorname{NNN}(5)+1$

GO TO 100

$80 \quad N N N(6)=N N N(6)+1$

$90 \quad I 3=I 3+K(3)$

$100 \quad I I=I I+K(1)$

110 I $2=I 2+K(2)$

$\operatorname{NNN}(1)=\operatorname{NNN}(5)+\operatorname{NNN}(6)$

IFS 692

IFS 694

IFS 698

IFS 700

IFS 702

IFS 704

IFS 706

IFS 710

IFS 720

IFS 730

IFS 740

IFS 750

IFS 760

IFS 770

IFS 780

IFS 790

IFS 800

IFS 810

IFS 820

$\operatorname{NNN}(2)=\operatorname{NNN}(4)+\operatorname{NNN}(6)$

IFS 830

$\operatorname{NNN}(7)=\operatorname{NNN}(2)$

IFS 835

$\operatorname{NNN}(3)=\operatorname{NNN}(4)+N N N(5)$

IFS 840

$\operatorname{IF}(\operatorname{NNN}(L 2-8)$.EQ .0) IF (INDEX (2,LEVEL) -INDEX (3, LEVEL) ) $130,130,140$

\section{RETURN}

IFS 845

IFS 850

IFS 860

C IF-COMMAND NOT AT END OF PERFORM LOOP, ADVANCE LOOP COUNT. IFS 870

IFS 880

$130 \quad \operatorname{INDEX}(2, \operatorname{LEVEL})=\operatorname{INDEX}(3$, LEVEL $)+1$

GO TO 120

IFS 890

IFS 900

IFS 910

IF-COMMAND IS AT END OF PERFORM LOOP, TERMINATE LOOP.

IFS 920

IFS 930

IFS 940

LEVEL $=$ LEVEL -1

IFS 950

GO TO 120

150 CALL ERROR (10)

RETURN

IFS 960

END

IFS 970

IFS 980 
SUBROUTINE INFERR (I)

C VERSION 5.00 INFERR $5 / 15 / 70$

C INFORMATIVE DIAGNOSTICS 200 AND UP

INF 10

COMMON / BLOCKC / KIO, INUNIT, I SCRAT , KBDOL

INF 20

COMMON/CONLB2 / ER, ISIGD

INF 30

ISCRUN=I SCRAT

INF 40

WR ITE (ISCRUN, 500)

500

FORMAT (/4IH* INFORMATIVE DIAGNOSTIC IN ABOVE COMMAND, 43X)

INF 45

INF 50

$I=I-200$

INF 60

GO

GO TO $(401,402,403,404,405,406,407,408,409,410,411,412$, INF 90

$1413,414,415,416,417,418,419,420$,

INF 100

$2421,422,423,424,425,426,427,428,429,430,431,432,433,434,435$, INF 105

$3436,437)$, I I

401 WRITE (ISCRUN, 201)

INF 110

201

FORMAT ( $52 \mathrm{H}^{*}$ TOO MUCH DATA IN SET, READ OR GENERATE, SPILL LOST,

132X)

GO TO 900

INF 120

INF 130

INF 140

WRITE (ISCRUN, 202)

INF 150

402

202 FORMAT $\left(61 H^{*}\right.$ COMMAND NOT ALLOWED IN REPEAT MODE. EXECUTED BUT NOT SINF 170

ITORED , 23X)

INF 180

GO TO 900

INF 190

403 WRITE( ISCRUN, 203 )

INF 200

203 FORMAT (61H* VALUE REQUESTED IN SHORTEN, ACOALESCE OR AAVERAGE NOT INF 210

IFOUND, 23X)

GO TO 900

INF 220

INF 230

404 WRITE( ISCRUN, 204 )

204 FORMAT (32H* BAD HEAD. COLUMN GT 50 OR NO $/, 52 X)$

INF 240

GO TO 900

INF 250

INF 260

405 WRITE ( ISCRUN, 205)

INF 270

205 FORMAT $\left(68 \mathrm{H}^{*}\right.$ THIS COMMAND WAS NOT EXECUTED BECAUSE ITS MEANING WAS INF 280 IQUESTIONABLE, 16X)

GO TO 900

406 WRITE (ISCRUN , 206)

206 FORMAT $\left(24 H^{*}\right.$ F LESS THAN 0, SET $\left.=0,60 X\right)$

GO TO 900

407 WRITE (ISCRUN , 207)

207 FORMAT (24H* NU1 OR NU2 LESS THAN $1,60 \mathrm{X})$

GO TO 900

408 WRITE (I SCRUN , 208)

208 FORMAT $\left(33 H^{*}\right.$ NUI OR NU2 TRUNCATED TO INTEGER,51X)

GO TO 900

409 WRITE (ISCRUN, 209)

209 FORMAT (34H* IMPROPER TITLE NUMBER, ASSUMED $1,50 \mathrm{X}$ )

INF 290

INF 300

INF 310

INF 320

INF 330

INF 340

INF 350

INF 360

INF 370

INF 380

INF 390

INF 400

INF 410

GO TO 900

INF 420

410 WRITE (ISCRUN, 210$)$
210 FORMAT $\left(54 H^{*}\right.$ NO OF

INF 430

$120 X)$

GO TO 900

INF 440

INF 450

INF 460

411 WRITE (ISCRUN, 211)

INF 470

211 FORMAT (52H* ASTERISK STRING IMPLYING ,THRU, INCORRECT, IGNORED,

$132 X)$

GO TO 900

INF 480

INF 490

INF 500

INF 510

INF 520

INF 530

INF 540

INF 550

INF 560

213 FORMAT (27H* PARTIAL STORAGE OF MATRIX,57X)

GO TO 900

INF 570

414 WRITE (ISCRUN, 214) 
415 WRITE (ISCRUN, 215)

INF 590

215 FORMAT (48H* NRMAX IS NOT LARGE ENOUGH TO ALLOW ITERATION, 36X) INF 600 GO TO 900

INF 610

INF 620

416 WRITE (ISCRUN, 216)

SINF 630

1 CONSTANT. , 16X)

INF 640

GO TO 900

417 WRITE (ISCRUN, 217)

INF 650

217 FORMAT (34H* ITERATION HAS FOUND NO VALUES. ,50X)

INF 660 GO TO 900

INF 670

418 WRITE (ISCRUN, 218)

INF 680

INF 690

218 FORMAT (81H* WORKSHEET IS TOO SHORT TO ACCOMMODATE ALL THE VALUES GINF 700 IENERATED BY THIS COMMAND. , $3 X$ )

INF 710 GO TO 900

INF 720

419 WRITE (ISCRUN, 219)

INF 730

219 FORMAT (3OH* MAXMIN HAS FOUND NO EXTREMA. ,54X)

GO TO 900

INF 740

INF 750

420 WRITE (ISCRUN, 220)

INF 760

220 FORMAT (84H* MAXMIN HAS FOUND AND IGNORED A TRIAD OF $X, S$ WITH AT LINF 770 IEAST TWO IDENTICAL VALUES.

INF 780 GO TO 900

INF 790

421 WRITE (ISCRUN, 221)

INF 800

221 FORMAT (59H* MORE THAN ONE ARGUMENT IN COMMAND. ONLY FIRST ONE IS UINF 810

ISED , 25X)

GO TO 900

INF 820

INF 830

422 WRITE (ISCRUN, 222)

222 FORMAT (43H* FORMAT NOT FOUND. READABLE FORMAT IS USED, 4IX)

INF 840 GO TO 900

423 HRITE (ISCRUN, 223)

223 FORMAT (38H* ONE, SOME OR ALL WEIGHTS ARE NEGATIVE, 46X) GO TO 900

424 WRITE (ISCRUN, 224)

INF 850

INF 860

INF 870

INF 880

INF 890

INF 900

224 FORMAT (48H* ALL WEIGHTS ARE ZERO. COMMAND IS NOT EXECUTED,36X)

GO TO 900

INF 910

INF 920

INF 930

425 WRITE (ISCRUN , 225)

FORMAT (81H* ARG FOR BESIN,BESJN,BESKN GIVES A RESULT TOO LARGE/SMAINF 940
1 LL. COMMAND NOT EXECÜTED.,3X)
INF 950

GO TO 900

INF 960

INF 970

426 WRITE (ISCRUN, 226)

FORMAT (73H* COLUMN NOT LONG ENOUGH TO STORE ALL ELEMENTS. ONLY NROINF 980
IW WILL BE STORED.,11X)
INF 990

GO TO 900

INF 1000

INF 1010

427 WRITE (ISCRUN, 227)

AINF 1020

IVAILABLE WILL BE USED.,6X)

INF 1025

INF 1030

GO TO 900

INF 1040

428 WRITE (ISCRUN, 228)

FORMAT (84H* SUM OF SQRS DO NOT ADD UP-ABS. VALUE OF (TOTAL-ROW-COLINF1050
1-RES.)/TOTAL EXCEEDS 5.E-7)
INFI060

GO TO 900

INF 1070

INF 1080

429 WRITE (ISCRUN, 229)

INF 1090

GO TO 900

INF 1100

WRITE (ISCRUN, 230)

INF 1110

430

FORMAT $\left(72 \mathrm{H}^{*}\right.$ ATTEMPT TO PROMOTE FROM BELOW NRMAX. FIRST ARGUMENT I

IS RESET TO NRMAX. , 12X)

I INF 1120

GO TO 900

INF 1130

INF 1140 
431 WRITE (ISCRUN, 231)

231 FORMAT (53H* ATTEMPT TO DEMOTE OFF THE WORKSHEET. SPILL IS LOST., INF 1160 $131 X)$

433 WRITE (ISCRUN, 233)

INF 1230

FORMAT (76H* NEGATIVE VALUE(S) WERE ENCOUNTERED BY PARTITION FUNCTIINF1240 ION. ZEROES STORED. , 8X)

INF 1250 GO TO 900

434 WRITE (ISCRUN, 234)

INF 1270

234 FORMAT( 45H* NEGATIVE ABSOLUTE TEMPERATURES CONVERTED . ,39X)

INF 1280 GO TO 900

INF 1290

435 WRITE (ISCRUN, 235)

INF 1300

235 FORMAT (76H* CAUTION, USE EXPERIMENTALLY ONLY. NOT OPTIMUM IN ORDEINF1310 IR TO MAKE IT MACHINE, $10 X / 84 \mathrm{H}$ INDEPENDENT. REFERENCES - J.B. KRUSINF1 320 2KAL, ACM $, 12,92$. AND J.H. HALTON, SIAM REV ., 12,1.)

INF 1330 GO TO 900

436 WRITE (ISCRUN, 236)

INF 1350

236 FORMAT (78H* COMMAND IGNORED - S BEFORE COMMAND NAME MEANINGLESS IINF 1360 IF NO STORAGE REQUESTED.)

INF 1370 GO TO 900

INF 1380

INF 1390

437 WRITE (ISCRUN , 237) IS IGD

237 FORMAT $\left(63 H^{*}\right.$ NUMBER OF SIGNIFICANT DIGITS AFTER DECIMAL PT HAS BEENINF 1400
1 SET TO,I3,18X)

900 RETURN

INF 1410

END

INF 1420 
COMMON /BLOCKA/ MODE, M, KARD (83), KARG, ARG, ARG2, NEWCD (80), KRDEND

INP 20

COMMON /BLOCKB/ NSTMT, NSTMTX, NSTMTH, NCOM, LCOM, IOVFL, COM(2000)

INP 30

COMMON /BLOCKC/ KIO, INUNIT, ISCRAT, KBDOUT, KRDKNT, LLIST

INP 40

C

THIS ROUTINE HANDLES THE READING OF INPUT RECORDS.

INP 50

INP 60

IF $K I O=0$, INPUT IS CARD IMAGE FROM CARD READER OR TAPE.

INP 70

IF $K I O=1$, INPUT IS REAL-TIME FROM A KEYBOARD.

INP 80

INP 90

INP 100

KRDKNT=KRDKNT+1

INP 110

IF (KIO.EQ.0) GO TO 20

INP 120

IF (MODE.EQ.3) GO TO 10

INP 130

WRITE (KBDOUT, 30)

INP 140

GO TO 20

INP 150

10 WRITE (KBDOUT, 40) NSTMT

INP 160

GO TO 20

INP 170

20 READ (INUNIT, 50) NEWCD

INP 180

$\operatorname{KARD}(1)=0$

$\operatorname{KARD}(2)=0$

INP 190

KARD $($ KRDEND +3$)=46$

INP 200

CALL OMCONV (NEWCD, KARD (3), KRDEND)

INP 210

RETURN

INP 220

INP 230

C

$\begin{array}{lll}30 & \text { FORMAT (9H READY) } \\ 40 & \text { FORMAT (9H READY, }\end{array}$

FORMAT (9H READY , I3,3H / )

INP 240

50 FORMAT (8OAl)

END

INP 250

INP 260

INP 270

INP 280 
COMMON /BLOCKD/ IARGS(100), KIND(100), ARGTAB (100), NRMAX, NROW, NCOL, NINT 90

IARGS, VWXYZ (8), NERROR

COMMON /BLOCKC/ KIO, INUNIT, ISCRAT, KBDOUT , KRDKNT, LLIST

DIMENSION ARGS $(100)$

INT 120

EQUIVALENCE (ARGS(1), RC (12501))

INT 130

COMMON /SCRAT/ NS, NS2, A(13500)

INT 140

INT 150

$\begin{array}{llll}\text { C CHECK TO SEE IF WE HAVE CORRECT NUMBER AND MODE OF ARGUMENTS } & \text { INT } 160 \\ \mathrm{C} & * & \text { INT } 170\end{array}$

$\begin{array}{ll}\text { IF (NARGS.NE.7) CALL ERROR (10) } & \text { INT } 180\end{array}$

$J=N A R G S$

INT 190

CALL CKIND (J)

IF (J.NE.O) CALL ERROR (3)

INT 200

INT 210

INT 220

$\begin{array}{lll}\text { C CHECK NO. OF POINTS LESS THAN OR EQUAL TO NRMAX AND POSITIVE } & \text { INT } 230 \\ \text { C } & *\end{array}$

IF (IARGS (3).LT.0.OR.IARGS(4).LT.0) CALL ERROR (3)

IF (IARGS (3).GT.NROW.OR.IARGS(4).GT.NRMAX) CALL ERROR (3)

INT 250

INT 260

INT 270

CHECK TO SEE IF WE HAVE MORE THAN TWO ENTRIES IN TABLE INT 280

COMPUTE COLUMN ADDRESSES

INT 290

INT 300

IF (IARGS (3).LT.2) CALL ERROR (3)

INT 310

$L X Y=I A R G S(3)$

$L X P=I A R G S(4)$

INT 320

$\operatorname{IARGS}(3)=\operatorname{IARGS}(5)$

INT 330

$\operatorname{IARGS}(4)=\operatorname{IARGS}(7)$

INT 340

NARGS $=4$

INT 350

CALL CHKCOL (J)

IF (J.NE.0) CALL ERROR (11)

INT 360

INT 370

INT 380

C *

CHECK TO SEE IF WE EXCEED SCRATCH AREA

INT 390

CHECK FOR PREVIOUS ERRORS

INT 400

INT 410

INT 420

INDRV $=0$

IF (IARGS (6)-LXY) $20,20,10$

INT 430

$\operatorname{IARGS}(6)=L X Y$

INDRV $=1$

INT 440

10

IF (IARGS $(6) * * 2+3 * \operatorname{IARGS}(6)+L X P$. LE.NS) GO TO 30

INT 450

INT 460

$C=1.0-4.0 * F L O A T$ (NS-LXP)

$I=F S Q R T(C)$

INT 470

INT 480

$\operatorname{IARGS}(6)=(-1+1) / 2$

INT 490

INDRV $=2$

INT 500

$I A I=I$ ARGS (1)

INT 510

IA2 =I ARGS (2)

INT 520

IA3 $=$ IARGS (3)

INT 530

$I A 4=3 * \operatorname{IARGS}(6)+L X P+1$

INT 540

CALL INTRP (RC (IA1) RC (IA2) LXY RC (IA3) A (1) , XP IARGS (6) A (LXP +1) INT 560

1, A ( IA4), IND)

C STORE RESULTS

INT 570

IA3 $=$ I ARGS (4) 
DO $40 \quad I=1, L X P$

INT 600

$\mathrm{RC}(\mathrm{IA} 3)=\mathrm{A}(\mathrm{I})$

INT 610

$I A 3=I A 3+1$

40 CONTINUE

INT 620

IF (INDRV.EQ.0.AND.IND.EQ.0) RETURN

INT 630

IF (INDRV-1) $70,50,60$

50 WRITE (ISCRAT, 80)

GO TO 70

INT 640

INT 650

INT 660

INT 670

60
70
IF ITE (IND.EQ.0) RETURN

INT 680

INT 690

WRITE (ISCRAT, 100)

INT 700

RETURN

INT 710

INT 720

C

80 FORMAT $(6 \mathrm{X}, 20(1 \mathrm{H}+), 39 H O R D E R$ OF INTERPOLATION EQUALS LIST SIZE, 19X) INT 730

90 FORMAT $(6 X, 20(1 H+), 53 H O R D E R$ OF INTERP WAS RESET DUE TO SIZE OF SCRINT 740 IATCH AREA, 5X)

INT 750

100 FORMAT $(6 \mathrm{X}, 20(1 \mathrm{H}+), 42 \mathrm{HEXTRAPOLATION}$ DONE FOR MORE THAN ONE DELTA, 1 INT 760 16X)

INT 770

END

INT 780 
SUBROUTINE INTRP $(X, Y, N L I S T, X I, R E S U L T, N X I, N O R D, S, S A, I N D) \quad$ INR 10

VERSION $5.00 \quad$ INTRP $5 / 15 / 70$

CALLING SEQUENCE

SUBROUTINE INTRP ( $X, Y, N L I S T, X 1, R E S U L T, N X 1, N O R D, S, S A, I N D)$

INR 20

INR 30

INR 40

INR 50

$X$ THE INDEPENDENT VALUE OF THE TABLE. MUST BE IN ASCENDING OR INR 60

DESCENDING ORDER. NEED NOT BE EVENLY SPACED

$Y$ THE DEPENDENT VALUE OF THE TABLE

NLIST LENGTH OF $X$ OR $Y$

$X I$ VALUES TO BE INTERPOLATED

RESULT RESULT FROM INTERPOLATION

NX1 LENGTH OF X1 VECTOR

NORD ORDER OF INTERPOLATION

S SCRATCH AREA S(3*NORD)

SA SCRATCH AREA SA (NORD, NORD)

IND INDICATOR

IND $=0$ EVERYTHING FINE

IND $=2$ EXTRAPOLATION AND MORE THEN ONE DELTA

INR 70

INR 80

INR 90

INR 100

INR 110

INR 120

INR 130

INR 140

INR 150

INR 160

INR 170

INR 180

INR 190

DIMENSION $X(1), Y(1), X 1(1), \operatorname{RESULT}(1), S(1)$, SA(NORD,NORD)

INR 200

INDA $=0$

IND $=0$

$N R D=N O R D-1$

NDI $R=1$

IF $(X(1) \cdot G E \cdot X(2)) \quad N D I R=2$

$I=1$

DO 220 II $I=1, N \times I$

INR 210

INR 220

INR 230

INR 240

INR 250

INR 260

INR 270

IC $=0$

$X A=X I$ (II)

GO TO $(10,180)$, NDIR

INR 280

INR 290

INR 300

INR 310

INR 320

INR 330

INR 340

INR 350

GO TO 80

30 DO $40 \quad I A=I, N L I S T$

IF $(X(I A)-X A) \quad 40,170,60$

40 CONTINUE

50 IF (ABS (X(NLIST)-XA).GT.ABS (X(NLIST)-X(NLIST-1))) INDA=2

$I E=1$

I $A=N L I S T-N R D$

$I \mathrm{C}=1$

GO TO 80

INR 360

INR 370

INR 380

INR 390

INR 400

INR 410

INR 420

INR 430

INR 440

$I A=I A-1$

IF $(X(I A)-X A) \quad 70,170,60$

70 IF (IA+NRD.LE.NLIST) GO TO 80

$I C=1$

I $A=N L I S T-N R D$

80 IF (NRD.GT.1) GO TO 90

TEMP $=(X A-X(I A)) /(\cdot X(I A+1)-X(I A))$

RESULT (II $)=Y(I A)+(Y(I A+I)-Y(I A)) * T E M P$

INR 450

INR 460

INR 470

INR 480

INR 490

INR 500

INR 510

GO TO 220

90 IF (IC.NE.0) GO TO 100

I $A=I A-N R D / 2$

INR 520

INR 530

INR 540

IF (IA.LE.0) I $A=1$

$100 \quad N A=I A+N R D$

INR 550

$P R O D=1$.

$I Z=1$

INR 560

INR 570

INR 580

$I Z A=N O R D+I$

INR 590 
DO 110 IB $=I A, N A$

INR 600

$S(I Z)=X(I B)$

$S(I Z A)=X A-X(I B)$

INR 610

PROD $=P R O D * S(I Z A)$

INR 620

$I Z=I Z+1$

$110 \quad I Z A=I Z A+1$

$N B=N R D+1$

$D O 120 \quad I A R=2, N B$

DO 120 IBR=IAR, NB

SA (IBR-1, IAR-1) =S (IAR-1)-S (IBR)

$120 S A(I A R-1, I B R)=(-S A(I B R-1, I A R-1))$

INR 630

INR 640

INR 650

INR 660

INR 670

INR 680

INR 690

$I Z B=I Z A$

$I Z C=N O R D+1$

DO $140 \quad$ IAR $=1$, NB

$S U M=S(I Z C)$

DO $130 \quad$ IBR $=1$, NRD

INR 700

INR 710

INR 720

INR 730

INR 740

INR 750

130 SUM=SUM*SA (IBR, IAR)

$S(I Z A)=P R O D / S U M$

INR 760

$I Z C=I Z C+I$

INR 770

INR 780

$140 \quad$ I $Z A=I Z A+1$

$\mathrm{R}=0.0$

I $A X=I A$

DO 150 I $X=1$, NORD

INR 790

INR 800

$R=R+S(I Z B) * Y(I A X)$

INR 810

INR 820

$I A X=I A X+1$

INR 830

INR 840

$150 \quad I Z B=I Z B+1$

INR 850

RESULT (II) $=R$

INR 860

GO TO 220

INR 870

160 RESULT (II) $=Y(1)$

INR 880

I $A=1$

INR 890

GO TO 220

INR 900

170 RESULT (II) $=Y$ (IA)

INR 910

GO TO 220

INR 920

180 IF $(X A-X(1)) \quad 190,160,30$

INR 930

190 DO 200 IA $=I, N L I S T$

INR 940

IF $(X A-X(I A)) \quad 200,170,210$

INR 950

200 CONTINUE

GO TO 50

INR 960

INR 970

210 I $A=I A-1$

IF $(X A-X(I A)) 70,170,210$

INR 980

INR 990

220 I $=I A$

INR 1000

IND = IND + INDA

INR 1010

RETURN

INRI 020

END

INR 1030 
SUBROUTINE INVCHK ( $A, M, N, A I N V, M 1, Y, L 2, E R R, I N D)$

INK 10

VERSION 5.00

INVCHK

$5 / 15 / 70$

INK 20

INVCHK FOR OMNITAB UNIVAC 1108 S. PEAVY 5/24/67

INK 30 THIS SUBROUTINE INVERTS A MATRIX AND PROVIDES ALL TH

IN PAC-1

INK 50

A IS THE MATRIX TO BE INVERTED

INK 60

A IS THE MATRIX TO BE INVERTED

INK 70

INK 90

INK 100

$N$ IS THE SI.ZE OF A TO BE INVERTED

INK 110 $N$ LESS THAN OR $=M-1$

INK 120

INK 130

AINV WILL CONTAIN THE INVERTED MATRIX IF INVERSION IS OBTAINABLE

MI IS THE SIZE OF AINV AS DIMENSIONED IN THE CALLING PROGRAM AINV (MI, 2*M1) MI MUST BE GREATER OR $=\mathrm{N}+1$

INK 140

INK 150

AINV MUST HAVE TWICE AS MANY COLUMNS AS ROWS

A AND AINV CANNOT BE SAME OR EQUIVALENT

INK 160

INK 170

INK 180

INK 190

INK 200

ERR WILL CONTAIN THE 3 WAYS OF EVALUATING NORM CHECKS

INK 210 ERR IS A DIMENSIONED AS ERR(3)

INK 220

INK 230

IND IS AN INDICATOR

IND $=0$ MATRIX INVERTED AND ERROR CHECKS MADE

INK 240

IND $=1$ MATRIX SINGULAR

INK 250

INK 260

INK 270

COLUMN AINV $(N+1, I) \quad I=1, \ldots, N$ WILL CONTAIN THE ERROR BOUND OF THE SUM CHECKS +1 .

INK 280

INK 290

INK 300

DIMENSION $A(M, M), \operatorname{AINV}(M I, M I), \operatorname{ERR}(3), \operatorname{ANORM}(2,3)$

DIMENSION $Y(N)$

INK 310

INK 320

DATA ZERO $/ 0.0 / .0 N E / 1.0 /$

$10 \quad \mathrm{NA}=\mathrm{N}$

DO $20 \quad I=1, N A$

INK 330

INK 340

INK 350

DO $20 \mathrm{~J}=1, \mathrm{NA}$

INK 360

$\operatorname{AINV}(J, I)=A(J, I)$

$N B=N A$

IF (L2.EQ.1) GO TO 40

INK 370

INK 380

INK 390

$\mathrm{NB}=\mathrm{NB}+1$

INK 400

DO $30 I=1$, NA

$\operatorname{AINV}(I, N A+1)=Y(I)$

$30 \quad \operatorname{AINV}(N A+1, I)=Z E R O$

$A$ INV $(N A+1, N A+1)=-0 N E$

INK 410

INK 420

INK 430

INK 440

$N A=N A+1$

40 DO $60 \quad I=1$, NA

SUM $=$ ZERO

A INV $(N A+1, I)=Z E R O$

DO $50 \mathrm{~J}=1$, NA

$50 \quad S U M=S U M+A I N V(I, J)$

$60 \quad \operatorname{AINV}(1, N A+1)=-S U M$

$A$ INV $(N A+1, N A+1)=0 N E$

INK 450

INK 460

INK 470

INK 480

INK 490

INK 500

INK 510

INK 520

$N B=N B+1$

INK 530

CALL SPINV (AINV,NB, MI, IND)

INK 540

IF (IND.NE.0) RETURN

DO $140 \mathrm{~K}=1,2$

DO $70 \quad I=1,3$

INK 550

INK 560

INK 570

$\operatorname{ANORM}(K, I)=Z E R O$

INK 580

DO $130 \quad I=1, N$

INK 590 
DO $120 \mathrm{~J}=1, \mathrm{~N}$

INK 610

GO TO $(80,90), K$

INK 620

$80 \operatorname{TEMP}=A B S(\operatorname{AINV}(I, J))$

INK 630

GO TO 110

INK 640

$90 \quad$ TEMP $=Z E R O$

INK 650

DO $100 \mathrm{~L}=1, \mathrm{~N}$

100 TEMP $=$ TEMP +A $(I, L) * A I N V(L, J)$

INK 660

IF $(I . E Q . J)$ TEMP $=0 N E-T E M P$

INK 670

$T E M P=A B S(T E M P)$

$110 \operatorname{ANORM}(K, 1)=\operatorname{ANORM}(K, 1)+\operatorname{TEMP} * * 2$

INK 680

INK 690

IF $(\operatorname{ANORM}(K, 2)$. LT. TEMP) ANORM $(K, 2)=T E M P$

INK 700

INK 710

INK 720

INK 730

IF $(\operatorname{ANORM}(K, 3) . L T . S U M) \operatorname{ANORM}(K, 3)=S U M$

INK 740

CONT INUE

$\operatorname{ANORM}(K, 1)=$ FSQRT $(\operatorname{ANORM}(K, 1))$

INK 750

$\operatorname{ANORM}(K, 2)=\operatorname{FLOAT}(N) * \operatorname{ANORM}(K, 2)$

INK 760

DO $150 \mathrm{~K}=1,3$

$150 \operatorname{ERR}(K)=(\operatorname{ANORM}(1, K) * \operatorname{ANORM}(2, K)) /(1 .-\operatorname{ANORM}(2, K))$

INK 770

RETURN

INK 780

END

INK 790

INK 800 
SUBROUTINE INVCOR ( $A, M, N, A I N V, M I, Y, L 2, E R R, I N D)$

VERSION $5.00 \quad$ INVCOR $5 / 15 / 70$

INC 10

INVCOR FOR OMNITAB UNIVAC 1108 S. PEAVY 5/24/67

INC 20

THIS SUBROUTINE INVERTS A MATRIX AND PROVIDES ALL THE CHECKS DESCRINC 40 IN PAC-1

INC 50

INC 60

$\begin{array}{ll}\text { A IS THE MATRIX TO BE INVERTED } & \text { INC } 70\end{array}$

$\begin{array}{rrr}\text { INC } & 80\end{array}$

$M$ IS THE SIZE OF A AS DIMENSIONEd IN THE CALLING PROGRAM A(M,M) INC 90

INC 100

$N$ IS THE SIZE OF A TO BE INVERTED

INC 110

$N$ LESS THAN OR $=M-1$

INC 120

INC 130

AINV WILL CONTAIN THE INVERTED MATRIX IF INVERSION IS OBTAINABLE INC 140

INC 150

MI IS THE SIZE OF AINV AS DIMENSIONED IN THE CALLING PROGRAM

INC 160 AINV (MI $2 * M 1) \quad M I$ MUST BE GREATER OR $=\mathrm{N}+1$

AINV MUST HAVE TWICE AS MANY COLUMNS AS ROWS

A AND AINV CANNOT BE SAME OR EQUIVALENT

ERR WILL CONTAIN THE 3 WAYS OF EVALUATING NORM CHECKS ERR IS A DIMENSIONED AS ERR(3)

IND IS AN INDICATOR

IND $=0$ MATRIX INVERTED AND ERROR CHECKS MADE

IND $=1$ MATRIX SINGULAR

INC 170

INC 180

INC 190

INC 200

INC 210

INC 220

INC 230

INC 240

INC 250

INC 260

INC 270

COLUMN AINV $(N+1, I) \quad I=1, \ldots, N$ WILL CONTAIN THE ERROR BOUND OF

INC 280

THE SUM CHECKS+1.

INC 290

INC 300

DIMENSION $A(M, M), \operatorname{AINV}(M 1, M 1), \operatorname{ERR}(3), \operatorname{ANORM}(2,3)$

DIMENSION $Y(N)$

DATA ZERO/0.0/.ONE/1.0/

10

$N A=N$

DO $20 \quad I=1, N A$

DO $20 \mathrm{~J}=1$, NA

INC 310

INC 320

INC 330

INC 340

INC 350

INC 360

$\operatorname{AINV}(J, I)=A(J, I)$

$N B=N A$

IF (L2.EQ.1) GO TO 40

$N B=N B+1$

DO $30 \quad I=1, N A$

$\operatorname{AINV}(I, N A+1)=Y(I)$

$30 \quad \operatorname{AINV}(N A+1, I)=Z E R O$

AINV $(N A+1, N A+1)=-O N E$

$N A=N A+1$

40 DO $60 \quad I=1, N A$

SUM $=$ ZERO

AINV $(N A+1, I)=Z E R 0$

DO $50 \mathrm{~J}=1$, NA

$50 \quad S U M=S U M+A I N V(I, J)$

$60 \quad \operatorname{AINV}(I, N A+1)=-S U M$

$A \operatorname{INV}(N A+1, N A+1)=0 N E$

$N B=N B+1$

CALL CSPINV (AINV, NB, MI, IND)

IF (IND.NE.0) RETURN

DO $140 \mathrm{~K}=1,2$

DO $70 \quad I=1,3$

INC 370

INC 380

INC 390

INC 400

INC 410

INC 420

INC 430

INC 440

INC 450

INC 460

INC 470

INC 480

INC 490

INC 500

INC 510

INC 520

INC 530

INC 540

INC 550

INC 560

INC 570

$\operatorname{ANORM}(K, I)=Z E R O$

INC 580

DO $130 \quad I=1, N$

INC 590 
SUM $=Z E R O$

DO $120 \mathrm{~J}=1, \mathrm{~N}$

GO TO $(80,90), K$

$80 \quad T E M P=A B S(A I N V(I, J))$

$$
\text { GO TO } 110
$$

90 TEMP $=$ ZERO

$$
\text { DO } 100 \quad L=1, N
$$

$100 T$ TEMP $=T E M P+A(I, L) * \operatorname{AINV}(L, J)$

IF (I.EQ.J) TEMP=ONE-TEMP

TEMP $=A B S(T E M P)$

$110 \quad \operatorname{ANORM}(K, 1)=\operatorname{ANORM}(K, 1)+\operatorname{TEMP} * * 2$

$$
\text { IF }(\operatorname{ANORM}(K, 2) . L T . T E M P) \text { ANORM }(K, 2)=\text { TEMP }
$$

120 SUM=SUM+TEMP

$$
\text { IF }(\operatorname{ANORM}(K, 3) . L T . S U M) \text { ANORM }(K, 3)=S U M
$$

130 CONTINUE

$$
\operatorname{ANORM}(K, 1)=F S Q R T(\operatorname{ANORM}(K, 1))
$$

$140 \operatorname{ANORM}(K, 2)=\mathrm{FLOAT}(N) * \operatorname{ANORM}(K, 2)$

$$
\text { DO } 150 \quad K=1,3
$$

$150 \operatorname{ERR}(K)=(\operatorname{ANORM}(1, K) * \operatorname{ANORM}(2, K)) /(1,-\operatorname{ANORM}(2, K))$ RETURN

END
INC 600

INC 610

INC 620

INC 630

INC 640

INC 650

INC 660

INC 670

INC 680

INC 690

INC 700

INC 710

INC 720

INC 730

INC 740

INC 750

INC 760

INC 770

INC 780

INC 790

INC 800 
SUBROUTINE INVERT

C VERSION 5.00 INVERT 5/15/70

C MATRIX INNVERSION, SOLUTION OF SYSTEM OF EQUATIONS

$S$ PEAVY $5 / 22 / 67$

MINVERT $(+++,+++)$ SIZE,++++++ STORE $(+++,+++)$

SOLVE $(+++,+++)$ SIZE,++++++ Y VECTOR +++ STORE +++

LARGEST MATRIX TO BE INVERTED OR SYSTEM TO BE SOLVED IS 50

$L 2=1 \quad$ INVERT

INV 110

$L 2=2$ SOLVE

INV 120

COMMON /SCRAT/ NS,NS2,A(13500)

COMMON /BLOCKE/ NAME (4), L1, L2, ISRFLG

INV 130

INV 140

COMMON /BLOCRC/ NRC, RC (12600)

INV 150

COMMON /BLOCKD/ IARGS(100), KIND(100), ARGTAB (100), NRMAX, NROW, NCOL, NINV 160

IARGS, VWXYZ (8), NERROR

INV 170

DIMENSION ARGS(100)

INV 180

EQUIVALENCE (ARGS (1), RC (12501))

COMMON /BLOCKC / KIO, INUNIT, ISCRAT, KBDOUT, KRDKNT, LLIST

INV 190

DIMENSION ERR (3)

INV 200

IF (NARGS.EQ.6) GO TO 10

INV 210

CALL ERROR (10)

INV 220

RETURN

10

$J=N A R G S$

INV 230

INV 240

CALL CKIND (J)

IF (J.NE.0) GO TO 100

IF (IARGS (3)-IARGS (4)) $105,20,107$

INV 250

INV 260

INV 270

INV 275

NARGS $=8$

$\operatorname{KIND}(7)=0$

$\operatorname{KIND}(8)=0$

INV 320

INV 330

IF (L2.EQ.1) GO TO 30

INV 340

$\operatorname{IARGS}(9)=\operatorname{IARGS}(6)$

INV 350

NARGS $=9$

INV 360

$\operatorname{KIND}(9)=0$

CALL ADRESS (NARGS, JE)

INV 370

INV 380

IF (JE.LE.0) GO TO 150

INV 390

$\operatorname{IARGS}(6)=\operatorname{IARGS}(5)$

$\operatorname{IARGS}(5)=1$

$\operatorname{IARGS}(8)=1$

GO TO 40

$30 \operatorname{IARGS}(8)=\operatorname{IARGS}(3)$

$40 \operatorname{IARGS}(7)=\operatorname{IARGS}(3)$

$J=2$

CALL MTXCHK (J)

IF (J.NE.O) GO TO 140

$J A=I$ ARGS (1)

$J B=I$ ARGS (5)

IF $(2 *((\operatorname{IARGS}(3)+2) * * 2)$.GT.NS) GO TO 120

IF (NERROR.NE.0) RETURN

$\mathrm{Ml}=I \operatorname{ARGS}(3)+1$

IF (L2.EQ.2) $M \mathrm{l}=\mathrm{Ml}+\mathrm{l}$

CALL INVCHK (RC (JA), NROW, IARGS (3), A, MI, RC (JB), L2, ERR, IND)

C CHECK TO SEE IF MATRIX WAS INVERTED. YES, IF IND=0

IF (IND.NE.0) GO TO 130

$I A=I A R G S(3)$

IF (L2.EQ.2) GO TO 70

C STORE INVERTED MATRIX

DO $60 \quad I=1$, IA

$\mathrm{JC}=\mathrm{JB}$

$J D=(I-1) * M I+1$

INV 400

INV 410

INV 420

INV 430

INV 440

INV 450

INV 460

INV 470

INV 480

INV 490

INV 500

INV 510

INV 520

INV 530

INV 540

INV 550

INV 560

INV 570

INV 580

INV 590

INV 600

INV 610

INV 620

INV 630

INV 640 


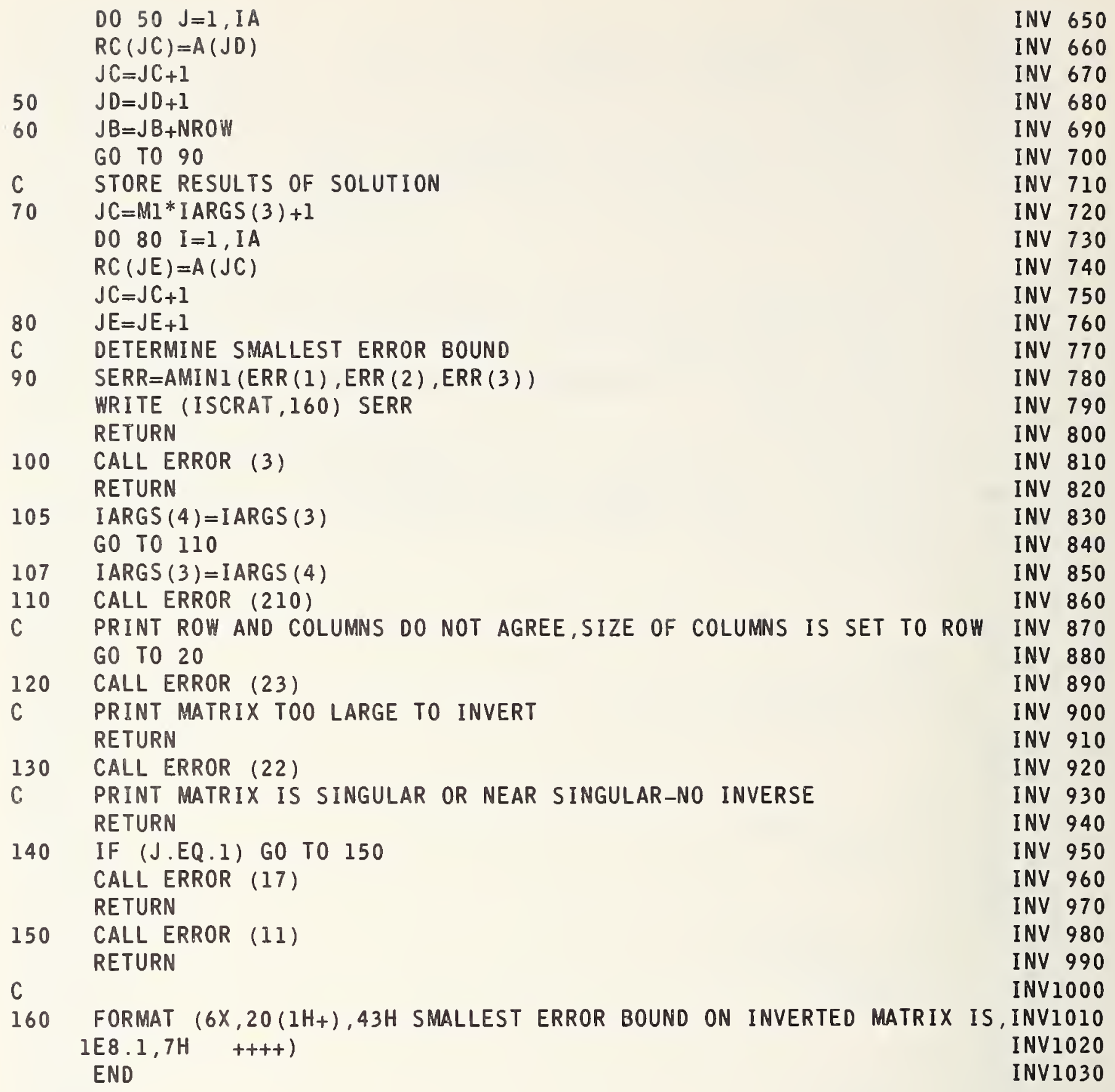


SUBROUTINE ITERAT

C VERSION 5.00 ITERAT 5/15/70

ITE 10

COMMON /BLOCRC/ NRC, RC (12600)

ITE 20

COMMON /SCRAT/ NS, NS2, A(13500)

ITE 30

ITE 40

COMMON /BLOCKD/ I ARGS(100), KIND (100), ARGTAB (100), NRMAX, NROW, NCOL, NITE 50

IARGS, VWXYZ (8), NERROR

ITE 60

DIMENSION ARGS(I00)

ITE 70

EQUIVALENCE (ARGS(I), RC (1250I))

COMMON /BLOCKE/ NAME (4), LI,L2, ISRFLG

ITE 80

ITE 90

$\mathrm{L} 2=1$

ITERATE $X$ IN ++, $Y$ IN ++, DESIRED $Y$ IN ++ START STORIN ITE 100

$\mathrm{L} 2=2$

ISETUP $X$ IN ++, $Y$ IN ++, DESIRED $Y$ IN ++ STORE STARTIN ITE IIO

COLUMN ++

ITE 120

IN COLUMN ++

ITE 130

$L 2=3$

ISOLATE X IN ++ FOR Y IN COL $++=* *$ STORE IN ++ AND ++ ISOLATE $X$ IN ++ FOR Y IN COL $++*^{* *}$ USE , POINTS, STORE

ITE 140 IN ++ AND ++

ITE 150

ITE 160

ITERATE AND ISETUP USE THREE COLUMNS AFTER THE INDICATED STORE ITE 170

STORAGE FOR ITERATE AND ISETUP ARE NEW $X$, AVERAGE BRACKETING $X$, ITE 180

AVERAGE BRACKETTING $Y$, AND SUCCESSFUL $Y$

CODE BY MRS. CARLA G. MESSINA NSRDS-NBS OCT 1967

ITE 190

INSERT $=3$

IF (NARGS-4) $10,40,80$

ITE 200

ITE 210

ITE 220

$K=10$

ITE 230

CALL ERROR ( $K$ )

RETURN

ITE 240

IF $(L 2-2) 50,50,10$

ITE 250

30

NARGS $=7$

ITE 260

DO $60 \quad I=5,7$

$\operatorname{KIND}(I)=0$

$60 \operatorname{IARGS}(\mathrm{I})=\operatorname{IARGS}(\mathrm{I}-1)+1$

IF (NROW-5) $70,180,180$

ITE 270

ITE 280

ITE 290

ITE 300

ITE 310

$\mathrm{K}=17$

GO TO 20

ITE 320

ITE 330

IF (L2-3) $10,90,10$

ITE 340

$\operatorname{IARGS}(7)=\operatorname{IARGS}(5)$

$\operatorname{KIND}(7)=\operatorname{KIND}(5)$

IF (NARGS-6) $100,110,10$

ITE 350

ITE 360

ITE 370

100 NARGS $=6$

$\operatorname{IARGS}(6)=\operatorname{IARGS}(5)$

$\operatorname{KIND}(6)=\operatorname{KIND}(5)$

$\operatorname{IARGS}(5)=\operatorname{IARGS}(4)$

$\operatorname{KIND}(5)=K \operatorname{IND}(4)$

GO TO 140

110 IF (KIND(4)) $120,130,120$

$120 \quad K=3$

GO TO 20

130 INSERT $=$ IARGS (4)

$\operatorname{IARGS}(4)=\operatorname{IARGS}(5)$

140 IF (INSERT-1) $120,150,150$

150 IF (NROW-INSERT-2) 70,160,160

160 IF (KIND(3)) $170,120,170$

170 POINT=ARGS (3)

$\operatorname{IARGS}(3)=\operatorname{IARGS}(2)$

$\operatorname{KIND}(3)=0$

180 CALL CHKCOL (J)

IF (J) $120,190,120$

190 IF (NERROR.NE.0) GO TO 30

IF (NRMAX-1) 200,210,220

$K=9$

ITE 380

ITE 390

ITE 400

ITE 410

ITE 420

ITE 430

ITE 440

ITE 450

ITE 460

ITE 470

ITE 480

ITE 490

ITE 500

ITE 510

ITE 520

ITE 530

ITE 540

ITE 550

ITE 560

ITE 570

ITE 580

ITE 590 
GO TO 20

ITE 600

$210 \quad K=215$

GO TO 20

ITE 610

220 IF $(\operatorname{L2}-2) \quad 300,230,230$
$230 \quad$ I $1=\operatorname{IARGS}(1)-1$

ITE 620

ITE 630

DO $240 \quad I=2$, NRMAX

ITE 640

$K=I I+I$

ITE 650

IF (RC (K-1)-RC (K)) $250,240,270$

ITE 660

CONTINUE

GO TO 290

250 DO 260 I=2, NRMAX

$K=I I+I$

IF (RC (K-1)-RC (K)) $260,260,290$

ITE 670

ITE 680

ITE 690

ITE 700

ITE 710

260 CONTINUE

ITE 720

ITE 730

ITE 740

270 DO $280 \quad I=2$, NRMAX

$K=I I+I$

ITE 750

IF (RC (K-1)-RC (K)) $290,280,280$

280 CONTINUE

GO TO 300

290 CALL ERROR (216)

300 DO $310 \quad I=1$, NRMAX

$I I=I$ ARGS $(1)+I-1$

$I 2=I$ ARGS $(2)+I-1$

$I 3=I \operatorname{ARGS}(3)+I-1$

$A(I)=R C$ (II)

$M=I+N R M A X$

$A(M)=R C(I 2)$

$M=M+N R M A X$

$310 \quad A(M)=R C(I 3)$

$M=0$

$\mathrm{MI}=0$

I OVFL $=0$

IND2 $=$ INSERT + 2

DIV $=$ INSER $T+1$

ITE 760

ITE 770

ITE 780

ITE 790

ITE 800

ITE 810

ITE 820

ITE 830

ITE 840

ITE 850

ITE 860

ITE 870

ITE 880

ITE 890

ITE 900

ITE 910

ITE 920

ITE 930

ITE 940

I D I $V=$ INSERT + I

ITE 950

I I =I ARGS (4) - I

ITE 960

$I 2=I$ ARGS $(5)-1$

ITE 970

$I 3=I$ ARGS $(6)-1$

ITE 980

$I 4=I$ ARGS $(7)-1$

ITE 990

I $5=$ I ARGS ( 4 ) -1

ITE 1000

IF (L2-2) $500,500,320$

ISOLATE

ITEI010

C

$320 \mathrm{Kl}=\mathrm{NRMAX}+1$

ITE 1020

$L 1=2$ *NRMAX

ITE 1030

ITE 1040

I $5=\operatorname{IARGS}(5)-1$

ITE1050

IF (POINT-A (KI)) $340,330,340$

ITE 1060

$M=M+1$

ITE 1070

I $2=12+1$

$R C(I 2)=A(1)$

$M 1=M 1+1$

ITE1080

ITE1090

I $3=13+1$

ITE 1100

$\mathrm{RC}(\mathrm{I} 3)=\mathrm{A}(1)$

ITEI110

ITE 1120

$\mathrm{K} I=\mathrm{K} 1+\mathrm{I}$

$\mathrm{I}=1$

DO $440 K=K 1$, LI

ITE 1130

ITE 1140

$\mathrm{I}=\mathrm{I}+1$

IF (POINT-A $(K-1)) \quad 350,440,360$

ITE 1150

ITE1160

ITE 1170

350 IF (POINT-A (K)) $440,390,370$

ITEI180 


\begin{tabular}{|c|c|c|}
\hline 360 & IF (POINT-A (K)) $370,390,440$ & ITEII90 \\
\hline 370 & IF (NROW-M-IND2) $380,400,400$ & ITE 1200 \\
\hline \multirow[t]{2}{*}{380} & IOVFL $=1$ & ITE 1210 \\
\hline & GO TO 430 & I TE 1220 \\
\hline \multirow[t]{2}{*}{390} & $A(I-1)=A(I)$ & I TE 1230 \\
\hline & IF (NROW-M-1) $430,420,420$ & ITE 1240 \\
\hline \multirow[t]{7}{*}{400} & $M=M+1$ & I TE 1250 \\
\hline & $I 2=I 2+1$ & ITE1260 \\
\hline & $R C(I 2)=A(I-1)$ & ITE1270 \\
\hline & $\mathrm{DELT}=(\mathrm{A}(\mathrm{I})-\mathrm{A}(\mathrm{I}-1)) / \mathrm{DIV}$ & ITE 1280 \\
\hline & DO 410 I I $=1$, INSERT & ITE 1290 \\
\hline & $M=M+1$ & ITE 1300 \\
\hline & $\mathrm{I} 2=\mathrm{I} 2+1$ & ITE 1310 \\
\hline 410 & $R C(I 2)=R C(I 2-1)+D E L T$ & I TE 1320 \\
\hline \multirow[t]{3}{*}{420} & $M=M+1$ & ITEI330 \\
\hline & $I 2=I 2+1$ & ITE 1340 \\
\hline & $R C(I 2)=A(I)$ & ITE 1350 \\
\hline \multirow[t]{4}{*}{430} & $M I=M I+1$ & ITE 1360 \\
\hline & $13=13+1$ & ITE 1370 \\
\hline & $R C(I 3)=(A(I-1)+A(I)) / 2.0$ & ITE 1380 \\
\hline & IF (NROW-MI) $470,470,440$ & ITE 1390 \\
\hline \multirow[t]{2}{*}{440} & CONTINUE & ITEI400 \\
\hline & IF (M) $480,480,450$ & ITEI410 \\
\hline 450 & NRMAX $=M$ & ITEI 420 \\
\hline 460 & IF (IOVFL) $470,30,470$ & ITEI 430 \\
\hline \multirow[t]{2}{*}{470} & $K=218$ & ITEI440 \\
\hline & GO TO 20 & ITE 1450 \\
\hline \multirow[t]{3}{*}{480} & $K=217$ & ITE 1460 \\
\hline & DO $490 I=1$, NRMAX & ITE 1470 \\
\hline & I $5=I 5+1$ & ITEI 480 \\
\hline \multirow[t]{2}{*}{490} & $R C(I 5)=A(I)$ & ITE 1490 \\
\hline & GO TO 20 & ITE 1500 \\
\hline \multirow[t]{4}{*}{500} & $K I=2 * N R M A X+1$ & ITEI510 \\
\hline & $L I=3 * N R M A X$ & ITEI 520 \\
\hline & DO $510 \mathrm{~K}=1$, NRMAX & ITE 1530 \\
\hline & IF $(A(L I)) 520,510,520$ & I TE 1540 \\
\hline \multirow[t]{2}{*}{510} & $L 1=L 1-1$ & I TEI550 \\
\hline & $L I=3 * N R M A X$ & ITEI560 \\
\hline \multirow[t]{2}{*}{520} & $N E W Y=L 1-K 1+1$ & ITE 1570 \\
\hline & IF $(L 2-2) 780,530,10$ & ITE 1580 \\
\hline C & I SETUP & ITE 1590 \\
\hline \multirow[t]{4}{*}{530} & DO $740 K=K 1, L 1$ & I TE 1600 \\
\hline & $I=1$ & ITEI6I0 \\
\hline & $L=N R M A X+1$ & ITE 1620 \\
\hline & IF $(A(K)-A(I)) \quad 590,540,590$ & ITE 1630 \\
\hline 540 & IF (NROW-M-IND2) $550,560,560$ & I TEI640 \\
\hline \multirow[t]{2}{*}{550} & IOVFL $=1$ & ITE 1650 \\
\hline & GO TO 580 & ITEI 660 \\
\hline \multirow[t]{3}{*}{560} & DO 570 I I =1, IND2 & ITE 1670 \\
\hline & $M=M+1$ & ITEI680 \\
\hline & $I I=I I+1$ & ITE 1690 \\
\hline 570 & $R C(I I)=A(I)$ & ITEI700 \\
\hline \multirow[t]{7}{*}{580} & $M 1=M 1+1$ & ITEI7I0 \\
\hline & $I 2=I 2+1$ & ITE 1720 \\
\hline & $R C(I 2)=A(I)$ & ITE 1730 \\
\hline & $I 3=I 3+1$ & ITE 1740 \\
\hline & $R C(I 3)=A(L)$ & ITE 1750 \\
\hline & $I 4=I 4+1$ & ITEI760 \\
\hline & $R C(I 4)=A(K)$ & ITE 1770 \\
\hline
\end{tabular}


$660 \quad M I=M I+1$

ITE 1900

$I 2=I 2+1$

ITE 1910

$R C(I 2)=A(I)$

ITE1920

$I 3=I 3+1$

ITEI930

$R C(I 3)=A(L)$

ITE 1940

GO TO 720

ITE 1950

670 IF (NROW-M-IND2) $680,690,690$

ITE 1960

680 IOVFL $=1$

GO TO 710

ITE 1970

ITE 1980

ITE 1990

690 DELT $=(A(I)-A(I-1)) / D I V$

ITE2000

$M=M+1$

$I \mathrm{I}=\mathrm{I} I+1$

$R C(I 1)=A(I-I)$

DO $700 \mathrm{~J}=1$, INSERT

$M=M+1$

$I I=I I+1$

$700 \quad \mathrm{RC}(\mathrm{I} I)=\mathrm{RC}(\mathrm{I} I-1)+\mathrm{DELT}$

$M=M+1$

$I I=I I+1$

$R C$ ( I I ) = A (I)

$710 \quad M 1=M 1+1$

$I 2=I 2+1$

$R C(I 2)=(A(I)+A(I-I)) / 2.0$

ITE 2010

ITE2020

ITE 2030

ITE 2040

ITE2050

ITE2060

ITE 2070

ITE2080

ITE2 090

ITE2 100

ITE 2110

ITE 2120

$I 3=\llbracket 3+1$

$R C(I 3)=(A(L)+A(L-1)) / 2.0$

ITE 2130

ITE2 140

ITE2 150

ITE2 160

I $4=I 4+1$

$\mathrm{RC}(\mathrm{I} 4)=\mathrm{A}(\mathrm{K})$

IF (NROW-MI) $470,470,740$

ITE 2170

ITE2 180

CONTINUE

ITE2 190

730 CONT INUE

ITE2200

750 IF (M) $480,480,760$

ITE2210

760 IF (M-NEWY) $770,770,450$

ITE2220

NRMAX $=$ NE WY

ITE2230

GO TO 460

ITE 2240

C

780 II $=$ IND2 2 (NRMAX/IND2)

\section{ITERATE}

ITE2250

ITE 2260

IF (II) $210,210,790$

ITE 2270

ITE22 20

DO $960 \mathrm{~K}=\mathrm{KI}, \mathrm{LI}$

ITE2290

D0 $950 \mathrm{~K} 3=1, \mathrm{II}, \mathrm{IND} 2$

ITE2 300

DO $830 \mathrm{~J}=1$, IDIV

ITE 2310

$\mathrm{I}=\mathrm{K} 3+\mathrm{J}$

ITE2320

$L=N R M A X+I$

ITE2 330

IF $(A(L-1)-A(K)) \quad 810,800,820$

$I=I-I$

ITE2 350

GO TO 840 


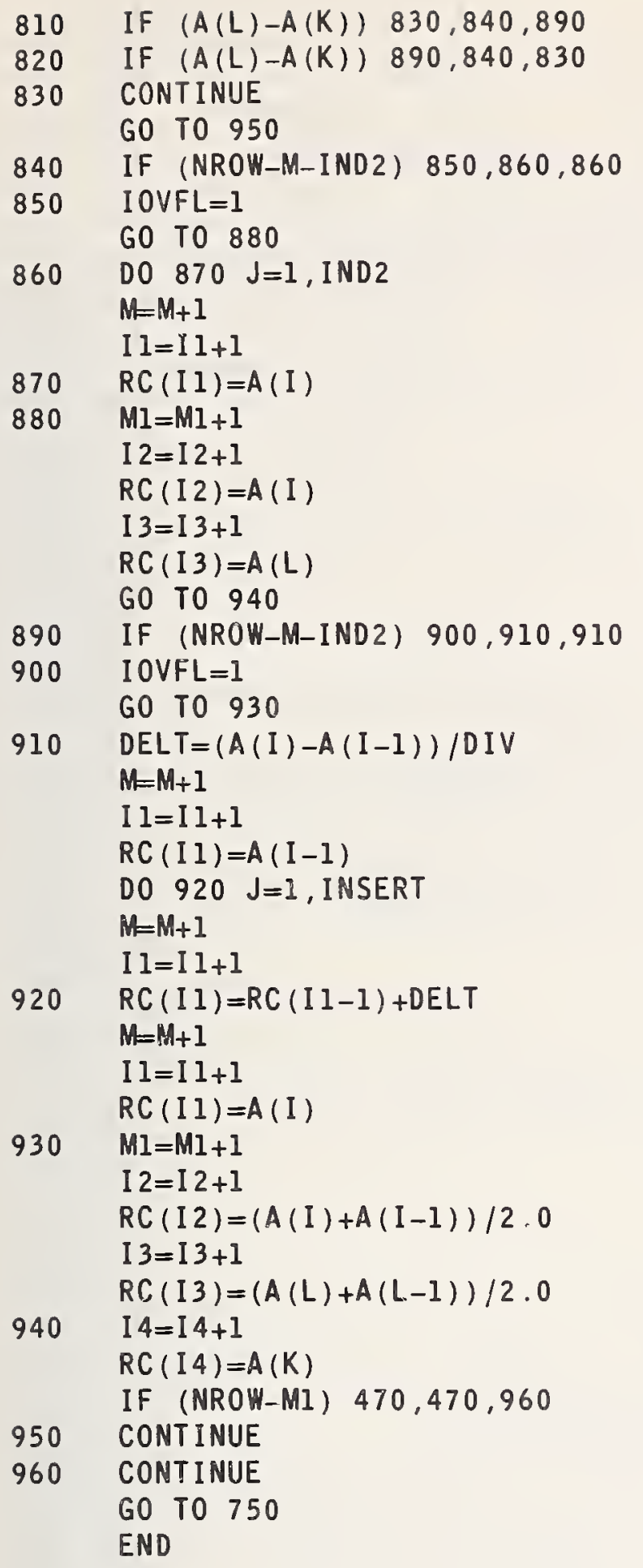




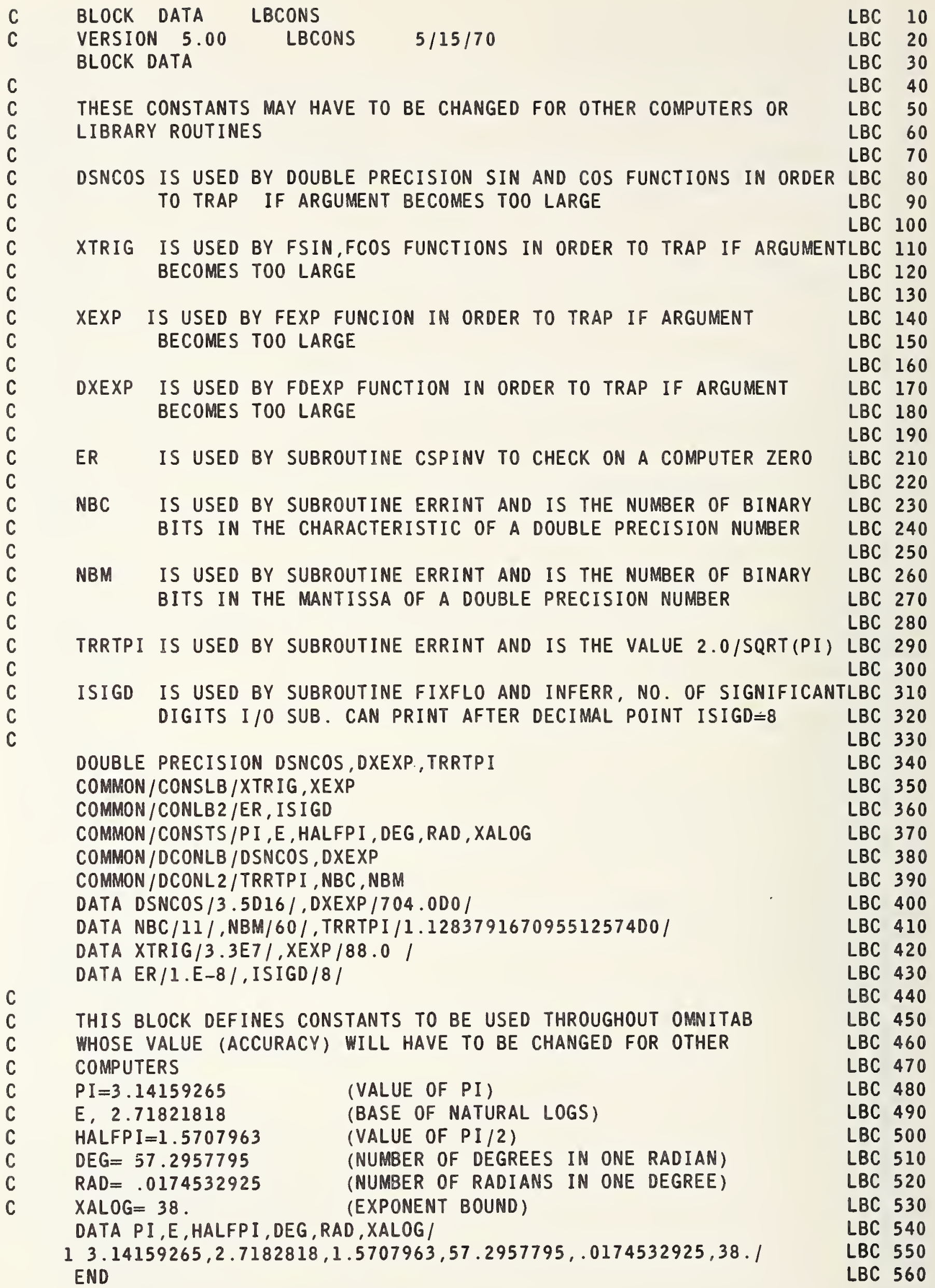


SUBROUTINE LIST (K)

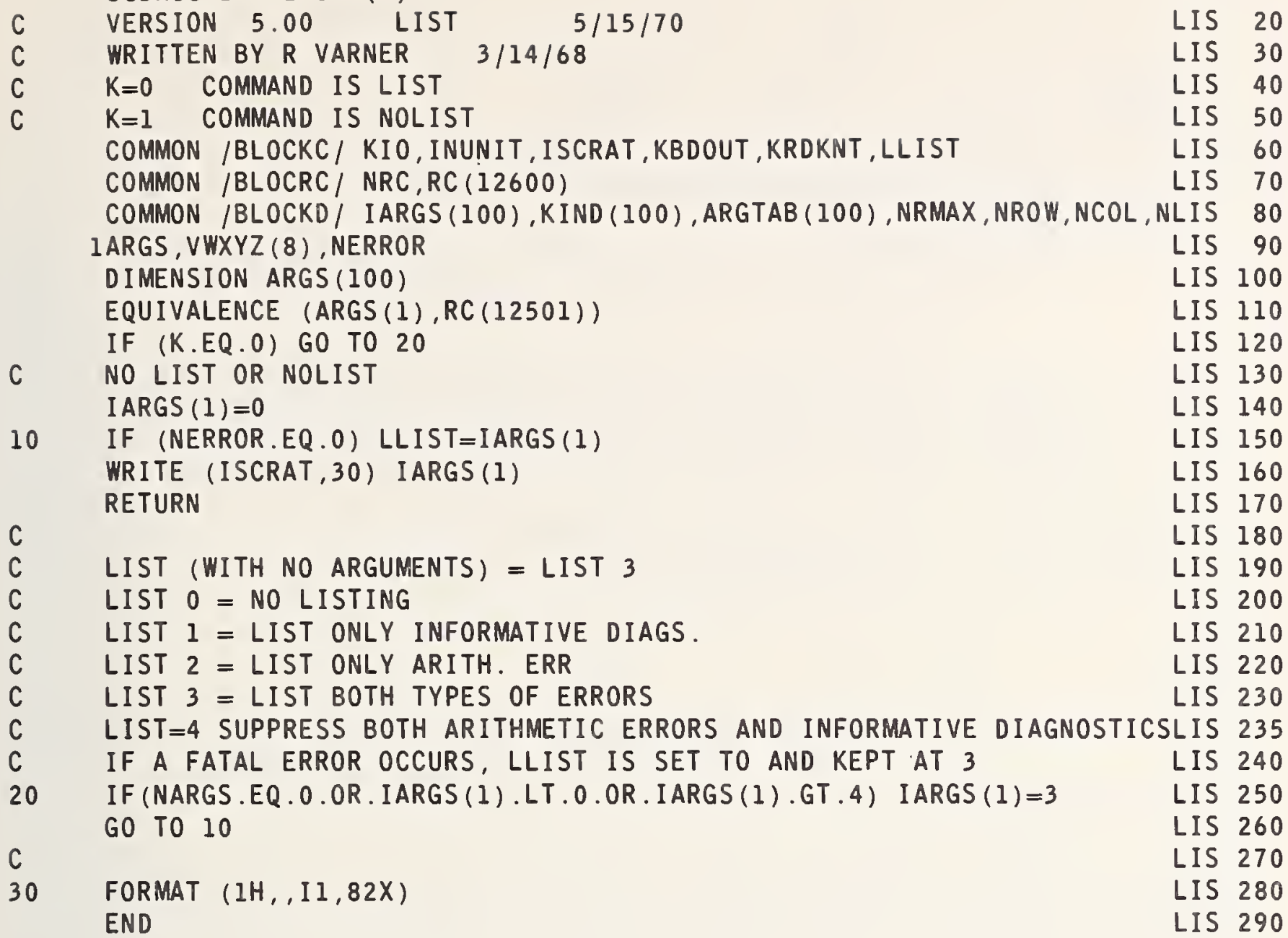

FUNCTION LOCATE (L) LOC 10

$\begin{array}{lllll}\text { C VERSION } 5.00 & \text { LOCATE } & 5 / 15 / 70 & \text { LOC } & 20 \\ & \text { COMMON /BLOCKB/ NSTMT, NSTMTX, NSTMTH, NCOM, LCOM, IOVFL, COM(2000) } & \text { LOC } & 30\end{array}$

$\begin{array}{llllll}\text { C VERSION } 5.00 & \text { LOCATE } & 5 / 15 / 70 & \text { LOC } & 20 \\ & \text { COMMON /BLOCKB/ NSTMT, NSTMTX, NSTMTH, NCOM, LCOM, IOVFL, COM(2000) } & \text { LOC } & 30\end{array}$

$\begin{array}{llllll}\text { C THIS FUNCTION SEARCHES THE LIST OF STORED COMMANDS TO SEE IF ONE LOC } & 40 \\ \text { C }\end{array}$

C WITH STATEMENT NUMBER L EXISTS. IF IT DOES, RETURN ITS LOCATION. LOC 60

C IF IT DOESN'T EXIST, RETURN NEGATIVE THE LOCATION OF THE NEXT LOC 70

C HIGHER STATEMENT NUMBER.

C $\quad$ LOC 90

$I=1$

$A L=L$

10 If $(\operatorname{COM}(I)-A L) 20,30,40$

$20 \quad I=I+\operatorname{IFIX}(\operatorname{COM}(I+1))$

GO TO 10

$30 \quad \angle O C A T E=I$

GO TO 50

$40 \quad$ LOCATE $=-I$

50 RETURN

END

LOC 100

LOC 105

LOC 110

LOC 120

LOC 130

LOC 140

LOC 150

LOC 160

LOC 170

LOC 180 
COMMON / ICODE / NIR,NID,NIRD, LIR, LID, LIRD

LOT 30

COMMON/CODE / IALPH (6), NALPH (5), ID $(9,3)$,

LOT 40

$1 \operatorname{IR}(300,4), \operatorname{IRD}(30,6)$

C **** THE FOLLOWING CARDS ARE NEEDED ONLY FOR TAPE OPERATIONS

LOT 50

THE FOLLOWING CARDS ARE
COMMON/ICODTP/NITP, LITP

LOT 60

COMMON/CODETP /ITP $(10,4)$

LOT 70

LOT 80

LOT 90

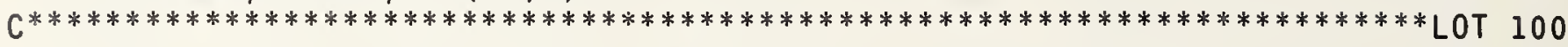

C ADD SUB SUBTRA MULT MULTIP DIV DIVIDE RAISE LOT 110

C

DATA IR(1,1), IR(1,2),IR(1,3), IR(1,4), IR(2,1), IR(2,2), IR(2,3), $\operatorname{LOT} 130$ $1 \operatorname{IR}(2,4), \operatorname{IR}(3,1), \operatorname{IR}(3,2), \operatorname{IR}(3,3), \operatorname{IR}(3,4), \operatorname{IR}(4,1), \operatorname{IR}(4,2), \operatorname{IR}(4,3), \operatorname{LOT} 140$ $2 \operatorname{IR}(4,4), \operatorname{IR}(5,1), \operatorname{IR}(5,2), \operatorname{IR}(5,3), \operatorname{IR}(5,4), \operatorname{IR}(6,1), \operatorname{IR}(6,2), \operatorname{IR}(6,3), \quad \operatorname{LOT} 150$ $3 \operatorname{IR}(6,4), \operatorname{IR}(7,1), \operatorname{IR}(7,2), \operatorname{IR}(7,3), \operatorname{IR}(7,4), \operatorname{IR}(8,1), \operatorname{IR}(8,2), \operatorname{IR}(8,3), \operatorname{LOT} 160$ $4 \operatorname{IR}(8,4) \quad /$

$5 \quad 841,0,11,1,14420,0,11,2,14420,15067,11,2$,

$610056,14580,11,3,10056,14839,11,3,3181,0,11,4$,

$73181,6674,11,4,13158,13986,11,51$

$\begin{array}{llllllll}\text { SIN } & \text { COS } & \text { TAN } & \text { COT } & & \text { ASIN } & & \text { ACOS } \\ \text { ATAN } & & \text { ACOT } & & & & & \\ \text { SIND } & \text { COSD } & \text { TAND } & \text { COTD } & \text { ASIND } & \text { ACOSD } & \text { ATAND } & \text { ACOTD } \\ & \text { SQRT } & \text { EXP } & \text { EXPONE } & \text { NEGEXP } & \text { LOG } & \text { LOGE } & \text { LOGTEN ANTILO } \\ \text { SINH } & \text { COSH } & \text { TANH } & \text { COTH } & \text { ASINH } & \text { ACOSH } & \text { ATANH } & \text { ACOTH ABS } \\ \text { ABSULO } & \text { INTEGE } & \text { FRACTI } & & & & & \end{array}$

DATA IR $(9,1), \operatorname{IR}(9,2), \operatorname{IR}(9,3), \operatorname{IR}(9,4), \operatorname{IR}(10,1), \operatorname{IR}(10,2)$, $1 \operatorname{IR}(10,3), \operatorname{IR}(10,4), \operatorname{IR}(11,1), \operatorname{IR}(11,2), \operatorname{IR}(11,3), \operatorname{IR}(11,4), \operatorname{IR}(12,1)$,

$2 \operatorname{IR}(12,2), \operatorname{IR}(12,3), \operatorname{IR}(12,4), \operatorname{IR}(13,1), \operatorname{IR}(13,2), \operatorname{IR}(13,3), \operatorname{IR}(13,4)$,

$3 \operatorname{IR}(14,1), \operatorname{IR}(14,2), \operatorname{IR}(14,3), \operatorname{IR}(14,4), \operatorname{IR}(15,1), \operatorname{IR}(15,2), \operatorname{IR}(15,3)$,

$4 \operatorname{IR}(15,4), \operatorname{IR}(16,1), \operatorname{IR}(16,2), \operatorname{IR}(16,3), \operatorname{IR}(16,4), \operatorname{IR}(17,1), \operatorname{IR}(17,2)$,

$5 \operatorname{IR}(17,3), \operatorname{IR}(17,4), \operatorname{IR}(18,1), \operatorname{IR}(18,2), \operatorname{IR}(18,3), \operatorname{IR}(18,4), \operatorname{IR}(19,1)$,

$6 \operatorname{IR}(19,2), \operatorname{IR}(19,3), \operatorname{IR}(19,4), \operatorname{IR}(20,1), \operatorname{IR}(20,2), \operatorname{IR}(20,3), \operatorname{IR}(20,4)$,

$7 \operatorname{IR}(21,1), \operatorname{IR}(21,2), \operatorname{IR}(21,3), \operatorname{IR}(21,4), \operatorname{IR}(22,1), \operatorname{IR}(22,2), \operatorname{IR}(22,3)$,

$8 \operatorname{IR}(22,4), \operatorname{IR}(23,1), \operatorname{IR}(23,2), \operatorname{IR}(23,3), \operatorname{IR}(23,4), \operatorname{IR}(24,1), \operatorname{IR}(24,2)$,

$9 \operatorname{IR}(24,3), \operatorname{IR}(24,4), \operatorname{IR}(25,1), \operatorname{IR}(25,2), \operatorname{IR}(25,3), \operatorname{IR}(25,4)$

A $14108,0,12,1,2611,0,12,2,14621,0,12,3$,

B $2612,0,12,4,0,0,0,0,1251,10206,12,5$,

C $\quad 0,0,0,0,825,13851,12,6,0,0,0,0$,

D $1270,10206,12,7,0,0,0,0,825,14580,12,8$,

E $14108,2916,12,9,2611,2916,12,10,14621,2916,12,11$,

F $2612,2916,12,12,1251,10314,12,13 /$

DATA IR $(26,1), \operatorname{IR}(26,2), \operatorname{IR}(26,3), \operatorname{IR}(26,4), \operatorname{IR}(27,1), \operatorname{IR}(27,2)$,

$1 \operatorname{IR}(27,3), \operatorname{IR}(27,4), \operatorname{IR}(28,1), \operatorname{IR}(28,2), \operatorname{IR}(28,3), \operatorname{IR}(28,4), \operatorname{IR}(29,1)$,

$2 \operatorname{IR}(29,2), \operatorname{IR}(29,3), \operatorname{IR}(29,4), \operatorname{IR}(30,1), \operatorname{IR}(30,2), \operatorname{IR}(30,3), \operatorname{IR}(30,4)$,

$3 \operatorname{IR}(31,1), \operatorname{IR}(31,2), \operatorname{IR}(31,3), \operatorname{IR}(31,4), \operatorname{IR}(32,1), \operatorname{IR}(32,2), \operatorname{IR}(32,3)$,

$4 \operatorname{IR}(32,4), \operatorname{IR}(33,1), \operatorname{IR}(33,2), \operatorname{IR}(33,3), \operatorname{IR}(33,4), \operatorname{IR}(34,1), \operatorname{IR}(34,2)$,

$5 \operatorname{IR}(34,3), \operatorname{IR}(34,4), \operatorname{IR}(35,1), \operatorname{IR}(35,2), \operatorname{IR}(35,3), \operatorname{IR}(35,4), \operatorname{IR}(36,1)$,

$6 \operatorname{IR}(36,2), \operatorname{IR}(36,3), \operatorname{IR}(36,4), \operatorname{IR}(37,1), \operatorname{IR}(37,2), \operatorname{IR}(37,3), \operatorname{IR}(37,4)$,

$7 \operatorname{IR}(38,1), \operatorname{IR}(38,2), \operatorname{IR}(38,3), \operatorname{IR}(38,4), \operatorname{IR}(39,1), \operatorname{IR}(39,2), \operatorname{IR}(39,3)$,

$8 \operatorname{IR}(39,4), \operatorname{IR}(40,1), \operatorname{IR}(40,2), \operatorname{IR}(40,3), \operatorname{IR}(40,4), \operatorname{IR}(41,1), \operatorname{IR}(41,2)$,

$9 \operatorname{IR}(41,3), \operatorname{IR}(41,4), \operatorname{IR}(42,1), \operatorname{IR}(42,2), \operatorname{IR}(42,3), \operatorname{IR}(42,4), \operatorname{IR}(43,1)$,

$\operatorname{A} \operatorname{IR}(43,2), \operatorname{IR}(43,3), \operatorname{IR}(43,4), \operatorname{IR}(44,1), \operatorname{IR}(44,2), \operatorname{IR}(44,3), \operatorname{IR}(44,4)$,

$\operatorname{B} \operatorname{IR}(45,1), \operatorname{IR}(45,2), \operatorname{IR}(45,3), \operatorname{IR}(45,4) /$

C $825,13959,12,14,1270,10314,12,15,825,14688,12,16$,

D

E $4309,0,12,18,4309,11318,12,18,10348,4309,12,19$,

LOT 170

LOT 180

LOT 190

LOT 200

LOT 210

LOT 220

LOT 230

LOT 240

LOT 250

LOT 260

LOT 270

LOT 280

LOT 290

LOT 300

LOT 310

LOT 320

LOT 330

LOT 340

LOT 350

LOT 360

LOT 370

LOT 380

LOT 390

LOT 400

LOT 410

LOT 420

LOT 430

LOT 440

LOT 450

LOT 460

LOT 470

LOT 480

LOT 490

LOT 500

LOT 510

LOT 520

LOT 530

LOT 540

LOT 550

LOT 560

LOT 570

LOT 590

LOT 600 
F 9160, $0,12,20,9160,3645,12,20,9160,14729,12,21$,

LOT 610

G $1127,6900,12,22,14108,5832,12,23,2611,5832,12,24$,

LOT 620

H $14621,5832,12,25,2612,5832,12,26,1251,10422,12,27$,

$825,14067,12,28,1270,10422,12,29$,

J $825,14796,12,30,802,0,12,31 /$

DATA IR $(46,1), \operatorname{IR}(46,2), \operatorname{IR}(46,3), \operatorname{IR}(46,4), \operatorname{IR}(47,1), \operatorname{IR}(47,2)$,

$1 \operatorname{IR}(47,3), \operatorname{IR}(47,4), \operatorname{IR}(48,1), \operatorname{IR}(48,2), \operatorname{IR}(48,3), \operatorname{IR}(48,4)$ /

2

$\mathrm{C}$

$36959,3839,12,32,4861,2736,12,331$

$802,11280,12,31$,

\section{GENERA SET FIXED FLOATI PLOT SPACE CGS SI}

DATA IR $(49,1), \operatorname{IR}(49,2), \operatorname{IR}(49,3), \operatorname{IR}(49,4), \operatorname{IR}(50,1), \operatorname{IR}(50,2)$,

$1 \operatorname{IR}(50,3), \operatorname{IR}(50,4), \operatorname{IR}(51,1), \operatorname{IR}(51,2), \operatorname{IR}(51,3), \operatorname{IR}(51,4), \operatorname{IR}(52,1)$,

$2 \operatorname{IR}(52,2), \operatorname{IR}(52,3), \operatorname{IR}(52,4), \operatorname{IR}(53,1), \operatorname{IR}(53,2), \operatorname{IR}(53,3), \operatorname{IR}(53,4)$,

$3 \operatorname{IR}(54,1), \operatorname{IR}(54,2), \operatorname{IR}(54,3), \operatorname{IR}(54,4), \operatorname{IR}(55,1), \operatorname{IR}(55,2), \operatorname{IR}(55,3)$,

$4 \operatorname{IR}(55,4), \operatorname{IR}(56,1), \operatorname{IR}(56,2), \operatorname{IR}(56,3), \operatorname{IR}(56,4), \operatorname{IR}(57,1), \operatorname{IR}(57,2)$,

$5 \operatorname{IR}(57,3), \operatorname{IR}(57,4), \operatorname{IR}(58,1), \operatorname{IR}(58,2), \operatorname{IR}(58,3), \operatorname{IR}(58,4) /$

$65252,4132,13,1,14006,0,13,2,4641,3753,13,3$;

$74713,1278,13,4,12003,14580,13,5,0,0,0,0$,

$814284,2322,13,9,2395,0,13,10,14094,0,13,11$,

$90,0,0,01$

C

C

$\mathrm{C}$

\section{BEGIN SCAN REPEAT EXECUT PERFOR INCREM RESTOR IFLT IFEQ \\ IFGT IFGE IFNE IFLE}

DATA IR $(59,1), \operatorname{IR}(59,2), \operatorname{IR}(59,3), \operatorname{IR}(59,4), \operatorname{IR}(60,1), \operatorname{IR}(60,2)$,

$1 \operatorname{IR}(60,3), \operatorname{IR}(60,4), \operatorname{IR}(61,1), \operatorname{IR}(61,2), \operatorname{IR}(61,3), \operatorname{IR}(61,4), \operatorname{IR}(62,1)$,

$2 \operatorname{IR}(62,2), \operatorname{IR}(62,3), \operatorname{IR}(62 ; 4), \operatorname{IR}(63,1), \operatorname{IR}(63,2), \operatorname{IR}(63,3), \operatorname{IR}(63,4)$,

$3 \operatorname{IR}(64,1), \operatorname{IR}(64,2), \operatorname{IR}(64,3), \operatorname{IR}(64,4), \operatorname{IR}(65,1), \operatorname{IR}(65,2), \operatorname{IR}(65,3)$,

$4 \operatorname{IR}(65,4), \operatorname{IR}(66,1), \operatorname{IR}(66,2), \operatorname{IR}(66,3), \operatorname{IR}(66,4), \operatorname{IR}(67,1), \operatorname{IR}(67,2)$,

$5 \operatorname{IR}(67,3), \operatorname{IR}(67,4), \operatorname{IR}(68,1), \operatorname{IR}(68,2), \operatorname{IR}(68,3), \operatorname{IR}(68,4), \operatorname{IR}(69,1)$,

$6 \operatorname{IR}(69,2), \operatorname{IR}(69,3), \operatorname{IR}(69,4), \operatorname{IR}(70,1), \operatorname{IR}(70,2), \operatorname{IR}(70,3), \operatorname{IR}(70,4)$,

$7 \operatorname{IR}(71,1), \operatorname{IR}(71,2), \operatorname{IR}(71,3), \operatorname{IR}(71,4) /$

$81600,6939,14,1,13933,10206,14,2,13273,3692,14,3$,

$94298,2774,14,3,11817,4797,14,3,6942,13270,14,6$,

A $13276,15003,14,8,6735,14580,14,9,6728,12393,14,10$,

B $6730,14580,14,11,6730,3645,14,12,6737,3645,14,13$,

C $6735,3645,14,141$

C

\section{MDEFIN ADEFIN AERASE MZERO AZERO MERASE MIDENT MSUBTR MDIAGO}

DATA IR $(72,1), \operatorname{IR}(72,2), \operatorname{IR}(72,3), \operatorname{IR}(72,4), \operatorname{IR}(73,1), \operatorname{IR}(73,2)$,

$1 \operatorname{IR}(73,3), \operatorname{IR}(73,4), \operatorname{IR}(74,1), \operatorname{IR}(74,2), \operatorname{IR}(74,3), \operatorname{IR}(74,4), \operatorname{IR}(75,1)$,

$2 \operatorname{IR}(75,2), \operatorname{IR}(75,3), \operatorname{IR}(75,4), \operatorname{IR}(76,1), \operatorname{IR}(76,2), \operatorname{IR}(76,3), \operatorname{IR}(76,4)$,

$3 \operatorname{IR}(77,1), \operatorname{IR}(77,2), \operatorname{IR}(77,3), \operatorname{IR}(77,4), \operatorname{IR}(78,1), \operatorname{IR}(78,2), \operatorname{IR}(78,3)$,

$4 \operatorname{IR}(78,4), \operatorname{IR}(79,1), \operatorname{IR}(79,2), \operatorname{IR}(79,3), \operatorname{IR}(79,4), \operatorname{IR}(80,1), \operatorname{IR}(80,2)$,

$5 \operatorname{IR}(80,3), \operatorname{IR}(80,4) /$

$69590,4631,15,1,842,4631,15,1,882,1247,15,2$,

$710184,13527,15,2,1436,13527,15,2,9630,1247,15,2$,

$89724,4043,15,3,10011,2016,18,2,9594,933,15,41$

C

\section{MINVER INVERT MMULTI}

C

DATA IR $(81,1), \operatorname{IR}(81,2), \operatorname{IR}(81,3), \operatorname{IR}(81,4), \operatorname{IR}(82,1), \operatorname{IR}(82,2)$,

$1 \operatorname{IR}(82,3), \operatorname{IR}(82,4), \operatorname{IR}(83,1), \operatorname{IR}(83,2), \operatorname{IR}(83,3), \operatorname{IR}(83,4)$ /

$29734,16191,16,1,6961,4151,16,1,9849,9297,17,1 /$
LOT 630

LOT 640

LOT 650

LOT 660

LOT 665

LOT 670

LOT 680

LOT 690

LOT 700

LOT 710

LOT 720

LOT 730

LOT 740

LOT 750

LOT 760

LOT 770

LOT 780

LOT 790

LOT 800

LOT 810

LOT 820

LOT 830

LOT 840

LOT 850

LOT 860

LOT 870

LOT 880

LOT 890

LOT 900

LOT 910

LOT 920

LOT 930

LOT 940

LOT 950

LOT 960

LOT 970

LOT 980

LOT 990

LOT1000

LOTI010

LOT 1020

LOT 1030

LOT 1040

LOT 1050

LOT 1060

LOT 1070

LOT 1080

LOT 1090

LOT 1100

LOT1110

LOT 1120

LOT 1130

LOT 1140

LOT 1150

LOT 1160

LOT 1170

LOT 1180 
DATA IR $(84,1), \operatorname{IR}(84,2), \operatorname{IR}(84,3), \operatorname{IR}(84,4), \operatorname{IR}(85,1), \operatorname{IR}(85,2)$,

$1 \operatorname{IR}(85,3), \operatorname{IR}(85,4) /$

$29849,9288,17,1,9964,7079,17,21$

$\mathrm{C}$
$\mathrm{C}$
$\mathrm{C}$

MADD MSUB MTRANS ATRANS AADD ASUB AMULT AMULTI ASUBTR MSCALA ADIVID ADIV ARAISE

DATA IR $(86,1), \operatorname{IR}(86,2), \operatorname{IR}(86,3), \operatorname{IR}(86,4), \operatorname{IR}(87,1), \operatorname{IR}(87,2)$, $6 \operatorname{IR}(87,3), \operatorname{IR}(87,4), \operatorname{IR}(88,1), \operatorname{IR}(88,2), \operatorname{IR}(88,3), \operatorname{IR}(88,4)$,

LOT 1260

LOT 1270

$7 \operatorname{IR}(89,1), \operatorname{IR}(89,2), \operatorname{IR}(89,3), \operatorname{IR}(89,4), \operatorname{IR}(90,1), \operatorname{IR}(90,2), \operatorname{IR}(90,3)$,

$8 \operatorname{IR}(90,4), \operatorname{IR}(91,1), \operatorname{IR}(91,2), \operatorname{IR}(91,3), \operatorname{IR}(91,4), \operatorname{IR}(92,1), \operatorname{IR}(92,2)$,

$9 \operatorname{IR}(92,3), \operatorname{IR}(92,4), \operatorname{IR}(93,1), \operatorname{IR}(93,2), \operatorname{IR}(93,3), \operatorname{IR}(93,4), \operatorname{IR}(94,1)$,

A IR $(94,2), \operatorname{IR}(94,3), \operatorname{IR}(94,4), \operatorname{IR}(95,1), \operatorname{IR}(95,2), \operatorname{IR}(95,3), \operatorname{IR}(95,4)$,

B IR $(96,1), \operatorname{IR}(96,2), \operatorname{IR}(96,3), \operatorname{IR}(96,4), \operatorname{IR}(97,1), \operatorname{IR}(97,2), \operatorname{IR}(97,3)$,

C $\operatorname{IR}(97,4), \operatorname{IR}(98,1), \operatorname{IR}(98,2), \operatorname{IR}(98,3), \operatorname{IR}(98,4) /$

D $9508,2916,18,1,10011,1458,18,2,10035,1126,18,3$,

E $1287,1126,18,3,760,2916,18,4,1263,1458,18,5$,

F $1101,9288,18,6,1101,9297,18,6,1263,2016,18,5$,

G $9993,1054,18,6,846,16285,18,7,846,16038,18,7$,

LOT1280

LOT 1290

LOT 1300

LOT1310

LOT 1320

LOT1330

LOT1340

LOT1350

LOT1360

LOT1370

LOT1380

H $1216,7079,18,8 /$

C
C
C

DATA IR $(99,1), \operatorname{IR}(99,2), \operatorname{IR}(99,3), \operatorname{IR}(99,4), \operatorname{IR}(100,1)$,

$1 \operatorname{IR}(100,2), \operatorname{IR}(100,3), \operatorname{IR}(100,4), \operatorname{IR}(101,1), \operatorname{IR}(101,2), \operatorname{IR}(101,3)$,

$2 \operatorname{IR}(101,4), \operatorname{IR}(102,1), \operatorname{IR}(102,2), \operatorname{IR}(102,3), \operatorname{IR}(102,4), \operatorname{IR}(103,1)$,

$3 \operatorname{IR}(103,2), \operatorname{IR}(103,3), \operatorname{IR}(103,4), \operatorname{IR}(104,1), \operatorname{IR}(104,2), \operatorname{IR}(104,3)$, $4 \operatorname{IR}(104,4) /$

$\begin{array}{lllllllll}5 & 0, & 0,0,0, & 0, & 0,0,0, & 0, & 0,0,0, \\ 6 & 0, & 0,0,0, & 0, & 0,0, & 0, & 0, & 0,0,0)\end{array}$

\section{PARSUM PARPRO RMS AVERAG SUM}

DATA IR $(105,1), \operatorname{IR}(105,2), \operatorname{IR}(105,3), \operatorname{IR}(105,4), \operatorname{IR}(106,1)$, $1 \operatorname{IR}(106,2), \operatorname{IR}(106,3), \operatorname{IR}(106,4), \operatorname{IR}(107,1), \operatorname{IR}(107,2), \operatorname{IR}(107,3)$,

$2 \operatorname{IR}(107,4), \operatorname{IR}(108,1), \operatorname{IR}(108,2), \operatorname{IR}(108,3), \operatorname{IR}(108,4), \operatorname{IR}(109,1)$,

$3 \operatorname{IR}(109,2), \operatorname{IR}(109,3), \operatorname{IR}(109,4) /$

$411709,14431,20,1,11709,12165,20,2,13492,0,20,3$,

$51328,13156,20,4,14431,0,20,51$

C

\section{ROWSUM PRODUC DEFINE MAX MAXIMU MIN MINIMU SORT ORDER ERASE EXCHAN FLIP CHANGE HIERAR LIST}

DATA IR(110,1), IR $(110,2), \operatorname{IR}(110,3), \operatorname{IR}(110,4), \operatorname{IR}(111,1)$, $1 \operatorname{IR}(111,2), \operatorname{IR}(111,3), \operatorname{IR}(111,4), \operatorname{IR}(112,1), \operatorname{IR}(112,2), \operatorname{IR}(112,3)$, $2 \operatorname{IR}(112,4), \operatorname{IR}(113,1), \operatorname{IR}(113,2), \operatorname{IR}(113,3), \operatorname{IR}(113,4), \operatorname{IR}(114,1)$, $3 \operatorname{IR}(114,2), \operatorname{IR}(114,3), \operatorname{IR}(114,4), \operatorname{IR}(115,1), \operatorname{IR}(115,2), \operatorname{IR}(115,3)$, $4 \operatorname{IR}(115,4), \operatorname{IR}(116,1), \operatorname{IR}(116,2), \operatorname{IR}(116,3), \operatorname{IR}(116,4), \operatorname{IR}(117,1)$, $5 \operatorname{IR}(117,2), \operatorname{IR}(117,3), \operatorname{IR}(117,4), \operatorname{IR}(118,1), \operatorname{IR}(118,2), \operatorname{IR}(118,3)$, $6 \operatorname{IR}(118,4), \operatorname{IR}(119,1), \operatorname{IR}(119,2), \operatorname{IR}(119,3), \operatorname{IR}(119,4), \operatorname{IR}(120,1)$, $7 \operatorname{IR}(120,2), \operatorname{IR}(120,3), \operatorname{IR}(120,4), \operatorname{IR}(121,1), \operatorname{IR}(121,2), \operatorname{IR}(121,3)$, $8 \operatorname{IR}(121,4), \operatorname{IR}(122,1), \operatorname{IR}(122,2), \operatorname{IR}(122,3), \operatorname{IR}(122,4), \operatorname{IR}(123,1)$, $9 \operatorname{IR}(123,2), \operatorname{IR}(123,3), \operatorname{IR}(123,4), \operatorname{IR}(124,1), \mathbb{I R}(124,2), \operatorname{IR}(124,3)$,

$A \operatorname{IR}(124,4), \operatorname{IR}(125,1), \operatorname{IR}(125,2), \operatorname{IR}(125,3), \operatorname{IR}(125,4), \operatorname{IR}(126,1)$,

B IR $(126,2), \operatorname{IR}(126,3), \operatorname{IR}(126,4) /$

C $13550,14431,21,1,12165,3486,21,2,3057,6944,21,3$,

D $9528,0,21,5,9528,6933,21,5,9734,0,21,6$,

E $9734,6933,21,6,14274,14580,21,8,11425,4131,21,9$,
LOT1390

LOT 1400

LOT1410

LOT 1420

LOT 1430

LOT1440

LOT1450

LOT1460

LOT1470

LOTI 480

LOT1490

LOT1500

LOT1510

LOT 1520

LOT 1530

LOT1540

LOT 1550

LOT1560

LOT1570

LOT1580

LOT 1590

LOT 1600

LOT1610

LOT 1620

LOT1630

LOT1640

LOT1650

LOT1660

LOT1670

LOT1680

LOT 1690

LOT 1700

LOT 1710

LOT 1720

LOT1730

LOT 1740

LOT1750

LOT1760

LOT 1770 
F $4132,13986,21,10,4296,5873,21,11,4707,11664,21,12$,

LOT 1780

G $2404,10400,21,13,6080,13167,21,14,9010,14580,21,15$,

H $\quad 0,0,0,0,10785,8748,21,17$ /

LOT1790

LOT 1800

LOT1810

POLYFI SPOLYF FIT SFIT SOLVE MORTHO

LOT 1820

LOT 1830

DATA IR $(127,1), I R(127,2), \operatorname{IR}(127,3), \operatorname{IR}(127,4), \operatorname{IR}(128,1)$,

LOT1840

$1 \operatorname{IR}(128,2), \operatorname{IR}(128,3), \operatorname{IR}(128,4), \operatorname{IR}(129,1), \operatorname{IR}(129,2), \operatorname{IR}(129,3)$,

LOT 1850

$2 \operatorname{IR}(129,4), \operatorname{IR}(130,1), \operatorname{IR}(130,2), \operatorname{IR}(130,3), \operatorname{IR}(130,4), \operatorname{IR}(131,1)$,

LOT 1860

$3 \operatorname{IR}(131,2), \operatorname{IR}(131,3), \operatorname{IR}(131,4), \operatorname{IR}(132,1), \operatorname{IR}(132,2), \operatorname{IR}(132,3)$,

$4 \operatorname{IR}(132,4)$ /

$512081,18396,22,1,14298,9429,22,2,4637,0,22,3$,

LOT 1870

LOT 1880

LOT 1890

$614022,14580,22,4,14268,16173,16,2,9900,14811,22,51$

C

C

$\mathrm{C}$

C

\section{MMOVE PROMOT DEMOTE DIMENS}

DATA IR $(133,1), \operatorname{IR}(133,2), \operatorname{IR}(133,3), \operatorname{IR}(133,4), \operatorname{IR}(134,1)$,

$1 \operatorname{IR}(134,2), \operatorname{IR}(134,3), \operatorname{IR}(134,4), \operatorname{IR}(135,1), \operatorname{IR}(135,2), \operatorname{IR}(135,3)$,

$2 \operatorname{IR}(135,4), \operatorname{IR}(136,1), \operatorname{IR}(136,2), \operatorname{IR}(136,3), \operatorname{IR}(136,4), \operatorname{IR}(137,1)$,

$3 \operatorname{IR}(137,2), \operatorname{IR}(137,3), \operatorname{IR}(137,4), \operatorname{IR}(138,1), \operatorname{IR}(138,2), \operatorname{IR}(138,3)$,

$4 \operatorname{IR}(138,4), \operatorname{IR}(139,1), \operatorname{IR}(139,2), \operatorname{IR}(139,3), \operatorname{IR}(139,4), \operatorname{IR}(140,1)$,

$5 \operatorname{IR}(140,2), \operatorname{IR}(140,3), \operatorname{IR}(140,4), \operatorname{IR}(141,1), \operatorname{IR}(141,2), \operatorname{IR}(141,3)$,

$6 \operatorname{IR}(141,4), \operatorname{IR}(142,1), \operatorname{IR}(142,2), \operatorname{IR}(142,3), \operatorname{IR}(142,4), \operatorname{IR}(143,1)$,

$7 \operatorname{IR}(143,2), \operatorname{IR}(143,3), \operatorname{IR}(143,4), \operatorname{IR}(144,1), \operatorname{IR}(144,2), \operatorname{IR}(144,3)$,

$8 \operatorname{IR}(144,4), \operatorname{IR}(145,1), \operatorname{IR}(145,2), \operatorname{IR}(145,3), \operatorname{IR}(145,4) /$

$9 \quad 0,0,0,0,0,0,0,0,2613,10746,23,2$,

A $14082,13667,23,3,4309,1111,23,4,3499,8994,23,5$,

B $9904,3645,23,6,3172,0,23,12,1095,16173,23,6$,

C $9843,16173,23,6,12165,9902,23,10,3064,11480,23,11$,

D $3172,4042,23,121$

C

C

C

\section{STATIS SSTATI RANKS ACCURA}

DATA IR $(146,1), \operatorname{IR}(146,2), \operatorname{IR}(146,3), \operatorname{IR}(146,4), \operatorname{IR}(147,1)$,

$1 \operatorname{IR}(147,2), \operatorname{IR}(147,3), \operatorname{IR}(147,4), \operatorname{IR}(148,1), \operatorname{IR}(148,2), \operatorname{IR}(148,3)$,

$2 \operatorname{IR}(148,4), \operatorname{IR}(149,1), \operatorname{IR}(149,2), \operatorname{IR}(149,3), \operatorname{IR}(149,4), \operatorname{IR}(150,1)$,

$3 \operatorname{IR}(150,2), \operatorname{IR}(150,3), \operatorname{IR}(150,4) /$

$414392,14842,24,1,14384,1278,24,2,13163,8532,24,3$.

$5813,15796,11,6,0,0,0,01$

C

C

C

\section{SELECT SEARCH CENSOR}

DATA IR $(151,1), \operatorname{IR}(151,2), \operatorname{IR}(151,3), \operatorname{IR}(151,4), \operatorname{IR}(152,1)$,

$1 \operatorname{IR}(152,2), \operatorname{IR}(152,3), \operatorname{IR}(152,4), \operatorname{IR}(153,1), \operatorname{IR}(153,2), \operatorname{IR}(153,3)$ 。

$2 \operatorname{IR}(153,4) /$

$313998,3746,25,1,13987,13211,25,2,2336,14274,25,31$

C

C

C

\section{MVECDI MVECMA MMATV}

DATA IR $(154,1), \operatorname{IR}(154,2), \operatorname{IR}(154,3), \operatorname{IR}(154,4), \operatorname{IR}(155,1)$,

$1 \operatorname{IR}(155,2), \operatorname{IR}(155,3), \operatorname{IR}(155,4), \operatorname{IR}(156,1), \operatorname{IR}(156,2), \operatorname{IR}(156,3)$,

$2 \operatorname{IR}(156,4), \operatorname{IR}(157,1), \operatorname{IR}(157,2), \operatorname{IR}(157,3), \operatorname{IR}(157,4), \operatorname{IR}(158,1)$,

$3 \operatorname{IR}(158,2), \operatorname{IR}(158,3), \operatorname{IR}(158,4), \operatorname{IR}(159,1), \operatorname{IR}(159,2), \operatorname{IR}(159,3)$,

$4 \operatorname{IR}(159,4) /$

$510076,2304,26,1,0,0,0,0,10076,2539,26,2$,

$60,0,0,0,9829,15179,26,3,0,0,0,01$

C

C
LOT1900

LOT1910

LOT 1920

LOT 1930

LOT 1940

LOT 1950

LOT1960

LOT1970

LOT1980

LOT 1990

LOT 2000

LOT 2010

LOT 2020

LOT 2030

LOT 2040

LOT2050

LOT 2060

LOT2070

LOT 2080

LOT 2090

LOT 2100

LOT 2110

LOT 2120

LOT2130

LOT 2140

LOT 2150

LOT2160

LOT 2170

LOT 2180

LOT 2190

LOT2200

LOT2210

LOT 2220

LOT2230

LOT2240

LOT 2250

LOT2260

LOT2270

LOT2280

LOT2290

LOT 2300

LOT2310

LOT2320

LOT2330

LOT2340

LOT2350

LOT2360 
$1 \operatorname{IR}(161,2), \operatorname{IR}(161,3), \operatorname{IR}(161,4), \operatorname{IR}(162,1), \operatorname{IR}(162,2), \operatorname{IR}(162,3)$,

C

$39792,11318,17,3,10035,6602,17,4,9621,5252,17,51$

\section{INTERP}

$\operatorname{DATA} \operatorname{IR}(163,1), \operatorname{IR}(163,2), \operatorname{IR}(163,3), \operatorname{IR}(163,4) /$
$16959,4147,25,4 /$

C

\section{ITERATE ISETUP ISOLATE} $2 \operatorname{IR}(170,4) /$

$37106,13169,28,1,7079,15163,28,2,7089,8795,28,3 /$

\section{EXTREMA SEPARATE INSERT MAXMIN}

DATA $2 \operatorname{IR}(173,4), \operatorname{IR}(174,1), \operatorname{IR}(174,2), \operatorname{IR}(174,3), \operatorname{IR}(174,4) /$

$34313,13270,29,4,14002,1216,29,2,6958,4151,29,3$,

$49528,9734,29,41$

\section{LAGUER NORMLA HERMIT UCHEBY TCHEBY LEGEND} $4 \operatorname{IR}(180,4) /$

$58782,15462,19,2,10629,9802,19,1,5985,9740,19,3$, $615398,3724,19,4,14669,3724,19,6,8890,4027,19,5 /$

\section{BJZERO BJONE BYZERO BYONE BIZERO BIONE BKZERO BKONE EXIZER EXIONE EXKZER EXKONE KBIZER KBIONE KBKZER KBKONE}

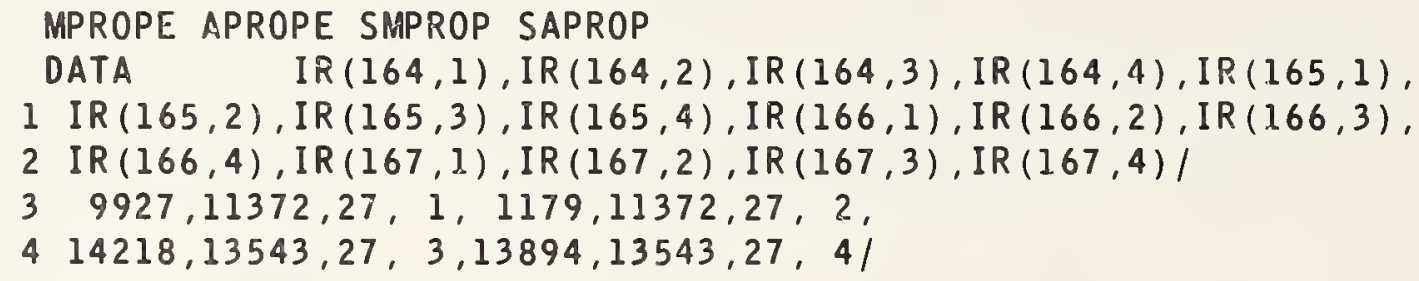

DATA IR $(168,1), \operatorname{IR}(168,2), \operatorname{IR}(168,3), \operatorname{IR}(168,4), \operatorname{IR}(169,1)$, $1 \operatorname{IR}(169,2), \operatorname{IR}(169,3), \operatorname{IR}(169,4), \operatorname{IR}(170,1), \operatorname{IR}(170,2), \operatorname{IR}(170,3)$, $\operatorname{IR}(171,1), \operatorname{IR}(171,2), \operatorname{IR}(171,3), \operatorname{IR}(171,4), \operatorname{IR}(172,1)$, $1 \operatorname{IR}(172,2), \operatorname{IR}(172,3), \operatorname{IR}(172,4), \operatorname{IR}(173,1), \operatorname{IR}(173,2), \operatorname{IR}(173,3)$,

DATA IR $(175,1), \operatorname{IR}(175,2), \operatorname{IR}(175,3), \operatorname{IR}(175,4), \operatorname{IR}(176,1)$, $1 \operatorname{IR}(176,2), \operatorname{IR}(176,3), \operatorname{IR}(176,4), \operatorname{IR}(177,1), \operatorname{IR}(177,2), \operatorname{IR}(177,3)$, $2 \operatorname{IR}(177,4), \operatorname{IR}(178,1), \operatorname{IR}(178,2), \operatorname{IR}(178,3), \operatorname{IR}(178,4), \operatorname{IR}(179,1)$, $3 \operatorname{IR}(179,2), \operatorname{IR}(179,3), \operatorname{IR}(179,4), \operatorname{IR}(180,1), \operatorname{IR}(180,2), \operatorname{IR}(180,3)$,

DATA IR $(181,1), \operatorname{IR}(181,2), \operatorname{IR}(181,3), \operatorname{IR}(181,4), \operatorname{IR}(182,1)$, $1 \operatorname{IR}(182,2), \operatorname{IR}(182,3), \operatorname{IR}(182,4), \operatorname{IR}(183,1), \operatorname{IR}(183,2), \operatorname{IR}(183,3)$, $2 \operatorname{IR}(183,4), \operatorname{IR}(184,1), \operatorname{IR}(184,2), \operatorname{IR}(184,3), \operatorname{IR}(184,4), \operatorname{IR}(185,1)$, $3 \operatorname{IR}(185,2), \operatorname{IR}(185,3), \operatorname{IR}(185,4), \operatorname{IR}(186,1), \operatorname{IR}(186,2), \operatorname{IR}(186,3)$, $4 \operatorname{IR}(186,4), \operatorname{IR}(187,1), \operatorname{IR}(187,2), \operatorname{IR}(187,3), \operatorname{IR}(187,4), \operatorname{IR}(188,1)$, $5 \operatorname{IR}(188,2), \operatorname{IR}(188,3), \operatorname{IR}(188,4), \operatorname{IR}(189,1), \operatorname{IR}(189,2), \operatorname{IR}(189,3)$, $6 \operatorname{IR}(189,4), \operatorname{IR}(190,1), \operatorname{IR}(190,2), \operatorname{IR}(190,3), \operatorname{IR}(190,4), \operatorname{IR}(191,1)$, $7 \operatorname{IR}(191,2), \operatorname{IR}(191,3), \operatorname{IR}(191,4), \operatorname{IR}(192,1), \operatorname{IR}(192,2), \operatorname{IR}(192,3)$, $8 \operatorname{IR}(192,4), \operatorname{IR}(193,1), \operatorname{IR}(193,2), \operatorname{IR}(193,3), \operatorname{IR}(193,4), \operatorname{IR}(194,1)$, $9 \operatorname{IR}(194,2), \operatorname{IR}(194,3), \operatorname{IR}(194,4), \operatorname{IR}(195,1), \operatorname{IR}(195,2), \operatorname{IR}(195,3)$,

A IR $(195,4), \operatorname{IR}(196,1), \operatorname{IR}(196,2), \operatorname{IR}(196,3), \operatorname{IR}(196,4) /$

B $1754,4146,30,1,1743,10341,30,2,2159,4146,30,3$, C $2148,10341,30,4,1727,4146,30,5,1716,10341,30,6$,
LOT 2400

LOT 2410

LOT 2420

LOT2430

LOT 2440

LOT2450

LOT2460

LOT2470

LOT2480

LOT 2490

LOT2500

LOT2510

LOT2520

LOT2530

LOT2540

LOT 2550

LOT 2560

LOT 2570

LOT2580

LOT 2590

LOT2600

LOT 2610

LOT2620

LOT2630

LOT2640

LOT2650

LOT 2660

LOT 2670

LOT2680

LOT 2690

LOT 2700

LOT 2710

LOT 2720

LOT 2730

LOT 2740

LOT 2750

LOT2760

LOT 2770

LOT 2780

LOT 2790

LOT 2800

LOT 2810

LOT 2820

LOT 2830

LOT 2840

LOT2850

LOT 2860

LOT 2870

LOT 2880

LOT 2890

LOT2900

LOT 2910

LOT2920

LOT 2930

LOT2940

LOT 2950 
C

C

C

C

C

C

C

C

C

C

C

C

C

C

C

C

C
D $1781,4146,30,7,1770,10341,30,8,4302,19107,30,9$,

E $4302,11318,30,10,4304,19107,30,11,4304,11318,30,12$,

F $8082,19107,30,13,8082,11318,30,14,8084,19107,30,15$,

G
LOT2960

LOT 2970

LOT 2980

LOT 2990

LOT 3000

LOT 3010

LOT 3020

LOT 3030

LOT 3040

LOT 3050

LOT 3060

LOT 3070

LOT 3080

LOT 3090

LOT 3100

LOT 3110

LOT 3120

LOT 3130

LOT 3140

LOT 3150

LOT 3160

LOT 3170

LOT 3180

LOT 3190

LOT 3200

LOT 3210

LOT 3220

LOT 3230

LOT 3240

LOT 3250

LOT 3260

LOT 3270

LOT 3280

LOT 3290

LOT 3300

LOT3310

LOT 3320

LOT3330

LOT3340

LOT3350

LOT3360

LOT3370

LOT 3380

LOT 3390

LOT 3400

LOT 3410

LOT3420

LOT3430

LOT3440

LOT3450

LOT3460

LOT3470

LOT 3480

LOT3490

LOT 3500

LOT3510

LOT3520

LOT3530

LOT3540 
DATA IR $(223,1), \operatorname{IR}(223,2), \operatorname{IR}(223,3), \operatorname{IR}(223,4), \operatorname{IR}(224,1)$,

LOT3570

I $\operatorname{IR}(224,2), \operatorname{IR}(224,3), \operatorname{IR}(224,4)$ /

$22610,13269,24,11,13947,13613,24,121$

LOT3580

LOT3590

LOT3600

LOT3610

COMPARE , ONEWAY, SONEWAY, ERROR, CERF , STWOWAY

LOT3620

LOT3630

LOT3640

DATA IR $(225,1), \operatorname{IR}(225,2), \operatorname{IR}(225,3), \operatorname{IR}(225,4), \operatorname{IR}(226,1), \operatorname{IR}(226,2), \operatorname{LOT} 3650$ $1 \quad \operatorname{IR}(226,3), \operatorname{IR}(226,4), \operatorname{IR}(227,1), \operatorname{IR}(227,2), \operatorname{IR}(227,3), \operatorname{IR}(227,4)$,

LOT3660

$\operatorname{IR}(228,1), \operatorname{IR}(228,2), \operatorname{IR}(228,3), \operatorname{IR}(228,4), \operatorname{IR}(229,1), \operatorname{IR}(229,2)$,

LOT3670

$\operatorname{IR}(229,3), \operatorname{IR}(229,4), \operatorname{IR}(230,1), \operatorname{IR}(230,2), \operatorname{IR}(230,3), \operatorname{IR}(230,4) / \operatorname{LOT} 3680$ $2605,11709,14,15,11318,16819,24,13,14270,4267,24,14$,

$5 \quad 4149,11421,21,18,2340,4374,21,19,14414,11557,24,7 /$

LOT3690

LOT3700

CTOF, FTOC , ATOMIC, MOLHT, EINSTEIN, PFTRANS, PFATOMIC, PARTFUNCT, BOLDISTLOT 3720

DATA IR $(231,1), \operatorname{IR}(231,2), \operatorname{IR}(231,3), \operatorname{IR}(231,4), \operatorname{IR}(232,1), \operatorname{IR}(232,2), \operatorname{LOT} 3730$

$1 \quad \operatorname{IR}(232,3), \operatorname{IR}(232,4), \operatorname{IR}(233,1), \operatorname{IR}(233,2), \operatorname{IR}(233,3), \operatorname{IR}(233,4), \operatorname{LOT} 3740$

$2 \quad \operatorname{IR}(234,1), \operatorname{IR}(234,2), \operatorname{IR}(234,3), \operatorname{IR}(234,4), \operatorname{IR}(235,1), \operatorname{IR}(235,2), \operatorname{LOT} 3750$

$3 \operatorname{IR}(235,3), \operatorname{IR}(235,4), \operatorname{IR}(236,1), \operatorname{IR}(236,2), \operatorname{IR}(236,3), \operatorname{IR}(236,4), \operatorname{LOT} 3760$

$4 \quad \operatorname{IR}(237,1), \operatorname{IR}(237,2), \operatorname{IR}(237,3), \operatorname{IR}(237,4), \operatorname{IR}(238,1), \operatorname{IR}(238,2), \operatorname{LOT} 3770$

$5 \quad \operatorname{IR}(238,3), \operatorname{IR}(238,4), \operatorname{IR}(239,1), \operatorname{IR}(239,2), \operatorname{IR}(239,3), \operatorname{IR}(239,4) / \operatorname{LOT} 3780$

$62742,4374,31,1,4929,2187,31,2,1284,9723,31,3,9894,17307 L 0 T 3790$

$7,31,4,3902,14396,31,5,11846,13163,31,6,11827,14998,31,7,11709 L 0 T 3800$

$8,14763,31,8,1875,3178,31,91$

LOT3810

LOT 3820

ROUND

LOT3830

LOT 3840

$\operatorname{DATA} \operatorname{IR}(240,1), \operatorname{IR}(240,2), \operatorname{IR}(240,3), \operatorname{IR}(240,4) / 13548,10314,13,14 / \quad \operatorname{LOT} 3850$

LOT3860

COMPLEX ARITHMETIC - CADD, CSUBTRACT, CMULTIPLY, CDIVIDE, LOT3870 CRECTANGULAR, CPOLAR
LOT3880

LOT 3890

DATA IR(241,1),IR(241,2), IR(241,3), IR(241,4), IR(242,1), IR(242,2), LOT3900

$1 \quad \operatorname{IR}(242,3), \operatorname{IR}(242,4), \operatorname{IR}(243,1), \operatorname{IR}(243,2), \operatorname{IR}(243,3), \operatorname{IR}(243,4), \operatorname{LOT} 3910$

$2 \operatorname{IR}(244,1), \operatorname{IR}(244,2), \operatorname{IR}(244,3), \operatorname{IR}(244,4), \operatorname{IR}(245,1), \operatorname{IR}(245,2), \operatorname{LOT} 3920$

$3 \quad \operatorname{IR}(245,3), \operatorname{IR}(245,4), \operatorname{IR}(246,1), \operatorname{IR}(246,2), \operatorname{IR}(246,3), \operatorname{IR}(246,4) / \operatorname{LOT} 3930$

$42218,2916,32,1,2721,2016,32,2,2559,9297,32,3, \quad$ LOT 3940

$5 \quad 2304,16285,32,4,2678,2728,32,5,2634,8793,32,61 \quad$ LOT 3950

C

$\mathrm{C}$

C

$\mathrm{C}$

C

C

C

C

C

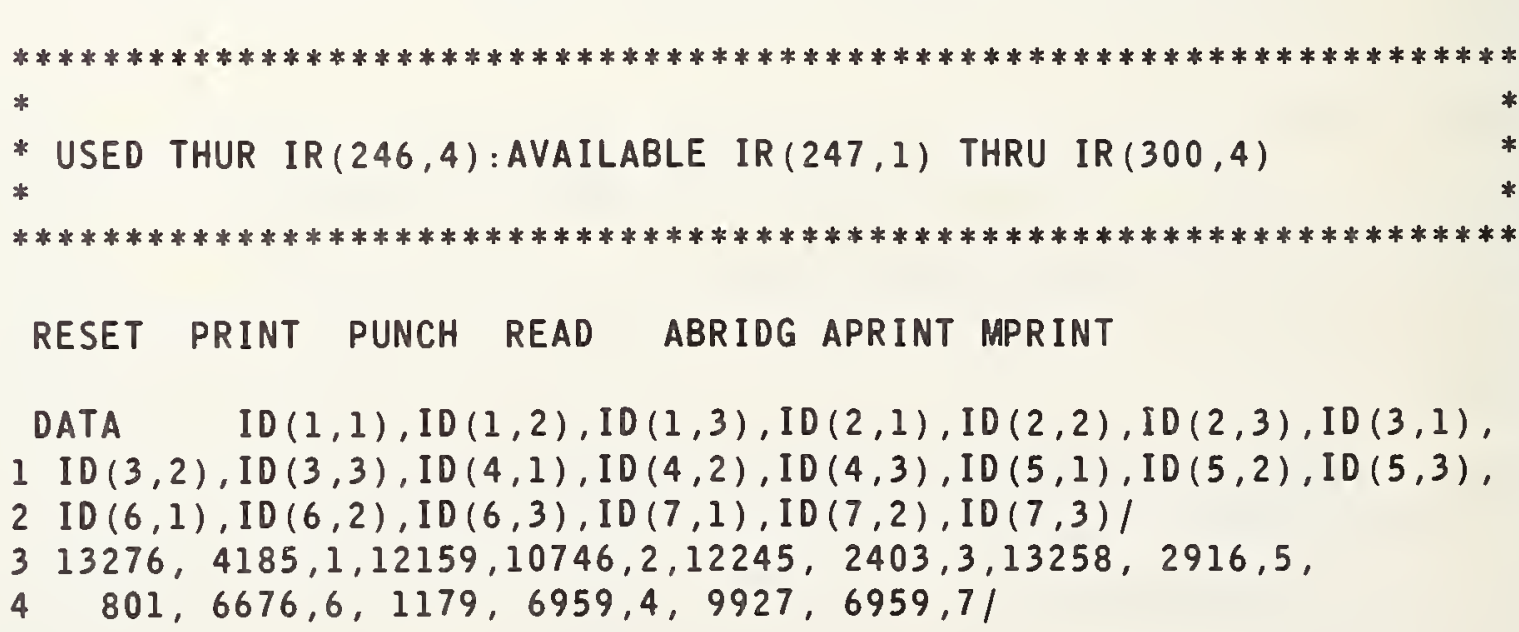

LOT3960

*LOT3970

*LOT3980

*LOT3990

* LOT4000

*LOT4010

LOT 4020

LOT 4030

LOT4040

LOT4050

LOT 4060

LOT4070

LOT4080

LOT 4090

LOT 4100

NPRINT

LOT4110

LOT4120

DATA

$\operatorname{ID}(8,1), I D(8,2), I D(8,3) /$

LOT 4130 
DATA IALPH(1), IALPH(2), IALPH (3), IALPH (4), IALPH (5), IALPH (6) / LOT 4160

LOT 4170

$1729,1458,2187,2916,3645,4374 /$

LOT 4180

LOT 4190

LOT 4200

$V W X Y Z$

LOT 4210

LOT 4220

DATA NALPH (1), NALPH (2), NALPH (3), NALPH ( 4 ), NALPH ( 5 ) /

LOT 4230

$116038,16767,17496,18225,18954 /$

LOT 4240

LOT 4250

NO LIST CLOSE UP NEW PAGE

$M(X X) \quad M,(X, A X)=M(X, X) \quad M(X A X) \quad M,(A D) \quad M(D A) \quad M(A V) \quad M(V, A)$

LOT 4260

LOT 4270

DATA IRD $(1,1), \operatorname{IRD}(1,2), \operatorname{IRD}(1,3), \operatorname{IRD}(1,4), \operatorname{IRD}(1,5)$,

1 IRD $(1,6), \operatorname{IRD}(2,1), \operatorname{IRD}(2,2), \operatorname{IRD}(2,3), \operatorname{IRD}(2,4), \operatorname{IRD}(2,5)$,

$2 \operatorname{IRD}(2,6), \operatorname{IRD}(3,1), \operatorname{IRD}(3,2), \operatorname{IRD}(3,3), \operatorname{IRD}(3,4), \operatorname{IRD}(3,5)$,

$3 \operatorname{IRD}(3,6), \operatorname{IRD}(4,1), \operatorname{IRD}(4,2), \operatorname{IRD}(4,3), \operatorname{IRD}(4,4), \operatorname{IRD}(4,5)$,

$4 \operatorname{IRD}(4,6), \operatorname{IRD}(5,1), \operatorname{IRD}(5,2), \operatorname{IRD}(5,3), \operatorname{IRD}(5,4), \operatorname{IRD}(5,5)$,

$5 \operatorname{IRD}(5,6), \operatorname{IRD}(6,1), \operatorname{IRD}(6,2), \operatorname{IRD}(6,3), \operatorname{IRD}(6,4), \operatorname{IRD}(6,5)$,

$6 \operatorname{IRD}(6,6), \operatorname{IRD}(7,1), \operatorname{IRD}(7,2), \operatorname{IRD}(7,3), \operatorname{IRD}(7,4), \operatorname{IRD}(7,5)$,

$7 \operatorname{IRD}(7,6), \operatorname{IRD}(8,1), \operatorname{IRD}(8,2), \operatorname{IRD}(8,3), \operatorname{IRD}(8,4), \operatorname{IRD}(8,5)$,

$8 \operatorname{IRD}(8,6), \operatorname{IRD}(9,1), \operatorname{IRD}(9,2), \operatorname{IRD}(9,3), \operatorname{IRD}(9,4), \operatorname{IRD}(9,5)$,

$9 \operatorname{IRD}(9,6), \operatorname{IRD}(10,1), \operatorname{IRD}(10,2), \operatorname{IRD}(10,3), \operatorname{IRD}(10,4), \operatorname{IRD}(10,5)$,

A $\operatorname{IRD}(10,6) /$

B $10611,0,9010,14580,21,16,2526,13986,15741$,

C 10364, $0,11698,3645,13,8,9477,0,18144$,

D 9477, $0,17496,0,51,2,9477,0,17547$,

E $9477,0,837,0,52,1,9477,0,2943$,

F 9477, $0,1323,0,53,1,9477,0,16038$,

$0,23,1$, $0,51,1$, $0,51,3$,

$\mathrm{C}$

\section{DUMMY A B C D}

DATA IRD $(11,1), \operatorname{IRD}(11,2), \operatorname{IRD}(11,3), \operatorname{IRD}(11,4), \operatorname{IRD}(11,5)$,

$1 \operatorname{IRD}(11,6), \operatorname{IRD}(12,1), \operatorname{IRD}(12,2), \operatorname{IRD}(12,3), \operatorname{IRD}(12,4), \operatorname{IRD}(12,5)$,

$3 \operatorname{IRD}(12,6), \operatorname{IRD}(13,1), \operatorname{IRD}(13,2), \operatorname{IRD}(13,3), \operatorname{IRD}(13,4), \operatorname{IRD}(13,5)$,

$3 \operatorname{IRD}(13,6), \operatorname{IRD}(14,1), \operatorname{IRD}(14,2), \operatorname{IRD}(14,3), \operatorname{IRD}(14,4), \operatorname{IRD}(14,5)$,

$4 \operatorname{IRD}(14,6), \operatorname{IRD}(15,1), \operatorname{IRD}(15,2), \operatorname{IRD}(15,3), \operatorname{IRD}(15,4), \operatorname{IRD}(15,5)$,

$5 \operatorname{IRD}(15,6) /$

$60,0,0,0,0,0,3496,10152,729,0,54,2$, $73496,10152,1458,0,54,3,3496,10152,2187,0,54,4$,

$83496,10152,2916,0,54,51$

LOT 4280

LOT 4290

LOT 4300

LOT 4310

LOT 4320

LOT 4330

LOT 4340

LOT4350

LOT 4360

LOT 4370

LOT 4380

LOT 4390

LOT 4400

LOT4410

LOT 4420

LOT 4430

LOT 4440

LOT 4450

LOT 4460

LOT 4470

LOT 4480

LOT4490

LOT 4500

LOT 4510

LOT 4520

LOT 4530

LOT 4540

LOT 4550

LOT 4560

LOT 4570

\section{ROW SUM F PROBAB}

DATA IRD $(16,1), \operatorname{IRD}(16,2), \operatorname{IRD}(16,3), \operatorname{IRD}(16,4), \operatorname{IRD}(16,5)$, $1 \operatorname{IRD}(16,6), \operatorname{IRD}(17,1), \operatorname{IRD}(17,2), \operatorname{IRD}(17,3), \operatorname{IRD}(17,4), \operatorname{IRD}(17,5)$,

$2 \operatorname{IRD}(17,6) /$

$313550,0,14431,0,21,1,4374, \quad 0,12165,1487,24,51$

\section{ELLIPT FIRST ELLIPT SECOND ZEROS BJZERO ZEROS BJONE} STRUVE ZERO STRUVE ONE

$\operatorname{DATA} \operatorname{IRD}(18,1), \operatorname{IRD}(18,2), \operatorname{IRD}(18,3), \operatorname{IRD}(18,4), \operatorname{IRD}(18,5)$, $1 \operatorname{IRD}(18,6), \operatorname{IRD}(19,1), \operatorname{IRD}(19,2), \operatorname{IRD}(19,3), \operatorname{IRD}(19,4), \operatorname{IRD}(19,5)$,

$2 \operatorname{IRD}(19,6), \operatorname{IRD}(20,1), \operatorname{IRD}(20,2), \operatorname{IRD}(20,3), \operatorname{IRD}(20,4), \operatorname{IRD}(20,5)$,

$3 \operatorname{IRD}(20,6), \operatorname{IRD}(21,1), \operatorname{IRD}(21,2), \operatorname{IRD}(21,3), \operatorname{IRD}(21,4), \operatorname{IRD}(21,5)$,

$4 \operatorname{IRD}(21,6), \operatorname{IRO}(22,1), \operatorname{IRD}(22,2), \operatorname{IRD}(22,3), \operatorname{IRD}(22,4), \operatorname{IRD}(22,5)$,

LOT 4580

LOT 4590

LOT4600

LOT4610

LOT4620

LOT 4630

LOT4640

LOT 4650

LOT 4660

LOT4670

LOT4680

LOT4690

LOT4700

LOT4710

LOT4720 
$5 \operatorname{IRD}(22,6), \operatorname{IRD}(23,1), \operatorname{IRD}(23,2), \operatorname{IRD}(23,3), \operatorname{IRD}(23,4), \operatorname{IRD}(23,5)$,

LOT 4730

$6 \operatorname{IRD}(23,6) /$

$73981,7013,4635,14391,30,30,3981,7013,13989,11317,30,31$,

$819107,11448,1754,4146,30,33,19107,11448,1743,10341,30,34$,
$\mathrm{C}$

$914409,15908,19107,10935,30,35,14409,15908,11318, \quad 0,30,36 /$

DATA

$1 \operatorname{IRD}(24,6) /$

$20,0,0,0,01$

C

C

C

C

C

C

C

C

C

C

C

C

C**** THE FOLLOWING CARD IS NEEDED ONLY FOR TAPE OPERATIONS

C

C

C

C

\section{PAGE PLOT}

\section{DATA}

$1 \operatorname{IRD}(25,6) /$

$211698,3645,12003,14580,13,61$

\section{GAUSS QUADRATURE}

$\operatorname{DATA} \operatorname{IRD}(26,1), \operatorname{IRD}(26,2), \operatorname{IRD}(26,3), \operatorname{IRD}(26,4), \operatorname{IRD}(26,5)$,

$1 \operatorname{IRD}(26,6) /$

$2 \quad 5151,14364,12961,3403,24,4 /$

\section{DUMMY E $F$ UNIFOR RANDOM}

DATA IRD $(27,1), \operatorname{IRD}(27,2), \operatorname{IRD}(27,3), \operatorname{IRD}(27,4), \operatorname{IRD}(27,5)$,

$1 \operatorname{IRD}(27,6), \operatorname{IRD}(28,1), \operatorname{IRD}(28,2), \operatorname{IRD}(28,3), \operatorname{IRD}(28,4), \operatorname{IRD}(28,5)$,

$2 \operatorname{IRD}(28,6), \operatorname{IRD}(29,1), \operatorname{IRD}(29,2), \operatorname{IRD}(29,3), \operatorname{IRD}(29,4), \operatorname{IRD}(29,5)$,

$3 \operatorname{IRD}(29,6) / 3496,10152,3645,0,54,6,3496,10152,4374$,

$454,7,15696,4797,13163,3334,24,151$

$* * * * * * * * * * * * * * * * * * * * * * * * * * * * * * * * * * * * * * * * * * * * * * * * * * * * * * * * * * * * * * * * * * *$

*

* USED THRU IRD $(29,6)$ : AVAILABLE $\operatorname{IRD}(30,1) \operatorname{THRU} \operatorname{IRD}(30,6)$
LOT 4740

LOT 4750

LOT4760

LOT4770

LOT 4780

LOT4790

LOT4800

LOT 4810

LOT 4820

LOT 4830

LOT 4840

LOT 4850

LOT 4860

LOT4870

LOT 4880

LOT 4890

LOT 4900

LOT4910

LOT 4920

LOT4930

LOT 4940

LOT 4950

LOT 4960

LOT 4970

LOT 4980

LOT 4990

LOT5000

LOT 5010

0, LOT5015

LOT5020

LOT5030

*LOT5040

*LOT5050

*LOT5060

*LOT5070

* LOT5080

LOT5090

LOT5100

LOT5110

LOT5120

LOT5130

LOT5140

LOT5 150

LOT5160

LOT5170

LOT5180

LOT5190

LOT5200

LOT5210

LOT5220

LOT5230

LOT 5240

LOT5250

LOT5260

C $* * * * * * * * * * * * * * * * * * * * * * * * * * * * * * * * * * * * * * * * * * * * * * * * * * * * * * * * * * * * * * * * * * * *)+\operatorname{LOT} 5270$

C ***** USED THRU ITP $(9,4):$ AVAILABLE ITP $(10,1) \operatorname{THRU} \operatorname{ITP}(10,4) * *$

C***********************************************************************LOT5290

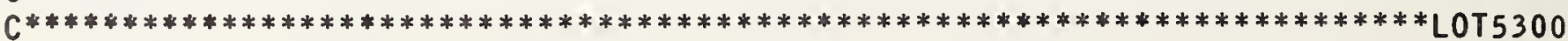

END 
SUBROUTINE LOOKUP

C VERSION 5.00 LOOKUP $5 / 15 / 70$

C WRITTEN BY S PEAVY $3 / 14 / 68$

COMMON / BLOCKE/ NAME (4),L1,L2, ISRFLG

COMMON /ICODE / NIR, NID,NIRD,LIR, LID, LIRD

COMMON /CODE/ IALPH (6), NALPH $(5), \operatorname{ID}(9,3), \operatorname{IR}(300,4), \operatorname{IRD}(30,6)$

C THE FOLLOWING CARDS ARE NEEDED ONLY FOR THE OPERATIONS

COMMON / ICODTP/ NITP,LITP

COMMON /CODETP/ ITP $(10,4)$

COMMON /TAPE/ NAME $4(2)$, NTPCT, IPUNCP, INUN IP, LITP

THIS SUBROUTINE CHECKS TO SEE IF FIRST WORD AND SOMETIMES SECOND

WORD ON COMMAND CARD IS A LEGITIMATE COMMAND

IF COMMAND IS FOUND LI AND L2 ARE ASSIGNED A VALUE

IF COMMAND IS NOT FOUND IN THE DICTIONARY LI IS SET EQUAL TO ZERO

$* * * * * * * * * * * * * * * * * * * * * * * * * * * * * * * * * * * * * * * * * * * * * * * * * * * * * * * * * * * * *$

$\mathrm{LI}=0$

DO $10 \quad I=1, N I R$

IF (NAME (1).NE.IR(I, i).OR.NAME (2).NE.IR(I, 2)) GO TO 10

$\operatorname{LI}=\operatorname{IR}(I, 3)$

$\operatorname{L} 2=\operatorname{IR}(I, 4)$

C THE FOLLOWING CARDS ARE NEEDED ONLY FOR TAPE OPERATIONS

IF (NAME (1).NE.IR $(50,1)$ ) RETURN

C NAME (3)=TAP NAME (4)=E

IF (NAME (3).EQ.14623. AND.NAME (4).EQ.3645) GO TO 40

C $\quad * * * * * * * * * * * * * * * * * * * * * * * * * * * * * * * * * * * * * * * * * * * * * * * * * * * * * * * * * * * * *)$

RETURN

10 CONTINUE

DO $20 \quad I=1, N I D$

IF (NAME (1).NE.ID(I,1).OR.NAME (2).NE.ID(I,2)) GO TO 20

$L I=I D(I, 3)$

GO TO 80

20 CONTINUE

DO $30 \quad I=1$, NIRD

LOU 10

LOU 20

LOU 30

LOU 40

LOU 50

LOU 60

LOU 70

LOU 80

LOU 90

LOU 100

LOU 102

LOU 104

LOU 106

LOU 108

LOU 110

LOU 120

LOU 130

LOU 140

LOU 150

LOU 160

LOU 170

LOU 180

LOU 190

LOU 200

LOU 210

LOU 220

LOU 230

LOU 240

LOU 250

LOU 260

LOU 270

LOU 280

LOU 290

IF (NAME (1).NE.IRD (I, I) .OR.NAME (2).NE.IRD(I, 2) . OR.NAME (3).NE. IRD (ILOU 300

$1,3)$. OR.NAME (4) .NE.IRD (I, 4)) GO TO 30

LOU 310

$\operatorname{LI}=\operatorname{IRD}(I, 5)$

$\operatorname{L2}=\operatorname{IRD}(I, 6)$

LOU 320

RETURN

30 CONTINUE

C THE FOLLOWING CARDS ARE NEEDED ONLY FOR TAPE OPERATIONS

LOU 330

LOU 340

LOU 350

LOU 360

DO $50 \quad I=1$, NITP

IF (NAME (1).NE.ITP(I, 1).OR.NAME (2).NE.ITP(I,2)) GO TO 50

LOU 370

$L I=I \operatorname{TP}(I, 3)$

LOU 380

$L 2=\operatorname{ITP}(I, 4)$

LOU 390

GO TO 60

LOU 400

LOU 410

CONT INUE

LOU 420

RETURN

60 IF (LI.GT.47) RETURN

DO $70 \quad I=1,6$

LOU 430

LOU 440

LOU 450

IF (NAME4(1).NE.IALPH (I)) GO TO 70

$L 2=I+1$

RETURN

LOU 460

LOU 470

LOU 480

70 CONTINUE LOU 490

C $\quad * * * * * * * * * * * * * * * * * * * * * * * * * * * * * * * * * * * * * * * * * * * * * * * * * * * * * * * * * * * * * * * * * * \operatorname{LOU} 500$

RETURN $\quad$ LOU 510

C THE FOLLOWING CARDS ARE NEEDED ONLY FOR TAPE OPERATIONS LOU 520

80 IF (LI.NE.5) GO TO $90 \quad$ LOU 530

C NAME (3)=TAP NAME (4)=E LOU 540

IF (NAME (3).EQ.14623.AND.NAME (4).EQ.3645) GO TO $40 \quad$ LOU 550 
C THIS CARD WAS $500 \quad$ L2=1 LOU 560

$90 \quad \mathrm{~L} 2=1 \quad$ LOU 570

C $\quad * * * * * * * * * * * * * * * * * * * * * * * * * * * * * * * * * * * * * * * * * * * * * * * * * * * * * * * * * * * * * * * * * *$ LOU 580

IF (LI.EQ.1) GO TO $110 \quad$ LOU 590

DO $100 \quad I=1,6 \quad$ LOU 600

IF (NAME (3).NE.IALPH(I)) GO TO $100 \quad$ LOU 610

$L 2=I+1 \quad$ LOU 620

RETURN $\quad$ LOU 630

100 CONTINUE $\quad$ LOU 640

IF (LI.NE.2) RETURN LOU 641

IF (L2.NE.1) RETURN LOU 642

IF (NAME (3).NE.10631. AND.NAME (4).NE.3645) RETURN LOU 643

THE COMMAND IS PRINT NOTE

$L l=13$

$L 2=13$

LOU 645

LOU 646

RETURN

LOU 650

LOU 660

LOU 670

LOU 680

LOU 690

LOU 700

LOU 710

LOU 720 
L2 $=1$ ADD MATRICES $A+B$ MADD $A() N, M$, TO $B() N,$,$M AND S$ ORE IN C(MAT 40

C L $2=2$ SUB MATRICES A-B MSUB $A(),) N, M$ FROM $B() N,$,$M AND STORE IN C(MAT 60$

C L2 $=3$ TRANSPOSE MATRIX MTRANS $A() \quad N,$,$M AND STORE IN C($,$) MAT 80$

C TRANSPOSE ARRAY ATRANS $A() \quad N,$,$M AND STORE IN C($,

C L2 $=4$ ARRAY ADD AADD

C L $2=5$ ARRAY SUBTRACT ASUB

C L2 $=6$ ARRAY MULTIPLY AMULT

C L2 $=7$ ARRAY DIVIDE ADIV

C L2 $=8$ ARRAY RAISE ARAISE

MAT 90

MAT 100

MAT 110

MAT 120

MAT 130

GENERAL FORMS FOR ARRAY OPERATIONS

MAT 140

C

C

$A() N,, M B() \quad N,$,$K STORE IN C($,$) ARRAY BY ARRAY MAT 160$

$A() N,, M B() \quad S T O R$,$E IN C($,$) ARRAY BY ARRAY MAT 170$

$A() N,, M K$ STORE IN $C($,$) ARRAY BY COLUMN MAT 180$

$A() \quad N,, M \quad X \quad$ STORE IN $C($,$) ARRAY BY CONSTANT MAT 200$

C

C

COMMON /BLOCRC/ NRC, RC (12600)

MAT 220

MAT 230

COMMON /BLOCKD/ IARGS(100), KIND(100), ARGTAB (100), NRMAX,NROW, NCOL, NMAT 240

IARGS , VHXYZ (8) , NERROR

DIMENSION ARGS $(100)$

EQUIVALENCE (ARGS (1), RC (12501))

COMMON /SCRAT/ NS, NS2, A(13500)

COMMON /BLOCKE/ NAME (4),L1,L2,ISRFLG

C

C CHECK TO SEE IF WE HAVE CORRECT NUMBER OF ARGUMENTS

C

$N P=N A R G S$

IF (L2-3) $10,20,30$

10 IF (NARGS.NE.8.AND.NARGS.NE.10) GO TO 40

GO TO 50

20 IF (NARGS.NE.6) GO TO 40

GO TO 50

30 IF (NARGS.LT.7.OR.NARGS.GT.10.OR.NARGS.EQ.9) GO TO 40

GO TO 50

40 CALL ERROR (10)

RETURN

MAT 250

MAT 260

MAT 270

MAT 280

MAT 290

MAT 300

MAT 310

MAT 320

MAT 330

MAT 340

MAT 350

MAT 360

MAT 370

MAT 380

MAT 390

MAT 400

MAT 410

MAT 415

MAT 420

MAT 430

CHECK TO SEE IF ALL ARGUMENTS ARE INTEGERS

MAT 440

MAT 450

MAT 460

MAT 470

MAT 480

MAT 490

MAT 500

MAT 510

MAT 520

MAT 530

MAT 540

MAT 550

MAT 560

MAT 570

C

GO TO 60

C

CHECK TO SEE IF DIMENSIONS ARE CORRECT IF THEY ARE GIVEN

MAT 580

MAT 590

MAT 600

80 IF (NP.NE.10) GO TO 90

IF (IARGS (3) .EQ.IARGS (7) . AND.IARGS (4) .EQ.IARGS (8)) GO TO 90

MAT 610

CALL ERROR (3)

MAT 620 
$100 \quad J=3$

$$
\operatorname{IARGS}(12)=\operatorname{IARGS}(4)
$$

130 IF (NP.EQ.8.OR. (NP.EQ.7.AND.KIND(NP).NE.0)) GO T0 190

MAT 780

IF (NP.EQ.6) GO TO 160

MAT 790

$\operatorname{IARGS}(6)=\operatorname{IARGS}(5)$

MAT 810

$\operatorname{IARGS}(8)=1$

MAT 860

$\operatorname{IARGS}(7)=\operatorname{IARGS}(3)$

MAT 870

$\operatorname{IARGS}(5)=1$

MAT 880

GO TO 210

MAT 890

MAT 900

$160 \operatorname{IARGS}(8)=\operatorname{IARGS}(3)$

MAT 910

$\operatorname{IARGS}(7)=\operatorname{IARGS}(4)$

MAT 920

GO TO 210

190 IF (NP.EQ.8) GO TO 200

MAT 930

$\operatorname{IARGS}(5)=\operatorname{IARGS}(6)$

$\operatorname{IARGS}(6)=\operatorname{IARGS}(7)$

$200 \operatorname{IARGS}(8)=\operatorname{IARGS}(4)$

$\operatorname{IARGS}(7)=\operatorname{IARGS}(3)$

MAT 1010

MAT 1020

MAT 1030

MAT 1040

MAT 1050

210 CALL MTXCHK (J)

IF (J-1) $240,220,230$

MAT 1060

MAT 1070

220 CALL ERROR (3)

RETURN

MAT 1080

MAT 1090

230 CALL ERROR (17)

MAT 1100

RETURN

MAT 1110

MAT 1120

CHECK TO SEE IF THERE WERE PREVIOUS ERRORS

MAT 1130

*

MAT 1140

MAT 1150

IF (NERROR.NE.O) RETURN

MAT 1160

SUM ELEMENTS IN SCRATCH AREA

MAT 1170

SUBTRACT ELEMENTS IN SCRATCH AREA

MAT 1180

PRODUCTS AND QUOTIENTS FORMED USING DOUBLE PRECISION IN SCRATCH ARMATII90 TRANSPOSE IN SCRATCH AREA

MAT 1200

MAT 1210

IROW=IARGS (3)

MAT 1220

$I C O L=I A R G S(4)$

MAT 1230

NROWPP $=$ NROW

MAT 1240

If $(L 2-3) 260,250,290$

MAT 1250

MAT1260

I I $=$ I COL

MAT 1270

$J J B=I R O W$

MAT 1280

$N R O W P P=0$

MAT 1290

$K=1$

MAT 1300

GO TO 280

MAT 1310

NROWP=NROW

MAT 1320

$I B P=I$ ARGS (5)

MAT 1330

$J \mathrm{JB}=I \mathrm{COL}$

$K=0$ 
$I A P=I A R G S(1)$

MAT 1370

GO TO 320

MAT 1380

290

IF (NP.GE.8) GO TO 260

IF (KIND(NP).EQ.1) GO TO 300

MAT 1390

$I B P=I A R G S(5)$

MAT 1400

GO TO 310

MAT 1410

$300 \operatorname{IARGS}(9)=\operatorname{IARGS}(5)$

MATI 420

MAT 1430

$310 \quad$ NROWP $=0$

GO TO 270

MAT 1440

320 DO $510 \mathrm{~J}=1$, JJB

C COMPUTE ADDRESSES

MAT 1450

$I A=I A P+(J-1) * K$

MAT 1460

$I B=I B P$

DO $500 \quad I=1, I I B$

GO TO $(330,340,370,380,390,400,410,420)$, L2

MAT 1465

MAT 1470

MAT 1480

MAT 1490

$330 \quad A($ IS $)=R C($ I A $)+R C$ (IB)

GO TO 470

MAT 1500

MAT 1510

MAT 1520

$340 \quad A($ IS $)=R C($ IA $)-R C$ (IB)

GO TO 470

MAT 1530

MAT 1540

$350 \quad A($ IS $)=R C($ IA $) * R C$ (IB)

GO TO 470

360 IF (RC (IB).EQ.0.0) GO TO 365

$A(I S)=R C$ (IA) $/ R C$ (IB)

GO TO 470

$365 \quad A(I S)=0.0$

GO TO 470

MAT 1550

MAT 1560

MAT 1562

MAT 1564

MAT 1566

MAT 1570

MAT 1580

$370 \quad A(I S)=R C$ (IA)

$I A=I A+N R O W$

MAT 1590

MAT 1600

GO TO 490

MAT 1610

MAT 1620

MAT 1630

$A(I S)=R C$ (IA) +ARGS (NP-2)

GO TO 480

390 IF (NP.GE.8.OR. (KIND(NP).EQ.0.AND.NP.LT.8)) GO TO 340 $A(I S)=R C(I A)-A R G S(N P-2)$

GO TO 480

400 IF (NP.GE.8.OR. (KIND (NP).EQ.0.AND.NP.LT.8)) GO T0 350 $A(I S)=R C(I A) * A R G S(N P-2)$

GO TO 470

410 IF (NP.GE.8.OR. (NP.LT.8.AND.KIND (NP).EQ.0)) GO TO 360 IF (ARGS (NP-2).EQ.0.0) GO TO 415

$A(I S)=R C(I A) / A R G S(N P-2)$

GO TO 470

$415 \quad A(I S)=0.0$

GO TO 470

420 IF (NP.GE.8.OR. (NP.LT.8.AND.KIND (NP).EQ.0)) GO T0 440 IF (RC (IA)) $430,460,430$

MAT 1640

MAT 1650

MAT 1660

MAT 1670

MAT 1680

MAT 1690

MAT 1700

MAT 1710

MAT 1712

MAT 1714

MAT 1716

MAT 1720

MAT 1730

MAT 1740

MAT 1750

MAT 1760

MAT 1770

MAT 1780

MAT 1790

MAT 1800

MAT 1810

MAT 1820

MAT 1830

MAT 1840

MAT 1850

MAT 1860

MAT 1870

$I B P=I B P+N R O W P$

MAT 1880 


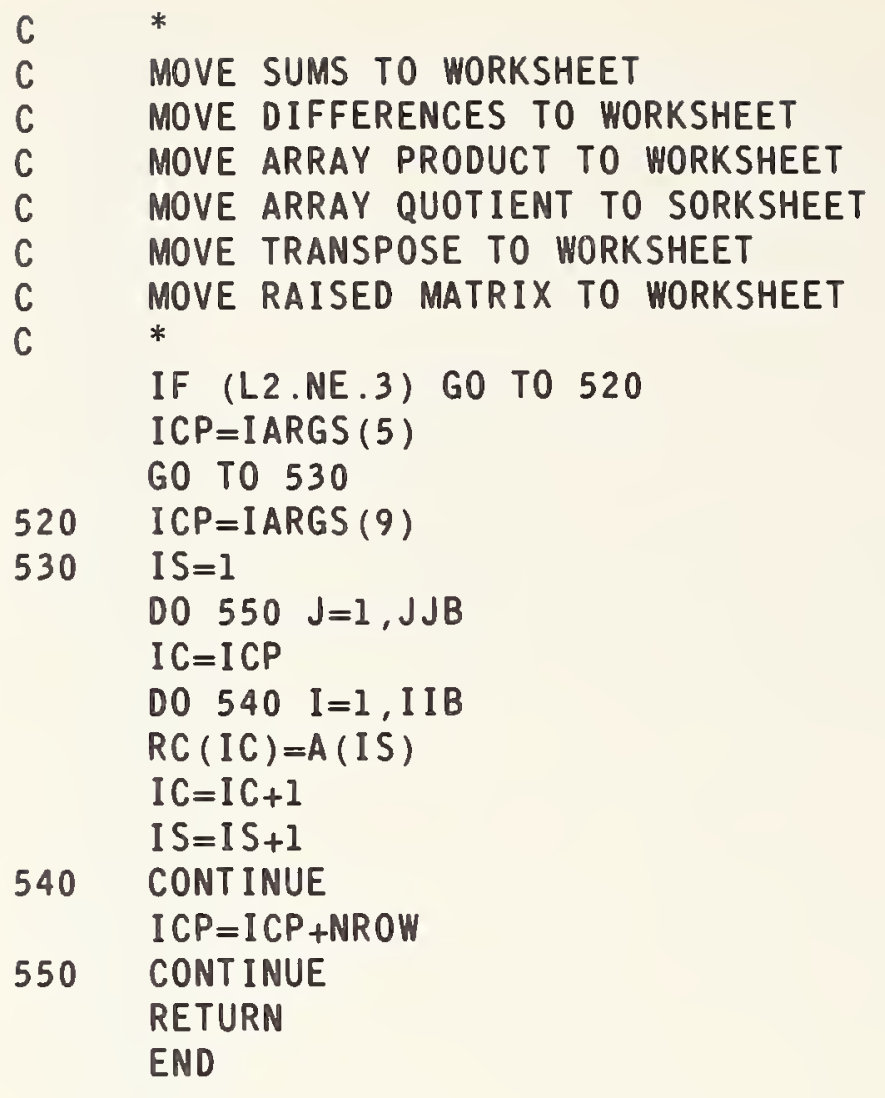


SUBROUTINE TO PRE OR POST MULTIPLY A MATRIX BY A DIAGONAL STORED AS A COLUMN

\section{$\mathrm{L} 2=1$} $M(A D)$

MATRIX A IS POSTMULTIPLIED BY THE DIAGONAL D STORED IN COL I GENERAL FORM OF COMMAND $M(A D) A() N, K,$,$D IN COL I STORE IN C($, $L 2=2 \quad M(A D)$

MATRIX A IS PREMULTIPLIED BY THE DIAGONAL D STORED IN COL I GENERAL FORM OF COMMAND $M(D A), A() N,, K \quad K$ IN COL I STORE IN $C($,

MDA 30

MDA 40

MDA 50

MDA 60

MDA 70

MDA 80

MDA 90

MDA 100

MDA 110

MDA 120

MDA 130

MDA 140 *

COMMON /SCRAT/ NS, NS2, A(13500)

MDA 150

COMMON /BLOCRC/ NRC, RC (12600)

MDA 160

COMMON /BLOCKD/ IARGS(100), KIND(100), ARGTAB (100), NRMAX, NROW, NCOL, NMDA 180

IARGS, VWXYZ (8), NERROR

DIMENSION ARGS(100)

EQUIVALENCE (ARGS(1),RC(12501))

COMMON /BLOCKE/ NAME (4),L1,L2, ISRFLG

C

C

C

C

C

C CHECK TO SEE IF DIMENSIONS ARE OUT OF RANGE

C COMPUTE ADDRESSES OF COLUMNS

C

CHECK FOR CORRECT NUMBER OF ARGUMENTS

IF (NARGS.NE.7) CALL ERROR (10)

CHECK TO SEE THAT ALL ARGUMENTS ARE INTEGERS

*

$J=N A R G S$

CALL CKIND (J)

IF (J.NE.0) CALL ERROR (3)

$\operatorname{IARGS}(12)=\operatorname{IARGS}(4)$

$\operatorname{IARGS}(11)=\operatorname{IARGS}(3)$

$\operatorname{IARGS}(10)=\operatorname{IARGS}(7)$

$\operatorname{IARGS}(9)=\operatorname{IARGS}(6)$

$\operatorname{IARGS}(8)=1$

GO TO $(10,20)$, L2

$10 \operatorname{IARGS}(7)=\operatorname{IARGS}(4)$

GO TO 30

$20 \operatorname{IARGS}(7)=\operatorname{IARGS}(3)$

$30 \operatorname{IARGS}(6)=\operatorname{IARGS}(5)$

$\operatorname{IARGS}(5)=1$

$J=3$

CALL MTXCHK (J)

IF (J-1) $60,40,50$

40 CALL ERROR (3)

RETURN

50 CALL ERROR (17)

RETURN

C

C

C

60 IF (NERROR.NE.0) RETURN

IP $=$ I ARGS (4)
MDA 190

MDA 200

MDA 210

MDA 220

MDA 230

MDA 240

MDA 250

MDA 260

MDA 270

MDA 280

MDA 290

MDA 300

MDA 310

MDA 320

MDA 330

MDA 340

MDA 350

MDA 360

MDA 370

MDA 380

MDA 390

MDA 400

MDA 410

MDA 420

MDA 430

MDA 440

MDA 450

MDA 460

MDA 470

MDA 480

MDA 490

MDA 500

MDA 510

MDA 520

MDA 530

MDA 540

MDA 550

MDA 560

MDA 570

MDA 580

MDA 590 
$J P=I$ ARGS (3)

GO TO $(80,70)$, L2

$70 \quad$ I $1=0$

$12=1$

GO TO 90

$80 \quad I I=1$

$$
12=0
$$

$90 \quad \operatorname{IA}=\operatorname{IARGS}(1)$

$I D P=I A R G S(5)$

$I B=I$ ARGS $(9)$

DO $110 \quad I=1$, IP

$I D=I D P$

DO $100 \mathrm{~J}=1, \mathrm{JP}$

$R C(I B)=R C(I D) * R C$ (IA)

$I D=I D+I 2$

I $A=I A+1$

$I B=I B+1$

100 CONTINUE

$I B=I B+N R O W-J P$

$I A=I A+N R O W-J P$

$I D P=I D P+I I$

110 CONTINUE

RETURN

END
MDA 600

MDA 610

MDA 620

MDA 630

MDA 640

MDA 650

MDA 660

MDA 670

MDA 680

MDA 690

MDA 700

MDA 710

MDA 720

MDA 730

MDA 740

MDA 750

MDA 760

MDA 770

MDA 780

MDA 790

MDA 800

MDA 810

MDA 820

MDA 830 
SUBROUTINE MEIGEN

$\begin{array}{llll}\text { C } & \text { VERSION } 5.00 & \text { MEIGEN } & 5 / 15 / 70 \\ \text { C } & \text { SUBROUTINE MEIGEN } & \\ C & * & & \end{array}$

$\begin{array}{llll}\text { C } & \text { VERSION } 5.00 & \text { MEIGEN } & 5 / 15 / 70 \\ \text { C } & \text { SUBROUTINE MEIGEN } & \\ C & * & & \end{array}$

ME I 10

SUBROUTINE TO COMPUTE EIGENVALUES AND EIGENVECTORS GENERAL FORMS OF COMMANDS

MEIGEN $A(,,++) R=,, C=$, STORE VALUES IN COL ++

MEIGEN $A(,++) R=, C=$, STORE VECTORS IN $B(,++)$

MEIGEN $A(,++) R=\ldots, C=$, STORE VALUES IN COL ++

$$
\text { STORE VECTORS IN } B(,++)
$$

BOTH R AND C MUST BE SPECIFIED

NARGS $=5$ COMPUTE ONLY EIGENVALUES

NARGS $=6$ COMPUTE ONLY EIGENVECTORS

NARGS $=7$ COMPUTE EIGENVALUES AND VECTORS

MEI 20

MEI 30

MEI 40

ME I 50

MEI 60

MEI 70

MEI 80

ME I 90

MEI 100

ME I 110

ME I 120

MEI 130

MEI 140

MEI 150

COMMON /BLOCRC/ NRC, RC (12600)

MEI 160

COMMON /BLOCKD/ IARGS $(100)$, KIND (100), ARGTAB (100), NRMAX, NROW, NCOL, NMEI 170

IARGS, VWXYZ (8), NERROR

DIMENSION ARGS $(100)$

MEI 180

MEI 190

EQUIVALENCE (ARGS(1), RC (12501))

MEI 200

COMMON /SCRAT/ NS, NS2, A(13500)

MEI 210

DIMENSION ISWCH (2)

MEI 220

MEI 230

$\begin{array}{llll}\text { C CHECK TO BE SURE THAT MATRIX IS NO BIGGER THAN } 54 \times 54 & \text { MEI } 240 \\ \text { C } & * & & \text { MEI } 250\end{array}$

MEI 250

IF (IARGS(3).NE.IARGS(4)) CALL ERROR (230)

MEI 260

IF (IARGS(3)**2.GT.NS2) CALL ERROR (23)

MEI 270

MEI 280

CHECK FOR CORRECT NUMBER OF ARGUMENTS

MEI 290

MEI 300

IF (NARGS.LT.5.OR.NARGS.GT.7) CALL ERROR (10)

MEI 310

MEI 320

CHECK TO SEE IF ARGUMENTS ARE ALL INTEGERS

MEI 330

MEI 340

$J=N A R G S$

CALL CKIND (J)

IF (J.NE.0) CALL ERROR (3)

MEI 350

MEI 360

MEI 370

MEI 380

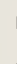

C CHECK TO SEE IF DIMENSIONS ARE OUT OF RANGE

MEI 390

COMPUTE ADDRESSES

MEI 400

MEI 410

I SWCH $(2)=$ NARGS -4

MEI 420

I SWCH $(1)=0$

IF (NARGS.EQ.6) GO TO 10

MEI 430

I $A D D=1$

MEI 440

CALL ADRESS $(5, J)$

IF (J.LE.0) CALL ERROR (11)

MEI 450

MEI 460

MEI 470

c

$J_{*}$ CONTAINS ADDRESS OF COLUMN

MEI 480

MEI 490

MEI 500

IF (NARGS.EQ.5) GO TO 20

MEI 510

$\operatorname{IARGS}(5)=\operatorname{IARGS}(6)$

$\operatorname{IARGS}(6)=\operatorname{IARGS}(7)$

10 IADD $=2$

$\operatorname{IARGS}(7)=\operatorname{IARGS}(3)$

MEI 520

MEI 530

MEI 540

$\operatorname{IARGS}(8)=\operatorname{IARGS}(4)$

MEI 550

MEI 560

GO TO 30

MEI 570

20 ISWCH $(1)=1$

MEI 580

30 CALL MTXCHK (IADD)

MEI 590 
IF (IADD-1) $60,40,50$

ME I 600

40 CALL ERROR (3)

RETURN

MEI 610

50 CALL ERROR (17)

MEI 620

ME I 630

RETURN

ME I 640

ME I 650

CHECK FOR PREVIOUS ERRORS

ME I 660

ME I 670

ME I 680

MEI 690

IF (NERROR.NE.0) RETURN

$I G P=I$ ARGS (5)

$I G=I$ ARGS (1)

ME I 700

CALL HDIAG (RC(IG), IARGS (3), ISWCH, A, RC(J), RC(IGP), NROW, A ( 3000$)$ )

MEI 710

MEI 720

MEI 730

RC(IG) IS LOCATION OF MATRIX TO BE DIAGONALIZED

MEI 740

IARG(3) GIVES SIZE OF MATRIX

ISWCH $(1)=1$

IF ONLY EIGENVALUES ARE TO BE COMPUTED

MEI 750

ISWCH $(1)=0$ COMPUTE EIGENVALUES AND EIGENVECTORS

MEI 760

ISWCH (2) = NARGS-4 AND IS USED FOR STORING RESULTS

MEI 770

A IS LOCATION OF SCRATCH AREA

RC(J) TELLS WHERE TO STORE EIGENVALUES

RC(IGP) IS WHERE EIGENVECTORS ARE STORED

MEI 780

MEI 790

MEI 800

MEI 810

RETURN

MEI 820

END

MEI 830 
SUBROUTINE MISC2

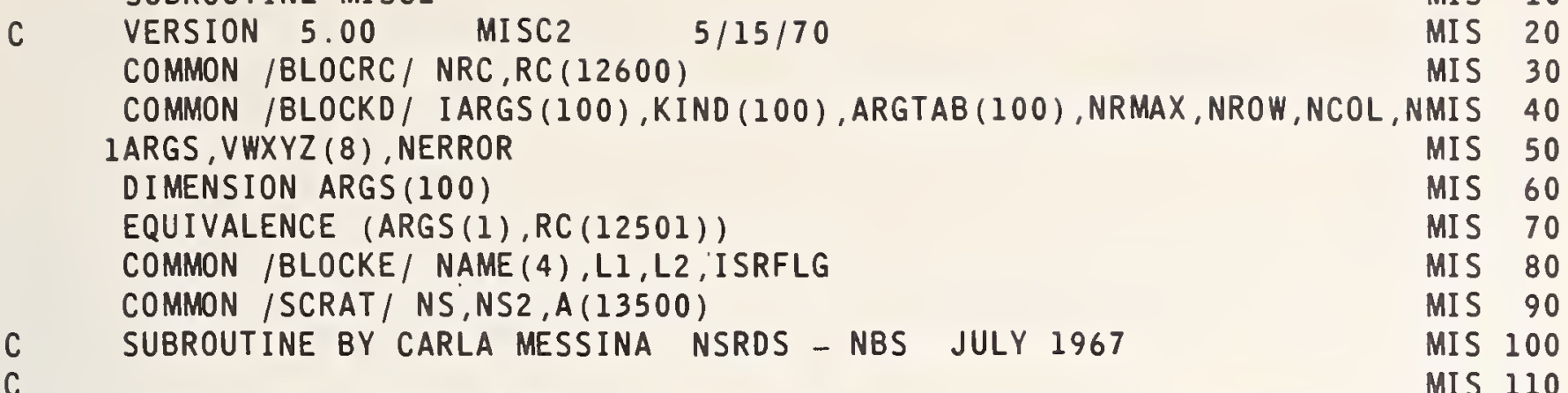

C

C

C

C

C

C

$10 \quad K=10$

L2=4 IS EXPAND $\$ \$$ TO , POWER IN INTERVALS OF , START STORE THE POWERS MAY BE INTEGER OR NOT

$L 2=1$ IS CLOSE UP ROWS HAVING ** IN ++,++, ETC

L2 $=2$ IS COUNT LENGTH OF COLUMN ++, STORE IN COLUMN ++ MIS 150

$\mathrm{L} 2=3$ IS SHORTEN COL ++ FOR COL $++=* *$ STORE IN COL ++ AND COLMIS 160

$L 2=5$ IS DUPLICATE, TIMES THE ARRAY IN , + $+\mathrm{R}=,, \mathrm{C}=$, , START MIS 170 STORING IN , + ++

MIS 180

MIS 190

IF (NARGS-2) $10,40,40$

MIS 200

MIS 210

CALL ERROR ( $K$ )

MIS 220

RETURN

MIS 230

GO TO $(50,80,50,340,540), \mathrm{L} 2$

MIS 240

IF (KIND(L2)) $60,60,70$

MIS 250

$K=3$

GO TO 20

$70 \quad \mathrm{KIND}(\mathrm{L} 2)=0$

$A R G 1=A R G S(L 2)$

$\operatorname{IARGS}(L 2)=\operatorname{IARGS}(L 2+1)$

MIS 260

MIS 270

MIS 280

MIS 290

MIS 300

80 CALL CHKCOL (J)

IF (J) $60,90,60$

MIS 310

MIS 320

90 DO $100 \mathrm{I}=1$, NARGS

MIS 330

$100 \operatorname{IARGS}(\mathrm{I})=\operatorname{IARGS}(\mathrm{I})-1$

IF (L2-2) $120,120,110$

MIS 340

MIS 350

110 IF (NARGS-5) $10,120,10$

120 IF (NERROR.NE.0) GO TO 30

MIS 360

IF (NRMAX) $130,130,140$

$130 \quad K=9$

GO TO 20

140 IF (L2-2) $150,210,250$

C CLOSE UP

150 DO $200 \mathrm{~J}=2$, NARGS

$K=I \operatorname{ARGS}(\mathrm{J})$

$M=1$

DO $170 \quad I=1$, NRMAX

$J I=K+I$

IF (RC (Jl)-ARGI) $160,170,160$

MIS 370

MIS 380

MIS 390

MIS 400

MIS 410

MIS 420

MIS 430

MIS 440

MIS 450

MIS 460

MIS 470

MIS 480

$160 \quad \mathrm{Kl}=\mathrm{K}+\mathrm{M}$

$R C(K I)=R C(J I)$

$M=M+1$

170 CONTINUE

IF (M-NRMAX) $180,180,200$

180 DO $190 I=M$, NRMAX

$\mathrm{J} I=\mathrm{K}+\mathrm{I}$

$190 \quad R C(J 1)=0.0$

200 CONTINUE

GO TO 30

MIS 490

MIS 500

MIS 510

MIS 520

MIS 530

MIS 540

MIS 550

MIS 560

MIS 570

MIS 580

C COUNT

MIS 590 


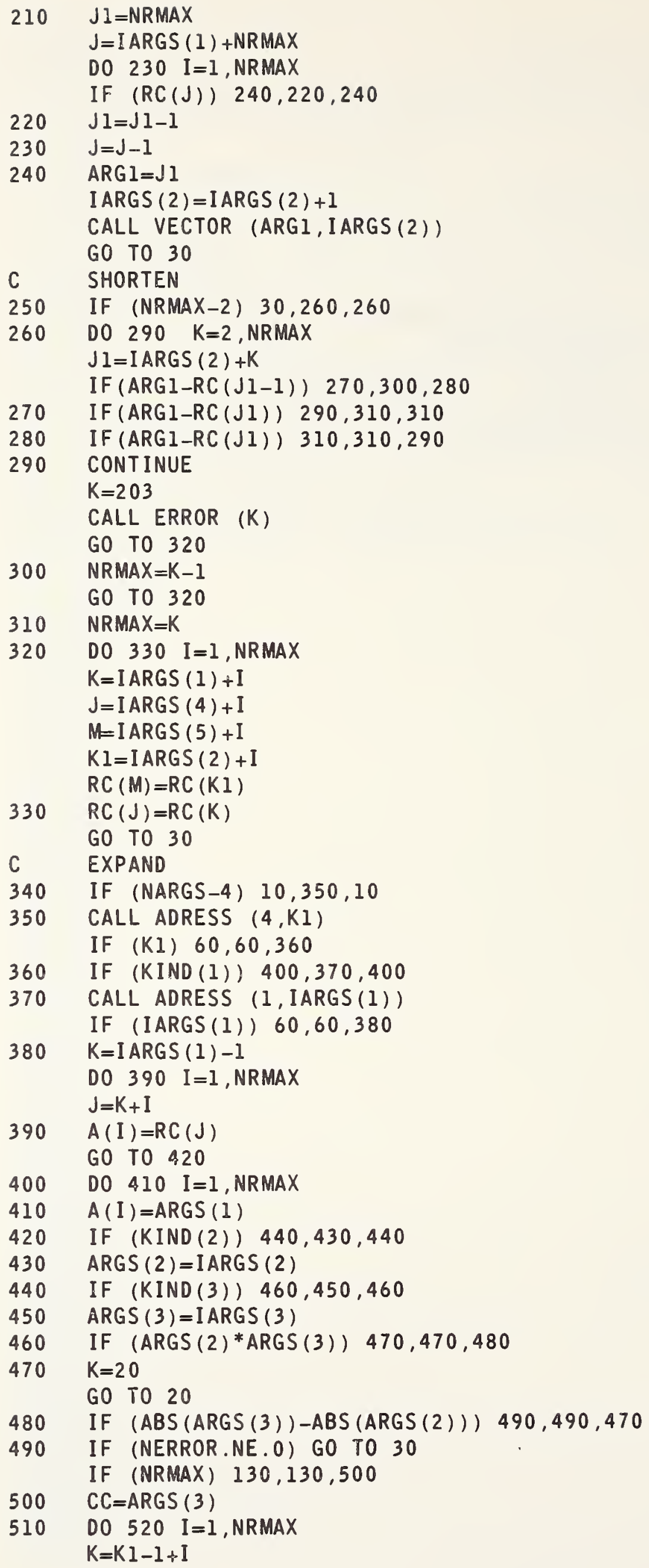


$520 \quad R C(K)=F E X P 2(A(I), C C)$

MIS 1190

IF (ABS (CC) +.5E-6-ABS (ARGS (2))) $530,30,30$

MIS 1200

$530 \quad C C=C C+A R G S(3)$

$\operatorname{IARGS}(4)=\operatorname{IARGS}(4)+1$

MIS 1210

CALL ADRESS $(4, K 1)$

MIS 1220

IF (KI) $60,60,510$

MIS 1230

$\begin{array}{ll}\text { C } & \text { DUPLICATE } \\ 540 & \text { IF (NARGS.NE.7) GO TO } 10\end{array}$

IF (IARGS(1).LE.0) GO TO 60

$K 1=\operatorname{MAX} 0(\operatorname{IARGS}(1) * \operatorname{IARGS}(4)+\operatorname{IARGS}(6)-1$, NRMAX)

MIS 1240

MIS 1250

MIS 1260

MIS 1270

IF (KI.GT.NROW) GO TO 590

$J=7$

CALL CKIND (J)

IF (J.NE.0) GO TO 60

NARGS $=6$

NDUP $=$ IARGS (1)

$\operatorname{IARGS}(61)=\operatorname{IARGS}(6)$

$\operatorname{IARGS}(62)=\operatorname{IARGS}(7)$

$\operatorname{IARGS}(63)=\operatorname{IARGS}(4)$

$\operatorname{IARGS}(64)=\operatorname{IARGS}(5)$

MIS 1280

MIS 1290

MIS 1300

MIS 1310

MIS 1320

MIS 1330

MIS 1340

MIS 1350

MIS 1360

MIS 1370

$\operatorname{IARGS}(65)=\operatorname{IARGS}(6)$

MIS 1380

$\operatorname{IARGS}(66)=\operatorname{IARGS}(7)$

MIS 1390

DO $550 \quad I=1,6$

$550 \operatorname{IARGS}(I)=\operatorname{IARGS}(I+1)$

MIS 1400

MIS 1410

CALL MOVE

MIS 1420

IF (NDUP.EQ.1) GO TO 580

MIS 1430

MIS 1440

DO $570 \quad I=2$, NDUP

MIS 1450

DO $560 \mathrm{~J}=1,6$

MIS 1460

MIS 1470

MIS 1480

$\operatorname{IARGS}(5)=\operatorname{IARGS}(65)+(I-1) * \operatorname{IARGS}(63)$

MIS 1490

MIS 1500

MIS 1510

MIS 1520

MIS 1530

MIS 1540 
SUBROUTINE MIST ( $M, B, L C H K, N L A$, IND)

C
VERSION 5.00
MIST
$5 / 15 / 70$

MST 10

DIMENSION B (1), NBC (12)

MST 20

IARGS, VWXYZ (8), NERROR

MST 30

COMMON /HEADER/ NOCARD $(80)$, ITLE $(60,6)$, LNCNT , IPRINT , NPAGE , IPUNCH

MST 50

$M M=M-2$

$N N=N R M A X-3$

$N C=10$

$M 1=(M-1) / N C+1$

$N L U=56$

$N C A=0$

$N R A=0$

DO $120 \mathrm{KEN}=1, \mathrm{Ml}$

$N C P=M I N O$ (NC, M-NCA)

$N R P=M-L C H K * N C A$

$N L P=(1+I N D / 7) * N R P+5$

IF (NLP.LT.NLU-NLA) GO TO 10

CALL PAGE (4)

$N L A=0$

10 DO 20 IYA=1, NCP

$I I=N C A+I Y A$

$20 \quad \mathrm{NBC}($ IYA $)=($ I ARGS $(I 1+1)-1) / N R O W+1$

GO TO $(30,40,50,60,70,80,90)$, IND

30 WRITE (IPRINT,130)

GO TO 100

40 WRITE (IPRINT, 140)

GO TO 100

50 WRITE (IPRINT, 150) MM

GO TO 100

60 WRITE (IPRINT, 160)

GO TO 100

70 WRITE (IPRINT, 170)

GO TO 100

80 I $1=($ IARGS $(N R A+3)-1) / N R O W+1$

$I 2=N C A * M+2$

WRITE (IPRINT, 180) NN, B(I2), I I, NBC (1)

GO TO 100

90 WRITE (IPRINT, 190)

100 WRITE (IPRINT, 200) (NBC (I), I=1,NCP)

WRITE (IPRINT, 210)

DO 110 NAGA $=1$, NRP

$N B R=N R A+N A G A$

I $I=N C A * M+N B R$

$I 2=I 1+(N C P-M A X O(0, L C H K *(N C P-N A G A))-1) * M$

NBR $=($ IARGS $($ NBR +1$)-1) / N R O W+1$

WRITE (IPRINT, 220) ,NBR, (B (I) , I=I 1 , I2, M)

IF (IND.NE.7) GO TO 110

I $I=I I+2 * M * M$

I $2=I 2+2 * M * M$

WRITE (IPRINT, 230) (B(I), I=I I, I 2, M)

WRITE (IPRINT, 210)

110 CONTINUE

$N L A=N L A+N L P$

$N R A=N R A+L C H K * N C P$

$120 N C A=N C A+N C P$

RETURN

MST 60

MST 70

MST 80

MST 90

MST 100

MST 110

MST 120

MST 130

MST 140

MST 150

MST 160

MST 170

MST 180

MST 190

MST 200

MST 210

MST 220

MST 230

MST 240

MST 250

MST 260

MST 270

MST 280

MST 290

MST 300

MST 310

MST 320

MST 330

MST 340

MST 350

MST 360

MST 370

MST 380

MST 390

MST 400

MST 410

MST 420

MST 430

MST 440

MST 450

MST 460

MST 470

MST 480

MST 490

MST 500

MST 510

MST 520

MST 530

MST 540

MST 550

MST 560

MST 570

MST 580

130 FORMAT (/1H, 44X,31HSIMPLE CORRELATION COEFFICIENTS)

MST 590 
140 FORMAT (/IH, 22X,75HSIGNIFICANCE LEVELS OF SIMPLE CORRELATION COEFMST 600 IFICIENTS (ASSUMING NORMALITY))

150 FORMAT (/IH ,25X,37HPARTIAL CORRELATION COEFFICIENTS WITH, I3,26H RMST 620 IEMAINING VARIABLES FIXED)

MST 630

160 FORMAT (/1H ,22X,76HSIGNIFICANCE LEVELS OF PARTIAL CORRELATION COEMST 640 IFFICIENTS (ASSUMING NORMALITY))

MST 650

170 FORMAT (/1H ,30X,58HSPEARMAN RANK CORRELATION COEFFICIENTS (ADJUSTMST 660 IED FOR TIES))

MST 670

180 FORMAT (/IH ,8X,79HSIGNIFICANCE LEVEL OF QUADRATIC FIT OVER LINEARMST 680 1 FIT BASED ON F RATIO WITH 1 AND, I5,19H DEGREES OF FREEDOM/1H ,7X, MST 690 213H(FOR EXAMPLE,F7.4,60H IS THE SIGNIFICANCE LEVEL OF THE QUADRATIMST 700 $3 C$ TERM WHEN COLUMN, $13,20 \mathrm{H}$ IS FITTED TO COLUMN, I3, IH))

MST 710

190 FORMAT (/1H ,17X,86HCONFIDENCE INTERVALS FOR SIMPLE CORRELATION COMST 720 IEFFICIENTS (USING FISHER TRANSFORMATION)/30X,68H95 PER CENT LIMITSMST 730

2 BELOW DIAGONAL, 99 PER CENT LIMITS ABOVE DIAGONAL)

200 FORMAT (/1H, 6HCOLUMN, 10I11) 210 FORMAT (IH)

MST 750

FORMAT (1H , I $4,4 X, 10 F 11.4)$

FORMAT (1H ,6X,10F 11.4$)$

END

MST 760

MST 770

MST 780

MST 790 
KRONECKER PRODUCT OF TWO MATRICES $A(N, C) * B(M, K)=D$

MKR 20

MKR 30

MKR 40

FIRST FOUR ARGUMENTS DEFINE MATRIX A STARTING POS AND SIZE

MKR 50

NEXT FOUR ARGUMENTS DEFINE MATRIX B STARTING POS AND SIZE

MKR 60

LAST TWO ARGUMENTS INDICATE WHERE RESULT IS TO BE STORED D

MKR 70

COMMAND IS:

MKRON $A(,,++), R=,, C=,,{ }^{*} B(,,++), R=, C=,, \operatorname{STORE~} D(,,++)$

MKR 80

MKR 90

MKR 100

MKR 110

MKR 120

MKR 130

COMMON /BLOCRC/ NRC, RC (12600)

MKR 140

COMMON /BLOCKD/ IARGS(100), KIND (100), ARGTAB (100), NRMAX, NROW, NCOL, NMKR 150

IARGS, VWXYZ (8), NERROR

DIMENSION ARGS $(100)$

MKR 160

EQUIVALENCE (ARGS(1), RC (12501))

MKR 170

COMMON /SCRAT/ NS, NS2, A(13500)

MKR 180

IF (NARGS.NE.10) CALL ERROR (10)

MKR 190

$J=N A R G S$

CALL CKIND (J)

IF (J.NE.O) CALL ERROR (3)

IF (NERROR.NE.0) RETURN

MKR 200

$\operatorname{IARGS}(11)=\operatorname{IARGS}(3) * \operatorname{IARGS}(7)$

$\operatorname{IARGS}(12)=\operatorname{IARGS}(4) * \operatorname{IARGS}(8)$

$\mathrm{J}=3$

CALL MTXCHK (J)

IF (J.EQ.0) GO TO 10

CALL ERROR (17)

RETURN

$10 \quad$ NRA $=\operatorname{IARGS}(3)$

$N C A=I A R G S(4)$

$N R B=I A R G S(7)$

$N C B=I A R G S(8)$

$N D S=1$

$K A=I A R G S(1)$

DO 40 ICA $=1, N C A$

$L A=I A R G S(5)$

DO 30 ICB $=1, N C B$

$K=K A$

DO 20 IRA $=1$, NRA

$\mathrm{T}=\mathrm{RC}(\mathrm{K})$

$K=K+1$

$\mathrm{L}=\mathrm{LA}$

DO 20 I RB $=1, N R B$

$A(N D S)=T^{*} R C(L)$

$\mathrm{L}=\mathrm{L}+1$

$20 \quad$ NDS $=$ NDS +1

$30 \quad L A=L A+N R O W$

$40 \quad \mathrm{KA}=\mathrm{KA}+\mathrm{NROW}$

$N C R=I$ ARGS (11)

$N C C=I$ ARGS (12)

NDS $=1$

$K A=I A R G S(9)$

DO $60 \quad I=1$, NCC

$K=K A$

MKR 210

MKR 220

MKR 230

MKR 240

MKR 250

MKR 260

MKR 270

MKR 280

MKR 290

MKR 300

MKR 310

MKR 320

MKR 330

MiKR 340

MKR 350

MKR 360

MKR 370

MKR 380

MKR 390

MKR 400

MKR 410

MKR 420

MKR 430

MKR 440

MKR 450

MKR 460

MKR 470

MKR 480

MKR 490

MKR 500

MKR 510

MKR 520

MKR 530

MKR 540

MKR 550

MKR 560

MKR 570

DO $50 \mathrm{~J}=1$, NCR

MKR 580

$R C(K)=A$ (NDS)

MKR 590 
$N D S=N D S+1$

MKR 600

$50 \quad K=K+1$

MKR 610

$60 \quad K A=K A+N R O W$

MKR 620

RETURN

MKR 630

END

MKR 040 
SUBROUTINE MMULT

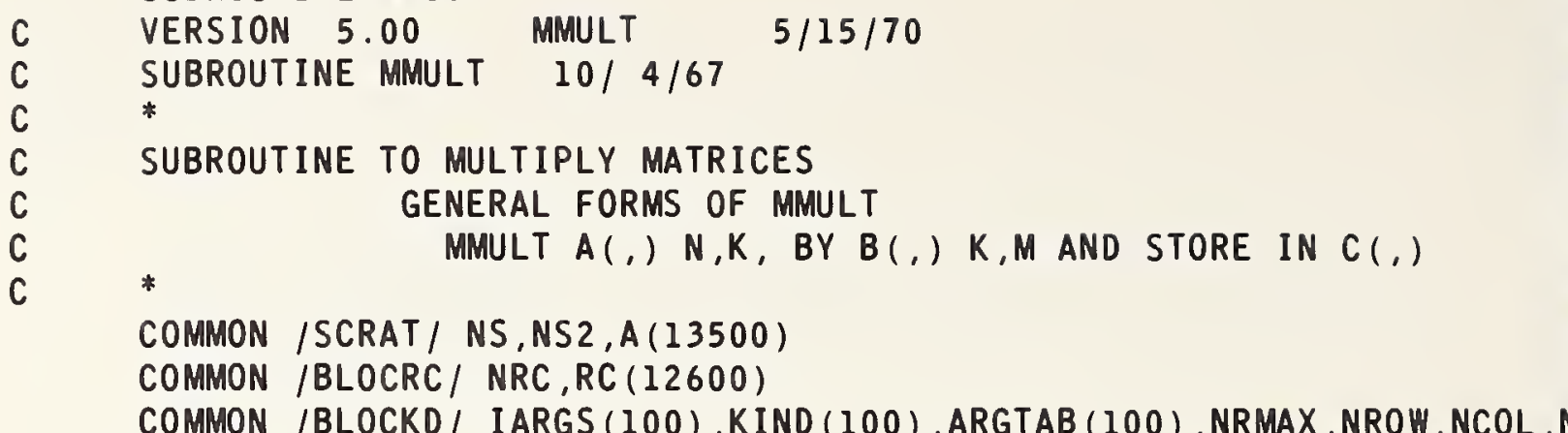

IARGS, VWXYZ (8), NERROR

DIMENSION ARGS $(100)$

EQUIVALENCE (ARGS (1), RC(12501))

DOUBLE PRECISION $X$, SUM

MMU 10

MMU 20

MMU 30

MMU 40

MMU 50

MMU 60

MMU 70

MMU 110

MMU 120

MMU 130

DIMENSION $X(1)$

EQUIVALENCE $(X, A)$

*

CHECK TO SEE IF WE HAVE CORRECT NUMBER OF ARGUMENTS

MMU 140

MMU 150

MMU 160

MMU 170

MMU 180

MMU 190

MMU 200

MMU 210

*

10 IF (NARGS.NE.10) CALL ERROR(10)

MMU 220

MMU 230

MMU 240

C

C CHECK TO SEE IF ALL ARGUMENTS ARE INTEGERS

MMU 250

C *

$J=N A R G S$

CALL CKIND (J)

IF (J.EQ.0) GO TO 20

CALL ERROR (3)

C

*

CHECK TO SEE IF DIMENSIONS ARE CORRECT

*

20 IF (IARGS (4).NE.IARGS (7)) CALL ERROR (26)

C

*

CHECK TO SEE IF ARGUMENTS ARE OUT OF RANGE

C FIND COLUMN ADDRESSES

C

70 IARGS ( 12$)=$ IARGS (NARGS-2)

$80 \quad \operatorname{IARGS}(11)=\operatorname{IARGS}(3)$

$100 \mathrm{~J}=3$

CALL MTXCHK (J)

IF $(J-1) \quad 130,110,120$

110 CALL ERROR (3)

RETURN

120 CALL ERROR (17)

RETURN

C *

C CHECK FOR PREVIOUS ERRORS

C *

130 IF (NERROR.NE.0) RETURN

IROWA =I ARGS (3)

ICOLA=IARGS (4)

ICOLB $=$ I ARGS ( 8 )

MMU 260

MMU 270

MMU 280

MMU 290

MMU 300

MMU 310

MMU 320

MMU 330

MMU 340

MMU 380

MMU 420

MMU 430

MMU 440

MMU 450

MMU 490

MMU 500

MMU 630

MMU 640

MMU 650

MMU 660

MMU 670

MMU 680

MMU 690

MMU 700

MMU 710

MMU 720

MMU 730

MMU 740

MMU 750

MMU 760

C BEGIN MULTIPLICATION

MMU 770

C

$$
\begin{aligned}
& \text { ISP }=1 \\
& \text { IBP }=\text { IARGS (5) } \\
& D O 160 \text { ICB }=1, \text { ICOLB } \\
& \text { IAP =IARGS (1) } \\
& D O 150 \text { IRA }=1, \text { I ROWA }
\end{aligned}
$$

MMU 780

MMU 790

MMU 800

MMU 810

MMU 820

MMU 830 


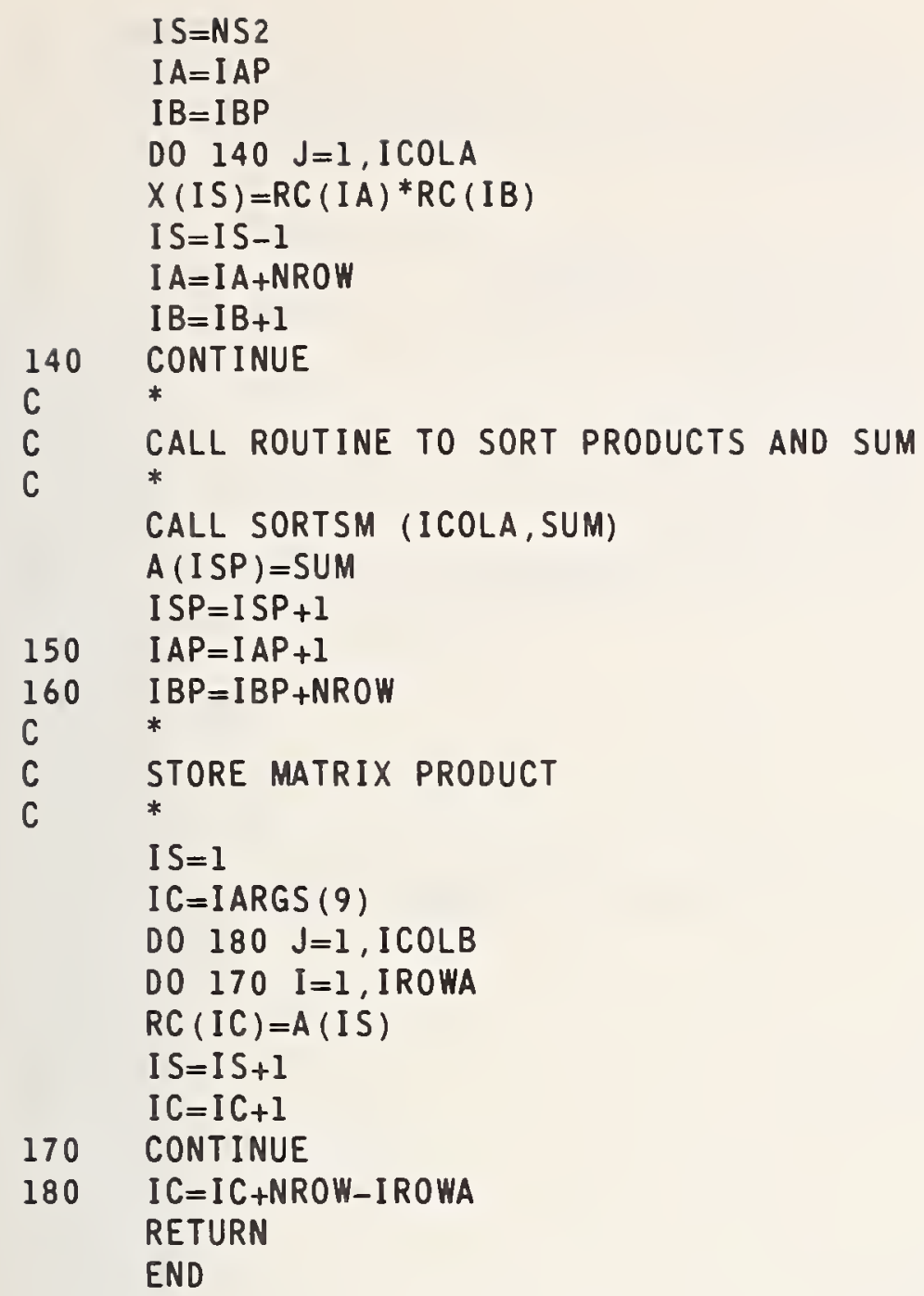

180 IC $=$ IC+NROW-IROWA RETURN

END

MMU 940

MMU 950

MMU 960

MMU 970

MMU 980

MMU 990

MMU 1000

MMU 1010

MMU 1020

MMU 1030

MMU 1040

MMU 1050

MMU 1060

MMU 1070

MMU 1080

MMU 1090

MMU 1100

MMU 1110

MMU 1120

MMU1 130

MMU 1140 
SUBROUTINE TO DO MDEFINE, ADEFINE, MZER

S. PEAVY FOR OMNITAB $1108 \quad 4 / 2 / 68$

COMMANDS ARE AS FOLLOWS

MDEFINE MATRIX IN $R, C$ SIZE $N \times M$ TO EQUAL $K$

ADEFINE ARRAY IN $R, C$ SIZE $N X M$ TO EQUAL $K$

MOP 30

C

COMMON /BLOCRC/ NRC, RC (12600)

MOP 140

MOP 280

MOP 290

MOP 300

MOP 310

MOP 320

MOP 330

MOP 340

COMMON /BLOCKD/ IARGS (100), KIND (100), ARGTAB (100), NRMAX, NROW, NCOL, NMOP 350

IARGS, VHXYZ (8), NERROR

DIMENSION ARGS (100)

EQUIVALENCE (ARGS (1), RC (12501))

COMMON /SCRAT/ NS, NS2, A(13500)

COMMON /BLOCKE/ NAME (4), L1, L2, ISRFLG

DATA ONE $/ 1.0 /$,ZERO $/ 0.0 /$

GO TO $(10,50,60,80)$, L2

10 IF (NARGS.NE.5) CALL ERROR (10)

IF (KIND (NARGS). NE.1) CALL ERROR (3)

IF (NARGS.EQ.4) IARGS (4)=IARGS (3)

CONST=ARGS (NARGS)

CONSTA=ARGS (NARGS)

$J=N A R G S-1$

20 CALL CKIND (J)

IF (J.NE.0) CALL ERROR (3)

$\mathrm{J}=1$

CALL MTXCHK (J)

IF (J.NE.O) CALL ERROR (17)

IF (NERROR.NE.0) RETURN

$J B=I$ ARGS (1)

$N=I$ ARGS (3)

$K=I A R G S(4)$

$J A=J B$

IF (L2.EQ.4) GO TO 90

DO $40 \mathrm{KA}=1, \mathrm{~K}$

$\mathrm{JC}=\mathrm{JB}$

DO $30 \quad N A=1, N$

$R C(J C)=C O N S T$

$30 \quad \mathrm{JC}=\mathrm{JC}+1$

IF (KA.GT.N) GO TO 40

$R C(J A)=$ CONSTA

$J A=J A+N R O W+1$

$40 \quad J B=J B+N R O W$

RETURN

50 IF (NARGS.NE.4) CALL ERROR (10)

CONST $=$ ZERO

CONSTA $=Z E R O$
MOP 360

MOP 370

MOP 380

MOP 390

MOP 400

MOP 410

MOP 420

MOP 430

MOP 440

MOP 450

MOP 460

MOP 470

MOP 480

MOP 490

MOP 500

MOP 510

MOP 520

MOP 530

MOP 540

MOP 550

MOP 560

MOP 570

MOP 580

MOP 590

MOP 600

MOP 610

MOP 620

MOP 630

MOP 640

MOP 650

MOP 660

MOP 670

MOP 680

MOP 690

MOP 700

MOP 710

MOP 720 
$J=N A R G S$

MOP 730

IF (NARGS.EQ.4) GO TO 20

MOP 740

$\operatorname{IARGS}(4)=\operatorname{IARGS}(3)$

MOP 750

$J=N A R G S-1$

MOP 760

GO TO 20

MOP 770

60 IF (NARGS.NE.4) CALL ERROR (10)

MOP 780

CONST $=$ ZERO

MOP 790

CONSTA $=$ ONE

MOP 800

$J=N A R G S$

MOP 810

GO TO 20

MOP 820

MOP 910

$J=N A R G S-1$

IF (NARGS.NE.5) CALL ERROR(10)

MOP 920

GO TO 20

MOP 930

MOP 970

MOP 980

DO $100 \quad N A=1, N$

$R C(J B)=A R G S$ (NARGS)

MOP 990

MOP 1000

$J B=J B+1+N R O W$

MOP 1010

RETURN

MOP 1020

$\operatorname{KIND}(5)=0$

MOP 1030

CALL ADRESS $(5, M)$

MOP 1040

IF (M.GT.O) GO TO 120

MOP 1050

CALL ERROR (11)

MOP 1060

RETURN

MOP 1070

MOP 1080

$A(N A)=R C(M)$

MOP 1090

MOP 1100

MOP 1110

MOP 1120

MOP 1130

MOP 1140 
SUBROUTINE MOVE

C VERSION 5.00

MOVE

$5 / 15 / 70$

MOV 10

COMMON /BLOCRC/ NRC, RC (12600)

MOV 20

COMMON /BLOCKD/ I ARGS(100), KIND(100), ARGTAB (100), NRMAX, NROW, NCOL, NMOV 40

IARGS, VHXYZ (8), NERROR

MOV 50

DIMENSION ARGS $(100)$

MOV 60

EQUIVALENCE (ARGS(1),RC(12501))

C THIS ROUTINE IS ALSO CALLED BLOCKTRANSFER

MOV 70

IF (NARGS.EQ.6) GO TO 50

MOV 80

$K=10$

10 CALL ERROR (K)

20 RETURN

$30 \quad K=20$

GO TO 10

$40 \quad K=11$

GO TO 10

$50 \operatorname{IARGS}(9)=\operatorname{IARGS}(1)+\operatorname{IARGS}(3)-1$

$\operatorname{IARGS}(13)=\operatorname{IARGS}(5)+\operatorname{IARGS}(3)-1$

IF (KIND(1)+KIND(3)+KIND (4)+KIND (5).NE.0) G0 T0 30

MOV 90

MOV 100

MOV 110

MOV 120

MOV 130

MOV 140

MOV 150

MOV 160

MOV 170

MOV 180

1. LE. NROW. AND. IARGS (13). LE.NROW) GO TO 60

MOV 190

$K=16$

GO TO 10

$60 \operatorname{IARGS}(10)=\operatorname{IARGS}(2)+\operatorname{IARGS}(4)-1$

$\operatorname{KIND}(10)=0$

$\operatorname{IARGS}(14)=\operatorname{IARGS}(6)+\operatorname{IARGS}(4)-1$

$\operatorname{KIND}(14)=0$

DO $70 \quad I=2,14,4$

CALL ADRESS (I, IARGS (I))

IF (IARGS (I)) $30,40,70$

$70 \quad$ IARGS (I) $=$ IARGS (I) -1

C

IF MOVE IS UP, IR $=-1$, IF DOWN, IR $=+1$

C IF MOVE IS LEFT, IC $=-1$, IF RIGHT, IC $=+1$

C DIRECTION OF MOVE IS SUCH THAT THE THO AREAS CAN BE OVERLAPPING

C AND IT WILL BE DONE PROPERLY.

C

$I R=I S I G N(1, \operatorname{IARGS}(5)-\operatorname{IARGS}(1))$

$I C=I S I G N(1, \operatorname{IARGS}(6)-\operatorname{IARGS}(2))$

$M M=I A R G S(4 * I R+5)+\operatorname{IARGS}\left(4^{*} I C+6\right)$

$N N=I$ ARGS $(4 * I R+9)+\operatorname{IARGS}\left(4^{*} I C+10\right)$

I C $=$ I C*NROW

MMM=IARGS (3)

$N N N=I A R G S(4)$

DO $90 \mathrm{~J}=1$, NNN

$M=M M$

$N=N N$

DO $80 \quad I=1$, MMM

$\mathrm{RC}(\mathrm{N})=\mathrm{RC}(\boldsymbol{M})$

$M=M-I R$

$80 \quad N=N-I R$

$M M=M M-I C$

$90 \quad N N=N N-I C$

GO TO 20

END

MOV 210

MOV 220

MOV 230

MOV 240

MOV 250

MOV 260

MOV 270

MOV 280

MOV 290

MOV 300

MOV 310

MOV 320

MOV 330

MOV 340

MOV 350

MOV 360

MOV 370

MOV 380

MOV 390

MOV 400

MOV 410

MOV 420

MOV 430

MOV 440

MOV 450

MOV 460

MOV 470

MOV 480

MOV 490

MOV 500

MOV 510

MOV 520

MOV 530

MOV 540

MOV 550 


\section{SUBROUTINE MPROP}

COMMAND IS AS FOLLOWS, $X=A$ OR M DEPENDING APROP OR MPROP IS REQUIRMPR 40

I XPROP OF MATRIX $(,+++$ NO OF ROWS, , NO OF COL $++\quad$ MPR 50 INFORMATION PRINTED AND NO STORAGE MPR 60

$\begin{array}{lll}\text { MPR } & 70\end{array}$

II XPROP MATRIX $(,,++)$ SIZE , , BY ++ PROPERTIES STORED ++ MPR 80 PROPERTIES PRINTED AND STORED MPR 90

MPR 100

III XPROP MATRIX $(,,++) \quad \mathrm{R}=,, \mathrm{C}=,, \operatorname{PROP}++\operatorname{COL}$ NORMS $(,,++)$ MPR 110 SAME AS II PLUS STORAGE OF COLUMN NORMS MPR 120

MPR 130

IV XPROP $(, \ldots++) R=,, C=,$, PROP ++ COL NORMS $(, \ldots++)$ ROW NORMS (MPR 140 SAME AS II I PLUS STORAGE OF ROW NORMS, ALSO $(R+1,++)$ OF NORM MPR 150 AVERAGES WILL CONTAIN GRAND AVERAGE, IF $X=A$.

MPR 160

MPR 170

$V \quad \operatorname{XPROP}(,,++) R=,, C=,, \operatorname{COL} \operatorname{NORMS}(,,++)$

SAME AS III EXCEPT PROPERTIES WILL NOT BE STORED

MPR 180

MPR 190

MPR 200

VI XPROP $(,,++) \quad R=,, C=,, \operatorname{COL}$ NORMS $(,,++)$ ROW NORMS $(,,++)$

MPR 210

SAME AS IV EXCEPT PROPERTIES WILL NOT BE STORED

MPR 220

MPR 230

VII SXPROP

IF COMMANDS I-VI ARE PREFACED WITH AN S PRINTOUT

MPR 240

OF PROPERTIES WILL BE SUPPRESSED

MPR 250

MPR 260

MPR 270

L2 OPTIONS:

L2= 1 MPROP: $\quad$ 2 $=3 \quad$ SMPROP

MPR 280

$L 2=2$ APROP: $L 2=4$ SAPROP

MPR 290

MPR 300

MPR 310

COMMON/HEADER/NOCARD $(80), \operatorname{ITLE}(60,6), \operatorname{LNCNT}$, IPR INT , NPAGE, IPUNCH

MPR 320

MPR 340

MPR 350

\section{COMMON /SCRAT/ NS, NS2,A (13500)}

COMMON /BLOCKD/IARGS (100), KIND (100), ARGTAB (100), NRMAX,NROW, NCOL, NMPR 360
IARGS, VWXYZ(8), NERROR

DIMENSION ARGS $(100)$

MPR 380

EQUIVALENCE (ARGS(1), RC (12501))

COMMON /BLOCKE/ NAME (4), L1, L2, ISRFLG

MPR 390

DIMENSION $\operatorname{IRSLT}(6), \operatorname{ERR}(3), \operatorname{IPROP}(5), \operatorname{IRSLTP}(11), \operatorname{IRSLTA}(2,5)$

MPR 400

DATA NO/3H NO/ IYES/3HYES/ IBLK/3H / LOWRA/3H LO/, OWRB/3HWER/4IMPR 420

IPPRA/3H UP/, IPPRB/3HPER/, IANDA/3H AN/, IANDB/3HD/, IRWA, IRWB/3H MPR 430

$2,3 \mathrm{HROW} /$, ICLMA, ICLMB/3HCOL, 3HUMN/, IBTHH, IBTHHA/3H ,3H T/, IBTHA, IMPR 440

$3 B T H B / 3 H W O-, 3 H W A Y /, N O A, N O A B / 3 H, 3 H$ NO/

IF (L2.LE.2.OR.NARGS.NE.4) GO TO 5

MPR 450

CALL ERROR (233)

RETURN

5 IF (NARGS.LT.4.OR.NARGS.GT.9) CALL ERROR (10)

LOC=IARGS (1)

$J=N A R G S$

CALL CKIND (J)

IF (J.NE.0) CALL ERROR (3)

$K=5$

$J=1$

IF (NARGS-5) $60,30,10$

10 IF (NARGS.EQ.6.OR.NARGS.EQ.8) GO TO 40

IS=IARGS (5)

$\mathrm{J}=2$

$K=9$

MPR 455

MPR 460

MPR 465

MPR 467

MPR 470

MPR 480

MPR 490

MPR 500

MPR 510

MPR 520

MPR 530

MPR 540

MPR 550

MPR 560

MPR 570 
IF (NARGS.EQ.7) GO TO 20

MPR 580

$\operatorname{IARGS}(11)=\operatorname{IARGS}(3)$

MPR 590

$\operatorname{IARGS}(12)=1$

$\operatorname{IARGS}(10)=\operatorname{IARGS}(9)$

MPR 600

$\operatorname{IARGS}(9)=\operatorname{IARGS}(8)$

MPR 610

$K=13$

$J=3$

IF (L2.GT.2) IARGS(11) =IARGS (11)+1

$20 \operatorname{IARGS}(5)=\operatorname{IARGS}(6)$

$\operatorname{IARGS}(6)=\operatorname{IARGS}(7)$

$\operatorname{IARGS}(7)=1$

$\operatorname{IARGS}(8)=\operatorname{IARGS}(4)$

$\operatorname{IARGS}(K)=I S$

30 CALL ADRESS ( $K, K P R O P$ )

IF (KPROP.LE.0) CALL ERROR (11)

MPR 620

MPR 630

MPR 640

MPR 650

MPR 660

MPR 670

MPR 680

MPR 690

MPR 700

MPR 710

MPR 720

GO TO 60

$40 \quad J=2$

IF (NARGS.EQ.6) GO TO 50

$\operatorname{IARGS}(9)=\operatorname{IARGS}(7)$

$\operatorname{IARGS}(10)=\operatorname{IARGS}(8)$

$\operatorname{IARGS}(11)=\operatorname{IARGS}(3)$

$\operatorname{IARGS}(12)=1$

$J=3$

IF (L2.GT.2) IARGS(1I) =IARGS (11)+1

$50 \operatorname{IARGS}(7)=1$

$\operatorname{IARGS}(8)=\operatorname{IARG} 5(4)$

60 CALL MTXCHK (J)

KARGS $=K$

IF (J.NE.0) CALL ERROR (17)

IF (NERROR.NE.O) RETURN

$K=I A R G S(1)$

IF (L2.EQ.2.OR.L2.EQ.4) GO T0 110

C COMMAND IS MPROP

C IS MATRIX A SQUARE ONE

IF (IARGS (3). NE. IARGS (4)) GO TO 90

C YES

CALL INVCHK (RC (K), NROW, I ARGS (3), A (100), I ARGS (3)+1, A, 1,ERR, IND)

MPR 730

MPR 740

MPR 750

MPR 760

MPR 770

MPR 780

MPR 790

MPR 800

MPR 810

MPR 820

MPR 830

MPR 840

MPR 850

MPR 860

MPR 870

MPR 880

MPR 890

MPR 900

MPR 910

MPR 920

MPR 930

MPR 940

$K A=K$

$M=100$

$L=I A R G S(3)$

DO $80 \mathrm{I}=1, \mathrm{~L}$

$K B=K A$

DO $70 \mathrm{~J}=1, \mathrm{~L}$

$A(M)=R C(K B)$

$K B=K B+1$

$70 \quad M=M+1$

$80 \quad K A=K A+N R O W$

CALL DETRNK (A $(100), L, L, D E T, R A N K)$

CALL PVTRI (RC (K), NROW, IARGS (3), INDU, INDB)

CALL PROCHK (RC (K),NROW, IARGS (3), IARGS (4), IPROP , A (1),NS2)

$A(30)=0$.

IF (INDU.EQ.0) $\quad A(30)=A(30)+1.0$

IF (INDB.EQ.0) $\quad A(30)=A(30)+2.0$

$A(19)=D E T$

$A(20)=$ RANK

$A(21)=E R R(1)$

$A(22)=E R R(2)$

$A(23)=E R R(3)$

$A(24)=0$.

MPR 950

MPR 960

MPR 970

MPR 980

MPR 990

MPR 1000

MPR 1010

MPR 1020

MPR 1030

MPR 1040

MPR 1050

MPR 1060

MPR 1070

MPR 1080

MPR 1090

MPR 1100

MPR 1110

MPR 1120

MPR 1130

MPR 1140

MPR 1150

MPR 1160 
IF (IPROP (3).EQ.0) $A(24)=1.0$

IF (IPROP (3).EQ.1) $\quad A(24)=2.0$

$A(25)=0$.

IF (IPROP (2).LT.2) $\quad A(25)=\operatorname{IPROP}(2)+1$

$A(26)=0$.

IF (IPROP (2).GT.2) $\quad A(26)=\operatorname{IPROP}(2)-2$

$A(27)=0$.

IF (IPROP(1).EQ.0) $A(27)=1$.

$A(28)=0.0$

IF (IPROP (4).LT.2) $A(28)=\operatorname{IPROP}(4)+1$

$A(29)=0$.

IF (IPROP (5).LT.2) $A(29)=\operatorname{IPROP}(5)+1$

GO TO 110

90

100

110

$$
\text { DO } 100 \quad I=19,31
$$

$A(I)=0.0$

CALL RCSUM (RC (K), NROW, IARGS (3), IARGS (4), A (101))

$L=I A R G S(3)$

IF (L.GT.IARGS (4)) L=IARGS (4)

ITRACE $=L$

I POS $=0$

IZERO $=0$

INEG $=0$

TRACE $=0.0$

$A M X=R C(K)$

$A M N=A M X$

$L A=I A R G S(3)+\operatorname{IARGS}(4)$

$A V G=A(L A+102) / F L O A T(\operatorname{IARGS}(3) * \operatorname{IARGS}(4))$

$A B S M X=A B S(A M X)$

$A B S M N=A B S(A M N)$

$A B S M N Z=A B S M N$

$S S Q=0.0$

$S R S Q=0.0$

$S C S Q=0.0$

IF (ABSMNZ.EQ.0.0) ABSMNZ $=1 . E 35$

$K A=K$

DO $120 \quad I=1, L$

$T R A C E=T R A C E+R C(K A)$

$120 \mathrm{KA}=\mathrm{KA}+\mathrm{NROW}+1$

IN $=$ I ARGS (3)

$J K=I A R G S(4)$

$K A=K$

$F I N=I N$

$\mathrm{FJK}=\mathrm{JK}$

DO $200 \mathrm{~J}=1, \mathrm{JK}$

$K B=K A$

DO $190 \quad I=1, I N$

$K C=I A R G S(4)+I$

$S S Q=S S Q+(R C(K B)-A V G) * * 2$

$S C S Q=S C S Q+(R C(K B)-A(J+100) / F I N) * * 2$

$S R S Q=S R S Q+(R C(K B)-A(K C+100) / F J K) * * 2$

IF (RC (KB)) $130,140,150$

130 INEG $=$ INEG+1

GO TO 160

140 IZERO $=I Z E R O+1$

GO TO 160

$150 \quad$ IPOS $=I P O S+1$

160 IF (AMX.GT.RC(KB)) GO TO 170

$A M X=R C(K B)$
MPR 1170

MPR 1180

MPR 1190

MPR 1200

MPR 1210

MPR 1220

MPR 1230

MPR 1240

MPR 1250

MPR 1260

MPR 1270

MPR 1280

MPR 1290

MPR 1300

MPR 1310

MPR 1320

MPR 1330

MPR 1340

MPR 1350

MPR 1360

MPR 1370

MPR 1380

MPR 1390

MPR 1400

MPR 1410

MPR 1420

MPR 1430

MPR 1440

MPR 1450

MPR 1460

MPR 1470

MPR 1480

MPR 1490

MPR 1500

MPR 1510

MPR 1520

MPR 1530

MPR 1540

MPR 1550

MPR 1560

MPR 1570

MPR 1580

MPR 1590

MPR 1600

MPR 1610

MPR 1620

MPR 1630

MPR 1640

MPR 1650

MPR 1660

MPR 1670

MPR 1680

MPR 1690

MPR 1700

MPR 1710

MPR 1720

MPR 1730

MPR 1740

MPR 1750 
GO TO 180

MPR 1760

170 IF (AMN.GT.RC (KB)) $A M N=R C(K B)$

$180 \quad R C A B=A B S(R C(K B))$

IF (ABSMX.LT.RCAB) ABSMX $=R C A B$

IF (ABSMN.GT.RCAB) $A B S M N=R C A B$

IF (ABSMNZ.GT.RCAB) IF (RCAB) $190,190,185$

GO TO 190

$185 \quad A B S M N Z=R C A B$

$190 \quad K B=K B+1$

$200 \quad K A=K A+N R O W$

IF (L2.EQ.2.OR.L2.EQ.4.OR.IARGS (3).NE.IARGS (4)) GO TO 250

I STOCR $=0$

ISTCHC $=0$

IF (AMN.LT.0.0) GO TO 240

DO $210 \mathrm{~J}=1, \mathrm{JK}$

IF $(A(J+100) . E Q .1 .0)$ GO TO 210

GO TO 220

210 CONTINUE

I STCHC $=2$

220 DO $230 \quad I=1, I N$

$M=I+J K$

IF $(A(M+100) . E Q .1 .0)$ GO TO 230

GO TO 240

230 CONTINUE

ISTOCR $=1$

$240 \quad A(31)=I S T C H C+I S T O C R$

$250 \quad A(1)=$ TRACE

$T R 2=0.0$

$K B=K$

$J K K=J K$

IF (JK.GT.IN) JKK=IN

DO $260 \mathrm{~J}=2$, JKK

$\mathrm{KA}=\mathrm{K}$

$\mathrm{KB}=\mathrm{KB}+\mathrm{NROW}+1$

$K C=K+J-1$

$K D=K+(J-1) * N R O W$

I I $=\mathrm{J}-\mathrm{I}$

DO $260 \quad I=1$, I I

$T R 2=T R 2+(R C(K A) * R C(K B)-R C(K C) * R C(K D))$

$K A=K A+N R O W+1$

$K C=K C+N R O W$

$260 \quad K D=K D+1$

$A(2)=$ TR2

$A(3)=A M X$

$A(4)=A M N$

$A(5)=A B S M X$

$A(6)=A B S M N$

$A(7)=A B S M N Z$

$A(8)=I P O S$

$A(9)=I$ ZERO

$A(10)=I N E G$

$A(11)=A(L A+101)$

$A(12)=A V G$

$A(13)=A(L A+103)$

$A(14)=S S Q$

$A(15)=S R S Q$

$A(16)=S C S Q$

$A(17)=A(L A+104)$

$A(18)=A(17) / F L O A T(I A R G S(3) * \operatorname{IARGS}(4))$

MPR 1770

MPR 1780

MPR 1790

MPR 1800

MPR 1805

MPR 1810

MPR 1820

MPR 1830

MPR 1840

MPR 1850

MPR 1860

MPR 1870

MPR 1880

MPR 1890

MPR 1900

MPR 1910

MPR 1920

MPR 1930

MPR 1940

MPR 1950

MPR 1960

MPR 1970

MPR 1980

MPR 1990

MPR 2000

MPR 2010

MPR2020

MPR2 030

MPR 2040

MPR 2050

MPR2060

MPR 2070

MPR 2080

MPR 2090

MPR 2100

MPR 2110

MPR 2120

MPR2 130

MPR 2140

MPR 2150

MPR 2160

MPR 2170

MPR 2180

MPR 2190

MPR 2200

MPR 2210

MPR 2220

MPR 2230

MPR2240

MPR2250

MPR 2260

MPR 2270

MPR 2280

MPR 2290

MPR 2300

MPR 2310

MPR 2320

MPR 2330 
IF (L2.GE.3) GO TO 570

MPR2340

CALL PAGE (4)

MPR 2350

IF (L2.NE. 1) GO TO 370

MPR 2360

WRITE (IPRINT,640) IARGS (3), IARGS (4), LOC, IARGS (2)

MPR2 2370

IF (MOD (NARGS, 2).EQ.0) GO TO 270

MPR 2380

WRITE (IPRINT,650) IARGS (KARGS)

MPR2390

270

WRITE (IPRINT, 660 ) ITRACE, (A (I) , I=1,7), IPOS, IZERO, INEG , (A (I) , I=11, MPR2400

116)

WRITE (IPRINT,670) A(17), A(18)

MPR2 410

WRITE (IPRINT, 680)

IF (IARGS (3).NE.IARGS (4)) GO T0 390

MPR2420

MPR2430

IRANK $=A(20)+.5 E-5$

MPR2440

WRITE (IPRINT,690) A(19), IRANK, (A (I), I=21, 23)

MPR2 450

DO $280 \quad I=1,6$

$\operatorname{IRSLT}(I)=\operatorname{IYES}$

$\operatorname{IRSLTP}(I)=A(I+23)$

IF $(A(I+23) \cdot E Q .0.) \quad \operatorname{IRSLT}(I)=N O$

280 CONTINUE

C SET IRSLT(I), I=1,6 FOR YES OR NO. ALSO A(I), I=24, 29

DO $290 \quad I=1,5$

$\operatorname{IRSLTA}(1, I)=I B L K$

$290 \operatorname{IRSLTA}(2, I)=I B L K$

$\operatorname{IRSLTA}(1,3)=N O A$

MPR2 460

MPR2470

MPR2480

MPR2490

MPR2500

MPR2510

MPR2520

MPR2530

MPR2 540

MPR2550

$\operatorname{IRSLTA}(2,3)=$ NOAB

MPR 2560

IF (INDU.NE.O.AND.INDB.NE.0) GO TO 320

IF (INDU.NE.0) GO TO 310

IF (INDB.EQ.0) GO TO 300

$\operatorname{IRSLTA}(1,1)=\operatorname{IPPRA}$

$\operatorname{IRSLTA}(2,1)=\operatorname{IPPRB}$

GO TO 320

$300 \operatorname{IRSLTA}(1,1)=\operatorname{IPPRA}$

$\operatorname{IRSLTA}(2,1)=\operatorname{IPPRB}$

MPR2 570

MPR2580

MPR2590

MPR2600

MPR2610

MPR2620

MPR2630

MPR2 640

$\operatorname{IRSLTA}(1,2)=\operatorname{IANDA}$

MPR2 650

$\operatorname{IRSLTA}(2,2)=\operatorname{IANDB}$

MPR2 660

MPR2 670

$310 \operatorname{IRSLTA}(1,3)=\operatorname{LOWRA}$

MPR2 680

$\operatorname{IRSLTA}(2,3)=\operatorname{LOWRB}$

MPR2 690

$320 \quad \operatorname{IRSLTA}(1,5)=$ NOA

MPR2700

$\operatorname{IRSLTA}(2,5)=$ NOAB

MPR2 710

IF (ISTOCR+ISTCHC.EQ.3) GO TO 340

MPR2720

IF (ISTOCR.EQ.O) GO TO 330

MPR2730

$\operatorname{IRSLTA}(1,5)=\operatorname{IRWA}$

$\operatorname{IRSLTA}(2,5)=\operatorname{IRWB}$

MPR2740

GO TO 350

330 IF (ISTCHC.EQ.0) GO TO 350

MPR2750

MPR2760

$\operatorname{IRSLTA}(1,5)=\operatorname{ICLMA}$

$\operatorname{IRSLTA}(2,5)=I$ CLMB

MPR2 2770

MPR2780

GO TO 350

$340 \operatorname{IRSLTA}(1,4)=\mathrm{IBTHH}$

MPR 2790

MPR2 800

$\operatorname{IRSLTA}(2,4)=$ IBTHHA

MPR2 810

$\operatorname{IRSLTA}(1,5)=\operatorname{IBTHA}$

MPR2820

$\operatorname{IRSLTA}(2,5)=\operatorname{IBTHB}$

MPR2830

MPR 2840

$350 \operatorname{IRSLTP}(7)=A(30)$

MPR2 850

$\operatorname{IRSLTP}(8)=A(31)$

MPR 2860

WRITE (IPRINT, 700) (IRSLT (I), IRSLTP (I), I=1,6)

MPR 2870

WRITE (IPRINT, 710) ((IRSLTA (I,J), I=1,2), J=1,3),IRSLTP(7), ((IRSLTA (MPR2880 $(I, J), I=1,2), J=4,5), I R S \operatorname{LTP}(8)$

DO $360 \quad I=1,2$

MPR2890

MPR2900

360 WRITE (IPRINT, 720)

MPR2910

WRITE (IPRINT, 730)

MPR2920 
GO TO 570

MPR2930

C APROP PRINT OUT
370 WRITE (IPRINT, 740) IARGS (3), IARGS (4), LOC, IARGS (2)

MPR2940

IF (MOD (NARGS, 2) .EQ.0) GO TO 380

MPR2950

WRITE (IPRINT,650) IARGS (KARGS)

MPR2960

MPR2970

380 WRITE (IPRINT, 660) ITRACE, (A (I) , I=1,7) , IPOS, IZERO, INEG, (A (I) , I=1 I, MPR2980

116)

WRITE (IPRINT,670) A(17),A(18)

GO TO 570

C MPROP PRINT OUT FOR A NON-SQUARE MATRIX

MPR2990

MPR 3000

MPR 3010

MPR 3020

390 DO $400 \quad I=1,2$

$\operatorname{IRSLTA}(1, I)=N O A$

$400 \quad \operatorname{IRSLTA}(2, I)=N O A B$

IF (IPROP (4).EQ.2) GO TO 550

IF (IABS (IPROP (4)) -4) 410,480,480

410 DO $420 \quad I=1,2$

$\operatorname{IRSLTA}(1, I)=\operatorname{IRWA}$

$420 \quad \operatorname{IRSLTA}(2, I)=I R W B$

IF (IPROP (4)) $430,430,440$

$430 \quad A(26)=2$

GO TO 450

$440 \quad A(26)=1$

450 IF (IPROP (5)) $460,460,470$

$460 \quad A(27)=2$

GO TO 550

$470 \quad A(27)=1$

GO TO 550

480 DO $490 \quad I=1,2$

$\operatorname{IRSLTA}(1, I)=I$ CLMA

$490 \quad \operatorname{IRSLTA}(2, I)=I$ CLMB

IF (IPROP (4)) $500,500,510$

$500 \quad A(26)=4$

GO TO 520

$510 \quad A(26)=3$

520 IF (IPROP(5)) $530,530,540$

$530 \quad A(27)=4$

GO TO 550

$540 \quad A(27)=3$

$550 \quad \operatorname{IRSLTP}(1)=A(26)$

$\operatorname{IRSLTP}(2)=A(27)$

WRITE (IPRINT, 750) ((IRSLTA $(J, I), J=1,2), \operatorname{IRSLTP}(I), I=1,2)$

MPR 3030

MPR 3040

MPR3050

MPR 3060

MPR 3070

MPR 3080

MPR3090

MPR3100

MPR 3110

MPR 3120

MPR 3130

MPR 3140

MPR 3150

MPR 3160

MPR 3170

MPR 3180

MPR 3190

MPR 3200

MPR 3210

MPR 3220

MPR 3230

MPR 3240

MPR 3250

MPR 3260

MPR 3270

MPR 3280

MPR 3290

MPR3 300

MPR3310

MPR 3320

MPR3330

DO $560 \quad I=1,22$

560 WRITE (IPRINT, 720)

WRITE (IPRINT, 760)

MPR 3340

MPR 3350

MPR3 360

MPR3370

IF (NARGS.EQ.4) RETURN

IF (MOD (NARGS, 2).EQ.0) GO TO 610

I $P=31$

MPR3380

MPR3390

MPR 3400

IF (IARGS (3).EQ.IARGS (4)) GO TO 590

I $P=27$

DO $580 \quad I=19,25$

$580 \quad A(I)=0.0$

590 IF $(M O D(L 2,2) \cdot E Q \cdot 0) \quad$ IP $=18$

IF (NROW.LT.IP) IP=NROW

DO $600 \quad I=1$, IP

$R C(K P R O P)=A(I)$

$600 \quad K P R O P=K P R O P+1$

IF (NARGS.EQ.5) RETURN

$610 \quad K A=I A R G S(5)$

MPR3410

MPR3420

MPR3430

MPR 3440

MPR 3450

MPR 3460

MPR 3470

MPR 3480

MPR 3490

MPR 3500

ANRMX $=\operatorname{IARGS}(3)$

MPR 3510 
DO $620 \mathrm{I}=1, \mathrm{JK}$

MPR 3520

$R C(K A)=A(I+100) / A N R M X$

MPR 3530

620

$K A=K A+N R O W$

MPR 3540

IF (NARGS.LT.8) RETURN

MPR 3550

$K A=I A R G S(9)$

MPR 3560

ANRMX $=I$ ARGS (4)

MPR 3570

$\mathrm{KB}=\mathrm{JK}+101$

MPR 3580

DO $630 \quad I=1, I N$

$R C(K A)=A(K B) / A N R M X$

MPR 3590

$K A=K A+1$

$630 \quad K B=K B+1$

IF (L2.GT.2) RC (KA) =AVG

RETURN

MPR 3600

MPR3610

MPR 3620

MPR3630

MPR 3640

MPR 3650

C

640 FORMAT (IHO,39X, 14HPROPERTIES OF , I3,3H X , I3,27H MATRIX STARTING ILOCATION $(, I 3,1 \mathrm{H}, \mathrm{I} 3, \mathrm{IH}))$

MPR3660

$\begin{array}{lll}650 & \text { FORMAT }(23 \mathrm{X}, 3 \mathrm{HCOL}, \mathrm{I} 7) & \text { MPR } 3680 \\ 660 & \text { FORMAT }(30 \mathrm{X}, 7 \mathrm{HGENERAL} / 23 \mathrm{X}, 1 \mathrm{HR} / 23 \mathrm{X}, 9 \mathrm{HI} \text { TRACE }(, 13,13 \mathrm{H} \text { VALUES USED }), \text { MPR } 3690\end{array}$

MPR3670

MPR3680 $17 X, 1 P E 15.6 / 23 X, 32 \mathrm{H} 2$ TRACE NO. 2

2 MAXIMUM ELEMENT

,E15.6//23X,32H3MPR3700 , E15.6/23X,20H4 MINUMUM ELEMENT ,MPR3710 $312 X \quad$ E $15.6 / 23 X, 32 \mathrm{H} 5$ MAXIMUM ELEMENT IN ABS VALUE, E15.6/23XMPR3720 4,32H6 MINUMUM ELEMENT IN ABS VALUE, E15.6/23X,32H7 MIN NON-ZERO EMPR3730 5LEM IN ABS VAL, E15.6//23X,32H8 NUMBER OF POSITIVE ELEMENTS, 10 MPR 3740 $6 \mathrm{X}, \mathrm{I} 5 /, 23 \mathrm{X}, 32 \mathrm{H} 9$ NUMBER OF ZERO ELEMENTS , 10X, I5/22X,33H10 NUMMPR 3750 7BER OF NEGATIVE ELEMENTS , 10X, I5//22X,33HII SUM OF TERMS MPR 3760 8 ,E15.6/22X,33H12 AVERAGE ,E15.6/22MPR3770 $9 X, 33 H 13$ SUM OF SQUARES ,E15.6/22X,33HI4 SUM OF SQUAMPR 3780 \$RES ABOUT MEAN ,E15.6/22X,33H15 WITHIN ROWS SUM OF SQUARES MPR 3790 $\$, E 15.6 / 22 X, 33 H 16$ WITHIN COLS SUM OF SQUARES ,E15.6) MPR3800

670 FORMAT (22X,33HI7 SUM OF ABSOLUTE VALUES

1 AVERAGE OF ABSOLUTE VALUES, E15.6)

, IPE15.6/22X,33H18MPR3810

MPR3820

$\begin{array}{lll}680 & \text { FORMAT } & (1 \mathrm{HO}, 29 \mathrm{X}, 8 \mathrm{HSPECIFIC} /) \\ 690 & \text { FORMAT } & (22 \mathrm{X}, 33 \mathrm{H} 19 \text { DETERMINANT }\end{array}$

1 RANK

$2 T$ OF SUM OF $B(I, J) * * 2$

,E15.1/22X,33H22 N*MAX (B (I ,J ))

MPR 3830

,IPE $15.6 / 22 X, 33 \mathrm{H} 18 \mathrm{MPR} 3840$ 3 ,E15.1/22X,33H23 MAX VAL OF ROW SUM

700 FORMAT ( $1 \mathrm{HO}, 21 \mathrm{X}, 32 \mathrm{H} 24$ NORMALITY

$1,1 \mathrm{H}) / 22 \mathrm{X}, 33 \mathrm{H} 25$ SYMMETRY

$22 X, 33$ H26 SKEW SYMMETRY

327 DIAGONALITY

4HOGONALITY: $\quad A, A=I$

5=DIAGONAL MATRIX, 7X,A3,2H*(, I I, IH) //)

$12 \mathrm{X}, 2 \mathrm{H}^{*}(\mathrm{I}, \mathrm{IH})(22 \mathrm{X}, 2 \mathrm{H} 29,17 \mathrm{X}, 19 \mathrm{HA}, \mathrm{AMPR} 3920$

MPR 3860

E 15.1$) \quad$ MPR 3870
$13 X, A 3,2 H^{*}(, 11 M P R 3880$ $, 12 X, A 3,2 H^{*}(, I 1, I H) / 2 M P R 3890$ $, 12 X, A 3,2 H^{*}(, I 1,1 H) / 22 X, 33 H M P R 3900$ $12 \mathrm{X}, \mathrm{A} 3,2 \mathrm{H}^{*}(, \mathrm{I} 1,1 \mathrm{H}) / 22 \mathrm{X}, 33 \mathrm{H} 28$ ORTMPR 3910

710 FORMAT $(22 \mathrm{X}, 13 \mathrm{H} 30$ TRIANGULAR, 20X,3A3, Al, 2A3,3H** $(, I 1,1 \mathrm{H}) / 22 \mathrm{X}, 33 \mathrm{H} 31$ MPR3940 ISTOCHASTIC (R AND/OR C SUMS $=1$ ) , 3X, 4A3, 4H***(,II, IH))

720 FORMAT (IH)

730 FORMAT $\left(9 X, 79 H^{*}\right.$ IF ANSWER IS YES, $(R, C)=1$ OR 2. (1, IF EXACT; 2, MPR3970 IIF TOLERANCE IS SATISFIED.)/ $11 X, 25 \mathrm{HIF}$ ANSWER IS NO, $(R, C)=0 . / / 8 \mathrm{X}, 1 \mathrm{MPR} 3980$ $20 H T R I A N G U L A R / 8 X, 69 H^{* *}(R, C)=0$, IF ANSWER IS NO; $(R, C)=1$, IF UPPER PMPR3990 3ART OF MATRIX IS ZERO;/ $11 X, 74 H(R, C)=2$, IF LOWER PART IS ZERO; ( $R, C M P R 4000$ $4)=3$, IF ALL OFF DIAGONAL ELEMENTS $=0 . / 17 \mathrm{X}, 10 \mathrm{HSTOCHASTIC} / 7 \mathrm{X}, 75 \mathrm{H}^{* * *} \mathrm{MPR} 4010$ $5(R, C)=0$, IF MATRIX IS NOT STOCHASTIC; $(R, C)=1$, IF SUM OF EACH ROWMPR 4020 $6=1 ; / 11 X, 75 H(R, C)=2$, IF SUM OF EACH COLUMN $=1 ;(R, C)=3$, IF SUM OFMPR 4030 7 EACH ROW AND COLUMN $=1$.)

MPR4040

740 FORMAT (IHO,39X, 14HPROPERTIES OF , I3,3H X I3,26H ARRAY STARTING LMPR 4050 IOCATION $(, I 3, I H, I 3, I H))$

750 FORMAT $(22 X, 32 \mathrm{H} 26$ ORTHOGONALITY: $A, A=I$ $9 X, 2 A 3,2 H^{*}(, I I, I H) / M P R 4070$

$122 \mathrm{X}, 2 \mathrm{H} 27,17 \mathrm{X}, 19 \mathrm{HA}, \mathrm{A}=\mathrm{DIAGONAL}$ MATRIX,3X2A3,2H*(,II, IH)) MPR 4080

760 FORMAT $\left(9 X, 86 H^{*}(R, C)=0\right.$, IF MATRIX IS NOT ORTHOGONAL; $(R, C)=1$ OR $2 M P R 4090$ I IF MATRIX IS ORTHOGONAL ROW WISE; $/ 11 X, 97 \mathrm{H}(\mathrm{R}, \mathrm{C})=3$ OR 4 , IF MATRIX MPR 4100 
2IS ORTHOGONAL COLUMN WISE. ( $(R, C)=I$, IF $I=1$ OR 3 ORTHOGONALITY ISMPR4IIO

3 EXACT; / 11X,5OHFOR I=2 OR 4 RELATIVE WITHIN ERROR BOUND OF .1E.6)/MPR 4L20 END

MPR 4130 
SUBROUTINE MRAISE

C VERSION 5.00 MRAISE 5/15/70

MRA 10

SUBROUTINE TO RAISE A MATRIX TO A POWER

GENERAL FORMS OF MRAISE

MRAISE $A() N,$,$N TO M POWER AND STORE IN C($,

$9 / 13 / 67$

MRA 20

MRA 30

$M$ MAY BE INTEGER OR REAL

IF $M=0 \quad C=I D E N T I T Y$ MATRIX

If $M=1 \quad C=A$

MRA 40

MRA 50

MRA 60

MRA 80

MRA 90

MRA 100

MRA 110

COMMON /BLOCRC/ NRC, RC(12600)

MRA 120

COMMON /BLOCKD/ I ARGS(100), KIND (100), ARGTAB (100), NRMAX, NROW, NCOL, NMRA 130

IARGS, VWXYZ (8), NERROR

DIMENSION ARGS $(100)$

EQUIVALENCE (ARGS(1), RC(12501))

COMMON /SCRAT/ NS,NS2,A(13500)

DOUBLE PRECISION $X$, SUM

DIMENSION $X(1)$

EQUIVALENCE $(X, A)$

C

C

CHECK NUMBER OF ARGUMENTS

*

IF (NARGS.NE.7) CALL ERROR (10)

MRA 140

MRA 150

MRA 160

MRA 170

MRA 180

MRA 190

MRA 200

MRA 210

MRA 220

MRA 230

MRA 240

$c$

C CHECK TO SEE IF ALL ARGUMENTS ARE INTEGER

MRA 250

C

$J=N A R G S$

CALL CKIND (J)

IF (J.EQ.0) GO TO 20

IF (KIND (NARGS-2).NE.0) GO TO 10

MRA 260

MRA 270

MRA 280

MRA 290

MRA 300

CALL ERROR (3)

MRA 310

GO TO 20

$10 \quad$ I ARGS (NARGS-2) =ARGS (NARGS-2)

C

C

CHECK TO SEE IF M (POWER) IS NEGATIVE

C *

20 IF (IARGS (NARGS-2).LT.0) CALL ERROR (3)

C

C CHECK TO SEE IF DIMENSIONS ARE CORRECT

C

C

IF (IARGS (3).NE.IARGS (4)) CALL ERROR (3)

MRA 320

MRA 330

MRA 340

MRA 350

MRA 360

MRA 370

MRA 380

MRA 390

MRA 400

MRA 410

MRA 430

MRA 440

CHECK TO SEE IF ARGUMENTS ARE OUT OF RANGE

MRA 450

MRA 460

$30 \quad$ NPOW $=$ I ARGS (NARGS -2) - I

$40 \operatorname{IARGS}(5)=$ I ARGS (NARGS-1)

MRA 470

MRA 500

$\operatorname{IARGS}(6)=\operatorname{IARGS}$ (NARGS)

$\operatorname{IARGS}(7)=\operatorname{IARGS}(3)$

$\operatorname{IARGS}(8)=\operatorname{IARGS}(4)$

$J=2$

CALL MTXCHK (J)

IF (J-1) $70,50,60$

50 CALL ERROR (3)

RETURN

MRA 510

MRA 520

MRA 530

MRA 540

MRA 550

MRA 560

MRA 570

MRA 580

MRA 590

MRA 600

RETURN

MRA 610

MRA 620

CHECK TO SEE IF PREVIOUS ERRORS

MRA 630 
MOVE ORIGINAL MATRIX TO SCRATCH AREA (COLUMNWISE)

MRA 680 *

MRA 690

MRA 700

IF (NPOW) $80,90,110$

MRA 710

MRA 720

$80 \quad$ IEXT $=1$

GO TO 100

MRA 730

$90 \quad$ IEXT $=2$

$100 \quad \operatorname{ISAV}=\operatorname{IARGS}(5)$

GO TO 120

$110 \quad$ IEXT $=3$

120 IP $=$ I ARGS (1)

I $C=1$

DO $220 \mathrm{~J}=1$, ISIZE

MRA 740

MRA 750

MRA 760

MRA 770

MRA 780

MRA 790

MRA 800

DO $180 \quad I=1$, ISIZE

MRA 810

GO TO $(130,160,170)$, IEXT

MRA 820

MRA 830

130 IF (I.EQ.J) GO TO 140

$R C($ I SAV $)=0.0$

GO TO 150

MRA 840

MRA 850

MRA 860

$140 \quad \mathrm{RC}($ ISAV $)=1.0$

$150 \quad$ I SAV $=I S A V+1$

GO TO 180

MRA 870

MRA 880

MRA 890

$160 \mathrm{RC}($ I SAV $)=\mathrm{RC}$ (IP)

$I P=I P+1$

GO TO 150

$170 \quad A(I C)=R C$ (IP)

I $C=I C+1$

$I P=I P+1$

180 CONTINUE

GO TO $(190,200,210)$, IEXT

MRA 900

MRA 910

MRA 920

MRA 930

MRA 940

MRA 950

MRA 960

MRA 970

MRA 980

$I S A V=I S A V+N R O W-I S I Z E$

GO TO 220

MRA 990

I SAV $=$ I SAV+NROW-IS IZE

MRA 1000

$I P=I P+N R O W-I S I Z E$

MRA 1010

CONTINUE

IF (IEXT.LE.2) RETURN

MRA 1020

$I X P=N S-I S I Z E * 2$

MRA 1030

DO $280 \mathrm{~K}=1$, NPOW

MRA 1040

ISAVP $=I$ ARGS (5)

MRA1050

$I M P=N S 2$

MRA1060

MRA1070

IF (K.GT.I) GO TO 230

MRA 1080

$I R P=I A R G S(1)$

MRA1090

GO TO 240

MRAI 100

MRAII10

$I R P=I$ ARGS (5)

240 DO $280 \quad I=1$, ISIZE

MRAI 120

I SAV $=$ I SAVP

MRAI130

I $C=1$

MRA1140

$I R=I R P$

MRAI 150

$I X=I X P$

C

$*$

SAVE ROW OF MATRIX

MRAI160

MRA1170

MRA1 180

MRA 1190

DO $250 \mathrm{~J}=1$, ISIZE

MRA1200

$A(I X)=R C(I R)$

MRA 1210

I $X=I X-1$

MRA1220 
IX $=$ IXP

MRA 1260

$I M=I M P$

DO $260 \mathrm{JP}=1$, ISIZE

MRA 1270

$X(I M)=A(I X) * A(I C)$

MRA 1280

$I M=I M-1$

MRA 1290

IX $=I X-1$

MRA 1300

MRA 1310

IC $=I C+1$

MRA 1320

CONTINUE

MRA 1330

CALL SORTSM (ISIZE, SUM)

MRA 1340

RC ( ISAV) $=$ SUM

MRA 1350

I SAV = I SAV + NROW

MRA 1360

MRA 1370

MRA 1380

I SAVP = I SAVP + I

MRA 1390

$I R P=I R P+1$

MRA 1400

CONTINUE

MRA 1410

RETURN

MRA1420 
COMMON /BLOCKD/IARGS(100), KIND(100), ARGTAB (100), NRMAX, NROW, NCOL, NMSC 40

IARGS, VWXYZ (8), NERROR

MSC 50

DIMENSION ARGS $(100)$

MSC 60

EQUIVALENCE (ARGS (1), RC (12501))

MSC 70

COMMON /BLOCKE/ NAME (4),L1,L2, ISRFLG

SUBROUTINE BY CARLA MESSINA 221.04

JUNE 1967

MSC 80

TYPE 1 IS

TYPE 2 IS

TYPE 3 IS

TYPE 4 IS

TYPE 5 IS
PARSUM OF COL ++ , STORE IN COL ++

PARPRODUCT OF COL ++ , STORE IN COL ++

ROOT MEAN SQUARE

RMS OF COL ++, STORE IN COL MSC 120

MSC 100

MSC 110

MSC 90 AVERAGE OF COL ++ , STORE IN COL ++ (DOWN TO NMSC 130 SUM COL ++, STORE IN COL ++ (DOWN TO NRMAX)

MSC 140 SUM COL ++ FROM ROW , TO ROW, STORE IN COL ++ MSC 150 SUM COL ++ FROM ROWS NUMBERED ,., ,., ETC STORE MSC 160

THE THREE TYPES OF SUM ARE IDENTIFIED BY THE NO. OF NARGS $=2,3$ ANDMSC 170 $E L E M=0.0$

MSC 180

IF (NARGS-2) $10,40,40$

MSC 190

$10 \quad K=10$

CALL ERROR (K)

RETURN

MSC 200

20

30

CALL ADRESS $(1, J 1)$

IF (JI) $50,50,60$

$50 \quad K=3$

GO TO 20

60 CALL ADRESS (NARGS, J2)

IF (J2) $50,50,70$

70 IF (NARGS-3) $210,80,80$

80 IF (L2-5) $10,90,10$

90 NARG $1=N A R G S-1$

DO $110 \quad I=2, N A R G 1$

IF (KIND(I).NE.0) GO TO 130

IF (IARGS (I)) $130,130,100$

100 IF (IARGS (I)-NROW) $110,110,130$

110 CONTINUE

IF (NERROR.NE.0) GO TO 30

IF (NARGS-4) $120,120,180$

C

C

C

SUM FROM ROW ,. TO ROW ,,

MSC 210

MSC 220

MSC 230

MSC 240

MSC 250

MSC 260

MSC 270

MSC 280

MSC 290

MSC 300

MSC 310

MSC 320

MSC 330

MSC 340

MSC 350

MSC 360

MSC 370

MSC 380

MSC 390

MSC 400

MSC 410

120 IF (IARGS (2)-IARGS (3)) $140,140,130$

$130 \quad I=I A R G S(2)$

$\operatorname{IARGS}(2)=\operatorname{IARGS}(3)$

$\operatorname{IARGS}(3)=\mathbb{I}$

140 IF (NRMAX) $150,150,160$

$150 \quad K=9$

GO TO 20

$160 \mathrm{~J}=\mathrm{J} 1+$ IARGS (2)

$E L E M=E L E M+R C(J-1)$

$\operatorname{IARGS}(2)=\operatorname{IARGS}(2)+1$

IF (IARGS (2)-IARGS (3)) $160,160,170$

MSC 420

MSC 430

MSC 440

MSC 450

MSC 460

MSC 470

MSC 480

MSC 490

MSC 500

MSC 510

MSC 520

CALL VECTOR (ELEM, J2)

170 CALL. VECTOF
GO TO 30

180 IF (NRMAX) $150,150,190$

MSC 530

MSC 540

MSC 550

MSC 560

SUM DISCRETE ROWS

MSC 570

MSC 580

MSC 590 


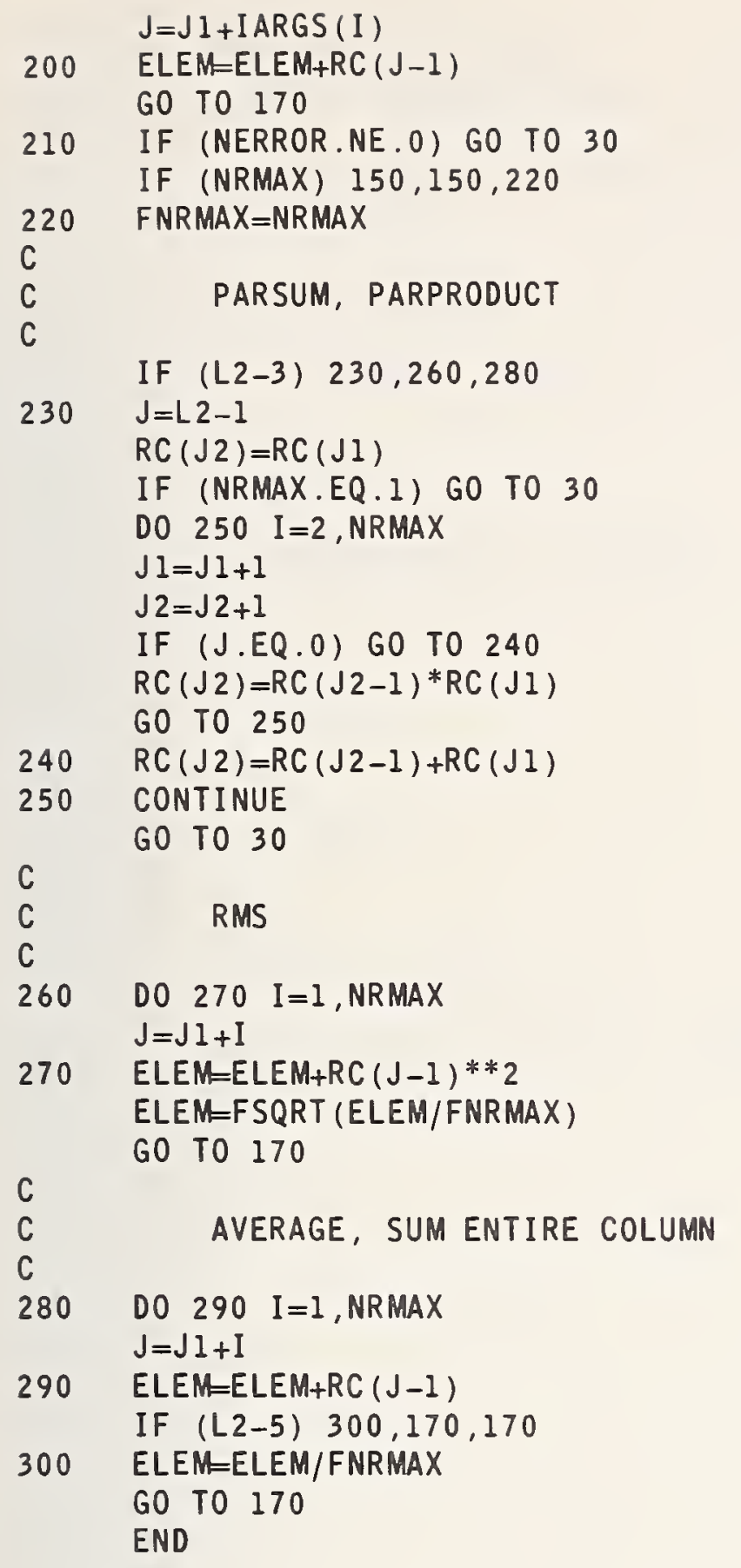

MSC 600

MSC 610

MSC 620

MSC 630

MSC 640

MSC 650

MSC 660

MSC 670

MSC 680

MSC 690

MSC 700

MSC 710

MSC 720

MSC 730

MSC 740

MSC 750

MSC 760

MSC 770

MSC 780

MSC 790

MSC 800

MSC 810

MSC 820

MSC 830

MSC 840

MSC 850

MSC 860

MSC 870

MSC 880

MSC 890

MSC 900

MSC 910

MSC 920

MSC 930

MSC 940

MSC 950

MSC 960

MSC 970

MSC 980

MSC 990 
TRIANGULARIZATION OF NON-SINGULAR, REAL SYMMETRIC MATRIX MTR 90

MTRIAN $A(,,++), R=,, C=$, STORE T IN $(,,++)$ AND T INVERSE $(,,++)$ MTR 100 THE UPPER TRIANGLE IS SET $=0$.

MTR 110

MTR 120

COMMON /SCRAT/ NS, NS2, A(13500)

MTR 130

COMMON /BLOCRC/ NRC, RC(12600)

MTR 140

COMMON /BLOCKD/ IARGS(100), KIND (100), ARGTAB (100), NRMAX, NROW, NCOL, NMTR 150

IARGS, VWXYZ (8), NERROR

MTR 160

DIMENSION ARGS $(100)$

MTR 170

EQUIVALENCE (ARGS(1), RC(12501))

MTR 180

DIMENSION $X(2)$

MTR 190

DOUBLE PRECISION $X$, SUM

MTR 200

EQUIVALENCE $(X, A)$

$K R R=7$

MTR 210

$\mathrm{KRRA}=7$

MTR 220

$\mathrm{KRRB}=7$

MTR 230

$K R R C=7$

MTR 240

$\mathrm{J}=2$

MTR 250

IF (NARGS.EQ.6.OR.NARGS.EQ.8) GO TO 10

MTR 260

CALL ERROR (10)

RETURN

10 IF (IARGS (3) .EQ.IARGS (4)) GO TO 20

CALL ERROR (KRR)

\section{RETURN}

20 IF (NARGS.EQ.6) GO TO 30

$\mathrm{J}=3$

$\operatorname{IARGS}(9)=\operatorname{IARGS}(7)$

$\operatorname{IARGS}(10)=\operatorname{IARGS}(8)$

$\operatorname{IARGS}(11)=\operatorname{IARGS}(3)$

MTR 270

MTR 280

MTR 290

MTR 300

MTR 310

MTR 320

MTR 330

MTR 340

MTR 350

MTR 360

$\operatorname{IARGS}(12)=\operatorname{IARGS}(4)$

MTR 370

MTR 380

$30 \operatorname{IARGS}(7)=\operatorname{IARGS}(3)$

MTR 390

$\operatorname{IARGS}(8)=\operatorname{IARGS}(4)$

MTR 400

CALL MTXCHK (J)

MTR 410

IF (J-1) $60,40,50$

MTR 420

40 CALL ERROR (3)

RETURN

MTR 430

MTR 440

50 CALL ERROR (17)

50 CALL ERROR (17)
RETURN

MTR 450

60 IF (NERROR.NE.0) RETURN

MTR 460

$I R=I$ ARGS ( 3 )

$I R M=I R-1$

MTR 470

MTR 480

$\mathrm{K}=\mathrm{I}$ ARGS $(1)$

MTR 490

MTR 500

D0 $70 \quad I=1, I R$

MTR 510

IF (RC(K).GT.0.0) GO TO 70

MTR 520

C *** ERRA - MATRIX CAN NOT BE TRIANLIZED SINCE ONE OF THE TERMS ON

MTR 530 THE DIAG. IS ZERO OR LESS.

CALL ERROR (KRRA)

MTR 540

MTR 550

MTR 560

RETURN

$70 \quad K=K+1+N R O W$

MTR 570

$K=I$ ARGS ( 1$)$

MTR 580

CALL SYMV (RC $(K), N R O W, I R, M)$

MTR 590 
IF (M.LE.1) GO TO 80

C $* * *$ NON-SYMMETRIC MATRIX

MTR 600

CALL ERROR (KRRC)

MTR 610

RETURN

$80 \quad M=2$

$A(1)=F S Q R T(R C(K))$

MTR 620

MTR 630

MTR 640

$\mathrm{K}=\mathrm{K}+1$

DO $90 \quad I=2, I R$

$A(M)=R C(K) / A(1)$

$\mathrm{K}=\mathrm{K}+1$

$90 \quad M=M+1$

$K A=I A R G S(1)$

$\mathrm{KB}=\mathrm{KA}+\mathrm{NROW}+1$

$M A=2$

DO $140 \quad I=2$, IR

$M B=M A$

$L=N S 2-1$

$X(N S 2)=R C(K B)$

$M=(I-I) * I R+I$

I I $=I-1$

DO $100 \mathrm{~J}=1$, I I

$X(L)=-(A(M B) * * 2)$

MTR 650

MTR 660

MTR 670

MTR 680

MTR 690

MTR 700

MTR 710

MTR 720

MTR 730

MTR 740

MTR 750

MTR 760

MTR 770

MTR 780

MTR 790

MTR 800

$\mathrm{L}=\mathrm{L}-1$

MTR 810

MTR 820

$100 M B=M B-I R$

CALL SORTSM (I, SUM)

MTR 830

MTR 840

IF (SUM.GT.0.0) GO TO 110

C *** ERRB-LEADING SUBMATRIX IS SINGULAR

MTR 850

MTR 860

CALL ERROR (KRRB)

RETURN

MTR 870

MTR 880

MTR 890

$S=S U M$

$S=F S Q R T(S)$

$A(M)=S$

MTR 900

MTR 910

$M=M+1$

IF (I.EQ.IR) GO TO 140

MTR 920

MTR 930

I $P=I+I$

MTR 940

$\mathrm{KC}=\mathrm{KB}+1$

MTR 950

DO $130 \mathrm{~J}=I \mathrm{P}, \mathrm{IR}$

MTR 960

$X(N S 2)=R C(K C)$

MTR 970

$\mathrm{KC}=\mathrm{KC}+1$

MTR 980

$L=N S 2-1$

MTR 990

$M C=J$

$M D=I$

MTR 1000

MTR 1010

DO $120 \mathrm{JJ}=1, I \mathrm{I}$

MTR 1020

$X(L)=-A(M C) * A(M D)$

MTR 1030

$M C=M C+I R$

MTR 1040

$M D=M D+I R$

MTR 1050

MTR 1060

CALL SORTSM (I, SUM)

MTR 1070

$A(M)=S U M / S$

MTR 1080

MTR 1090

$M=M+1$

$M A=M A+I R+1$

MTR 1100

MTR 1110

MTR 1120

MTR 1130

MTR 1140

MTR 1150

MTR 1160

MTR 1170

MTR 1180 
$R C(K A)=A(M)$

MTR 1190

$K A=K A+1$

$150 \quad M=M+1$

IF (I:EQ.I) GO TO 170

MTR1200

I I $=I-1$

DO $160 \mathrm{~J}=1, \mathrm{II}$

$R C(K C)=0.0$

$160 \quad K C=K C+1$

$170 \quad K B=K B+N R O W$

$180 K=K+N R O W$

MTR 1210

MTR 1220

MTR 1230

MTR 1240

MTR 1250

MTR 1260

MTR 1270

MTR 1280

IF (NARGS.EQ.6) RETURN

MTR 1290

$K C=I A R G S(5)$

DO $210 \quad I=1, I R$

MTR 1300

MTR 1310

$M=(I-I) * I R+I$

$A(M)=1.0 / R C(K C)$

IF (I.EQ.IR) GO TO 210

MTR 1320

MTR 1330

MTR 1340

$M=M+1$

$I P=I+I$

$K B=K C+N R O W+1$

$\mathrm{JC}=1$

DO $200 \mathrm{~J}=\mathrm{IP}, I R$

$K A=K C+J-I$

$M A=(I-1) * I R+I$

$\mathrm{L}=\mathrm{NS} 2$

DO $190 \mathrm{JA}=1, \mathrm{JC}$

$X(L)=R C(K A) * A(M A)$

$M A=M A+1$

$K A=K A+N R O W$

MTR1350

MTR 1360

MTR 1370

MTR 1380

MTR 1390

MTR 1400

MTR 1410

MTR 1420

MTR1430

MTR 1440

MTR 1450

MTR 1460

MTR 1470

$\mathrm{L}=\mathrm{L}-1$

CALL SORTSM (JC, SUM)

MTR1480

$S=S U M$

$A(M)=-S / R C(K B)$

$K B=K B+N R O W+1$

$M=M+1$

$200 \quad \mathrm{JC}=\mathrm{JC}+1$

$210 \quad K C=K C+N R O W+1$

$K=\operatorname{IARGS}(9)-1$

$K B=I$ ARGS ( 9 )

DO $250 \quad I=1$, IR

$K A=K+I$

$M=(I-I) * I R+I$

$K C=K B$

DO $220 \mathrm{~J}=\mathrm{I}, \mathrm{IR}$

MTR 1490

MTR 1500

MTR 1510

MTR 1520

MTR 1530

MTR 1540

MTR 1550

MTR 1560

MTR 1570

MTR 1580

MTR 1590

MTR1600

MTR 1610

$\mathrm{RC}(\mathrm{KA})=\mathrm{A}(\mathrm{M})$

MTR 1620

$K A=K A+1$

$220 \quad M=M+1$

MTR 1630

MTR 1640

IF (I.EQ.I) GO TO 240

MTR 1650

I I $=I-1$

DO $230 \mathrm{~J}=1$, I I

$R C(K C)=0$.

$230 \quad K C=K C+1$

$240 \quad K B=K B+N R O W$

MTR 1660

MTR 1670

MTR 1680

MTR 1690

MTR 1700

MTR 1710

$K=K+N R O W$

MTR 1720

RETURN

MTR 1730 


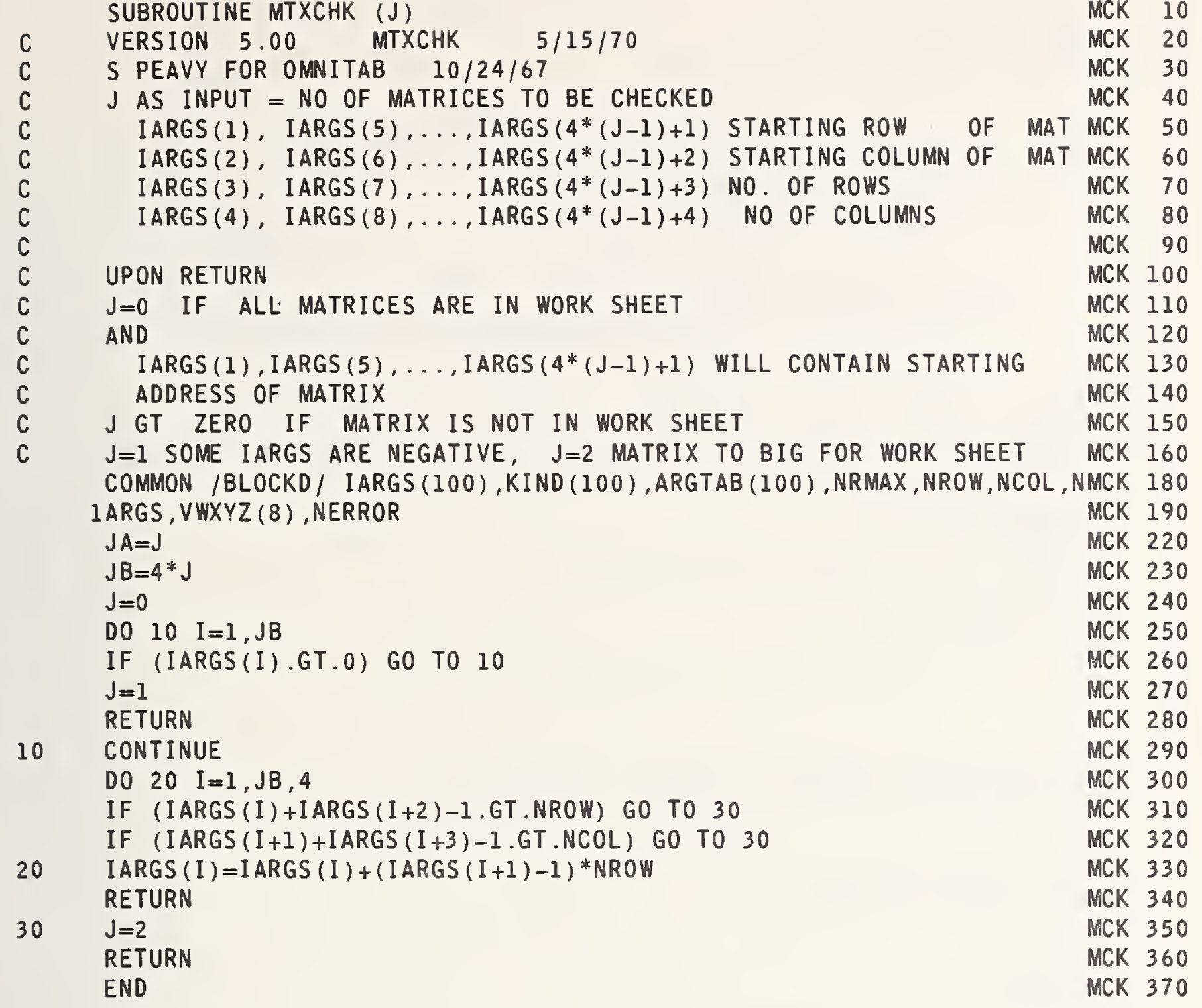



GENERAL FORM OF COMMAND

$$
M(X X T) \quad A(,) N, K, \text { STORE IN } C(,) \quad N, K \text { DEFINE } X
$$

MXT 60

MXY 70

MXT 80

$L 2=2$ MULTIPLY TRANSPOSE OF MATRIX BY ITSELF

MXT 90 GENERAL FORM OF COMMAD

$$
M(X T X) \quad A(,) N, K \text { STORE IN } C(,) \quad N, K \text { DEFINE } X
$$

MXT 110

MXT 120

MXT 130

MXT 150

COMMON /SCRAT/ NS, NS2, A(13500)

MXT 160

COMMON /BLOCRC/ NRC, RC (12600)

MXT 170

COMMON /BLOCKD/ IARGS (100), KIND(100), ARGTAB (100), NRMAX, NROW, NCOL, NMXT 180 IARGS, VWXYZ (8), NERROR

DIMENSION ARGS (100)

MXT 190

EQUIVALENCE (ARGS(1), RC (12501))

COMMON /BLOCKE/ NAME (4),L1,L2, ISRFLG

MXT 200

MXT 210

DOUBLE PRECISION AP $(3000)$

EQUIVALENCE (A, AP)

MXT 220

MXT 230

MXT 240

MXT 250

CHECK FOR CORRECT NUMBER OF AGRUMENTS

MXT 260

*

*

DECIDE WHETHER COMMAND IS $M\left(X A X^{\prime}\right)$ OR $M\left(X^{\prime} A X\right)$

MXT 270

MXT 280

$L 2=3$ MEANS $M\left(X A X^{\prime}\right) \quad L 2=2$ NARGS.GT. 6 MEANS $M\left(X^{\prime} A X\right)$

MXT 290

*

IF (L2-2) $30,10,20$

MXT 300

MXT 310

MXT 320

IF (NARGS.LE.6) GO TO 30

MXT 330

$\mathrm{L} 2=4-\mathrm{L} 2$

CALL TRANSF

RETURN

30 IF (NARGS.NE.6) CALL ERROR (10)

C

CHECK TO SEE IF ALL ARGUMENTS ARE INTEGERS

*

$J=N A R G S$

CALL CKIND (J)

IF (J.NE.O) CALL ERROR (3)

C CHECK TO SEE IF DIMENSIONS ARE OUT OF RANGE COMPUTE ADDRESSES

C

C

40 GO TO $(50,60)$, L2

$50 \operatorname{IARGS}(8)=\operatorname{IARGS}(3)$

$\operatorname{IARGS}(7)=\operatorname{IARGS}(3)$

GO TO 70

MXT 340

MXT 350

MXT 360

MXT 370

MXT 380

MXT 390

MXT 400

MXT 410

MXT 420

MXT 430

MXT 440

MXT 450

MXT 460

MXT 470

MXT 520

MXT 530

MXT 540

MXT 550

$60 \operatorname{IARGS}(8)=\operatorname{IARGS}(4)$

MXT 560

$\operatorname{IARGS}(7)=\operatorname{IARGS}(4)$

MXT 570

$70 \quad J=2$

CALL MTXCHK (J)

MXT 580

MXT 590

IF $(\mathrm{J}-1) 100,80,90$

80 CALL ERROR (3)

RETURN

MXT 600

MXT 610

MXT 620

90 CALL ERROR (17)

RETURN 
C CHECK FOR PREVIOUS ERRORS

MXT 660

C *

100 IF (NERROR.NE.0) RETURN

MXT 670

I $G=$ IARGS (I)

CALL MXTXP (RC (IG), NROW, IARGS (3), IARGS (4), A, L2, NS2, AP)

MXT 680

GO TO $(110,120)$, L2

$110 \quad N R O W P=I A R G S(3)$

GO TO 130

MXT 690

MXT 700

MXT 710

MXT 720

$120 \quad$ NROWP $=$ IARGS (4)

MXT 730

MXT 740

$I G=I$ ARGS (5)

MXT 750

CALL STORMT (RC (IG), NROW, NROWP, NCOLP, A)

MXT 760

MXT 770

C *

C MOVE FROM SCRATCH AREA TO STORAGE

MXT 780

MXT 790

MXT 800

RETURN

MXT 810

END

MXT 820 
SUBROUTINE MXTXP (X,N,NP, K, A, L2, NASIZE, XP)

MXP 10

$$
\text { VERSION } 5.00 \quad \text { MXTXP } 5 / 15 / 70
$$

$2 / 12 / 68$

MXP 20

SUBROUTINE MXTXP

R VARNER

MXP 30

$X$ IS MATLIX TO BE USED

MXP 40

$N$ IS DIMENSIONED SIZE OF A

NP IS NUMBER OF ROWS IN A

MXP 50

$K$ IS NUMBER OF COLUMNS IN A

MXP 60

MXP 70

$L 2=1$ MULTIPLY $X$ TIMES $X$ TRANSPOSED

MXP 80

$L 2=2$ MULTIPLY $X$ TRANSPOSED TIMES $X$

MXP 90

NASIZE IS SIZE OF A DIVIDED BY 2

MXP 100

A IS SCRATCH AREA WHERE MATRIX IS TO BE STORED

MXP 110

C

C

C *

DOUBLE PRECISION XP, SUM

DIMENSION $X(N, 1), A(1), X P(1)$

I $\mathrm{C}=1$

GO TO $(10,40)$, L2

C COMPUTE $X$ TIMES $X$ TRANSPOSED

C *

10 DO $30 \quad K K=1, N P$

DO $30 \mathrm{I}=1$, NP

IS=NASIZE

DO $20 \mathrm{~J}=1, \mathrm{~K}$

$X P(I S)=X(I, J) * X(K K, J)$

20 IS $=I S-1$

CALL SORTSM (K, SUM)

$A(I C)=S U M$

MXP 120

MXP 130

MXP 140

MXP 150

MXP 160

MXP 170

MXP 180

MXP 190

MXP 200

MXP 210

MXP 220

MXP 230

MXP 240

MXP 250

MXP 260

MXP 270

MXP 280

MXP 290

RETURN

MXP 300

MXP 310

MXP 320

MXP 330

MXP 340

MXP 350

MXP 360

MXP 370

MXP 380

MXP 390

MXP 400

MXP 410

MXP 420

MXP 430 
SUBROUTINE NNAME (NAME)

NNA 10

VERSION 5.00 NNAME $5 / 15 / 70$

NNA 20

COMMON /BLOCKA/ MODE,M,KARD (83), KARG, ARG, ARG2, NEWCD (80), KRDEND NNA 30

DIMENSION NAME (2), MISC (6)

NNA 40

C

THIS SUBROUTINE ASSEMBLES A NAME UP TO THE FIRST NON-LETTER OR UP NNA 60

SIX LETTER, WHICHEVER IS FIRST. THE INDEX, M, IS INITIALLY POINTINNNA 70

THE FIRST LETTER, IT IS LEFT POINTING AT THE FIRST NON-LETTER. NNA 80

SPACE OUT SO THAT TABLE LIES ALL ON ONE PAGE

NNA 90

C

C

C

C

C

C

$\mathrm{C}$

C

C

C

THE FIRST THREE CHARACTERS GO INTO THE FIRST WORD OF NAME

THE SECOND THREE CHARACTERS GO INTO THE SECOND WORD OF NAME

CONVERSION TABLE FOR ALPHABETIC TO NUMERIC AS USED BY OMNITAB.

$\begin{array}{rrrr}\text { A } & 729 & 27 & 1 \\ \text { B } & 1458 & 54 & 2 \\ \text { C } & 2187 & 81 & 3 \\ \text { D } & 2916 & 108 & 4 \\ \text { E } & 3645 & 135 & 5 \\ \text { F } & 4374 & 162 & 6 \\ \text { G } & 5103 & 189 & 7 \\ \text { H } & 5832 & 216 & 8 \\ \text { I } & 6561 & 243 & 9 \\ \text { J } & 7290 & 270 & 10 \\ \text { K } & 8019 & 297 & 11 \\ \text { L } & 8748 & 324 & 12 \\ \text { M } & 9477 & 351 & 13 \\ \text { N } & 10206 & 378 & 14 \\ \text { O } & 10935 & 405 & 15 \\ \text { P } & 11664 & 432 & 16 \\ \text { Q } & 12393 & 459 & 17 \\ \text { R } & 13122 & 486 & 18 \\ \text { S } & 13851 & 513 & 19 \\ \text { T } & 14580 & 540 & 20 \\ \text { U } & 15309 & 567 & 21 \\ \text { V } & 16038 & 594 & 22 \\ \text { W } & 16767 & 621 & 23 \\ \text { X } & 17496 & 648 & 24 \\ \text { Y } & 18225 & 675 & 25 \\ \text { Z } & 18954 & 702 & 26\end{array}$

NNA 100

NNA 110

NNA 120

NNA 130

NNA 140

NNA 150

NNA 160

NNA 170

NNA 180

NNA 190

NNA 200

NNA 210

NNA 220

NNA 230

NNA 240

NNA 250

NNA 260

NNA 270

NNA 280

NNA 290

NNA 300

NNA 310

NNA 320

NNA 330

NNA 340

NNA 350

NNA 360

NNA 370

NNA 380

NNA 390

NNA 400

NNA 410

NNA 420

NNA 430

NNA 440

NNA 450

NNA 460

NNA 470

NNA 480

NNA 490

NNA 500

NNA 510

NNA 520

NNA 530

NNA 540

NNA $\mathbf{5 5 0}$

NNA 560

NNA 570

NNA 580

NNA 590

NNA 600 
FUNCTION NONBLA (I)

C VERSION 5.00 NONBLA $5 / 15 / 70$

NON 10

$\mathrm{C}$

SCAN KARD STARTING AT KARD(I) UNTIL A NON-BLANK CHARACTER IS

NON 20

FOUND. POINT M AT IT AND ALSO RETURN IT AS FUNCTION VALUE.

NON 30

COMMON /BLOCKA/ MODE,M,KARD (83), KARG, ARG, ARG2, NEWCD (80), KRDEND

NON 40

$M=I$

IF (KARD(M).NE.44) GO TO 20

NON 50

NON 60

NON 70

NON 80

$M=M+1$

GO TO 10

20

NONBLA $=$ KARD (M)

RETURN

NON 90

NON 100

NON 110

END

NON 120

NON 130

SUBROUTINE NOTEPR (J)

C VERSION 5.00 NOTEPR $5 / 15 / 70$

NOT 10

WRITTEN BY STP $4 / 21 / 70$

NOT 20

NOT 30

NOT 40

IF $\mathrm{J}=0$ BLANK OUT NOTE VARIABLE

NOT 50

IF J=1 STORE IN NOTE(1) THRU NOTE(60) FROM NEWCD (M-2) 60 CHAR NOT 60

IF $J=2$ STORE IN NOTE(61) THRU NOTE(120) FROM NEWCD (M-2) 60 CHAR NOT 70

IF $J=3$ PRINT OUT NOTE(1) THRU NOTE(120)

NOT 80

COMMON / BLOCKA / MODE , M, KARD (83), KARG, ARG , ARG2, NEWCD (80), KRDEND

NOT 90

COMMON / HEADER / NOCARD $(80)$, ITLE $(60,6)$, LNCNT , IPR INT , NPAGE , I PUNCH

NOT 100

COMMON / NOTE / NOTE (120)

DATA IBLANK/IH /

NOT 110

NOT 120

NOT 130

IF (J.NE.O) GO TO 20

NOT 140

DO $10 \quad I=1,120$

10 NOTE (I ) =IBLANK

\section{RETURN}

20 IF (J.NE.3) GO TO 40

IF (NPAGE.EQ.0) CALL PAGE(0)

WRITE (IPRINT, 30) (NOTE (I), I=2,120)

RETURN

NOT 150

NOT 160

NOT 170

NOT 180

NOT 190

NOT 200

NOT 210

FORMAT ( IX, 119AI)

NOT 220

30 FORMAT

NOT 230

$M=M+1$

IF (MA.GT.82) $\quad M A=82$

NOT 240

NOT 250

$M B=(J-1) * 60+1$

$M C=M B+59$

NOT 260

IF (J.NE.1.AND.J.NE.2) RETURN

NOT 270

DO $100 \quad I=M B, M C$

100 NOTE (I ) = IBLANK

$\mathrm{I}=\mathrm{MB}$

DO 110 IC $=M$, MA

$\operatorname{NOTE}(I)=\operatorname{NEWCD}(I C-2)$

$110 \quad \mathrm{I}=\mathrm{I}+1$

RETURN

NOT 280

NOT 290

NOT 300

NOT 310

NOT 320

NOT 330

NOT 340

NOT 350

END

NOT 360 
SUBROUTINE OANOVA (YSUM, SU,ND9,FM,M,N,ND7,SSQ, IHC,NSU,B) OAN 10

$\begin{array}{llll}C & \text { VERSION } 5.00 & \text { OANOVA } 5 / 15 / 70 & \text { OAN } 20\end{array}$

C COMPUTE AND PRINT ANALYSIS OF VARIANCE $\quad$ OAN 30

C WRITTEN BY DAVID HOGBEN, SEL, NBS. 10/09/69.

C ***** $\quad$ OAN 50

COMMON/BLOCKD/IARGS (100), KIND (100), ARGTAB (100), NRMAX,NROW,NCOL, OAN 60

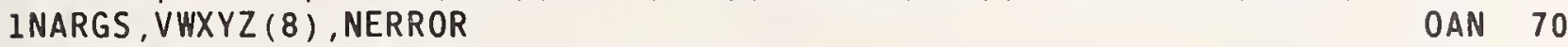

COMMON/BLOCKE / NAME (4),L1,L2, ISRFLG $\quad$ OAN 90

COMMON/HEADER/NOCARD $(80)$, I TLE $(60,6), \operatorname{LNCNT}, \operatorname{IPRINT}$, NPAGE, IPUNCH OAN 100

COMMON/SCRAT/NS, NS2, A $(13500) \quad$ OAN 110

DIMENSION B(1),IHC(1) $\quad$ OAN 160

DOUBLE PRECISION YSUM $\quad$ OAN 170

$\begin{array}{ll}C & * * * * * \\ 1850 * \text { OAN } 180\end{array}$

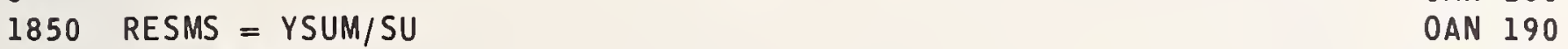

$\begin{array}{ll}\text { NSUA }=\text { NSU } & \text { OAN } 200\end{array}$

$\begin{array}{ll}\mathrm{IT}=1 & \text { OAN } 210\end{array}$

IF (L2.EQ.3) IT=3 $\quad$ OAN 220

WRITE (IPRINT, 1860) IHC (IT), IHC (IT+1) OAN 230

1860 FORMAT (////50X,20HANALYSIS OF VARIANCE/24X,73H-DEPENDENT ON ORDEROAN 240

1 VARIABLES ARE ENTERED, UNLESS VECTORS ARE ORTHOGONAL-// OAN 250

$21 X, 2 A 3,4 X, \quad 21 \mathrm{H}$ SS=RED. DUE TO COEF.,21H CUM. MS REDUCTIOOAN 260

$3 N, 6 H$ D.F.,2IH CUM. RESIDUAL MS ,6H D.F.,1IH F (COEF $=0), 6 H$ POAN 270

$4(F), 11 H \quad F(C O E F S=0), 6 H \quad P(F) /)$

IND $9=N D 9+M$

ASUM $=0.0$

$V R=S U-F M$

RESSS $=V R * S S Q$

IND7 $=$ ND7+M

$A(I N D 7)=$ RESSS

IF (M.EQ.1) GO TO 1866

DO 1865 I $1=2, M$

IND7 = IND7-1

$A($ IND7 $)=A($ IND7+1) $+A($ IND9 $)$

OAN 280

OAN 290

OAN 300

OAN 340

OAN 350

OAN 400

OAN 410

OAN 415

OAN 420

OAN 430

OAN 440

1865 IND9 = IND9-1

1866 VIF2 $=F M+1.0$

$B(1)=A($ IND9-1)

$B(2)=A($ IND9-2)

$A($ IND9-1) $=$ YSUM

$A($ IND9-2) $=$ RESSS

CALL RFORMT (A(IND9-2), $M+2,8, N$ HI, NDEC $1,18, A(1), A(1), 0,0)$

$A$ (IND9-1) $=B(1)$

$A($ IND9-2) $=B(2)$

CALL RFORMT (A(IND9), $M, 8, N W 2, N D E C 2,18, A(1), A(1), 0,0$ )

$S S U=S U$

DO $1867 I=1, M$

$S S U=S S U-1.0$

$B(I)=A($ IND7) $/ S S U$

OAN 450

OAN 460

OAN 470

OAN 480

OAN 482

OAN 484

OAN 490

OAN 492

OAN 494

OAN 496

OAN 498

OAN 500

OAN 505

OAN 510

OAN 515

OAN 520

IND7 $=$ IND7 $-M$

CALL RFORMT $(B(1), M, 8, N W 3, N D E C 3,18, A(1), A(1), 0,0)$

OAN 525

$S S U=S U$

DO $1950 \quad I=1, M$

OAN 530

OAN 540

NSUA $=$ NSUA-1

$A S U M=A S U M+A($ IND9)

$S S U=S S U-1.0$

OAN 550

OAN 560

$C R=A S U M / F L O A T(I)$

IF (ABS(SSU).GT.0.0) GO TO 1880

OAN 570

OAN 580

RESMS $=0.0$

OAN 590

OAN 600

$F 1=0.0$

OAN 610 
$F 2=0.0$

OAN 620

$\mathrm{PFI}=1.0$

OAN 630

$P F 2=1.0$

OAN 640

GO TO 1890

OAN 650

1880 RESMS $=A($ IND7 $) / S S U$

OAN 660

VIF $2=V I F 2-1.0$

IF (ABS (RESMS).LE.0.0) GO TO 1870

OAN 670

NEVER POOL

$F I=A($ IND9) $/ S S Q$

CALL PROB ( 1 ., VR,F $1, P F 1$ )

OAN 680

C

TEST HIGHER SUB-HYPOTHESES

$F 2=((A(I N D 7)+A($ IND9) - RESSS) $/$ VIF2) /SSQ

OAN 690

OAN 700

C

CALL PROB (VIF2, VR, F2,PF2)

$1890 \quad I I=I A B S(I-1)$

CALL RFORMT (A(1),1,8,NW1,NDEC1, 0,A(IND9),B( 1),21-NW1, 1)

OAN 710

CALL RFORMT ( $A(1), 1,8, N W 2, N D E C 2,0, C R \quad, B(22), 21-N W 2,1)$

OAN 720

OAN 730

OAN 740

OAN 750

CALL RFORMT (A(1),1,8,NW3,NDEC3,0, RESMS ,B (43),21-NW3,1)

OAN 760

IF (L2.EQ.1) GO TO 1920

OAN 770

\begin{tabular}{l} 
OAN 790 \\
\hline
\end{tabular}

WR ITE (IPRINT, 1900) IARGS (I+3), (B (I I), I I=1,42), I, (B (I 2), I 2=43,63), OAN 800 INSUA, F1, PF1, F2, PF2

1900 FORMAT (IX,I4,6X,42AI, I6,21A1, I6,2(OPF11.3,F6.3))

GO TO 1940

OAN 810

OAN 820

OAN 830

1920 WRITE (IPRINT, 1900) II , ( $($ ( I 1$), I 1=1,42), I,(B(I 2), I 2=43,63$ ),

OAN 840

INSUA, F1,PF $1, F 2$, PF 2

1940 IND7 $=$ IND7+1

1950 IND9 $=$ IND9+1

1951 FORMAT (/,1X, 1OHRESIDUAL ,21A1,21X, I6)

1952 FORMAT (1X, IOHTOTAL ,21A1,21X, I6)

$\mathrm{FI}=$ RESSS

CALL RFORMT (A(1), 1,8,NW1,NDEC $1,0, F 1$

WR ITE (IPRINT, 1951) (B(I), I=1,21), NSUA

$B(1), 21-N W 1,1)$

OAN 850

OAN 860

OAN 870

OAN 880

OAN 890

OAN 900

OAN 910

OAN 920

$F 2=$ YSUM

CALL RFORMT ( $A(1), 1,8, N W 1, N D E C 1,0, F 2, B(1), 21-N W 1,1)$

WR ITE (IPRINT, 1952) (B(I), I=1,21),NSU

RETURN

END

OAN 930

OAN 940

OAN 950

OAN 960

OAN 970 
SUBROUTINE OCOEFF (MI,N,ND18,ND17, IND19S, IND18S, IHC,B, IND7S, NSU, SSOCO 10 1, SSOLD, YSUM)

C VERSION 5.00 OCOEFF $5 / 15 / 70$

C WRITTEN BY DAVID HOGBEN, SEL, NBS. $10 / 14 / 69$.

C

COMMON /BLOCKD / I ARGS (100), KIND (100), ARGTAB (100), NRMAX,NROW,NCOL , INARGS, VWXYZ ( 8 ) , NERROR

COMMON / BLOCKE / NAME (4), L 1, L2, ISRFLG

COMMON/HEADER/NOCARD $(80)$, I TLE $(60,6)$, LNCNT, IPR INT , NPAGE , IPUNCH

COMMON/SCRAT/NS, NS2, A (13500)

DIMENSION B(1), IHC (1)

DOUBLE PRECISION YSUM

C

IT $=1$

IF (L2.EQ.3) IT=3

OCO 20

OCO 30

OCO 40

OCO 50

OCO 60

OCO 70

OCO 90

OCO 100

OCO 110

OCO 160

OCO 165

OCO 170

OCO 180

OCO 190

$M=M 1+1$

WRITE (IPRINT, 1960) IHC (IT), IHC (IT+1), IHC (IT), IHC (IT+1)

OCO 200

OCO 210

1960 FORMAT (////20X,32HESTIMATES FROM LEAST SQUARES FIT,38X, 18HFIT OMIOCO 220

1TTING LAST ,2A3//1X,2A3,5X,11HCOEFFICIENT, 8X,14HS.D. OF COEFF . 4X, OCO 230 25HRATI0,3X,12H*ACC. DIGITS,9X, 11HCOEFFICIENT, 7X, 14HS.D. OF COEFF. OCO 240 $35 X, 5$ HRATIO/)

INDI $8=N+N D 18+1$

$0 \mathrm{CO} 250$

IND $17=$ NDI7+1

OCO 260

INDI $1=$ INDI9S+1

OCO 270

IND $19=$ IND $185+1$

OCO 280

IND7 $=$ IND7S +1

OCO 290

CALL RFORMT (A(IND19), M1+1,8,NW1,NDEC1,18,A(1),A(1),0,0)

OCO 300

CALL RFORMT (A(INDI), $M 1+1,8, N W 2, N D E C 2,18, A(1), A(1), 0,0$ )

OCO 310

IF (M1.EQ.0) GO TO 2005

CALL RFORMT (A(IND18), M1 , 8, NW3, NDEC3,18,A(1),A(1),0,0)

OCO 320

OCO 330

CALL RFORMT (A(INDI7),M1 , $8, N W 4, N D E C 4,18, A(1), A(1), 0,0$ )

0 CO 340

DO $2000 \mathrm{~J}=1, M 1$

IF $(A($ INDI)) $1982,1981,1982$

OCO 350

OCO 360

OCO 370

$1981 \mathrm{Fl}=0.0$

GO TO 1983

OCO 380

OCO 390

$1982 F 1=A($ INDI9) $/ A(I N D I)$

1983 IF (A(IND17)) 1985,1984,1985

OCO 400

OCO 410

$F 2=0.0$

GO TO 1986

$1985 \mathrm{~F} 2=A($ IND18)/A(IND17)

1986 CALL RFORMT (A(1), 1,8,NW1,NDEC1,0,A(IND19), B (1),20-NW1, 1)

OCO 420

OCO 430

OCO 440

CALL RFORMT (A (1), 1,8,NW2, NDEC2,0,A(IND1),B(21),20-NW2,1)

OCO 450

$0 \mathrm{CO} 460$

CALL RFORMT (A (1), 1,8,NW3,NDEC3,0,A (IND 18), B (41), 20-NW3,1)

OCO 470

CALL RFORMT (A (1), 1,8,NW4, NDEC4,0,A (IND17), B (61), 20-NW4, 1)

OCO 480

$J J=L 2 / 3$

$\mathrm{J} J=\operatorname{IABS}(\mathrm{J}-1) *(1-\mathrm{J} J)+\operatorname{IARGS}(\mathrm{J}+3) * \mathrm{~J} J$

OCO 490

OCO 500

WRITE (IPRINT, 1990) JJ,(B(I I), I I=1,40), FI, A(IND7), (B(I2), I $2=41,80) 0 C 0510$

$1, \mathrm{~F} 2$

1990 FORMAT (IX, I $4,2 X, 40 A 1,0 P F 7.2,6 X, F 5.2,8 X, 40 A 1, F 7.2)$

$0 \mathrm{CO} 520$

$0 \mathrm{CO} 530$

IND7 $=$ IND7 +1

INDI9 $=$ INDI9+1

$0 \mathrm{CO} 540$

INDI $17=$ IND $17+1$

OCO 550

INDI $=I N D I+1$

$0 \mathrm{CO} 560$

OCO 570

OCO 580

2000 INDI8 $=$ INDI8+1

2005 CALL RFORMT ( $A(1), 1,8, N W 1, N D E C 1,0, A(I N D 19), B(1), 20-N W 1,1$ )

OCO 590

CALL RFORMT ( $A(1), 1,8, N W 2, N D E C 2,0, A$ (IND1), B (21),20-NW2,1)

$F I=A($ INDI9) $/ A($ INDI $)$

NSUA $=$ NSU-M

OCO 600

OCO 610

OCO 620

$N R M I=N S U-M I$

OCO 630 


\begin{tabular}{|c|c|c|c|}
\hline & & & \\
\hline & $J J=I A B S(M I) *(I-J J)+I A R G S(M I+4) * J J$ & $\mathrm{Cl}$ & \\
\hline & WRITE (IPRINT, 1990) JJ, (B(I), I=1,40), FI, A(IND7) & $\mathrm{OCO}$ & 660 \\
\hline & CALL RFORMT (SSOLD 1,8, NW2, NDEC $2,18, A(1), A(1), 0,0)$ & $\mathrm{OCO}$ & 670 \\
\hline & $B(52)=S S$ & $\mathrm{OCO}$ & 672 \\
\hline C & ADJUST FOR $M l=0$ & $\mathrm{OCO}$ & 674 \\
\hline & IF (MI.NE.0) GO TO 2009 & $0 \mathrm{CO}$ & 676 \\
\hline & $B(52)=$ YSUM/FLOAT (N) & $\mathrm{CO}$ & 678 \\
\hline & $B(52)=F S Q R T(B(52))$ & $\mathrm{CO}$ & 680 \\
\hline 2009 & CALL RFORMT $(B(52), 1,8, N H 4, N D E C 4,18, A(1), A(1), 0,0)$ & $0 \mathrm{CO}$ & 682 \\
\hline & CALL RFORMT (A (1), 1,8,NW2,NDEC2,0,SSOLD,B $(1), 20-N W 2,0)$ & $\mathrm{OCO}$ & 690 \\
\hline & CALL RFORMT (A(1), 1,8,NW4,NDEC 4,0,B(52),B(21),20-NW4,0) & $0 \mathrm{CO}$ & 700 \\
\hline & WRITE (IPRINT, 2010) (B(I), I=1,40), NSU, M, NSUA, NSU, MI, NRMI & $0 \mathrm{CO}$ & 710 \\
\hline 10 & FORMAT $(/ 1 \mathrm{X}, 3$ OHRESIDUAL STANDARD DEVIATION $=, 3 \mathrm{X}, 20 \mathrm{Al}, 26 \mathrm{X}$, & $0 \mathrm{CO}$ & 720 \\
\hline & 128 HBASED ON DEGREES OF FREEDOM , 9X, I $4,1 \mathrm{H}-, \mathrm{I} 2,3 \mathrm{H}=, \mathrm{I} 3,33 \mathrm{X}, \mathrm{I} 4,1 \mathrm{H}-$, & OCO & 730 \\
\hline & $2 \mathrm{I} 2,3 \mathrm{H}=, \mathrm{I} 3 / / 120 \mathrm{H} *$ THE NUMBER OF CORRECTLY COMPUTED DIGITS IN EAC & $0 \mathrm{OCO}$ & 740 \\
\hline & 3H COEFFICIENT USUALLY DIFFERS BY LESS THAN I FROM THE NUMBER GIVEN & & 750 \\
\hline & 4 HERE) & $\mathrm{OCO}$ & 760 \\
\hline & RETURN & $\mathrm{OCO}$ & 770 \\
\hline & END & $0 \mathrm{CO}$ & 780 \\
\hline
\end{tabular}


SUBROUTINE OCOVAR (M,ND7,MDI,IHC,B,IHT) $\quad$ OCV 10

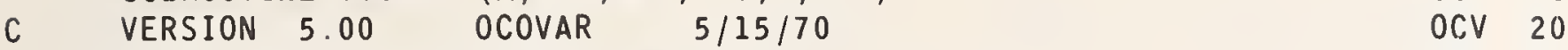

C PRINT VARIANCE-COVARIANCE MATRIX $\quad$ OCV 30

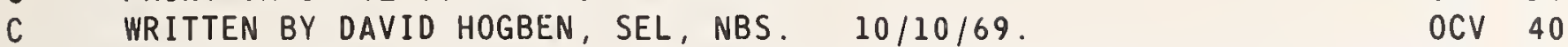

C $* * * * * \quad$ OCV 50

COMMON/BLOCKD / I ARGS (100), KIND (100), ARGTAB (100), NRMAX,NROW, NCOL, OCV 60

INARGS, VWXYZ(8), NERROR $\quad$ OCV 70

COMMON/BLOCKE / NAME (4),L1,L2, ISRFLG $\quad$ OCV 80

COMMON/HEADER/NOCARD $(80), \operatorname{ITLE}(60,6), \operatorname{LNCNT}$, IPR INT, NPAGE, IPUNCH OCV 90

COMMON/SCRAT/NS, NS2, A (13500) $\quad$ OCV 100

DIMENSION IHC (1),B(1),IHT(1) $\quad$ OCV 110

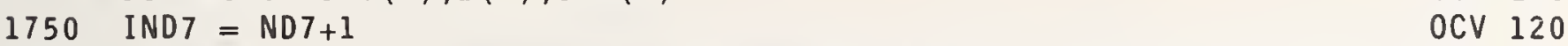

1791 FORMAT (////31X,56HVARIANCE-COVARIANCE MATRIX OF THE ESTIMATED COEOCV 130 IFFICIENTS)

1792 FORMAT $(/ 1 X, 2 A 3,1 X, 7(6 X, I 5,4 X))$

1793 FORMAT (IX,I4,3X,106A1)

WRITE (IPRINT, 1791)

CALL RFORMT (A (IND7), MD 1,8,NW1,NDECl, 13,A(1),A(1),0,0)

IF (L2.EQ.1) I $6=-1$

IF (L2.EQ.3) I $6=3$

C IIEND = NUMBER OF BLOCKS OF PRINTING

I IEND $=(M+6) / 7$

DO 1820 I $1=1$, I IEND

I $3 B E G=7 *(I 1-1)+1$

$\mathrm{I} 2 \mathrm{BEG}=\mathrm{I} 3 \mathrm{BEG}+\mathrm{I} 6$

$I 2 E N D=M I N O \quad(M+I 6, I 2 B E G+6)$

IF (L2-2) 1811,1811,1813

1811 I $7 E N D=I 2 E N D+1-I 2 B E G$

DO $1812 \quad I 7=1, I 7 E N D$

1812 IHT (I7) $=$ I $2 B E G-1+I 7$

WRITE (IPRINT, 1792) IHC (L2), IHC (L2+1), (IHT (I 7), I 7=1, I 7END)

GO TO 1814

OCV 140

OCV 150

OCV 160

OCV 180

OCV 190

OCV 200

OCV 210

OCV 220

OCV 230

OCV 240

OCV 250

OCV 260

OCV 270

OCV 280

OCV 290

OCV 300

OCV 310

OCV 320

OCV 330

1813 WRITE (IPRINT, 1792) IHC(L2), IHC(L2+1), (IARGS(I2), I 2=I2BEG, I 2END) OCV 340

1814 WRITE (IPRINT, 1793)

OCV 350

$\mathrm{LOCl}=\mathrm{IND} 7+(\mathrm{I} 3 \mathrm{BEG} *(\mathrm{I} 3 \mathrm{BEG}+1)) / 2-1$

C I3 IS FOR LOOP ON ROWS

DO 1820 I $3=I 3 B E G, M$

$I 4 E N D=M I N O(I 3, I 3 B E G+6)+1-I 3 B E G$

C I IS FOR LOOP ON COLUMNS

DO 1815 I4 $=1$, I4END

OCV 360

OCV 370

OCV 380

OCV 390

OCV 400

OCV 410

CALL RFORMT (A (1), 1,8,NW1, NDEC1,0,A(LOC1),B(15*I4-14),15-NW1,0) OCV 420

$1815 \quad \mathrm{LOCl}=\mathrm{LOC} 1+1$

I5END $=15 *$ I 4END

IF $(L 2 . E Q .1)$ I $8=13-1$

IF (L2.EQ.3) I $8=\operatorname{IARGS}(13+3)$

WRITE (IPRINT, 1793) I8, (B (I5), I $5=1$, I 5 END)

1820 LOCI $=$ LOCI-I $4 E N D+I 3$

RETURN

END

OCV 430

OCV 440

OCV 450

OCV 460

OCV 470

OCV 480

OCV 490

OCV 500 


\begin{tabular}{|c|c|c|}
\hline & SUBROUTINE OMCONV (NWCD, KRD, KRDEND) & OMC \\
\hline C & VERSION $5.00 \quad$ OMCONV & OMC \\
\hline & COMMON /ABCDEF/ L (48) & OMC \\
\hline$C$ & & OMC \\
\hline C & ARRAY L CONTAINS THE ALPHABET FORMATTED & OMC \\
\hline C & & OMC \\
\hline $\mathrm{C}$ & THIS ROUTINE CONVERTS INPUT CARD IMAGES TO A STANDARD CODE SO & OMC \\
\hline C & THAT OMNITAB CAN DEAL WITH THE CHARACTERS AS INTEGERS. & OMC \\
\hline C & & OMC \\
\hline C & & OMC 100 \\
\hline C & THIS ROUTINE IS INCLUDED ONLY FOR COMPLETENESS. IT SHOULD BE & OMC 110 \\
\hline C & REWRITTEN IN ASSEMBLY LANGUAGE FOR EACH COMPUTER. ALSO, IT & OMC 120 \\
\hline C & CANNOT MEET ASA STANDARDS BECAUSE ASA DOES NOT REQUIRE THAT DATA & OMC 130 \\
\hline $\mathrm{C}$ & READ WITH FORMAT AI BE STORED THE SAME AS HOLLERITH DATA SETUP & OMC 140 \\
\hline C & WITH IH ALTHOUGH THEY WILL BE THE SAME ON MOST COMPUTERS. & OMC 150 \\
\hline C & & OMC 160 \\
\hline C & ALSO, ASA DOES NOT RECOGNIZE THE CHARACTER ' APOSTROPHE & OMC 170 \\
\hline C & & OMC 210 \\
\hline & DIMENSION NWCD(1), KRD(1) & OMC 220 \\
\hline & DO $30 \mathrm{I}=1, \mathrm{KRDEND}$ & OMC 230 \\
\hline & $K=N W C D(I)$ & OMC 240 \\
\hline C & SPECIAL CASE TO CHECK FOR BLANKS & OMC 250 \\
\hline & IF (K.NE.L(45)) GO TO 10 & OMC 260 \\
\hline & $J=45$ & OMC 270 \\
\hline & GO TO 30 & OMC 280 \\
\hline$C$ & & OMC 290 \\
\hline C & THE UPPER BOUND OR LIMIT ON J MUST BE CHANGED IF MORE CHARACTERS & OMC 300 \\
\hline $\mathrm{C}$ & ARE ADDED TO THE VECTOR L IN LABEL COMMON ABCDEF & OMC 310 \\
\hline & & OMC 320 \\
\hline 10 & $0020 \mathrm{~J}=1,48$ & OMC 330 \\
\hline & IF (K.EQ.L (J)) GO TO 30 & OMC 340 \\
\hline 20 & CONT INUE & OMC 350 \\
\hline & $J=47$ & OMC 360 \\
\hline 30 & $\operatorname{KRD}(I)=J-1$ & OMC 370 \\
\hline & RETURN & OMC 380 \\
\hline & END & OMC 410 \\
\hline
\end{tabular}


COMMON/BLOCKA/ MODE,M,KARD (83), KARG, ARG,ARG2 NEWD

OMN 20

COMMON /BLOCKB/ NSTMT, NSTMTX, NSTMTH,NCOM, LCOM, IOVFL,COM(2000) OMN 50

COMMON /BLOCKC/ KIO, INUNIT, ISCRAT, KBDOUT, KRDKNT, LLIST $\quad 0 M N 60$

COMMON /BLOCRC/ NRC,RC(12600)

COMMON /BLOCKD/ IARGS(100), KIND(100), ARGTAB(100), NRMAX,NROW, NCOL, NOMN 80

IARGS, VWXYZ (8), NERROR

OMN 90

DIMENSION ARGS (100)

OMN 100

EQUIVALENCE (ARGS(1), RC (12501))

COMMON /BLOCKE/ NAME (4),L1,L2, ISRFLG

COMMON/HEADER/NOCARD $(80)$, ITLE $(60,6)$, LNCNT , IPRINT, NPAGE , IPUNCH

OMN 110

THE FOLLOWING CARDS ARE NEDDED ONLY FOR TAPE OPERATIONS

OMN 120 COMMON /TAPE/ NAME4 (2), NTPCT, IPUNCP, INUNIP, LITP

OMN 130

OMN 150

$* * * * * * * * * * * * * * * * * * * * * * * * * * * * *$

DATA IBLANK/IH /,LETSGO/-1/

OMN 160

THIS IS THE MAIN OMNITAB PROGRAM

OMN 180

OMN 190

SUBROUTINES CALLED BY THIS PROGRAM. .

OMN 200

OMN 210

OMN 220

SETUP, INPUT, ERROR, STMT , NNAME, AARGS, ASTER, SETQ, READQ, STORE, XECUTE AERR , XOMNIT , XFORMT , LOOKUP

OMN 230

OMN 240

OMN 250

OMN 260

\section{$M O D=1$ INTERPRETIVE MODE}

$=2$ DATA MODE (READ SET)

$=3$ STORAGE MODE (BETWEEN BEGIN AND FINISH)

$=4$ IMPLIED STORAGE MODE (STATEMENT NUMBER GIVEN)

OMN 270

OMN 280

OMN 290

OMN 300

OMN 310

OMN 320

OMN 330

$0=0,1=1, E T C ., 9=9, A=10, B=11, E T C, Z=35, /=360 \mathrm{MN} 340$

. $=37,-=38,+=39, *=40,(=41)=42,,,=43 \quad$ OMN 350

BLANK $=44,==45, \$$ AND OTHERS $=46$

OMN 360

CALL SETUP

10 IF (MODE.EQ.3) NSTMT=NSTMT +10

IF (MODE.EQ.4) $M O D E=1$

OMN 370

OMN 460

OMN 470

$\operatorname{NAME}(1)=0$

NAME $(2)=0$

OMN 480

$\operatorname{NAME}(3)=0$

OMN 490

NAME $(4)=0$

OMN 500

NARGS $=0$

OMN 510

$J=0$

OMN 520

OMN 530

OMN 540

OMN 550

CHECK FOR ACCUMULATED ERRORS DURING LAST EXECUTED COMMAND

OMN 560

CALL AERR (0)

OMN 570

OMN 580

CALL INPUT

OMN 590

OMN 600

SCANNING BEGINS WITH THE THIRD CHARACTER. THE FIRST TWO ARE DUMMY OMN 610 TO KEEP THE PROGRAM OUT OF TROUBLE. SCANNING TERMINATES WITH A $\$$ OMN 620 A \$ HAS BEEN PLANTED IN THE (KRDEND+1)-TH POSITION.

$M=2$ 
A NUMBER IS THE FIRST ALPHANUMERIC CHARACTER ENCOUNTERED, ERROR IFOMN 700

CALL OUTPUT

35 CALL ERROR (2)

IF (MODE.NE.3) GO TO 50

OMN 720

OMN 730

OMN 740

GO TO 20

40 IF (MODE.NE.4) CALL OUTPUT

OMN 750

OMN 760

GO TO 10

C CHECK FOR * OR ,

45 IF $(K-40) \quad 30,190,30$

50 CALL STMT (NSTMT)

IF (KARG.NE.0) IF (MODE-2) 35,185,35

OMN 770

OMN 780

OMN 790

OMN 800

OMN 810

OMN 815

IF AN ILLEGAL STATEMENT NUMBER WAS FOUND, KARG $=1$ (KARG $=0$ IF

OMN 820 LEGAL)

$M O D E=4$

C

M IS POINTING AT THE FIRST LETTER ON THE CARD, ASSEMBLE NAME.

$\mathrm{C}$

CALL NNAME (NAME (1))

C

C

CHECK THE FIRST NAME FOR SPECIAL NAMES...

OMNITAB, FORMAT, NOTE, FOOTNOTE, HEAD, TITLE

OMN I TAB

IF (NAME (1).NE.11300.OR.NAME (2).NE.7102) IF(LETSG0) 65,67,67

OMN 830

OMN 840

OMN 850

OMN 860

OMN 870

OMN 880

OMN 890

OMN 900

OMN 910

OMN 920

OMN 930

OMN 940

OMN 950

OMN 960

OMN 970

OMN 975

OMN 980

IF NOT THE FIRST OMNITAB CARD, WRITE EOF RECORD.

OMNN 990

OMN 1000

IF (LETSGO.NE. $(-1)$ ) WRITE (ISCRAT, 390)

OMN1010

OMN1020

LETSGO $=$ LETSGO +1

OMN 1030

IF (LETSGO.NE. (-1)) GO TO 10

OMN 1040

OMN 1050

LETSGO $=0$

OMN 1060

OMN 1070

FINISH

OMN 1080

OMN 1090

OMN 1100

$M O D E=1$

GO TO 40

C

FORMAT

C

70 IF (MODE.NE.4) CALL OUTPUT

IF (NAME (1).NE.4797.OR.NAME (2).NE.9524) GO TO 90

OMN 1110

OMN 1120

ONAN 1130

OMN 1140

OMN 1150

OMN 1160

CALL XFORMT

80 IF (MODE.GE.3) CALL ERROR (202)

IF (MODE. NE .3) MODE $=1$

GO TO 10

C

NOTE

C

90 IF (NAME (1).NE.10631.0R.NAME (2).NE.3645) GO TO 100

OMN 1170

OMN1180

OMN 1190

OMN1 200

OMN1210

OMN 1220

OMN 1230

OMN 240 
$K=K A R D(M)$

OMN 1243

IF (K.EQ.1.OR.K.EQ.2) GO TO 95

OMN 1245

IF (NPAGE.EQ.0) CALL PAGE (0)

OMN 1247

WRITE (IPRINT, 400) (NEWCD (I-2), I=M, 82)

OMN 1250

LNCNT $=$ LNCNT +1

GO TO 80

95 CALL NOTEPR (K)

GO TO 80

OMN 1260

OMN 1270

OMN 1273

OMN 1275

OMN 1280

HEAD

OMN 1290

OMN 1300

IF (NAME (1).NE.5968.OR.NAME (2).NE.2916) GO TO 110

CALL XHEAD

GO TO 80

OMN 1310

OMN 1320

OMN 1330

OMN 1340

TITLES. TITLEX $=$ TITLE5, TITLEY $=$ TITLE6

OMN 1350

OMN 1360

OMN 1370

IF (NAME (1).NE.14843) GO TO 160

CHECK NAME TITLE

IF (NAME (2).EQ.8883) GO TO 120

OMN 1380

OMN 1390

CHECK TITLEX, TITLEY

OMN 1400

$\mathrm{K}=5$

$M=M+1$

IF (NAME (2), NE .8908) IF (NAME (2)-8907) 160,130,160

OMN 1410

OMN 1420

$\mathrm{K}=6$

GO TO 130

OMN 1425

OMN 1430

OMN 1440

OMN 1450

OMN 1460

IF (K.GE.1.AND.K.LE.4) GO TO 130

CALL ERROR (209)

$\mathrm{K}=1$

OMN 1470

OMN 1480

OMN 1490

$M M=M I N O(M+59,81)$

DO $140 \quad I=1,60$

OMN 1500

OMN 1510

OMN 1520

OMN 1530

OMN 1540

OMN 1550

OMN1560

OMN 1570

OMN 1580

OMN 1590

OMN 1600

OMN 1610

OMNI 620

OMN 1630

OMN 1640

OMN 1650

OMN 1660

OMN 1670

OMN 1680

OMN 1685

OMN 1690

OMN 1700

OMN 1710

OMN 1720

OMN 1730

OMN 1740

OMN 1750

OMN 1760 
SKIP ONE CHARACTER (') IF FIRST NAME $=(M)$

THE FOLLOWING CARD IS NEEDED ONLY FOR TAPE OPERATIONS

IS NAME (3) EQUAL TO TAP AND NAME (4)=E

IF (NAME (3).NE.14623.OR.NAME (4).NE.3645) GO TO 180

CALL TAPOP

OMN 1780

OMN 1790

OMN 1800

OMN 1810

OMN 1820

OMN 1830

OMN 1840

GO TO 190

OMN 1850

C

180 IF (NAME (1).EQ.9477) $M=M+1$

180 IF (NAME (1).EQ.9477) $M=M+1$
GO TO 190

OMN 1860

C

C

SCAN FOR ARGUMENTS AND END OF CARD

C

$185 \quad M=3$

$190 \mathrm{~J}=\mathrm{J}+1$

GO TO 210

$200 \quad M=M+1$

$210 \quad K=K A R D(M)$

IF (K.GE.10) IF (K-40) 200,255,315

OMN 1870

OMN 1880

OMN 1890

OMN 1900

OMN 1910

OMN 1920

OMN 1930

OMN1940

OMN 1950

OMN 1960

OMN 1965

C

C

NUMBER FOUND, CONVERT ARGUMENT. IF KARG RETURNED $=0$, NUMBER IS

OMN 1970

INTEGER, IF KARG $=1$, NUMBER IS FLOATING POINT, IF KARG $=-1$, ERROROMN1990

CALL AARGS

IF (KARG) $10,230,220$

$220 \quad \operatorname{ARGTAB}(\mathrm{J})=0$.

$\mathrm{J}=\mathrm{J}+1$

GO TO 240

OMN 2000

OMN2010

OMN 2020

OMN 2030

OMN 2040

OMN 2050

C

ARGUMENT IS AN INTEGER. ADD A BIAS OF 8192 THEN CHECK THAT IT IS

OMN 2060

C .GT. 0

OMN 2070

OMN 2080

OMN 2090

$A R G=A R G+8192$.

IF (ARG.GT.0.) GO TO 240

OMN 2100

OMN 2110

OMN 2120

CALL ERROR (18)

OMN 2130

GO TO 10

OMN 2140

OMN 2150

OMN2160

OMN 2170

OMN 2180

OMN 2190

OMN 2200

IF BRACKETED BY SINGLE ASTERISKS, QUANTITY IS TO BE USED AS A

FLOATING POINT ARGUMENT. IF BRACKETED BY DOUBLE ASTERISKS, QUANTITYOMN 2210
IS TO BE TRUNCATED AND USED AS AN INTEGER ARGUMENT.

OMN2230

OMN2240

$K A R G=1$

$M=M+1$

OMN 2250

IF (KARD (M).NE.40) GO TO 260

OMN 2260

$K A R G=0$

OMN 2270

$M=M+1$

OMN 2280

$M S=M$

CALL ASTER

OMN 2290

OMN 2300

OMN 2310

THE TERMINAL ASTERISK(S) HAVE BEEN CHECKED TO BE THE SAME AS THE OMN2320 INTITAL SET (IF NO ERROR) AND M IS POINTING AT THE FIRST CHARACTEROMN 2330 AFTER THE LAST ASTERISK. 
C

A STRING OF THREE OR MORE ASTERISKS IMPLIES -THRUEXAMPLE. .

$\begin{array}{lllllllllllll}\text { ERASE } & 1 & 2 & 3 & 4 & 12 & 13 & 14 & 15 & 16 & 20 & \text { IS EQUIVALENT TO }\end{array}$

ERASE $1 * * * 4,12 * * * 16,20$

$\begin{array}{lllllllll}\text { PRINT } & 1 & 20 & 19 & 18 & 17 & 16 & 15 & 14\end{array}$

IS EQUIVALENT TO

PRINT $1,20 * * * 14$

Z.B. *PI*

$3=$ INTEGER NAMED VARIABLE, Z.B. **NRMAX**

$4=\mathrm{FL}$. PT. NAMED VARIABLE, Z.B. *NRMAX*

$5=$ INTEGER ROW-COLUMN,

$6=$ FL. PT. ROW-COLUMN,

7 = STRING OF ASTERISKS
Z.B. **3, 40**

Z.B. ***
Z.B. $* 1,2 *$

GO TO $(270,220,280,280,290,290,300)$, KARG

OMN 2520

OMN 2530

OMN 2540

GO TO 210

280

$A R G T A B(J)=-2 . * A R G-F L O A T(K A R G-3)$

GO TO 250

OMN2550

OMN 2560

OMN2570

OMN 2580

OMN 2590

OMN 2600

OMN2610

OMN 2620

OMN 2630

OMN 2640

OMN 2650

OMN 2660

OMN 2670

OMN 2680

OMN 2690

OMN 2700

OMN 2710

OMN 2720

OMN 2730

OMN 2740

TO WHICH A BIAS OF 8192 HAS BEEN ADDED. THIS IS TO SAY THAT A

OMN 2750

OMN 2760

OMN 2770

OMN 2780

OMN 2790

OMN 2800

OMN 2810

OMN 2820

OMN 2830

OMN 2840

OMN 2850

OMN 2860

OMN 2870

OMN 2880

$V, W, X, Y, Z$, ARE FOR PROGRAMMING CONVENIENCE ONLY AND DO NOTOMN 2890 AFFECT THE OPERATION OF OMNITAB

OMN 2900

OMN 2910

IF ENTRY IS EVEN, CURRENT VALUE TO BE TRUNCATED AND USED OMN2920 AS AN INTEGER ARGUMENT. 
IF ENTRY .GT. 16, IT IS A WORKSHEET REFERENCE (ROW, COLUMN) TO OMN 2960 HAS BEEN ADDED.

OMN 2990 TO BE TRUNCATED AND USED AS AN INTEGER ARGUMENT.

OMN 3030

C

THE TERMINATION OF CARD FOUND ( \$ ENCOUNTERED)

OMN 3040

OMN 3050

OMN 3060

OMN 3070

OMN 3080

OMN 3090

OMN 3100

OMN 3110

320 IF (J.EQ.0) J=1

IF (MODE.NE.2.OR.NAME (1).NE.0) GO TO 350

OMN 3120

OMN 3130

OMN 3140

IN INPUT MODE AND NO POSSIBLE NAME, RETURN TO SET OR READ ROUTINE OMN3150

CALL SETQ

GO TO 10

OMN 3180

OMN 3190

CALL READQ

GO TO 10

OMN 3200

LOOK UP NAME (AND POSSIBLE QUALIFIER) IN DICTIONARY. RETURN COORDINATES OF ENTRY. IF $L I=0$, NAME NOT FOUND

OMN 3210

OMN 3220

C

IF (LI.NE.0) GO TO 360

IF (MODE.EQ.2) GO TO 330

OMN 3230

OMN 3240

OMN 3250

OMN 3260

OMN 3270

OMN 3280

CALL ERROR (1)

OMN 3290

GO TO 10

OMN 3300

OMN 3310

OMN 3320

NAME FOUND

OMN 3330

OMN 3340

THE FOLLOWING CARDS ARE NEDDED ONLY FOR TAPE OPERATIONS

OMN 3350

STATEMENT WAS 220 IF (MODE.EQ.2) MODE $=1$

OMN 3360

OMN 3370

360 IF (MODE.EQ.2) GO TO 370

* OMN 3380

IF (MODE.EQ.1) GO TO 380

OMN 3390

CALL STORE (J)

OMN 3400

GO TO 10

OMN 3410

THE FOLLOWING CARDS ARE NEDDED ONLY FOR TAPE OPERATIONS

OMN 3420

OMN 3430 $M O D E=1$

C I NUN I T $=$ INUN I P

380 CALL EXPAND (J,ARGTAB)

OMN 3460

CALL XECUTE

OMN3470

GO TO 10

C 
$* * * * * * * * * * * * * * * * * * * * * * * * * * * * * * * * * * * * * * * * * * * * * * * * * * * * * * * * * * * * * * * * * * 0 \mathrm{NE} \quad 40$

OMNITAB * ONEWAY STAT. ANALYSIS.

ONE 50

WRITTEN BY DAVID HOGBEN, SEL, NBS. 10/25/69.

ONE 60

ONEWAY ANALYSIS OF DATA IN ++ WITH TAG IN ++ STORE IN ++,++, . $\quad$ ONE 70

TAG NUMBERS DIFFERENTIATE BETWEEN GROUPS

ONE 80

WHEN TAG IS NON-POSITIVE ZERO WEIGHT IS GIVEN TO MEASUREMENTS ONE 90 NUMBER OF GROUPS MUST BE GREATER THAN I AND MUST NOT EXCEED NLNTH2ONE 100 NRMAX MUST NOT EXCEED NLNTHI

ONE 110

SLOPE IN ANOVA IS ONLY GIVEN IF FPROB FOR BETHEEN IS LESS THAN . I0ONE 120

$* * * * * * * * * * * * * * * * * * * * * * * * * * * * * * * * * * * * * * * * * * * * * * * * * * * * * * * * * * * * * * * * * * 0 \mathrm{NE} 130$

COMMON /BLOCKD/IARGS (100), KIND(100), ARGTAB (100), NRMAX, NROW, NCOL, NONE 140

IARGS, VWXYZ ( 3 ) , NERROR

COMMON /BLOCRC/ NRC, RC (12600)

COMMON /BLOCKE/ NAME (4),L1,L2, ISRFLG

ONE 150

DIMENSION ARGS $(100)$

ONE 160

EQU IVALENCE (ARGS (1), RC (12501))

COMMON /HEADER/ NOCARD $(80), \operatorname{ITLE}(60,6), \operatorname{LNCNT}$, IPRINT, NPAGE, IPUNCH

ONE 170

ONE 180

ONE 190

COMMON /SCRAT/ NS, NS2,A(13500)

COMMON /ABCDEF/ L (48)

EQUIVALENCE (BLANK,L(45)), (SLO,L(22)), (HIGH,L (18))

C

NLNTHI = LENGTH OF ARRAYS $=2700$, MUST BE CHANGED IF NS CHANGED

ONE 200

ONE 210

ONE 220

ONE 230

ONE 240

$5 *$ NLNTHI MUST BE LE NS

DIMENSION A3 (NLNTHI)

ONE 250

DIMENSION A2(2700), A3(2700), A4(2700), A5(2700)

ONE 260

EQUIVALENCE (A2(1),A(2701))

ONE 270

EQUIVALENCE (A3(1),A(5401)), (A4 (1),A(8101)), (A5 (1),A(10801))

ONE 280

C

NLNTH2 = LENGTH OF ARRAYS $=540$, MUST BE CHANGED IF NS CHANGED

ONE 290

$10 *$ NLNTH2 LE NLNTH1

DIMENSION BI (NLNTH2)

ONE 300

DIMENSION B1 $(540), B 2(540), B 3(540), B 4(540), B 5(540), B 6(540), B 70 N E 310$

$1(540), B 8(540), B 9(540), B 10(540)$

ONE 320

EQUIVALENCE (B1(1), $A(1)),(B 2(1), A(541)),(B 3(1), A(1081)),(B 4(1)$, ONE 330

$1 A(1621)),(B 5(1), A(2161)),(B 6(1), A(2701)),(B 7(1), A(3241)),(B 8(10 N E 340$

$2), A(3781)),(B 9(1), A(4321)),(B 10(1), A(4861))$

ONE 350

$C$

EXECUTION TIME CAN BE CONSIDERABLY SHORTENED USING LESS ACCURATE VERSION OF FPPT.

ONE 360

C

C

$* * * * * * * * * * * * * * * * * * * * * * * * * * * * * * * * * * * * * * * * * * * * * * * * * * * * * * * * * * * * * * * * * * 0$

NLNTHI $=N S / 5$

ONE 370

ONE 380

ONE 390

NLNTH2 $=$ NLNTH $1 / 5$

C ERROR CHECKING

IF (NRMAX.GT.NLNTHI) GO TO 50

IF (NRMAX.EQ.0) CALL ERROR ( 9 )

IF (NARGS.EQ.6) GO TO 40

IF (NARGS.EQ.2.AND.L2.EQ.13) GO TO 40

IF (NARGS.EQ.2.AND.L2.EQ.14) GO TO 10

IF (NARGS.EQ.3) GO TO 20

CALL ERROR (10)

RETURN

10 CALL ERROR (236)

RETURN

20 DO $30 \quad I=4,6$

$\operatorname{IARGS}(I)=\operatorname{IARGS}(3)+1$

$30 \operatorname{KIND}(I)=0$

NARGS $=6$

ONE 410

ONE 420

ONE 430

ONE 440

ONE 450

ONE 460

ONE 470

ONE 480

ONE 490

ONE 500

ONE 510

ONE 520

ONE 530

ONE 540

ONE 550

ONE 560

ONE 570

ONE 580

CALL CHKCOL (J) 
C MOVE $Y$ AND TAG TO SCRATCH AREA MOVING TO BOTTOM IF TAG

ONE 620

C

$M 11=I$ ARGS $(1)-1$

M12=I ARGS $(2)-1$

ONE 630

$\mathrm{K}=0$

$N Z W=0$

DO $80 I=1$, NRMAX

ONE 640

ONE 650

ONE 660

ONE 670

ONE 680

$M 02=M 1 I+I$

ONE 690

$M 03=M 12+I$

ONE 700

IF (RC(M03).GE.1.0) GO TO 70

ONE 710

$N Z W=N Z W+1$

ONE 720

MOI=NRMAX $-N Z W+I$

ONE 730

$A 3(M 01)=R C(M 02)$

ONE 740

A4 (MO I) $=0.0$

GO TO 80

$70 \quad J=I-N Z W$

A3 $(\mathrm{J})=R C(\mathrm{MO} 2)$

A4 $(\mathrm{J})=$ AINT $(R C(M 03)+1 . E-8)$

$80 \quad K=\operatorname{MAX} 0(K, I N T(A 4(J)+1.0 E-6))$

ONE 750

ONE 760

ONE 770

ONE 780

ONE 790

$N Z W=N R M A X-N Z W$

IF (NZW.LE.K) GO TO 50

IF (K.LT.2) GO TO 50

IF (K.GT.NLNTH2) GO TO 50

M3 $4=N Z W+1$

C COMPUTE NI,MEAN,S(R), SETUP MIN + MAX, IBAR, FOR $I=1, K$

ONE 800

ONE 810

ONE 820

ONE 830

ONE 840

ONE 850

ONE 860 DO $90 \mathrm{I}=1$, NLNTHI

$90 \quad A(I)=0.0$

CALL RANKO (NZW,A3 (1),A2 (1),A5 (1),A (49))

$A(49)=12.0 * A(49)$

$A(133)=N Z W$

DO $100 \quad I=1, N Z W$

$\mathrm{M} 40=\mathrm{A} 4$ (I)

$\mathrm{B} 2(\mathrm{M} 40)=\mathrm{B} 2(\mathrm{M} 40)+1.0$

$B 3(M 40)=B 3(M 40)+A 3(I)$

ONE 870

ONE 880

ONE 890

ONE 900

ONE 910

ONE 920

ONE 930

ONE 940

ONE 950

$B 5(M 40)=B 5(M 40)+A 5(I)$

ONE 960

ONE 970

$B 6(M 40)=A 3(I)$

ONE 980

$B 7(M 40)=A 3(I)$

ONE 990

$A(21)=A(21)+A 3(I)$

ONE 1000

$A(101)=A(101)+A 4(I)$

ONE 1010

ONE 1020

$A(21)=A(21) / A(133)$

ONE 1030

$A(101)=A(101) / A(133)$

ONE 1040

DO $110 \quad I=1, K$

ONE 1050

IF $(B 2$ (I).GT.0.0) B3(I) $=B 3$ (I) $/ B 2$ (I)

ONE 1060

C COMPUTE MIN,MAX,SD, S,SS,DF,MS, F, FPROB,S(I/NI),S(NI**3),S(R**2/NONE 1070

$110 \quad B 9(I)=B 2$ (I)

DO $120 \quad I=1, N Z W$

ONE 1080

ONE 1090

$\mathrm{M} 40=\mathrm{A} 4(\mathrm{I}) \div .0001$

ONE 1100

$B 6(M 40)=A M I N I(B 6(M 40), A 3(I))$

ONE 1110

$B 7(M 40)=A M A X I(B 7(M 40), A 3(I))$

ONE 1120

$B 4(M 40)=B 4(M 40)+(A 3(I)-B 3(M 40)) * * 2$

ONE 1130

$A(1)=A(1)+(B 3(M 40)-A(21)) * * 2$

ONE 1140

$A(4)=A(4)+(A 3(I)-B 3(M 40)) * * 2$

ONE 1150

$A(5)=A(5)+(A 3(I)-A(2 I)) * * 2$

ONE 1160

$A(17)=0.0$

ONE 1170

$A(22)=B 3(1)$

ONE 1180 
$A(23)=B 3(1)$

ONE 1190

$A(24)=F S Q R T(B 4(1))$

ONE 1200

$A(25)=0$.

$A(26)=B 6(1)$

$A(27)=B 7(1)$

$A(48)=0.0$

DO $150 \quad I=1, K$

$\mathrm{B} 10(\mathrm{I})=(\mathrm{B} 2(\mathrm{I}) *(\mathrm{~B} 2(\mathrm{I})-1.0)) / \mathrm{B} 4$ (I)

ONE 1210

$A(126)=A(126)+B 10(I) * B 3$ (I)

ONE 1220

ONE 1230

ONE1240

ONE 1250

ONE 1260

$A(127)=A(127)+B 10(I)$

IF (B2 (I)-1.) $150,140,130$

130

$B 4(I)=F S Q R T(B 4(I) /(B 2(I)-1.0))$

$A(121)=A(121)+(B 2(I)-1.0) * F L O G(B 4(I) * B 4(I))$

ONE 1270

ONE 1280

ONE 1290

ONE 1300

ONE 1310

$A(131)=A(131)+1$.

$A(25)=A M A X I(A(25), B 4(I))$

ONE 1320

ONE 1330

$A(24)=A M I N I(A(24), B 4(I))$

ONE 1340

$A(120)=A(120)+1.0 /(B 2(I)-1.0)$

$140 \quad A(2)=A(2)+B 2(I) *(F L O A T(I)-A(101)) *(B 3(I)-A(21))$

ONE 1350

$A(114)=A(114)+B 2(I) *((F L O A T(I)-A(101)) * * 2)$

ONE 1360

$A(22)=A M I N I(A(22), B 3(I))$

$A(23)=A M A X I(A(23), B 3(I))$

$A(26)=A M I N I(A(26), B 6(I))$

$A(27)=A M A X I(A(27), B 7(I))$

$A(17)=A(17)+B 5(I) * * 2 / B 2(I)$

$A(18)=A(18)+1 . / B 2(I)$

$A(48)=A(48)+(B 3(I)-A(21)) * * 2$

$A(122)=A(122)+B 4(I) * * 2$

$A(129)=A(129)+B 2(I) * * 2$

$150 \quad A(118)=A(118)+B 2(I) * * 3$

$A(126)=A(126) / A(127)$

$A(2)=A(2) * 2 / A(114)$

$A(3)=A(1)-A(2)$

C DEGREES OF FREEDOM FOR ANOVA

$M \mathrm{l}=\mathrm{K}-\mathrm{l}$

$A(136)=F L O A T(M 1)$

$\mathrm{M} 2=1$

$M 3=K-2$

$M 4=N Z W-K$

$A(134)=F L O A T(M 4)$

$M 5=N Z W-1$

C MEAN SQUARES

$A(6)=A(1) / A(136)$

$A(7)=A(2) / F L O A T(M 2)$

$A(8)=A(3) / F L O A T(M 3)$

$A(9)=A(4) / F L O A T(M 4)$

ONF 1370

ONE 1380

ONE 1390

ONE 1400

ONE1410

ONE 1420

ONE 1430

ONE 1440

ONE 1450

ONE 1460

ONE 1470

ONE 1480

ONE 1490

ONE 1500

ONE 1510

ONE 1520

ONE 1530

ONE 1540

ONE 1550

ONE 1560

ONE 1570

ONE 1580

ONE 1590

ONE 1600

ONE 1610

ONE 1620

ONE 1630

ONE 1640

$A(10)=A(5) / F L O A T$ (M5)

ONE 1650

$A(11)=A(6) / A(9)$

$A(12)=A(7) /((A(3)+A(4)) /(A(133)-2)$.

ONE 1660

$A(13)=A(8) / A(9)$

CALL PROB (A(136), FLOAT (M4), A(11), A(14))

ONE 1670

ONE 1680

CALL PROB (FLOAT (M2), FLOAT (M4),A(12),A(15))

ONE 1690

CALL PROB (FLOAT (M3), FLOAT (NZW-2),A(13),A(16))

ONE 1700

COMPUTE FOR KRUSKAL-WALLIS TEST

$A(117)=N Z W^{*}(N Z W+1)$

$A(17)=(12 . * A(17)) / A(117)-3 .{ }^{*} F L O A T(N Z W+1)$

ONE 1710

ONE 1720

ONE 1730

$A(102)=1.0-A(49) / F L O A T\left(N Z W^{* *} 3-N Z W\right)$

ONE 1740

$A(17)=A(17) / A(102)$

$A(106)=($ FLOAT $(N Z W * * 3)-A(118)) / A(117)$

ONE 1750

ONE 1760

$A(105)=$ FLOAT $(2 * M 1)-.4 *$ FLOAT $\left(3 * K * M 3+N Z W^{*}\left(2 * K^{*}(K-3)+1\right)\right) / A(117)-6 .{ }^{* A}($ ONE 1770 
$118) / 5$

ONE 1780

$A(103)=A(136) *(A(136) *(A(106)-A(136))-A(105)) /\left(.5^{*} A(105) * A(106)\right)$

$A(104)=(A(106)-A(136)) * A(103) / A(136)$

$A(19)=A(17) *(A(106)-A(136)) /(A(136) *(A(106)-A(17)))$

CALL PROB (AINT $(A(103)+.5)$, AINT $(A(104)+.5), A(19), A(20))$

C COMPUTE TOTAL STATISTICS

$A(31)=F S Q R T(A(9))$

$A(32)=F S Q R T(A(48) / A(136))$

$A(33)=F S Q R T(A(10))$

$A(34)=A(31) / F S Q R T(A(133))$

$A(35)=A(32) / F S Q R T(F L O A T(K))$

$A(36)=A(33) / F S Q R T(A(133))$

CALL TPCTPT (FLOAT (M4), A(37))

CALL TPCTPT (A(136), A(38))

CALL TPCTPT (FLOAT (M5), A(39))

$A(41)=A(21)-A(34) * A(37)$

$A(42)=A(21)-A(35) * A(38)$

$A(43)=A(21)-A(36) * A(39)$

$A(44)=A(21)+A(34) * A(37)$

$A(45)=A(21)+A(35) * A(38)$

$A(46)=A(21)+A(36) * A(39)$

C SORT XBAR FOR MULTIPLE COMPARISIONS OF MEANS

DO $160 \quad I=1, M I$

M37 $=\mathrm{K}-\mathrm{I}$

DO $160 \mathrm{~J}=1, \mathrm{M} 37$

IF $(B 8(\mathrm{~J}) . \mathrm{LE} . B 8(\mathrm{~J}+1))$ G0 T0 160

$A(113)=B 8(\mathrm{~J})$

$B 8(\mathrm{~J})=B 8(\mathrm{~J}+1)$

$B 8(J+1)=A(113)$

$A(113)=89(\mathrm{~J})$

$B 9(J)=B 9(J+1)$

$B 9(J+1)=A(113)$

160 CONTINUE

CALL FPPT (A(136), FLOAT (M4),.05,A(115))

$A(116)=A(31) * F S Q R T(A(136) * A(115))$

ONE 1790

ONE 1800

ONE 1810

ONE 1820

ONE 1830

ONE 1840

ONE 1850

ONE 1860

ONE 1870

ONE 1880

ONE 1890

ONE 1900

ONE 1910

ONE 1920

ONE 1930

ONE 1940

ONE 1950

ONE 1960

ONE 1970

ONE 1980

ONE1990

ONE2 000

ONE2 010

ONE2 2020

ONE 2030

ONE 2040

ONE 2050

ONE 2060

ONE 2070

ONE 2080

ONE2 090

ONE 2100

ONE 2110

ONE 2120

ONE 2130

ONE 2140

$A(51)=(A(25) * A(25)) / A(122)$

ONE 2150

$A(123)=A I N T(A(133) / F L O A T(K)-0.5)$

CALL PROB (A(123),A(123)*(A(131)-1.), (A(131)-1.)*A(51)/(1.-A(51)), ONE 2155
O $(52))$
ONE2 160

$A(52)=(A(131)-1) * A.(52)$

ONE 2165

IF $(A(52) . G T .1) \quad A.(52)=1.0$

ONE 2170

$A(57)=(A(25) / A(24)) * * 2$

$A(121)=A(134) * F L O G(A(9))-A(121)$

$A(124)=(A(120)-1.0 / A(134)) /\left(3 .{ }^{*} A(136)\right)$

ONE 2180

$A(125)=(A(131)+1) /.(A(124) * A(124))$

ONE 2190

$A(53)=(A(125) * A(121)) /((A(131)-1.0) *(A(125) /(1.0-A(124)+2.0 / A(125) 0 N E 2220$

1) $-A(121))$ )

CALL PROB (A(131)-1.0,AINT (A (125)+.5),A(53),A(54))

ONE2230

$A(130)=(A(133)-A(129) / A(133)) / A(136)$

ONE 2240

$A(47)=(A(6)-A(9)) / A(130)$

ONE 2250

ONE 2260

C

COMPUTATIONS ARE NOW COMPLETE

$M 0=L 2-12$

GO TO $(170,480)$, MO

C AUTOMATIC PRINTING WHEN L2 $=13$

C FORMAT STATEMENTS

170 CALL PAGE (4)

C PRINT ANOVA

ONE 2270

ONE 2280

ONE2 290

ONE 2300

ONE2310

ONE 2320

WRITE (IPRINT, 520)

ONE2330

ONE2340 
WRITE (IPRINT,530) $M 1, A(1), A(6), A(11), A(14)$

ONE2350

IF (K.LT.3) GO TO 180

IF (A(14).GE.10) GO TO 180

WRITE (IPRINT,540) $M 2, A(2), A(7), A(12), A(15)$

ONE 2360

WRITE (IPRINT,550) M3,A(3),A(8),A(13),A(16)

ONE 2370

WRITE (IPRINT,560) M4,A(4),A(9)

WRITE (IPRINT, 570) M5,A(5)

ONE 2380

ONE 2390

ONE 2400

ONE2 410

C PRINT KRUSKAL-WALLIS TEST

ONE2 420

WRITE (IPRINT, 580) A(17), A(20)

C PRINT ESTIMATES

ONE 2430

WRITE (IPRINT, 590)

ONE 2440

DO $220 \quad I=1, K$

$A(107)=B L A N K$

$A(108)=B L A N K$

IF $(B 2(I)-1.0) 220,190,190$

190 IF (B3(I).LE.A(22)) $A(107)=S L O$

IF (B3(I).GE.A(23)) $A(107)=\mathrm{HIGH}$

ONE 2450

ONE 2460

ONE 2470

ONE 2480

ONE 2490

IF (B4 (I).LE.A(24)) $A(108)=S L O$

ONE 2500

IF (B4 (I) .GE.A(25)) $A(108)=\mathrm{HIGH}$

ONE 2510

$M 8=B 2(I)$

ONE 2520

ONE 2530

IF (M8-1) $220,200,210$

200 WRITE (IPRINT,610) I, M8,B3(I),A(107),B6(I),B7 (I),B5(I)

ONE 2540

GO TO 220

$210 \quad A(109)=B 4$ (I) /FSQRT (B2 (I))

CALL TPCTPT (FLOAT (M8-1), A(112))

ONE 2550

ONE 2560

ONE 2570

ONE2 580

$A(110)=B 3(I)-A(109) * A(112)$

ONE2 2590

$A(111)=B 3(I)+A(109) * A(112)$

ONE 2600

ONE 2610

WRITE (IPRINT,600) I, M8,B3 (I),A(107),B4(I),A(108),A(109),B6(I),B7(ONE2620

II), $B 5(I), A(110), A(111)$

220 CONTINUE

ONE 2630

CONTINUE ONE 2640

WRITE (IPRINT,620) NZW, A(21), A(26), A(27), A(31), A(34), A(41), A(44), AONE2650

$1(32), A(35), A(42), A(45), A(33), A(36), A(43), A(46)$

C COMPUTE AND PRINT FOR MULTIPLE COMPARISIONS

ONE2 660

IF $(A(14)-0.10) 230,450,450$

ONE 2670

ONE 2680

230 IF (M4.LT.4) GO TO 340

ONE 2690

WRITE (IPRINT, 630)

C NEWMAN-KEULS-HARTLEY

ONE 2700

WRITE (IPRINT, 640)

ONE 2710

$J=1$

$M 28=0$

ONE 2720

ONE2 2730

ONE 2740

ONE 2750

$240 \quad I=K$

250 IF $(I-M 28) 330,330,260$

ONE 2760

260 IF (I.EQ.J) GO TO 280

ONE 2770

$A(135)=A B S(B 8(I)-B 8(J))$

ONE 2780

ONE2790

MANDEL APPROXIMATION TO PERCENT POINT OF STUDENTIZED RANGE

ONE 2800

$A(137)=I-J+1$

$R X=-.283917+2.63532 *(A(134)-1.00123) * *(-.95862)$

ONE 2810

$\mathrm{Ul}=-.314115+2.38301 *(A(134)-1.03428) * *(-.864005)$

ONE2 820

$U 2=3.65961 * U 1 * 2-1.00891 * U 1-0.166346$

ONE2 830

$\mathrm{C}=2.3849867-2.9051857^{*}(\mathrm{~A}(137)-0.57583164) *(-.069648109)$

ONE2 840

$V I=1.30153-1.95073 *(A(137)+.394915) * *(-.139783)$

ONE2 850

$V_{2}=4.72863 * V_{1} * 2+0.404271 * V 1-0.135104$

ONE 2860

$A(119)=6.15075+4.441409 * R X+6.7514569 * C+7.4671282 * U 1 * V 1-.157537 * U 2 * 0 N E 2870$ IV2

ONE2 880

$A(119)=A(119) * F S Q R T(.5 *(1.0 / B 9(I)+1.0 / B 9(J))) * A(31)$

ONE 2890

IF $(A(135)-A(119)) 280,280,270$

$270 \quad \mathrm{I}=\mathrm{I}-1$

GO TO 250

280 IF (J.EQ.1) GO TO 310

ONE2 2900

ONE2910

ONE2920

ONE2930 
IF (J-M28) 290,290,300

ONE2940

WRITE (IPR INT, 670)

ONE2950

GO TO 310

ONE2 290

$\begin{array}{llll}300 & \text { WRITE } & \text { (IPRINT, 680) } \\ 310 & \text { WRITE } & \text { (IPRINT, 660) } & (\mathrm{B} 8(\mathrm{M} 29), M 29=\mathrm{J}, \mathrm{I})\end{array}$

ONE 2970

IF (I-K) $320,340,340$

ONE2 2980

$\begin{array}{ll}320 & M 28=I \\ 330 & J=J+1\end{array}$

ONE2990

ONE 3000

GO TO 240

ONE 3010

ONE 3020

C SCHEFFE METHOD

ONE 3030

340 WRITE (IPRINT, 650)

ONE 3040

$\mathrm{J}=1$

$M 28=0$

ONE 3050

$350 \quad I=K$

360 IF (I-M28) $440,440,370$

ONE 3060

ONE 3070

370 IF (I.EQ.J) GO TO 390

ONE 3080

$A(135)=A B S(B 8(I)-B 8(J))$

$A(119)=A(116) * F S Q R T(1.0 / B 9(I)+1.0 / B 9(J))$

ONE 3090

ONE 3100

IF $(A(135)-A(119)) \quad 390,390,380$

$380 \quad I=I-1$

GO TO 360

390 IF (J.EQ.1) GO TO 420

IF (J-M28) 400,400,410

ONE 3110

ONE 3120

ONE 3130

ONE 3140

ONE 3150

ONE 3160

400 WRITE (IPRINT,670)

GO TO 420

410 HRITE (IPRINT,680)

420 HRITE (IPRINT,660) (B8 (M29), M29=J, I)

IF (I -K) $430,450,450$

$430 \quad M 28=I$

$440 \mathrm{~J}=\mathrm{J}+1$

GO TO 350

450 IF (A(131).LT.2.0) GO TO 480

WRITE (IPRINT,690) A(51),A(52),A(53), A(54), A(57)

IF $(A(52) . G T \ldots 10$. AND.A(54).GT . 10) GO T0 470

DO $460 \quad I=1, K$

$A(55)=A(55)+B 10(I) *(B 3(I)-A(126)) * 2$

$460 \quad A(128)=A(128)+(1 .-B 10$ (I) $/ A(127)) * * 2 /(B 2(I)-1$.

$A(128)=($ FLOAT $(K * * 2)-1) /.\left(3 .{ }^{*} A(128)\right)$

$A(55)=(A(55) / F L O A T(M I)) /\left(1 .+\left(2 .{ }^{*}\right.\right.$ FLOAT $\left.\left.(M 3)\right) /\left(3 .{ }^{*} A(128)\right)\right)$

ONE 3170

ONE 3180

ONE 3190

ONE 3200

ONE 3210

ONE 3220

ONE 3230

ONE3 240

ONE3 250

ONE3 360

ONE3270

ONE 3280

ONE 3290

ONE 3300

ONE3310

CALL PROB (FLOAT (MI), AINT (A (128)+.5), A (55), A (56))

ONE 3320

ONE 3330

WRITE (IPRINT, 700) A(55), A(56)

ONE3340

ONE3350

470 WRITE (IPRINT,710) A(47)

ONE 3360

AUTOMATIC PRINTOUT IS FINISHED - NOW STORE RESULTS

ONE 3370

ONE3 380

IF (NARGS.EQ.2) GO TO 510

ONE3390

$M 13=I$ ARGS (3)

ONE 3400

$M 14=I$ ARGS (4)

ONE 3410

MI5=IARGS (5)

ONE 3420

$M 16=\operatorname{IARGS}(6)$

ONE 3430

DO $500 \quad \mathrm{I}=1, \mathrm{~K}$

ONE 3440

DONT STORE IF $\mathrm{N}=0$

IF (B2(I).LE.0.0) GO TO 490

ONE 3450

TAG

ONE 3460

$R C(M 13)=I$

ONE 3470

$\mathrm{N}$

ONE 3480

$\mathrm{RC}(\mathrm{M14})=\mathrm{B} 2$ (I)

ONE 3490

XBAR

ONE 3500

$R C(M 15)=B 3$ (I)

ONE 3510

STANDARD DEVIATION

ONE 3520 
FORMAT (// , 48X,20HANALYSIS OF VARIANCE //17X,6HSOURCE, $14 \mathrm{X}, 4 \mathrm{HD} . \mathrm{F} ., 4$ XONE3610 $1,14 \mathrm{HSUM}$ OF SQUARES, 5X, $12 \mathrm{HMEAN}$ SQUARES, 9X,7HF RATI0,4X,7HF PROB. /) ONE 3620

580 FORMAT ( $11 X, 65$ HKRUSKAL-WALLIS RANK TEST FOR DIFFERENCE BETWEEN GROONE 3680 IUP MEANS $* \mathrm{H}=$, OPF $9.3,10 \mathrm{H}, \mathrm{F}$ PROB $=, \mathrm{F} 6.3,10 \mathrm{H}($ APPROX.) $/)$

ONE 3690

590 FORMAT (55X,9HESTIMATES//1X,5HGROUP, 5X,3HNO . 6X, 4HMEAN, 7X, 11HWITHIONE 3700 IN S.D. ,2X,12HS.D. OF MEAN, 5X,7HMINIMUM, 7X,7HMAXIMUM,6X,4HS (R) ,4X,20NE 3710 23H95PCT CONF INT FOR MEAN/)

ONE 3720

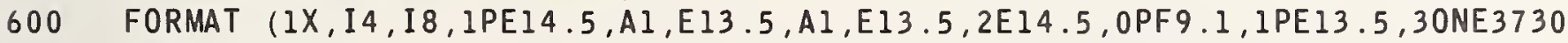
IH TO,E12.5)

ONE 3740

610 FORMAT (IX,I4,I8,1PE14,5,AI,3X,24H ESTIMATE NOT AVAILABLE ,IP2E14.0NE3750 15$, OPF $.1,3 \mathrm{X}, 25 \mathrm{H} * * * * * * * * * *$ TO $* * * * * * * * * *)$

ONE 3760

620 FORMAT (/,1X,5HTOTAL, I7, IPE14.5,28X,2E14.5/7X,20HFIXED EFFECTS MODONE3770 IEL ,2E14.5,37X,E13.5,3H T0,E12.5/7X,20HRANDOM EFFECTS MODEL, ,2E14.0NE3780 $25,37 X, E 13.5,3 \mathrm{H}$ TO,E12.5/7X,14HUNGROUPED DATA,6X,2E14.5,37X,E13.5,30NE3790 $3 \mathrm{H}$ TO,E12.5/)

ONE3 3800

630 FORMAT (IX, 120HPAIRWISE MULTIPLE COMPARISON OF MEANS. THE MEANS AONE 3810 IRE PUT IN INCREASING ORDER IN GROUPS SEPARATED BY *****. A MEAN IONE3820 $2 S / 120 \mathrm{H}$ ADJUDGED NON-SIGNIFICANTLY DIFFERENT FROM ANY MEAN IN THEONE3830 3 SAME GROUP AND SIGNIFICANTLY DIFFERENT AT THE .05 LEVEL FROM / $1200 N E 3840$ 4H ANY MEAN IN ANOTHER GROUP. ********** INDICATES ADJACENT GROUPONE3850 5S HAVE NO COMMON MEAN.

ONE 3860

640 FORMAT (/3X,89HNEWMAN-KEULS TECHNIQUE, HARTLEY MODIFICATION. (APPRONE 3870 IOXIMATE IF GROUP NUMBERS ARE UNEQUAL.))

ONE 3880

650 FORMAT (/3X,18HSCHEFFE TECHNIQUE .)

660 FORMAT $(3 X, 9(1 \mathrm{PE} 12.5,1 \mathrm{H})$, 670 FORMAT $(6 \mathrm{X}, 5 \mathrm{H} * * * * *)$

ONE 3890

ONE3 3900 FORMAT $(3 \mathrm{X}, 11 \mathrm{H} * * * * * * * * * *)$ ONE 3910

FORMAT (/,36H TESTS FOR HOMOGENEITY OF VARIANCES. /7X, 13HCOCHRAN,S ONE3930 $I \mathrm{C}=31 \mathrm{H}$ MAX. VARIANCE/SUM(VARIANCES) $=, \mathrm{F} 6.4,5 \mathrm{H}, \mathrm{P}=, \mathrm{F} 6.3,10 \mathrm{H}$ (APPROONE3940 $2 \mathrm{X}.) / 7 \mathrm{X}, 16 \mathrm{HBARTLETT}-\mathrm{BOX} \mathrm{F}=, \mathrm{F} 9.3,5 \mathrm{H}, \mathrm{P}=, \mathrm{F} 6.3 / 7 \mathrm{X}, 37 \mathrm{HMAXIMUM}$ VAR I ANCONE3950 $3 E$ / MINIMUM VARIANCE $=, F 10.3$ )

ONE 3960

700 FORMAT ( $7 X, 7$ OHAPPROX BETHEEN MEANS F-TEST IN PRESENCE OF HETEROGENONE 3970 IEOUS VARIANCE. $F=, F 8.3,5 \mathrm{H}, \mathrm{P}=, \mathrm{F} 6.3$ ) ONE3980

710 FORMAT (/,1X,35HMODEL II - COMPONENTS OF VARIANCE. /7X,29HESTIMATEONE3990 1 OF BETWEEN COMPONENT, IPE 15.7) ONE 4000 END 
SUBROUTINE OPONE ( $N, M, M X, N X, N D 2, N D 3, N D 19, B, S S Q, I X) \quad$ OPO 10

VERSION 5.00 OPONE 5/15/70

C SUBROUTINE TO PRINT PAGE 1 OF POLYFIT AND FIT

OPO 20

$* * * * *$

WRITTEN BY DAVID HOGBEN, SEL, NBS. $10 / 14 / 69$.

OPO 30

COMMON / BLOCRC / NRC, RC (12600)

OPO 40

COMMON / BLOCKE / NAME (4), L1, L 2, I SRFLG

OPO 50

COMMON / HEADER/NOCARD $(80)$, I TLE $(60,6)$, LNCNT, IPR INT , NPAGE, IPUNCH

OPO 60

COMMON/SCRAT/NS, NS2, A(13500)

COMMON / KF MT / KFMT (100)

COMMON / FMAT / I FMTX (6), IOSWT , IFMTS (6), LHEAD (96)

OPO 70

COMMON /BLOCKD/I ARGS (100), KIND (100), ARGTAB (100), NRMAX, NROW, NCOL,

INARGS, VWXYZ (8), NERROR

DIMENSION ARGS $(100)$, IIRGS $(100)$

EQUIVALENCE (ARGS(1), RC (12501)), (IIRGS(1),KFMT (1))

DIMENSION B(1)

$\begin{array}{ll}\mathrm{C} & * * * * * \\ \mathrm{C} & * * * * * \\ \mathrm{C} & * * * * * \\ \mathrm{C} & * * * * *\end{array}$

IF (L2.EQ.1) GO TO 1620

IF (MX.GT.I) GO TO 1640

OPO 80

OPO 90

OPO 100

OPO 110

OPO 120

OPO 130

OPO 140

OPO 150

OPO 160

OPO 170

OPO 180

OPO 190

OPO 200

OPO 210

OPO 220

1620 WRITE (IPRINT, 1625) (LHEAD(I 1), I l=13,24), (LHEAD(I 2), I 2=1, 12),

OPO 230

1 IARGS (IX), IARGS (1)

OPO 240

1625 FORMAT $(/ / 5 X, 2(4 X, 12 A 1,4 X), 6 X, 9 H P R E D I C T E D, 8 X, 12 H S T D$. DEV. OF, 25X, OPO 250

1 4HSTD./2X,3HROW, 3X, 9HIN COLUMN, I5,6X, 9HIN COLUMN, I5, 10X,6HVALUEOPO 260

$2 S, 10 X, 12$ HPRED. VALUES, $8 X, 9$ HRESIDUALS , 8X, 4HRES . 3X, 7HWEIGHTS/) $\quad$ OPO 270

GO TO 1660

1640 IF (MX.GT.2) GO TO 1650

WRITE (IPRINT, 1645) (LHEAD (I), I =1, 12), IARGS (NX+4), IARGS (NX+5), $\quad$ OPO 300

1 IARGS ( 1 )

OPO 310

1645 FORMAT (//8X,22HPREDICTOR VARIABLES IN,6X,12A1,8X,9HPREDICTED,6X, OPO 320

1 12HSTD. DEV. OF, 22X,4HSTD. /2X,3HROW, 3X, 4HCOL., I 4,6X,4HCOL., I $4,8 X, 0 P 0330$

$24 \mathrm{HCOL}$. . I $4,11 X, 6 \mathrm{HVALUES}, 8 \mathrm{X}, 12 \mathrm{HPRED}$. VALUES, 6X, 9HRESIDUALS, $7 X, 4 \mathrm{HRES} .0 \mathrm{PO} 340$

$3,3 X, 7$ HWEIGHTS / )

GO TO 1660

WRITE (IPRINT, 1655) (LHEAD (I), I=1,12), IARGS $(N X+4)$, IARGS $(N X+5), \quad$ OPO 370

$1 \quad \operatorname{IARGS}(N X+6), \operatorname{IARGS}(1)$

OPO 380

1655 FORMAT (//12X,22HPREDICTOR VARIABLES IN,9X, 12A1,6X,9HPREDICTED,4X OPO 390

$1,12 \mathrm{HSTD}$. DEV. OF, 19X,4HSTD . $2 \mathrm{X}, 3 \mathrm{HROW}, 2 \mathrm{X}, 3$ (4HCOL., I $4,4 \mathrm{X}), 2 \mathrm{X}, 4 \mathrm{HCOL} ., 0 \mathrm{OPO} 400$

$2 I 4,9 X, 6$ HVALUES , 6X, 12 HPRED. VALUES, 4X, 9HRESIDUALS, 6X, 4HRES . ,3X, $\quad$ OPO 410

37 HWE IGHTS /)

1660 IX $=$ IIRGS (IX)

$I Y=\operatorname{IIRGS}(1)$

IND3 $=N D 3+1$

IND2 $=N D 2+1$

IND4 $=$ NDI9+1

OPO 420

$L L=0$

$N S D=8$

NWM $=18$

IF (L2.EQ.1 .OR. MX.EQ.1) GO TO 1666

OPO 430

OPO 440

OPO 450

OPO 460

OPO 470

OPO 480

OPO 490

$I X 2=I I R G S(N X+5)$

NWM $=2^{*}(2 / M X)$

$\mathrm{LL}=4-\mathrm{NWM}$

$N S D=4+N K M$

$N W M=N W M+10$

CALL RFORMT (RC (IX2), N,NSD,NW2,NDEC2,NWM, $A(1), A(1), 0,0)$

OPO 500

OPO 510

OPO 520

OPO 530

OPO 540

OPO 550

OPO 560

IF (MX.EQ.2) GO TO 1666

OPO 570

$I X 3=\operatorname{IIRGS}(N X+6)$ 
CALL RFORMT (RC (IX3),N,NSD,NW3,NDEC $3, N W M, A(1), A(1), 0,0)$

1666

CALL RFORMT

CALL RFORMT

CALL RFORMT

CALL RFORMT

$(R C(I X), N, N S D, N W 1, N D E C 1, N W M, A(1), A(1), 0,0)$

(RC (IY), N

$8, N H 4, N D E C 4,18-L L, A(1), A(1), 0,0)$

$(A(I N D), N$,

$8, N H 5, N D E C 5,17-L L, A(1), A(1), 0,0)$

$(A(I N D 2), N$,

$8, N W 6, N D E C 6,17-L L, A(1), A(1), 0,0)$

CALL RFORMT (A (IND4), N, $8, N W 7, N D E C 7,17-L L, A(1), A(1), 0,0$ )

IF (KIND(2).EQ.1) GO TO 1667

$I H=I I R G S(2)$

CALL RFORMT (RC ( I W) , N , 4, NW9, NDEC 9, 9, A (1), A (1), 0,0)

GO TO 1680

1667 CALL RFORMT (ARGS(2), 1,4,NW9,NDEC 9,9,A(1),A(1),0,0)

CALL RFORMT (A(1), 1,4,NW9,NDEC9,0,ARGS(2),B (98),11-NW9, 1)

WT=ARGS (2)

1670 FORMAT (IX, I 4, 97AI, OPF7.2,11A1)

1680 DO $1745 \quad I=1, N$

CALL RFORMT ( $A(1), 1, N S D, N W 1, N D E C 1,0, R C(I X), B(1), N W M+2-N W 1,1)$

IF (L2.EQ.1 .OR. MX.EQ.1) GO TO 1685

CALL RFORMT (A (1), 1,NSD,NW2,NDEC2, $0, R C(I X 2), B(N W M+3), N W M+2-N W 2,1)$

$I X 2=I X 2+1$

IF (MX.EQ.2) GO TO 1685

CALL RFORMT ( $A(1), 1, N S D, N W 3, N D E C 3,0, R C(I X 3), B(25), 12-N H 3,1)$

$I X 3=I X 3+1$

1685 CALL RFORMT (A(1), 1,8, NH4, NDEC4,0,RC (IY),B (4*LL+21), 20-LL-NW4, 1)

CALL RFORMT ( $A(1), 1,8, N W 5, N D E C 5,0, A($ IND 3$\left.), B\left(3^{*} L L+41\right), 19-L L-N W 5,1\right)$

CALL RFORMT ( $A(1), 1,8, N W 6, N D E C 6,0, A\left(\right.$ IND2) $\left., B\left(2^{*} L L+60\right), 19-L L-N W 6,1\right)$

CALL RFORMT (A(1),1,8,NW7,NDEC7,0,A(IND4),B(LL + 79),19-LL-NW7,1)

IF (KIND(2).EQ.1) GO TO 1730

CALL RFORMT (A(1), 1,4,NH9,NDEC9,0,RC(IW),B (98),11-NW9, 1)

$W T=R C(I W)$

$I W=I W+1$

1730 IF (WT.GT.0.0) STDRES=A (IND4)/FSQRT (SSQ/WT-A (IND2)**2)

IF (WT . LE.0.0) STDRES $=0.0$

WRITE (IPRINT, 1670) I , ( $($ ( I I ) , I I =1,97) , STDRES , (B ( I I ) , I I =98, 108)

$1740 \quad I X=I X+1$

$I Y=I Y+1$

IND $3=$ IND $3+1$

IND2 = IND2 +1

1745 IND4 $=$ IND4+1

RETURN

END
OPO 600

OPO 610

OPO 620

OPO 630

OPO 640

OPO 650

OPO 660

OPO 670

OPO 680

OPO 690

OPO 700

OPO 710

OPO 715

OPO 720

OPO 730

OPO 740

OPO 750

OPO 760

OPO 770

OPO 780

OPO 790

OPO 800

OPO 810

OPO 820

OPO 830

OPO 840

OPO 850

OPO 860

OPO 865

OP0 870

OP0 875

OPO 880

OPO 890

OPO 900

OPO 910

OPO 920

OPO 930

OPO 940

OPO 950

OPO 960 
COMMON /BLOCKD/ IARGS (100), KIND(100), ARGTAB (100), NRMAX, NROW, NCOL, NORT 70

IARGS, VWXYZ (8), NERROR

DIMENSION ARGS $(100)$

EQUIVALENCE (ARGS(1), RC (12501))

COMMON /BLOCKE/ NAME (4),L1,L2, ISRFLG

COMMON /HEADER/ NOCARD $(80), \operatorname{ITLE}(60,6)$, LNCNT , IPRINT, NPAGE, IPUNCH

ORT 80

ORT 90

ORT 100

ORT 110

COMMON /SCRAT/ NS, NS2,A(13500)

COMMON /KFMT/ KFMT $(100)$

COMMON /FMAT/ IFMTX (6), IOSWT, IFMTS (6), LHEAD (96)

DIMENSION IIRGS $(100)$

EQUIVALENCE (IIRGS(1), KFMT (1)), (B (1), IB)

DIMENSION IMTRXA $(2,3)$

DIMENSION B(120), IHC (4), IHT (8)

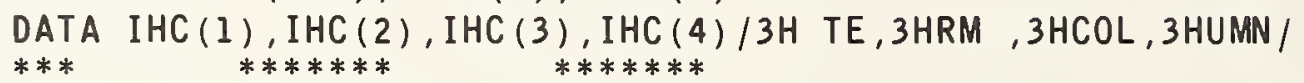

ORTHONORMALIZATION PROGRAM BY PHILIP J. WALSH JULY 1,1967

ORT 120

ORT 130

ORT 140

ORT 150

ORT 160

ORT 170

ORT 180

ORT 190

ORT 200

ORT 210

REVISED BY S. PEAVY 5/28/68

REVISED BY DAVID HOGBEN AND SALLY PEAVY, SEL, NBS. $9 / 23 / 69$.

LEAST SQUARES PROGRAM USING GRAM SCHMIDT PROCESS

POLYFIT Y IN COL ++ WITH WEIGHTS (ALL EQUAL TO **)

(IN COL ++)

USING POLYNOMIAL OF DEGREE, TO $X$ IN COL ++

STORE: COEFFICIENTS IN COL ++

DEVIATIONS IN COL ++

STANDARD DEV OF PREDICTED VALUES IN COL ++

FOURIER COEFFICIENTS IN COL ++

VARIANCE COVARIANCE MATRIX STARTING IN $(,,++)$

ONLY FIRST 4 ARGUMENTS MUST BE SPECIFIED.

STORAGE WILL TAKE PLACE FOR ONLY THE STORAGE ARGUMENTS PROVIDED

$$
\text { L2=1 POLYFIT: L2=2 SPOLYFIT }
$$

FIT Y IN COL ++ WITH WEIGHTS(ALL EQUAL TO**) AS A

$$
\text { ( IN COL ++) }
$$

ORT 220

ORT 230

ORT 240

ORT 250

ORT 260

ORT 270

ORT 280

ORT 290

ORT 300

ORT 310

ORT 320

ORT 330

ORT 340

ORT 350

ORT 360

ORT 370

ORT 380

ORT 390

ORT 400

ORT 410

FUNCTION OF $\mathrm{K}=++$ VARIABLES IN COLS $++,++,++, \ldots,+++$

STORE: COEFFICIENTS IN COL ++

DEVIATIONS IN COL ++

STANDARD DEV OF PREDICTED VALUES IN COL ++

FOURIER COEFFICIENTS, ETC. IN COL ++

VARIANCE COVARIANCE MATRIX STARTING IN $(++,$,

MINIMUM OF 4 ARGUMENTS IS NEEDED BEFORE COMMAND IS EXEC. $L 2=3$ FIT : L2=4 SFIT

MORTHO $X(,,++) \quad R=,, C=$, WITH WEIGHTS ( ALL EQUAL TO **)

STORE IN $M(,,++)$ A MATRIX IN $(,,++)$ ( IN COL ++ ) L2 $=5$ MORTHO :

ORT 420

ORT 430

ORT 440

ORT 450

ORT 460

ORT 470

ORT 480

ORT 490

ORT 500

ORT 510

ORT 520

ORT 530

ORT 540

ORT 550

ORT 560

$I X I(I, J, I N)=I N+(I *(I-I)) / 2+J$

ORT 570

ORT 580

PRECHECKING SECTION

ORT 582

IF (L2.EQ.1.OR.L2.EQ.3) G0 T0 8

ORT 584 
IF (NARGS.NE.IARGS (3)+3) GO TO 8

6 CALL ERROR (236)

RETURN

8 IREF IT $=0$

IF (NARGS.GT.0) GO TO 10

CALL ERROR (10)

RETURN

10 IF (NRMAX.NE.0) GO TO 20

CALL ERROR (9)

RETURN

$20 \quad N M U I=1$

C IF $L 2=5$ THEN COMMAND IS MORTHO

IF (L2.EQ.5) GO TO 1850

IF (NARGS.LT.4) GO TO 1830

C COMMAND IS POLYFIT OR FIT

CALL ADRESS (1,IIRGS(1))

IF (IIRGS(1).LE.0) CALL ERROR (11)

IF (KIND (2).EQ.1) GO TO 30

CALL ADRESS (2,IIRGS(2))

IF (IIRGS (2).LE.0) CALL ERROR (11)

GO TO 40

30 SU $=$ NRMAX

WSUM=SU

IF (ARGS(2).LE.0.0) CALL ERROR (24)

NMU I $=2$

$40 \quad$ NST $=1$

$\operatorname{IF}(\operatorname{KIND}(3), E Q .1) \quad \operatorname{IARGS}(3)=\operatorname{ARGS}(3)$

NEND $=$ NARGS

$J=N A R G S-4$

IF (L2.GT.2) J=J-IARGS (3)+1

IF (J.LE.4.AND.J.GE.0) GO TO 50

IF (J.NE.6) GO TO 1830

$45 \quad \mathrm{NEND}=$ NARGS -2

NST $=2$

50 DO $60 \quad I=4$, NEND

CALL ADRESS (I, IIRGS (I))

IF (IIRGS (I).LE.0) CALL ERROR (11)

60 CONTINUE

$M=I A R G S(3)$

IF (L2.LE. 2) $M=M+1$

$N=N R M A X$

$\mathrm{FN}=\mathrm{N}$

GO TO $(100,70)$, NST

70 CALL ADRESS (NARGS, IST)

IF (IST.GT.0) GO TO 80

CALL ERROR (11)

RETURN

$80 \quad M M T X R=M$

MMT XC $=M$

IST $=I S T-1+I$ ARGS (NARGS-1)

IF (IARGS (NARGS) +M-1.GT.NCOL) MMTXC=NCOL-IARGS(NARGS) +1

IF (IARGS (NARGS-1)+M-1.GT. NROW) MMTXR=NROW-I ARGS (NARGS-1) +1

IF (MMTXR.GT.0) GO TO 90

ORT 586

ORT 588

ORT 590

ORT 595

ORT 600

ORT 610

ORT 620

ORT 630

ORT 640

ORT 650

ORT 660

ORT. 670

ORT 680

ORT 690

ORT 700

ORT 710

ORT 720

ORT 730

ORT 740

ORT 750

ORT 760

ORT 770

ORT 780

ORT 790

ORT 800

ORT 810

ORT 815

ORT 820

ORT 825

ORT 830

ORT 835

ORT 837

ORT 840

ORT 850

ORT 860

ORT 870

ORT 880

ORT 890

ORT 900

ORT 910

ORT 920

ORT 930

ORT 940

ORT 950

ORT 960

ORT 970

ORT 980

ORT 990

ORT 1000

ORT 1010

ORT1020

ORT 1030

ORT 1040

ORT 1050

ORT 1060

CALL ERROR (213)

ORT 1070

ORT 1080

ORT1090

ORTI100 


\begin{tabular}{|c|c|c|}
\hline & WSUM $=0.0$ & ORT 1110 \\
\hline & L22=IIRGS (2) & ORT 1120 \\
\hline & $L 22 A=L 22$ & ORT 1130 \\
\hline & DO $130 \quad I=1, N$ & ORT 1140 \\
\hline & IF $(R C(L 22 A)) 1840,130,120$ & ORT 1150 \\
\hline 120 & $S U=S U+1.0$ & ORT 1160 \\
\hline & $W S U M=W S U M+R C(L 22 A)$ & ORT 1170 \\
\hline 130 & $\mathrm{~L} 22 \mathrm{~A}=\mathrm{L} 22 \mathrm{~A}+1$ & ORT 1180 \\
\hline 140 & $F M=M$ & ORT 1190 \\
\hline & IF (SU-FM) $150,160,170$ & ORT 1200 \\
\hline 150 & CALL ERROR (24) & ORT 1210 \\
\hline & RETURN & ORT 1220 \\
\hline 160 & DENOM=1.0 & ORT 1230 \\
\hline & GO TO 180 & ORT 1240 \\
\hline 170 & DENOM=FSQRT $(S U-F M)$ & ORT 1250 \\
\hline 180 & $N P M=N+M$ & ORT 1260 \\
\hline & $M 1=M-1$ & ORT 1270 \\
\hline & $M 2=M+1$ & ORT 1280 \\
\hline & $\mathrm{Nl}=\mathrm{N}-\mathrm{l}$ & ORT 1290 \\
\hline & $N 2=N+1$ & ORT 1300 \\
\hline & $M D I=\left(M^{*}(M 2)\right) / 2$ & ORT 1310 \\
\hline C & & ORT 1320 \\
\hline C & & ORT 1330 \\
\hline & $N D 1=M 2 * N P M$ & ORT 1340 \\
\hline C & $X$ REQUIRES NDI CELLS & ORT 1350 \\
\hline C & GET A (NDI + 1) FOR START OF PK & ORT 1360 \\
\hline & $N D 2=M * N P M$ & ORT 1370 \\
\hline & $M D 3=N D 2+N$ & ORT 1380 \\
\hline & ND3 $=$ ND1 & ORT 1390 \\
\hline C & ADD NPM TO REACH XP & ORT 1400 \\
\hline & $N D 4=N D 3+N P M$ & ORT 1410 \\
\hline C & ADD NPM TO REACH QK & ORT 1420 \\
\hline & ND5 $=$ ND $4+N P M$ & ORT 1430 \\
\hline C & ADD $(M+1)$ TO REACH CV & ORT 1440 \\
\hline & $N D 6=N D 5+M 2$ & ORT 1450 \\
\hline C & $\operatorname{ADD}\left(M^{*}(M+1)\right) / 2+M$ TO REACH VCV & ORT 1460 \\
\hline & $N D 66=M D I+M$ & ORT 1470 \\
\hline & ND7 $=$ ND $6+N D 66$ & ORT 1480 \\
\hline C & ADD THE SAME AMOUNT TO REACH Q & ORT 1490 \\
\hline & ND $8=N D 7+N D 66$ & ORT 1500 \\
\hline C & Q IS (M+1) CELLS LONG THEN COMES Q2 & ORT 1510 \\
\hline & $N D 9=N D 8+M 2$ & ORT 1520 \\
\hline C & Q2 E AND EP ARE EACH M CELLS LONG & ORT 1530 \\
\hline & $N D 10=N D 9+M$ & ORT 1540 \\
\hline & $N D I I=N D I 0+M$ & ORT 1550 \\
\hline & $N D 12=N D 11+M$ & ORT 1560 \\
\hline C & THE A MATRIX IS NEXT & ORT 1570 \\
\hline & ND1 $3=N D 12+M D 1$ & ORT 1580 \\
\hline C & GRAM FACTOR STORAGE & ORTI590 \\
\hline & $N D 14=N D 13+M 2$ & ORT 1600 \\
\hline C & ENF & ORT 1610 \\
\hline C & CV DIAGONALS & ORT 1620 \\
\hline & ND1 $6=N D 14+M$ & ORT 1630 \\
\hline C & VCV DIAGONALS & ORT 1640 \\
\hline & ND $17=N D 16+M$ & ORT 1650 \\
\hline & ND1 $8=N D 17+M$ & ORT 1660 \\
\hline & $N D 19=N D 18+N P M$ & ORT 1670 \\
\hline & $\mathrm{ND20}=\mathrm{ND} 19+\mathrm{N}$ & ORT 1680 \\
\hline & IF (IREFIT.EQ.1) GO TO 190 & ORT 1690 \\
\hline
\end{tabular}


IF (ND20.GT.NS) CALL ERROR (23)

ORT1700

IF (NERROR.NE.O) RETURN

ORT 1710

190

NRBAR $=1$

ORT 1720

I=I IRGS (1)

ORT 1730

$\mathrm{L} 22 \mathrm{~A}=\mathrm{L} 22$

ORT 1732

CONS3 $=R C(L 22)$

ORT 1733

$\operatorname{IF}(K I N D(2) . E Q .1) \quad C O N S 3=A R G S(2)$

ORT1735

CONSI $=R C$ (I)

ORT1736

CONS2 $=R C$ (I)

ORT 1737

DO 195 I $I=1$, NRMAX

ORT1738

IF (KIND (2) .EQ.0) CONS3 $=R C(L 22 A)$

ORT 1740

IF (CONS.3) $192,192,191$

ORT1741

191 CONS=RC (I)

IF (CONS.LT.CONSI) CONSI $=$ CONS

ORT 1742

IF (CONS.GT. CONS2) CONS $2=$ CONS

ORT1743

ORT 1745

$192 \quad \mathrm{I}=\mathrm{I}+1$

$195 \quad \mathrm{~L} 22 \mathrm{~A}=\mathrm{L} 22 \mathrm{~A}+1$

$\mathrm{YCONS}=($ CONS $2+\mathrm{CONS} 1) / 2.0$

GO TO $(200,200,240,240,240)$, L2

ORT1746

ORT 1747

ORT1748

ORT 1750

C THIS IS POLYFIT

$200 \quad$ L33 $=\operatorname{IIRGS}(4)$

MXARGS $=5$

ORT1760

$\mathrm{L} 33 \mathrm{~A}=\mathrm{L} 33$

ORT1770

$K=N P M+1$

ORT 1780

ORT 1790

DO $210 \quad I=1, N$

ORT1800

$A(I)=1.0$

ORT 1810

$A(K)=R C(L 33 A)$

ORT 1820

$K=K+1$

$210 \quad L 33 A=L 33 A+I$

IF (M.EQ.2) GO TO 320

DO $230 \mathrm{~K}=2, \mathrm{MI}$

$\mathrm{L} 33 \mathrm{~A}=\mathrm{L} 33$

$K 2=K * N P M+1$

$\mathrm{K} 1=\mathrm{K} 2-\mathrm{NPM}$

DO $220 \quad I=1, N$

$A(K 2)=A(K 1) * R C(L 33 A)$

$K 2=K 2+1$

$K I=K I+1$

$220 \quad \mathrm{~L} 33 \mathrm{~A}=\mathrm{L} 33 \mathrm{~A}+1$

230 CONTINUE

GO TO 320

$240 \quad I=4$

C FIND OUT IF ALL $X(I)=1.0$, IF SO SET NX=1 AND MX=M-1.

L33 =I IRGS (4)

$N X=1$

D0 250 NW6 $=1, N$

IF (ABS (RC $(L 33)-1.0)$.LE.1.E-6) GO TO 250

$N X=0$

GO TO 260

ORT 1830

ORT 1840

ORT 1850

ORT 1860

ORT1870

ORT 1880

ORT 1890

ORT1900

ORT1910

ORT1920

ORT1930

ORT1940

ORT1950

ORT1960

ORT1970

ORT1980

ORT1990

ORT2000

ORT 2010

ORT 2020

ORT 2030

ORT 2040

ORT 2050

ORT 2060

$\begin{array}{ll}250 & L 33=L 33+1 \\ 260 & M X=M A X O(1, M-N X)\end{array}$

IF (NX.EQ.0.OR.L2.EQ.5) YCONS $=0.0$

ORT 2070

ORT 2075

MXARGS $=M+4$

L44=MXARGS-1

ORT 2080

$\mathrm{J}=1$

270 DO $310 \quad I I=I, L 44$

$\mathrm{Kl}=\mathrm{J}$

L33=I IRGS (II)

$K 2=K 1$

ORT 2090

ORT2100

ORT 2150

ORT 2160

ORT2170

ORT2180 


\begin{tabular}{|c|c|c|}
\hline \multirow{3}{*}{290} & DO $300 \quad I 2=1, N$ & ORT2190 \\
\hline & $A(K 2)=R C(L 33)$ & ORT2220 \\
\hline & $K 2=K 2+1$ & ORT 2230 \\
\hline \multirow[t]{2}{*}{300} & $L 33=L 33+1$ & ORT 2240 \\
\hline & I JKLM=2 & ORT 2250 \\
\hline 310 & $J=J+N P M$ & ORT2260 \\
\hline C & GENERATE IDENTITY MATRIX AUGMENTATION & ORT 2270 \\
\hline \multirow[t]{5}{*}{320} & $\mathrm{~K} 1=\mathrm{N} 2$ & ORT 2280 \\
\hline & DO $340 \mathrm{~K}=1, \mathrm{M}$ & ORT 2290 \\
\hline & $K 2=K 1$ & ORT2300 \\
\hline & DO $330 I=1, M$ & ORT2310 \\
\hline & $A(K 2)=0$ & ORT2320 \\
\hline \multirow[t]{3}{*}{330} & $K 2=K 2+1$ & ORT2330 \\
\hline & $K 2=K 1+K-1$ & ORT2340 \\
\hline & $\mathrm{Kl}=\mathrm{Kl}+\mathrm{NPM}$ & ORT2350 \\
\hline 340 & $A(K 2)=1.0$ & ORT 2360 \\
\hline \multirow[t]{10}{*}{ C } & BEGIN THE G.S. PROCESS & ORT 2370 \\
\hline & NBE $I=1$ & ORT2380 \\
\hline & NRH I =1 & ORT2390 \\
\hline & $118=1+N D 13$ & ORT2400 \\
\hline & NGAI $=2$ & ORT2410 \\
\hline & NSI I $=2$ & ORT 2420 \\
\hline & NDE $I=1$ & ORT 2430 \\
\hline & NNUI $=1$ & ORT2440 \\
\hline & $\lfloor Z 1=1$ & ORT 2450 \\
\hline & $L Z 2=1$ & ORT2460 \\
\hline \multirow[t]{2}{*}{ C } & K CONTROLS WHOLE LOOP & ORT2470 \\
\hline & $K=1$ & ORT2480 \\
\hline 350 & NTHI $=1$ & ORT2490 \\
\hline \multirow[t]{5}{*}{360} & NAL I =1 & ORT2500 \\
\hline & $\mathrm{NOMI}=1$ & ORT2510 \\
\hline & $\mathrm{NJ}=\mathrm{ND} 3+\mathrm{N}+1$ & ORT2520 \\
\hline & $00370 \mathrm{~J}=1, \mathrm{M}$ & ORT2530 \\
\hline & $A(N J)=0$ & ORT2540 \\
\hline 370 & $\mathrm{NJ}=\mathrm{NJ}+1$ & ORT2550 \\
\hline C & BOX 6 . & ORT2560 \\
\hline \multirow[t]{6}{*}{380} & $K D l=(K-1) * N P M$ & ORT2570 \\
\hline & $\mathrm{I} I=\mathrm{ND} 3+1$ & ORT 2580 \\
\hline & $12=K D I+1$ & ORT2590 \\
\hline & $\mathrm{L} 22 \mathrm{~A}=\mathrm{L} 22$ & ORT 2600 \\
\hline & DO $420 \quad I=1, N$ & ORT 2610 \\
\hline & GO TO $(390,400)$, NMUI & ORT 2620 \\
\hline C & PK (I) & ORT2630 \\
\hline \multirow[t]{3}{*}{390} & $A(I 1)=A(I 2) * R C(L 22 A)$ & ORT2640 \\
\hline & $\mathrm{L} 22 \mathrm{~A}=\mathrm{L} 22 \mathrm{~A}+1$ & ORT2650 \\
\hline & GO TO 410 & ORT2660 \\
\hline 400 & $A(I 1)=A(I 2) * A R G S(2)$ & ORT2670 \\
\hline 410 & $I 1=I 1+1$ & ORT2680 \\
\hline \multirow[t]{2}{*}{420} & $\mathrm{I} 2=\mathrm{I} 2+1$ & ORT2690 \\
\hline & GO TO $(430,460)$, NOMI & ORT2700 \\
\hline \multirow[t]{9}{*}{430} & $I A I=1$ & ORT2710 \\
\hline & I A $2=N D 5+1$ & ORT2720 \\
\hline & $D 0450 \quad I=1, K$ & ORT 2730 \\
\hline & $I 2=I A I$ & ORT 2740 \\
\hline & $S U M=0.0$ & ORT 2750 \\
\hline & $\mathrm{J} 2=\mathrm{ND} 3+1$ & ORT2760 \\
\hline & DO $440 \mathrm{~J}=1, N P M$ & ORT 2770 \\
\hline & $S U M=S U M+A(J 2) * A(I 2)$ & ORT2780 \\
\hline & $12=12+1$ & ORT2790 \\
\hline
\end{tabular}




\begin{tabular}{|c|c|c|}
\hline \multirow{4}{*}{$\begin{array}{l}440 \\
C\end{array}$} & $\mathrm{~J} 2=\mathrm{J} 2+1$ & ORT2800 \\
\hline & QK (I ) & ORT2810 \\
\hline & $A(I A 2)=S U M$ & ORT 2820 \\
\hline & $I A I=I A I+N P M$ & ORT2830 \\
\hline \multirow[t]{2}{*}{450} & $\mathrm{IA} 2=\mathrm{IA} 2+1$ & ORT 2840 \\
\hline & GO TO 490 & ORT2850 \\
\hline \multirow[t]{6}{*}{460} & DK $2=0$ & ORT 2860 \\
\hline & $I I=(K-1) * N P M+1$ & ORT 2870 \\
\hline & IND $3=N D 3+1$ & ORT 2880 \\
\hline & D0 $470 I=1, N P M$ & ORT2890 \\
\hline & $\mathrm{DK} 2=\mathrm{DK} 2+\mathrm{A}(\mathrm{IND} 3) * \mathrm{~A}(\mathrm{II})$ & ORT 2900 \\
\hline & $I I=I I+I$ & ORT 2910 \\
\hline \multirow[t]{2}{*}{470} & IND $3=$ IND $3+1$ & ORT2920 \\
\hline & $\mathrm{DK}=\mathrm{FDSQRT}(\mathrm{DK} 2)$ & ORT 2930 \\
\hline \multirow[t]{6}{*}{ C } & GRAM FACTORS & ORT2940 \\
\hline & $A(I 18)=D K$ & ORT 2950 \\
\hline & I $18=I 18+1$ & ORT2960 \\
\hline & $K I=(K-1) * N P M+1$ & ORT2970 \\
\hline & D0 $480 \quad I=1, N P M$ & ORT 2980 \\
\hline & $A(K 1)=A(K 1) / D K$ & ORT2990 \\
\hline \multirow[t]{3}{*}{480} & $\mathrm{Kl}=\mathrm{Kl}+\mathrm{l}$ & ORT 3000 \\
\hline & NOMI $=1$ & ORT3010 \\
\hline & GO TO 380 & ORT 3020 \\
\hline C & $\mathrm{B} 0 \times 8$ & ORT 3030 \\
\hline 490 & GO TO $(500,560)$, NDEI & ORT3040 \\
\hline \multirow[t]{2}{*}{500} & $L Z 1=-L Z 1$ & ORT3050 \\
\hline & IF (LZ1) $550,510,510$ & ORT 3060 \\
\hline C & $B 0 \times 8 A$ & ORT3070 \\
\hline \multirow{4}{*}{510} & $K I=K-1$ & ORT 3080 \\
\hline & IRUTH=ND $5+1$ & ORT 3090 \\
\hline & DO $520 \mathrm{I}=1, \mathrm{KI}$ & ORT 3100 \\
\hline & $A(I R U T H)=-A($ I RUTH $)$ & ORT3110 \\
\hline \multirow[t]{11}{*}{520} & IRUTH=IRUTH+I & ORT 3120 \\
\hline & IRUTH=K+ND 5 & ORT 3130 \\
\hline & $A($ IRUTH $)=1.0$ & ORT 3140 \\
\hline & $\mathrm{J} 2=N D 4+1$ & ORT3150 \\
\hline & DO $540 \quad I=1, N P M$ & ORT 3160 \\
\hline & $S U M=0.0$ & ORT 3170 \\
\hline & $\mathrm{J} 1=\mathrm{I}$ & ORT 3180 \\
\hline & $\mathrm{J} 3=\mathrm{ND} 5+1$ & ORT 3190 \\
\hline & DO $530 \mathrm{~J}=1, \mathrm{~K}$ & ORT 3200 \\
\hline & $S U M=S U M+A(J I) * A(J 3)$ & ORT 3210 \\
\hline & $J I=J I+N P M$ & ORT3220 \\
\hline \multirow{3}{*}{$\begin{array}{l}530 \\
C\end{array}$} & $\sqrt{ } 3=\sqrt{ } 3+1$ & ORT3230 \\
\hline & $X P(I)$ & ORT 3240 \\
\hline & $A(J 2)=S U M$ & ORT 3250 \\
\hline \multirow[t]{2}{*}{540} & $\mathrm{~J} 2=\mathrm{J} 2+1$ & ORT 3260 \\
\hline & GO TO 640 & ORT 3270 \\
\hline & GET QK (I 18) & ORT3280 \\
\hline & I $S A L=I 18+M 2$ & ORT3290 \\
\hline & IRUTH $=$ ND $5+K$ & ORT3300 \\
\hline & $A(I S A L)=F S Q R T(A(I R U T H))$ & ORT3310 \\
\hline & GO TO 510 & ORT 3320 \\
\hline C & NDE 1 & ORT 3330 \\
\hline \multirow[t]{2}{*}{560} & $L Z 2=-L Z 2$ & ORT3340 \\
\hline & IF (LZ2) $570,510,510$ & ORT 3350 \\
\hline C & GET E AMD OTHER VECTORS & ORT3360 \\
\hline & D0 $580 I=1, M$ & ORT 3370 \\
\hline & IND $5=N D 5+I$ & ORT3380 \\
\hline
\end{tabular}


IND9 $=N D 9+I$

ORT 3390

I ND $8=N D 8+I$

ORT 3400

$A($ IND8 $)=A($ IND5)

580

$A($ IND9 $)=A($ IND 5$) * A($ IND 5$)$

ORT 3410

$A($ IND $8+1)=A($ IND $5+1)$

ORT 3420

$A(N D 10+1)=A($ IND $8+1)-A(N D 9+1)$

ORT 3430

IND $10=N D 10+1$

I ND $9=N D 9+1$

ORT 3440

ORT 3450

DO $590 \mathrm{~J}=2, \mathrm{M}$

ORT 3460

INDI $0=$ INDI $10+1$

ORT3470

I ND $9=1 N D 9+1$

ORT 3480

ORT3490

$590 \quad A($ IND10) $=A($ INDI0-1) $-A($ IND9)

ORT 3500

$\mathrm{F} I=1.0$

ORT 3510

IND $10=N D 10$

ORT 3520

IND $11=$ ND 11

ORT 3530

DO $635 \mathrm{I}=1, \mathrm{M}$

ORT 3540

I NDI $0=$ IND $10+1$

ORT 3550

$I N D I 1=I N D I 1+1$

ORT 3560

IF (FN-FI) $630,630,600$

ORT 3570

600 IF (A(IND10)) $610,620,620$

ORT 3580

$610 \quad A($ INDII) $=-F S Q R T(A B S(A(I N D 10)) /(F N-F I))$

ORT 3590

GO TO 635

$620 A(I N D I 1)=F S Q R T(A(I N D 10) /(F N-F I))$

GO TO 635

ORT 3600

ORT 3610

$630 \quad A($ INDI0) $=-1.0$

$635 \quad F I=F I+1.0$

GO TO 510

C B0X9

640 GO TO $(650,670,800)$, NTHI

$650 \quad \mathrm{Kl}=(\mathrm{K}-1) * \mathrm{NPM}+1$

IND $4=N D 4+1$

D0 $660 \quad I=1, N P M$

$A(K 1)=A(I N D 4)$

$\mathrm{Kl}=\mathrm{Kl}+\mathrm{l}$

660 IND4 $=$ IND4 +1

GO TO 760

670 IND 18=ND18+1

!ND4 $=$ ND $4+1$

D0 $680 \quad I=1, N$

ORT 3620

ORT 3630

ORT 3640

ORT 3650

ORT 3660

ORT 3670

ORT 3680

ORT 3690

ORT 3700

ORT 3710

ORT 3720

ORT 3730

ORT 3740

ORT 3750

ORT 3760

ORT 3770

ORT 3780

$A($ IND18) $=A($ IND4 $)$

ORT 3790

IND $18=$ IND $18+1$

ORT 3800

I ND $4=$ IND $4+1$

ORT 3810

$\mathrm{NI}=\mathrm{N}+\mathrm{I}$

DO $690 \quad I=1, M$

ORT 3820

$K K I=N D I 8+N I$

ORT3830

IND 4 =ND4+N I

ORT 3840

$A(K K I)=-A($ IND 4$)$

ORT 3850

$\mathrm{NI}=\mathrm{N} \mathrm{I+1}$

ORT 3860

I ND $4=$ ND 4

ORT3870

IND $19=N D 19$

ORT 3880

DO $700 \quad I=1, N$

ORT 3890

IND $4=$ IND $4+1$

ORT3900

IND $19=$ IND $19+1$

ORT 3910

ORT3920

ORT3930

ORT3940

ORT3950

ORT3960

ORT3970 
D0 $720 \quad I=1, N$

ORT3980

$\mathrm{L} 66 \mathrm{~A}=\mathrm{L} 66 \mathrm{~A}+1$

ORT 3990

I ND 4=I ND 4+1

720

$R C($ L66A)'=A (IND 4)

ORT 4000

730

$L 55=I$ IRGS (MXARGS)

ORT 4010

$\mathrm{L} 55 \mathrm{~A}=\mathrm{L} 55$

$\mathrm{NI}=\mathrm{N}+\mathrm{ND} 4+\mathrm{I}$

$R C(L 55 A)=-A(N I)+Y C O N S$

ORT 4020

ORT 4030

IF (M.EQ.1) GO TO 750

ORT 4040

DO $740 \quad I=2, M$

ORT4050

$\mathrm{NI}=\mathrm{NI}+1$

$L 55 A=L 55 A+1$

ORT 4060

ORT 4070

ORT 4080

$740 \quad R C(L 55 A)=-A(N I)$

ORT 4090

750 NTHI $=3$

GO TO 650

ORT 4100

ORT 4110

C B0X10

ORT 4120

760 GO TO $(770,780)$, NALI

770 NOMI $=2$

NALI $=2$

GO TO 380

780 IF (K-M) $790,830,830$

$790 \quad K=K+1$

GO TO 350

800 GO TO $(810,820)$, NNUI

ORT 4130

ORT 4140

ORT 4150

ORT 4160

ORT 4170

ORT 4180

ORT 4190

ORT 4200

ORT 4210

$810 \quad$ NNUI $=2$

GO TO 920

ORT4220

ORT 4230

$820 \quad S S=D K / D E N O M$

ORT 4240

$S S Q=S S * S S$

ORT4250

GO TO 920

ORT4260

830 GO TO $(840,800)$, NBEI

ORT 4270

C

C GET THE A MATRIX

ORT 4280

ORT 4290

$840 \quad \mathrm{Kl}=1$

D0 $860 \quad I=I, M$

$I I=I * N+(I-I) * M$

ORT4300

ORT4310

D0 $850 \mathrm{~J}=1$, I

ORT 4320

ORT 4330

$I 2=J+I I$

$\mathrm{K} 2=\mathrm{K} 1+\mathrm{ND} 12$

ORT 4340

ORT 4350

$A(K 2)=A(I 2)$

ORT 4360

ORT4370

ORT4380

ORT 4390

ORT 4400

ORT 4410

ORT 4420

ORT 4430

ORT 4440

ORT 4450

ORT 4460

ORT 4470

ORT 4480

ORT 4490

ORT 4500

ORT 4510

ORT 4520

ORT 4530

ORT 4540

ORT 4550

ORT 4560 
C

THE ARGUMENT IN THE FOLLOWING SQRT OCCASIONALLY IS NEGATIVORT4620 $A(J)=F S Q R T(A(J))$

ORT 4630

$\mathrm{J}=\mathrm{J}+1$

ORT 4640

$\begin{array}{ll}900 & \mathrm{Jl}=\mathrm{Jl}+\mathrm{I}+1 \\ 910 & \mathrm{NGAI}=1\end{array}$

ORT 4650

ORT 4660

GO TO 800

ORT 4670

920 GO TO $(930,990), \mathrm{NRHI}$

ORT4680

930 IF (NRBAR) 940,1030,940

ORT 4690

$940 \quad N R B A R=N R B A R-1$

NTHI $=2$

ORT 4700

NRHI $=2$

ORT 4710

LI I=I IRGS (I) -1

ORT 4720

$L I I A=L 11+1$

ORT 4730

I $1=N D 2+1$

ORT 4740

DO $970 \quad I=1, N$

IF (L2.NE.5) GO TO 960

ORT 4750

GO TO $(960,950)$, NMUI

ORT4760

ORT 4770

$950 \quad A(I 1)=\operatorname{ARGS}(2)$

GO TO 970

ORT4780

ORT 4790

$960 \quad A(I I)=R C(L I I A)-Y C O N S$

ORT 4800

$L 11 A=L 11 A+1$

$970 \quad I I=I I+1$

I $1=M D 3+1$

DO $980 \quad I=1, M$

$A(I I)=0$.

$980 \quad \mathrm{I} l=\mathrm{I} 1+1$

GO TO 360

ORT 4810

ORT 4820

ORT 4830

ORT 4840

ORT 4850

ORT 4860

ORT 4870

ORT 4880

990 GO TO $(930,1000)$, NSII

ORT 4890

C GET VCV AND DEV AND COEF

ORT 4900

$1000 \quad$ IND $7=N D 7+1$

I ND $6=$ ND $6+1$

ORT 4910

DO $1010 \quad I=1, M D I$

ORT 4920

ORT 4930

$A($ IND7 $)=S S Q^{*} A($ IND6 $)$

ORT 4940

IND $7=$ IND $7+1$

1010 IND $6=$ IND $6+1$

ORT 4950

ORT 4960

IND1 $6=N D 16+1$

ORT 4970

IND $17=$ ND $17+1$

ORT 4980

DO $1020 \quad I=1, M$

ORT 4990

$A\left(\right.$ INDI7) $=S S^{*} A($ IND 16)

ORT5000

I ND $16=$ IND $16+1$

ORT5010

ORT5 020

INDI $7=$ INDI $7+1$

ORT5 030

ORT5040

THE CALCULATIONS ARE COMPLETED. NOH OUTPUT THE RESULTS

ORT5050

ORT5060

ORT5070

IND $18=N D 18+N+1$

ORT5080

$A($ IND 18$)=A($ IND 18) + YCONS

ORT5090

IF (IREFIT.EQ.1) GO TO 1640

ORT5100

IF (L2.EQ.5) GO TO 1960

ORT5110

ORT5120

$L 55 A=L 55+M$

ORT5130

ISF $=1$

ORT5140

$L 55 B=L 55 A+M-1$

ORT5150 
IF (M.GE.NROW) GO TO 1190

ORT5 160

I $S F=2$

ORT5170

$\mathrm{L} 55 \mathrm{~B}=\mathrm{L} 55+\mathrm{NROW}-1$

ORT 5180

1040 IND $17=$ ND $17+1$

ORT 5190

DO $1050 \quad I=L 55 A, L 55 B$

ORT5200

$R C(I)=A$ (INDI7)

ORT 5210

1050 IND $17=$ IND $17+1$

ORT5220

GO TO $(1060,1190)$, ISF

ORT5230

ORT5240

ORT5250

IF (IMS.GT.6) IMS $=6$

ORT5260

GO TO $(1180,1170,1160,1150,1140,1070)$, IMS

ORT5270

ORT5280

ORT5290

ORT5 300

ORT5 510

ORT5 320

ORT5330

ORT5340

ORT5 550

ORT5 360

ORT5 570

ORT5 380

ORT5390

ORT5 400

ORT5410

ORT5420

ORT5430

ORT5440

ORT5450

ORT5460

ORT5470

ORT5480

ORT5490

ORT5500

ORT5510

ORT5520

ORT5530

ORT5540

ORT5550

ORT5560

ORT5570

ORT5580

ORT5590

ORT5600

ORT5610

ORT5620

ORT5630

ORT5640

ORT5650

ORT5660

ORT5670

ORT5680

ORT5690

ORT5700

$1200 \quad I P=I P+N P M$

ORT5710

$A(I N D 2)=F S Q R T(S P) * S S$

ORT5720

IPIC $=$ IP IC +1

ORT5730

IND $2=$ IND2 +1

ORT5740 
$\mathrm{L} 22 \mathrm{~A}=\mathrm{L} 22 \mathrm{~A}+1$

ORT 5790

GO TO 1230

ORT 5800

1220 YSUM $=Y S U M+R C$ (IY) **2*ARGS (2)

ORT 5810

$1230 \quad$ I $Y=I Y+1$

ORT 5820

C CHECK TO SEE IF RESULTS ARE TO BE PUT IN WORK SHEET

ORT5 830

IF (NARGS.LE.MXARGS+1) G0 TO 1460

ORT 5840

IF (NARGS- $($ MXARGS +3$)) 1440,1390,1240$

C STORE VARIANCE COVARIANCE MATRIX IN WORK SHEET

ORT5850

1240 LSWT $=0$

ORT5860

IND $7=N D 7+1$

ORT 5870

1250 ISTR=IST

ORT 5880

ISTC $=$ IST

ORT5890

MSTOP=MINO (MMTXC, MMTXR)

ORT5900

DO $12.70 \mathrm{I}=1$, MSTOP

ORT5910

ISTRR $=$ I STR

I STCC $=$ I STC

DO $1260 \mathrm{~J}=1, \mathrm{I}$

$R C($ ISTRR $)=A($ IND7)

$R C($ ISTCC $)=A($ IND7)

IND $7=$ IND $7+1$

$I S T R R=I S T R R+N R O W$

ORT 5920

ORT5930

ORT5940

ORT5950

ORT 5960

ORT5970

ORT5980

1260 ISTCC $=I S T C C+1$

ISTR $=I S T R+1$

1270 ISTC $=$ ISTC+NROW

IF (MMTXC.EQ.MMTXR) GO TO 1330

C VARIANCE COVARIANCE MATRIX IS STORED AS A RECTANGULAR MATRIX MSTP $=$ MAXO (MMTXC, MMTXR) -MSTOP

IF (MMTXC-MMTXR) $1280,1280,1290$

1280 ICONA $=$ NROW

I $\mathrm{CONB}=1$

ISTART $=$ ISTR

GO TO 1300

1290 ICONA $=1$

$I$ CONB $=$ NROW

ISTART $=$ I STC

1300 DO $1320 \mathrm{I}=1$, MSTP

ISTRC $=$ I START

DO $1310 \mathrm{~J}=1$, MSTOP

ORT 5990

ORT 6000

ORT 6010

ORT6020

ORT6030

ORT 6040

ORT 6050

ORT6060

ORT6070

ORT 6080

ORT6090

ORT6100

ORT6110

ORT6120

ORT6130

ORT6140

ORT 6150

ORT 6160

$R C($ ISTRC) $=A($ IND7)

ORT6170

IND $7=$ IND 7 + I

ORT 6180

ORT 6190

1310 ISTRC $=$ ISTRC + I CONA

ORT6200

IND $7=I N D 7+I$

1320 ISTART $=$ ISTART + ICONB

C STORE GRAM FACTORS, VECTOR NORMS AND GRAM DETERMINANTS

ORT6210

ORT 6220

ORT 6230

ORT 6240

ORT 6250

IF (MSTOP.LT.(-1)) GO TO 1380

ORT 6260

IND I $4=$ NDI $14+1$

ORT6270

GMDT $=1.0$

I STR $=I S T+M$

ORT 6280

DO $1370 \quad I=1$, MMTXC

IF (MSTOP) $1360,1350,1340$

ORT 6290

ORT 6300

ORT 6310

GMDT $=\operatorname{GMDT}^{*}(\operatorname{A}($ INDI3 $) / A($ IND I 4$)) * * 2$

ORT 6320

$R C(I S T R+2)=G M D T$

ORT6330 
IND $13=$ IND $13+1$

$1360 \mathrm{RC}($ ISTR+I) $=A($ IND 14)

IND I $4=$ IND $14+1$

1370 ISTR=ISTR+NROW

C STORE FOURIER COEFFICIENTS

1380 IF (LSWT.EQ.1) RETURN

1390 LST $=$ IIRGS (MXARGS+3)

IND9 $=$ ND 9

DO $1400 \quad I=1, M$

IND9 $=$ I ND9+1

$R C(L S T)=A($ IND9)

$1400 \quad$ LST $=$ LST +1

IF (NROW- $(M+1)) \quad 1440,1420,1410$

$1410 \quad \mathrm{RC}($ LST +1$)=\mathrm{YSUM}$

$1420 R C(L S T)=(S U-F M) * S S Q$

$\mathrm{LST}=\mathrm{LST}+2$

IF (M+2.GE.NROW) GO TO 1440

$L S T A=L S T+M-1$

IF $(2 * M+2 . G T . N R O W) \quad L S T A=I I R G S(M X A R G S+3)+N R O W-1$

IND $8=N D 8$

DO $1430 I=L S T$, LSTA

IND $8=$ IND $8+1$

$1430 R C(I)=A$ (IND8)

C STORE S.D. OF PREDICTED VALUES

1440 LSTOR=IIRGS (MXARGS+2)

IP I $\mathrm{C}=1$

IND2 $=$ ND2 +1

DO $1450 \quad I=1, N$

$R C(L S T O R)=A(I N D 2)$

IND $2=$ IND2 +1

1450 LSTOR=LSTOR +1

C START PRINTING

1460 GO TO $(1480,1470,1480,1470,1470)$, L2

1470 RETURN

1480 IT ITLE=1

IF (L2.EQ.3) ITITLE=2

I $P G=1$

$N S U=S U+.5 E-5$

CALL PREPAK (5,NWI, NWI, IARGS (1), LHEAD)

IF (NWI.EQ.0) GO TO 1500

DO $1490 \quad I=1,4$

LHEAD (I) $=L(45)$

1490 LHEAD $(I+8)=L(45)$

$\operatorname{LHEAD}(5)=\mathrm{L}(14)$

$\operatorname{LHEAD}(6)=\mathrm{L}(11)$

$\operatorname{LHEAD}(7)=\mathrm{L}(30)$

$\operatorname{LHEAD}(8)=\mathrm{L}(11)$

1500 CALL PREPAK (5,NW1,NW1, I ARGS (4), LHEAD(13))

IF (NW1.EQ.0) GO TO 1510

$\operatorname{LHEAD}(13)=\mathrm{L}(45)$

$\operatorname{LHEAD}(14)=\mathrm{L}(32)$

$\operatorname{LHEAD}(15)=\mathrm{L}$ (11)

$\operatorname{LHEAD}(16)=\mathrm{L}(28)$

$\operatorname{LHEAD}(17)=\mathrm{L}(19)$

$\operatorname{LHEAD}(18)=\mathrm{L}$ (11)

$\operatorname{LHEAD}(19)=\mathrm{L}(12)$

$\operatorname{LHEAD}(20)=\mathrm{L}(22)$

$\operatorname{LHEAD}(21)=\mathrm{L}(15)$

$\operatorname{LHEAD}(22)=\mathrm{L}(45)$
ORT6340

ORT6350

ORT6360

ORT6370

ORT6380

ORT6390

ORT 6400

ORT 6410

ORT 6420

ORT6430

ORT6440

ORT 6450

ORT6460

ORT6470

ORT 6480

ORT 6490

ORT6500

ORT 6510

ORT 6520

ORT 6530

ORT 6540

ORT 6550

ORT 6560

ORT 6570

ORT 6580

ORT 6590

ORT6600

ORT6610

ORT6620

ORT 6630

ORT 6640

ORT6650

ORT6660

ORT 6670

ORT6680

ORT 6690

ORT6700

ORT 6710

ORT 6720

ORT 6730

ORT 6740

ORT6750

ORT6760

ORT 6770

ORT6780

ORT 6790

ORT 6800

ORT6810

ORT 6820

ORT 6830

ORT 6840

ORT 6850

ORT 6860

ORT 6870

ORT6880

ORT6890

ORT6900

ORT 6910

ORT6920 
1520 WRITE (IPRINT,2000) IARGS(3), (LHEAD (I), I=13,24), IARGS (4)

ORT 6980

1530 GO TO $(1540,1550)$, NMUI

ORT6990

$1540 \quad N Z W=N R M A X-N S U$

WR ITE (IPRINT, 2010) NSU,NZW, I ARGS (2)

GO TO 1560

1550 CALL RFORMT (ARGS(2), $1,8, N W 1, N D E C 1,10, A(1), A(1), 0,0)$

ORT 7000

ORT7010

ORT7 020

ORT 7030

CALL RFORMT ( $A(1), 1,8, N W 1, N D E C 1,0, \operatorname{ARGS}(2), B(1), 0,0)$

ORT7040

WRITE (IPRINT, 2020) NSU, (B (I), I=1,10)

1560 GO TO $(1570,1580,1610,1650)$, IPG

1570 IXA=4

IF (L2.EQ.3) IXA=IXA+(M-MX)

IX $=I X A$

CALL OPONE ( $N, M, M X, N X, N D 2, N D 3, N D 19, B, S S Q, I X)$

$I P G=2$

1580 IF (NSU.GE.3) GO TO 1590

WR ITE (IPRINT, 2030)

GO TO 1600

1590 CALL ORTPLT (ND19,ND2,N,SSQ,ND3, IB, I IRGS (IXA), I IRGS (2))

ORT 7050

ORT7060

ORT 7070

ORT7080

ORT 7090

ORT 7100

ORT 7110

ORT 7120

ORT 7130

ORT 7140

ORT 7150

ORT7 160

$1600 \quad I P G=3$

GO TO 1510

1610 CALL OCOVAR (M,ND7, MD 1, IHC, B, IHT)

C PRINT ANALYSIS OF VARIANCE

CALL OANOVA (YSUM, SU, ND9, FM, M, N, ND7, SSQ, IHC, NSU, B)

GO TO 1660

C REFIT FOR $M=M-1$

1620 IREF IT $=1$

$M=M-1$

$F M=M$

$\mathrm{M} 1=\mathrm{M}-1$

$M 2=M+1$

SSOLD $=$ SS

IND $17=$ ND $17+1$

ORT 7170

ORT7 180

ORT 7190

ORT 7200

ORT 7210

ORT 7220

ORT 7240

ORT 7250

ORT 7260

ORT7280

ORT7290

ORT7300

ORT7310

IND18S $=N D 18+N$

ORT7320

ORT7330

IND19=ND19+1

ORT7340

IND19S=ND19

ORT7350

DO $1630 \mathrm{~J}=1, M 2$

ORT7360

$A($ IND19) $=A($ IND17)

ORT7370

IND $19=$ INDI $9+1$

ORT7380

ORT7390

1630 IND $17=$ IND $17+1$

ORT7395

IF (M.EQ.0) GO TO 1640

ORT7400

GO TO 170

ORT 7410

C BEGIN REFIT TO PREDICTED VALUES

ORT7430

$1640 \mathrm{Ml}=\mathrm{M}$

ORT7440

REFIT FOR $M=M-1$ COMPLETE-OUTPUT PAGE 3

ORT7450

I $P G=4$

ORT7455

$M=M+1$

ORT7 460

GO TO 1510

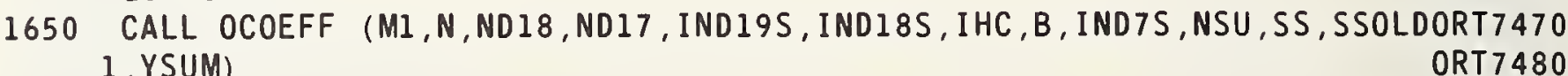
RETURN

1660 IW $=$ I IRGS (2) 
$1670 A($ IND2) $=A($ IND3) $* R C$ (IW)

$I \mathrm{~W}=\mathrm{I} W+1$

GO TO 1690

ORT7580

ORT7590

$1680 \quad A($ IND2) $=A($ IND 3$) * A R G S(2)$

ORT7600

1690 IND $3=I N D 3+1$

1700 IND2 $=$ IND $2+1$

ORT7610

ORT7620

IND $5=N D 5+1$

DO $1720 \mathrm{~J}=1, M$

ORT7630

IF $=$ IF I

A SUM $=0.0$

IND $2=N D 2+1$

DO $1710 \quad I=1, N$

ORT7640

ORT7650

ORT7660

ORT7670

$A S U M=A($ IF $) * A($ IND 2) + ASUM

ORT7680

IND $2=I N D 2+1$

1710 IF $=I F+1$

$A($ IND 5$)=$ ASUM

ORT7690

ORT 7700

ORT7710

ORT7720

I F I $=I F I+N P M$

ORT7730

1720 IND 5 =IND 5+1

ORT 7740

$A D E V=0.0$

ORT7750

$I W=I$ IRGS (2)

ORT7760

IF $I=1$

ORT7770

IND $2=N D 3+1$

ORT 7780

DO $1770 \quad I=1, N$

ORT 7790

IND $5=N D 5+1$

ORT 7800

IF $=$ IF I

ORT 7810

ASUM $=0.0$

ORT7820

DO $1730 \mathrm{~J}=1, M$

ORT7830

$A S U M=A S U M+A(I F) * A(I N D 5)$

ORT7840

$I F=I F+N P M$

1730 IND 5 =IND 5+1

$D E V=A($ IND 2) $-A S U M$

GO TO $(1740,1750)$, NMUI

ORT 7850

ORT7860

ORT7870

ORT7880

$1740 \mathrm{DEV}=\mathrm{DEV} * * 2 * \mathrm{RC}$ (IW)

$I \mathrm{~W}=\mathrm{I} W+1$

ORT 7890

ORT 7900

GO TO 1760

1750 DEV $=D E V * * 2 * A R G S(2)$

ORT7910

ORT 7920

ORT7930

$A D E V=A D E V+D E V$

IND2 $=$ IND $2+1$

ORT7940

ORT7950

ORT7960

IND I $8=N D 18+1+N$

ORT7970

$I M=N D 12$

ORT7980

IND7S=ND $18+N-M$

ORT7990

IND $7=I N D 7 S+1$

ORT 8000

DO $1810 \quad I=1, M$

ORT8010

$I M=I M+I$

ORT 8020

$\mathrm{COEF}=0.0$

ORT 8030

IS $=I M$

ORT 8040

IND $5=N D 5+I$

ORT 8050

DO $1780 \mathrm{~J}=\mathrm{I}, \mathrm{M}$

ORT 8060

$\mathrm{COEF}=\mathrm{COEF}+\mathrm{A}($ IS $) * A($ IND 5$)$

ORT 8070

$I S=I S+J$

1780 IND $5=$ IND5 +1

ORT 8080

$D I F=A(I N D I 8)-C O E F$

ORT 8090

IF (ABS(DIF).GT.0.0) GO TO 1790

ORT 8100

C 8.0 EQUAL NUMBER OF DIGITS IN COMPUTER

ORT 8110 


\begin{tabular}{|c|c|c|}
\hline & $D I G=8.0$ & ORT 8120 \\
\hline & GO TO 1800 & ORT 8130 \\
\hline \multirow[t]{3}{*}{1790} & $D I G=-F L O G I 0(A B S(D I F))+F L O G 10(A B S(C O E F))$ & ORT 8140 \\
\hline & $D I G=A M I N I(8.0, D I G)$ & ORT 8150 \\
\hline & $D I G=A M A X I(-8,0, D I G)$ & ORT 8160 \\
\hline \multirow[t]{2}{*}{1800} & $A($ IND7 $)=D I G$ & ORT 8170 \\
\hline & IND $7=$ IND $7+1$ & ORT 8180 \\
\hline 1810 & IND $18=I N D 18+1$ & ORT 8190 \\
\hline & DELETE GRAM FACTORS, VECTOR NORMS, GRAM DETERMINANT & ORT 8200 \\
\hline & GO TO 1620 & ORT 8210 \\
\hline C & TITLE FOR PRINT & ORT 8220 \\
\hline \multirow[t]{7}{*}{1820} & $I I=I \operatorname{ARGS}(3)+3$ & ORT 8230 \\
\hline & $I B A=I I$ & ORT 8233 \\
\hline & I $B C=L(44)$ & ORT 8235 \\
\hline & IF (II.GT.II) II =II & ORT 8237 \\
\hline & IF (M.GT.I) GO TO 1823 & ORT 8240 \\
\hline & WRITE (IPRINT,2050) M, IARGS (4) & ORT 8241 \\
\hline & GO TO 1530 & ORT 8242 \\
\hline \multirow[t]{10}{*}{1823} & IF (IBA.EQ.I I ) IBC $=\mathrm{L}(45)$ & ORT 8243 \\
\hline & WRITE (IPRINT, 2050) M, I ARGS (4), (L (44), I ARGS (I) , I=5, I I), IBC & ORT 8244 \\
\hline & $00 \quad 1827 \quad J=1,4$ & ORT 8245 \\
\hline & IF (M.LE. $\left.24^{*}(\mathrm{~J}-1)+8\right)$ GO TO 1530 & ORT 8246 \\
\hline & $I I=24 * J+1 I$ & ORT 8247 \\
\hline & III $=$ II -23 & ORT 8248 \\
\hline & I I $=$ MINO (I I , I ARGS $(3)+3)$ & ORT 8249 \\
\hline & IF (II.NE.III) GO TO 1825 & ORT 8250 \\
\hline & WRITE (IPRINT, 2060) I ARGS (I I) & ORT 8252 \\
\hline & GO TO 1530 & ORT 8254 \\
\hline \multirow[t]{2}{*}{1825} & I I I $=$ I I I +1 & ORT 8256 \\
\hline & IF (I I .EQ. IBA) IBC $=L(45)$ & ORT 8257 \\
\hline \multirow[t]{2}{*}{1827} & WRITE (IPRINT, 2060) I ARGS(I I I-1), (L (44), IARGS(I), I=I I I , I I) , IBC & ORT 8258 \\
\hline & GO TO 1530 & ORT 8260 \\
\hline \multirow[t]{2}{*}{1830} & CALL ERROR (10) & ORT 8270 \\
\hline & RETURN & ORT 8280 \\
\hline \multirow[t]{2}{*}{1840} & CALL ERROR (25) & ORT 8290 \\
\hline & RETURN & ORT 8300 \\
\hline C & MORTHO CHECK & ORT 8310 \\
\hline \multirow[t]{3}{*}{1850} & IF (NARGS.EQ.7.OR.NARGS.EQ.9) GO TO 1860 & ORT 8320 \\
\hline & CALL ERROR (10) & ORT 8330 \\
\hline & RETURN & ORT 8340 \\
\hline \multirow[t]{3}{*}{1860} & IF (IARGS (3).GE.IARGS(4)) GO T0 1870 & ORT 8350 \\
\hline & CALL ERROR (26) & ORT 8360 \\
\hline & RETURN & ORT 8370 \\
\hline \multirow[t]{8}{*}{1870} & CALL ADRESS $(2, I X M)$ & ORT 8380 \\
\hline & IF (IXM.LE.0) CALL ERROR (11) & ORT 8390 \\
\hline & IF (IARGS (1)+IARGS(3)-1.GT.NROW) CALL ERROR (17) & ORT 8400 \\
\hline & IF (IARGS (2)+IARGS (4)-1.GT.NCOL) CALL ERROR (17) & ORT 8410 \\
\hline & $I X M=I X M-1+I A R G S(1)$ & ORT 8420 \\
\hline & IF (NERROR.GT.0) RETURN & ORT 8430 \\
\hline & $\mathrm{J}=7$ & ORT 8440 \\
\hline & $\mathrm{J} J=1$ & ORT 8450 \\
\hline \multirow[t]{2}{*}{1880} & CALL ADRESS $(J, I M T R X A(J J, 1))$ & ORT 8460 \\
\hline & IF (IMTRXA(JJ, I).GT.0) GO TO 1900 & ORT 8470 \\
\hline \multirow[t]{2}{*}{1890} & CALL ERROR (11) & ORT 8480 \\
\hline & RETURN & ORT 8490 \\
\hline \multirow[t]{4}{*}{1900} & $\operatorname{IMTRXA}(\mathrm{JJ}, 2)=\operatorname{IARGS}(3)$ & ORT 8500 \\
\hline & IF (JJ.EQ.2) IMTRXA (JJ, 2) $=\operatorname{IARGS}(4)$ & ORT 8510 \\
\hline & $\operatorname{IMTRXA}(\mathrm{JJ}, 3)=\operatorname{IARGS}(4)$ & ORT 8520 \\
\hline & IF (IARGS(J-1).GT.NROW) GO TO 1890 & ORT 8530 \\
\hline
\end{tabular}


$\operatorname{IMTRXA}(J J, 1)=\operatorname{IMTRXA}(J J, 1)-1+\operatorname{IARGS}(J-1)$

ORT 8540

IF (IARGS (J)+IARGS (4)-1.GT.NCOL) IMTRXA (JJ,3)=NCOL $-I A R G S(J)+1 \quad 0 R T 8550$

IF (IARGS $(J-1)+\operatorname{IARGS}(3)-1 . G T$.NROW) IMTRXA (JJ,2)=NROW-IARGS $(\mathrm{J}-1)+1$ ORT 8560

IF (JJ.EQ.2.OR.NARGS.EQ.7) GO TO 1910

ORT 8570

$J=9$

$J J=2$

ORT 8580

ORT 8590

GO TO 1880

1910 IF (NERROR.NE.0) RETURN

ORT 8600

IF (IMTRXA $(1,2) \cdot \operatorname{NE}$. IARGS (3).OR. IMTRXA $(1,3) \cdot \operatorname{NE}$. IARGS (4)) CALL ERRORORT8620

1 (213)

IF (NARGS.EQ.7) GO TO 1920

ORT 8630

ORT 8640

IF (IMTRXA $(2,2)$.NE.IARGS(4).OR.IMTRXA $(2,3)$ ).NE.IARGS(4)) CALL ERRORORT8650 $1(213)$

1920 IF (KIND(5).EQ.1) GO TO 1930

CALL ADRESS $(5, \operatorname{IIRGS}(2))$

IIRGS ( I) =I IRGS (2)

IF (IIRGS (2).GT.0) GO TO 1940

CALL ERROR (11)

RETURN

1930 SU=IARGS (3)

IF (ARGS (5).LE.0.0) CALL ERROR (25)

NMU I $=2$

$\operatorname{KIND}(2)=1$

$\operatorname{ARGS}(2)=\operatorname{ARGS}(5)$

$1940 \quad M=I A R G S$ (4)

DO $1950 \quad I=1, M$

$1950 \operatorname{IIRGS}(I+3)=I X M+(I-1) *$ NROW

$N=I$ ARGS (3)

$\mathrm{FN}=\mathrm{N}$

GO TO 100

C START STORING RESULTS FOR MORTHO

ORT 8660

ORT 8670

ORT 8680

ORT 8690

ORT 8700

ORT 8710

ORT 8720

ORT 8730

ORT 8740

ORT 8750

ORT 8760

ORT 8770

ORT 8780

ORT 8790

ORT 8800

ORT 8810

ORT 8820

ORT 8830

ORT 8840

1960 IST=IMTRXA $(1,1)$

$K=1$

MMTXC $=\operatorname{IMTRXA}(1,3)$

ORT 8850

ORT 8860

ORT 8870

MMT XR =I MTRXA $(1,2)$

ORT 8880

DO $1980 I=1$, MMTXC

ORT 8890

$\mathrm{KK}=\mathrm{K}$

ORT 8900

ISTRR $=$ I ST

ORT 8910

DO $1970 \mathrm{~J}=1$, MMTXR

ORT 8920

$R C($ ISTRR $)=A(K K)$

ORT 8930

$K K=K K+1$

ORT 8940

ORT 8950

ORT 8960

ORT 8970

ORT 8980

ORT 8990

ORT 9000

ORT9010

ORT 9020

ORT9030

ORT 9040

ORT 9050

C

$X C=I M T R X A(2,3)$

1990 FORMAT (/35X,22HLEAST SQUARES FIT FOR, 12A1,11H IN COLUMN, I4) ORT9060
2000 FORMAT (25X,26HAS A POLYNOMIAL OF DEGREE, I2,4H IN, 12A1,11H IN COORT 9070 ILUMN , I4)

ORT 9080

2010 FORMAT (20X,6HUSING, I4,22H NON-ZERO WEIGHTS AND, I 4,24H ZERO WEIGORT9090 IHTS IN COLUMN, I 4$)$

2020 FORMAT (35X,6HUSING, I4, 19H NON-ZERO WEIGHTS $=, 10 \mathrm{AI}$ )

ORT9100

ORT9110

2030 FORMAT (6OHO PLOTS ARE NOT PRINTED BECAUSE NO. OF POINTS IS LESS TORT 9120 
IHAN 3)

ORT9130

2050 FORMAT (23X,24HAS A LINEAR FUNCTION OF, I2,31H PREDICTOR VARIABLESORT9150 1 IN COLUMNS, I $4,8(A 1$, I 4$)$ )

ORT9160

2060 FORMAT (I $4,24(A 1, I 4))$

ORT 9165

END

ORT 9170 
SUBROUTINE ORTHRV ( $A$, NROW, $N, N C O L, I N D, X, N A S I Z E, X P$ )

ORV 10

C VERSION 5.00 ORTHRV 5/15/70

ORV 20

SUBROUTINE ORTHRV ( $A$, NROW, N, NCOL, IND , X, NASIZE, XP)

ORV 30

C SUBROUTINE TO CHECK TO SEE IF MATRIX IS ORTHOGONAL

ORV 40

DIMENSION A (NROW, 1$), X(1)$, IND (1)

ORV 50

DOUBLE PRECISION XP(1)

ORV 60

C IF NUMBER OR ROWS IS GREATER THAN NUMBER OF COLUMNS COMPUTE A'A ORV 70

C OTHERWISE AA'

IF (N.GT.NCOL) GO TO 10

$L 2 P=1$

$M P=N$

GO TO 20

$10 \quad \mathrm{~L} 2 \mathrm{P}=2$

$M P=N C O L$

20 CALL MXTXP (A,NROW, N,NCOL, X, L2P , NASIZE, XP)

I $C=1$

IND $(1)=0$

$\operatorname{IND}(2)=0$

DO $80 \quad I=1, M P$

DO $80 \mathrm{~J}=1, M P$

IF (I.EQ.J) GO TO 40

IF $(X(I C)) 30,80,30$

30 IF (ABS(X(IC)) $-1 . E-7) \quad 60,60,90$

40 IF (X (IC) -1.0$) 50,80,50$

50 IF (ABS(X(IC)-1.0)-1.E-7) $60,60,70$

$60 \quad$ IND $(2)=1$

GO TO 80

ORV 80

ORV 90

ORV 100

ORV 110

ORV 120

ORV 130

ORV 140

ORV 150

ORV 160

ORV 170

ORV 180

ORV 190

ORV 200

ORV 210

ORV 220

ORV 230

ORV 240

ORV 250

ORV 260

ORV 270

ORV 280

ORV 290

ORV 300

ORV 310

ORV 320

ORV 330

ORV 340

ORV 350

ORV 360

ORV 370

ORV 380

ORV 390

ORV 400

ORV 410

ORV 420

ORV 430

ORV 440

ORV 450

ORV 460

ORV 470

ORV 480

ORV 490

ORV 500

ORV 510

ORV 520

ORV 530

ORV 540

ORV 550

ORV 560

ORV 570

ORV 580

ORV 590 
C IND (2)=1 RELATIVE ORTHOGONAL NORMALIZED

ORV 600

IND $(2)=2$ NON-ORTHOGONAL

ORV 610

IND $(2)=-3$ RELATIVE ROWWISE (NORMALIZED)

ORV 620

IND $(2)=3$ EXACT ROWWISE (NORMALIZED)

ORV 630

IND ( 2$)=-4$ RELATIVE COLUMNWISE (NORMALIZED)

ORV 640

IND $(2)=4$ EXACT COLUMNWISE (NORMALIZED)

ORV 650

ORV 660

ORV 670

ORV 680

ORV 690

ORV 700

150 RETURN

ORV 710 
SUBROUT INE ORTPLT( NDI9,ND2,N,SSQ,ND3, IB, IXA, IWS) $\quad$ ORP 10

$\begin{array}{llll}C & \text { VERSION } 5.00 & \text { ORTPLT } 5 / 15 / 70 & \text { ORP } 20\end{array}$

C THIS PROGRAM IS USED BY ORTHO TO GENERATE PLOTS $\quad$ ORP 30

C WRITTEN BY S PEAVY 10/11/69 $\quad$ ORP 40

DIMENSION IU (1), IB(1) $\quad$ ORP 50

EQUIVALENCE (IU,A) $\quad$ ORP 60

$\begin{array}{lll}\text { C } * * * * * & \text { ORP } 70\end{array}$

COMMON/BLOCKD/IARGS (100), KIND (100), ARGTAB (100), NRMAX,NROW,NCOL, ORP 80

INARGS, VWXYZ ( 8$)$, NERROR

COMMON / BLOCRC / NRC, RC (12600)

COMMON/HEADER/NOCARD $(80), I T L E(60,6)$, LNCNT, IPRINT, NPAGE, IPUNCH ORP 110

COMMON / SCRAT /NS, NS2, A (13500)

COMMON / FMAT / I FMTX (6) , IOSWT, IFMTS (6), LHEAD (96)

DIMENSION ARGS(100)

EQUIVALENCE (ARGS(1), RC(12501))

COMMON/ABCOEF/L (48)

COMMON/CONSTS/PI , E , HALFPI , DEG, RAD , XALOG

C

IW $W *$ IWS

$I W S T=1$

IF (KIND(2).EQ.0) GO TO 18310

IWST $=2$

WT =ARGS (2)

$\begin{array}{ll}\text { ORP } & 80 \\ \text { ORP } & 90\end{array}$

ORP 100

ORP 120

ORP 130

ORP 140

ORP 150

ORP 160

ORP 170

ORP 180

ORP 182

ORP 184

ORP 186

ORP 187

ORP 188

18310 IND4 $=$ ND19+1

IND2 $=$ ND $2+1$

$\mathrm{NZW}=\mathrm{N}$

DO $18320 \quad I=1, N$

GO TO $(18312,18314)$, IHST

ORP 190

ORP 200

ORP 205

ORP 210

ORP 211

$18312 W T=R C$ (IW)

$I W=I W+I$

18314 IF (WT.NE.0.0) GO TO 18316

IU $($ IND4) $=27$

$N Z W=N Z W-1$

GO TO 18318

$18316 Z=A$ (IND4) /FSQRT (SSQ/WT-A (IND2)**2)

$I Z=Z \quad / .3$

IF $(Z . G T .0 .0 . A N D . A M O D(Z, .3)$.NE.0.0) IZ $=I Z+1$

IU $($ IND 4$)=I Z+13$

IF (IU (IND 4$) \cdot$ LE.0) IU (IND 4$)=1$

IF (IU (IND4).GT.26) IU (IND4) $=26$

18318 IND2 $=$ IND $2+1$

18320 IND $4=$ IND $4+1$

IND3 $=N D 3+1$

$Y M A X=A($ IND 3$)$

$Y M I N=A($ IND3)

DO $18340 \quad I=2, N$

IND $3=$ IND $3+1$

IF(YMIN.LE.A(IND3)) GO TO 18330

$Y M I N=A$ ( IND 3)

GO TO 18340

18330 IF (YMAX.LT.A (IND3)) YMAX $=A($ IND3)

18340 CONTINUE

YMM=ABS (YMAX-YMIN) $/ 50$.

$Y M X=F L O A T(N-1) / 50$.

CALL PAGE (0)

IPLOT $=1$

WR ITE (IPR INT , 18350)

18350 FORMAT (15X,36HSTANDARDIZED RESIDUALS VS ROW NUMBER, 22X,

1 42HSTANDARDIZED RESIDUALS VS PREDICTED VALUES)

ORP 212

ORP 213

ORP 214

ORP 215

ORP 216

ORP 218

ORP 220

ORP 230

ORP 240

ORP 250

ORP 260

ORP 270

ORP 280

ORP 290

ORP 300

ORP 310

ORP 320

ORP 330

ORP 340

ORP 350

ORP 360

ORP 370

ORP 380

ORP 390

ORP 400

ORP 410

ORP 420

ORP 430

ORP 440

ORP 450

ORP 460 
18355 WRITE(IPRINT, 18360) (L (39), I=1,88)

ORP 470

18360 FORMAT $\left(7 X, 2\left(1 H_{+}, 9 A 1\right), 1 H_{+}, 4 A 1,1 H X, 4 A 1,2(1 H+9 A 1), 1 H_{+}, 10 X, 2\left(1 H_{+}, 9 A 1\right)\right.$ $1,1 \mathrm{H}+, 4 \mathrm{Al}, 1 \mathrm{HX}, 4 \mathrm{Al}, 2(1 \mathrm{H}+, 9 \mathrm{Al}), 1 \mathrm{H}+)$ YYPR $=3.75$

$$
\text { LINE }=26
$$

ORP 480

ORP 490

ORP 500

ORP 510

18390 D0 $20050 \quad I=1,5$

ORP 520

DO 18400 I J I $=1,102$

ORP 530

$18400 \mathrm{IB}(\mathrm{I} \mathrm{J} \mathrm{I})=\mathrm{L}(45)$

GO TO $(18410,19000)$, IPLOT

ORP 540

ORP 550

18410 IND $3=N D 3+1$

ORP 560

IND $4=$ ND $19+1$

ORP 570

DO 18430 I J I $=1, N$

ORP 580

IF (IU(IND4).NE.LINE) GO TO 18420

ORP 590

$I Z=F L O A T(I J I-1) / Y M X+.5$

ORP 600

$I Z=I Z+1$

ORP 610

IF (IZ.LE.0) IZ $=1$

ORP 620

IF (IZ.GT.51) IZ $=51$

ORP 630

$I B(I Z)=L(41)$

$I Z=(A(I N D 3)-Y M I N) / Y M M$

ORP 640

$\mathrm{IZ}=\mathrm{IZ}+1$

ORP 650

IF (IZ.LE.0) IZ $=1$

IF (IZ.GT.5I) IZ $=51$

ORP 660

$I B(I Z+5 I)=L(41)$

18420 IND $4=$ IND $4+1$

18430 IND $3=$ IND3 +1

GO TO 20000

19000 IND $4=N D 19+1$

I $X=I X A$

DO 19010 IJ I $=1, N$

IF (IU(IND4).NE.LINE) GO T0 19005

$I Z=(R C(I X)-X M I N) / X M M$

$I Z=I Z+1$

IF (IZ.LE.0) IZ $=1$

IF (IZ.GT.51) IZ $=51$

$I B(I Z)=L(41)$

RATI $0=($ AN-GAMMA $) /$ FDEN

YMM $=4.91 *($ RATI $* * * .14-(1 .-$ RATIO $) * * .14)$

$A N=A N-1.0$

ORP 670

ORP 680

ORP 690

ORP 700

ORP 710

ORP 720

ORP 730

ORP 740

ORP 750

ORP 760

ORP 770

ORP 780

ORP 790

ORP 800

ORP 810

ORP 820

ORP 830

ORP 840

IF (AN.LT.2. AND. NZW.LE.10) GAMMA $=1.13$.

ORP 850

$I \bar{L}=Y M M / . I$

ORP 860

$I Z=I Z+26$

IF (IZ.LE.0) IZ $=1$

$I F(I Z . G T .51) \quad I Z=51$

$I B(I Z+5 I)=L(4 I)$

19005 I $X=I X+1$

19010 IND $4=$ IND $4+1$

ORP 870

ORP 880

ORP 890

ORP 900

ORP 910

ORP 920

ORP 930

20000 IF (I-1) $20010,20010,20030$

ORP 940

20010 WRITE (IPRINT, 20020 ) YYPR, ( IB ( I J I), I J I=1,51), YYPR, (IB ( I J I ), I J I =52, 1102 )

ORP 950

ORP 960

20020 FORMAT $\left(1 X, F 5.2,1 H_{+}, 51 \mathrm{Al}, 1 \mathrm{H}_{+}, 3 \mathrm{X}, \mathrm{F} 5.2,1 \mathrm{H}+, 51 \mathrm{Al}, 1 \mathrm{H}_{+}\right)$

ORP 970

GO TO 20045

ORP 980

20030 WRITE (IPRINT, 20040) (IB (I J I) , I J I=1, 102)

ORP 990

20040 FORMAT $\left(6 X, 1 H_{-}, 51 \mathrm{Al}, 1 \mathrm{H}_{-}, 8 \mathrm{X}, 1 \mathrm{H}_{-}, 51 \mathrm{Al}, 1 \mathrm{H}-\right)$

ORP 1000

20045 LINE $=$ LINE-1

ORP 1010

IF (LINE.EQ.0) GO TO 20060

ORP 1020

20050 CONTINUE

ORP 1030

$Y Y P R=Y Y P R-1.5$

ORP 1040

GO TO 18390

ORP 1050

20060 WRITE (IPRINT, 18360) (L (39), I=1,88) 
G0 T0 $(20070,21100)$, IPLOT

ORP 1060

$20070 \quad Y M M=Y M X * 25.0+1.0$

ORP 1070

$Y M M Y=(Y M A X-Y M I N) / 2+Y M I N$

ORP 1080

WRI TE (IPRINT, 20080) YMM, N, YMIN, YMMY, YMAX

ORP 1090

20080 FORMAT (6X,3H1.0,18X,F9.4,16X, I5,2H.0 ,1PE15.4,E26.4,10X,E10.4)

ORPI100

WRITE (IPRINT, 20090)

ORPII10

20090 FORMAT (IH )

I PLOT $=2$

ORP 1120

$I X=I X A$

ORP 1130

$X M A X=R C(I X)$

ORP 1140

ORP 1150

$X M I N=R C(I X)$

ORP 1160

DO $21000 \quad I=2, N$

ORP 1170

$I X=I X+1$

ORP 1180

IF (RC (IX) . GT .XMAX) XMAX $=R C(I X)$

ORP 1190

IF (XMIN.GT.RC (IX)) XMIN=RC (IX)

ORP 1200

21000 CONTINUE

$X M M=A B S(X M A X-X M I N) / 50$.

ORP 1210

ORP 1230

$G A M M A=P I / 8.0$

ORP 1240

$A N=N Z W$

ORP 1245

$\mathrm{FDEN}=\mathrm{AN}-2 .{ }^{*} \mathrm{GAMMA}+1.0$

ORP 1250

WR ITE (IPRINT , 21010) (LHEAD (I) I I=13,24)

ORP 1270

21010 FORMAT (14X,26HSTANDARDIZED RESIDUALS VS, 12AI, 21X,

ORP 1280

142 HPROBABILITY PLOT OF STANDARDIZED RESIDUALS)

ORP 1290

GO TO 18355

$21100 \quad Y M M Y=(X M A X-X M I N) \quad 12,+X M I N$

ORP 1300

ORP 1310

WRITE (IPRINT, 21110) XMIN, YMMY, XMAX

21110 FORMAT(IPE13.4,14X, E12.4, 8X, E12.4,7X,4H-2.5,22X,3H0.0,22X,

ORP 1320

ORP 1330

$13 \mathrm{H} 2.5)$

RETURN

ORP 1340

ORP 1350

END

ORP 1360 
C VERSION 5.00 OUTPUT $5 / 15 / 70$

COMMON /BLOCKA/ MODE, M,KARD (83), KARG, ARG, ARG2, NEWCD (80), KRDEND

COMMON /BLOCKB/ NSTMT, NSTMTX,NSTMTH, NCOM, LCOM, IOVFL, COM(2000)

COMMON /BLOCRC/ NRC, RC (12600)

COMMON /BLOCKD/ IARGS(100), KIND (100), ARGTAB (100), NRMAX, NROW, NCOL, NOUT 60

IARGS, VWXYZ (8), NERROR

OUT 70

DIMENSION ARGS $(100)$

OUT 80

EQUIVALENCE (ARGS (1), RC (12501))

COMMON /BLOCKC / KIO, INUNIT, ISCRAT , KBDOUT, KRDKNT, LLIST

OUT 90

OUT 100

C

C WRITE RECORD ON SCRATCH UNIT

OUT 110

OUT 120

C

IF (NERROR.EQ.0.AND.LLIST.EQ.0) GO TO 10

OUT 130

IF (MODE.EQ.3) GO TO 20

OUT 140

WRITE (ISCRAT, 30) NEWCD

OUT 150

10 RETURN

OUT 160

OUT 170

20 I =NSTMT / 10

OUT 180

WRITE (ISCRAT, 40) I, NEWCD

OUT 190

GO TO 10

OUT 200

\section{C}

30 FORMAT (4X,80AI)

40 FORMAT $(1 \mathrm{H}+,[3,80 \mathrm{AI})$

END

OUT 210

OUT 220

OUT 230

OUT 240 
SUBROUTINE PACK (NWORD, MWORD, NO, IP)

PAC 10

VERSION 5.00 PACK $5 / 15 / 70$

PAC 20

WRITTEN BY S PEAVY $9 / 17 / 69$

NWORD CONTAINS CHARACTERS TO BE PACKED OR UNPACKED

PAC 30

MWORD THE PACKED CHARACTERS IN CODED FORM (SEE BELOW) OR THE

PAC 40

C

C

C

NO NO OF CHARACTERS TO BE PACKED OR UNPACKED

IP IP $=0 \quad P A C K$

IP IP=1 UNPACK

PAC 50

PAC 60

PAC 70

PAC 80

PAC 90

PAC 100

THE CHARACTERS ARE PACKED IN A CODED FORM. EACH CHARACTER HAS BEENPAC 110 ASSIGNED A VALUE IN OMCONV. THIS VALUE IS I LESS THAN THE

PAC 120 SUBSCRIPT OF L (IN LABELED COMMON ABCDEF) FOR THAT PARTICULAR

PAC 130 CHARACTER. THESE VALUES ARE STORED IN KARD. THE VALUES OF THE

PAC 140 CHARACTERS ARE PACKED AS FOLLOWS

PAC 150 MWORD $(I)=(\operatorname{KARD}(K)+1) * 2 * * 16+(\operatorname{KARD}(K-1)+1) * 2 * * 8+\operatorname{KARD}(K-2)+1$ PAC 160

COMMON /ABCDEF/ L (48)

PAC 170

DIMENSION NWORD (1), MWORD (1)

PAC 180

$\mathrm{KB}=1$

$K A=1$

PAC 190

IF (IP.EQ.1) GO TO 30

PAC 200

PAC 210

C PACK

PAC 220

$10 \quad M W O R D(K A)=0$

PAC 230

DO $20 \quad I=1,3$

PAC 240

MWORD $(K A)=$ MWORD $(K A) * 256+N$ WORD $(K B)+1$

PAC 250

$K B=K B+1$

PAC 260

IF (KB.GT.NO) GO TO 22

PAC 270

CONTINUE

PAC 280

20

$K A=K A+1$

PAC 290

PAC 300

GO TO 10

PAC 310

PAC 311

PAC 312

IF (ICE.EQ.0) RETURN

PAC 313

ICE $=3-I C E$

PAC 314

PAC 315

ICE $=$ ICE-I

PAC 316

IF (ICE.EQ.0) RETURN

PAC 317

GO TO 24

PAC 320

PAC 330

$30 \quad$ I $C A=N$ WORD (KB)

PAC 340

ICD $=65536$

PAC 350

DO $40 \quad I=1,3$

PAC 360

$I C B=I C A / I C D$

PAC 370

IF (ICB.EQ.0) GO TO 40

PAC 380

MWORD $(K A)=L(I C B)$

PAC 390

$K A=K A+1$

PAC 400

IF (KA.GT.NO) RETURN

PAC 410

ICA $=I C A-I C B * I C D$

$40 \quad I C D=I C D / 256$

PAC 420

$K B=K B+1$

PAC 430

GO TO 30

PAC 440

END

PAC 450 
BRING UP A NEW PAGE AND PRINT OMNITAB CARD AND PAGE NUMBER THEN, IF $J=0$, DONE

$$
\begin{aligned}
& \mathrm{J}=1, \text { PRINT TITLEI } \\
& \mathrm{J}=2, \text { PRINT TITLEI, } 2 \\
& \text { ETC. FOR } \mathrm{J}=3,4
\end{aligned}
$$

PAG 30

PAG 40

PAG 50

PAG 60

PAG 70

PAG 80

THIS ROUTINE ASSUMES THAT THE EXECUTIVE SYSTEM LEAVES

PAG 90

PAG 100 COMMON / HEADER / NOCARD $(80), \operatorname{ITLE}(60,6)$, LNCNT, IPR INT , NPAGE , IPUNCH

PAG 110 NPAGE $=N P A G E+1$

WRITE (IPRINT, 20) NOCARD, NPAGE

PAG 150

PAG 160

IF (J.LE.O.OR.J.GT.4) GO TO 10

PAG 180

WRITE (IPRINT, 30) ((ITLE (I, I I) , I=1,60), I I=1, J)

PAG 190

10 RETURN

PAG 200

C

20 FORMAT $(1 \mathrm{H} 1,19 X, 80 \mathrm{Al}, 10 \mathrm{X}, 4 \mathrm{HPAGE}, \mathrm{I} 4)$

PAG 210

PAG 220

FORMAT $(1 X, 120 A 1 / 1 X, 120 A 1)$

END

PAG 230

PAG 240 


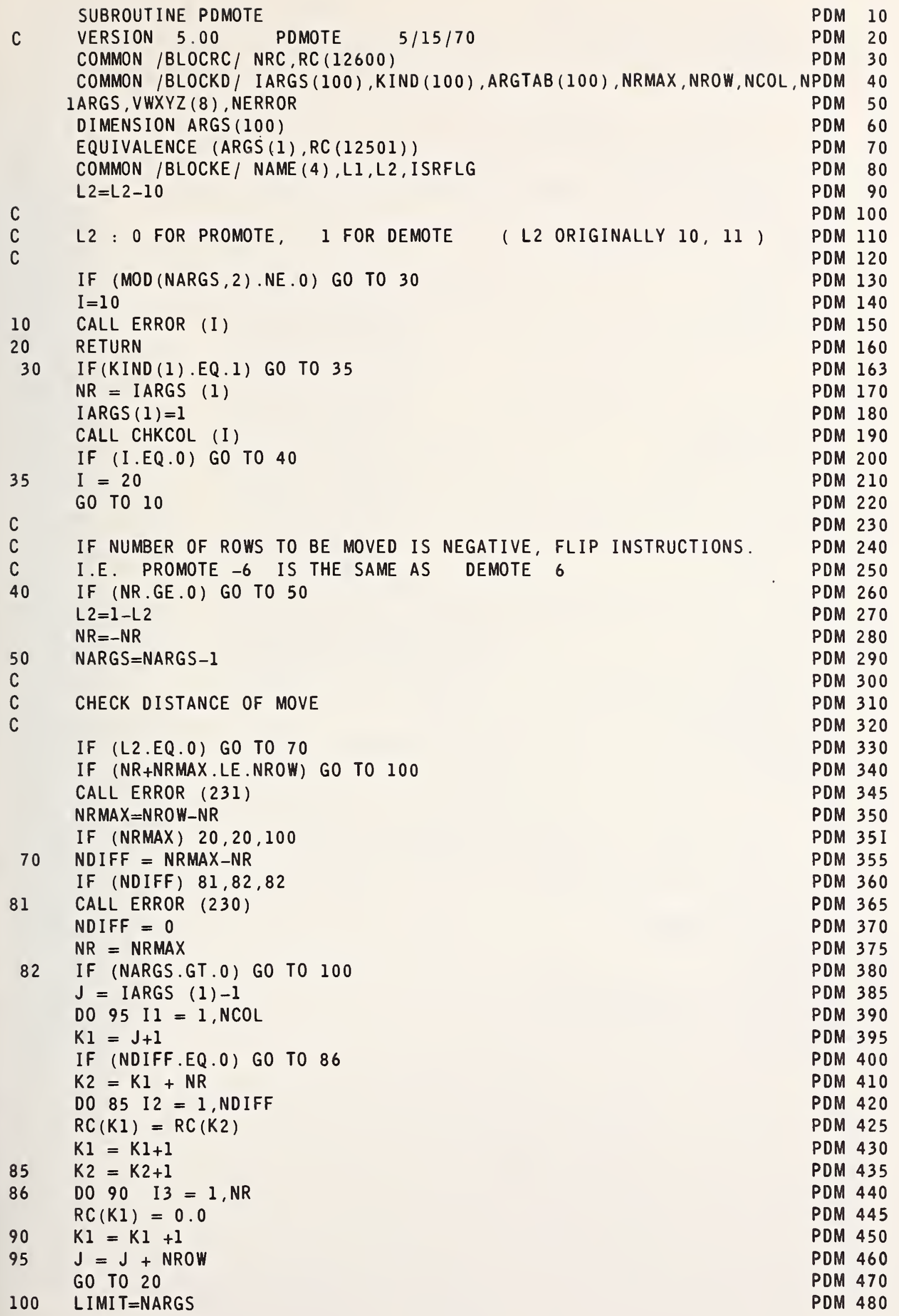


IF (LIMIT.EQ.0) LIMIT $=2 * N C O L$

PDM 490

IF (NERROR.NE.0) GO TO 20

PDM 500

IF (NRMAX.NE.0) GO TO 110

PDM 510

GO TO 10

PDM 520

C

START PROMOTING OR DEMOTING

PDM 530

PDM 540

PDM 550

PDM 560

DO 180 I=1,LIMIT , 2

IF (NARGS.NE.0) GO TO 120

PDM 570

$K I=I A R G S(1)$

$K 2=K 1$

$\operatorname{IARGS}(1)=\operatorname{IARGS}(1)+$ NROW

PDM 580

PDM 590

PDM 600

GO TO 130

PDM 610

PDM 620

$K I=I$ ARGS $(I+1)$

$K 2=\operatorname{IARGS}(I+2)$

PDM 630

PDM 640

PDM 650

DEMOTE COL AT KI TO COL AT K2

PDM 660

PDM 670

$K 1=K 1+N R M A X$

$K 2=K 2+N R M A X+N R$

PDM 680

PDM 690

DO $140 \mathrm{~J}=1$, NRMAX

PDM 700

$\mathrm{Kl}=\mathrm{K} 1-1$

$K 2=K 2-1$

$140 \quad \mathrm{RC}(\mathrm{K} 2)=\mathrm{RC}(\mathrm{K} 1)$

GO TO 180

PDM 710

PDM 720

PDM 730

PDM 740

C

PROMOTE COL AT K1 TO COL AT K2

PDM 750

PDM 760

PDM 770

C

$150 \mathrm{JJ}=N R M A X-N R$

IF (JJ.EQ.0) GO TO 165

$\mathrm{K} I=\mathrm{K} 1+\mathrm{NR}$

DO $160 \mathrm{~J}=1, \mathrm{JJ}$

$R C(K 2)=R C(K 1)$

$\mathrm{Kl}=\mathrm{K} 1+\mathrm{l}$

$160 \quad K 2=K 2+1$

C

IF PROMOTE ARRAY, FILL REST OF COLUMN WITH ZEROES.

POM 780

POM 785

PDM 790

PDM 800

PDM 810

PDM 820

PDM 830

PDM 840

PDM 850

PDM 860

IF (NARGS.NE.0) GO TO 180

PDM 870

PDM 880

$\mathrm{JJ}=\mathrm{J} J+1$

DO $170 \mathrm{~J}=\mathrm{JJ}$, NRMAX

PDM 890

$\mathrm{RC}(\mathrm{K} 2)=0$.

PDM 900

$\mathrm{K} 2=\mathrm{K} 2+1$

PDM 910

PDM 920

CONTINUE

PDM 930

IF (L2.NE.0) NRMAX =NRMAX +NR

PDM 940

GO TO 20

PDM 950 
SUBROUTINE PHYCON (NAME)

PHYCON

$5 / 15 / 70$

PHY 10

VERSION 5.00

COMMON /BLOCKA/ MODE, M, KARD (83), KARG, ARG , ARG2, NEWCD (80), KRDEND

PHY 20

COMMON / PCONST / JPC, P (40), N (40)

REMOVE

PHYSICAL CONSTANT LIST

PHY 30

PHY 40

PHY 50

PHY 60

PHY 70

PHY 80

ENTRIES ARE IN PAIRS, FIRST MKS VALUE, THEN CGS (ELECTROMAGNETIC)

PHY 90

PHY 100

PHY 110

PI PI

E BASE OF NATURAL LOGS

C SPEED OF LIGHT IN VACUUM

Q ELEMENTARY CHARGE

N AVOGADRO CONSTANT

ME ELECTRON REST MASS

MP PROTON REST MASS

F FARADAY CONSTANT

H PLANCK CONSTANT

ALPHA FINE STRUCTURE CONSTANT

QME CHARGE TO MASS RATIO FOR ELECTRON

RINF RYDBERG CONSTANT

GAMMA GYROMAGNETIC RATIO OF PROTON (CORRECTED FOR H2O)

MUB BOHR MAGNETON

$R \quad$ GAS CONSTANT

K BOLTZMANN CONSTANT

CONE FIRST RADIATION CONSTANT

CTWO SECOND RADIATION CONSTANT

SIGMA STEPHAN-BOLTZMANN CONSTANT

G

GRAVITATIONAL CONSTANT

IF NAME .LE. 0, NAME = INDEX FROM MKS, CGS $0=$ CGS, $-1=$ MKS

PHY 120

PHY 130

PHY 140

PHY 150

PHY 160

PHY 170

PHY 180

PHY 190

PHY 200

PHY 210

PHY 220

PHY 230

PHY 240

PHY 250

PHY 260

PHY 270

PHY 280

PHY 290

PHY 300

PHY 310

PHY 320

PHY 330

PHY 340

PHY 350

PHY 355

PHY 360

PHY 370

PHY 380

PHY 390

PHY 400

PHY 410

PHY 420

PHY 430

PHY 440

PHY 450

PHY 460

PHY 470 
(THEIR VALUES AND NUMBER REPRESENTATIGN)

PHC 30

BLOCK DATA

COMMON / PCONST / JPC , P (40), N (40)

PCONST DEFINES PHYSICAL CONSTANT VALUES SI UNITS CGS UNITS

SEE BELOW FOR FURTHER COMMENTS

$\begin{array}{ll}P I & P(1)=3.1415926535 \\ E & P(3)=2.718281828459 \\ C & P(5)=2.997925 E 8 \\ Q & P(7)=1.60210 E-19 \\ N & P(9)=6.02252 E 23 \\ \text { ME } & P(11)=9.1091 E-31 \\ \text { MP } & P(13)=1.67252 E-27 \\ F & P(15)=9.64870 E 4 \\ \text { H } & P(17)=6.6256 E-34 \\ \text { ALPHA } & P(19)=7.29720 E-3 \\ \text { QME } & P(21)=1.758796 E 11 \\ \text { RINF } & P(23)=10973731 . \\ \text { GAMMA } & P(25)=2.67519 E 8 \\ \text { MUB } & P(27)=9.2732 E-24 \\ \text { R } & P(29)=8.3143 \\ \text { K } & P(31)=1.38054 E-23 \\ \text { CONE } & P(33)=3.7415 E-16 \\ \text { CTWO } & P(35)=1.43879 E-2 \\ \text { SIGMA } & P(37)=5.6697 E-8 \\ \text { G } & P(39)=6.670 E-11\end{array}$

$P(2)=3.1415926535$

$P(4)=2.718281828459$

PHC 40

PHC 50

PHC 60

PHC 70

PHC 80

PHC 90

PHC 100

PHC 110

$P(6)=2.997925 E 10$

$P(8)=1.60210 E-20$

PHC 120

PHC 130

$P(10)=6.02252 E 23$

$P(12)=9.1091 E-28$

$P(14)=1.67252 E-24$

$P(16)=9648.70$

$P(18)=6.6256 E-27$

$P(20)=7.29720 E-3$

$P(22)=17587960$.

$P(24)=109737.31$

$P(26)=26751.9$

$P(28)=9.2732 E-21$

$P(30)=8.3143 E 7$

PHC 140

PHC 150

PHC 160

PHC 170

PHC 180

PHC 190

PHC 200

PHC 210

PHC 220

PHC 230

PHC 240

PHC 250

$P(32)=1.38054 E-16$

$P(34)=3.7415 E-5$

PHC 260

PHC 270

PHC 280

$P(36)=1.43879$

PHC 290

DATA $P(1), P(2), P(3), P(4), P(5), P(6), P(7), P(8), P(9), P(10)$ /

PHC 300

$12 * 3.1415926535,2 * 2.718281828459,2.997925 E 8,2.997925 E 10$,

$21.60210 \mathrm{E}-19,1.60210 \mathrm{E}-20,2 * 6.02252 \mathrm{E} 23 /$

PHC 310

PHC 320

DATA P(11),P(12),P(13), P(14),P(15),P(16),P(17),P(18),P(19),P(20)/ PHC 330

$19.1091 \mathrm{E}-31,9.1091 \mathrm{E}-28,1.67252 \mathrm{E}-27,1.67252 \mathrm{E}-24,9.64870 \mathrm{E} 4,9648.70, \mathrm{PHC} 340$

$26.6256 E-34,6.6256 E-27,2 * 7.29720 E-3 /$

PHC 350

DATA $P(21), P(22), P(23), P(24), P(25), P(26), P(27), P(28), P(29), P(30) / P H C 360$

$11.758796 E 11,17587960 \ldots 10973731 ., 109737.31,2.67519 E 8,26751.9$,

PHC 370

$29.2732 E-24,9.2732 E-21,8.3143,8.3143 E 7 /$

PHC 380

DATA $P(31), P(32), P(33), P(34), P(35), P(36), P(37), P(38), P(39), P(40) / P H C ~ 390$

$11.38054 E-23,1.38054 E-16,3.7415 E-16,3.7415 E-5,1.43879 E-2,1.43879$, PHC 400

$25.6697 E-8,5.6697 E-5,6.670 E-11,6.670 E-8 /$

DATA $N(1), N(2), N(3), N(4), N(5), N(6), N(7), N(8), N(9), N(10) /$

$111907,3645,2187,12393,10206,9612,9909,4374,5832,1069 /$

PHC 410

PHC 420

PHC 430

PHC 440

DATA $N(11), N(12), N(13), N(14), N(15), N(16), N(17), N(18), N(19), N(20) /$

$112749,13379,5143,10046,13122,8019,2606,2750,14101,5103 /$

C PHYSICAL CONSTANTS INTEGER REPRESENTATION

C N $(1)=11907=P I \quad$ (PI)

C N $(2)=3645=E$

(BASE OF NATURAL LOGS)

C N $(3)=2187=C$

(SPEED OF LIGHT IN VACUUM)

C N $(4)=12393=Q$

(ELEMENTARY CHARGE)

C $N(5)=10206=N$

(AVOGADRO CONSTANT)

C N $(6)=9612=$ ME

(ELECTRON REST MASS)

C N $(7)=9909=M P$

(PROTON REST MASS)

C N $(8)=4374=F$

(FARADAY CONSTANT)

C. $N(9)=5832=H$

(PLANCK CONSTANT)

C $N(10)=1069=$ ALPHA

C $N(11)=12749=$ QME

C $N(12)=13379=$ RINF

(FINE STRUCTURE CONSTANT)

(CHARGE TO MASS RATIO FOR ELECTRON)

(RYDBERG CONSTANT)

PHC 450

PHC 460

PHC 470

PHC 480

PHC 490

PHC 500

PHC 510

PHC 520

PHC 530

PHC 540

PHC 550

PHC 560

PHC 570

PHC 580

PHC 590 


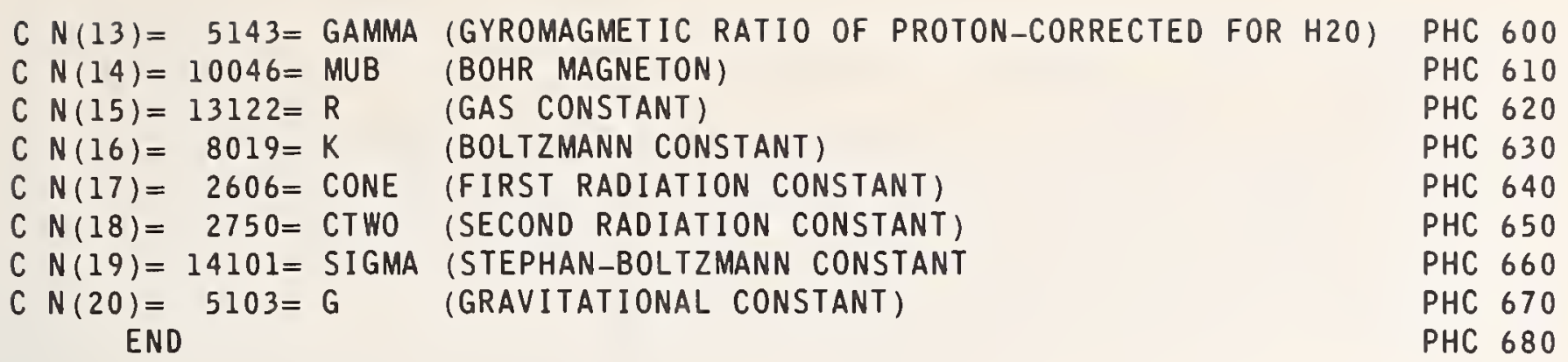


THIS ROUTINE PLOTS MAX. OF 5 CURVES. IF MORE THEN ONE POINT FALLS PLO 40 ON THE SAME POSITION A TALLY IS KEPT AND THE NUMBER IS PRINTED. PLO 50 THE USER MAY PROVIDE THE BOUNDS ON THE $X, Y$ COORDINATES. $\quad$ PLO 60 IF BOUNDS ARE PROVIDED, THEY MUST APPEAR IN PAIRS AS READ NOS. IF APLO 70 PAIR OF REAL NOS ARE EQUAL THE PROGRAM ASSUMES THERE ARE NO BOUNDSPLO 80 COMMANDS FOR USING THIS PLOT ARE AS FOLLOWS

PLO 90

FOR THE AXIS THAT PAIR REPRESENTS AND THE BOUNDS WILL BE CALCULAT-PLO 100

ED.

COMMANDS FOR USING PLOT ARE AS FOLLOWS

PLO 110

I PLOT $Y+++,+++, \ldots X+++$

II PLOT $Y+++,+++\ldots \ldots,(Y M I N, Y M A X) X+++(X M I N, X M A X)$

PLO 120

II I PLOT $Y+++,+++\ldots \ldots$ (YMIN, YMAX) $X++1$

PLO 130

IV PLOT $Y+++,+++, \ldots ., V_{+++}$(XMIN, XMAX)

PLO 140

$\checkmark \quad P L O T Y+++,+++\ldots X$ (XMIN, XMAX) (YMIN, YMAX)

PLO 150

PLO 160

PLO 170

PLO 180

\section{ERRORS}

I WHEN TYPE II COMMAND IS USED THERE MUST BE TWO PAIRS OF REAL PLO 200 NOS. OTHERWISE THE FOLLOWING MESSAGE IS PRINTED ' $Y$ BOUNDS ARE NOT SET UP CORRECTLY'

I IF BOUNDS ARE PROVIDED, THEN THERE MUST BE FOUR REAL NOS. PLO 230

II IF A SINGLE REAL NO. APPEARS AHEAD OF COLUMN NOS., THE FOLLOW-PLO 240 ING MESSAGE WILL BE PRINTED AND NO PLOTTING WILL TAKE PLACE PLO 250 ' $Y$ BOUNDS ARE NOT SET UP CORRECTLY'

PLO 260

II I IF A PLOT COMMAND ENDS WITH ONE REAL NO, THE FOLLOWING MESSAGEPLO 270 WILL BE PRINTED AND PLOTTING WILL BE TERMINATED $' X$ BOUNDS ARE NOT SET UP CORRECTLY'

PLO 290

IV IF MORE THEN 5 PLOTS ARE REQUESTED PER GRAPH, NO GRAPH WILL BEPLO 300 PRODUCED AND FOLLOWING MESSAGE WILL BE PRINTED.

PLO 310 ' MORE THEN 5 PLOTS WERE REQUISTED PER GRAPH'

PLO 320

COMMON /BLOCKE/ NAME (4),L1,L2, ISRFLG

PLO 330 COMMON /BLOCRC/ NRC, RC (12600)

PLO 340

COMMON /BLOCKD/ IARGS(100), KIND(100), ARGTAB (100), NRMAX,NROW, NCOL, NPLO 350 IARGS, VWXYZ (8), NERROR

DIMENSION ARGS $(100)$

EQUIVALENCE (ARGS(1),RC(12501))

COMMON /SCRAT/ NS,NS2, A(13500)

COMMON /HEADER/ NOCARD $(80)$, ITLE $(60,6)$, LNCT, IPRINT , NPAGE, IPUNCH COMMON /FMAT / IFMTX (6), IOSWT, IFMTS (6), LHEAD (96)

DIMENSION TIT $(60), \operatorname{TITX}(60)$

EQUIVALENCE (TIT, ITLE $(1,6))$, (TITX, ITL.E $(1,5)$ )

DIMENSION $X(1), \operatorname{KCCL}(6), \operatorname{PRINT}(101), X P(6), \operatorname{BOOL}(5), \operatorname{IDGT}(9)$

EQUIVALENCE (RC $(1), X(1)),(P R I N T, A)$

INTEGER PRINT, BLANK

EQUIVALENCE (XO,XMIN), (XI,XMAX), (YO,YMIN), (Y $1, Y M A X)$

DIMENSION IH $(12,8), \operatorname{IPR}(101)$

PLO 360

PLO 370

PLO 380

PLO 390

PLO 400

PLO 410

PLO 420

PLO 430

PLO 440

PLO 450

PLO 460

PLO 470

PLO 480

EQUIVALENCE (LHEAD, IH), (IPR, A(200))

INTEGER BOOL

PLO 490

PLO 500

DATA BOOL (1), BOOL (2),BOOL (3),BOOL (4),BOOL (5)/IH . , IH*, IH+, IH, , IH- /,PLO 510 IBLANK/IH /

PLO 520

DATA IDGT (1), IDGT (2), IDGT(3), IDGT (4), IDGT (5), IDGT (6), IDGT (7), IDGT(PLO 530

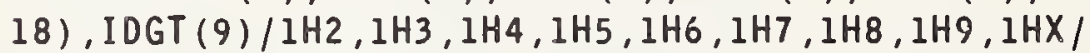

INITIAL SWITCHES

DATA IXPR/IHX/

IF (NRMAX.GT.0) GO TO 10

CALL ERROR (9)

RETURN

PLO 540

PLO 550

PLO 560

PLO 570

PLO 580

PLO 590 
$Y U P=1 . E+35$

PLO 630

$Y D O W N=-1 . E+35$

PLO 640

$\mathrm{NCN}=0$

IPT $=100$

IPTX $=101$

IF (L2.NE.6) GO TO 20

PLO 650

PLO 660

PLO 670

PLO 680

IPT $=60$

PLO 690

IPTX $=61$

PLO 700

PLO 710

PLO 720

IF (KIND(NARGS).EQ.0) GO TO 40

IF (KIND (NARGS)-KIND (NARGS-1). NE.0) GO T0 680

PLO 730

PLO 740

PLO 750

PLO 760

IF (KIND (NARGS-2).EQ.0) GO TO 30

PLO 770

IF (KIND(NARGS-3).EQ.0) GO TO 680

PLO 780

I $S W T=5$

YUP $=$ ARGS (NARGS)

YDOWN=ARGS (NARGS-1)

$X U P=A R G S(N A R G S-2)$

PLO 790

PLO 800

PLO 810

$X D O W N=A R G S$ (NARGS-3)

PLO 820

NARGS=NARGS -4

GO TO 50

C $X$ BOUNDS ARE PROVIDED

PLO 830

PLO 840

PLO 850

PLO 860

PLO 870

$X U P=A R G S$ (NARGS)

XDOWN=ARGS (NARGS-1)

NARGS=NARGS -2

IF (NARGS.EQ.2) GO TO 50

C CHECK TO SEE IF THERE ARE $Y$ BOUNDS

40 IF (KIND (NARGS-1)-KIND (NARGS-2).NE.0) GO TO 670

IF (KIND (NARGS-1).EQ.0) GO TO 50

C Y LIMITS ARE PROVIDED

I SWT $=I S W T+1$

YUP $=$ ARGS $($ NARGS-1)

YDOWN=ARGS (NARGS-2)

I ARGS (NARGS-2) =I ARGS (NARGS)

KIND (NARGS -2$)=0$

NARGS=NARGS -2

50 DO $60 \quad I=1$, NARGS

$60 \operatorname{KCCL}(\mathrm{I})=\operatorname{IARGS}(\mathrm{I})$

$M=N A R G S-1$

IF (NARGS.GT.6) GO TO 710

PLO 880

PLO 890

PLO 900

PLO 910

PLO 920

PLO 930

PLO 940

PLO 950

PLO 960

PLO 970

PLO 980

PLO 990

PLO1000

PLO1010

PL01020

PLO1030

PLO1040

CALL CHKCOL (J)

IF (J.GT.O) GO TO 690

C NO ERROR FOUND IN COLUMN NOS.

PL01050

PLO1060

IF (NERROR.GE. I) RETURN

C SEARCH FOR MAX AND MIN ON AXIS, IF BOUNDS ARE NOT PROVIDED,

PLO1070

PLO1080

PLO1090

PLO1100

OTHERWISE TALLY NO OF POINTS THAT FALL OUTSIDE OF BOUNDS.

IF (XUP.GE.XDOWN) GO TO 70

$X A P=X D O W N$

$X A N=X U P$

GO TO 80

PLO1110

PLO1120

PLO1130

PLO1140

$X A P=X U P$

$X A N=X D O W N$

PLO1150

PLO1160

IF (YUP.GE. YDOWN) GO TO 90

PL01170

$Y A P=Y D O W N$

PL01180 


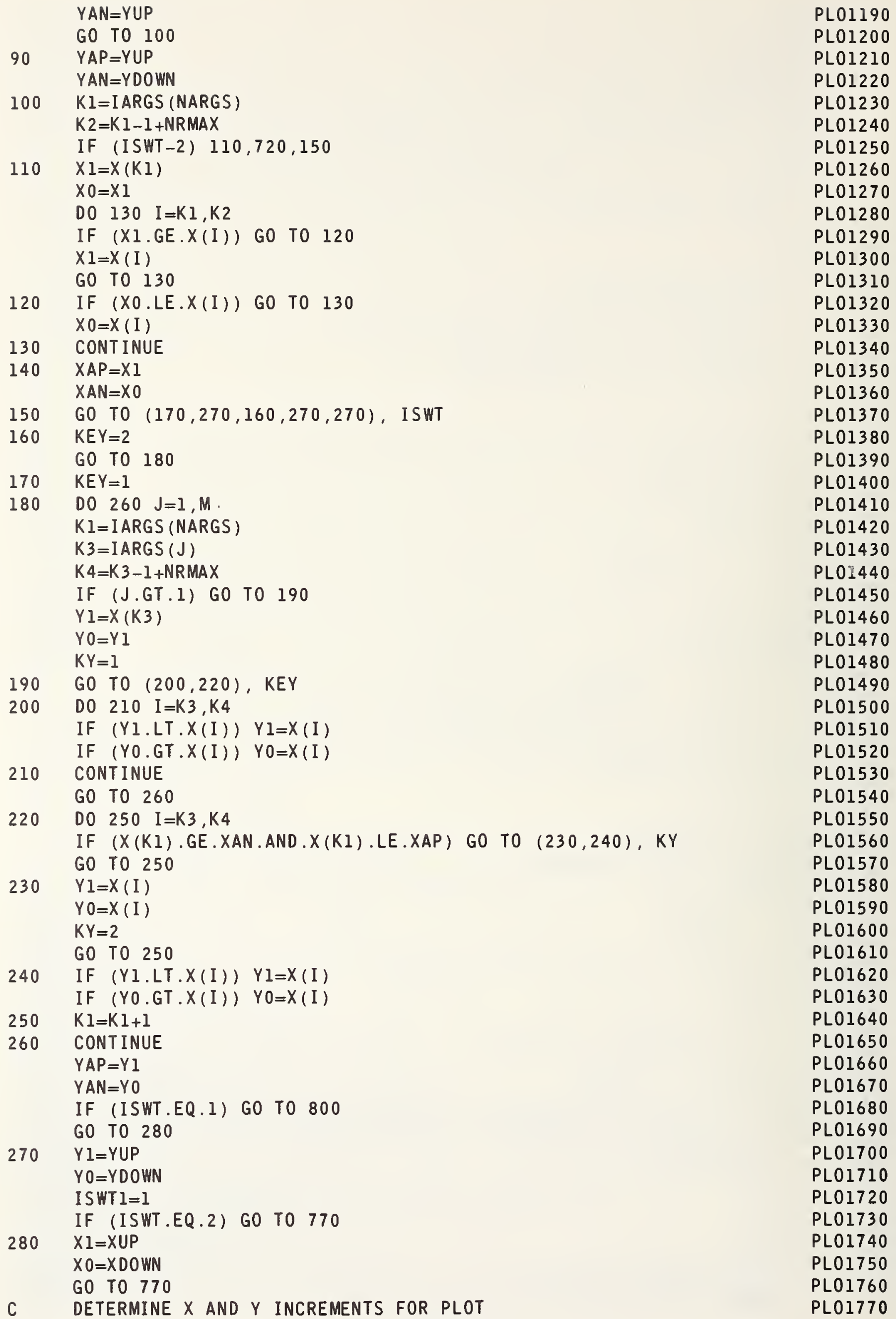


$Y L=Y M A X-Y D E L T A / 2$.

PL01810

$Y T=Y M A X$

PL01820

IF (ISWT.LE. I) GO TO 820

PL01830

IF (L2.EQ.6) GO TO 300

PL01840

WRITE (IPRINT, 1050) NTOT, NCN

PL01850

GO TO 820

PL01860

300 WRITE (IPRINT,940) NTOT, NCN

PL01870

GO TO 820

PL01880

$\mathrm{KYTL}=1$

PL01890

IF (YMAX.LT.YMIN) KYTL $=2$

PLO1900

$\mathrm{KXTL}=1$

PL01910

IF (XMAX.LT.XMIN) KXTL $=2$

PL01920

I TB $=1$

PL01930

PLO1940

THE I LOOP CONTROLS THE 5 DIVISIONS OF THE $Y$ ORDINATE

PL01950 DO $620 \quad I=1,6$

$L=1$

C THE J LOOP IS FOR EACH LINE OF PRINT WITHIN THE DIVISIONS

PL01960

DO $620 \mathrm{~J}=1,10$

PL01970

PLO1980

PL01990

BLANK OUT PRINT BUFFER LINE.

PL02000

DO $320 \mathrm{~K}=1$, IPTX

PL02010

320 PRINT $(K)=B L A N K$

C THE KK INDEX IS FOR EACH CURVE. KK LESS THAN 6.

PLO2020

DO $500 \mathrm{KK}=1, M$

PL02030

$K 3=\operatorname{IARGS}(K K)$

PL02040

$K 4=K 3-1+N R M A X$

PL02050

$\mathrm{K} 5=\mathrm{Kl}$

C THIS DETERMINES IF $Y(K)$ VALUE IS ON THE PRESENT PRINT LINE

PL02060

DO $490 \quad K=K 3, K 4$

PL02070

PL02080

GO TO $(330,350)$, KYTL

330 IF $(X(K)-Y T) \quad 340,340,490$

PL02090

PL02100

IF $(X(K)-Y L) \quad 490,490,370$

PL02110

IF $(X(K)-Y L) \quad 360,360,490$

PL02120

IF $(X(K)-Y T) \quad 490,490,370$

PL02130

YES. $Y(K)$ BELONGS ON THIS PRINT LINE

c

THEREFORE DETERMIND WHERE ALL THE $X(K 5)$ FALL ON THE $X$-AXIS

PL02140

PL02150

$X L=X M I N$

$X T=X M I N+X D E L T A / 2$.

PL02160

PL02170

DO $480 \mathrm{KA}=1$, IPTX

PL02180

GO TO $(400,380), K X T L$

380 IF (X(K5)-XT) $470,470,390$

PL02190

IF $(X(K 5)-X L) 420,420,470$

PL02200

390

IF $(X(K 5)-X L) \quad 470,410,410$

PL02210

400

IF $(X(K 5)-X T) \quad 420,470,470$

PL02220

410

IF (PRINT (KA)-BLANK) $440,430,440$

PL02230

420

PRINT $(K A)=B O 0 L(K K)$

PL02240

GO TO 490

PL02250

PL02260

C IF MORE THEN ONE POINT FALLS ON THE PRINT POSITION, TALLY THE NO. PL02270

C OF POINTS.

PL02280

DO $450 \quad K K K=1,9$

PLO2290

IF (PRINT (KA)-IDGT (KKK)) $450,460,450$

PLO2300

CONT INUE

PLO2310

$\operatorname{PRINT}(K A)=I D G T(1)$

PLO2320

GO TO 490

PLO2330

IF (PRINT (KA).NE.IDGT(9)) PRINT (KA) $=I D G T(K K K+1)$

PL02340

GO TO 490

PL02350

$470 \quad X L=X T$

PL02360 


\begin{tabular}{|c|c|c|}
\hline 480 & $X T=X T+X D E L T A$ & PL02370 \\
\hline 490 & $K 5=K 5+1$ & PL02380 \\
\hline \multirow[t]{5}{*}{500} & CONTINUE & PL02390 \\
\hline & $Y P=Y T^{*} Y L$ & PLO2400 \\
\hline & $Y T=Y L$ & PL02410 \\
\hline & $Y L=Y L-Y D E L T A$ & PL02420 \\
\hline & GO TO $(510,550), \mathrm{L}$ & PL02430 \\
\hline 510 & IF (I-5) $520,520,630$ & PL02440 \\
\hline \multirow[t]{2}{*}{520} & $\mathrm{~L}=2$ & PL02450 \\
\hline & $Y S=Y T+Y D E L T A / 2$ & PL02460 \\
\hline C & THIS PATH IS EXECUTED ONCE IN EVERY DIVISION OF THE Y-AXIS. EVERY & PLO2470 \\
\hline \multirow[t]{3}{*}{ C } & TENTH LINE, STARTING WITH ZERO LINE & PL02480 \\
\hline & IF $(L 2 . E Q .6)$ GO TO 570 & PL02490 \\
\hline & IF (YP) $530,530,540$ & PL02500 \\
\hline \multirow[t]{2}{*}{530} & WRITE (IPRINT,960) TIT (ITB), YS, PRINT & PL02510 \\
\hline & GO TO 620 & PL02520 \\
\hline \multirow[t]{2}{*}{540} & HRITE (IPRINT,950) TIT(ITB), YS,PRINT & PL02530 \\
\hline & GO TO 620 & PL02540 \\
\hline \multirow[t]{2}{*}{550} & IF $(L 2 . E Q .6)$ GO TO 590 & PL02550 \\
\hline & IF (YP) $560,560,610$ & PL02560 \\
\hline$C$ & PRINTS LINE & PL02570 \\
\hline \multirow[t]{2}{*}{560} & WRITE (IPRINT,970) TIT(ITB), PRINT & PL02580 \\
\hline & GO TO 620 & PL02590 \\
\hline \multirow[t]{3}{*}{570} & IF (YP.GT.0.0) GO TO 580 & PL02600 \\
\hline & WRITE (IPRINT,980) TIT (ITB), YS, (PRINT (K),K=I, IPTX) & PL02610 \\
\hline & GO TO 620 & PL02620 \\
\hline \multirow[t]{2}{*}{580} & WRITE (IPRINT,990) TIT (ITB), YS, (PRINT (K),K=1, IPTX) & PL02630 \\
\hline & GO TO 620 & PL02640 \\
\hline \multirow[t]{3}{*}{590} & IF (YP.GT.0.0) GO TO 600 & PL02650 \\
\hline & WRITE (IPRINT, 1000) TIT (ITB), (PRINT (K),K=1, IPTX) & PL02660 \\
\hline & GO TO 620 & 2670 \\
\hline \multirow[t]{2}{*}{600} & WR ITE (IPRINT, 1010) TIT(ITB), (PRINT (K),K=1, IPTX) & PL02680 \\
\hline & GO TO 620 & PL02690 \\
\hline 610 & WRITE (IPRINT, 1020) TIT(ITB),PRINT & PL02700 \\
\hline 620 & $I T B=I T B+1$ & PL02710 \\
\hline \multirow[t]{2}{*}{630} & IF (L2.EQ.6) GO TO 910 & PL02720 \\
\hline & IF (YP) $640,640,650$ & PL02730 \\
\hline \multirow[t]{2}{*}{640} & HRITE (IPRINT,960) TIT(51), YMIN, PRINT & PL02740 \\
\hline & GO TO 660 & PL02750 \\
\hline \multirow{6}{*}{$\begin{array}{l}650 \\
C \\
660\end{array}$} & WRITE (IPRINT, 950) TIT (51), YMIN, PRINT & PL02760 \\
\hline & LAST LINE OF PRINT OUT PLUS $X$ VALUES ALONG $X$-AXIS & PL02770 \\
\hline & WRITE (IPRINT, 1060) IPR & PL02780 \\
\hline & WRITE (IPRINT, 1030) XP & PL02790 \\
\hline & WRITE (IPRINT, 1070) TITX & PL02800 \\
\hline & RETURN & 810 \\
\hline$v$ & THIS PRINTS OUT THAT 'Y BOUNDS ARE NOT SET UP CORRECTLY'. & PL02820 \\
\hline 670 & CONTINUE & PL02830 \\
\hline C & THIS PRINTS OUT THAT ' $X$ BOUNDS ARE NOT SET UP CORRECTLY'. & PL02840 \\
\hline 680 & CONT INUE & PL02850 \\
\hline & NOS. APPEAR AS ARGUMENTS'. & PL02860 \\
\hline 690 & CALL ERROR (20) & 870 \\
\hline \multirow[t]{2}{*}{700} & NERROR $=$ NERROR -1 & PL02880 \\
\hline & RETURN & PL02890 \\
\hline$v$ & THIS PRINTS THAT 'MORE THEN 5 PLOTS WERE REQUISTED PER GRAPH'. & PLO2900 \\
\hline 710 & CALL ERROR (10) & PL02910 \\
\hline & GO TO 700 & PL02920 \\
\hline 72 & $K E Y=1$ & PL02930 \\
\hline & DO $760 \quad I K=1, M$ & PL02940 \\
\hline & $I K K=I$ ARGS $(I K)$ & PL02950 \\
\hline
\end{tabular}


DO $750 \quad I=K 1, K 2$

IF (X (IKK) .GE.YAN.AND.X(IKK).LE.YAP) GO TO $(730,740)$, KEY

PL02960 GO TO 750

PL02970

$X I=X(I)$

PL02980

730

$X_{0}=X_{1}$

PL02990

$\mathrm{KEY}=2$

PL03000

GO TO 750

PL03010

PL03020

IF $(X I . L T . X(I)) \cdot X I=X(I)$

PL03030

IF $\left(X_{0}\right.$.GT.X(I)) $\quad X 0=X(I)$

PLO3040

$I K K=I K K+I$

PL03050

CONT INUE

PL03060

IF (KEY.EQ.2) GO TO 140

PL03070

$X 0=X D O W N$

PL03080

$\mathrm{XI}=\mathrm{XUP}$

PL03090

GO TO 140

PLO3100

D0 $790 \mathrm{~J}=1, M$

PLO3110

$K I=I$ ARGS (NARGS)

PLO3120

$K 3=\operatorname{IARGS}(\mathrm{J})$

PL03130

$K 4=K 3-1+N R M A X$

PLO3140

DO $790 \quad I=K 3, K 4$

PLO3150

IF (X(I).GT.YAP.OR.X(I).LT.YAN) GO TO 780

PLO3160

IF $(X(K I)$.LE.XAP.AND.X(KI).GE.XAN) GO TO 790

PL03170

$\begin{array}{ll}780 & N C N=N C N+1 \\ 790 & K I=K I+1\end{array}$

PL03180

PLO3190

NTOT $=$ M*NRMAX-NCN

PLO3200

PL03210

PL03220

PL03230

PL03240

PL03250

PL03260

IF (L2.EQ.6) GO TO 810

WRITE (IPRINT, 1040) (IH ( I,NARGS), I=1, 12), ( ( IH (I,J), I=1, 12), BOOL (J)PL03270
PL03280

PLO3290

810 WRITE (IPRINT, 1080) (IH(I,NARGS), I=1, 12), ( (IH (I,J), I=I, I2), BOOL (J)PL03300 $1, J=1, M)$

GO TO 290

PL03310

PLO3320

$X P(1)=X M I N$

PL03330

$X P(6)=X M A X$

PL03340

$X R=20 . * X D E L T A$

PL03350

DO $830 \quad I=2,5$

PL03360

PL03370

$\mathrm{XP}(\mathrm{I})=\mathrm{XP}(\mathrm{I}-1)+\mathrm{XR}$

PLO3380

DO $840 \mathrm{~J}=1,100$

PL03390

DO $850 \quad I=1,101,10$

PL03400

PL03410

$I P R(I)=B O O L(3)$

IF (XMIN*XMAX.GE.0.0) GO TO 900

PL03420

$\mathrm{J}=0$

PL03430

D0 $860 \quad I=2,5$

PL03440

IF $(X P(I-1) * X P(I)) \quad 870,890,860$

PL03450

CONTINUE

PL03460

$N=$ I PTX

PL03470

GO TO 890

PL03480

$X X P=X P(1-1)+X D E L T A$

PL03490

DO $880 \mathrm{~J}=1,20$

PL03500

IF $(X P(I-1) * X X P . L E .0 .0) \quad$ GO TO 890

PL03510

$X X P=X X P+X D E L T A$

PL03520

$J=20$

PL03530

$N=(I-2) * 20+J$

PL03540 
$\operatorname{IPR}(N)=I X P R$

900 WRITE (IPRINT, 1060) (IPR (K), K=1, IPTX)

PL03550

PL03560

IF (YP.GT.0.0) GO TO 920

PL03570

910

WRITE (IPRINT, 980) TIT (51), YMIN, (PRINT (K), K=1, IPTX)

PL03580

GO TO 930

920

WRITE (IPRINT, 990) TIT (51), YMIN, (PRINT (K), K=1, IPTX)

PL03590

PL03600

PL03610

WRITE (IPRINT, 1060) (IPR (K),K=1, IPTX)

PL03620

WRITE (IPRINT, 1030) (XP(K), K=1,4)

PL03630

WRITE (IPRINT, 1090) TITX

PL03640

RETURN

PL03650

PL03660

940 FORMAT (2IH NO. OF PTS. PLOTTED, I5,33H NO. NOT PLOTTED (OUT OF BOPL03670 IUNDS) , I5)

950 FORMAT (1X,Al, IPE $\left.11.4,1 \mathrm{H}_{+}, 101 \mathrm{Al}, 1 \mathrm{H}+\right)$

PL03680

960 FORMAT (1X,Al, IPE $11.4,1 \mathrm{HX}, 101 \mathrm{Al}, 1 \mathrm{HX})$

PL03690

PL03700

FORMAT $(1 X, A 1,11 X, 1 H X, 101 A 1,1 H X)$

PL03710

FORMAT (1X,Al, IPE $11.4,1 \mathrm{HX}, 61 \mathrm{Al}, 1 \mathrm{HX})$

PL03720

FORMAT (IX,AI, IPE $11.4,1 \mathrm{H}+, 61 \mathrm{Al}, 1 \mathrm{H}+)$

PL03730

FORMAT (1X,A1, 11X, 1HX,61A1, 1HX)

PL03740

FORMAT $(1 X, A 1,11 X, 1 H-, 61 A 1,1 H-)$

PL03750

FORMAT (IX,AI, 11X, IH-, 101Al, IH-)

PL03760

FORMAT $(6(7 X, 1 P E 13.4))$

PL03770

FORMAT (6H ABS-, 12Al,6H,ORD- ,5(12Al,2H (,Al,3H), ))

PL03780

1050 FORMAT (29H TOTAL NO. OF PTS. PLOTTED IS, I5,6OH AND NO. NOT PLOTTEPL03790 ID BECAUSE THEY FALL OUTSIDE OF BOUNDS IS, I5)

1060 FORMAT (14X,101A1)

1070 FORMAT $(34 X, 60 \mathrm{Al})$

1080 FORMAT (6H ABS- ,12A1/6H ORD - ,5(12A1,2H $(, A 1,3 H), 1)$

1090 FORMAT $(14 \times, 60 A 1)$

END

PL03800

PL03810

PL03820

PL03830

PL03840

PL03850 
SUBROUTINE PREPAK (N, IND, IA, LOC,LH)

PRE 10

VERSION 5.00 PREPAK $5 / 15 / 70$
S. PEAVY
$8 / 5 / 69$

THIS SUBROUTINE DOES THE FOLLOWING:

$N=1$ PACK FORMAT IN IFMT

PRE 20

PRE 30

PRE 40

$N=2$ PACK HEAD IN IHEAD. IF MORE THAN 50 HEADINGS ARE STORED, STACK OF HEADINGS IS PUSHED DOWN AND BOTTOM ONES DISCARDED

$\mathrm{N}=3$ CLEAR VARIABLES IFMT AND IHEAD

$\mathrm{N}=4$ PICK UP PROPER FORMAT NO. IN IA AND STORE IN LH

$N=5$ UNPACKS THE HEADING OF LOC. INTO LH IA AI LEFT JUSTIFIED

IND INDICATOR. IF IND $=0$ CALL TO PREPAK WAS O.K.

IF IND $=1$ A FLAG THAT RESULTS WERE NOT OBTAINED

IA COL NUMBER FOR THE HEADING TO BE PACKED OR FORMAT DESIRED

LOC LOC CONTAINS THE COLUMN NUMBER WHOSE HEADING THE SUBROUTINE IS TRYING TO FIND.

LH IS WHERE THE HEADING WILL BE STORED AS AI LEFT JUSTIFIED AFTER IT IS UNPACKED, IF THE TITLE IS FOUND. OR

PRE 50

PRE 60

PRE 70

PRE 80

PRE 90

PRE 100

PRE 110

PRE 120

PRE 130

PRE 140

PRE 150

PRE 160

PRE 170

PRE 180

PRE 190

PRE 200

PRE 210

PRE 220

WHERE FORMAT WILL BE STORED IF $\mathrm{N}=4$

PRE 230

PRE 240

PRE 250

PRE 260

PRE 270

COMMON /BLOCKC/ KIO, INUNIT, ISCRAT, KBDOUT, KRDKNT, LLIST

COMMON /SCRAT/ NS,NS2, A(13500)

PRE 280

DIMENSION IAA(80)

PRE 290

EQUIVALENCE (A, IAA)

PRE 300

DIMENSION LH (1)

PRE 310

C

$* * * * * * * * * * * * * * *$

PRE 320

PRE 330

THE VARIABLE IFMT CONTAINS THE INFORMATION FOR 6 FORMATS

PRE 340

(I E. FORMAT A THUR F). THE MAXIMUM LENGTH FOR EACH FORMAT IS PRE 350

72 CHARACTERS INCLUDING LEFT AND RIGHT PARENTASIS. IF FORMATS ARE PRE 360

PACKED DIFFERENTLY THEN STATED BELOW, THE DIMENSION SIZE OF PRE 370

THE FIRST (12) CONSTANT MUST BE CHANGED TO BE EQUAL OR GREATER PRE 380

THAN $72 /$ (NO. OF CHARACTERS PER HORD) +M. SEE NOTE BELOW FOR M VALUEPRE 390

CAUTION: FORMATS MUST BE PACKED IF NH CONVERSION IS PERMITTED PRE 400

PRE 410

THE VARIABLE IHEAD $(5, L A)$ CONTAINS THE HEADINGS FOR LA COLUMNS. PRE 420

MAXIMUN NO. OF CHARACTERS PER HEADING IS 12.

PRE 430

PRE 440

DIMENSION IFMT $(12,6), \operatorname{IHEAD}(5,50)$

PRE 450

PRE 460

IF THE VARIABLES IFMT $(I I, 6), \operatorname{IHEAD}(5$, LA ARE REDIMENSIONED SO THAT PRE 470 II DOES NOT $=12$

AND LA DOES NOT $=50$

PRE 480

PRE 490

THEN THE FOLLOWING DATA STATEMENT MUST BE CHANGED

PRE 500

PRE 510

DATA II / 12/,LA/50/

PRE 520

PRE 530

FORMAT 90 MUST BE CHANGED IF MORE OR FEWER CHARACTERS CAN BE PRE 540
PACKED INTO A WORD. 90 FORMAT (IIAK) WHERE II IS DEFINED ABOVE ANDPRE 550 $K=12 /($ CHARACTERS PER WORD $)+M$.

PRE 560

WHERE $M=0$ IF 12 /(NO. OF CHAR/WORD) HAS NO REMAINDER

PRE 570

AND $M=1$ IF $12 /$ (NO. OF CHAR/WORD) HAD A REMAINDER

PRE 580

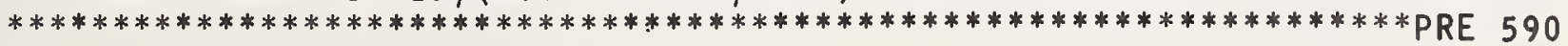


IF (N.GT.5) GO TO 60

IND $=0$

GO TO $(10,70,150,180,200), N$

C THIS PART OF PROGRAM PACKS AND STORES FORMAT

$10 \quad K=K A R D(M)$

$15 \quad M=M+1$

IF (KARD (M).NE.41) IF (KARD (M)-46) 15,60,15

$K K=1$

$K A=0$

$M A=M+1$

$K R=K R D E N D+3$

DO $20 \quad I=M A, K R$

IF (KARD (I) .EQ.41) KK=KK+1

IF (KARD(I).NE.42) GO TO 20

PRE 600

PRE 610

PRE 620

PRE 630

PRE 640

PRE 650

PRE 655

PRE 660

PRE 670

PRE 680

PRE 690

PRE 700

PRE 710

$K A=K A+1$

PRE 720

IF (KA.EQ.KK) GO TO 30

20 CONTINUE

GO TO 60

$30 \quad M B=M-2$

$I M=I-M+1$

D0 $40 \mathrm{JA}=1,80$

$40 \quad \operatorname{IAA}(\mathrm{JA})=\mathrm{L}(45)$

DO $50 \mathrm{JA}=1, \mathrm{IM}$

$\operatorname{IAA}(\mathrm{JA})=\operatorname{NEWCD}(\mathrm{MB})$

$50 \quad M B=M B+1$

WRITE (ISCRAT, 240) (IAA (JA), JA $=1,80)$

BACKSPACE ISCRAT

READ (ISCRAT, 230) (IFMT (JA,K-9), JA=1, I I)

BACKSPACE ISCRAT

RETURN

60 IND $=I N D+1$

RETURN

C THIS PACKS HEADS

70 IF (IHCNT.GE.IHTP) GO TO 140

IF (IHCNT.EQ.0) GO TO 110

C IHEAD $(1, I)=C O L$ NUMBER FOR THAT HEADING

DO $80 \quad I=1$, IHCNT

IF (IA.EQ.IHEAD( $1, I)) \quad$ GO TO 130

80 CONTINUE

$K B=I H C N T$

90 DO $100 \quad I=1, K B$

$K A=K B-I+2$

DO $100 \mathrm{~K}=1,5$

$100 \operatorname{IHEAD}(K, K A)=\operatorname{IHEAD}(K, K A-1)$

$110 \quad I H C N T=I H C N T+1$

$120 \operatorname{IHEAD}(1,1)=I A$

I CHAR $=12$

PRE 730

PRE 740

PRE 750

PRE 760

PRE 770

PRE 780

PRE 790

PRE 800

PRE 810

PRE 820

PRE 830

PRE 840

PRE 850

PRE 860

PRE 870

PRE 880

PRE 890

PRE 900

PRE 910

PRE 920

PRE 930

PRE 940

PRE 950

PRE 960

PRE 970

PRE 980

PRE 990

PRE 1000

PRE 1010

PRE 1020

PRE 1030

PRE 1040

PRE 1042

PRE 1044

PRE 1046

PRE 1050

PRE 1052

PRE 1054

PRE 1060

PRE 1070

PRE 1080

PRE 1090

PRE 1100

PRE 1105

PRE 1110 


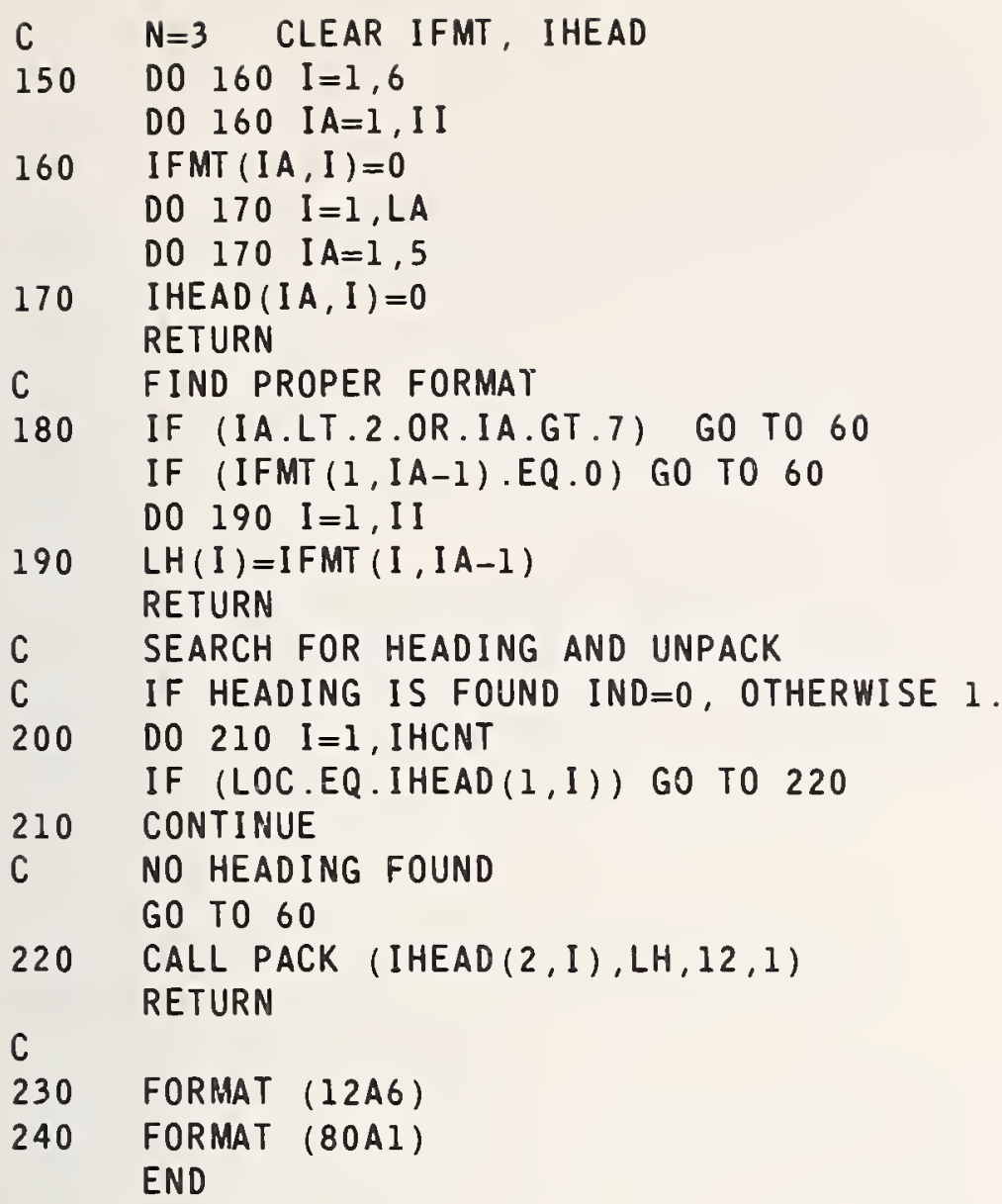


SUBROUTINE PRINTX

C VERSION 5.00 PRINTX 5/15/70

PRI 10

COMMON /BLOCKE/ NAME (4),L1,L2, ISRFLG

PRI 20

COMMON /BLOCRC/ NRC, RC (12600)

PRI 30

COMMON /BLOCKD/ IARGS(100), KIND(100), ARGTAB (100), NRMAX,NROW, NCOL, NPRI 50

IARGS, VWXYZ (8), NERROR

DIMENSION ARGS $(100)$

EQUIVALENCE (ARGS (1), RC (12501))

COMMON / HEADER / NOCARD $(80), \operatorname{ITLE}(60,6), \operatorname{LNCNT}$, IPR INT , NPAGE , IPUNCH

COMMON /FMAT/ IFMTX (6), IOSWT, IFMTS (6), LHEAD (96)

COMMON /KFMT/ KFMT (100)

C

$\mathrm{C}$

$\mathrm{C}$

$\mathrm{C}$

$\mathrm{C}$

$\mathrm{C}$

C

C LI=2 PRINT

C LI=8 NPRINT

IF (NARGS.NE.0) GO TO 45

10 CALL ERROR (205)

20 RETURN

30 CALL ERROR (222)

40 IF (NPAGE.EQ.0 .AND.LI.EQ.8) CALL PAGE (0)

CALL RPRINT

RETURN

45 IF (L2.EQ.1) IF (IOSWT) 40,40,230

CALL PREPAK (4, IND,L2, IND , KFMT)

IF (IND.NE.0) GO TO 30

I $P=1$

50 CALL CHKCOL (I)

IF (I.NE.0) GO TO 10

IF (NERROR.NE.0) RETURN

$\mathrm{I} B=0$

I $A=1$

ICP $=0$

GO TO $(60,70)$, IP

60 IB $=$ NARGS

GO TO 100

$70 \quad$ IBB $=$ NARGS

80 IF (IBB.GT.8) GO TO 90

$I B=I B B+I B$

I $C=I B B$

$\mathrm{IBB}=0$

GO TO 100

$90 \quad \mathrm{IBB}=\mathrm{IBB}-8$

$I B=8+I B$

IC $=8$

$100 \quad L L=N R M A X$

110 IF (LL.GT.51) GO TO 120

$J=L L$

$L L=0$

GO TO 130

$120 \quad L L=L L-50$

$J=50$

130 IF (L1.EQ.8) IF (NPAGE) $155,260,155$

CALL PAGE (4)

GO TO $(150,140)$, IP
PRI 60

PRI 70

PRI 80

PRI 90

PRI 100

PRI 110

PRI 120

PRI 130

PRI 140

PRI 150

PRI 160

PRI 170

PRI 180

PRI 183

PRI 187

PRI 190

PRI 200

PRI 210

PRI 220

PRI 225

PRI 230

PRI 240

PRI 249

PRI 250

PRI 260

PRI 270

PRI 280

PRI 290

PRI 300

PRI 305

PRI 310

PRI 320

PRI 330

PRI 340

PRI 350

PRI 360

PRI 370

PRI 380

PRI 390

PRI 400

PRI 410

PRI 420

PRI 430

PRI 440

PRI 450

PRI 460

PRI 470

PRI 480

PRI 490

PRI 500

PRI 510

PRI 520

PRI 530

PRI 540 
140 CALL HEADS (KFMT (IA), IC, ICP,0)

PRI 550

150 WRITE (IPRINT, 250)

155 D0 $200 \quad M=1, J$

DO $160 \quad I=I A$, IB

PRI 560

$\mathrm{K}=\mathrm{I}$ ARGS (I)

$\operatorname{IARGS}(I)=\operatorname{IARGS}(I)+1$

PRI 570

PRI 580

PRI 590

$160 \quad \operatorname{ARGS}(\mathrm{I})=\mathrm{RC}(\mathrm{K})$

GO TO $(170,180)$, IP

PRI 600

PRI 610

PRI 620

170 WRITE (IPRINT,KFMT) (ARGS (I), I=1, NARGS)

PRI 630

GO TO 200

180 WRITE (IPRINT, IFMTX) (ARGS (I), I=IA, IB)

PRI 640

190 IF (MOD (M, 10).EQ.0) WRITE (IPRINT, 250)

PRI 650

200 CONT INUE

I $C P=1$

PRI 660

PRI 670

IF (LL) $210,210,110$

210 GO TO $(20,220)$, IP

220 IF (IBB.EQ.0) GO TO 20

IF (LI.EQ.8) WRITE (IPRINT, 250)

$I A=I B+1$

$\mathrm{I} C \mathrm{P}=0$

GO TO 80

C USE STANDARD OR SPECIFIED FORMAT

PRI 680

PRI 690

PRI 700

PRI 710

PRI 713

PRI 715

PRI 720

PRI 730

$230 \quad \mathrm{IP}=2$

DO $240 \quad I=1$, NARGS

$240 \operatorname{KFMT}(\mathrm{I})=\operatorname{IARGS}(\mathrm{I})$

GO TO 50

C

250 FORMAT (IX)

260 CALL PAGE (0)

GO TO 155

END

PRI 820

PRI 830

PRI 840

PRI 850

PRI 860

PRI 870

PRI 880

PRI 890

PRI 900

PR I 910 
SUBROUTINE PROB (VNU1, VNU2, $F, Q$ )

C VERSION 5.00 PROB $5 / 15 / 70$

PRB 10

DOUBLE PRECISION FDSIN, FDCOS, FDEXP, FDLOG

PRB 20

DOUBLE PRECISION C, A, $X, W, O N E, B, T A, T B, G$

PRB 30

DATA C/.6366197723675814D0/,EP/I.E-5/,ONE/1.D0/.TWO/2.0/.ONEP/1./,PRB 50

$1 P 5 / .5 /$

$N U I=V N U 1+E P$

PRB 60

$N U 2=V N U 2+E P$

PRB 70

$\mathrm{VI}=\mathrm{NUI}$

$\mathrm{V} 2=\mathrm{NU} 2$

IF (ABS (VI-VNUI).GT.EP) GO TO 200

PRB 80

PRB 90

PRB 100

IF (ABS(V2-VNU2).GT.EP) GO T0 200

PRB 110

IF (F) $5,6,10$

PRB 120

$F=0.0$

C ERROR , SET $F=0$ SINCE $F$ LESS THEN 0 ,

PRB 130

CALL ERROR (206)

PRB 140

$6 \quad \begin{aligned} & \text { Q } \\ & \text { RETURN }\end{aligned}$

PRB 150

PRB 160

10 IF (NUI.LT.O.OR.NU2.LT.0) GO TO 190

PRB 162

PRB 164

$20 \quad M N U 1=M O D(N U 1,2)$

MNU2 $=$ MOD $(N U 2,2)$

IF (MNU2.NE.0) GO TO 70

I $1=N \cup 2 / 2-1$

$X=V 2 /(V 2+V 1 * F)$

$V_{4}=\mathrm{VI} / \mathrm{T}$ WO

I $4=$ NUI

$30 \quad A=0 N E$

IF (II.EQ.0) GO TO 50

$W=A$

DO $40 \quad I=I, I 1$

$T=I$

$W=((V 4+T-O N E P) / T) * X * W$

$40 \quad A=A+W$

$50 Q Q=A * F D E X P(V 4 * F D L O G(O N E-X))$

IF (I4.EQ.NUI) $Q=O N E P-Q$

60 IF (Q.LT.0.) $Q=0$

IF (Q.GT.ONEP) $Q=0 N E P$

RETURN

PRB 170

PRB 180

PRB 190

PRB 200

PRB 210

PRB 220

PRB 230

PRB 240

PRB 250

PRB 260

PRB 270

PRB 280

PRB 290

PRB 300

PRB 310

PRB 320

PRB 330

PRB 340

PRB 350

PRB 360

PRB 370

PRB 380

$I I=N U 1 / 2-1$

$X=0 N E P-V 2 /(V 2+V 1 * F)$

$V_{4}=V_{2} / T$ WO

I $4=$ NU 2

GO TO 30

80 IF (NU2.NE.I) GO TO 130

IF (NUI.NE.I) GO TO 90

$Q=C * A T A N($ ONEP/FSQRT $(F))$

GO TO 60

$90 X=\operatorname{ATAN}\left(F S Q R T\left(V_{2} /\left(V_{1} * F\right)\right)\right)$

$I I=(N U I-3) / 2$

IS $=1$

$100 \operatorname{TB}=\mathrm{FDSIN}(X)$

$A=F D \operatorname{COS}(X)$

IF (II.EQ.0) GO TO 120

$\mathrm{T} A=A * 2$

$W=A$

PRB 390

PRB 400

PRB 410

PRB 420

PRB 430

PRB 440

PRB 450

PRB 460

PRB 470

PRB 480

PRB 490

PRB 500

PRB 510

PRB 520

PRB 530

PRB 540

DO $110 \quad I=1, I I$

PRB 550

V $3=I$

PRB 560

$W=V 3 /(V 3+P 5) * T A * W$

PRB 570 


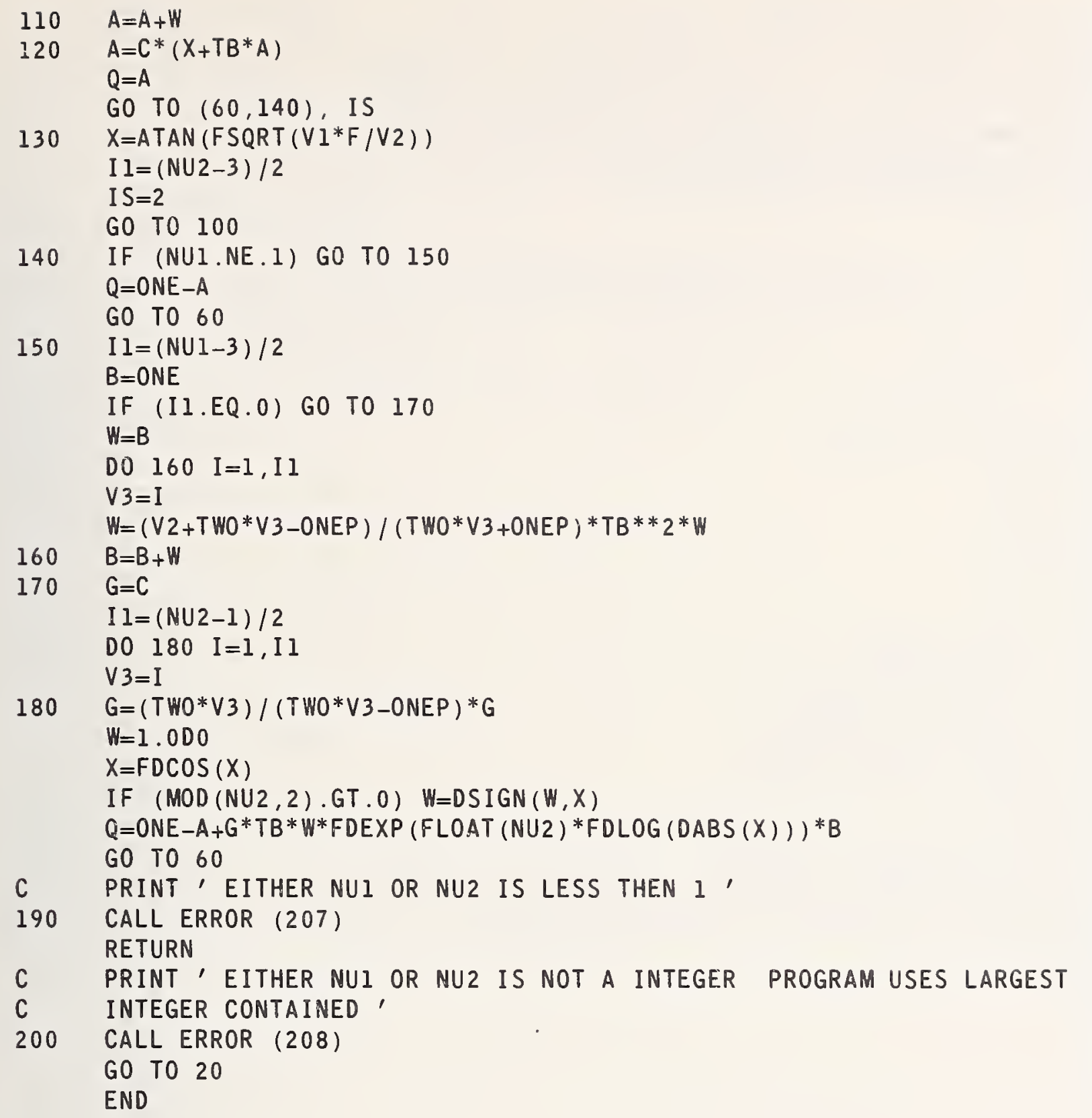


SUBROUTINE PROCHK ( $A, N R O W, N, N C O L$, I VEC , $X, N S I Z E$ )

PRK 10

C VERSION 5.00 PROCHK 5/15/70

C SUBROUTINE PROCHK R VARNER 5/7/68

C R VARNER SUBROUTINE TO CHECK FOR DIAGONAL, NORMAL, SYMMETRIC,

PRK 20

PRK 30

C SKEW-SYMMETRIC AND ORTHOGONAL MATRIX

DIMENSION A(NROW,1), IVEC (1)

C SET ALL INDICATORS TO NO CONDITION

DO $10 \quad I=1,5$

$10 \quad \operatorname{IVEC}(\mathrm{I})=2$

C TEST TO SEE IF WE HAVE A DIAGONAL MATRIX

C IF YES IVEC (1)=0 IF NO IVEC (1)=2

DO $30 \quad I=1, N$

DO $30 \mathrm{~J}=1, \mathrm{~N}$

IF $(I-J) 20,30,20$

20 IF $(A(I, J)) 40,30,40$

30 CONTINUE

$\operatorname{IVEC}(1)=0$

$\operatorname{IVEC}(2)=0$

$\operatorname{IVEC}(3)=0$

GO TO 50

$40 \quad \operatorname{IVEC}(1)=2$

C CHECK FOR SUMMETRY

CALL SYMV (A, NROW, N, IVEC (2))

C CHECK FOR SKEW SYMMETRY

CALL SKSYMV (A, NROW, N, IRV)

IF (IRV.GE.3) IVEC (2) =IRV

IF (IVEC (2).EQ.2) GO TO 50

$\operatorname{IVEC}(3)=0$

C CHECK FOR ORTHOGONAL MATRIX

C IF A IS ORTHOGONAL IVEC (4)=0 OTHERWISE IVEC $(4)=2$

50 CALL ORTHRV ( $A, N R O W, N, N C O L, I V E C(4), X, N S I Z E, X)$

RETURN

END

PRK 40

PRK 50

PRK 60

PRK 70

PRK 80

PRK 90

PRK 100

PRK 110

PRK 120

PRK 130

PRK 140

PRK 150

PRK 160

PRK 170

PRK 180

PRK 190

PRK 200

PRK 210

PRK 220

PRK 230

PRK 240

PRK 250

PRK 260

PRK 270

PRK 280

PRK 290

PRK 300

PRK 310

PRK 320

PRK 330 
SUBROUTINE PROROW

C VERSION 5.00 PROROW 5/15/70

PRO 10

PROGRAMMED BY CARLA MESSINA MAY, 1967

PRO 20

$\mathrm{L} 2=1$, ROWSUM $L 2=2$, PRODUCT

PRO 30

COMMON /BLOCRC/ NRC, RC (12600)

PRO 40

PRO 50

COMMON /BLOCKD/ IARGS(100), KIND(100), ARGTAB (100), NRMAX, NROW, NCOL, NPRO 60

IARGS, VWXYZ (8), NERROR

DIMENSION ARGS $(100)$.

EQUIVALENCE (ARGS (1), RC (12501))

COMMON /BLOCKE/ NAME (4), L1,L2, ISRFLG

PRO 70

PRO 80

COMMON /SCRAT / NS, NS2, A(13500)

PRO 90

IF (NARGS-3) $10,40,40$

PRO 100

10 IF (NARGS.EQ.1.AND.KIND(1).EQ.0) GO TO 230

10 IF (NARGS.EQ.1.AND.KIND(1).EQ.0) GO TO 230

PRO 110

PRO 120

CALL ERROR (K)

RETURN

CALL CHKCOL (J)

IF (J) $50,60,50$

PRO 130

PRO 140

PRO 150

PRO 160

$\begin{array}{ll}50 & K=3 \\ & \text { GO TO } 20 \\ 60 & \text { IF (NRMAX) }\end{array}$

$\begin{array}{ll}50 & K=3 \\ & \text { GO TO } 20 \\ 60 & \text { IF (NRMAX) }\end{array}$

PRO 170

PRO 180

PRO 190

PRO 200

60 IF (NRMAX) $70,70,80$

PRO 210

$\mathrm{K}=9$

GO TO 20

80 IF (NERROR.NE.0) RETURN

DO $100 \quad I=1$, NRMAX

PRO 220

PRO 230

PRO 240

$A(I)=0.0$

GO TO $(100,90)$, L2

PRO 250

PRO 260

PRO 270

$90 \quad A(I)=1.0$

100 CONTINUE

IF (NARGS-4) $110,190,190$

110 IF (IARGS(1)-IARGS (2)) $120,120,50$

$120 \quad K=I A R G S(1)$

DO $150 \mathrm{I}=1$, NRMAX

$\mathrm{J}=\mathrm{K}+\mathrm{I}-1$

GO TO $(130,140)$, L2

PRO 280

PRO 290

PRO 300

PRO 310

PRO 320

PRO 330

PRO 340

PRO 350

$130 \quad A(I)=A(I)+R C(J)$
$G O$ TO 150

$140 \quad A(I)=A(I) * R C(J)$

150 CONTINUE

IF (IARGS (1)+NROW-IARGS (2)) $160,160,170$

$160 \operatorname{IARGS}(1)=\operatorname{IARGS}(1)+$ NROW

GO TO 120

$170 K=I$ ARGS (NARGS)

DO $180 \quad I=1$, NRMAX

$\mathrm{J}=\mathrm{K}+\mathrm{I}-\mathrm{l}$

$180 \quad R C(J)=A(I)$

GO TO 30

190 I I =NARGS - I

DO $220 \quad L=1$, I I

$K=I$ ARGS $(L)$

DO 220 I=1, NRMAX

$\mathrm{J}=\mathrm{K}+\mathrm{I}-1$

GO TO $(200,210)$, L2

$200 \quad A(I)=A(I)+R C(J)$

GO TO 220

PRO 360

PRO 370

PRO 380

PRO 390

PRO 400

PRO 410

PRO 420

PRO 430

PRO 440

PRO 450

PRO 460

PRO 470

PRO 480

PRO 490

PRO 500

PRO 510

PRO 520

PRO 530

PRO 540

PRO 550

PRO 560

PRO 570

PRO 580

PRO 590 
IF (J.LE.0) CALL ERROR (3)

PRO 600

DO $250 I=1$, NRMAX

PRO 610

$I R=I$

PRO 620

SUM $=0$.

PRO 630

DO $240 \mathrm{~K}=1, \mathrm{NCOL}$

PRO 640

$S U M=S U M+R C(I R)$

PRO 650

PRO 660

PRO 670

PRO 680

PRO 690

$R C(J)=S U M$

PRO 700 
SUBROUTINE PUNCH

THE COMMAND PUNCH MAY BE USED IN THE FOLLOWING WAYS PUNCH COL,,,++++++++ (4 COLUMN LIMIT) PUNCH // COL,,,,++++++++ ECT (ACCORDIND TO FORMAT //)

THIS SUBROUTINE IS USED TO EXECUTE WRITE TAPE WRITE TAPE T FROM COL,,,++++++++ (4 COLUMN LIMIT) WRITE TAPE T // FROM COL,,++++ ETC (USE FOLMAT//) COMMON /BLOCKD/ IARGS (100), KIND $(100)$, ARGTAB $(100)$, NRMAX, NROW, NCOL, NPUN 120 I ARGS, VWXYZ (8), NERROR

COMMON /BLOCRC/ NRC, RC (12600)

PUN 130

DIMENSION ARGS $(100)$

PUN 140

EQUIVALENCE (ARGS(1), RC $(12501)$ )

PUN 150

COMMON /KFMT/ KFMT (100)

COMMON /FMAT/ IFMTX (6), IOSWT, IFMTS (6), LHEAD (96)

PUN 160

PUN 170

COMMON /TAPE/ NAME4 (2), NTPCT, IPUNCP, INUN IP, LITP

PUN 180

COMMON /HEADER/ NOCARD $(80), \operatorname{ITLE}(60,6)$, LNCNT, IPRINT, NPAGE , IPUNCH

PUN 190

PUN 200

$L 1=3 \quad$ PUNCH $L l=47$ WRITE TAPE

PUN 210

C

C

C

$I X=1$

IF (LI.EQ.47) IX=2

IF (NARGS.NE.0) GO TO 20

PUN 220

PUN 230

PUN 240

PUN 250

PUN 260

CALL ERROR (205)

RETURN

PUN 270

PUN 280

PUN 290

IF L2=1 ONLY 4 COLUMNS CAN BE PRINTED

PIJN 300

PUN 310

IF (L2.NE. 1) GO TO 25

PUN 320

NARGS $=$ MI NO (NARGS , 4)

PUN 330

GO TO 30

25 CALL PREPAK (4, IND, L2, IND, KFMT)

IF (IND.NE.0) GO TO 90

PUN 340

PUN 345

PUN 350

30 CALL CHKCOL (I)

IF (NERROR.NE.0) GO TO $(110,100)$, IX

PUN 360

PUN 370

DO $60 \quad I=1$, NRMAX

PUN 380

PUN 390

DO $40 \mathrm{~J}=1$, NARGS

PUN 400

$K=\operatorname{IARGS}(\mathrm{J})$

$\operatorname{IARGS}(J)=K+1$

$40 \quad \operatorname{ARGS}(\mathrm{J})=\mathrm{RC}(\mathrm{K})$

IF (L2.NE.1) GO TO 50

WRITE (IPUNCH, IFMTX) (ARGS $(K), K=1, N A R G S$ )

GO TO 60

50 WRITE (IPUNCH, KFMT) (ARGS (K), K=1, NARGS)

60 CONTINUE

IF (L1.NE.47) RETURN

DO $70 \mathrm{~J}=1$, NARGS

$70 \quad \operatorname{ARGS}(\mathrm{J})=0.0$

IF (L2.NE.1) GO TO 80

WRITE (IPUNCH, IFMTX) (ARGS(K), $K=1$, NARGS)

GO TO 100

80 WRITE (IPUNCH,KFMT) (ARGS $(K), K=1, N A R G S$ )

GO TO 100

90 CALL ERROR (222)

PUN 410

PUN 420

PUN 430

PUN 440

PUN 450

PUN 460

PUN 470

PUN 480

PUN 490

PUN 500

PUN 510

PUN 520

PUN 530

PUN 540

PUN 550

PUN 560

PUN 570

$L 2=1$

PUN 580 
SUBROUTINE PVTRI (A, NROW, N, INDU, INDB)

PVT 10
VERSION 5.00
PVTRI
$5 / 15 / 70$

PVT 20

PVT 30

TO DETERMINE IF A IS AN UPPER OR LOWER TRIANGULAR MATRIX

PVT 40

$S$ PEAVY FOR UNIVAC $1108 \quad 2 / 7 / 68$

PVT 50

PVT 60

A MATRIX TO BE CHECKED

NROW- DIMENSION SIZE OF A

PVT 70

N- PRESENT SIZE OF A

PVT 80

PVT 90

INDU INDICATOR INDU $=0$, UPPER TRIANGLE $=0$, INDU $=1$, UPPER TRIANGLE NOPVT 100 INDB $=0$, BOTTOM TRIAL $=0$, INDB $=1, B O T T O M$

NOT ZEPVT 110

DIMENSION A (NROW, NROW)

PVT 120

I NDU $=1$

INDB $=1$

$\mathrm{NN}=\mathrm{N}-1$

DO $50 \quad I=1, N N$

$I I=I+1$

PVT 130

PVT 140

PVT 150

PVT 160

PVT 170

DO $50 \mathrm{~J}=\mathrm{II}, \mathrm{N}$

GO TO $(10,20)$, INDU

10 IF $(A(I, J) . N E .0) \quad I N D U=$.

20 GO TO $(30,40)$, INDB

30 IF $(A(J, I) . N E .0$.$) \quad INDB =2$

40 IF (INDU.EQ.2.AND.INDB.EQ.2) GO TO 60

50 CONTINUE

60 INDU $=$ INDU-1

PVT 180

PVT 190

PVT 200

PVT 210

PVT 220

PVT 230

PVT 240

PVT 250

PVT 260

INDB $=$ INDB -1

PVT 270

RETURN

PVT 280

END

PVT 290 
SUBROUTINE RANKO (N, X, H, R, T)

C VERSION $45.0 \quad$ RANKO $3 / 6 / 70$
DIMENSION $X(1), H(1), R(1)$
C

PUTS RANK OF $N$ X'S IN VECTOR R. VECTOR H IS USED FOR STORAGE. $X, H$ AND $R$ MUST BE DIMENSIONED $N$ OR GREATER.

STORES CORRECTION FOR TIES IN $T=(1 / 12) * \operatorname{SUM}(T-1) * T^{*}(T+1)$. $T=0$ MEANS NO TIES.

MRITTEN BY DAVID HOGBEN, SEL, NBS. 4/9/69.

MOVE $X$ TO $R$ AND PUT I IN $H$

10 DO $20 \quad I=1, N$

$H(I)=I$

$20 \quad R(I)=X(I)$

C SORT $X$ IN $R$, CARRY ALONG I IN H TO OBTAIN HIERARCHY IN $H$.

C SORT USES 'PUSH-DOWN' METHOD. SEE ORGANICK, PAGE 84.

$$
\mathrm{K} l=\mathrm{N}-\mathrm{l}
$$

DO $30 \quad I=1, K I$

$\mathrm{K} 2=\mathrm{N}-\mathrm{I}$

DO $30 \mathrm{~J}=1, \mathrm{~K} 2$

IF $(R(J) . L E \cdot R(J+1))$ GO TO 30

$W=R(J)$

$R(\mathrm{~J})=R(\mathrm{~J}+1)$

$R(J+1)=W$

$W=H(J)$

$H(J)=H(J+1)$

$H(J+1)=W$

30 CONTINUE

C REPLACE R(I) BY I*.

C LET K BE SUCH THAT $R(I)=R(I-J+1), J=1, K$. THEN $I *=I-(K-1) / 2$.

$$
K=1
$$

$T=0$

DO $70 \quad I=2, N$

IF $(R(I)-R(I-1)) 50,40,50$

$40 \quad K=K+1$

GO TO 70

50 DO $60 \mathrm{~J}=1, \mathrm{~K}$

$I \mathrm{~J}=\mathrm{I}-\mathrm{J}$

$60 \quad \mathrm{R}(\mathrm{IJ})=\mathrm{FLOAT}(\mathrm{I}-1)-\mathrm{FLOAT}(\mathrm{K}-1) / 2$.

IF (K.GT.1) $T=T+(F L O A T(K-1) * F L O A T(K) * F L O A T(K+1)) / 12.0$

$\mathrm{K}=1$

70 CONTINUE

$T=T+($ FLOAT $(K-1) * F L O A T(K) * F L O A T(K+1)) / 12.0$

DO $80 \quad I=1, K$

$\mathrm{K} 2=\mathrm{N}+1-\mathrm{I}$

$80 \quad \mathrm{R}(\mathrm{K} 2)=\mathrm{FLOAT}(\mathrm{N})-\mathrm{FLOAT}(\mathrm{K}-1) / 2.0$

C SORT H CARRY ALONG $R$ TO OBTAIN RANKS IN $R$

DO $90 \mathrm{I}=1, \mathrm{KI}$

$\mathrm{K} 2=\mathrm{N}-\mathrm{I}$

DO $90 \mathrm{~J}=1, \mathrm{~K} 2$

IF $(H(J) . L E . H(J+1))$ GO TO 90

$\mathrm{H}=\mathrm{H}(\mathrm{J})$

$H(J)=H(J+1)$

$H(J+1)=W$

$W=R(J)$

$R(J)=R(J+1)$

$R(J+1)=W$

90 CONTINUE

RETURN

END

RKO 10

RKO 20

RKO 30

RKO 40

RKO 50

RKO 60

RKO 70

RKO 80

RKO 90

RKO 120

RKO 130

RKO 140

RKO 150

RKO 160

RKO 170

RKO 180

RKO 190

RKO 200

RKO 210

RKO 220

RKO 230

RKO 240

RKO 250

RKO 260

RKO 270

RKO 280

RKO 290

RKO 300

RKO 310

RKO 320

RKO 330

RKO 340

RKO 350

RKO 360

RKO 370

RKO 380

RKO 390

RKO 400

RKO 410

RKO 420

RKO 430

RKO 440

RKO 450

RKO 460

RKO 470

RKO 480

RKO 490

RKO 500

RKO 510

RKO 520

RKO 530

RKO 540

RKO 550

RKO 560

RKO 570

RKO 580

RKO 590

RKO 600

RKO 610 
, NCOL, NRAS 60

RAS 70

RAS 80

RAS 90

RAS 100

RAS 110

RAS 120

RAS 130

RAS 140

RAS 150

RAS 160

RAS 170

RAS 180

RAS 190

20 CALL ADRESS $(1, J 1)$

CALL ADRESS $(2, J 2)$

RAS 200

IF (J1.GT.0.AND.J2.GT.0) GO TO 30

RAS 210

CALL ERROR (3)

RETURN

30 IF (NRMAX,GT.0) GO TO 40

CALL ERROR (9)

RETURN

40 IF (NERROR.NE.0) RETURN

CALL RANKX (NRMAX, RC (J1),A(2), RC (J2), A(1))

IF (NRMAX.GE.NROW) RETURN

$J A N R=J 2+N R M A X$

$R C(J A N R)=A(1)$

RETURN

RAS 220

RAS 230

RAS 240

RAS 250

RAS 260

RAS 270

RAS 280

RAS 290

RAS 300

RAS 305

RAS 310

END

RAS 320

RAS 330 
SUBROUTINE RANKX (N, $X, H, R, T)$

C VERSION 5.00 RANKX 5/15/70

DIMENSION $X(1), H(1), R(1)$

\section{$* * * * *$}

PUTS RANK OF $N X^{\prime} S$ IN VECTOR R. VECTOR H IS USED FOR STORAGE.

$X, H$ AND $R$ MUST BE DIMENSIONED $N$ OR GREATER.

STORES CORRECTION FOR TIES IN T $=(1 / 12) * \operatorname{SUM}(T-1) * T *(T+1)$.

$T=0$ MEANS NO TIES.

WRITTEN BY DAVID HOGBEN, SEL, NBS. $4 / 9 / 69$.

COMPUTATION OF T CORRECTED $8 / 26 / 69$

$* * * * *$

MOVE $X$ TO $R$ AND PUT I IN H

DO $20 \quad I=1, N$

$H(I)=I$

$20 \quad R(I)=X(I)$

C SORT $X$ IN R, CARRY ALONG I IN H TO OBTAIN HIERARCHY IN $H$.

C SORT USES 'PUSH-DOWN' METHOD. SEE ORGANICK, PAGE 84.

$\mathrm{Kl}=\mathrm{N}-\mathrm{I}$

DO $30 \mathrm{I}=1, \mathrm{KI}$

$\mathrm{K} 2=\mathrm{N}-\mathrm{I}$

DO $30 \mathrm{~J}=1, \mathrm{~K} 2$

IF $(R(J) . L E . R(J+1))$ GO TO 30

$W=R(J)$

$R(J)=R(J+1)$

$R(J+1)=W$

$W=H^{\prime}(J)$

$H(J)=H(J+1)$

$H(J+1)=W$

30 CONTINUE

C REPLACE R(I) BY I*.

C LET K BE SUCH THAT R(I)=R(I-J+l),J=l,K. THEN I* $=I-(K-1) / 2$.

$K=1$

$\mathrm{T}=0$

DO $70 \quad I=2, N$

IF $(R(I)-R(I-1)) \quad 50 ; 40,50$

$40 \quad K=K+1$

GO TO 70

$50 \quad$ DO $60 \mathrm{~J}=1, \mathrm{~K}$

$I J=I-J$

$60 \quad R(I J)=F L O A T(I-1)-F L O A T(K-1) / 2$.

IF (K.GT.1) $T=T+(F L O A T(K-1) * F L O A T(K) * F L O A T(K+1)) / 12.0$

$K=1$

70 CONTINUE

$T=T+($ FLOAT $(K-1) *$ FLOAT $(K) * F L O A T(K+1)) / 12.0$

DO $80 \quad I=1, K$

$\mathrm{K} 2=\mathrm{N}+1-\mathrm{I}$

$80 \quad \mathrm{R}(\mathrm{K} 2)=\mathrm{FLOAT}(\mathrm{N})-\mathrm{FLOAT}(\mathrm{K}-1) / 2.0$

C SORT H CARRY ALONG $R$ TO OBTAIN RANKS IN $R$

DO $90 \mathrm{I}=1, \mathrm{KI}$

$\mathrm{K} 2=\mathrm{N}-\mathrm{I}$

DO $90 \mathrm{~J}=1, \mathrm{~K} 2$

IF $(H(J) . L E . H(J+1))$ GO TO 90

$W=H(J)$

$H(J)=H(J+1)$

$H(J+1)=H$

$W=R(J)$

$R(J)=R(J+1)$

$R(J+1)=W$

90 CONTINUE

RONTINUE

END

RAX 10

RAX 20

RAX 30

RAX 40

RAX 50

RAX 60

RAX 70

RAX 80

RAX 90

RAX 95

RAX 100

RAX 110

RAX 120

RAX 130

RAX 140

RAX 150

RAX 160

RAX 170

RAX 180

RAX 190

RAX 200

RAX 210

RAX 220

RAX 230

RAX 240

RAX 250

RAX 260

RAX 270

RAX 280

RAX 290

RAX 300

RAX 310

RAX 320

RAX 330

RAX 340

RAX 350

RAX 360

RAX 370

RAX 375

RAX 380

RAX 390

RAX 400

RAX 410

RAX 415

RAX 420

RAX 430

RAX 440

RAX 450

RAX 460

RAX 470

RAX 480

RAX 490

RAX 500

RAX 510

RAX 520

RAX 530

RAX 540

RAX 550

RAX 560

RAX 570

RAX 580 
SUBROUTINE RCSUM ( $A, N R O W, N, K, R$ )

$C$
$C$
$C$
$C$
$C$
$C$
$C$
$C$
$C$
$C$
$C$
$C$
$C$
$C$
$C$
$C$

VERSION 5.00 RCSUM $5 / 1$

$5 / 15 / 70$

RCS 10

WRITTEN BY S PEAVY $11 / 22 / 67$

WHERE :

A IS LOCATION OF MATRIX TO BE SUMMED ROW AND COLUMN WISE NROW SIZE OF A IN DIMENSION STATEMENT A (NROW,NROW)

$N$ NO OF ROWS IN A

$K$ NO OF COLS IN A

R RESULTS.

$$
\begin{array}{ll}
R(I) \ldots R(K) \quad C O L \text { SUMS } \\
R(K+1) \ldots R(K+N) \text { ROW SUMS } \\
R(K+N+2) \quad \text { GRAND SUM } \\
R(K+N+1) \quad \text { S A (I,J) FOR ALL I,J. } \\
R(K+N+3) \quad S \quad A(I, J) * * 2 \text { FOR ALL I,J. } \\
R(K+N+4) \text { SUM OF ABSOLUTE VALUES OF ALL A I I J) }
\end{array}
$$

DIMENSION A (NROW, NROW), R(N)

$$
L=1
$$

ASUM $=0$.

DO $20 \mathrm{~J}=1, \mathrm{~K}$

$S U M=0$.

DO $10 \quad I=1, N$

$A S U M=A S U M+A B S(A(I, J))$

$10 S S U M=S U M+A(I, J)$

$R(L)=S U M$

$20 \quad L=L+1$

$S=0.0$

$S S=0.0$

DO $40 \quad I=1, N$

SUM $=0.0$

DO $30 \mathrm{~J}=1, \mathrm{~K}$

$S U M=S U M+A(I, J)$

$30 \quad S S=S S+A(I, J) * * 2$

$S=S+S U M$

$R(L)=S U M$

$40 \quad L=L+1$

$R(L)=S$

$R(L+2)=S S$

$R(L+1)=S$

$R(L+3)=A S U M$

RETURN

END
RCS 20

RCS 30

RCS 40

RCS 50

RCS 60

RCS 70

RCS 80

RCS 90

RCS 100

RCS 110

RCS 120

RCS 130

RCS 140

RCS 150

RCS 160

RCS 170

RCS 180

RCS 190

RCS 200

RCS 210

RCS 220

RCS 230

RCS 240

RCS 250

RCS 260

RCS 270

RCS 280

RCS 290

RCS 300

RCS 310

RCS 320

RCS 330

RCS 340

RCS 350

RCS 360

RCS 370

RCS 380

RCS 390

RCS 400

RCS 410

RCS 420

RCS 430 
$\begin{array}{llll} & \text { SUBROUTINE READQ } & \text { REQ } & 10 \\ \text { C } & \text { VERSION 5.00 READQ } & \text { REQ } & 20 \\ & \text { COMMON /BLOCRC/ NRC,RC }(12600) & \text { REQ } & 30\end{array}$ COMMON /BLOCKD/ IARGS (100), KIND(100), ARGTAB (100), NRMAX,NROW, NCOL, NREQ 40

IARGS, VWXYZ(8), NERROR $\quad$ REQ 50

DIMENSION ARGS $(100) \quad$ REQ 60

EQUIVALENCE (ARGS (1), RC(12501)) $\quad$ REQ 70

COMMON /QRS/ NDROW, IFLAG, J,NNARG $\quad$ REQ 80

COMMON /BLOCKA/ MODE, M, KARD (83), KARG, ARG, ARG2, NEWCD (80), KRDEND REQ 90

COMMON /BLOCKC/ KIO, INUNIT, ISCRAT, KBDOUT, KRDKNT, LLIST REQ 100

COMMON / T.APE / NAME4 (2), NTPCT, IPUNCP, INUNIP, LITP

COMMON / KFMT / KFMT (100)

IF (IFLAG.NE.0) GO TO 80

REQ 110

REQ 115

REQ 120

IF (J.LT.NROW) GO TO 10

REQ 130

IFLAG $=1$

REQ 140

CALL ERROR (201)

REQ 150

GO TO 40

C NNARG CONTAINS NARGS OF READ COMMAND

C KFMT (I)THRU KFMT (NNAGR) CONTAINS ADDRESSES OF COLUMN TOPS

C THESE CORRECTIONS ARE NEEDED FOR TAPE OPERATIONS

10 IF (LITP.NE.45) GO TO 50

DO $30 \quad I=1$, NNARG

REQ 160

REQ 170

REQ 180

REQ 190

REQ 200

REQ 210

$K=K F M T(I)+J$

REQ 220

IF (KIND(I).EQ.0) GO TO 20

REQ 230

IF (ARGS (I).NE.0.) GO TO 50

REQ 240

GO TO 30

REQ 250

IF (IARGS (I).NE.0) GO TO 50

REQ 260

CONTINUE

REQ 270

INUN I T=INUN I P

REQ 280

$M O D E=1$

REQ 290

GO TO 80

50 IF (NARGS.GE.NNARG) GO TO 55

REQ 300

NNS $=$ NARGS +1

REQ 305

REQ 307

DO $52 I=$ NNS, NNARG

REQ 310

KIND (I) $=0$

REQ 315

$\operatorname{IARGS}(\mathrm{I})=0$

REQ 320

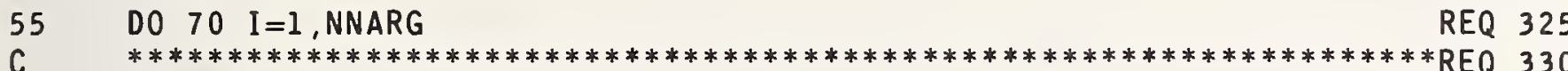

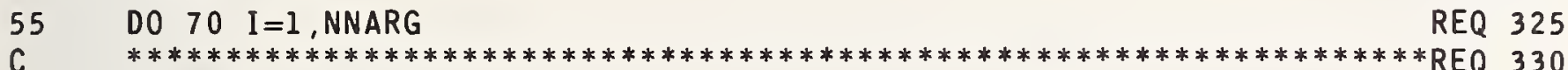

$\mathrm{K}=\mathrm{KFMT}(\mathrm{I})+\mathrm{J}$

REQ 340

IF (KIND(I).EQ.0) GO TO 60

REQ 350

$R C(K)=A R G S(I)$

REQ 360

GO TO 70

REQ 370

$60 \quad R C(K)=I A R G S(I)$

REQ 380

CONTINUE

C J IS CARD COUNT. IT COUNTS FROM ZERO.

REQ 390

$\mathrm{J}=\mathrm{J}+1$

NRMAX $=$ MAXO (NRMAX, J)

C THESE CORRECTIONS ARE NEEDED FOR TAPE OPERATIONS

REQ 400

REQ 410

REQ 420

IF (LITP.NE.46) GO TO 80

REQ 430

NTPCT $=$ NTPCT -1

REQ 440

IF (NTPCT.EQ.0) GO TO 40

REQ 450

REQ 460

C

RETURN

REQ 470

80

END

REQ 480

REQ 490 
C VERSION 5.00 READX 5/15/70

REX 10

COMMON /BLOCKA/ MODE,M,KARD (83), KARG, ARG, ARG2, NEWCD (80), KRDEND

REX 20

COMMON /BLOCRC/ NRC, RC (12600)

REX 30

COMMON /BLOCKD/ IARGS(100), KIND(100), ARGTAB (100), NRMAX, NROW, NCOL, NREX 50

IARGS, VHXYZ (8), NERROR

DIMENSION ARGS (100)

REX 60

EQUIVALENCE (ARGS (i),RC (12501))

REX 70

COMMON /BLOCKE/ NAME (4),L1,L2, ISRFLG

REX 80

COMMON /QRS/ NDROW, IFLAG,J, NNARG

REX 90

COMMON /BLOCKC/ KIO, INUNIT, ISCRAT, KBDOUT, KRDKNT, LLIST

REX 100

C

THE FOLLOWING CARD IS NEEDED ONLY FOR TAPE OPERATIONS

REX 110

COMMON /TAPE / NAME4 (2), NTPCT, IPUNCP, INUNIP, LITP

REX 120

*************************************************

REX 130

C

COMMON / KFMT / KFMT (100)

REX 140

IF (L2.NE.1) GO TO 90

REX 150

I $S R F L G=0$

REX 170

IF (NARGS.GT.0) GO TO 20

REX 180

10 CALL ERROR (10)

REX 190

GO TO 70

REX 200

$20 \quad M O D E=2$

CALL CHKCOL (I)

IF (I.EQ.0) GO TO 40

REX 210

REX 220

REX 230

30 CALL ERROR (3)

GO TO 70

REX 240

REX 250

IF (NERROR.NE.0) GO TO 70

REX 260

DO $50 \mathrm{I}=1$, NARGS

$\operatorname{KFMT}(\mathrm{I})=\operatorname{IARGS}(\mathrm{I})$

$\operatorname{IARGS}(I)=0$

$50 \quad \operatorname{ARGS}(I)=0$.

I FLAG $=0$

$J=0$

REX 270

REX 280

REX 290

NNARG $=$ NARGS

REX 300

REX 310

REX 320

GO TO 80

$60 \mathrm{MODE}=2$

70 IFLAG $=1$

THE FOLLOWING CARDS ARE NEEDED ONLY FOR TAPE OPERATIONS

REX 330

REX 340

REX 350

REX 360

REX 370

IF (LITP.EQ.46.OR.LITP.EQ.45) GO TO 200

REX 380

REX 390

C

$* * * * * * * *$

RETURN

REX 400

REX 410

REX 430

FORMATTED READ

REX 435

READ $\times N C \subset C C$

REX 440

REX 450

$N=$ NUMBER OF CARDS TO READ. IF $N=0$, READ UNTIL $A$

REX 460

BLANK CARD IS FOUND

REX 470

$X$ IS THE FORMAT IDENTIFIER, $A, B, C, D, E, F$

REX 480

REX 490

IF (NARGS.LE.1) GO TO 10

SETUP FORMAT

CALL PREPAK (4, IND , L 2, I , KFMT)

IF (IND.NE.0) CALL ERROR (27)

IF (NERROR.NE.O) GO TO 60

C CHECK AND CONVERT ARGUMENTS

DO $100 \quad I=2$, NARGS

REX 500

REX 510

REX 520

REX 525

REX 530

REX 540

REX 550

CALL ADRESS (I, IARGS (I))

IF (IARGS (I).LE.0) GO TO 185

100 CONTINUE

IF (IARGS (1)) $30,110,120$

REX 560

REX 565

REX 567

REX 570 


\begin{tabular}{|c|c|c|}
\hline 110 & $N=N R C$ & REX 580 \\
\hline & GO TO 130 & REX 590 \\
\hline 120 & $N=\operatorname{IARGS}(1)$ & REX 600 \\
\hline \multirow[t]{2}{*}{130} & DO $170 \quad I=1, N$ & REX 610 \\
\hline & READ (INUNIT, KFMT) (ARGS $(\mathrm{J}), \mathrm{J}=2$, NARGS) & REX 620 \\
\hline \multirow[t]{4}{*}{$C$} & CHECK IF LOOKING FOR BLANK CARD & REX 630 \\
\hline & IF (IARGS(1).NE.0) GO TO 150 & REX 640 \\
\hline & DO $140 \mathrm{~J}=2$, NARGS & REX 650 \\
\hline & IF (ARGS (J).NE.0.) GO TO 150 & REX 655 \\
\hline 140 & CONTINUE & REX 657 \\
\hline \multirow[t]{2}{*}{ C } & BLANK CARD FOUND, TERMINATE READ. & REX 660 \\
\hline & GO TO 180 & REX 670 \\
\hline 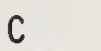 & IF THERE IS TOO MUCH DATA, DO NOT ENTER EXCESS & REX 680 \\
\hline \multirow[t]{4}{*}{150} & IF (I.GT.NROH) GO TO 170 & REX 690 \\
\hline & DO $160 \mathrm{~J}=2$, NARGS & REX 700 \\
\hline & $K=\operatorname{IARGS}(\mathrm{J})$ & REX 710 \\
\hline & $\operatorname{IARGS}(\mathrm{J})=\mathrm{K}+1$ & REX 720 \\
\hline 160 & $R C(K)=A R G S(J)$ & REX 730 \\
\hline \multirow[t]{2}{*}{170} & CONTINUE & REX 740 \\
\hline & $I=N+1$ & REX 750 \\
\hline \multirow[t]{4}{*}{180} & $\mathrm{I}=\mathrm{I}-\mathrm{I}$ & REX 760 \\
\hline & NRMAX $=$ MAXO (NRMAX, MINO (I, NROW) $)$ & REX 770 \\
\hline & WRITE (ISCRAT, 210) I & REX 780 \\
\hline & IF (I.GT.NROW) CALL ERROR (201) & REX 790 \\
\hline C & THE FOLLOWING CARDS ARE NEEDED ONLY FOR TAPE OPERATIONS & REX 800 \\
\hline & THE CARD WAS GO TO 80 & REX 810 \\
\hline & GO TO 190 & REX 820 \\
\hline $\mathrm{C}$ & $* * * * * * * * * * * * * * * * * * *$ & REX 830 \\
\hline 185 & CALL ERROR (11) & REX 840 \\
\hline C & THE FOLLOWING CARD IS NEEDED ONLY FOR TAPE OPERATIONS & REX 850 \\
\hline C & THE CARD WAS GO TO 80 & REX 860 \\
\hline 190 & IF (LITP.NE . 45.AND.LITP.NE . 46) & REX 870 \\
\hline \multirow[t]{3}{*}{200} & INUN I T=INUN IP & REX 880 \\
\hline & $\mathrm{MODE}=1$ & REX 890 \\
\hline & RETURN & REX 900 \\
\hline C & $* * * * * * * * * * * * * * * * * * * * * * * * * * * * * * * * *$ & REX 910 \\
\hline C & & REX 920 \\
\hline \multirow[t]{2}{*}{210} & FORMAT $(5 X, 14,33 \mathrm{H}$ DATA CARD(S) READ BUT NOT LISTED, 42X) & REX 930 \\
\hline & END & REX 940 \\
\hline
\end{tabular}


SUBROUTINE REPINC (IJSWT)

REP 10

$\begin{array}{lllll}\text { C VERSION } 5.00 & \text { REPINC } & 5 / 15 / 70 \\ C & \text { WRITTEN BY R VARNER } 4 / 9 / 68\end{array}$

REP 20

COMMON/BLOCKE/ NAME (4),L1,L2, ISRFLG

REP 30

COMMON /BLOCKB/ NSTMT, NSTMTX, NSTMTH, NCOM, LCOM, IOVFL, COM(2000)

REP 40

COMMON /BLOCKX/INDEX $(6,8)$, LEVEL

REP 50

COMMON /BLOCRC/ NRC, RC (12600)

REP 60

COMMON /BLOCKD/ IARGS(100), KIND(100), ARGTAB (100), NRMAX, NROW, NCOL, NREP 80

IARGS, VWXYZ (8), NERROR

DIMENSION ARGS $(100)$

REP 90

EQUIVALENCE (ARGS(1), RC(12501))

REP 100

$\mathrm{C}$

C IJSWT=I COMMAND IS REPEAT INITIALIZE THINGS

REP 110

IJSWT $=2$ IN REPEAT MODE

REP 120

REP 130

IJSWT $=3$ COMMAND IS INCREMENT OR RESTORE

REP 140

$\mathrm{L} 2=6 \quad$ INCREMENT

REP 150

$L 2=8$ RESTORE

REP 160

REP 170

REP 180

GO TO $(350,490,10)$, IJSWT

REP 190

IF (L2.EQ.6) GO TO 20

REP 200

10

$T=0.0$

GO TO 30

$20 \quad T=1.0$

30 IF (NARGS.GE.2) GO TO 50

$40 \quad K=10$

GO TO 320

REP 210

REP 220

REP 230

REP 240

REP 250

REP 260

REP 270

GET STATEMENT NUMBER. CAN BE FLOATING OR INTEGER.

REP 280

c

C

IF (KIND(1).EQ.0) GO TO 60

REP 290

REP 300

$J=10 . * A R G S(1)+.5$

REP 310

GO TO 70

$60 \mathrm{~J}=10 *$ IARGS (1)

70 IF (J.GT.NSTMTH) GO TO 80

$J=L O C A T E(J)$

REP 320

REP 330

REP 340

REP 350

REP 360

REP 370

$J$ HAS LOCATION OF COMMAND TO BE MODIFIED

REP 380

REP 390

IF $(J . G T .0)$ GO TO 90

REP 400

$K=13$

REP 410

GO TO 320

REP 420

REP 430

JJ IS FIRST LOCATION OF THE NEXT STORED COMMAND.

REP 440

REP 450

$J J=J+I F I X(\operatorname{COM}(J+1))$

REP 460

REP 470

REP 480

CHECK THAT COMMAND HAS THE PROPER NUMBER OF ARGUMENTS

REP 490

IF (NARGS-1.NE.MOD (IFIX $(\operatorname{COM}(\mathrm{J}+2)), 64))$ GO TO 40

REP 500

$\mathrm{J}=\mathrm{J}+3$

REP 510

REP 520

REP 530

SKIP OVER HEADER

REP 540

REP 550

REP 560

CHECK IF THIS COMMAND IS STORED. IF SO, PULL OUT INTO ARGTAB.

REP 570

(ALL BUT FIRST ARG WHICH IS STATEMENT NUMBER)

REP 580

IF (LEVEL.EQ.0) GO TO 110

REP 590 
$\mathrm{K}=2 *$ NARGS

DO $100 \quad I=2, K$

REP 600

$\operatorname{ARGTAB}(I)=\operatorname{COM}(I 2+4)$

REP 610

$I 2=I 2+1$

REP 620

100

\section{I2 IS LOCATION OF THIS COMMAND}

REP 630

REP 640

REP 650

REP 660

REP 670

REP 680

PERFORM INCREMENT OR RESTORE. PICK UP ARGUMENT FROM

REP 690

COMMAND TO BE MODIFIED AND EXAMINE IT.

REP 700

REP 710

REP 720

REP 730

FLOATING POINT CONST.

REP 740

REP 750

130 IF (ARGTAB (I)) $140,160,310$

REP 760

REP 770

C INCR. FL. PT. CONST. BY 'STATEMENT'

REP 780

140 IF (ARGTAB (I).EQ. (-1.)) GO TO 310

CALL XPND (ARGTAB (I), K, Y,KND)

IF (K.LT.O) GO TO 220

IF (KND.EQ.0) GO TO 310

$\operatorname{COM}(\mathrm{J}+1)=\mathrm{T}^{*} \operatorname{COM}(\mathrm{J}+1)+\mathrm{Y}$

$\mathrm{J}=\mathrm{J}+2$

$150 \quad \mathrm{I}=\mathrm{I}+\mathrm{K}+1$

GO TO 190

$160 \operatorname{COM}(\mathrm{J}+1)=\mathrm{T}^{*} \operatorname{COM}(\mathrm{J}+1)+\operatorname{ARGTAB}(\mathrm{I}+1)$

$170 \quad \mathrm{~J}=\mathrm{J}+2$

$180 \quad \mathrm{I}=\mathrm{I}+2$

190 IF (J-JJ) $120,330,330$

C

COLUMN NUMBER

C

200 IF (ARGTAB(I)) $210,310,230$

C

INTEGER CONST MODIFIED BY : STATEMENT:

REP 790

REP 800

REP 810

REP 820

REP 830

REP 840

REP 850

REP 860

REP 870

REP 880

REP 890

REP 900

REP 910

REP 920

REP 930

REP 940

REP 950

REP 960

REP 970

REP 980

REP 990

REP 995

REP 1000

REP 1010

REP 1020

REP 1030

REP 1040

REP 1050

REP 1060

REP 1070

REP 1080

REP 1090

REP 1100

REP 1110

REP 1120

REP 1130

REP 1140

REP 1150

REP 1160

REP 1170 
$$
\text { IF (ARGTAB (I)+16.0) } 270,310,310
$$

260 IF (ARGTAB (I $)+16.0) 270,310,310$
$270 \quad \operatorname{COM}(\mathrm{J})=T^{*}(\operatorname{COM}(\mathrm{J})+8208)+,A R G T A B(I)$

IF (COM(J).GT.(-16.)) GO TO 310

IF $(\operatorname{COM}(\mathrm{J}+1) * A R G T A B(I+1)) \quad 310,310,280$

$280 Y=T *(A B S(\operatorname{COM}(J+1))-8192)+.A B S(A R G T A B(I+1))$

IF (Y) $310,310,290$

REP 1200

REP 1210

REP 1220

REP 1230

REP 1240

REP 1250

REP 1260

$290 \operatorname{COM}(\mathrm{J}+1)=\operatorname{SIGN}(Y, \operatorname{COM}(J+1))$

REP 1270

REP 1280

GO TO 170

$300 \quad K=18$

GO TO 320

$310 \quad K=20$

320 CALL ERROR (K)

330 RETURN

REP 1290

REP 1300

REP 1310

REP 1320

REP 1330

REP 1340

*** (=THRU) IGNORE. INCREM. OR RESTORE MAY OR MAY NOT

REP 1350

HAVE CORRESPONDING ***

REP 1360

REP 1370

REP 1380

IF (ARGTAB (I) .EQ. $(-1).) \quad I=I+1$

$J=J+1$

GO TO 190

C

C

NESTED PERFORMS UP TO EIGHT LEVELS ARE ALLOWED.

CURRENT LEVEL IS STORED IN LEVEL

INDEX ( 1 , LEVEL) CONTAINS LOCATION OF COMMAND AT ARGI (FIRST)

REP 1390

REP 1400

REP 1410

REP 1420

REP 1430

REP 1440

REP 1450

REP 1460

REP 1470

INDEX (2,LEVEL) CONTAINS RUNNING INDEX FROM ARGI TO ARG2

REP 1480

INDEX ( 3, LEVEL) CONTAINS LOCATION OF COMMAND AT ARG2 (LAST)

REP 1490

INDEX ( 4, LEVEL) CONTAINS THIRD ARG (REPEAT COUNT)

INDEX (5, LEVEL) CONTAINS CURRENT LEVEL COUNTER (1 TO ARG3)

REP 1500

INDEX (6, LEVEL) CONTAINS STATEMENT NUMBER OF STATEMENT CURRENTLY

REP 1510

REP 1520 BEING EXECUTED.

REP 1530

REP 1540

REP 1550

IF (NARGS-3) $360,400,390$

IF (NARGS-1) $390,370,380$

REP 1560

REP 1570

SECOND ARG MISSING, MAKE SAME AS FIRST ARG

REP 1580

REP 1590

REP1600

REP1610

REP 1620

$\operatorname{KIND}(2)=\operatorname{KIND}(1)$

REP 1630

THIRD ARG MISSING, SET TO INTEGER 1

REP 1640

REP 1650

REP 1660

REP 1670

REP 1680

REP 1690

REP 1700

REP 1710

REP 1720

REP 1730

REP 1740

REP 1750

REP 1760 
420 IARGS (I) $=10 *$ I ARGS (I)

430 IF (IARGS (I).GT.NSTMTH) GO TO 440

IARGS (I) =LOCATE (IARGS (I))

IF (IARGS (I).GT.0) GO TO 450

440 CALL ERROR (13)

GO TO 500

450 CONTINUE

IF (LEVEL.LT.8) GO TO 460

REP 1770

REP 1780

REP 1790

REP 1800

REP 1810

REP 1820

REP 1830

CALL ERROR (19)

REP 1840

GO TO 500

REP 1850

REP 1860

460 IF (IARGS (2).LT.IARGS (1)) CALL ERROR (3)

REP 1870

IF (NERROR.NE.0) GO TO 500

REP 1880

LEVEL $=$ LEVEL +1

REP 1890

$\operatorname{INDEX}(1$, LEVEL $)=\operatorname{IARGS}(1)$

$\operatorname{INDEX}(3, \operatorname{LEVEL})=\operatorname{IARGS}(2)$

REP 1900

$\operatorname{INOEX}(4, \operatorname{LEVEL})=\operatorname{IARGS}(3)$

REP 1910

$\operatorname{INDEX}(5, \operatorname{LEVEL})=0$

REP 1920

REP 1930

C

OUTER LOOP

REP 1940

REP 1950

REP 1960

$470 \quad \operatorname{INDEX}(5, \operatorname{LEVEL})=\operatorname{INDEX}(5, \operatorname{LEVEL})+1$

IF (INDEX (5,LEVEL). LE. INDEX ( 4, LEVEL) ) GO TO 480

REP 1970

REP 1980

REP 1990

FINISHED OUTER LOOP, REDUCE LEVEL BY 1

REP 2000

REP 2010

LEVEL=LEVEL -1

IF (LEVEL.GT.0) GO TO 490

REP 2020

REP 2030

RETURN

480 INDEX $(2$, LEVEL $)=\operatorname{INDEX}(1, \mathrm{LEVEL})$

490 I $2=\operatorname{INDEX}(2$, LEVEL $)$

IF (I2.GT.INDEX (3,LEVEL)) GO TO 470

REP 2040

REP 2050

REP 2060

REP 2070

$\operatorname{INDEX}(6, \operatorname{LEVEL})=\operatorname{COM}(12)$

REP 2080

$\mathrm{K}=\operatorname{COM}(I 2+1)$

REP 2090

INDEX $(2$, LEVEL $)=\operatorname{INDEX}(2$, LEVEL $)+K$

REP 2100

$\mathrm{L} 2=\operatorname{COM}(\mathrm{I} 2+2)$

REP 2110

$\mathrm{L} 1=\mathrm{L} 2 / 64$

REP 2120

NARGS $=\mathrm{L} 2-64 * \mathrm{LI}$

REP 2130

$\mathrm{L} 2=\mathrm{L} 1 / 64$

REP 2140

REP 2150

$\mathrm{L} I=\mathrm{L} 1-64 * \mathrm{~L} 2$

CALL EXPAND $(\mathrm{K}-2, \mathrm{COM}(\mathrm{I} 2+3))$

REP 2160

RETURN

REP 2170

REP 2180

REP 2190

REP 2200 
COMMON /BLOCRC/ NRC, RC (12600) IARGS, VWXYZ ( 8 ), NERROR

DIMENSION ARGS $(100)$

EQUIVALENCE (ARGS (1), RC (12501))

COMMON /BLOCKE/ NAME (4),L1,L2, ISRFLG

IF (NARGS.EQ.1) IF (L2-2) 25,20,40

RES 30

$K=10$

10

CALL ERROR (K)

RETURN

RESET NRMAX

C

IF (KIND (1).NE.0) IARGS (1)=ARGS (1)

IF (IARGS (1).GE.0.AND.IARGS (1).LE.NROW) GO TO 30

NRES

RES 50

RES 60

$\mathrm{K}=3$

GO TO 10

$30 \quad$ NRMAX $=$ I ARGS (1)

GO TO 20

RES 70

RES 80

RES 85

RES 90

RES 100

RES 110

RES 120

RES 130

RES 140

RES 150

RES 160

RES 170

RES 180

C RESET $V, W, X, Y, Z$

RES 190

40 IF (KIND (1).EQ.0) ARGS (1)=IARGS (1)

RES 200

VWXYZ (L2-2)=ARGS (1)

RES 210

GO TO 20

END

RES 220

RES 230 
SUBROUTINE RFORMT ( $X, N, N S, N W, N D, N X, X V A L, A R R A Y, N B, N C) \quad$ RFO 10 $\begin{array}{lllll}\text { VERSION } 5.00 & \text { RFORMT } 5 / 15 / 70 & \text { RFO } & 20\end{array}$

WRITTEN BY DAVID HOGBEN, SEL, NBS. 4/18/69. RFO 30

$\begin{array}{lll}* * * * * & \text { RFO } & 40\end{array}$

REWRITE AND COMBINING OF FXFARG(2/1/69) AND FXFORM(2/7/69). $\quad$ RFO 50

FLOATING FORMAT IPE NW.NS-1 IS GIVEN IF N LT 0 AND NX LT $0 . \quad 60$

FIXED FORMAT $F$ NW.ND IS GIVEN IF $N$ GE 0 AND NX LT 0. SET NS $=8 . \quad$ RFO 70

PERIOD NOT PRINTED IF NDECS $=0$

FXFARG SET *** XVAL $=X$ (OR ANY REAL VARIABLE)

$A R R A Y=A$ (OR ANY DIMENSIONED VECTOR)

NBLANK $=0$ (OR ANY OTHER INTEGER)

INPUT $* * * X, N, N S I G D S$, NWMAX.

OUTPUT *** NWIDTH, NDECS.

FXFORM SET *** $X=X$ (OR ANY REAL VARIABLE)

$N=1$ (OR ANY INTEGER)

NWMAX $=0$

INPUT *** XVAL, NSIGDS, NWIDTH, NDECS, NBLANK.

OUTPUT $* * *$ ARRAY

RFO 80

RFO 90

RFO 100

RFO 110

RFO 120

RFO 130

RFO 140

RFO 150

RFO 160

RFO 170

RFO 180

$* * * * *$

RFO 190

NUMBER IS CENTERED IF NC=1 AND NOT CENTERED IF NC $=0$ (RIGHT JUST'D) RFO 195

THIS REVISION WRITTEN $10 / 09 / 69$

RFO 197

COMMON /ABCDEF / L (48)

RFO 200

DIMENSION $X(1), \operatorname{ARRAY}(1), C(10)$

RFO 210

EQUIVALENCE (C(1),L(1)), (BLANK,L(45)), (PERIOD,L(38)), (CPLUS,L (4RFO 220

10)), (CMINUS,L (39)), (CASTER,L (41))

RFO 230

DOUBLE PRECISION Z

RFO 240

10

NSIGDS $=$ MINO $(8, N S)$

RFO 250

NSIGDS=MAXO ( 1 , NSIGDS )

RFO 260

IF (NX) $50,60,20$

RFO 270

FXFARG SUBROUTINE

RFO 280

INPUT FOR THE SUBROUTINE FXFORM. NHMAX IS THE MAXIMUM ALLOWABLE

VALUE OF NWIDTH. X MUST BE DIMENSIONED AND $N$ IS ITS LENGTH.

RFO 290

NWMAX MUST BE GREATER THAN NSIGDS PLUS FOUR

RFO 300

RFO 310

NWIDTH $=$ MIN (MMAX-MMIN+NSIGDS +2, NWMAX)

RFO 320

NDECS=NSIGDS-MMIN-1, NDECS=MINO (MAXO (NDECS,NSIGDS+2), NWMAX-3), IF RFO 340

NWIDTH EXCEEDS NHMAX.

IF NSIGDS GT 8, IT IS SET = TO 8, IF LT 1 SET = T0 1.

RFO 350

REFERENCE *** SEL NOTE N-68-3, SEPTEMBER, 1968.

WRITTEN BY DAVID HOGBEN, SEL, NBS. $2 / 1 / 69$.

$* * * * *$

RFO 360

RFO 370

RFO 380

RFO 390

NWMAX $=$ MAXO $(N S I G D S+5, N X)$

$Y=A B S(X(1))$

IF (Y.LE.0.) $Y=1.0$

$Y 1=Y$

$Y 2=Y$

IF (N.LT.1) $\mathrm{N}=1$

DO $30 \quad I=1, N$

$Y=A B S(X(I))$

IF (Y.LE.0.) $Y=1.0$

IF $(Y . L T . Y I) \quad Y I=Y$

IF $\left(Y . G T . Y_{2}\right) \quad Y_{2}=Y$

30 CONTINUE

MMIN $=F L O G 10(Y 1)$

IF (YI.LT.1.) MMIN=MMIN-I

MMAX $=F L O G 10(Y 2)$

IF (Y2.LT.1.) MMAX $=$ MMAX -1

RFO 400

RFO 410

RFO 420

RFO 430

RFO 440

RFO 445

RFO 450

RFO 460

RFO 470

RFO 480

RFO 490

RFO 495

RFO 500

RFO 510

RFO 520

RFO 530

$Z=Y 1$

RFO 540

$L L=Z * 10 . D 0 *$ (NSIGDS-MMIN ) +5. ODO

RFO 550 
IF (LL.GE . 10**(NSIGDS+1)) MMIN=MMIN+1

RFO 560

IF (LL.LT.10**NSIGDS) MMIN=MMIN-1

RFO 570

$Z=Y 2$

$L L=2 * 10 . D 0 * *(N S I G D S-M M A X)+5.000$

RFO 580

IF (LL.GE.10**(NSIGDS+1)) MMAX $=$ MMAX +1

RFO 590

IF (LL.LT.10**NSIGDS) MMAX = MMAX-1

RF0 600

NDECS $=$ NS I GDS-MMIN-1

RFO 610

NDECS $=\operatorname{MAX} 0(0, N D E C S)$

RFO 620

NHIDTH $=$ MMAX $+3+N D E C S$

RF0 630

IF (MMAX.LT.0) NWIDTH $=N D E C S+2$

RFO 640

IF (NWIDTH.LE.NWMAX) GO TO 40

RFO 650

NDECS $=$ MAXO (NDECS, NSIGDS+2)

RFO 660

NDECS = MINO (NDECS, NWMAX-3)

RFO 670

NWI DTH=NWMAX

IF (NDEC.S.LT.0) NDECS $=0$

RFO 680

RF0 690

$N D=N D E C S$

RFO 700

NWI $=$ NWIDTH

RF0 710

RETURN

RFO 720

$* * * * *$

RFO 730

FXFORM SUBROUTINE

RFO 740

SUBROUTINE FXFORM ALLOWS PRINTING OF REAL NUMBERS $X$ IN FIXED

RF0 750

C

C

FORMAT WITH DECIMAL POINT IN CONSTANT POSITION. NWIDTH $=$ WIDTH

RFO 760

FIELD, NDECS = NUMBER OF PLACES TO RIGHT OF DECIMAL POINT, $X$ IS RFO 780

THE NUMBER, ARRAY IS THE VECTOR WHERE X IS RETURNED TO BE PRINTED RFO 790

ACCORDING TO A FORHAT, NSIGDS = NUMBER OF DIGITS GIVEN WITH BLANKSRFO 800

ON THE RIGHT, NBLANK IS THE NUMBER OF BLANKS TO BE PUT ON THE RFO 810

LEFT OF THE FIELD. IF $X$ IS TOO LARGE OR TOO SMALL IT IS RETURNED RFO 820

AS A FLOATING POINT NUMBER. ARRAY MUST BE DIMENSIONED.

RF0 830

A PERIOD IS NOT GIVEN IF $X$ IS GREATER THAN OR EQUAL TO 10**NSIGDS RFO 840

ZERO IS WRITTEN 0 .

IF NDECS=NWIDTH, BLANKS ARE RETURNED

SUBROUTINE MAY BE USED IN CONJUCTION WITH SUBROUTINE FXFARGS.

RF0 850

RF0 860

IF NSIGDS GT 8, IT IS SET $=$ T0 8, IF LT 1 SET $=$ T0 1 .

RF0 870

NWIDTH IS ADJUSTED IF NECESSARY SO THAT IT IS GE NDECS+2,

RFO 880

GE NSIGDS+2 IF FIXED AND GE NSIGDS+5 IF FLOATING

RFO 890

REFERENCE *** SEL NOTE N-68-3, SEPTEMBER, 1968.

RF0 900

WRITTEN BY DAVID HOGBEN, SEL, NBS. $2 / 7 / 69$.

RFO 910

RF0 920

$* * * * *$

RF0 930

RFO 940

NWIDTH=NW

GO TO 70

60 NWIDTH=MAXO (NW,NSIGDS +2)

$70 \quad \operatorname{NDECS}=\operatorname{MAXO}(0, N D)$

IF (NWIDTH.LT.NDECS) NWIDTH=NDECS +2

RFO 950

RFO 960

RFO 970

RFO 980

$N B=\operatorname{MAXO}(0, N B)$

RF0 985

IF (NC.NE.0) NC=1

$N B L A N K=N B-(N B / 2) * N C$

RFO 990

RFO 995

$M F=0$

$Y=A B S(X V A L)$

$80 \quad$ NDIFF $=$ NWIDTH-NDECS

RF0 1000

RFO1010

RFO1020

NWMAX $=N$ WI DTH+NBLANK

RF01030

$N P O N E=N D I F F+N B L A N K$

RF01040

$I E N D=N W I D T H+N B$

RF01045

RF01050

DO $90 \mathrm{I}=1, \mathrm{IEND}$

$90 \quad$ ARRAY (I $)=B L A N K$

IF (NDECS.EQ.NWIDTH) RETURN

RF01060

RF01070

IF (Y.GT.O.) GO TO 110

RF01080

RFO 1090

$X V A L=0$. IS SPECIAL CASE UNLESS FIXED OR FLOATING

RF01100

IF (NX.GE.0) GO TO 100

RFO 1110 
$M=0$

RFO1120

GO TO 130

100 IF (NDECS.NE.0) ARRAY (NPONE)=PERIOD

RF01130

ARRAY (NPONE -1$)=C(1)$

RF01140

RETURN

$110 \quad \mathrm{M}=\mathrm{FLOGIO}(\mathrm{Y})$

IF (Y.LT.1.) $M=M-1$

$Z=Y$

$Z=Z * 10 . D 0 * *(N S I G D S-M)$

$X_{1}=Z$

$\mathrm{LLI}_{1}=\mathrm{X} \mathrm{I}$

$X 2=Z-D B L E(X 1)$

$\mathrm{LL2}=\mathrm{X} 2$

$L L=L L 1+L L 2+5$

IF (LL.LT.10**(NSIGDS+1)) GO TO 120

RF01150

RF01160

RF01170

RF01180

RF01190

RF01193

RF01196

RF01200

RF01205

RF01210

RF01215

RF01220

$M=M+1$

RF01230

$L L=L L / 10$

RF01240

GO TO 130

RF01250

RF01260

IF (LL.GE.10**NSIGDS) GO TO 130

RF01270

in $=\mathrm{M}-1$

RF0 1280

$L L=10 * L L$

130 IF (NX.EQ.0) GO TO 170

RF0 1290

IF (N.LT.O) GO TO 180

RF0 1300

C FIXED

IF (M.LT.NDIFF-2) GO TO 150

IF (M.EQ.NDIFF-2.AND.XVAL.GE.0.) GO TO 150

RF01310

RFO 1320

RF0 1330

NSIGDS $=$ MAXO $(0, N H I D T H-5)$

RF01340

IF (NSIGDS.GT.0) GO TO 180

C PUT IN ASTERISKS

DO $140 \quad I=1$, NWIDTH

$I R V S P=I+N B L A N K$

140 ARRAY (IRVSP) $=$ CASTER RETURN

150 NSIGDS $=$ MINO $(8, N D E C S+M+1)$

NSIGDS $=$ MAXO $(0, N S I G D S)$

$L L=(L L-5) /(10 * *(8-N S I G D S))+5$

IF (NSIGDS.GT.0) GO TO 170

IF (XVAL.LT.0.0) ARRAY (NPONE-1)=CMINUS

DO $160 \quad I=N P O N E$, NWMAX

$160 \quad \operatorname{ARRAY}(\mathrm{I})=\mathrm{C}(1)$

ARRAY (NPONE) $=$ PERIOD

IF (NDECS +1.EQ. (-M) .AND .LL.GT.10) ARRAY (NWMAX) =C (2)

RETURN

170 MREAL $=0$

IF (M.GE.NSIGDS-1-NDECS.AND.M.LT.NDIFF-2) GO TO 190

RF0 1350

RF01360

RF0 1370

RF01375

RF01380

RF01390

RF01400

RF01410

RF01420

RF01430

RF01440

RF01450

RF01460

RF01470

RF01480

RF01490

RF01500

RF01510

IF (M.EQ.NDIFF-2.AND.XVAL.GT.0.) GO TO 190

RF01520

C FLOATING

160 MREAL $=M$

$M=0$

$\mathrm{MF}=1$

$Y=Y^{*} 10 * *(-M R E A L)$

RF0 1530

RFO1540

RF01550

RF01560

RF01570

RF01580

IF (M.LT.NSIGDS.AND.NDECS.NE.0) ARRAY (NPONE)=PERIOD

RFO 1590

NINT $=$ NPONE $-1-M$

IF (M.LT.0) NINT=NINT+1

RF01600

NEND $=N I N T+N S I G D S-1$

IF (M.GE.O.AND.M.LT.NSIGDS-I) NEND $=$ NEND +1

RFO1610

DO $200 \mathrm{~J}=$ NINT, NEND

RF01620

$I=N E N D+N I N T-J$

RF01630

IF (I.EQ.NPONE) GO TO 200 
$L L=L L / 10$

RF01660

$N N=$ MOD $(L L, 10)$

RF01670

ARRAY $(I)=C(N N+1)$

RFO1680

CONT INUE

IF (MF.EQ.0) GO TO 220

C PUT IN EXPONENT FOR FLOATING POINT NUMBER

RF01690

RF01700

IF (NWIDTH.GE.NSIGDS+5) GO TO 210

NWI DTH $=N S I G D S+5$

GO TO 80

210 IF (MREAL.LT.0) ARRAY (NEND +1$)=$ CMINUS

IF (MREAL.GE.0) ARRAY (NEND +1$)=$ CPLUS

RF01710

RF01720

RF01730

RF01740

RF01750

MREALA $=$ I ABS (MREAL)

$M I=M R E A L A / 10$

$M 2=M O D(M R E A L A, 10)$

ARRAY $(N E N D+2)=C(M l+1)$

ARRAY $(N E N D+3)=C(M 2+1)$

220 IF (XVAL.LT.0.AND.M.GE.0) ARRAY (NINT-1)=CMINUS

IF (XVAL.LT.0.AND.M.LT.0) ARRAY (NPONE-1)=CMINUS

RF01760

RF0 1770

RF01780

RF01790

RF01800

RF01810

IF (M.GE.(-1)) GO TO 240

I $1=N P O N E+1$

I $2=N I N T-1$

DO $230 \mathrm{I}=\mathrm{I} 1, \mathrm{I} 2$

RF01820

RF01830

RF01840

RF01850

RF01860

RF01870

$\operatorname{ARRAY}(I)=C(1)$

RF01880

RETURN

C PUT IN NON-SIGNIFICANT ZEROS

240 IF (M.LT.NSIGDS.OR.MF.NE.0) RETURN

RF01890

RF01900

I $I=N I N T+N S I G D S$

I $2=N P O N E-1$

DO $250 \mathrm{I}=\mathrm{I} 1, \mathrm{I} 2$

$250 \operatorname{ARRAY}(I)=C(1)$

RETURN

RF01910

RF01920

RF01930

RF01940

RF01950

RF01960

END

RF01970 
IF AN ERROR IS MADE IN A STORED STATEMENT, THIS ROUTINE PRINTS

RND 40 OUT EXACTLY WHEN AND WHERE IT OCCURRED.

RND 50

RND 60

RND 70

RND 80

$A=F L O A T(I N D E X(6, L E V E L)) / 10$.

RND 90

WRITE (ISCRAT, 50) A.

RND 100

$\mathrm{N}=$ LEVEL -1

10 IF (N) $40,30,20$

$20 \quad A=F L O A T(\operatorname{INDEX}(6, N)) / 10$.

WRITE (ISCRAT,60) INDEX $(5, N+1), \operatorname{INDEX}(4, N+1), A$

RND 110

RND 120

RND 130

$\mathrm{N}=\mathrm{N}-1$

GO TO 10

30 WRITE (ISCRAT, 70) INDEX $(5,1), \operatorname{INDEX}(4,1)$

40 RETURN

RND 140

RND 150

RND 160

RND 170

RND 180

RND 190

FORMAT ( 3 IH IN COMMAND AT STATEMENT NUMBER, F $6.1,47 \mathrm{X}$ )

RND 200

60 FORMAT ( $10 \mathrm{H}$ CYCLE NO.,I4,3H OF, I4,24H OF PERFORM AT STATEMENT,F6. IRND 210 $1,23 X)$

RND 220

70 FORMAT ( $10 \mathrm{H}$ CYCLE NO.,I $4,3 \mathrm{H}$ OF, I4,3IH OF EXTERNAL PERFORM STATEMENRND 230 IT. 32X)

END

RNJ 10

C VERSION 5.00 RNJBK $5 / 15 / 70$

DATA M,FLM/8192,8192./

RNJ 20

C $\quad 8192=2 * 13$

RNJ 30

RNJ 40

C JB KRUSKAL (1969) ACM, 12, 93-94.

RNJ 50

$* * * * *$

RNJ 60

RETURNS RANDOM NUMBER $(0,1)$ IN RNJBK.

RNJ 70

SET NSTART $=1$ TO START AT BEGINNING, OTHERWISE PREVIOUS NFINSH.

RNJ 80

RETURNS NFINSH FOR LAST NUMBER GENERATED.

WRITTEN BY DAVID HOGBEN, SEL, NBS. 3/24/69.

RNJ 90

RNJ 100

******

RNJ 110

$\mathrm{K}=\mathrm{NSTART}$

RNJ 120

DO $10 \quad I=1,3$

RNJ 130

$K=\operatorname{MOD}(5 * K, M)$

RNJ 140

RNO $=F L O A T(K) / F L M$

RNJ 150

NF INSH $=K$

RNJ 160

RETURN

RNJ 170

END

RNJ 180 
VERSION $5.00 \quad$ RPRINT $5 / 15 / 70 \quad$ RPR 20

$* * * * * * * * * * * * * * * * * * * * * * * * * * * * * * * * * * * * * * * * * * * * * * * * * * * * * * * * * * * * * * * * * * \mathrm{RPR} \quad 30$

RPRINT COLS ++, ++, ...++ (MAXIMUM OF 50 COLS) (NO OF SD IS 8 ) RPR 40

RPRINT COLS ++, ++, ...++ WITH ** SIGNIFICANT DIGITS (49 COL MAX)RPR 50

RPRINT COLS $++\ldots++$ WITH $* *$ SD,$\ldots(N W M A X=13$, NWIDTH+NBLANK=15) RPR 60

RPRINT ** COLS, COL ++ WITH , SD .. (NWMAX $=22$, NBLANK=3) RPR 80

RPRINT ** COLS, ++ WITH, SD AND NWMAX $=\ldots \ldots \quad \ldots \quad(N B L A N K=3) \quad$ RPR 90

RPRINT ** COLS, ++ WITH , SD NWMAX , NBLANK , ... RPR 100

RPR 110

FLOATING IPEW.D IS OBTAINED IN LAST TWO OPTIONS IF NWMAX $=0 . \quad$ RPR 120 $W=N S I G D S+5$ AND $D=N S I G D S-1$

FIXED FW.D IS OBTAINED IN LAST TWO OPTIONS IF NWMAX LT $0 . \quad$ RPR 140 $W=-N H M A X \quad$ AND $D=N S I G D S \quad$ RPR 150

IW IS OBTAINED IN LAST TWO OPTIONS IF NSIGDS=0 AND NWMAX $=-W . \quad$ RPR 160

$* * * * * * * * * * * * * * * * * * * * * * * * * * * * * * * * * * * * * * * * * * * * * * * * * * * * * * * * * * * * * * * * * * \mathrm{RPR} 170$

REPLACES TRAILING ZEROS BY BLANKS IF COUNT LT NRMAX RPR 180

IF COLUMN CONTAINS ALL ZEROS AND FIRST ARGUMENT IS A COLUMN NUMBERRPR 190 NO COLUMN HEADING IS GIVEN

RPR 200

IF FIRST ARGUMENT IS NOT A COLUMN NUMBER (1) NO HEADING IF WIDTH RPR 210 LESS THAN 6, (2) COL NO IF 6 LE WIDTH LT 12, (0) COLUMN XXX. IFRPR 220 WIDTH GREATER THAN OR RQUAL TO 12.

IF NUMBER IS FLOATED ASTERISK IS PUT TO RIGHT OF FIELD.

NUMBERS ARE PRINTED IN BLOCKS OF 5 IF NRMAX IS LESS THAN 49.

RPR 230

WRITTEN BY DAVID HOGBEN, SEL, NBS. 4/17/69.

RPR 240

COMMON /ABCDEF/ L (48)

RPR 250

COMMON /BLOCRC/ NRC, RC (12600)

RPR 270

COMMON /BLOCKD/ IARGS(100), KIND (100), ARGTAB $(100)$,NRMAX, NROW, NCOL, NRPR 300

IARGS, VWXYZ (8), NERROR

DIMENSION ARGS $(100)$

EQUIVALENCE (ARGS(1),RC(12501))

COMMON /BLOCKE/ NAME (4),L1,L2, ISRFLG

COMMON /SCRAT/ NS, NS2, A(13500)

COMMON /HEADER/ NOCARD $(80), \operatorname{ITLE}(60,6)$, LNCNT, IPRINT, NPAGE , IPUNCH COMMON /FMAT/IFMTX (6), IOSWT, IFMTS (6), LHEAD (96)

RPR 310

RPR 320

RPR 330

RPR 340

RPR 350

RPR 360

RPR 370

$* * * * * * * * * * * * * * * * * * * * * * * * * * * * * * * * * * * * * * * * * * * * * * * * * * * * * * * * * * * * * * * * * * \mathrm{RPR} \quad 380$

DIMENSION NHIDTH $(100), \operatorname{NDECS}(100), \operatorname{NBLANK}(100), \operatorname{IRGS}(100), \operatorname{NCOUNT}(\mathrm{RPR} 390$ $1100), \operatorname{AWMAX}(100), \operatorname{NSIGDS}(100), \mathrm{AL}(48), \operatorname{NF}(100), \mathrm{NWM}(100) \quad$ RPR 400

EQUIVALENCE (NHIDTH(1),A(1001)), (NDECS(1),A(1101)), (NBLANK(1),A(RPR 410 $11201)),(\operatorname{IRGS}(1), A(1301)),(\operatorname{NCOUNT}(1), A(1401)),(\operatorname{NWMAX}(1), A(1601)) R P R 420$

2. $(\operatorname{NSIGDS}(1), A(1701)),(A L(1), L(1)),(N F(1), A(1801)),(N W M(1), A(19 R P R 430$ $301))$

IRGS(I) NEEDED FOR HEADS BECAUSE CHKCOL IS USED

RPR 450

LINES 20 TO 70 DO ERROR CHECKING

RPR 460

IF (LI.NE.6) GO TO 20

RPR 470

NARGS $=$ NARGS -1

IF (KIND(1).EQ.1) GO TO 50

IF (IARGS (1).LE.0.OR.IARGS (1).GT.NROW) GO TO 60

RPR 480

RPR 490

RPR 500

NRJ $=$ IARGS $(1)-1$

IF (NARGS.LT.I) GO TO 30

DO $10 \quad I=1$, NARGS

$\operatorname{KIND}(I)=\operatorname{KIND}(I+1)$

$\operatorname{IARGS}(I)=I$ ARGS $(I+1)$

$10 \operatorname{ARGS}(I)=\operatorname{ARGS}(I+1)$ 
GO TO 400

RPR 600

IF (NRMAX.GT.0) GO TO 70

RPR 610

CALL ERROR (?)

GO TO 400

RPR 620

RPR 630

50 CALL ERROR (3)

GO TO 400

60 CALL ERROR (11)

GO TO 400

RPR 640

RPR 650

RPR 660

C ALL ARGUMENTS ARE INTEGERS.

RPR 670

RPR 680

$N P A R=1$

IF (KIND(1).EQ.1.0R.KIND (NARGS).EQ.1) GO T0 90

RPR 690

DO $80 \quad I=1$, NARGS

RPR 700

$\operatorname{NSIGDS}(I)=8$

RPR 710

$N F(I)=1$

$N W M(I)=0$

RPR 720

RPR 730

$\operatorname{NWMAX}(I)=13$

80 IRGS (I) =I ARGS (I)

CALL CHKCOL (J)

IF (J.NE.0) GO TO 50

$N A=N A R G S$

GO TO 160

C LAST ARGUMENT IS NOT AN INTEGER, NSIGDS IS GIVEN.

90 IF (KIND (1).EQ.1) GO TO 130

$\mathrm{LL}=0$

DO $100 \quad I=I$, NARGS

IF (KIND(I).EQ.0) GO TO 100

$\mathrm{LL}=\mathrm{LL}+1$

$\operatorname{ARGS}(L L)=\operatorname{ARGS}(I)$

100 CONTINUE

$N L=0$

DO $120 \quad I=1$, NARGS

IF (KIND(I).EQ.0) GO TO 110

$\mathrm{NL}=\mathrm{NL}+1$

GO TO 120

$110 \quad \mathrm{I} 2=\mathrm{I}-\mathrm{NL}$

$\operatorname{IARGS}($ I 2$)=I$ ARGS (I)

$\operatorname{NSIGDS}(I 2)=A R G S(N L+1)$

NWMAX (I 2) $=13$

NF $(12)=1$

NWM $(I 2)=0$

$\operatorname{IRGS}($ I 2$)=I$ ARGS (I 2$)$

$120 \quad \mathrm{KINO}$ (I 2$)=0$

$N A=N A R G S-N L$

NARGS=NA

CALL CHKCOL (J)

IF (J.NE.0) GO TO 50

GO TO 160

C FIRST ARGUMENT IS NOT AN INTEGER, PARAMETERS ARE GIVEN.

130 IF (ARGS(1).LE.0.) GO TO 50

IRVSP $=$ ARGS (1)

NPAR $=($ NARGS-1) $/$ IRVSP

IF (NPAR.NE.2.AND.NPAR.NE.3.AND.NPAR.NE.4) GO TO 30

$A(1)=A B S(F L O A T(N P A R) * A R G S(1)+1 .-F L O A T$ (NARGS))

IF $(A(1) . G T .0 .0)$ GO TO 30

$N A=A R G S(1)$

DO $150 \quad I=1, N A$

$I S U B=2+N P A R^{*}(I-1)$

$\operatorname{IRGS}(I)=I$ ARGS (ISUB)

$\operatorname{IARGS}(I)=\operatorname{IARGS}($ ISUB $)$

RPR 740

RPR 750

RPR 760

RPR 770

RPR 780

RPR 790

RPR 800

RPR 810

RPR 820

RPR 830

RPR 840

RPR 850

RPR 860

RPR 870

RPR 880

RPR 890

RPR 900

RPR 910

RPR 920

RPR 930

RPR 940

RPR 950

RPR 960

RPR 970

RPR 980

RPR 990

RPR 1000

RPR 1010

RPR 1020

RPR 1030

RPR 1040

RPR 1050

RPR 1060

RPR 1070

RPR 1080

RPR 1085

RPR 1090

RPR 1100

RPR 1110

RPR 1120

RPR 1130

RPR 1140

RPR 1150

RPR 1160

RPR 1170 
$N F(I)=1$

$\operatorname{NWM}(I)=0$

IF (NWMAX (I).GT.0) GO TO 150

RPR 1220

IF (NWMAX (I).LT.0) GO TO 140

RPR 1230

RPR 1240

RPR 1250

FLOATING

c

NWIDTH (I ) $=$ NSIGDS (I $)+5$

RPR 1260

$\operatorname{NDECS}(I)=\operatorname{NSIGDS}(I)+2$

RPR 1270

NF (I) $=-1$

RPR 1280

$N W M(I)=-1$

RPR 1290

GO TO 150

C FIXED

140 NWIDTH (I ) =-NWMAX (I)

$\operatorname{NDECS}(I)=\operatorname{NSIGDS}(I)$

RPR 1300

RPR 1310

RPR 1320

$\operatorname{NSIGDS}(I)=8$

$\mathrm{NWM}(\mathrm{I})=-1$

$150 \operatorname{NBLANK}($ I $)=$ I ARGS $($ ISUB +3$) *($ NPAR $/ 4)+3 *(1-N P A R / 4)$

RPR 1330

IF (NPAR . EQ . 4. AND.NBLANK (1).LT.1) NBLANK (1)=1

RPR 1340

RPR 1350

RPR 1360

C LINES 160 TO 240 INITIALIZE AND CALL FXFARG

RPR 1370

RPR 1375

RPR 1380

160 IF (NERROR.NE.0) GO TO 400

RPR 1390

IF (LI.NE.6) GO TO 210

RPR 1400

I $1=1$

I $2=0$

RPR 1405

$\mathrm{LL}=1$

$I 2=\operatorname{MINO}(8, N A)+I 2$

RPR 1410

165

$\mathrm{NA}=\mathrm{NA}-8$

DO $200 \quad I=I 1, I 2$

RPR 1415

RPR 1420

RPR 1425

$K=I$ ARGS (I)+NR J

RPR 1430

IF (NWMAXX(I).LE.0) GO TO 170

RPR 1440

RPR 1450

CALL RFORMT (RC (K), I, NSIGDS (I), NWIDTH (I), NDECS (I), NWMAX (I), A (I), A (RPR1460 11) $, 0,0)$

170 IF (NPAR.EQ.1) NBLANK (I) $=15-N W I D T H(I)$

RPR 1470

RPR 1480

CALL RFORMT (A,NF (I) ,NSIGDS (I),NWIDTH (I),NDECS (I), NWM(I) , RC (K) , A (LRPR1490 IL), NBLANK (I) , 0)

$L L=L L+N W I D T H(I)+N B L A N K(I)$

IF (NWIDTH (I).LT.NWMAX (I).OR.NWMAX (I).LE.0) GO TO 200

IF (NBLANK (I).EQ.0) GO TO 200

I $5=$ LL-NDECS $(I)+N S I G D S(I)-1$

IF (A(I5) $-A L(39)) \quad 180,190,180$

180 IF $(A(I 5)-A L(40)) \quad 200,190,200$

$190 \quad \mathrm{~K}=\mathrm{LL}-\mathrm{NWIDTH}(\mathrm{I})-1$

$A(K)=A L(41)$

200 CONTINUE

$N L=M I N O(L L-1,120)$

WRITE (IPRINT, 410) (A (I), I=2,NL)

IF (NA.LE.0) GO TO 400

I $1=I 2+1$

GO TO 165

$210 \quad$ I $1=1$

DO $240 \mathrm{I}=1$, NA

$\mathrm{K}=\mathrm{I}$ ARGS $(\mathrm{I})$

C DETERMINE COUNT OF COL I

NCOUNT (I) =NRMAX

DO $220 \mathrm{~J}=1$, NRMAX

$K 1=K+N R M A X-J$

IF (ABS (RC (K1)).GT.0.) GO TO 230

RPR 1500

RPR 1510

RPR 1520

RPR 1530

RPR 1540

RPR 1550

RPR 1560

RPR 1570

RPR 1580

RPR 1590

RPR 1600

RPR 1610

RPR 1615

RPR 1620

RPR 1625

RPR 1630

RPR 1640

RPR 1650

RPR 1660

RPR 1670

RPR 1680

RPR 1690

RPR 1700 
230 IF (NCOUNT (I) .GT.NRMAX - 3) NCOUNT (I) =NRMAX

IF (NCOUNT (I).EQ.0) NWIDTH (I) $=$ NWMAX (I)

RPR 1720

IF (NCOUNT (I) .EQ.0) GO TO 240

RPR 1730

IF (NWMAX (I).LE.0) GO TO 240

RPR 1740

$1, A(1), A(1), 0,0)$

240

C

250

IF (NPAR.EQ.1) NBLANK (I) =15-NWIDTH (I)

LINES 250 TO 390 CALL FXFORM AND PRINT IN READABLE FORMAT

IF (LI.NE.8) CALL PAGE (4)

$I 4=0$

DO $260 \quad I=I 1$, NA

I 4=I 4+NWIDTH (I ) +NBLANK (I)

IF (I 4 .LE.120) GO TO 260

I $4=I-I 1$

GO TO 270

260 IF (I.EQ.NA) I $4=N A-I I+1$

270 I $2=I 4+I 1-1$

NARGS $=12-11+1$

C FROM HERE TO 290 PUTS IN COL HEADING IF FIRST ARG NOT A COL NO. IF (NPAR.EQ.1) GO TO 290

CALL RFORMT $(A, 1,1,119,119,0,1.0, A(1), 0,0)$

$L L=1$

DO $280 \quad I=I 1, I 2$

$L L=L L+N W I D T H(I)+N B L A N K(I)$

IF (NWIDTH(I).LT.6.OR.NCOUNT (I).EQ.0) GO TO 280

$A(200)=\operatorname{IRGS}(I)$

I $5=F L O G I 0(A(200))+1.0$

CALL RFORMT $(A, 1, I 5,6,0,0, A(200), A(L L-6), 0,0)$

$A(L L-1)=A L(45)$

IF (NWIDTH(I).LT.12) GO TO 280

$A(L L-12)=A L(13)$

$A(L L-11)=A L(25)$

$A(L L-10)=A L(22)$

$A(L L-9)=A L(31)$

$A(L L-8)=A L(23)$

$A(L L-7)=A L(24)$

280 CONTINUE

WRITE (IPRINT, 410) (A (I) , I=2,LL)

GO TO 320

290 IF (LI.EQ.8) GO T0 325

CALL HEADS (IRGS (II), I $4,0,1$ )

DO $310 \quad I=I 1, I 2$

IF (NCOUNT (I).GT.0) GO TO 310

I $5=12 *(I-I I)+1$

DO $300 \quad$ I $6=1,12$

$\operatorname{LHEAD}($ I 5$)=\mathrm{L}(45)$

$300 \quad$ I $5=I 5+1$

310 CONTINUE

I $5=12 *$ I 4

WRITE (IPRINT, 420) (LHEAD(I6), I $6=1,15$ )

320 WRITE (IPRINT, 410)

325 DO $390 \mathrm{~J}=1$, NRMAX

$L L=1$

DO $350 \quad I=I 1, I 2$

$K=I$ ARGS $(I)+J-I$

C PRINT BLANKS IF NCOUNT (I) LT NRMAX

IF (J.GT.NCOUNT (I)) NDECS (I) $=\operatorname{NWIDTH}$ (I)

CALL RFORMT (A,NF (I),NSIGDS (I),NWIDTH (I),NDECS (I),NWM (I), RC (K), A (LRPR2290
RPR 1770

RPR 1780

RPR 1790

RPR 1800

RPR 1810

RPR 1820

RPR 1830

RPR 1840

RPR 1850

RPR 1860

RPR 1870

RPR 1880

RPR 1890

RPR 1900

RPR 1910

RPR 1920

RPR 1930

RPR 1940

RPR 1950

RPR 1960

RPR 1970

RPR 1980

RPR 1990

RPR 2000

RPR 2010

RPR 2020

RPR 2030

RPR 2040

RPR2050

RPR 2060

RPR 2070

RPR 2080

RPR 2090

RPR 2100

RPR 2110

RPR 2120

RPR2 130

RPR 2140

RPR 2150

RPR 2160

RPR 2170

RPR 2180

RPR 2190

RPR2200

RPR 2210

RPR2220

RPR2230

RPR 2240

RPR 2250

RPR2 260

RPR 2270

RPR2280 
IL), NBLANK (I), 0 )

$L L=L L+N W I D T H(I)+N B L A N K(I)$

IF (NWIDTH(I).LT.NWMAX (I).OR.NWMAX (I).LE.0) GO TO 350

IF (NBLANK (I).EQ.O) GO TO 350

C PUT IN ASTERISK IF READABLE RETURNS FLOATING

RPR2300

RPR2310

RPR 2320

RPR 2330

RPR 2340

I $5=$ LL-NDECS (I ) +NSI GDS ( I ) - I

IF (A(I5)-AL (39)) $330,340,330$

330 IF $(A(I 5)-A L(40)) \quad 350,340,350$

RPR2350

RPR 2360

RPR 2370

RPR 2380

$K=L L-N W I D T H(I)-I$

$A(K)=A L(41)$

350 CONTINUE

C I $=2$ COMPENSATES FOR $I X$

$\mathrm{NL}=\mathrm{LL}-1$

WRITE (IPRINT, 410) (A(I), I=2,NL)

C PRINT IN BLOCKS OF FIVE

IF (J.EQ.NRMAX) GO TO 355

IF (MOD $(J, 5)$.EQ.0.AND.NRMAX.LE.48) WRITE (IPRINT, 410)

IF (MOD (J,10).EQ.0.AND.NRMAX.GT.48) WRITE (IPRINT, 410)

RPR 2390

RPR 2400

RPR 2410

RPR 2420

RPR 2430

RPR 2440

RPR 2445

RPR 2450

RPR 2460

C CALL NEW PAGE IF NRMAX GT 50

RPR 2470

RPR2 480

IF (MOD $(J, 50)$.NE.0) GO TO 390

IF (J.EQ.NRMAX) GO TO 390

IF (LI.NE.8) CALL PAGE (4)

IF (LI.EQ.8) GO TO 390

I $5=12 *$ I 4

IF (NPAR.GT.1) GO TO 360

WR ITE (IPRINT, 420) (LHEAD (I 6), I $6=1$, I 5)

GO TO 380

C FROM HERE TO 380 PUTS IN COL HEADING IF FIRST ARG NOT A COL NO.

RPR 2485

RPR2490

RPR2500

RPR2510

RPR 2520

RPR 2530

RPR 2540

RPR 2550

CALL RFORMT $(A, 1,1,119,119,0,1.0, A(1), 0,0)$

$\mathrm{LL}=1$

DO $370 \quad I=I 1, I 2$

$L L=L L+N W I D T H(I)+N B L A N K(I)$

IF (NHIDTH(I).LT.6.OR.NCOUNT(I).EQ.0) GO TO 370

$A(200)=I R G S(I)$

I $5=F L O G I 0(A(200))+1.0$

CALL RFORMT (A, I, I $5,6,0,0, A(200), A(L L-6), 0,0)$

$A(L L-1)=A L(45)$

IF (NWIDTH(I).LT.12) G0 TO 370

$A(L L-12)=A L(13)$

$A(L L-11)=A L(25)$

$A(L L-10)=A L(22)$

$A(L L-9)=A L(31)$

$A(L L-8)=A L(23)$

$A(L L-7)=A L(24)$

370 CONTINUE

WRITE (IPRINT, 410) (A(I), I=2,LL)

380 WRITE (IPRINT, 410)

390 CONTINUE

C ADJUST FOR MORE THAN 8 COLUMNS

IF (I2.EQ.NA) GO TO 400

IF (LI.NE.8) GO TO 145

WR ITE (IPRINT, 410)

$145 \quad I 1=I 2+1$

GO TO 250

400 RETURN

C

410 FORMAT (IX, 119AI)

420 FORMAT $(8(3 X, 12 A 1))$

END

RPR 2560

RPR 2570

RPR 2580

RPR 2590

RPR 2600

RPR 2610

RPR 2620

RPR 2630

RPR 2640

RPR 2650

RPR 2660

RPR 2670

RPR 2680

RPR 2690

RPR 2700

RPR 2710

RPR 2720

RPR 2730

RPR 2740

RPR 2750

RPR 2760

RPR 2770

RPR 2772

RPR 2778

RPR 2780

RPR 2790

RPR 2810

RPR 2820

RPR 2830

RPR 2840

RPR 2850 
SUBROUTINE SELECT

C VERSION 5.00 SELECT $5 / 15 / 70$

SEL $\quad 10$

COMMON /BLOCRC/ NRC, RC(12600)

SEL 20

COMMON /BLOCKD/ IARGS(100), KIND(100), ARGTAB(100), NRMAX, NROW, NCOL, NSEL 40

IARGS, VWXYZ (8), NERROR

SEL 50

DIMENSION ARGS (100)

EQUIVALENCE (ARGS(1), RC (12501))

SEL 60

COMMON /BLOCKE/ NAME (4),L1,L2, ISRFLG

SEL 70

COMMON /SCRAT/ NS, NS2, A (13500)

SEL 80

SEL 90

C

ITYPE $=1$ SELECT IN COL ++ VALUES APPROX COL ++ TO WITHIN **,

SEL 100

C

C

C

C

C

$\mathrm{C}$

C

C

C

C

C

c

c

ITYPE $=1$ SELECT IN COL ++ VALUES APPROX COL ++ TO WITHIN **, SEL 130

STORE IN COL ++

SEL 110

SEL 120

STORE IN COL ++ TO COL ++ SEL 140

ITYPE $=1$ SELECT IN COL ++ VALUES APPROX COL ++ TO WITHIN **, SEL 150 STORE ++ TO ++, STORE NUMBER FALLING WITHIN TOL IN COSEL 160

ITYPE $=2$ SEARCH IN COL ++ FOR NUMBERS IN ++, TRANSFER CORRESP VSEL 180 FROM ++ INTO ++, ++ INTO ++, ETC SEL 190

SEL 200

ITYPE $=3$ CENSOR COL ++ FOR \$\$, REPLACING BY \$\$, STORE IN COL ++SEL 210

SEL 220

ITPE $=5$ MATCH COLUMN (C) WITH (E), EXTRACT (E), PUT IN COLUMN (C) SEL 225 WRITTEN BY DAVID HOGBEN SEL, NBS. 2/28/70. (CENSOR REVISED) SEL 230

G0 TO $(10,100,130,40,130), \mathrm{L} 2$

SEL 235

IF (KIND(3)) $50,20,50$

$\begin{array}{ll}10 & I F \\ 20 & K=3\end{array}$

30 CALL ERROR (K)

40 RETURN

$50 \quad \operatorname{IARGS}(3)=\operatorname{IARGS}(2)$

$\operatorname{KIND}(3)=0$

IF (NARGS-4) $60,70,80$

$60 \quad K=10$

GO TO 30

$65 \quad K=11$

GO TO 30

$70 \quad \operatorname{IARGS}(5)=\operatorname{IARGS}(4)$

NARGS $=$ NARGS +1

$\operatorname{KIND}(5)=\operatorname{KIND}(4)$

80 IF (NARGS-6) $90,90,60$

90 IF (IARGS (4)-IARGS (5)) 230,230,20

100 IF (NARGS-4) $60,110,110$

110 IF (2* (NARGS/2)-NARGS) $60,120,60$

120 CALL CHKCOL (J)

IF (J) $20,200,20$

130 IF (NARGS-4) $60,140,60$

140 CALL ADRESS ( $1, I 1)$

IF (II) $20,65,150$

150 CALL ADRESS (2, I2)

IF (I2) $160,65,170$

$160 \quad \mathrm{I} 2=-\mathrm{I} 2$

170 CALL ADRESS (3, I3)

IF (I3) $180,65,190$

$180 \quad \mathrm{I} 3=-\mathrm{I} 3$

190 CALL ADRESS (4, I4)

IF (I4) $20,65,200$

200 IF (NRMAX) $210,210,220$

SEL 240

SEL 250

SEL 260

SEL 270

SEL 280

SEL 290

SEL 300

SEL 310

SEL 320

SEL 330

SEL 335

SEL 337

SEL 340

SEL 350

SEL 360

SEL 370

SEL 380

SEL 390

SEL 400

SEL 410

SEL 420

SEL 430

SEL 440

SEL 450

SEL 460

SEL 470

SEL 480

SEL 490

SEL 500

SEL 510

SEL 520

SEL 530

SEL 540

$210 \quad K=9$

SEL 550 
GO TO 30

SEL 555

GO TO $(250,450,520,40,520), \mathrm{L} 2$

SEL 565

230

IF (IARGS (5)-IARGS (4)-NRMAX+1) 120,120,240

SEL 570

$240 \operatorname{IARGS}(5)=\operatorname{IARGS}(4)+N R M A X-1$

SEL 580

GO TO 120

C SELECT

250 DO $280 \quad I=1$, NRMAX

SEL 590

$L=I$ ARGS ( 1 ) $+\mathrm{I}-1$

SEL 600

$K=I$ ARGS $(2)+I-1$

SEL 610

$J=I$ ARGS $(4)+I-1$

SEL 620

SEL 630

$M=N R M A X+I$

SEL 640

$A(I)=R C(K)$

SEL 650

$A(M)=R C(L)$

SEL 660

SEL 670

$260 \quad R C(J)=0.0$

SEL 680

IF $(J-I-I A R G S(5)+1) \quad 270,280,280$

SEL 690

$270 \mathrm{~J}=\mathrm{NROW}+\mathrm{J}$

GO TO 260

SEL 700

SEL 710

280 CONTINUE

ARG $3=A B S(\operatorname{ARGS}(3))$

SEL 720

DO $440 \quad I=1$, NRMAX

SEL 730

$K=N R M A X+1$

SEL 740

$L=2$ *NRMAX

SEL 750

$M=3 *$ NRMAX

SEL 760

$N=4$ * NRMAX

SEL 770

$I 1=\operatorname{IARGS}(4)+I-1$

SEL 780

$J I=I \operatorname{ARGS}(6)+I-1$

SEL 790

DO $300 \quad \mathrm{~J}=\mathrm{K}, \mathrm{L}$

$A T=A B S(A(I)-A(J))$

SEL 800

SEL 810

SEL 820

IF (ARG3-AT) $300,290,290$

SEL 830

SEL 840

SEL 850

$A(M)=A T$

$\mathrm{N}=\mathrm{N}+\mathrm{l}$

$A(N)=A(J)$

300 CONTINUE

IF (M-3*NRMAX-1) $310,330,350$

310 IF (NARGS-5) $440,440,320$

$320 \quad \mathrm{RC}(\mathrm{Jl})=0.0$

GO TO 440

$330 \quad \mathrm{RC}(\mathrm{I}$ I $)=A(\mathrm{~N})$

IF (NARGS-5) $440,440,340$

$340 \quad R C(J I)=1.0$

GO TO 440

$350 \quad M 1=3 * N R M A X+2$

$360 \quad \mathrm{~K} 2=0$

DO $380 \quad J=M 1, M$

IF $(A(J)-A(J-1)) \quad 370,380,380$

SEL 860

SEL 870

SEL 880

SEL 890

SEL 900

SEL 910

SEL 920

SEL 930

SEL 940

SEL 950

SEL 960

SEL 970

SEL 980

SEL 990

SEL 1000

SEL 1010

SEL1020

$A(J)=A(J-1)$

SEL 1030

$A(J-1)=A T$

SEL 1040

$\mathrm{N}=\mathrm{J}+\mathrm{NRMAX}$

SEL 1050

$A T=A(N)$

SEL 1060

$A(N)=A(N-1)$

SEL 1070

$A(N-1)=A T$

SEL 1080

$\mathrm{K} 2=\mathrm{K} 2+1$

SEL 1090

CONTINUE

SEL 1100

IF (K2) $390,390,360$

SEL 1110

$390 \quad \mathrm{~N}=4$ *NRMAX +1

SELI120 


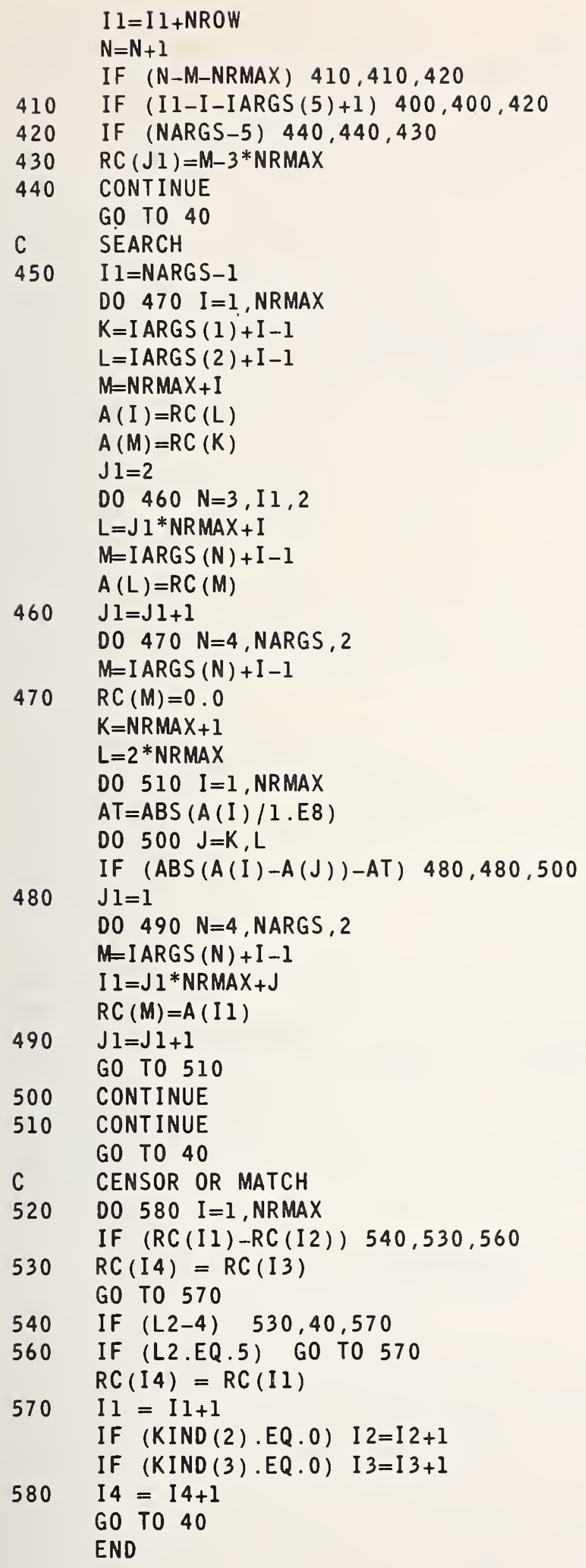


COMMON /BLOCKD/IARGS(100), KIND(100), ARGTAB (100), NRMAX, NROW, NCOL, NSET 50

IARGS, VHXYZ (8), NERROR

DIMENSION ARGS $(100)$

SET 60

EQUIVALENCE (ARGS(1),RC(12501))

SET 70

COMMON /BLOCKE/ NAME (4),L1,L2, ISRFLG

SET 80

COMMON /QRS/ NDROW, IFLAG,J,NNARG

C THE FOLLOWING CARDS ARE NEDDED ONLY FOR TAPE OPERATIONS

SET 90

SET 100 COMMON /TAPE/ NAME4 (2), NTPCT, IPUNCP, INUNIP,LITP

SET 110

COMMON /BLOCKC/ KIO, INUNIT, ISCRAT, KBDOUT, KRDKNT LLIST

SET 120

SET 130

C

I SRFLG $=1$

IF (NARGS.EQ.1.OR.NARGS.EQ.2) GO TO 10

SET 140

SET 150

CALL ERROR (10)

SET 160

GO TO 70

SET 170

$10 \quad M O D E=2$

SET 180

CALL ADRESS (NARGS,J)

SET 190

IF (J) $20,30,40$

20 CALL ERROR (3)

GO TO 70

SET 200

SET 210

30 CALL ERROR (11)

SET 220

SET 230

SET 240

GO TO 70

SET 250

$40 \quad$ NDROW $=J+N R O W-1$

SET 260

IF (NARGS.EQ.1) GO TO 60

IF (KIND (1).NE.0) GO TO 20

SET 270

IF (IARGS (1).LE.NROW.AND.IARGS (1).GT.0) GO T0 50

SET 280

CALL ERROR (16)

SET 290

GO TO 70

SET 300

SET 310

$50 \quad J=J+I A R G S(1)-1$

SET 320

IFLAG $=0$

SET 330

$M O D E=2$

SET 340

GO TO 80

SET 350

70 IFLAG $=1$

SET 360

THE FOLLOWING CARDS ARE NEDDED ONLY FOR TAPE OPERATIONS

SET 370 IF (LITP.NE.48.AND.LITP.NE.49) RETURN

SET 380 MODE $=1$

SET 390

INUNI T =INUNIP

SET 400

C

RETURN

END 
COMMON /BLOCRC/ NRC,RC(12600) $\quad$ STQ 30

COMMON /BLOCKD/ IARGS(100), KIND(100), ARGTAB (100), NRMAX, NROW, NCOL, NSTQ 40

IARGS, VWXYZ (8), NERROR $\quad$ STQ 50

DIMENSION ARGS $(100) \quad$ STQ 60

EQUIVALENCE (ARGS(1), RC(12501)) $\quad$ STQ 70

COMMON /QRS/ NDROW, IFLAG,J,NNARG $\quad$ STQ 80

COMMON /BLOCKA/ MODE, M, KARD (83), KARG, ARG, ARG2, NEWCD (80), KRDEND STQ 90

COMMON /BLOCKC/ KIO, INUNIT, ISCRAT, KBDOUT, KRDKNT, LLIST

COMMON /TAPE/ NAME4 (2), NTPCT, IPUNCP, INUNIP,LITP STQ 110

C CHECK IF END OF ROW HAS BEEN EXCEEDED PREVIOUSLY IN THIS SET. STQ 120

IF (IFLAG.NE.O.OR.NARGS.EQ.0) GO TO $80 \quad$ STQ 130

C J IS WHERE NEXT DATA ITEM IS TO GO IN COLUMN

C JJ IS WHERE LAST DATA ITEM OF THIS SET IS TO GO

C NDROW IS ADDRESS OF LAST ELEMENT OF ROW. $\quad$ STQ 160

$J J=J+N A R G S-1$

IF (JJ.LE.NDROH) GO TO 10

STQ 170

CALL ERROR (201)

IFLAG 1 (

IF (J.GT.NDROW) GO TO 80

STQ 190

STQ 200

$J J=N D R O W$

C THE FOLLOWING CARDS ARE NEEDED ONLY FOR TAPE OPERATIONS $\quad$ STQ 230

10 IF (LITP.NE.48) GO TO $50 \quad$ STQ 240

$\mathrm{K}=1$

$0030 \quad I=J, J J$

IF (KIND(K).EQ.0) GO TO 20

STQ 250

STQ 260

IF (ARGS (K) . ME OO.) GO TO $50 \quad S T Q 270$

IF (ARGS $(K)$.NE.O.) GO TO 50

STQ 280

STQ 290

GO TO 30

STQ 300

IF (IARGS (K).NE.0) GO TO 50

STQ 310

CONTINUE

STQ 320

INUN I T=INUN IP

STQ 330

$M O D E=1$

STQ 340

RETURN

$\begin{array}{llll}\text { C THIS STATEMENT WAS } 10 \mathrm{~K}=1 & \text { STQ } 350 \\ 50 \quad K=1 & \text { STQ } 360\end{array}$

C $\quad * * * * * * * * * * * * * * * * * * * * * * * * * * * * * * * * * * * * * * * * * * * * * * * * * * * * * * * * * * * * * * * * * *$ STQ 370

DO $70 I=\mathrm{J}, \mathrm{JJ} \quad$ STQ 380

IF (KIND(K).EQ.0) GO TO $60 \quad$ STQ 390

$R C(I)=A R G S(K) \quad$ STQ 400

GO TO $70 \quad$ STQ 410

$60 \quad \operatorname{RC}(I)=\operatorname{IARGS}(K) \quad$ STQ 420

$\begin{array}{ll}70 \quad K=K+1 & \text { STQ } 430\end{array}$

$\begin{array}{lr}J=J J+1 & \text { STQ } 440\end{array}$

NRMAX =MAXO (NRMAX, JJ-NDROW+NROW) $\quad$ STQ 450

C THE FOLLOWING CARDS ARE NEEDED ONLY FOR CSET TAPE $\quad$ STQ 460

IF (LITP.NE.49) RETURN $\quad$ STQ 470

$\begin{array}{ll}\text { NTPCT }=\text { NTPCT-1 } & \text { STQ } 480\end{array}$

IF (NTPCT.EQ.0) GO TO $40 \quad$ STQ 490

C $\quad * * * * * * * * * * * * * * * * * * * * * * * * * * * * * * * * * * * * * * * * * * * * * * * * * * * * * * * * * * * * * * * * * *$ STQ 500

80 RETURN STQ 510

$\begin{array}{lr}\text { END } & \text { STQ } 520\end{array}$ 
COMMON /BLOCKA/ MODE, M, KARD (83), KARG, ARG, ARG2, NEWCD (80) ,KRDEND

COMMON /BLOCKC / KIO, INUNIT, ISCRAT, KBDOUT, KRDKNT, LLIST

COMMON /BLOCRC/ NRC,RC(12600)

STP 50

COMMON /BLOCKD/ IARGS $(100)$, KIND $(100)$, ARGTAB $(100)$, NRMAX, NROW, NCOL, NSTP 60

IARGS, VWXYZ (8), NERROR

DIMENSION ARGS $(100)$

STP 70

EQUIVALENCE (ARGS (1), RC (12501))

STP 80

COMMON/HEADER/NOCARD $(80), \operatorname{ITLE}(60,6), \operatorname{LNCNT}$, I PR INT , NPAGE, I PUNCH

STP 90

COMMON / PKSWT / IHCNT, IHTP

COMMON /SCRAT/ NS, NS2, A (13500)

COMMON / ICODE / NIR, NID, NIRD, LIR, LID, LIRD

STP 100

STP 110

STP 120

COMMON /BLOCKX/ INDEX $(6,8), \operatorname{LEVEL}$

STP 130

COMMON / PCONST / JPC, P (40), N (40)

COMMON / CONSTS/ PI ,E , HALFPI , DEG, RAD, XALOG

THE FOLLOWING CARD IS NEEDED ONLY FOR TAPE OPERATIONS

STP 140

STP 150

STP 160

COMMON / ICODTP / NITP,LITP

STP 170

COMMON /TAPE / NAME4 (2), NTPCT, IPUNCP, INUN IP, LITP

STP 180

STP 190

C

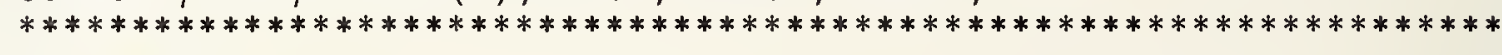

$\mathrm{P}(1)=\mathrm{PI}$

STP 200

$P(2)=P I$

STP 210

$P(3)=E$

STP 220

$P(4)=E$

STP 230

KRDEND $=80$

STP 240

NERROR $=0$

STP 250

LEVEL $=0$

STP 260

$M O D E=1$

STP 270

I PR I NT $=6$

STP 280

I PUNCH $=3$

STP 290

I NUN I T $=5$

STP 300

I SCRAT $=45$

STP 310

NS $=13500$

STP 320

$\mathrm{KI} 0=0$

STP 330

CALL AERR (-1)

STP 340

$N R C=12500$

STP 350

NS $2=N S / 2$

STP 360

STP 370

C

THESE VARIABLES MUST BE REDEFINED IF A NEW COMMAND IS ADDED

STP 380

$N$ I $R=246$

STP 390

$N I R D=29$

STP 400

N I D $=8$

STP 410

$L I R=300$

STP 420

$L I D=9$

STP 430

$L I R D=30$

STP 440

STP 450

THE FOLLOWING CARD IS NEEDED ONLY FOR TAPE OPERATIONS

STP 460

NITP $=9$

STP 470

LITP $=10$

STP 480

INUN I $P=$ INUN I T

STP 490

IPUNCP $=$ IPUNCH

** THESE SWITCHES MUST BE SET BEFORE COMPILING. NEEDED INFORMATIONSTP 520 FOR PACKING HEADS AND FORMATS.

IHTP $=$ NO. OF HEADINGS PERMITTED.

THIS IS SET $=50$, HOWEVER IN ORDER TO SAVE SPACE ONE MAY DESIRE 
c

RETURN

STP 620

END

STP 630

STP 640

SUBROUTINE SKSYMV (A,NROW, N,K)

$c$
$c$
$c$
$c$
$c$
$c$
$c$
$c$
$c$
$c$
$c$

VERSION 5.00 SKSYMV $5 / 15 / 70$

SKS 10

SKS 20

SKS 30

SKS 40

A MATRIX TO BE TESTED FOR SKEW SYMMETRY

SKS 50

NROW DIMENSION OF A

SKS 60

$N$ PRESENT SIZE OF MATRIX

SKS 70

$K$ STATUS

$K=2$ NO SYMMETRY

$K=3$ EXACT SKEW SYMMETRY

$\mathrm{K}=4$ RELATIVE (1.E-7) SKEW SYMMETRY

SKS 80

SKS 90

SKS 100

SKS 110

SKS 120

DIMENSION A (NROW, NROW)

SKS 130

$\mathrm{K}=3$

$\mathrm{NN}=\mathrm{N}-1$

DO $40 \mathrm{~J}=1$, NN

IF $(A(J, J) . E Q .0 .0)$ GO TO 10

$\mathrm{K}=2$

RETURN

$10 \quad \mathrm{I}=\mathrm{J}+1$

DO $40 \mathrm{~L}=\mathrm{I}, \mathrm{N}$

IF $(A(L, J) . N E .0$. $)$ GO TO 20

$T=A B S(A(J, L))$

GO TO 30

$20 \quad T=A B S(1.0+A(L, J) / A(J, L))$

30 IF (T.EQ.0.0) GO TO 40

$\mathrm{K}=4$

IF (T.LE.1.E-7) GO TO 40

$\mathrm{K}=2$

RETURN

40 CONTINUE

IF $(A(N, N) . N E .0 .0) \quad K=2$

RETURN

END

SKS 140

SKS 150

SKS 160

SKS 170

SKS 180

SKS 190

SKS 200

SKS 210

SKS 220

SKS 230

SKS 240

SKS 250

SKS 260

SKS 270

SKS 280

SKS 290

SKS 300

SKS 310

SKS 320

SKS 330

SKS 340 
COMMON /BLOCKD/ I ARGS (100), KIND(100), ARGTAB (100), NRMAX, NROW, NCOL, NSOD 40

IARGS, VWXYZ (8), NERROR

DIMENSION ARGS $(100)$

SOD 50

EQUIVALENCE (ARGS(1),RC(12501))

COMMON /BLOCKE/ NAME (4),L1,L2, ISRFLG

COMMON /SCRAT/ NS, NS2, A(13500)

SUBROUTINE BY CARLA MESSINA 221.04 JUNE 1967

SOD 60

SOD 70

SOD 80

SOD 90

$L 2=8$ FOR SORT, $L 2=9$ FOR ORDER, L2 $=14$ FOR HEIRARCHY

SOD 100

SOD 110

TYPE 1 IS HEIRARCHY OF COL ++, STORE IN COL ++

SOD 120

SOD 130

HEIRARCHY GIVES THE ROW LOCATION OF THE SMALLEST NO. OF THE SOD 140

THE FIRST COLUMN IN THE FIRST ROW OF THE SECOND COLUMN

SOD 150

THE ROW NO. OF THE SECOND LOWEST NO. OF THE FIRST COLUMN IS STOREDSOD 160

IN THE SECOND ROH OF THE SECOND COLUMN, ..... THE ROW NO. OF THE SOD 170

LARGEST NO. OF THE FIRST COL IS STORED IN THE NRMAX ROW OF THE 2NDSOD 180

COLUMN. THE FIRST COLUMN IS UNCHANGED BY THIS COMMAND.

SOD 190

TYPE 2 IS ORDER COLUMNS,,,++++++ ETC

ORDER PLACES EACH ONE OF THE GIVEN COLUMNS IN NUMERICALLY

SOD 200 INCREASING ORDER.

TYPE 3 IS SORT COL ++ CARRY ALONG COLUMNS,,++++ ETC

SOD 210

C

SORT PLACES THE FIRST COLUMN IN NUMERICALLY INCREASING ORDER

SOD 220

WHILE PRESERVING THE ROW RELATIONSHIPS AMONG THE GIVEN COLUMNS

SOD 230

SOD 240

C

C

THESE INSTRUCTIONS CAN BE DONE FASTER IF A MACHINE LANGUAGE

SOD 250

SOD 260 PROGRAM IS SUBSTITUTED FOR THIS ONE.

SOD 270

SOD 280

IF (NARGS) $10,10,40$

$10 \quad \mathrm{~K}=10$

20 CALL ERROR (K)

30 RETURN

40 CALL CHKCOL (J)

IF (J) $50,60,50$

$50 \quad K=3$

GO TO 20

60 IF (L2-9) $80,80,70$

70 IF (NARGS-2) $10,80,10$

80 IF (NERROR) $30,90,30$

90 IF (NRMAX-1) $100,110,120$

$100 \quad K=9$

GO TO 20

110 IF (L2-9) $30,30,210$

$120 \quad K 3=1$

$K=\operatorname{IARGS}(1)-1$

130 DO $140 I=1$, NRMAX

$J=K+I$

$L=N R M A X+I$

$A(I)=R C(J)$

$140 \quad A(L)=I$

$\mathrm{K} I=N R M A X$

$150 \quad \mathrm{Kl}=\mathrm{Kl}-1$

$K 2=0$

IF (KI-1) $160,160,170$

$160 \quad \mathrm{Kl}=2$

170 DO $190 \quad I=1, K I$

IF $(A(I)-A(I+1)) 190,190,180$

$180 \quad C C=A(I)$

SOD 290

SOD 300

SOD 310

SOD 320

SOD 330

SOD 340

SOD 350

SOD 360

SOD 370

SOD 380

SOD 390

SOD 400

SOD 410

SOD 420

SOD 430

SOD 440

SOD 450

SOD 460

SOD 470

SOD 480

SOD 490

SOD 500

SOD 510

SOD 520

SOD 530

SOD 540

SOD 550

SOD 560

SOD 570

SOD 580

SOD 590 


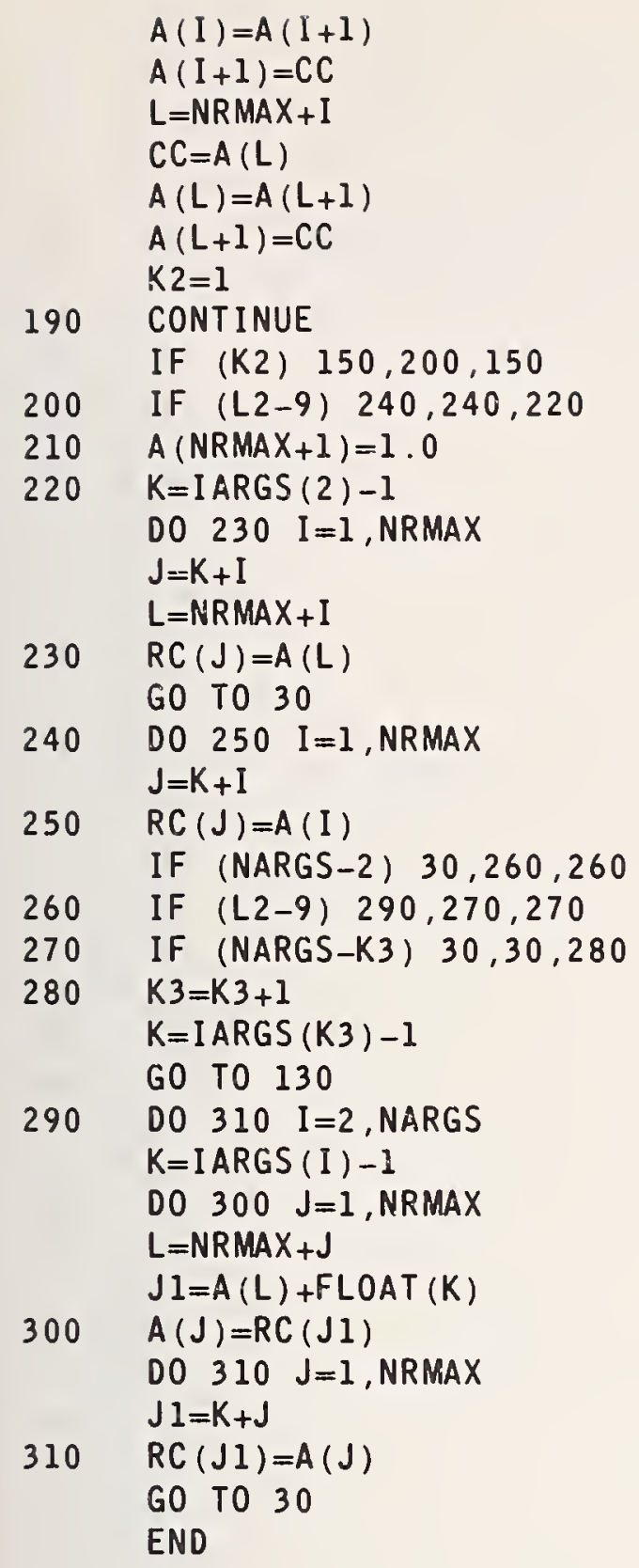


DIMENSION $X(1)$

DOUBLE PRECISION $X$, SAVE, SUM

SOM 60

SOM 70

SOM 80

EQUIVALENCE $(X, A)$

SOM 90

IF (N.NE.I) GO TO 10

SOM 100

SUM $=X$ (NS2)

SOM 110

SOM 120

RETURN

$10 \quad K=0$

I $S=N S 2$

DO $30 \quad I=2, N$

IF $(X($ IS $)-X($ IS -1$)) \quad 20,30,30$

SOM 130

SOM 140

SOM 150

SOM 160

SOM 170

SOM 180

SOM 190

$X($ IS-I $)=X($ IS $)$

SOM 200

$X(I S)=S A V E$

SOM 210

$30 \quad$ IS $=I S-1$

IF (K.NE.0) GO TO 10

SOM 220

SOM 230

$N P=N / 2$

IF $(M O D(N, 2) . E Q .0) \quad$ GO TO 40

SOM 240

$N P A=N S 2-N P-1$

$N P B=N P A+2$

$N P C=N S 2-N P$

$S U M=X(N P C)$

GO TO 50

$40 \quad S U M=0 . D 0$

$N P A=N S 2-N P$

$N P B=N P A+1$

50 D0 $60 \quad I=1, N P$

$S U M=S U M+X(N P A)+X(N P B)$

$N P A=N P A-1$

$N P B=N P B+1$

SOM 250

SOM 260

SOM 270

SOM 280

SOM 290

SOM 300

SOM 310

SOM 320

SOM 330

SOM 340

SOM 350

SOM 360

SOM 370

60 CONTINUE

SOM 380

RETURN

SOM 390

END 
SUBROUTINE SPACE

C

VERSION $5.00 \quad$ SPACE

COMMON /BLOCRC/ NRC, RC (12600)

$5 / 15 / 70$

SPA 10

COMMON /BLOCKD/ IARGS $(100)$, KIND (100), ARGTAB $(100)$, NRMAX,NROW, NCOL, NSPA 40

IARGS, VWXYZ (8), NERROR

DIMENSION ARGS $(100)$

SPA 50

EQUIVALENCE (ARGS(1),RC (12501))

SPA 60

COMMON/HEADER/NOCARD $(80)$, I TLE $(60,6)$, LNCNT , IPR INT , NPAGE , IPUNCH

SPA 70

IF (NARGS-1) $40,32,10$

SPA 80

$I=10$

SPA 100

SPA 110

CALL ERROR (I)

RETURN

SPA 120

SPA 130

IF (KIND(1).EQ.0) IF(IARGS(1)) 35,30,45

SPA 135

$\mathrm{I}=20$

GO TO 20

SPA 140

SPA 150

$\mathrm{I}=3$

GO TO 20

SPA 160

SPA 170

$\operatorname{IARGS}(1)=1$

$J=M I N O(60, \operatorname{IARGS}(1))$

IF (NERROR.NE.0) GO TO 30

SPA 180

SPA 190

DO $50 \quad I=1, J$

SPA 200

SPA 210

SPA 220

SPA 230

SPA 240

SPA 250

60 FORMAT (IX)

SPA 260 
SUBROUTINE SPINV (A,M,KK, ISIG)

SPI 10

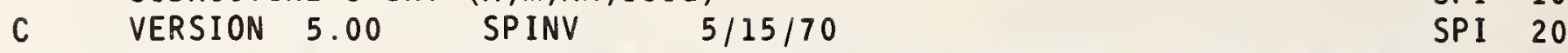

C 7058MI MATRIX INVERSION WITH MINIMUM ROUNDOFF ERROR ACCUMULATION.SPI 30

DATA ONE $/ 1.0 /, Z E R 0 / 0.0 /, E R / 1 . E-8 / \quad$ SPI 40

DIMENSION A(1)

SPI 50

IS I G $=0$

$N=M$

$N N=K K$

SPI 60

$\mathrm{N} 2=\mathrm{N}+\mathrm{N}$

SPI 70

DO $30 \mathrm{~J}=1, \mathrm{~N}$

SPI 80

$\mathrm{NJCOL}=(\mathrm{N}+\mathrm{J}-1) * \mathrm{NN}$

SPI 90

DO $30 \quad I=1, N$

SPI 100

$K I N J=N J C O L+I$

SPI 110

IF (I-J) $10,20,10$

SPI 120

SPI 130

SPI 140

$10 \quad A(K I N J)=Z E R O$

SPI 150

GO TO 30

SPI 160

$20 \quad A(K I N J)=0 N E$

SPI 170

30 CONTINUE

SPI 180

C DETERMINE MAXIMUM ABS OF VARIABLE BEING ELIMINATED. THIS BECOMES SPI 190 $L=0$

SPI 200

$40 \quad L=L+1$

$L C O L=N N * L-N N$

SPI 210

$K L L=L C O L+L$

SPI 220

IF (L-N) $50,100,200$

SPI 230

SPI 240

C FIND THE LARGEST ELEMENT IN THE LTH COLUMN.

SPI 250

$50 \quad \mathrm{~J} I=\mathrm{L}$

$C=A B S(A(K L L))$

$\mathrm{LI}=\mathrm{L}+1$

DO $70 \quad I=L 1, N$

$K I L=L C O L+I$

$X=A B S(A(K I L))$

IF (C-X) $60,70,70$

C RECORD THE NUMBER OF THE ROW HAVING THE GREATER ELEMENT.

SPI 260

SPI 270

SPI 280

SPI 290

SPI 300

SPI 310

SPI 320

SPI 330

$60 \mathrm{Jl}=\mathrm{I}$

C C BECOMES THE GREATER.

SPI 340

$C=X$

SPI 350

SPI 360

SPI 370

70 CONTINUE

C INTERCHANGE ROW JI WITH ROW L. JI IS THE ROW WITH THE LARGEST ELEMSPI 380
C TEST TO SEE IF INTERCHANGING IS NECESSARY.

IF (Jl-L) $80,100,80$

80 DO $90 \mathrm{~J}=\mathrm{L}, \mathrm{N} 2$

SPI 400

JCOL=NN*J-NN

SPI 410

$K J I J=J C O L+J I$

SPI 420

$H O L D=A(K J I J)$

SPI 430

$K L J=J C O L+L$

SPI 440

$A(K J I J)=A(K L J)$

$A(K L J)=H O L D$

SPI 450

SPI 460

SPI 470

90 CONTINUE $\quad$ SPI 480

C IF THE LARGEST ABSOLUTE ELEMENT IN A COLUMN IS ZERO WE HAVE A SINSPI 490

100 IF (ABS (A (KLL))-ER) $110,110,120 \quad$ SPI 500

GO TO 200

SPI 510

SPI 520

C ZERO ALL THE ELEMENTS IN THE LTH COLUMN BUT THE PIVOTAL ELEMENT. SPI 530

$120 \quad$ Ll $=1$

$\mathrm{L} 2=\mathrm{L}-1$

IF (L2) $130,130,150$

SPI 1540

SPI 550

SPI 560

IF (L-N) $140,170,140$

SPI 570

$\mathrm{LI}=\mathrm{L}+\mathrm{l}$

SPI 580

$\mathrm{L} 2=\mathrm{N}$

SPI 590 
$150 \quad$ D0 $160 \quad I=L 1, L 2$

$\mathrm{KIL}=\mathrm{LCOL}+\mathrm{I}$

$Z=-A(K I L) / A(K L L)$

SPI 600

DO $160 \mathrm{~J}=\mathrm{L}, \mathrm{N} 2$

$J C O L=N N^{*} J-N N$

$\mathrm{KIJ}=\mathrm{JCOL}+\mathrm{I}$

$\mathrm{KLJ}=J \mathrm{COL}+\mathrm{L}$

$160 \quad A(K I J)=A(K I J)+Z^{*} A(K L J)$

IF (N-L2) $40,40,130$

C DIVIDE BY DIAGONAL ELEMENTS.

$170 \quad$ DO $180 \quad I=1, N$

$\mathrm{KKK}=\mathrm{NN} * \mathrm{I}-\mathrm{NN}+\mathrm{I}$

$Z Z=A(K K K)$

DO $180 \mathrm{~J}=1, \mathrm{~N} 2$

$\mathrm{KKI}=\mathrm{NN} * \mathrm{~J}-\mathrm{NN}+\mathrm{I}$

$180 \quad A(K K I)=A(K K I) / Z Z$

C RETURA AFTER PUTTING A INVERSE INTO B

DO $190 \mathrm{~J}=1, \mathrm{~N}$

$J C O L=N N^{*} J-N N$

$\mathrm{NJCOL}=\mathrm{NN} * \mathrm{~N}+\mathrm{JCOL}$

DO $190 \quad I=1, N$

$K I J=J C O L+I$

$K I N J=N J C O L+I$

$190 \quad A(K I J)=A(K I N J)$

200 RETURN

END

SPI 610

SPI 620

SPI. 630

SPI 640

SPI 650

SPI 660

SPI 670

SPI 680

SPI 690

SPI 700

SPI 710

SPI 720

SPI 730

SPI 740

SPI 750

SPI 760

SPI 770

SPI 780

SPI 790

SPI 800

SPI 810

SPI 820

SPI 830

SPI 840

SPI 850 
I WITH WEIGHTS

STA 30

A. STATIS COL +++ WEIGHTS +++ START STORING RESULTS +++ STA 60

(RESULTS WILL BE STORED IN THE NEXT 4 COL)

B. STATIS COL +++ WHTS +++ RESULTS,,,$++++++++++++\quad$ STA 80

II WITHOUT WHTS

A. SAME AS I. A. EXCEPT WHTS COL OMITTED

STA 90

B. SAME AS I. B. EXCEFT WHTS COL OMITTED

STA 100

COMMON /BLOCRC/ NRC, RC (12600)

STA 110

COMMON /BLOCKD/ IARGS(100), KIND(100), ARGTAB (100), NRMAX,NROW, NCOL, NSTA 130

IARGS, VWXYZ ( 8 ), NERROR

DIMENSION ARGS(100)

STA 140

EQUIVALENCE (ARGS(1), RC (12501))

STA 150

COMMON /SCRAT/ NS, NS2,A(13500)

STA 160

COMMON/HEADER/NOCARD $(80)$, ITLE $(60,6)$, LNCNT , IPRINT , NPAGE , I PUNCH

STA 170

COMMON /BLOCKE/ NAME (4),L1,L2, ISRFLG

STA 180

DIMENSION SA $(3125,3)$, ISA(3125)

STA 200

DIMENSION IB (10)

STA 210

EQUIVALENCE (A(101), ISA), (A(3226), SA)

STA 220

DIMENSION BCON(4), BKCON(4), AKCON(4), AT5 (6), CKI(6), DK2 (6), XKISTA 240 $1(7), \operatorname{YK} 2(7)$

STA 250

$\operatorname{DATA} \operatorname{BCON}(1), \operatorname{BCON}(2), \operatorname{BCON}(3), \operatorname{BCON}(4) / 3.6948,-1.6561, .406,2.7764 /, B S T A 260$ $1 K C O N(1), \operatorname{BKCON}(2), \operatorname{BKCON}(3), \operatorname{BKCON}(4) / 7.45894,-.89082, .61522,2.56706 / 5 T A 270$ $2, \operatorname{AKCON}(1), \operatorname{AKCON}(2), A K C O N(3), A K C O N(4) /-.51732,-.61863,-.04122, .5589 S T A 280$ 37/,AT5(1),AT5 (2),AT5(3),AT5(4),AT5 (5),AT5(6)/1.9599640,2.3722712,2STA 290 $4.8224986,2.5558497,1.5895341, .7328982 /, C K 1(1), C K 1(2), C K 1(3), C K 1(4)$ STA 300 $5, C K I(5), C K I(6) /-.70285,-.02006,-.01687,-.01447,-.01263, .67839 /$, DK2STA 310 6(1),DK2 (2),DK2 (3),DK2(4),DK2(5),DK2(6)/-1.49016,.13384,.09764,.074STA 320 $776, .05931,1.68641 /, X K I(1), X K I(2), X K I(3), X K I(4), X K I(5), X K I(6), X K I(7 S T A 330$ 8) $/-40.343875,14.1365,-2.743342, .84143957, .001066,-6.3701507 \mathrm{E}-6,1.7$ STA 340 $949484 E-8 /, Y K 2(1), Y K 2(2), Y K 2(3), Y K 2(4), Y K 2(5), Y K 2(6), Y K 2(7) / 50.2982 S T A 350$ $\$ 33,-11.395210,6.0537922,1.1542370,-9.8051279 \mathrm{E}-4,5.5609437 \mathrm{E}-6,1.458$ STA 360 $\$ 4433 \mathrm{E}-8 /$, CONK/1.959964/

STA 370

DATA ZERO/0.0/,ONE/1.0/,TWO/2.0/

STA 380

IF (L2.EQ.1.OR.NARGS.NE.1) GO T0 5

STA 385

CALL ERROR (236)

RETURN

5 DO $10 \quad \mathrm{I}=1,60$

$10 \quad A(I)=0.0$

$N X C O L=I$ ARGS (1)

STA 387

NXWT =IARGS (2)

STA 390

I STORE $=$ I

STA 395

$N A R=N A R G S$

STA 400

I $W T=1$

IF (NARGS.EQ.1) GO TO 30

STA 410

IF (NARGS.EQ.3.AND. IARGS(NARGS).LT.0) GO TO 20

STA 420

GO TO 40

STA 430

STA 440

STA 450

STA 460

STA 470

STA 480

NARGS=NARGS -1

STA 490

I $W T=2$

30 ISTORE $=2$

STA 500

GO TO 50

STA 510

STA 520

IF (NARGS.NE.2.AND.NARGS.NE.3.AND.NARGS.NE.5.AND.NARGS.NE.6) CALL IERROR (10) 
CALL CHKCOL (J)

IF (J.NE.O) CALL ERROR (11)

STA 590

IF (NRMAX*4.LE.NS) GO TO 60

STA 600

CALL ERROR (214)

RETURN

STA 610

60 IF (NERROR.NE.0) RETURN

STA 620

STA 630

I XN $=$ NR MAX

STA 640

$A(1)=N R M A X$

STA 650

$\mathrm{K}=\mathrm{I}$ ARGS $(1)$

STA 660

$M=1$

STA 670

IF (NAR.EQ.3.OR.NAR.EQ.6) GO TO 80

STA 680

$N Z W=N R M A X$

STA 690

$S U M=0.0$

STA 700

$\mathrm{S} 2=0$.

STA 710

$W T=0.0$

STA 720

ASUMWT $=0$.

STA 730

DO $70 \quad I=1, I X N$

STA 740

$S A(I, 2)=R C(K)$

STA 750

$S A(I, 3)=1.0$

STA 760

$I S A(I)=M$

STA 770

$\mathrm{SA}(\mathrm{I}, 1)=\mathrm{RC}(\mathrm{K})$

STA 780

$\mathrm{K}=\mathrm{K}+1$

STA 790

$M=M+1$

STA 800

$W T=W T+1$

STA 810

$S U M=S U M+S A(I, 2)$

ASUMWT $=A S U M W T+A B S(S A(I, 2))$

STA 820

STA 830

STA 840

STA 850

SUMWT $=S U M$

STA 860

GO TO 110

STA 870

STA 880

WT $=0$.

STA 890

SUMWT $=0.0$

$M A=I A R G S(2)$

STA 900

$S 2=0.0$

STA 910

I $W T=2$

STA 920

$N E G W T=0$

STA 930

ASUMWT $=0$.

DO $100 \quad I=1, I X N$

IF (RC (MA).EQ.0.) GO TO 90

STA 940

STA 950

STA 960

STA 970

IF (RC (MA).LT.0.0) NEGHT $=N E G H T+1$

STA 980

$S A(M, 2)=R C(K)$

STA 990

STA 1000

ISA $(M)=M$

$\mathrm{SA}(M, 1)=\mathrm{RC}(\mathrm{K})$

$S 2=S 2+S A(M, 2) * * 2 * R C$ (MA)

STA 1010

STA1020

$S U M=S U M+R C(K)$

STA1030

$W T=W T+R C(M A)$

STA1040

SUMWT $=S A(M, 2) * R C(M A)+S U M W T$

ASUMWT $=$ ASUMWT $+A B S(S A(M, 2)) * R C(M A)$

STA 1050

STA 1060

$M=M+1$

STA 1070

STA1080

STA1090

STA1100

STAIIIO

STA1120

STA1130

STAII 40

STA 1150

$110 \quad A(2)=N Z W$

STA 1160 
$A(4)=$ SUMWT $/$ WT

STA1 170

$A(24)=(2.0 * A(2)-1) /$.

STA 1180

$A(25)=F S Q R T((16 . * A(2)-29) / 90$.

STAL190

$A(39)=S 2$

$A(42)=$ ASUMWT

STA 200

$A(43)=A S U M W T / W T$

STA1210

$I X N=N Z W$

STA1220

$I X N M I=I X N-1$

STA 1230

$120 \quad \mathrm{IST}=0$

STA 240

DO $130 \quad I=2, I X N$

IF $(S A(I-1,1) . L E . S A(I, 1)) \quad$ GO TO 130

STA1250

$K=I S A(I-I)$

STA1260

ISA (I-I) $=$ I SA ( I )

STA1270

I SA ( I ) $=\mathrm{K}$

STA 1280

$T=S A(I-1,1)$

STA1290

STA 1300

$S A(I-1,1)=S A(I, 1)$

STA 1310

$S A(I, 1)=T$

$I S T=1$

130 CONTINUE

IF (IST.NE.0) GO TO 120

NALPHA $=.25 * A(2)$

I $X A=N A L P H A+1$

I XNA $=$ I XN-NALPHA

TSUME $=0$.

TWSUM $=0$

STA1 320

STA 1330

STA1340

STA1350

STA 1360

STA 1370

STA 1380

STA1390

STA 400

$T W T=0$

DO $140 I=I X A$, IXNA

STA1410

STA 1420

$M=I S A$ (I)

TWSUM $=$ TWSUM+SA $(I, 1) * S A(M, 3)$

STA1430

STA1440

$T W T=T W T+S A(M, 3)$

$140 \quad T S U M=T S U M+S A(I, 1)$

$A(7)=$ TSUM/ $\left(A(2)-2 .{ }^{*} F L O A T(N A L P H A)\right)$

STA1450

STA1460

STA 1470

A $(8)=$ T WSUM $/$ T WT

STA1480

$N 2=(N Z W+1) / 2$

STA1490

STA 1500

$A(5)=S A(N 2,1)$

STA1510

IF $(M O D(N Z W, 2) \cdot E Q, 0) \quad A(5)=(A(5)+S A(N 2+1,1)) / T W O$

STA1520

$A(6)=(S A(1,1)+S A(I X N, 1)) / T W O$

STA1530

$A(11)=S A(I X N, 1)-S A(1,1)$

STA 1540

$A(34)=S A(1,1)$

STA1550

$A(35)=S A(I X N, 1)$

STA 1560

$D E L X=A(11) / 10$.

STA 1570

$X B=S A(1,1)$

STA1580

$X T=X B+D E L X$

STA 1590

$L=1$

DO $170 \quad I=1,10$

STA 1600

STA1610

I $C=0$

STA1620

IF $(S A(L, I), G E . X T)$ GO TO 160

STA 1630

I $\mathrm{C}=\mathrm{I} \mathrm{C}+\mathrm{I}$

STA 1640

$L=L+1$

IF (L.NE.IXN) GO TO 150

STA1650

STA1660

STA 1670

STA1680

STA 1690

STA1700

$\begin{array}{ll}\text { IF } & (L . G T . I X N) \\ \text { DO } & 180 \quad I=L, I X N\end{array}$

IF $(S A(I, 1), G E \cdot X T-D E L X) \quad A(60)=A(60)+1$.

STA1710

180 CONTINUE

STA1720

DO $200 \quad I=1, I X N M I$

STA1730

$S A(I, 3)=S A(I+1,1)-S A(I, 1)$

STA1740

$\mathrm{LA}=1$

STA1750 
DO $210 \quad I=1, I X N$

$\mathrm{K}=\mathrm{ISA}$ (I)

$S A(K, 1)=L A$

STA1760

STA 1770

$210 \quad \mathrm{~L} . \mathrm{A}=\mathrm{LA}+1$

$K=0$

STA1780

RNS $=0$.

STA 1790

RNSS $=0$ NE

STA 800

$L R=0$

DO $250 \quad I=1$, IXNMI

STA1810

STA 1820

STA 1830

IF $(S A(I, 3) \cdot N E \cdot 0.0 . A N D \cdot K . E Q .0)$ GO TO 240

STA 1840

IF $(S A(I, 3) . N E .0 .0) \quad$ GO TO 220

STA 1850

RNS $=$ RNS + RNSS

$\mathrm{K}=\mathrm{K}+1$

GO TO 250

$220 \quad K=K+1$

RNS $=$ RNS +RNSS

STA1860

STA1 870

STAI 880

STA 1890

STA 1900

RNS $=$ RNS / FLOAT $(K)$

DO $230 L=1, K$

$L R=L R+1$

$L R R=I S A$ (LR)

$230 \quad S A(L R R, 1)=R N S$

$L R=L R-1$

RNS $=0$.

$K=0$

$240 \quad L R=L R+1$

250 RNSS $=$ RNSS +ONE

I C I $=0$

I PLUS $=0$

STA1910

STA1920

STA 930

STA1940

STA1950

STA 1960

STA 1970

STA1980

STA 1990

STA2000

STA2010

STA2020

I MINUS $=0$

STA2030

I DRUNS $=0$

STA2040

I $C=0$

STA2050

$A D E V=0.0$

STA2060

$D E V 3=0.0$

STA2070

$D E V 2=0.0$

STA2080

STA2090

$D E V=0.0$

STA2100

$D E V I=0.0$

STA2110

$D E V W T=0$.

STA2120

DEV $4=0.0$

STA2130

$A K=1$.

STA2 140

$K W T=I A R G S(2)$

STA2 150

$N R X X=K W T+N R M A X-1$

STA2160

$T A=1.0$

STA2 170

DO $320 \quad I=1, I X N$

STA2 180

$T=S A(I, 2)-A(4)$

STA2190

$S A(I, 3)=T$

$D E V=T+D E V$

STA2200

$A D E V=A D E V+A B S(T)$

STA2210

STA2220

$D E V 2=D E V 2+T * * 2$

STA2230

DEV $3=D E V 3+T * * 3$

STA2240

$D E V 4=D E V 4+T * * 4$

STA2250

$D E V I=A K * T+D E V I$

STA2260

$A K=A K+1.0$

STA2270

IF (IWT.EQ.I) GO TO 280

STA2280

IF (RC(KWT).NE.0.) GO TO 270

STA2290

IF (KWT.GE.NRXX) GO TO 290

STA2300

$K W T=K W T+1$

STA2310

GO TO 260

STA2320

$T A=R C(K W T)$

STA2330

$280 \quad D E V W T=D E V W T+T A * T * * 2$

STA2340 
290 IF (T.LT.0.0) GO TO 300

ICI $=+1$

GO TO 310

$300 \quad$ IMINUS $=$ IMINUS +1

STA2380

IC I $=-1$

310 IF (IC.EQ.ICI) GO T0 320

STA2390

I $C=I C I$

I DRUNS $=$ I DRUNS +1

STA2400

STA2410

STA2420

STA2430

$320 \quad K W T=K W T+1$

STA2 440

$A(13)=\operatorname{DEVWT} /(A(2)-1$.

STA2450

$A(9)=F S Q R T(A(13))$

STA2460

$A(10)=A(9) / F S Q R T(W T)$

STA2470

$A(14)=100 .{ }^{*} A(9) / A(4)$

STA2480

$A(28)=I P L U S$

STA2490

$A(29)=I$ MINUS

STA2500

$A(31)=1 .+(2 . * A(28) * A(29) / A(2))$

STA2510

$A(32)=F S Q R T((2 . * A(28) * A(29) *(2 . * A(28) * A(29)-A(28)-A(29))) /((A(28)+S T A 2520$

$1 A(29)) * * 2 *(A(2)-1))$.

$A(36)=(D E V 3 / A(2)) * * 2 /((A(2)-1.) / A(2) * A(13)) * * 3$

STA2530

$A(37)=(D E V 4 / A(2)) /((A(2)-1.) / A(2) * A(13)) * * 2$

STA2540

$A(38)=$ SUMWT

STA2550

$A(40)=D E V W T$

STA2560

$A(30)=I D R U N S$

STA2570

$A(33)=(A(30)-A(31)) / A(32)$

STA2580

$A(19)=12 . * D E V I /(A(2) *(A(2) * * 2-1)$.

STA2590

$A(20)=F S Q R T\left((1 . /(A(2)-2)) *.\left(12 .{ }^{*} D E V 2 /(A(2) *(A(2) * * 2-1))-.A(19) * * 2\right)\right.$ STA2610

1)

$A(21)=A(19) / A(20)$

STA2620

STA2630

CALL PROB (ONE, $A(2)-O N E, A(21) * A(21), A(22)$ )

STA2640

$D I F=0$

STA2650

IRUN $=1$

STA2660

DO $325 I=2, I X N$

STA2665

$T A=S A(1,2)-S A(1-1,2)$

STA2670

IF (TA) $326,325,326$

STA2675

CONTINUE

STA2680

326 DO $330 \quad I=2, I X N$

STA2685

$T=S A(I, 2)-S A(I-1,2)$

STA2690

$D I F=D I F+T * * 2$

STA2700

IF (TA*T.GE.0.0) GO TO 330

STA2710

$T A=T$

I RUN $=$ IRUN +1

STA2720

STA2730

CONTINUE

STA2740

STA2750

$A(23)=I R U N$

$A(26)=D I F /(A(2)-1$.

STA2760

$A(27)=A(26) / A(13)$

$A(41)=A(4) * F S Q R T(H T) / A(9)$

$A(12)=A D E V / A(2)$

$N U=N Z W-1$

$\mathrm{VNU}=N \mathrm{~N}$

$T=Z E R O$

STA2770

STA2780

STA2790

STA2800

STA2810

STA2820

TK $1=Z E R O$

STA2830

TK2 $=$ ZERO

STA2840

IF (NU.GE.5) GO TO 350

STA2850

DO $340 \quad I=1,4$

STA2860

$V=1 / N U$

STA2870

$T=T+B \operatorname{CON}(I) * V$

TK2 $=B K C O N(I) * V+T K 2$

STA2880

STA2890

$T K 1=T K I+A K C O N(I) * V$

STA2900 
GO TO 400

$350 \mathrm{~T}=((((\operatorname{AT5}$ (6) $/ \mathrm{VNU}+\mathrm{AT} 5(5)) / \mathrm{VNU}+\mathrm{AT} 5(4)) / \mathrm{VNU}+\mathrm{AT} 5$ (3))/VNU+AT5 (2))/VNU+ASTA2920

$1 T 5(1)$

IF (NU.GT.10) GO TO 370

STA2 230

DO $360 \quad \mathrm{I}=1,6$

STA2940

$\mathrm{V}=(\mathrm{I}+4) / \mathrm{NU}$

STA2950

$T K I=T K I+C K I(I) * V$

STA2960

360

$T K 2=T K 2+D K 2(I) * V$

STA2970

GO TO 400

STA2980

IF (NU.GT.100) GO TO 390

STA2990

370

DO $380 \quad \mathrm{I}=1,7$

STA 3000

$\mathrm{V}=\mathrm{VNU}^{* *}(\mathrm{I}-4)$

STA 3010

$T K I=T K I+X K I(I) * V$

STA 3020

$T K 2=T K 2+Y K 2$ (I) *V

STA 3030

380

GO TO 400

STA 3040

STA3050

$390 \quad V 2=F S Q R T$ ( TWO *VNU)

STA 3060

$\mathrm{V} 2 \mathrm{Ml}=\mathrm{FSQRT}$ ( $T$ HO*VNU-ONE)

STA 3070

$\mathrm{TK} 1=\mathrm{V} 2 /(\mathrm{CONK}+\mathrm{V} 2 \mathrm{Ml})$

STA 3080

$\mathrm{TK} 2=\mathrm{V} 2 /(-\mathrm{CONK}+\mathrm{V} 2 \mathrm{Ml})$

STA 3090

$400 \quad A(15)=A(4)-T^{*} A(10)$

STA 3100

$A(16)=A(4)+T^{*} A(10)$

STA 3110

$A(17)=T K 1 * A(9)$

STA 3120

$A(18)=$ TK $2 * A(9)$

STA 3130

C START PRINT OUT

STA 3140

IF (L2.EQ.2) GO TO 560

STA 3150

CALL PAGE (4)

STA 3160

IF (IWT.EQ.2) GO TO 410

STA 3170

WRITE (IPRINT, 640) NXCOL, NZW

STA 3180

GO TO 440

410 IF (NZW.NE.NRMAX) GO TO 420

STA 3190

WRITE (IPRINT, 650) NXCOL, NXWT, NZW

STA 3200

GO TO 430

420 WRITE (IPRINT, 660) NXCOL, NXWT, NZW, NRMAX

STA 3210

STA 3220

STA 3230

WRITE (IPRINT, 670)

STA 3240

DO $450 \quad I=1,10$

STA 3250

$I B(I)=A(I+50)$

STA 3260

WRITE (IPRINT, 680) (IB (I), I=1,10)

STA 3270

WRITE (IPRINT, 690)

HRITE (IPRINT, 700) (A $(I+2), A(I+8), I=1,6)$

STA 3280

WRITE (IPRINT, 710) (A(I), I=15,18)

STA 3290

WRITE (IPRINT, 720) (A(I), A (I+15), I=19,22), (A (I), I=38,41)

STA 3300

$I B(1)=A(23)$

$I B(2)=A(28)$

STA 3310

STA 3320

STA 3330

$I B(3)=A(29)$

STA 3340

STA 3350

$I B(4)=A(30)$

WRITE (IPRINT, 730) IB(I), A (42), A (24), A(43), (A (I), I=25, 27), (IB (I), ISTA3360
STA3370

WRITE (IPRINT, 740)

STA 3380

$K B=I S A(1)$

STA3390

$T=S A(K B, 2)$

STA 3400

LINEP $=40$

STA3410

LINE $=0$

STA 3420

$L W=I A R G S(2)$

DO 520 I=1, IXNMI

STA 3430

IF (LINEP.LT.40) GO TO 470

STA 3440

LINEP $=0$

STA 3450

CALL PAGE (4)

WRITE (IPRINT, 750)

STA3460

STA 3470

IF (IWT.EQ.I) GO TO 460 
WRITE (IPRINT, 760)

STA 3500

GO TO 470

STA 3510

460

WRITE (IPRINT, 770)

STA 3520

$470 K=I S A(I+1)$

$\mathrm{TA}=\mathrm{SA}(\mathrm{K}, 2)-\mathrm{T}$

STA 3530

GO TO $(500,480)$, I HT

480 IF (RC(LW).NE.0.0) GO T0 490

STA 3540

STA 3550

$L W=L W+1$

STA 3560

GO TO 480

STA 3570

STA 3580

490 WRITE (IPRINT, 790) I, SA (I, 2), SA (I, 1), SA (I, 3), RC (LW), ISA(I), T, TA

STA 3590

$L \mathrm{H}=\mathrm{L} W+1$

GO TO 510

500 WRITE (IPRINT, 780) I, SA (I, 2), SA (I, I), SA (I, 3), ISA (I), T, TA

STA 3600

STA3610

STA3620

$T=S A(K, 2)$

LINE $=L I N E+1$

IF (LINE.NE.10) GO TO 520

$L I N E=0$

L INEP $=$ L INEP +10

WRITE $(6,800)$

520 CONTINUE

IF (IWT.EQ. I) GO TO 550

530 IF (RC(LW).NE.0.0) GO TO 540

$L W=L W+1$

GO TO 530

STA 3630

STA 3640

STA 3650

STA3660

STA3670

STA3680

STA3690

STA 3700

STA 3710

STA 3720

STA 3730

540 WRITE (IPRINT, 790) NZW, SA (NZW, 2), SA(NZW, 1), SA (NZW, 3) , RC (LW), ISA (NZSTA3740

IW),$T$

GO TO 560

STA 3750

STA 3760

550 WRITE (IPRINT, 780) NZW, SA(NZW, 2), SA (NZW, 1), SA (NZW, 3), ISA (NZW), T

STA 3770

560 IF (ISTORE.EQ.2) RETURN

IF (NARGS.EQ.2.OR.NARGS.EQ.3) GO TO 570

STA 3780

$L=I$ ARGS (NARGS -3)

STA3790

$M=I$ ARGS (NARGS -2 )

STA 3800

$K=$ I ARGS (NARGS-1)

STA 3810

STA 3820

$J=I$ ARGS (NARGS)

STA 3830

GO TO 580

STA 3840

$L=I$ ARGS (NARGS)

STA3850

$M=L+N R O W$

STA3860

$K=M+N R O W$

STA 3870

$\mathrm{J}=\mathrm{K}+\mathrm{NROW}$

STA3880

STA3890

STA3900

$M B=I S A(I)$

STA3910

$R C(K)=S A(M B, 2)$

STA3920

$R C(M)=S A(I, I)$

STA3930

$R C(J)=S A(I, 3)$

STA 3940

$M=M+1$

STA3950

$\mathrm{K}=\mathrm{K}+1$

STA3960

$\mathrm{J}=\mathrm{J}+1$

STA3970

IF (NZW.EQ.NRMAX) GO TO 610

STA3980

$N Z W 1=N Z W+1$

STA3990

DO 600 I=NZWI, NRMAX

STA 4000

$R C(M)=0$

$R C(K)=0$.

STA 4010

$R C(J)=0$.

STA4020

$M=M+1$

STA 4030

$\mathrm{K}=\mathrm{K}+1$

STA 4040

$\mathrm{J}=\mathrm{J}+1$

STA4050

STA4060

$N T O P=60$

STA4070

IF (NROW.LT.NTOP) NTOP=NROW

STA 4080 
$R C(L)=0$.

STA4130

630

$L=L+1$

STA 4140

RETURN

STA4150

STA4 160

C

FORMAT (IHO, 4X,28HSTATISTICAL ANALYSIS OF COL ,I $4,33 X, 4 H \mathrm{~N}=$, I 4$)$ STA4I70

FORMAT (IHO $4 X, 28 H S T A T I S T I C A L$ ANALYSIS OF COL , I $4,8 X, 15 H W E I G H T S$ INSTA 4180

$1 \mathrm{COL}, \mathrm{I} 4,6 \mathrm{X}, 4 \mathrm{HN}=$, I4)

STA 4190

660 FORMAT (1HO, 4X,28HSTATISTICAL ANALYSIS OF COL , I $4,8 X, 15 H W E I G H T S$ INSTA4200

$1 \mathrm{COL}, I 4,6 \mathrm{X}, 4 \mathrm{HN}=, \mathrm{I} 4,33 \mathrm{H}$ (NO OF NON-ZERO WTS) COL LENGTH =, I4) STA4210

670 FORMAT (1HO,24X,64HALL COMPUTATIONS ARE BASED ON OBSERVATIONS WITHSTA4220 1 NON-ZERO WEIGHTS)

680 FORMAT (1HO/15X,28HFREQUENCY DISTRIBUTION (1-6),7X,10 I6)

STA4230

690 FORMAT (IHO/5X,26HMEASURES OF LOCATION (2-2),34X,28HMEASURES OF DISTA4250 ISPERSION $(2-6))$

700 FORMAT ( $1 \mathrm{HO}$,

$19 X, 26 H U N W E I G H T E D$ MEAN

2 26HSTANDARD DEVIATION

$310 X, 26 H W E$ IGHTED MEAN

4 26HS.D. OF MEAN

$510 X, 26 H M E D I A N$

6 26HRANGE

$710 X, 26 H M I D-R A N G E$

8 26HMEAN DEVIATION

$910 \mathrm{X}, 26 \mathrm{H} 25$ PCT UNHTD TRIMMED MEAN=,

A 26HVARIANCE

B10X,26H25 PCT WTD TRIMMED MEAN =,

C 26HCOEFFICIENT OF VARIATION $=$,

$=$ IPE15.7,20X,

$=\quad$ El5.7 I

$=$ E15.7,20X,

$=$ E E15.7 /

$=$ El5.7,20X,

$=, \quad$ E15.7 /

$=$, El5.7,20X,

$=$ E15.7 /

E15.7,20X,

E15.7 /

E15.7,20X,

E15.7 )

STA 4260

STA 4265

STA 4270

STA 4275

STA4 280

STA4290

STA4295

STA 4300

STA4305

STA 4310

STA 4315

STA 4320

STA4325

STA 4330

710 FORMAT (1HO//20X,50HA TWO-SIDED 95 PCT CONFIDENCE INTERVAL FOR MEASTA4350 IN ISIPEII.4,3H TO,E11.4,6H (2-2)/20X,50HA TWO-SIDED 95 PCT CONFIDESTA4360 2NCE INTERVAL FOR S.D. IS,E $11.4,3 \mathrm{H}$ TO,E $11.4,6 \mathrm{H}(2-7)$ )

STA 4370

720 FORMAT (1HO//5X,30HLINEAR TREND STATISTICS (5-1), 30X, 16HOTHER STASTA4380 ITISTICS / / 10X, 5HSLOPE , 20X, IH =, IPE 15.7,20X,7HMINIMUM, 18X, IH=, E15.7/1STA4390 20X,13HS.D. OF SLOPE, 12X,1H=,E15.7,20X,7HMAXIMUM, 18X, IH=, E15.7/10X, STA4400 326HSLOPE/S.D. OF SLOPE $=T=, E 15.7,20 X, 8 \mathrm{HBETA}$ ONE, $17 \mathrm{X}, 1 \mathrm{H}=, \mathrm{E} 15.7 / 1 \mathrm{STA} 4410$ $40 X, 35 H P R O B$ EXCEEDING ABS VALUE OF OBS $T=, 0 P F 6.3,20 X, 8 H B E T A$ TWO, 17STA4420 $5 X, 1 H=, 1 P E 15.7 / 71 X, 17 H W T D$ SUM OF VALUES $, 8 X, 1 H=, E 15.7 / 71 X, 18 H W T D$ SUMSTA 4430 6 OF SQUARES $, 7 X, 1 H=, E 15.7 / 5 X, 24 H T E S T S$ FOR NON-RANDOMNESS, 42X, 26HWTDSTA 4440 7 SUM OF DEVS SQUARED $=, E 15.7 / 71 X, 11$ HSTUDENT, $\mathrm{T}, 14 \mathrm{X}, 1 \mathrm{H}=, E 15.7$ ) STA4450

730 FORMAT (10X,26HNO OF RUNS UP AND DOWN $=, 15,30 X, 26 H W T D$ SUM ABSOLUSTA4460 ITE VALUES $=, 1$ PE $15.7 / 10 X, 26$ HEXPECTED NO OF RUNS =,OPF7.1,28X, STA4470 226HWTD AVE ABSOLUTE VALUES =,IPE15.7/10X,26HS.D. OF NO OF RUNS STA4480 $3=, 0 P F 8.2 / 10 X, 26 H M E A N$ SQ SUCCESSIVE DIFF $=, 1$ PE $16.7 / 10 X, 26$ HMEANSTA 4490 4 SQ SUCC DIFF/VAR =.OPF9.3///10X,24HDEVIATIONS FROM WTD MEAN//1STA4500 $55 X, 21 H N O O F+$ SIGNS $=, I 5 / 15 X, 21 H N O O F-$ SIGNS $=15 / 15 X$, STA45 10 610HNO OF RUNS, 10X, IH=, I5/15X,21HEXPECTED NO OF RUNS $=, F 7.1 / 15 X, 12 H S T A 4520$ 7S.D. OF RUNS, 8X,1H=,F8.2/15X,2IHDIFF./S.D. OF RUNS =F9.3) STA4530

740 FORMAT (////68H NOTE - ITEMS IN PARENTHESES REFER TO PAGE NUMBER STA4540 IIN NBS HANDBOOK 91)

750 FORMAT (//27X,12HOBSERVATIONS,47X,20HORDERED OBSERVATIONS) STA4560

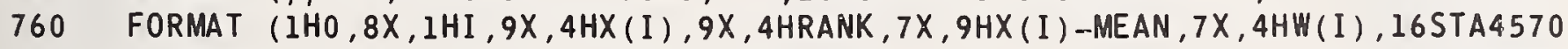
$1 X, 3 H N 0 ., 8 X, 4 H X(J), 10 X, 11 H X(J+1)-X(J)) \quad$ STA4580

770 FORMAT (1HO,8X, 1HI ,9X,4HX(I) ,9X,4HRANK, 7X, 9HX(I) -MEAN, 27X, 3HNO . 8XSTA4590 $1,4 H X(J), 10 X, 11 H X(J+1)-X(J))$

780 FORMAT (I 10,1PE17.7,OPF9.1,IPE17.7,22X,I6,1P2E17.7)

790 FORMAT (I 10,1PE17.7, OPF9.1, IPE17.7, IPE 12.3,10X, 16, IP2E17.7)

800 FORMAT (IH)

STA4600

END 


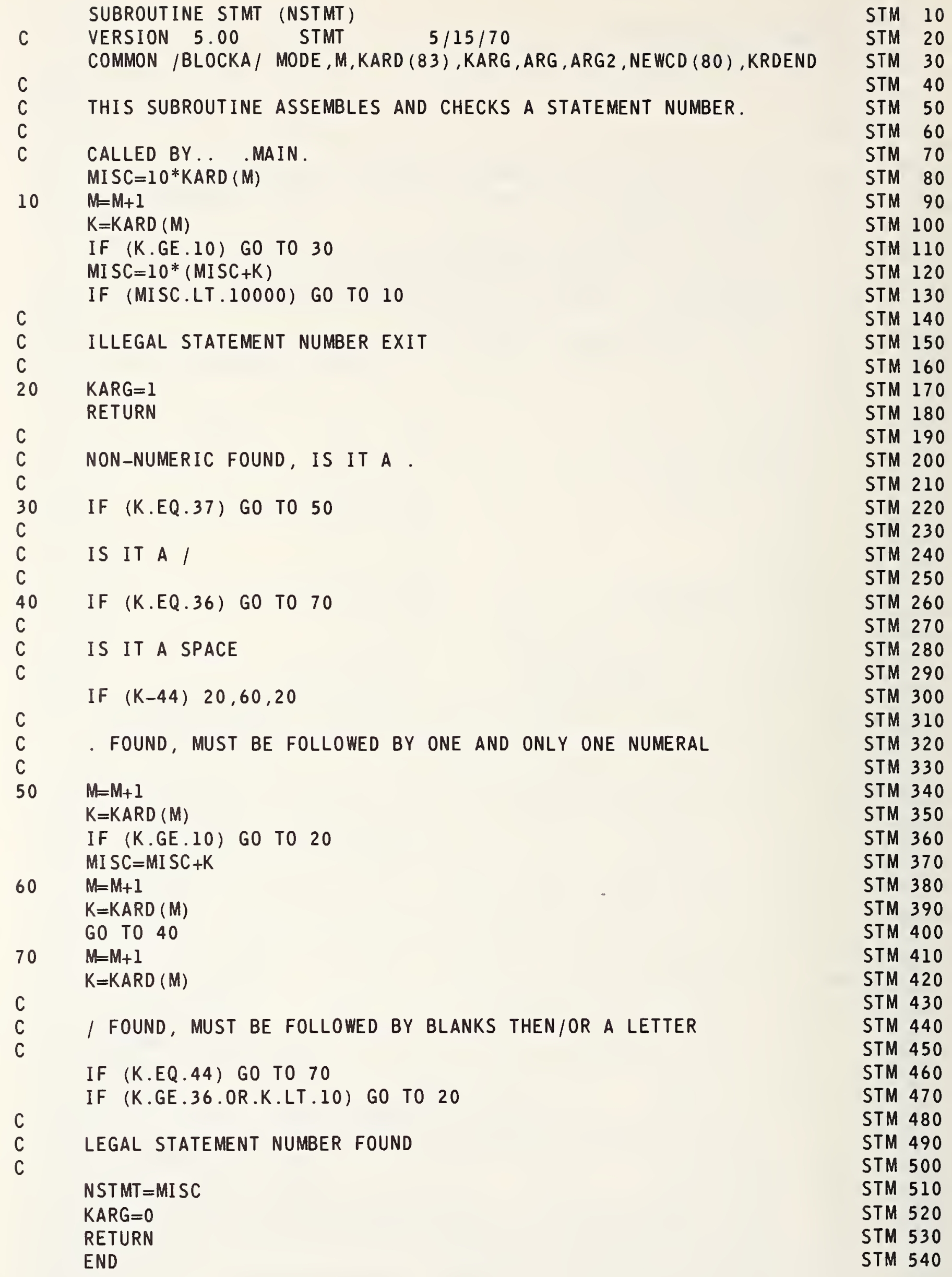


SUBROUTINE STORE (J)

VERSION $5.00 \quad$ STORE

$5 / 15 / 70$

STO 20

COMMON /BLOCKA/ MODE,M,KARD (83), KARG, ARG, ARG2, NEWCD (80), KRDEND STO 30

COMMON /BLOCKB/ NSTMT, NSTMTX, NSTMTH, NCOM, LCOM, IOVFL, COM(2000) STO 40

COMMON /BLOCRC/ NRC, RC (12600)

STO 50

COMMON /BLOCKD/ IARGS(100), KIND (100), ARGTAB (100), NRMAX, NROW, NCOL, NSTO 60

IARGS, VWXYZ ( 8$)$, NERROR

STO 70

DIMENSION ARGS $(100)$

STO 80

EQUIVALENCE (ARGS (1), RC (12501))

COMMON /BLOCKE/ NAME (4),L1,L2, ISRFLG

STO 90

STORAGE LAYOUT. .

STATEMENT NUMBER

NUMBER OF WORDS IN ENTRY

STO 100

C

C

C

ALL ITEMS ARE STORED IN

NARGS $+64 *(L 1+64 * L 2)$

STO 110

STO 120

FLOATING POINT TO ALLOW

( ENTRY 1 )

STO 130

STO 140

CONVERSION TO DOUBLE-

PRECISION .

( LAST WORD )

STO 150

STO 160

STO 170

IF (IOVFL.NE.0) RETURN

STO 180

$I Z E=J+2$

IF (NSTMT.GT.NSTMTH) GO TO 90

STO 190

STO 200

STO 210

STATEMENT IS AN INSERTION OR A REPLACEMENT

STO 220

STO 230

STO 240

$L=L O C A T E$ (NSTMT)

IF (L.GT.O) GO TO 30

STO 250

STO 260

$\mathrm{L}=-\mathrm{L}$

IDIF $=12 E$

$L L=N C O M$

10

STATEMENT IS AN INSERTION, OPEN GAP

STO 270

STO 280

STO 290

STO 300

$I I=L L+I D I F$

IF (II.GE.LCOM) GO TO 100

STO 310

STO 320

STO 330

DO $20 \quad \mathrm{I}=\mathrm{L}, \mathrm{NCOM}$

STO 340

$\operatorname{COM}($ II $)=\operatorname{COM}(\mathrm{LL})$

STO 350

I I $=$ I I - I

STO 360

$\mathrm{LL}=\mathrm{LL}-1$

STO 370

GO TO 60

STO 380

STO 390

\section{STATEMENT IS REPLACEMENT}

STO 400

STO 410

IDIF $=$ IZE-IF IX $(\operatorname{COM}(L+1))$

STO 420

IF (IDIF) $40,60,10$

STO 430

STO 440

NEW STATEMENT SMALLER THAN OLD, CLOSE UP GAP.

STO 450

STO 460

STO 470

$I=L-I D I F$

STO 480

I $I=L$

DO 50 I $A=I, N C O M$

STO 490

$\operatorname{COM}(I I)=\operatorname{COM}($ I A )

STO 500

$I I=I I+1$

STO 510

STO 520

INSERT STATEMENT

STO 530

STO 540

STO 550

STO 560

$\operatorname{COM}(L)=N S T M T$

$\operatorname{COM}(L+1)=I Z E$

STO 570

$\operatorname{COM}(L+2)=N A R G S+64 *(L 1+64 * L 2)$

STO 580

$\mathrm{NCOM}=\mathrm{NCOM}+\mathrm{IDIF}$

STO 590 


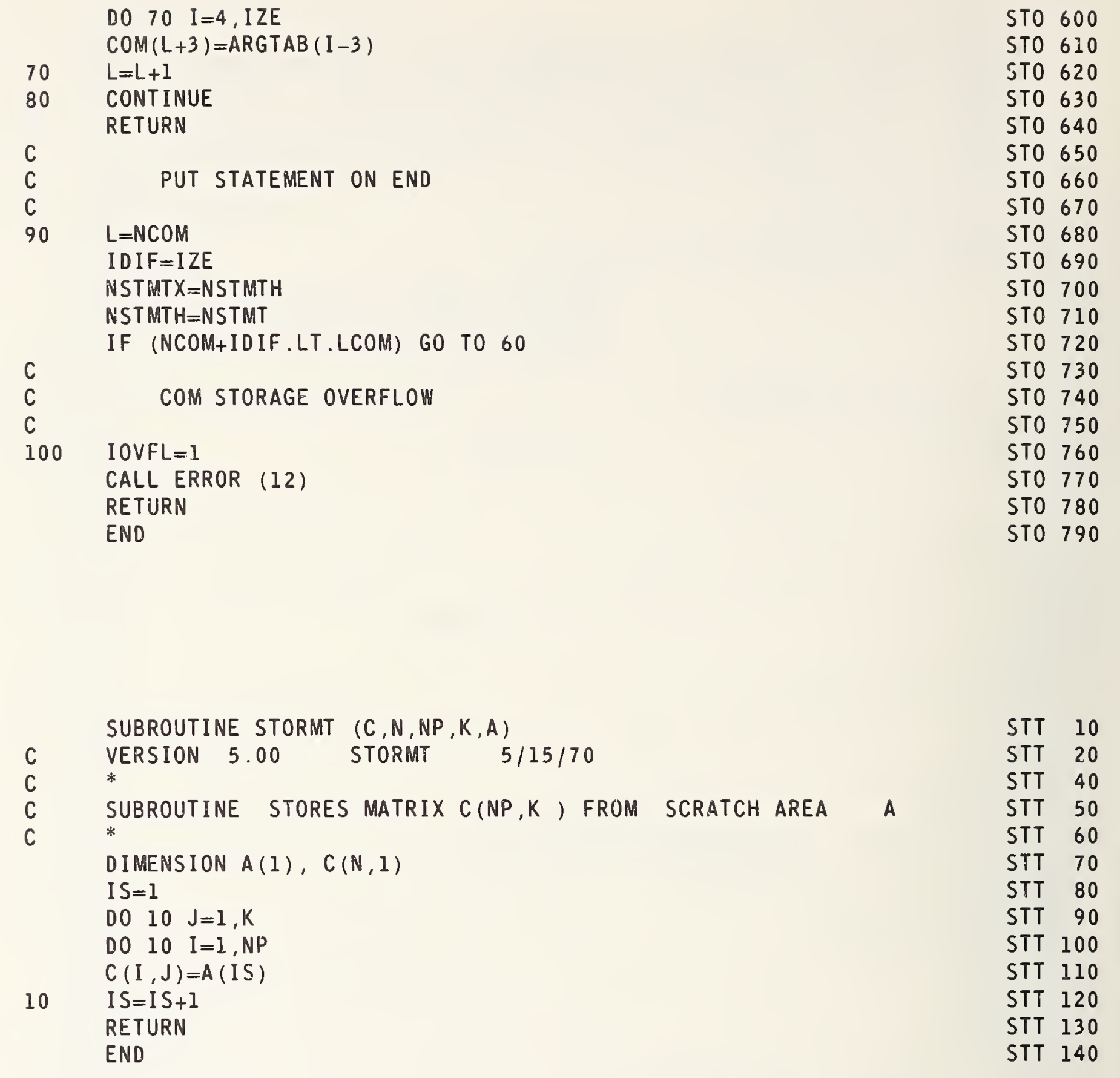


SUBROUTINE STRUVE $(Z, A, B, C)$

C VERSION 5.00 STRUVE 5/15/70

DIMENSION C ( 1 )

DOUBLE PRECISION $Z, A, B, C, X, P, Q, R, S, D B E J$

$X=D A B S(Z)$

IF $(X . G T \ldots O D O)$ GO TO 10

$A=.0 D 0$

$\mathrm{B}=.0 \mathrm{DO}$

GO TO 40

10 IF (X.GT.70.D0) GO TO 30

CALL BEJN $(0, C, X)$

$P=.000$

$Q=.000$

DO $20 \quad \mathrm{~N}=1,49$

$\mathrm{J}=2 * \mathrm{~N}$

$\mathrm{K}=\mathrm{J}+1$

$R=J-1$

$S=4 * N * 2-1$

$P=P+C(J) / R$

$20 \quad Q=Q+C(K) / S$

$A=P / .7853981633900$

$B=\left(2 . D 0^{*} Q+1 . D 0-C(1)\right) / 1.5707963268 D 0$

GO TO 40

$30 \quad S=1.00 / X^{* * 2}$

$P=1 . D 0-S *(1 . D 0-9 . D 0 * S *(1 . D 0-25 . D 0 * S *(1 . D 0-49 . D 0 * S)))$

$A=D B E J(X, 0,5)+P /(X * 1.5707963268 D 0)$

$Q=1.00+S *(1 . D 0-3.00 * S *(1 . D 0-15.00 * S *(1.00-35.00 * S)))$

$B=\operatorname{DBEJ}(X, 1,5)+Q /(1.5707963268 D 0)$

40 RETURN

END

SUBROUTINE SYMV (A, NROW, N, K)

VERSION 5.00 SYMV $5 / 15 / 70$

$C$
$C$
$C$
$C$
$C$
$C$
$C$
$C$
$C$
$C$

C A

A-FIRST ELEMENT OF MATRIX A

NROW - NO. OF ROWS IN A AS DEFINED IN A DIMENSION ST

$N$-PRESENT SIZE OF A

$K$-STATUS FOR SYMMETRY

$K=0$ EXACT SYMMETRY A $A(I, J) / A(J, I))=1$

$K=1$ SYMM TO A RELATIVE RROR ABS $(I-A(I, J) / A(J, I))=$ OR LESS $1 . E-7$

$K=2$ NO SYMMETRY

DIMENSION A (NROW, NROW)

$\mathrm{K}=0$

$\mathrm{NN}=\mathrm{N}-\mathrm{I}$

DO $40 \mathrm{~J}=1, \mathrm{NN}$

$\mathrm{I}=\mathrm{J}+1$

DO $40 \mathrm{~L}=\mathrm{I}, \mathrm{N}$

IF $(A(J, L)) 20,10,20$

$10 \quad T=A B S(A(L, J))$

GO TO 30

$20 \quad T=A B S(1.0-A(L, J) / A(J, L))$

30 IF (T.EQ.0.) GO TO 40

$K=1$

IF (T.LE.1.E-7) GO TO 40

$K=2$

RETURN

40

CONTINUE

RETURN

END
STR 10

STR 20

STR 30

STR 40

STR 50

STR 60

STR 70

STR 80

STR 90

STR 100

STR 110

STR 120

STR 130

STR 140

STR 150

STR 160

STR 170

STR 180

STR 190

STR 200

STR 210

STR 220

STR 230

STR 240

STR 250

STR 260

STR 270

STR 280

STR 290

STR 300

SYM 10

SYM 20

SYM 30

SYM 40

SYM 50

SYM 60

SYM 70

SYM 80

SYM 90

SYM 100

SYM 110

SYM 120

SYM 130

SYM 140

SYM 150

SYM 160

SYM 170

SYM 180

SYM 190

SYM 200

SYM 210

SYM 220

SYM 230

SYM 240

SYM 250

SYM 260

SYM 270

SYM 280

SYM 290 
COMMON /BLOCKA/ MODE, M,KARD (83), KARG, ARG, ARG2, NEWCD (80), KRDEND

TAP 55

COMMON /BLOCKE/ NAME (4),L1,L2, ISRFLG

TAP 60

COMMON /CODETP/ ITP $(10,4)$

TAP 70

NAME $4(1)=0$

TAP 80

$\operatorname{NAME} 4(2)=0$

TAP 90

NTPCT $=0$

TAP 100

$10 \quad K=K A R D(M)$

TAP 110

C THE TAPE ID MAY BE A NUMBER OR A LETTER

TAP 120

IF (K.LT.36) IF $(K-10) 20,30,30$

TAP 130

$M=M+1$

TAP 135

GO TO 10

TAP 140

TAP 150

20 ITAPE $=$ KARD $(M)+6$

TAP 160

$M P=M-1$

GO TO 50

TAP 170

$30 \quad M P=M-1$

TAP 180

CALL NNAME (NAME4 (1))

TAP 190

I TAPE $=0$

TAP 200

DO $40 \quad I=1,6$

IF (NAME4 (1).NE.IALPH (I)) GO TO 40

TAP 210

I TAPE $=I+6$

GO TO 50

40 CONTINUE

50 IF(ITAPE.GT.9) GO TO 60

$\mathrm{KARD}($ MP) $=L(45)$

$\operatorname{KARD}(M P+1)=I$ TAPE

GO TO 80

$60 \quad \operatorname{KARD}(M P)=1$

KARD $(M P+1)=I T A P E-10$

TAP 220

TAP 230

TAP 240

TAP 250

TAP 260

TAP 270

TAP 280

TAP 290

TAP 300

TAP 310

TAP 320

C CREAD READ WRITE

TAP 340

80 IF (NAME (1).NE .ITP $(2,1)$. AND.NAME (1).NE . ITP $(1,1)$. AND. NAME (1). NE . ITPTAP 350 $1(3,1))$ GO TO 110

$\operatorname{NAME} 4(1)=0$

TAP 360

NAME $4(2)=0$

TAP 370

$M=M+1$

TAP 380

$90 \quad K=K A R D(M)$

IF (K.LT.36) IF $(K-10) 110,100,100$

TAP 390

$M=M+1$

TAP 400

TAP 410

GO TO 90

100 CALL NNAME (NAME4(1))

$110 \quad M=M P$

RETURN

END

TAP 420

TAP 430

TAP 440

TAP 450

TAP 460

TAP 470 
SUBROUTINE TAPOP2

C VERSION $5.00 \quad$ TAPOP2 $5 / 15 / 70$

THIS SUBROUTINE IS NEDDED ONLY FOR TAPE OPERATIONS

TP2 10

$L I=45 \quad L 2=1,7$

READ TAPE A-F A-F (FORMAT) INTO COLUMNS,,,,++++++++ ETC.

READ UNTIL A RECORD OF A ZEROS ARE ENCOUNTERED

TP2 20

TP2 30

TP2 40

TP2 50

IF NO FORMAT IS GIVEN, CARDS ARE READ AS IN READ COMMAND 10

$L I=46 \quad L 2=1,7$

CREAD TAPE A-F A-F (FORMAT) , CARDS INTO COLUMNS,,,++++++ ETP2 90

CREAD TAPE A-F , CARDS INTO COLUMNS ++, ++,++,ETC. TP2 100

READ USING A COUNTER

$\mathrm{Ll}=47 \quad \mathrm{~L} 2=1,7$

WRITE TAPE A-F A-F (FORMAT) FROM COLUMNS,,,,$++++++++ E T C$.

TP2 110

TP2 120

TP2 130

A RECORD OF ZEROS IS WRITTEN AFTER NRMAX VALUES

TP2 140

$L I=48 \quad L 2=1$

SET TAPE A-F INTO COLUMNS ++

SET TAPE A-F INTO ROW , OF COLUMN ++

TP2 150

TP2 160

TP2 170

READ UNTIL A RECORD OF ZEROS IS ENCOUNTERED

TP2 180

$L I=49 \quad L 2=1$

CSET TAPE A-F, VALUES INTO COLUMN ++

CSET TAPE A-F, , VALUES INTO ROW, OF COLUMN ++

TP2 190

TP2 200

READ USING A COUNTER

TP2 210

TP2 220

$L I=50 \quad L 2=1$

TP2 230

ENDFILE TAPE A-F

TP2 240

$L I=50 \quad L 2=2$

REWIND TAPE A-F

TP2 250

$L I=50 \quad L 2=3$

SKIP TAPE A-F FORWARD , RECORDS

TP2 260

TP2 270

TP2 280

$L I=50 \quad L 2=4$

TP2 290

BACKSPACE TAPE A-F, RECORDS

COMMON /BLOCKC/ KIO, INUNIT, ISCRAT, KBDOUT, KRDKNT, LLIST

TP2 300

TP2 310

COMMON /BLOCKD/ IARGS(100), KIND(100), ARGTAB (100), NRMAX, NROW, NCOL, NTP2 320

IARGS, VWXYZ (8), NERROR

COMMON /BLOCKE/ NAME (4), L1,L2, ISRFLG

TP2 330

COMMON/HEADER/NOCARD $(80)$, I TLE $(60,6)$, LNCNT, I PR INT, NPAGE, IPUNCH

TP2 340

COMMON /SCRAT/ NS, NS2, A(13500)

COMMON /TAPE/ NAME4 (2), NTPCT, IPUNCP, INUNIP, LITP

C CHECK FOR CORRECT NUMBER OF ARGUMENTS

IF (LI.LT.50) IF (NARGS-2) 5,30,30

TP2 350

TP2 370

TP2 380

TP2 390

GO TO $(10,10,20,20), L 2$

TP2 395

TP2 400

CALL ERROR (10)

GO TO 30

TP2 410

IF (NARGS.NE.1) CALL ERROR (10)

GO TO 40

TP2 420

TP2 430

TP2 440

20 IF (NARGS.NE.2) CALL ERROR (10)
C ALL ARGUMENTS SHOULD BE INTEGERS

TP2 450

J=NARGS

CALL CKIND (J)

IF (J.NE.O) CALL ERROR (3)

$C$ IS TAPE NUMBER CORRECT

TP2 460

TP2 470

TP2 480

TP2 490

TP2 500

IF (IARGS (1).LT.7.OR.IARGS(1).GT.12) CALL ERROR (28)

IF (NERROR.NE.0) RETURN

TP2 510

LIP $=$ LI -44

GO TO $(50,70,90,60,80,170)$, LIP

50 IF (L2.EQ.1) GO TO 60

I NUN I T=I ARGS ( 1 )

$\operatorname{IARGS}(1)=0$

GO TO 130

TP2 520

TP2 530

TP2 540

TP2 550

TP2 560

TP2 570

TP2 580 


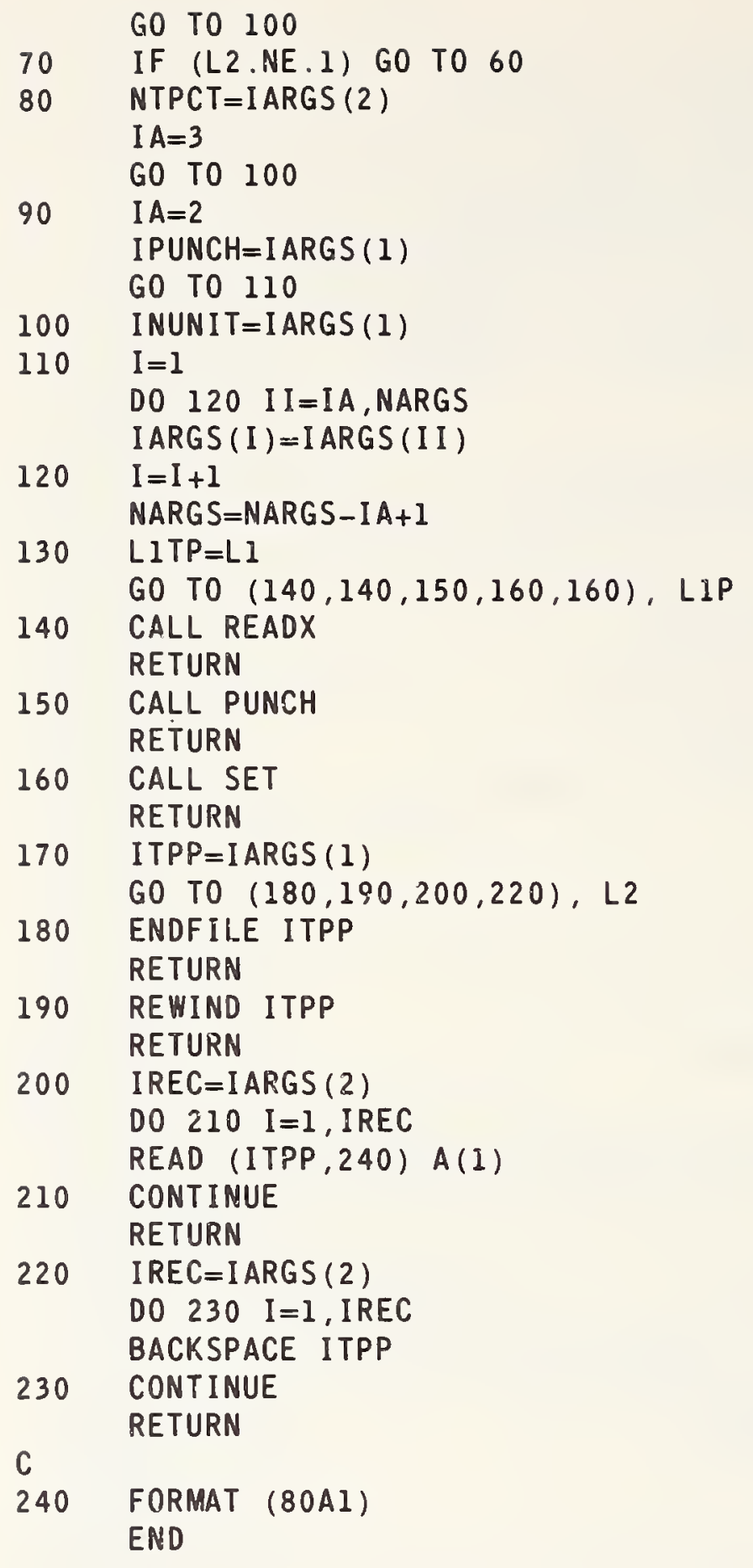

TP2 690

TP2 700

TP2 710

TP2 720

TP2 730

TP2 740

TP2 750

TP2 760

TP2 770

TP2 780

TP2 790

TP2 800

TP2 810

TP2 820

TP2 830

TP2 840

TP2 850

TP2 860

TP2 870

TP2 880

TP2 890

TP2 900

TP2 910

TP2 920

TP2 930

TP2 940

TP2 950

TP2 960

TP2 970

TP2 980

TP2 990

TP21000 
SUBROUTINE THERMO

VERSION $5.00 \quad$ THERMO $5 / 15 / 70$

THE 10

THE 20

IT NOW CONTAINS THE COMMANDS CTOF, FTOC, ATOMIC, MOLHT, EINSTEIN, THE 30

PFTRANSLATIONAL, PFATOMIC, AND PARTFUNCTION THE 40

THERMODYNAMIC PACKAGE WRITTEN BY R. MCCLENON, NSRDS-NBS, NOV. 69 THE 50

THE VALUES FOR L2 ARE --

1 -CTOF (CENTIGRADE TO FAHRENHEIT)

2 - FTOC (FAHRENHEIT TO CENTIGRADE)

3 - ATOMIC MASS TABLE

4 - MOLWT (MOLECULAR WEIGHT)

5 - EINSTEIN FUNCTION

6 - PFTRANS ( PARTITION FUNCTION TRANSLATIONAL)

7 - PFATOM ( P.F. ATOMIC)

8 - PARTFUNCTION

9 - BOLDISTRIBUTION (BOLZMAN DISTRIBUTION)

WRITTEN BY R. MCCLENON, NSRDS-NBS, DEC. 1969

THE 60

THE 70

THE 80

THE 90

THE 100

THE 110

THE 120

THE 130

THE 140

THE 150

THE 160

THE 170

THE 180

COMMAND FORMATS ARE AS FOLLOWS --

THE 190

CTOF OF \$\$ STORE IN COL ++

THE 200

FTOC OF \$\$ STORE IN COL ++

ATOMIC MASSES STORE IN COL ++

THE 210

THE 220

, $Z=$, AMOUNT $=, \ldots$.. STORE SUM IN COL ++

EINSTEIN TEMP IN \$ VIB FREQ IN WAVE NO IN \$\$ START STORING IN ++THE 250

OR EINSTEIN TEMP IN $\$$ FREQ IN $\$$ GAS CONST $R=$, START IN ++ THE 260

PFTRANS TEMP IN \$\$ MOL WT M IN \$\$ START STORING IN \$\$

THE 270

PFATOH TEMP IN \$\$ MOL WT M IN \$\$ WAVE NO IN ++ DEGEN G IN ++ START STORING IN COL ++

PARTFUNC TEMP IN \$\$ WAVE NO IN ++ G IN ++ START STORING IN ++

THE 280

THE 290

THE, 300

VIBOIST TEMP IN \$\$ WAVE NO IN ++ G IN ++ START STORING IN ++

THE 310

THE 320

SEE HANDBOOK 101 FOR DETAILS ON STORAGE BY ALL COMMANDS EXCEPT

THE 330

VIBDIST (WHICH IS NEW)

THE 340

VIBOIST STORES THE PERCENTAGE OF MOLECULES IN EACH OF THE

THE 350

VIBRATIONAL ENERGY LEVELS. IF THERE ARE N ENERGY LEVELS VIBDIST THE 360

WILL USE N COLUMNS FOR STORAGE

THE 370

THE 380

COMMON /BLOCKD/ IARGS(100), KIND(100), ARGTAB(100), NRMAX, NROW, NCOL, NTHE 390

IARGS, YWXYZ (8), NERROR

EQUIVALENCE (ARGS(1), RC (12501))

COMMON /BLOCKE/ NAME (4), L1,L2, ISRFLG

DIMENSION ARGS(100)

COMHON /BLOCRC/ NRC, RC (12600)

DOUBLE PRECISION X,EXX, EXDIF, FDEXP, FDLOG, QO Q Q1,Q2, G, QQ

COMMON /SCRAT/ NS, NS2,A(13500)

C

DIMENSION QQ (NS2)

DIMENSION QQ(6750)

EQUIVALENCE (A(1), QQ(1))

DIMENSION ATWT (103)

THE 400

THE 410

THE 420

THE 430

THE 440

THE 450

THE 460

THE 470

THE 480

THE 490

THE 500

DATA ATWT (1), ATWT (2),ATHT (3), ATWT (4),ATWT (5), ATWT (6)/1.00800,4.002THE 510 $160,6.94100,9.01218,10.81000,12.01100 /$

THE 520

DATA ATWT (7), ATWT (8), ATWT (9),ATWT (10), ATWT (11), ATWT (12)/14.00670,1THE 530 $15.99940,18.99840,20.17900,22.98980,24.30500 /$

THE 540

DATA ATWT (13), ATWT (14), ATWT (15), ATWT (16), ATWT (17), ATWT (18)/26.9815THE 550 $10,28.08600,30.97380,32.06000,35.45300,39.48000 /$

THE 560

DATA ATWT (19), ATWT (20), ATWT (21), ATWT (22), ATWT (23), ATWT (24)/39. 1020THE 570 $10,40.08000,44.95590,47.90000,50.94140,51.99600 /$

THE 580

DATA ATWT (25), ATWT (26), ATWT (27), ATHT (28),ATWT (29),ATWT (30)/54.9380THE 590 
$10,55.84700,58.93320,58.71000,63.54600,65.37000 /$

THE 600

DATA ATWT (31),ATWT (32), ATWT (33), ATWT (34), ATWT (35), ATWT (36)/69.7200THE 610 $10,72.59000,74.92160,78.96000,79.90400,83.80000 /$

THE 620

DATA ATWT (37), ATWT (38), ATWT (39), ATWT (40), ATWT (41), ATWT (42)/85.4678THE 630 $10,87.62000,88.90590,91.22000,92.90640,95.94000 /$

THE 640

DATA ATWT (43), ATWT (44), ATWT (45), ATWT (46), ATWT (47), ATWT (48)/98.9062THE 650 $10.101 .07000,102.90550,106.40000,107.86800,112.40000 / \quad$ THE 660

DATA ATWT (49), ATWT (50),ATWT (51), ATWT (52),ATWT (53), ATWT (54)/114.820THE 670 $100,118.69000,121.75000,127.60000,126.90450,131.30000 / \quad$ THE 680

DATA ATWT (55),ATWT (56),ATWT (57), ATWT (58), ATWT (59), ATWT (60)/132.905THE 690 $150,137.34000,138.90550,140.12000,140.90770,144.24000 / \quad$ THE 700

DATA ATWT (61), ATWT (62), ATWT (63), ATWT (64), ATWT (65), ATWT (66)/147.000THE 710 $100,150.40000,151.96000,157.20000,158.92540,162.50000 / \quad$ THE 720

DATA ATWT (67), ATWT (68), ATWT (69), ATWT (70), ATWT (71), ATWT (72)/164.930THE 730 $130,167.26000,168.93420,173.04000,174.97000,178.49000 / \quad$ THE 740

DATA ATWT (73), ATWT (74), ATWT (75), ATWT (76), ATWT (77), ATWT (78)/180.947THE 750 $190,183.85000,186.20000,190.20000,192.22000,195.09000 / \quad$ THE 760

DATA ATWT (79), ATWT (80), ATWT (81), ATWT (82), ATWT (83), ATWT (84)/196.966THE 770 $150,200.59000,204.37000,207.20000,208.98060,210.00000 / \quad$ THE 780

DATA ATWT (85), ATWT (86), ATWT (87), ATWT (88), ATWT (89), ATWT (90)/210.000THE 790 $100,222.00000,223.00000,226.02540,227.02000,232.03810 / \quad$ THE 800 DATA ATWT (91), ATWT (92), ATWT (93), ATWT(94), ATWT(95), ATWT (96)/231.035THE 810 $190,238.02900,237.04820,239.00000,243.00000,247.00000 / \quad$ THE 820

DATA ATWT (97), ATWT (98), ATWT (99), ATWT (100),ATWT(101), ATWT (102)/247.THE 830 $100000,249.00000,254.00000,253.00000,255.00000,257.00000 / \quad$ THE 840 DATA ATHT (103)/255.0/

GO TO $(10,250,260,380,490,590,640,710,760)$, L2

C THIS IS CTOF

$10 \quad M=1$

20 IF (NARGS-2) $1210,30,1210$

30 CALL ADRESS $(2, I 2)$

IF (I2) $1220,1230,40$

40 CALL ADRESS $(1, I 1)$

IF (I I) $50,1230,50$

50 IF (NRMAX) $1240,1240,60$

60 IF (NERROR) $1180,70,1180$

70 IF (II) $160,1230,80$

$80 \quad \mathrm{IE}=0$

DO $150 \mathrm{~J}=1$, NRMAX

I I $1=I 1+\mathrm{J}-1$

I I $2=I 2+\mathrm{J}-1$

IF $(M-1) 1180,100,90$

$90 \quad R C($ I I 2$)=(R C($ II 1$)-32.0) / 1.8$

IF (RC(II $)+273.15) \quad 110,150,150$

100 IF (RC(III) +273.15) $110,140,140$

110 IF (IE) $130,120,130$

120 CALL ERROR (230)

$I E=I$

130 IF $(M-1) \quad 1180,140,150$

$140 \quad \mathrm{RC}($ I I 2$)=(1.8 * R C($ I I I $))+32.0$

150 CONTINUE

GO TO 1180

160 IF (M-1) $1180,180,170$

$170 T=(\operatorname{ARGS}(1)-32) /$.

IF $(T+273.15) 190,230,230$

180 IF (ARGS(1)+273.15) $190,220,220$

190 IF (IE) $210,200,210$

200 CALL ERROR (230)

$I E=1$

THE 850

THE 860

THE 870

THE 880

THE 890

THE 900

THE 910

THE 920

THE 930

THE 940

THE 950

THE 960

THE 970

THE 980

THE 990

THE 1000

THEI010

THE 1020

THE 1030

THE 1040

THE 1050

THE 1060

THE 1070

THE 1080

THE 1090

THE 1100

THE 1110

THE 1120

THE 1130

THE 1140

THE 1150

THE 1160

THE 170

THE 180 
210 IF (M-1) $1180,220,230$

$220 \quad \mathrm{~T}=(1.8 *$ ARGS $(1))+32.0$

THE 1200

230 DO $240 \mathrm{~J}=1$, NRMAX

I I $2=$ I $2+\mathrm{J}-1$

$240 \quad \mathrm{RC}(\mathrm{II} 2)=\mathrm{T}$

GO TO 1180

C. THIS IS FTOC

THE 1210

THE 1220

THE 1230

THE 1240

$M=2$

GO TO 20

C THIS IS ATOMIC WEIGHT

THE 1250

260 IF (NARGS-1) $1210,280,270$

THE 1260

THE 1270

THE 1280

270 CALL ERROR (221)

280 CALL ADRESS $(1, I 1)$

IF (II) $1220,1230,290$

290 IF (NROW-92) 300,310,310

300 CALL ERROR (226)

310 IF (NERROR) $1180,320,1180$

THE 1290

THE 1300

THE 1310

THE 1320

THE 1330

THE 1340

320 IF (NROW-103) $330,340,340$

THE 1350

$L=N R O H$

GO TO 350

THE 1360

THE 1370

$\begin{array}{ll}340 & \mathrm{~L}=103 \\ 350 & \mathrm{D} 0360 \quad \mathrm{~J}=1, \mathrm{~L}\end{array}$

THE 1380

I I I $=$ I I $+\mathrm{J}-\mathrm{I}$

360 RC (III) =AT WT (J)

IF (NRMAX-L) $370,1180,1180$

THE 1390

THE 1400

THE 1410

THE 1420

NRMAX $=\mathrm{L}$

THE 1430

GO TO 1180

C THIS IS MOLWT

380 I=NARGS

CALL CKIND (I)

IF (I - I ) $390,1220,1220$

THE 1440

THE 1450

THE 1460

THE 1470

THE 1480

THE 1490

THE 1500

THE 1510

IF (NARGS-2*N) $400,1210,400$

THE 1520

400 IF (NRMAX) $1240,1240,410$

THE 1530

CALL ADRESS (NARGS,I)

THE 1540

IF (I) $1220,1230,420$

THE 1550

THE 1560

IF (N-1) $1210,430,430$

THE 1570

THE 1580

THE 1590

THE 1600

THE 1610

THE 1620

THE 1630

THE 1640

THE 1650

THE 1660

THE 1670

THE 1680

THE 1690

THE 1700

THE 1710

THE 1720

THE 1730

THE 1740

THE 1750

THE 1760

GO TO 560

THE 1770 
IF (I) $1220,1230,540$

540 IF (KIND(3)-1) $1220,550,1220$

$550 \quad R=A R G S(3)$

I I = I ARGS (4)

IF (R) $1250,1250,560$

560 CALL ADRESS (2,IFQ)

IF (IFQ) $570,1230,580$

$570 \quad F=A R G S(2)$

IF (F) $1250,1250,580$

580 IF (NCOL-II-7) $1230,800,800$

590 IF (NARGS-3) $1210,600,1210$

600 CALL ADRESS $(3,1)$

IF (I) $1220,1230,61 \mathrm{C}$

610 II $=\operatorname{IARGS}(3)$

CALL ADRESS (2, IWT)

IF (IWT) $620,1230,630$

620 WT=ARGS (2)

IF (WT) $1250,1250,630$

630 IF (NCOL-II-6) $1230,800,800$

640 IF (NARGS-5) $1210,650,1210$

650 CALL ADRESS $(5, I)$

IF (I) $1220,1230,660$

660 I I $=$ I ARGS (5)

CALL ADRESS $(2, I W T)$

IF (IWT) $670,1230,680$

$670 W T=A R G S(2)$

IF (WT) $1250,1250,680$

680 CALL ADRESS (3,IFQ)

IF (IFQ) $1220,1230,690$

690 CALL ADRESS $(4, I G)$

IF (IG) $1220,1230,700$

700 IF (NCOL-II-6) $1230,800,800$

710 IF (NARGS-4) $1210,720,1210$

720 CALL ADRESS $(4, I)$

IF (I) $1220,1230,730$

730 I I $=$ I ARGS (4)

CALL ADRESS (2,IFQ)

IF (IFQ) $1220,1230,740$

740 CALL ADRESS $(3, I G)$

IF (IG) $1220,1230,750$

$750 \quad I W T=0$

If (NCOL-II-3) $1230,800,800$

760 IF (NARGS-4) $1210,770,1210$

770 CALL ADRESS $(4, I)$

IF (I) $1220,1230,780$

$780 \quad$ I WT $=0$

I I =I ARGS (4)

CALL ADRESS (2, IFQ)

IF (IFQ) $1220,1230,790$

790 CALL ADRESS (3,IG)

IF (IG) $1220,1230,800$

800 CALL ADRESS ( 1, ITP)

IF (ITP) $810,1230,820$

810 T=ARGS $(1)$

IF (T) $1250,1250,820$

$820 \quad \mathrm{IE}=0$

IF (NRMAX) $1240,1240,830$

830 IF (NERROR) $1180,840,1180$

840 IF (L2-8) $890,850,850$
THE 1780

THE 1790

THE 1800

THE 1810

THE 1820

THE 1830

THE 1840

THE 1850

THE 1860

THE 1870

THE 1880

THE 1890

THE 1900

THE 1910

THE 1920

THE 1930

THE 1940

THE 1950

THE 1960

THE 1970

THE 1980

THE 1990

THE 2000

THE 2010

THE2020

THE2030

THE 2040

THE2050

THE 2060

THE 2070

THE 2080

THE 2090

THE 2100

THE 2110

THE 2120

THE 2130

THE 2140

THE 2150

THE 2160

THE 2170

THE 2180

THE2 190

THE 2200

THE 2210

THE2 220

THE 2230

THE 2240

THE 2250

THE 2260

THE 2270

THE 2280

THE 2290

THE2300

THE 2310

THE 2320

THE 2330

THE 2340

THE 2350

THE 2360 
850 D0 $880 \mathrm{~J}=\mathrm{NROW}, 1,-1$

I I $G=I G+J-1$

THE 2370

THE 2380

IF (RC(IIG)) $1260,880,860$

$860 \quad K K=J$

IF (KK-NS2) $890,890,870$

THE2390

THE2 400

THE2410

870 IF (L2-8) $890,890,1190$

THE 2420

880 CONTINUE

GO TO 1270

THE 2430

THE 2440

890 DO $1170 \mathrm{~J}=1$, NRMAX

IF (ITP-1) $910,900,900$

$900 \quad$ I IT $=I T P+J-1$

$T=R C$ ( I IT)

IF (T) $1150,910,910$

910 IF (IWT) $940,920,930$

THE 2450

THE 2460

THE2470

THE2480

THE 2490

THE 2500

$920 W T=1.0$

GO TO 940

930 I I W=I WT + J-I

THE 2510

THE 2520

$W T=R C$ (II $W$ )

THE 2530

IF (WT) $1150,940,940$

940 IF (L2-8) $1000,950,950$

THE2540

THE 2550

THE2 260

$950 \quad Q 0=0$.

$\mathrm{Q} 1=0$.

THE 2570

THE 2580

Q2 $=0$.

THE 2590

DO $990 \mathrm{JJ}=1, K K$

THE 2600

I I $=I F Q+J J-1$

THE 2610

II $G=I G+J J-1$

THE 2620

$E=R C($ I IF )

THE2630

$\mathrm{G}=\mathrm{RC}$ (IIG)

THE2640

IF (G) $1260,960,960$

THE 2650

960 IF (E) $1130,970,970$

THE2 660

THE 2670

THE 2680

$\operatorname{EXX}=F \operatorname{FDXP}(-X)$

$\mathrm{Q} 0=\mathrm{Q} 0+\mathrm{G}^{*} \mathrm{EXX}$

$\mathrm{Q} 1=\mathrm{Q} 1+\mathrm{G} * X^{*} E X X$

$Q 2=Q 2+G * X * X * E X X$

IF (L2-8) $990,990,980$

$980 \quad Q Q(J J)=G^{*} E X X$

990 CONTINUE

GO TO 1010

$1000 \quad Q 0=1.0$

$\mathrm{Q} 1=0$.

$Q 2=0$.

IF (L2-6) $1020,1010,1010$

THE2690

THE 2700

THE2710

THE 2720

THE 2730

THE 2740

THE 2750

THE2760

THE 2770

THE2780

THE 2790

THE 2800

THE2 210

$H E=2.500+Q 1 / Q 0$

$\mathrm{S}=\mathrm{FE}+\mathrm{HE}$

$C P=2.5 D 0+Q 2 / Q 0-(Q 1 / Q 0) *(Q 1 / Q 0)$

$H B Y T=H E * T$

GO TO 1050

1020 IF (IFQ-1) $1040,1030,1030$

THE 2820

THE 2830

THE2840

THE 2850

THE 2860

THE 2870

THE 2880

$E=R C$ (IIF)

THE 2890

IF (E) $1130,1040,1040$

THE 2900

THE 2910

THE 2920

THE 2930

THE 2940

THE 2950 


\begin{tabular}{|c|c|c|}
\hline & $\mathrm{S}=\mathrm{FE}+\mathrm{HE}$ & THE 2960 \\
\hline & $H B Y T=H E * T$ & THE 2970 \\
\hline \multirow{2}{*}{1050} & $K=I+J-I$ & THE2980 \\
\hline & IF (L2-8) $1060,1090,1100$ & THE 2990 \\
\hline 1060 & IF (L2-6) $1070,1080,1080$ & THE 3000 \\
\hline \multirow[t]{2}{*}{1070} & $R C(K)=E$ & THE3010 \\
\hline & $\mathrm{K}=\mathrm{K}+\mathrm{NROW}$ & THE 3020 \\
\hline \multirow[t]{12}{*}{1080} & $R C(K)=T$ & THE 3030 \\
\hline & $K=K+N R O W$ & THE 3040 \\
\hline & $R C(K)=F E$ & THE 3050 \\
\hline & $\mathrm{K}=\mathrm{K}+\mathrm{NROW}$ & THE3060 \\
\hline & $R C(K)=H E$ & THE 3070 \\
\hline & $\mathrm{K}=\mathrm{K}+\mathrm{NROW}$ & THE3080 \\
\hline & $R C(K)=S$ & THE 3090 \\
\hline & $\mathrm{K}=\mathrm{K}+\mathrm{NROW}$ & THE 3100 \\
\hline & $R C(K)=C P$ & THE3110 \\
\hline & $\mathrm{K}=\mathrm{K}+\mathrm{NROW}$ & THE 3120 \\
\hline & $R C(K)=H B Y T$ & THE 3130 \\
\hline & GO TO 1170 & THE 3140 \\
\hline \multirow[t]{6}{*}{1090} & $R C(K)=Q 0$ & THE 3150 \\
\hline & $\mathrm{K}=\mathrm{K}+\mathrm{NROW}$ & THE 3160 \\
\hline & $R C(K)=Q 1$ & THE 3170 \\
\hline & $\mathrm{K}=\mathrm{K}+\mathrm{NROW}$ & THE 3180 \\
\hline & $\mathrm{RC}(\mathrm{K})=\mathrm{Q} 2$ & THE 3190 \\
\hline & GO TO 1170 & THE 3200 \\
\hline 1100 & IF (NCOL-II-KK) $1200,1110,1110$ & THE 3210 \\
\hline \multirow[t]{2}{*}{1110} & DO $1120 \mathrm{JJ}=1, K K$ & THE 3220 \\
\hline & $R C(K)=Q Q(J J) / Q 0$ & THE 3230 \\
\hline \multirow[t]{2}{*}{1120} & $K=K+N R O W$ & THE 3240 \\
\hline & GO TO 1170 & THE 3250 \\
\hline \multirow[t]{4}{*}{1130} & $Q 0=1.0$ & THE 3260 \\
\hline & $Q 1=0$. & THE 3270 \\
\hline & $Q 2=0$. & THE 3280 \\
\hline & IF (IE) $1010,1140,1010$ & THE 3290 \\
\hline \multirow[t]{3}{*}{1140} & CALL ERROR (229) & THE 3300 \\
\hline & $I E=1$ & THE 3310 \\
\hline & GO TO 1010 & THE 3320 \\
\hline \multirow[t]{6}{*}{1150} & $\mathrm{FE}=0$ & THE 3330 \\
\hline & $H E=0$. & THE 3340 \\
\hline & $C P=0$. & THE 3350 \\
\hline & $S=0$ & THE 3360 \\
\hline & $H B Y T=0$. & THE 3370 \\
\hline & IF (IE) $1050,1160,1050$ & THE 3380 \\
\hline \multirow[t]{3}{*}{1160} & CALL ERROR (229) & THE3390 \\
\hline & $I E=1$ & THE 3400 \\
\hline & GO TO 1050 & THE3410 \\
\hline 1170 & CONTINUE & THE 3420 \\
\hline 1180 & RETURN & THE 3430 \\
\hline \multirow[t]{2}{*}{1190} & CALL ERROR (23) & THE 3440 \\
\hline & GO TO 1180 & THE 3450 \\
\hline \multirow[t]{2}{*}{1200} & CALL ERROR (17) & THE 3460 \\
\hline & GO TO 1180 & THE 3470 \\
\hline \multirow[t]{2}{*}{1210} & CALL ERROR (10) & THE 3480 \\
\hline & GO TO 1180 & THE 3490 \\
\hline \multirow[t]{2}{*}{1220} & CALL ERROR (20) & THE 3500 \\
\hline & GO TO 1180 & THE 3510 \\
\hline \multirow[t]{2}{*}{1230} & CALL ERROR (11) & THE 3520 \\
\hline & GO TO 1180 & THE3530 \\
\hline 1240 & CALL ERROR (9) & THE 3540 \\
\hline
\end{tabular}


1270 CALL ERROR (224)

THE 3600

GO TO 1180

THE3610

END

THE3620

SUBROUTINE TPCTPT $(V, T)$

TPC 10

C VERSION 5.00 TPCTPT

TPC 20

IF (V.LE.0.) GO TO 30

TPC 30

IF (V-AINT (V)) $30,10,30$

TPC 40

10 IF (V.GT.4.) GO TO 20

TPC 50

$T=3.6948 * \operatorname{AINT}(1 . / \mathrm{V})-1.6561 * \operatorname{AINT}(2 . / \mathrm{V})+.406 * \mathrm{AINT}(3 . / \mathrm{V})+2.7764 *$ AINT (TPC 60 14. (V) RETURN

TPC 70

TPC 80

$20 \quad T=1.959964+2.3722712 / \mathrm{V}+2.8224986 / \mathrm{V} * 2+2.5558497 / \mathrm{V*} * 3+1.5895341 / \mathrm{V} *$ TPC 90 $14+.73289821 / \mathrm{V} * 5$

C 25 FORMAT (IX/10X, * INFORMATIVE DIAGNOSTIC * V IMPROPER,) TPC 110

C 30 WRITE (IPRINT, 25)

30 RETURN

TPC 120

TPC 130

END

TPC 140 
COMMON /BLOCKD/ IARGS (100), KIND(100), ARGTAB (100), NRMAX, NROW, NCOL, NTRA 170

IARGS, VWXYZ ( 8), NERROR

DIMENSION ARGS $(100)$

TRA 180

EQUIVALENCE (ARGS(1), RC (12501))

COMMON /BLOCKE/ NAME (4),L1,L2, ISRFLG

TRA 190

DIMENSION $X(3000)$

DOUBLE PRECISION $X$, SUM

TRA 200

EQUIVALENCE $(X, A)$

$*$

CHECK TO SEE IF WE HAVE CORRECT NUMBER OF ARGUMENTS

TRA 210

TRA 220

TRA 230

TRA 240

TRA 250

*

IF (NARGS.NE.10) CALL ERROR (10)

TRA 260

TRA 270

TRA 280

TRA 290

CHECK TO SEE IF ALL ARGUMENTS ARE INTEGERS

TRA 300

*

TRA 310

$J=N A R G S$

TRA 320

CALL CKIND (J)

IF (J.NE.0) CALL ERROR (3)

TRA 330

TRA 340

TRA 350

CHECK TO SEE IF DIMENSIONS ARE CORRECT

TRA 360

*

TRA 370

GO TO $(30,40)$, L2

TRA 380

30 IF (IARGS (3).NE.IARGS (4).OR.IARGS (3).NE.IARGS (8)) CALL ERROR (3) GO TO 50

TRA 440

TRA 450

40 IF (IARGS (3).NE.IARGS (4).OR.IARGS (3).NE.IARGS (7)) CALL ERROR (3)

TRA 460

TRA 470

CHECK TO SEE IF DIMENSIONS ARE OUT OF RANGE

TRA 480

COMPUTE ADORESSES

TRA 490

TRA 500

IF (NARGS.EQ.10) G0 TO 60

TRA 510

$\operatorname{IARGS}(12)=\operatorname{IARGS}(L 2+5)$

TRA 520

$\operatorname{IARGS}(11)=\operatorname{IARGS}(L 2+5)$

TRA 530

GO TO 70

$60 \quad \operatorname{IARGS}(12)=\operatorname{IARGS}(L 2+6)$

TRA 540

$\operatorname{IARGS}(11)=\operatorname{IARGS}(L 2+6)$

TRA 550

GO TO 80

$70 \operatorname{IARGS}(10)=\operatorname{IARGS}$ (NARGS)

TRA 560

TRA 570

TRA 580

$\operatorname{IARGS}(9)=\operatorname{IARGS}($ NARGS -1$)$

TRA 590

$\operatorname{IARGS}(8)=\operatorname{IARGS}($ NARGS -2$)$

TRA 600

$\operatorname{IARGS}(7)=\operatorname{IARGS}($ NARGS -3$)$

TRA 610

$\operatorname{IARGS}(6)=\operatorname{IARGS}(5)$

$\operatorname{IARGS}(5)=\operatorname{IARGS}(4)$

$\operatorname{IARGS}(4)=\operatorname{IARGS}(3)$

$80 \quad J=3$

TRA 620

TRA 630

TRA 640

TRA 650

CALL MTXCHK (J)

TRA 660 


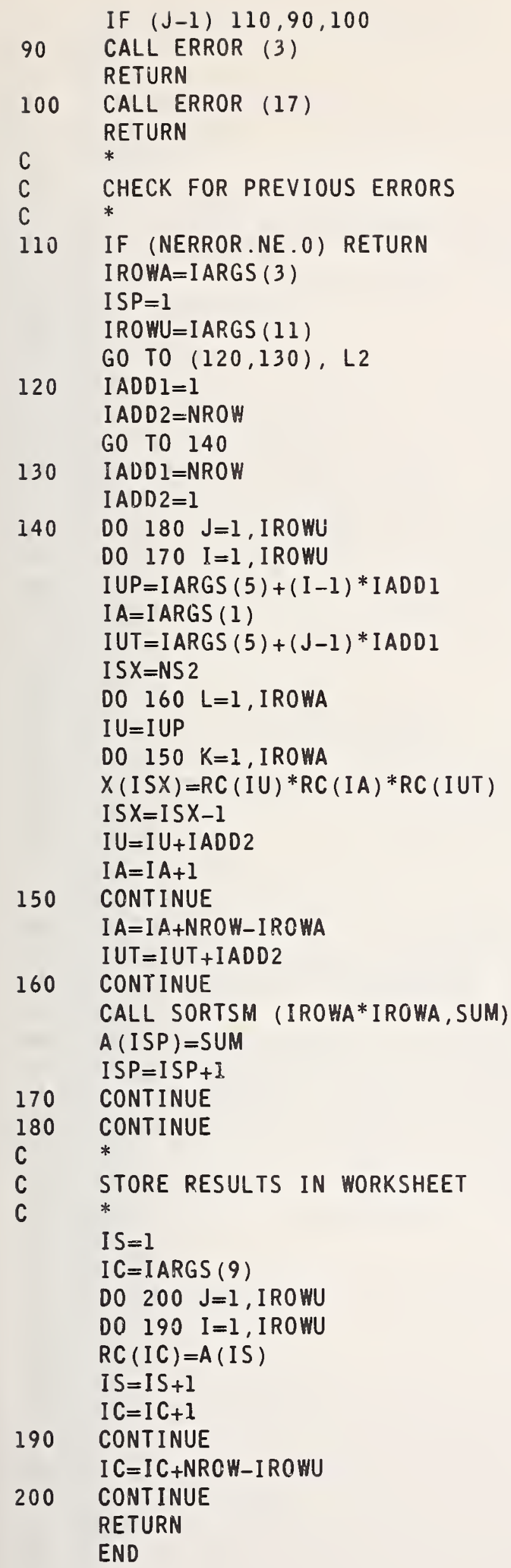

TRA 750

TRA 760

TRA 770

TRA 780

TRA 790

TRA 800

TRA 810

TRA 820

TRA 830

TRA 840

TRA 850

TRA 860

TRA 870

TRA 880

TRA 890

TRA 900

TRA 910

TRA 920

TRA 930

TRA 940

TRA 950

TRA 960

TRA 970

TRA 980

TRA 990

TRAI000

TRA1010

TRA1020

TRA1030

TRA1040

TRA1050

TRA 1060

TRA1070

TRA1080

TRA1090

TRAII00

TRAIIIO

TRAI 120

TRA1130

TRA1140

TRA1150

TRA1160

TRA1170

TRA1 180

TRA1 190

TRA 1200

TRA 1210 
SUBROUTINE THOWAY (LL)

C VERSION 5.0 TWOHAY 5/15/70

TWO 10

COMMON /BLOCRC/ NRC, RC(12600)

TWO 20

THO 30

COMMON /BLOCKD/ IARGS(100), KIND (100), ARGTAB (100), NRMAX, NROW, NCOL, NTWO 40

IARGS, VWXYZ (8), NERROR

TWO 50

DIMENSION ARGS (100)

THO 60

EQUIVALENCE (ARGS(1), RC (12501))

TWO 70

COMMON /HEADER/ NOCARD $(80), \operatorname{ITLE}(60,6)$, LNCNT, IPRINT, NPAGE, IPUNCH TWO 80

COMMON /KFMT/ KFMT (100)

COMMON /SCRAT/ NS,NS2,A(13500)

TWO 90

COMMON /ABCDEF/ L (48)

TWO 100

DIMENSION IIRGS (100)

TWO 110

EQUIVALENCE (KFMT, I IRGS)

TWO 120

TWO 130

DOUBLE PRECISION FDSQRT, DK2, SUM

TWO 140

DIMENSION ASTO(120)

TWO 150

EQUIVALENCE (ND1,KIND(100)), (ND2,KIND(99)), (ND3,KIND(98)), (ND4, TWO 160 IKIND(97)), (ND5,KIND(96)), (ND6,KIND(95)), (ND7,KIND(94)), (ND8, KITWO 170 2ND(93)), (ND9, KIND(92)), (ND10,KIND(91)), (ND11,KIND(90)), (ND12,KTHO 180 3IND(89)), (ND13,KIND(88)), (ND14,KIND(87)), (ND16,KIND(86)), (ND17TWO 190 $4, \operatorname{KIND}(85)),(\operatorname{ND} 18, \mathrm{KIND}(84))$, (ND19,KIND(83)) TWO 200

OMNITAB TWOWAY ANALYSIS OF VARIANCE SUBROUTINE

WRITTEN BY DAVID HOGBEN, SEL, NBS. 4/17/69 VERSION.

TWO 210

TWO 220

THOWAY ANALYSIS FOR R = , $C=$, DATA IN ++ VECTORS START IN ++ (WEIGHTS IN COL ++)

MEASUREMENTS ARE STORED IN THE COLUMN ROW BY ROW

THO 230

TWO 240

THO 250

TWO 260

LAST ARGUMENT USED ONLY IF WEIGHTS ARE UNEQUAL (E.G. IF ZERO WTS

FOR MISSING OBSERVATIONS OR REJECTED OUTLIERS)

COEFFICIENTS ARE STORED IN COL $(X+R+C-1)$

RESIDUALS ARE STORED IN COL $(X+R+C)$

STANDARD DEVIATIONS OF PREDICTED VALUES ARE IN COL $(X+R+C+1)$

SUMS OF SQUARES ARE STORED IN COLUMN $(X+R+C+2)$

TWO 270

$R^{*} C$ MUST = NRMAX WHICH MUST BE LESS THAN OR EQUAL TO NO. OF ROWS

$(X+R+C+2)$ MUST BE LESS THAN OR EQUAL TO THE NUMBER OF COLUMNS

$R+C+6$ MUST BE LESS THAN OR EQUAL TO NCOL

TWO 280

TWO 290

TWO 300

TWO 310

TWO 320

TWO 330

THO 340

TWO 350

TUKEY,S TEST FOR NON-ADDITIVITY IS NOT DONE IF WTS ARE SPECIFIED

TWO 360

IF ZERO WTS ARE USED FOR M.0. THE ESTIMATES GIVEN ARE THE SAME AS THO 380

THOSE OBTAINED FROM DATA AUGMENTED USING THE M.0. FORMULA TWO 390

SIZE OF TABLED CONSTRAINED BY NS AND ORTHO

MID-RANGE SUBTRACTED BEFORE DOING FIT

AUTOMATIC OUTPUT USING READABLE FORMAT

TABLE OF STANDARDIZED RESIDUALS IS GIVEN ON PAGE THO.

FORMAT STATEMENT NUMBERS ARE *

TWO 400

TWO 410

TWO 420

TWO 430

THO 440

THO 450

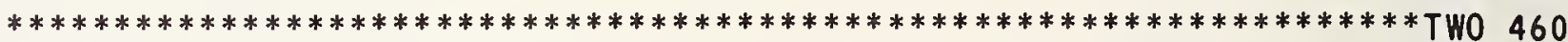

LINES 10 TO 20 GIVE CONSTANTS

THO 470

$\operatorname{IXI}(I, J, I N)=I N+(I *(I-1)) / 2+J$

$M I=\operatorname{IARGS}(1)$

$M 2=\operatorname{IARGS}(2)$

$M 3=I A R G S(3)$

$M 4=M 1 * M 2$

$M 5=M 1+M 2-1$

$M 6=M 1-1$

$M 7=M 1+1$

$M 8=M 2-1$

$M 9=M 6 * M 8$

$M 10=M 4-1$

MI $1=\operatorname{IARGS~(4)~}$

TWO 480

TWO 490

TWO 500

TWO 510

TWO 520

THO 530

TWO 540

TWO 550

TWO 560

TWO 570

THO 580

TWO 590 
IF (NARGS.EQ.5) $M 12=I A R G S$ (5)

TWO 850

TWO 860

TWO 870

TWO 880

THO 890

THO 900

TWO 910

THO 920

TWO 930

THO 940

THO 950

THO 960

THO 970

TWO 980

TWO 990

TWO 1000

TWO1010

TWO 1020

TW01030

TWO1040

$160 \quad \mathrm{RC}(\mathrm{M19})=1.0$

TW01050

DO $180 \quad I=2, M 1$

TW01060

$K 1=I A R G S(I)$

TW01070

DO $170 \mathrm{~J}=1, \mathrm{M}$

TW01080

$K 2=K 1+M 2 *(K-1)+J-1$

TW01090

$\mathrm{RC}(\mathrm{K} 2)=0$.

TW01100

TW01110

TW01120

DO $180 \mathrm{~J}=1, M 2$

TW01130

$K 2=K 1+M 2 *(M 1-1)+J-1$

TW01140

TW01150

DO $200 \quad I=M 7$, MS

TW01160

DO $200 \mathrm{~K}=1$, MI

TW01170

DO $190 \mathrm{~J}=1$, M8

TWO1180 
$R C(K 2)=0.0$

TWO1190

IF (J.EQ.I-MI) $R C(K 2)=1.0$

TWO1200

$K 2=I$ ARGS $(I)+M 2 * K-1$

TW0 1210

$\begin{array}{ll}200 & R C(K 2)=-1.0 \\ C & \text { LINES } 300 \text { T0 } 325 \text { CALL NEW ORTHO }\end{array}$

TW01220

$N A R G S=M 1+M 2+6$

TWO 1230

$\operatorname{IARGS}(1)=M 3$

TW01240

$\operatorname{IARGS}(2)=M 11$

TWO1250

DO $210 \mathrm{I}=4$, NARGS

TWO1260

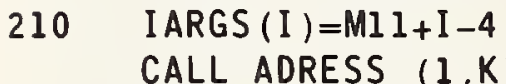

TWO 1270

IF (M13.EQ.4) GO TO 220

TWO 1280

TWO 1290

$\operatorname{IARGS}(2)=M 12$

CALL ADRESS $(2, J)$

TWO 1300

TWO 1310

$\mathrm{J}=\mathrm{J}-\mathrm{I}$

TWO1320

TWO 1330

CONS $1=R C(K)$

CONS2 $=R C(K)$

DO $240 \quad I=1, M 4$

THO 1340

TWO 1350

IF (M13.EQ.4) GO TO 230

THO 1360

$\mathrm{J}=\mathrm{J}+1$

IF (RC(J)) $240,240,230$

TW01370

TW01380

TW01390

IF (RC $(K) . L T . C O N S 1) \quad C O N S 1=R C(K)$

TW01400

IF $(R C(K) . G T . C O N S 2) \quad C O N S 2=R C(K)$

TW01410

$K=K+1$

$K=K-M 4$

$B=($ CONS $1+\operatorname{CONS} 2) / 2.0$

DO $250 \mathrm{I}=1, \mathrm{M} 4$

$M 19=K-1+I$

$250 \quad \mathrm{RC}(\mathrm{M} 19)=\mathrm{RC}(\mathrm{M} 19)-\mathrm{B}$

DO $260 \quad I=1, N A R G S$

$260 \quad \operatorname{KIND}(\mathrm{I})=0$

$L 2=4$

$\operatorname{IARGS}(3)=M 1+M 2-1$

GO TO 690

270 IF (NERROR.NE.0) RETURN

C LINES 330 TO 360 COMPUTE COEFFICIENTS AND THEIR STD. DEVIATIONS CALL ADRESS (NARGS $-3, K$ )

$A S T 0(M 1+11)=0$.

D0 $280 \quad I=1, M 6$

$M 19=I+K$

ASTO $(I+11)=R C(M 19)$

ASTO $(M 1+11)=A S T O(M 1+11)-R C(M 19)$

$K 3=12+M 1+M 2+I$

$\mathrm{K} 4=\mathrm{K}+\mathrm{Ml}+\mathrm{M} 8+\mathrm{I}$

$280 \quad$ ASTO $(K 3)=R C(K 4)$

$\mathrm{K} 3=\mathrm{Ml}+\mathrm{Ml}+\mathrm{M} 2+\mathrm{l} 1$

ASTO $(K 3+1)=$ ASTO $(K 3)$

$K 4=K+M 6+M 2$

ASTO $(M 5+13)=R C(K 4)$

ASTO $(M 5+12)=0$.

DO $2 \dot{90} \quad I=1, M 8$

$K 4=I 1+M 1+I$

$K 5=K+M 6+I$

ASTO $(K 4)=R C(K 5)$

$K 4=K 5+M 8+M 1$

$K 6=K 3+1+1$

ASTO $(K 6)=R C(K 4)$

$290 \quad$ ASTO $(M 5+12)=A S T 0(M 5+12)-R C(K 5)$

ASTO $(M 21+11)=A S T 0(M 21+10)$

TW01420

TW01430

TW01440

TW01450

TW01460

TW01470

TWO1480

TW01490

TWO 1500

THO1510

TWO 1520

TW01530

TWO1540

TWO1550

TW01560

TW01570

TWO 1580

TWO1590

TW01600

TW01610

TWO 1620

TWO 1630

TW0 1640

THO 1650

TW01660

THO1670

TWO1680

TWO 1690

TW01700

TW0 1710

TW01720

TW01730

TW0 1740

TW01750

TW01760

TW01770 
$A(1)=0$.

TW01850

$A(2)=0$.

TWO1860

DO $310 \quad I=1, M 6$

TWO1870

$M 19=K+I$

TW01880

TW01890

$A(1)=A(1)+R C$ (M19)

TW01900

D0 $320 \quad I=1, M 8$

TW01910

$K 4=M 6+I+K$

TW01920

$A(2)=A(2)+R C(K 4)$

TW01930

$K 4=K+M 5$

TW01940

$A(3)=R C(K 4)$

$A(4)=R C(K 4+1)-R C(K)$

TW01950

$A(5)=A(1) / F L O A T(M 6)$

TW01960

TW01970

$A(6)=A(2) / F L O A T(M 8)$

TW01980

$A(7)=A(3) / F L O A T$ ( M9)

TW01990

$A(8)=A(5) / A(7)$

$A(9)=A(6) / A(7)$

TW02000

CALL PROB (FLOAT (M6), FLOAT (M9), A(8), A(10))

TW02010

TW0 2020

CALL PROB (FLOAT (M8), FLOAT (M9),A(9),A(11))

CALL RFORMT ( $A(1), 4,8, N W 1, N D E C 1,20, A(1), A(1), 0,0)$

TW02030

CALL RFORMT (A(5),3,8,NW2,NDEC2,20,A(1),A(1),0,0)

TW02040

TW02050

CALL PAGE (4)

WRITE (IPRINT, 1680) M1, M2

IF (M13.EQ.5) GO TO 510

CALL RFORMT (A(1), 1,8,NW1,NDEC1,0,A(1),A(101),25-NW1,0)

CALL RFORMT (A (1), 1,8, NW2, NDEC2,0,A (5), A (126), 25-NW2,0)

TW02060

TW0 2070

TW02080

TW02090

TW02100

TW02110

TW02120

WRITE (IPRINT,1690) M6, (A (I), I=101,150),A(8),A(10)

CALL RFORMT ( $A(1), 1,8, N W 1, N D E C 1,0, A(2), A(101), 25-N W 1,0)$

CALL RFORMT ( $A(1), 1,8, N W 2, N D E C 2,0, A(6), A(126), 25-N W 2,0)$

TW02130

TW02140

WRITE (IPRINT,1700) M8, (A (I), I=101,150),A(9), A(11)

CALL RFORMT (A(1), 1,8,NH1, NDEC1,0,A(3),A(201),25-NW1, 0 )

CALL RFORMT (A (1), 1,8,NW2, NDEC2,0,A(7),A(226), 25-NW2,0)

WRITE (IPRINT, 1710) M9, (A(I), I=201,250)

CALL RFORMT ( $A(1), 1,8, N W 1, N D E C 1,0, A(4), A(101), 25-N W 1,0)$

WRITE (IPRINT, 1720) MI0, (A(I), I=101,125)

C LINES 600 TO 645 COMPUTE AND PRINT FOR TUKEY'S TEST

CALL ADRESS $(1, J)$

$A(12)=0$.

DO $330 \quad$ I $1=1, M I$

DO $330 \quad$ I $2=1, M 2$

$\mathrm{J} 1=\mathrm{J}+\mathrm{M} 2 *(\mathrm{I} 1-1)+12-1$

$M 19=M 1+11+I 2$

$330 \quad A(12)=A(12)+A S T O(I 1+11) * A S T O(M 19) * R C(J 1)$

$A(12)=(A(12) * A(12)) /((A(1) /$ FLOAT $(M 1)) *(A(2) /$ FLOAT (M2)) )

$A(13)=A(3)-A(12)$

$A(6)=A(13) / F L O A T(M 9-1)$

$A(16)=A(12) / A(6)$

CALL PROB (1., FLOAT (M9) -1, A $(16), A(17)$ )

$A(5)=A(12)$

$A(14)=A(3)$

CALL RFORMT (A(12),3,8,NW1,NDEC1, 20,A(1),A(1),0,0)

TW0 2150

TW02160

TW02170

TW02180

TW02190

TW02200

TW02210

TW02220

TW02230

TW02240

TW02250

TW02260

TW02270

TW02280

TW02290

TW02300

TW02310

TW02320

TW02330

TW02340

TW02350

TW02360 
CALL RFORMT (A (5),3,8,NW2,NDEC2,20,A(1),A(1),0,0)

CALL RFORMT (A(1),1,8,NW1,NDEC1,0,A(12),A(101),25-NW1,0)

CALL RFORMT ( $A(1), 1,8, N W 2, N D E C 2,0, A(5), A(126), 25-N W 2,0)$

WRITE (IPRINT,1740) MI4, (A(I), I=101,150),A(16),A(17)

CALL RFORMT (A(1), 1,8,NW1,NDEC1, 0,A (13),A(101),25-NW1, 0)

CALL RFORMT ( $A(1), 1,8, N W 2, N D E C 2,0, A(6), A(126), 25-N H 2,0)$

WRITE (IPRINT,1750) MI5, (A(I), I=101,150)

CALL RFORMT (A(1), 1,8,NW1,NDEC1,0,A(14),A(101),25-NW1,0)

CALL RFORMT (A(1),1,8,NW2,NDEC2,0,A(7), A(126),25-NW2,0)

WRITE (IPRINT, 1710) $M 9,(A(I), I=101,150$ )

WRITE (IPRINT, 1760)

DO $340 \quad I=1,4$

$340 \quad$ ASTO (I) $=A(I)$

C LINES 650 TO 690 PRINT COEFFICIENTS AND THEIR STD. DEVIATIONS

350 CALL ADRESS $(1, K)$

DO $360 \quad I=1, M 4$

$\mathrm{K} 5=\mathrm{K}-1+\mathrm{I}$

$360 \quad R C(K 5)=R C(K 5)+B$

$J=K$

CALL ADRESS (NARGS $-3, K$ )

$\mathrm{K} 5=\mathrm{K}+\mathrm{M} 2 \mathrm{I}+7$

DO $370 \quad I=1,6$

$K 5=K 5-1$

$370 \quad \mathrm{RC}(\mathrm{K} 5)=\mathrm{RC}(\mathrm{K} 5-4)$

DO $380 \quad I=1, M 21$

$K 5=K+I$

$380 \quad R C(K 5)=A S T O(I+11)$

$R C(K)=R C(K)+B$

$K 5=K+M 2 l+1$

$A(1000)=R C(K 5)$

$R C(K 5)=R C(K 5+3)$

CALL ADRESS $(2, J 1)$

CALL ADRESS (NARGS, J2)

$A(1002)=0.0$

$S U M=0.000$

DO $390 \quad I=1, M 4$

$A(1002)=A(1002)+R C(J 1)$

SUM $=S U M+R C(J 1) * R C(J) * * 2$

$\mathrm{J}=\mathrm{J}+1$

$390 \quad J 1=J 1+1$

$J 1=J 2+M 1+M 2$

$R C(J 1)=S U M$

$R C(J l+1)=R C(J l+1)+B * F S Q R T(A(1002))$

$R C(J 2)=R C(J 1+1) * * 2$

IF (LL.EQ.6) GO TO 400

$R C(K 5)=A(1000)$

RETURN

$400 \quad K 5=K+M 5+2$

CALL RFORMT ( $R C(K), M 5+2,8, N W 3, N D E C 3,20, A(1), A(1), 0,0)$

CALL RFORMT (RC(K5),M5+3,8,NW4,NDEC4,20,A(1),A(1),0,0)

CALL RFORMT (A (1), 1,8,NW3,NDEC 3,0,RC(K),A(101),25-NW3,0)

CALL RFORMT (A(1),1,8,NW4,NDEC4,0,RC(K5),A(126),25-NW4, 0)

WRITE (IPRINT, 1770) (A(J), J=101,150)

$K 5=K+M 21+1$

$R C(K 5)=A(1000)$

DO $410 \quad I=1, M 1$

$\mathrm{K} 5=\mathrm{K}+\mathrm{I}$

$K 6=K 5+M 5+2$

CALL RFORMT ( $A(1), 1,8, N W 3, N D E C 3,0, R C(K 5), A(101), 25-N W 3,0$ )
TW02370

TWO 2380

TW02390

TW02400

TW02410

TW02420

TW02430

TW02440

TW02450

TW02460

TW02470

TW02480

TWO2490

TW02500

TW02510

TW02520

TW02530

TW02540

TW02550

TW02560

TW02570

TW02580

TW02590

TW02600

TW02610

TW02620

TW02630

TW02640

TW02650

TW02 660

TW02670

TW02 680

TW02690

TW0 2700

TW027 10

TW02720

TW02730

TW0 2740

TW02750

TW02760

TW02770

TW02780

TW02790

TW02800

TW02810

TW02 820

TW02830

TW02840

TW02850

TW02860

TW02870

TW02880

TW02890

TW02900

TW02910

TW02920

TW02930

TW02940

TW02950 
CALL RFORMT (A (1), 1,8,NW4, NDEC4, 0, RC (K6), A (126), 25-NW4, 0)

WRITE (IPRINT, 1780) I, (A (J),J=101,150)

TW02970

WRITE (IPRINT, 1760)

THO2980

DO $420 \quad I=1, M 2$

$\mathrm{K} 5=\mathrm{K}+\mathrm{Ml}+\mathrm{I}$

TW02990

TW03000

$K 6=K 5+M 5+2$

TW03010

CALL RFORMT (A (1), 1,8,NW3,NDEC $3,0, R C(K 5), A(101), 25-N W 3,0$ )

TW03020

CALL RFORMT (A (1),1,8,NW4,NDEC 4,0,RC (K6), A (126), 25-NW4, 0 )

TW03030

TW03040

TW0 3050

$K 6=K+M 2 I+4$

CALL RFORMT (A (1), 1,8,NW4,NDEC4, 0,RC (K6), A (101), 50-NH4, 0)

TW03060

WRITE (IPRINT, 1760)

WRITE (IPRINT, 1800) (A (J), J=101,150)

ASTO $(1)=$ ASTO $(1)+$ ASTO $(2)+$ ASTO $(3)$

TW0 3070

TW03080

TW03090

ASTO (2) =ABS (ASTO (4)-ASTO (1))/ASTO (4)

TW03100

IF (ASTO(2).GT.5.E-7) CALL ERROR (228)

TW03110

GO TO 630

C LINES 700 TO 780 GIVE ANOVA WHEN WEIGHTS ARE SPECIFIED

TW03120

THO3130

TW0 3140

TW03150

TW03160

TW0 3170

TW0 3180

TW0 3190

TW03200

TW03210

TW0 3220

TW0 3230

TW03240

TW03250

TW0 3260

TW03270

TW03280

TW03290

TW03300

TW03310

TW03320

TH03330

TH03340

TW03350

TH03360

TH03370

TW03380

TH03390

TW03400

TH03410

TW03420

TW03430

TW0 3440

TW03450

TW03460

TW03470

TW03480

TW03490

TW03500

TW03510

TW03520

TH03530

TW03540 
CALL ADRESS $(2, K)$

TW03550

DO $550 \quad I=1, M 17$

TW03560

$K 5=J-1+I$

$K 6=J+M 4-I$

$A(20)=R C(K 5)$

$R C(K 5)=R C(K 6)$

$R C(K 6)=A(20)$

$K 5=K-1+I$

$K 6=K+M 4-I$

$A(20)=R C(K 5)$

$R C(K 5)=R C(K 6)$

550

$R C(K 6)=A(20)$

GO TO 690

560

CALL ADRESS $(1, J)$

CALL ADRESS $(2, K)$

CALL ADRESS (NARGS-2,KI)

CALL ADRESS (NARGS-1,K2)

DO $570 \quad \mathrm{I}=1, \mathrm{MI} 7$

$\mathrm{K} 5=\mathrm{J}-1+\mathrm{I}$

$K 6=J+M 4-I$

TW03570

TW03580

THO 3590

TW03600

TW03610

TW03620

TW03630

TW03640

TW03650

TW03660

TW03670

TW03680

TW03690

TW03700

TW03710

TW03720

TW03730

TW0 3740

TW03750

$A(20)=R C(K 5)$

TW03760

$R C(K 5)=R C(K 6)$

TW03770

$R C(K 6)=A(20)$

TW0 3780

$K 5=K 2-1+I$

TW03790

$K 6=K 2+M 4-I$

TW03800

$A(20)=R C(K 5)$

TW03810

$R C(K 5)=R C(K 6)$

TW0 3820

$R C(K 6)=A(20)$

TW03830

$K 5=K 1-1+I$

TW03840

$K 6=K 1+M 4-I$

$A(20)=R C(K 5)$

TW03850

TW03860

TW03870

TW03880

$R C(K 6)=A(20)$

TW03890

$K 5=K-1+I$

TW03900

$K 6=K+M 4-I$

$A(20)=R C(K 5)$

TW03910

$R C(K 5)=R C(K 6)$

TW03920

TW03930

TW03940

$A(18)=0$.

CALL ADRESS (NARGS, K)

TW03950

TW03960

DO $580 \quad \mathrm{I}=1$, M8

TW03970

$K 5=I+K$

$580 \quad A(18)=A(18)+R C(K 5)$

TW03980

$A(17)=0$.

DO $590 \quad I=1, M 6$

$K 5=M 2+I+K-1$

$590 \quad A(17)=A(17)+R C(K 5)$

$J l=M 21-2$

$K 5=201$

$K 6=K+1$

D0 $600 \quad I=1, J I$

$A(K 5)=R C(K 6)$

$K 5=K 5+1$

$600 \quad K 6=K 6+1$

$K 5=K+1$

$K 6=201+M 8$

$\mathrm{J} I=K+M 16+4$

$\mathrm{J} 2=204+M 16+M 8$

DO $610 \quad I=1, M 6$

TW03990

TW04000

TW04010

TW04020

TW04030

TW04040

TW04050

TW04060

TW0 4070

TW0 4080

TW0 4090

TW04100

TWO4110

TWO4120

TW04130 
CALL RFORMT (A(1), 1,8,NW1,NDEC1,0,A(17),A(101),25-NW1,0)

TW04280

TW04290

TW04300

TW04310

CALL RFORMT (A (1), 1,8,NW2, NDEC2,0,A(19), A (126), 25-NW2,0)

TW04320

WRITE (IPRINT, 1690) M6, (A(I), I=101,150),A(21),A(22)

TW04330

CALL RFORMT (A (1), 1,8,NW1,NDEC1,0,A(18),A(101),25-NW1,0)

TW04340

CALL RFORMT ( $A(1), 1,8, N W 2, N D E C 2,0, A(20), A(126), 25-N W 2,0)$

TW04350

WRITE (IPRINT, 1700) M8,(A(I), I=101,150)

CALL RFORMT (A (1), 1,8,NW1,NDEC1,0,ASTO (3),A(101),25-NW1, 0)

TW04360

TW0 4370

CALL RFORMT (A (1), 1,8,NW2,NDEC2,0,ASTO (7), A (126), 25-NW2, 0 )

TW04380

WRITE (IPRINT,1710) M9,(A(I), I=101,150)

CALL RFORMT (A(1), 1,8,NW1,NDEC1,0,ASTO(4),A(101),25-NW1,0) WRITE (IPRINT, 1720) $M 10,(A(I), I=101,125$ )

CALL RFORMT (A (1), 1,8,NW1,NDEC1,0,ASTO(1),A(101),25-NW1,0)

CALL RFORMT (A (1), 1,8,NW2,NDEC2,0,ASTO (5), A (126), 25-NW2, 0 )

WRITE (IPRINT, 1690) M6, (A(I), I=101,150)

CALL RFORMT (A (1), 1,8,NW1,NDEC1,0,ASTO (2),A(101),25-NW1,0)

CALL RFORMT (A (1), 1,8,NW2, NDEC2, 0, ASTO (6), A (126), 25-NW2, 0 )

WRITE (IPRINT, 1700) M8, (A (I), I=101,150),ASTO (9),ASTO(11)

CALL RFORMT (A(1), 1,8,NW1,NDEC1, 0,ASTO (3),A(101),25-NW1, 0)

CALL RFORMT (A(1), 1,8,NW2,NDEC2, 0,ASTO (7), A (126), 25-NW2, 0 )

WRITE (IPRINT, 1710) M9, (A(I), I=101,150)

CALL RFORMT (A (1), 1,8,NW1, NDEC1, 0,ASTO(4), A(101),25-NW1,0)

WRITE (IPRINT, 1720) MI0,(A(I), I=101,125)

$M 31=M 5+M 9$

$M 32=M 4-M 31$

WRITE (IPRINT, 1730) M3 1, M32, M12

CALL ADRESS (NARGS-3,K)

$K 5=K+M 6+M 2+M 2$

$K 6=K+M 1+M 2$

$K 3=M 1+M 1+M 2+12$

$\mathrm{ASTO}\left(\mathrm{K}_{3}\right)=\mathrm{RC}(\mathrm{K} 5)$

ASTO $(M 21+11)=R C(K 6)$

GO TO 350

630 CALL PAGE (4)

WRITE (IPRINT, 1810) M1, M2

TW04390

TW04400

TW04410

TW04420

TW04430

TW04440

TW04450

TW04460

TW04470

TW04480

TW04490

TW04500

TW04510

TW04520

TW04530

TW04540

TW04550

TW04560

TW04570

TW04580

TW04590

TW04600

TW04610

TW04620

TW0 4630

TW04640

TW04650

$M 31=M I N O(15, M 2)$

TW04660

D0 $640 \quad I=1, M 31$

TW04670

TW04680

TW04690

WRITE (IPRINT, 1820) (KIND (I) I I=1,M3 I)

TW04700

WRITE (IPRINT, 1830)

TW04710

CALL ADRESS (NARGS-2,J)

TW04720 
IF (M13.EQ.5) KIND(2)=0

IF (M13.EQ.5) CALL ADRESS (2,M32)

TW0 4730

DO $670 \quad I=1, M 4$

IF (M13.EQ.5) GO T0 650

TH0 4740

$A(I)=R C(J) / F S Q R T(R C(K 6) * * 2-R C(K 3) * * 2)$

TW04750

GO TO 660

TW04760

TW04770

TW0 4780

650 IF (RC(M32).GT.0.0) A (I) =RC (J)/FSQRT((RC (K6)**2)/RC (M32)-RC (K3)**2TW04790 1)

IF (RC (M32).LE.0.0) $A(I)=0.0$

TW04800

$M 32=M 32+1$

$660 \mathrm{~J}=\mathrm{J}+1$

$670 \quad K 3=K 3+1$

DO $680 \quad I=1, M I$

WRITE (IPRINT, 1760)

TW04810

TW04820

TW04830

THO 4840

TW04850

$M 31=M 2 *(I-1)$

$K A=M 31+M 2$

TW04860

$K B=M 31+1$

TW04870

TW04880

TW04890

680 WRITE (IPRINT, 1840) I, ( $(K), K=K B, K A)$

TW04900

RETURN

C THIS IS NEW ORTHO - SFIT PORTION

TW04910

TW04920

$690 \quad$ NMUI $=1$

CALL ADRESS (1,IIRGS(1))

TW04930

CALL ADRESS $(2, I I R G S(2))$

TW04940

NST $=1$

NEND=NARGS

DO $700 \quad I=4$, NENO

700 CONTINUE

$M=I$ ARGS (3)

$\mathrm{N}=\mathrm{NRMAX}$

$\mathrm{FN}=\mathrm{N}$

$\mathrm{SU}=0.0$

L22=IIRGS (2)

$\mathrm{L} 22 \mathrm{~A}=\mathrm{L} 22$

DO $730 \quad I=1, N$

IF (RC(L22A)) $710,730,720$

710 CALL ERROR (25)

RETURN

TW04950

TW04960

TW04970

TW04980

TW04990

TW05000

TW0 5010

TW05020

TW05030

TW0 5040

TW05050

TW05060

TW05070

TW05080

TW05090

TW0 5100

TW05 110

$720 \quad S U=S U+1.0$

$730 \quad \mathrm{~L} 22 \mathrm{~A}=\mathrm{L} 22 \mathrm{~A}+1$

$F M=M$

TW05 120

TW05130

IF (SU-FM) $740,750,760$

740 CALL ERROR (24)

RETURN

750 DENOM $=1.0$

GO TO 770

760 DENOM=FSQRT (SU-FM)

$770 \quad N P M=N+M$

$M 31=M-1$

$M 32=M+1$

TW05140

TW05150

TW05160

TW0 5170

TW0 5180

TW05190

TW05200

TW05210

TW05220

$\mathrm{N} 1=\mathrm{N}-\mathrm{l}$

THO5230

$\mathrm{N} 2=\mathrm{N}+1$

TW05240

$M D 1=\left(M^{*}(M 32)\right) / 2$

TW05250

ND $1=M 32 * N P M$

TH05260

ND2 $=M^{*} N P M$

TW05270

$\mathrm{MD} 3=\mathrm{ND} 2+\mathrm{N}$

TW05280

$N D 3=N D 1$

TW05290

$N 04=N D 3+N P M$

TW05300

ND5 $=$ ND $4+N P M$

TW05310 
$N D 6=N D 5+M 32$

$N D 66=M D 1+M$

$N D 7=N D 6+N D 66$

ND $8=N D 7+N D 66$

$N D 9=N D 8+M 32$

$N D 10=N D 9+M$

$N D 11=N D 10+M$

$N D 12=N D 11+M$

$N D 13=N D 12+M D 1$

$N D 14=N D 13+M 32$

$N D 16=N D 14+M$

$N D 17=N D 16+M$

$N D 18=N D 17+M$

$N D 19=N D 18+N P M$

$N D 20=N D 19+N$

IF (ND20.GT.NS) CALL ERROR (23)

IF (NERROR.NE.O) RETURN

$N R B A R=1$

$I=4$

$M X A R G S=M+4$

L44=MXARGS -1

$J=1$

DO $790 \quad I I=I, L 44$

$\mathrm{KI}=\mathrm{J}$

L33=I IRGS (II)

$\mathrm{K} 2=\mathrm{K} 1$

DO $780 \quad$ I $2=1, N$

$A(K 2)=R C(L 33)$

$\mathrm{K} 2=\mathrm{K} 2+1$

$780 \quad \mathrm{~L} 33=\mathrm{L} 33+1$

$790 \quad \mathrm{~J}=\mathrm{J}+\mathrm{NPM}$

$\mathrm{K} l=\mathrm{N} 2$

DO $810 K=1, M$

$\mathrm{K} 2=\mathrm{K} 1$

DO $800 \quad I=1, M$

$A(K 2)=0$.

$800 \quad K 2=K 2+1$

$K 2=K 1+K-1$

$K l=K 1+N P M$

$810 \quad A(K 2)=1.0$

NBE $I=1$

$N R H I=1$

I $18=1+N D 13$

$N G A I=2$

NSI I $=2$

NDE $I=1$

NNUI $=1$

$L Z 1=1$

$L Z 2=1$

$K=1$

820 NTHI $=1$

830 NALI $=1$

NOMI $=1$

$\mathrm{NJ}=\mathrm{ND} 3+\mathrm{N}+1$

DO $840 \mathrm{~J}=1, \mathrm{M}$

$A(N J)=0$.

$840 \quad \mathrm{NJ}=\mathrm{NJ}+1$

$850 \quad K D I=(K-1) * N P M$

$\mathrm{I} I=\mathrm{ND} 3+1$
TW05320

TW0 5330

TW0 5340

TW0 5350

TW05360

TW05370

TH05380

TW05390

TW05400

TW05410

TW05420

TW05430

TW05440

TW05450

TW05460

TW05470

TW05480

TW0 5490

TW05500

TW05510

TW05520

TW05530

TW05540

TW05550

TW05560

TW05570

TW0 5580

TH0 5590

TW0 5600

TW05610

TW05620

TW05630

TW05640

TW05650

TH05660

TW05670

TW05680

TW05690

TW05700

TW057 10

TW05720

TW05730

TW05740

TW05750

TW05760

THO 05770

TW05780

TW05790

TW05800

TW05810

TW05820

TW0 5830

TW05840

TW05850

TW05860

TW0 5870

TW0 5880

TW05890

TW05900 
$870 \quad I A I=1$

TW05990

$\mathrm{IA} 2=\mathrm{ND} 5+1$

D0 $890 \quad I=1, K$

TW06000

I $2=I A I$

TW06010

$\mathrm{SUM}=0.0$

TW06020

$\mathrm{J} 2=\mathrm{ND} 3+1$

TW06030

DO $880 \mathrm{~J}=1$, NPM

TW06040

$S U M=S U M+A(J 2) * A(I 2)$

TW06050

$\mathrm{I} 2=\mathrm{I} 2+1$

TW06060

TW06070

$\mathrm{J} 2=\mathrm{J} 2+1$

$A(I A 2)=S U M$

TW06080

$I A I=I A I+N P M$

TW06090

TW06 100

$I A 2=I A 2+1$

TW06110

GO TO 930

TW06 120

$900 \quad$ DK2 $=0$.

$I I=(K-1) * N P M+1$

TW06130

IND $3=N D 3+1$

DO $910 \quad I=1, N P M$

TW06140

TW06150

$D K 2=D K 2+A($ IND 3$) * A(I 1)$

TH06160

$I I=I I+1$

910 IND3 $=$ IND3 +1

$D K=F D S Q R T(D K 2)$

$A$ ( I 18$)=D K$

$118=\llbracket 18+1$

$\mathrm{K} I=(\mathrm{K}-1) * \mathrm{NPM}+1$

DO $920 I=1, N P M$

$A(K 1)=A(K 1) / D K$

$920 \quad K l=K l+1$

NOMI $=1$

GO TO 850

930 GO TO $(940,1000)$, NDE I

TW06170

TW06180

TW06190

TW06200

TH06210

TW06220

TW06230

TW06240

TH06250

TW06260

TW06270

TW06280

TW06290

TW06300

$L Z 1=-L Z 1$

IF (LZ1) $990,950,950$

TW06310

$\mathrm{Kl}=\mathrm{K}-\mathrm{l}$

TW06320

I RUTH $=$ ND $5+1$

TW06330

DO $960 \quad I=1, K 1$

TW06340

$A($ IRUTH $)=-A($ I RUTH $)$

960 IRUTH $=$ IRUTH +1

TW06350

TW06360

I RUTH $=K+N D 5$

TW06370

A ( I RUTH $)=1.0$

TW06380

$\mathrm{J} 2=\mathrm{ND} 4+1$

TW06390

DO $980 \quad I=1, N P M$

TW06400

SUM $=0.0$

TW06410

$\mathrm{J} I=\mathrm{I}$

$\mathrm{J} 3=\mathrm{ND} 5+1$

TW06420

TW06430

DO $970 \mathrm{~J}=1, \mathrm{~K}$

TW06440

$S U M=S U M+A(J I) * A(J 3)$

TW06450

$J l=J l+N P M$

TW06460

$\mathrm{J} 3=\mathrm{J} 3+1$

TW06470

$A(J 2)=S U M$

TW06480

$\mathrm{J} 2=\mathrm{J} 2+1$

TW06490 


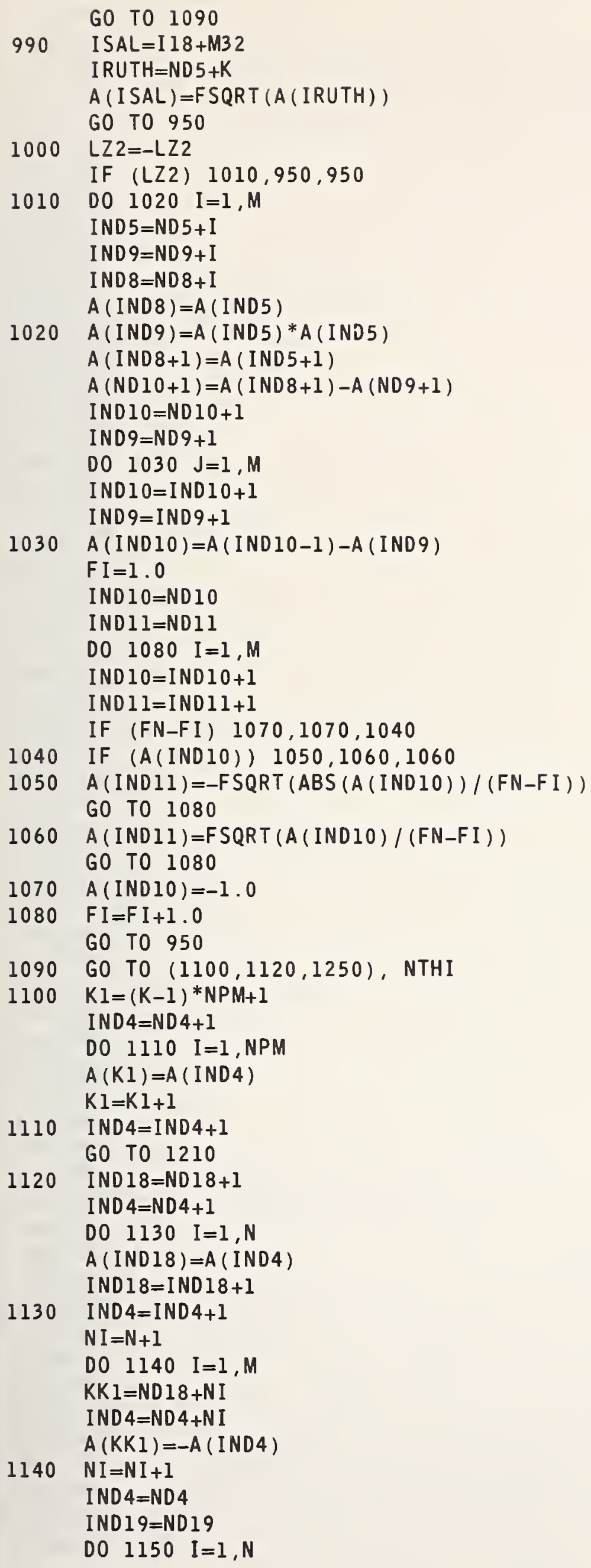

TW06750

TW06760

TW06770

TW06780

TW06790

TW06800

TW06810

TW06820

TW06830

TW06840

TW06850

TH06860

TW06870

TW06880

TW06890

TW06900

TH06910

TW06920

TW06930

TW06940

TW06950

TW06960

TW06970

TW06980

TW06990

TW07 000

TW07010

TW07020

TW07030

TW07040

TW07050

TW07060

TW07070

TW07080 


\begin{tabular}{|c|c|c|}
\hline & IND4 $=$ IND $4+1$ & TW07090 \\
\hline & IND19=IND19+1 & TW07100 \\
\hline 1150 & $A($ IND19) $=A($ IND 4$)$ & TW07110 \\
\hline & IF (NARGS-MXARGS) $1200,1180,1160$ & TW07120 \\
\hline 1160 & L66 $=$ I IRGS $($ MXARGS +1$)-1$ & TW07130 \\
\hline & $L 66 A=L 66$ & TW07140 \\
\hline & IND $4=$ ND 4 & TW07150 \\
\hline & DO $1170 \quad I=1, N$ & TW07160 \\
\hline & $L 66 A=L 66 A+1$ & TW07170 \\
\hline & IN D $4=$ I ND4 +1 & TW07180 \\
\hline 1170 & $R C(L 66 A)=A(I N D 4)$ & TW07190 \\
\hline 1180 & L55=I IRGS (MXARGS) & TW07200 \\
\hline & $L 55 A=L 55-1$ & TW07210 \\
\hline & $N I=N+N D 4$ & TW07220 \\
\hline & DO $1190 I=1, M$ & TW07230 \\
\hline & $N I=N I+1$ & TW07240 \\
\hline & $L 55 A=L 55 A+1$ & TW07250 \\
\hline 1190 & $R C(L 55 A)=-A(N I)$ & TH07260 \\
\hline 1200 & NTHI $=3$ & TW07270 \\
\hline & GO TO 1100 & TW07280 \\
\hline 1210 & GO TO $(1220,1230)$, NALI & TW07290 \\
\hline 1220 & NOMI $=2$ & TW07300 \\
\hline & NAL I $=2$ & TW07310 \\
\hline & GO TO 850 & TW07320 \\
\hline 1230 & IF (K-M) $1240,1280,1280$ & TW07330 \\
\hline 1240 & $K=K+1$ & TW07340 \\
\hline & GO TO 820 & TW07350 \\
\hline 1250 & GO TO $(1260,1270)$, NNUI & TW07360 \\
\hline 1260 & NNU $I=2$ & TH07370 \\
\hline & GO TO 1370 & TW07380 \\
\hline 1270 & $S S=D K / D E N O M$ & TW07390 \\
\hline & $S S Q=S S * S S$ & TW07400 \\
\hline & GO TO 1370 & TW07410 \\
\hline 1280 & GO TO $(1290,1250)$, NBE I & TW07420 \\
\hline 1290 & $\mathrm{~K} \mathrm{l}=1$ & TW07430 \\
\hline & DO $1310 \quad I=1, M$ & TW07440 \\
\hline & $I I=I * N+(I-1) * M$ & TW07450 \\
\hline & DO $1300 \mathrm{~J}=1, \mathrm{I}$ & TW07460 \\
\hline & $I 2=\mathrm{J}+I 1$ & TW07470 \\
\hline & $K 2=K 1+N D 12$ & TW07480 \\
\hline & $A(K 2)=A(I 2)$ & TW07490 \\
\hline 1300 & $K 1=K 1+1$ & TW07500 \\
\hline 1310 & CONTINUE & TW07510 \\
\hline & NDE $I=2$ & TW07520 \\
\hline & NBE $I=2$ & TW07530 \\
\hline & NTHI $=2$ & TW07540 \\
\hline & $K=K+1$ & TW07550 \\
\hline & GO TO $(1250,1320)$, NGAI & TW07560 \\
\hline 1320 & CONTINUE & TW07570 \\
\hline & DO $1340 \quad \mathrm{IL}=1, \mathrm{M}$ & TW07580 \\
\hline & $L O C=I X I(I L, 0, N D 6)+1$ & TW07590 \\
\hline & DO $1340 \mathrm{~J}=1, \mathrm{IL}$ & TW07600 \\
\hline & SUM $=0$. & TW07610 \\
\hline & DO $1330 \mathrm{KK}=\mathrm{IL}, \mathrm{M}$ & TW07620 \\
\hline & $L O C I=I X I(K K, I L, N D 12)$ & TW07630 \\
\hline & $L O C 2=I \times I(K K, J, N D 12)$ & TW07640 \\
\hline 1330 & $S U M=S U M+A(L O C 1) * A(L O C 2)$ & TW07650 \\
\hline & $A(L O C)=S U M$ & TH07660 \\
\hline 1340 & $L O C=L O C+1$ & TW07670 \\
\hline
\end{tabular}




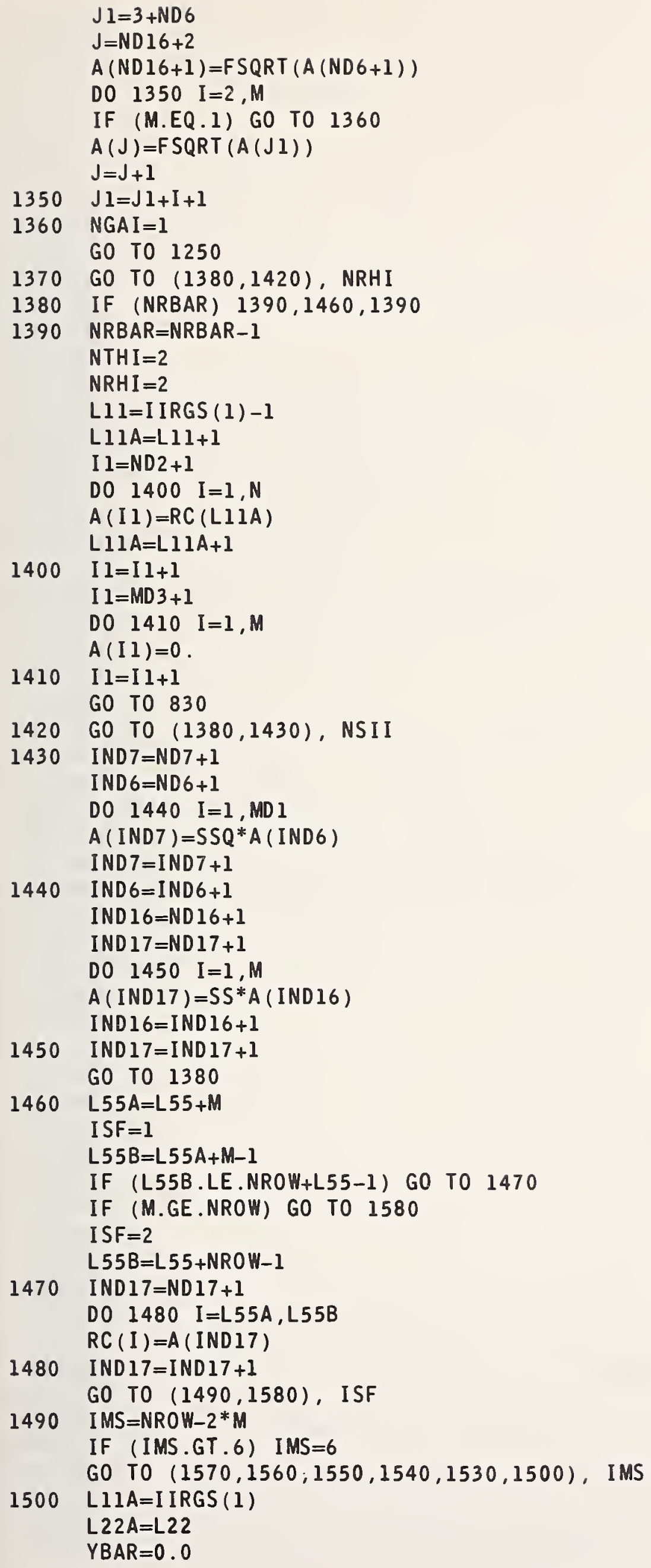

TW07720

TW07730

TW07740

TW07750

TW07760

TW07770

TW07780

TW07790

TW07800

TW07810

TW07820

TW07830

TW07840

TW07850

TW07860

TW07870

TW07880

TW07890

TW07900

TW07910

TW07920

TW07930

TW07940

TW07950

TH07 960

TW07970

TW07980

TW07990

TW0 8000

TW08010

TW0 8020

TW08030

TW0 8040

TH08050

TW08060

TW08070

TH08080

TW08090

TW08100

TH0 8110

TW08 120

THO 8130

TH0 8140

TH08150

THO 8160

TH08170

THO 8180

TW08190

TW0 8200

TW08210

TW08220

TW08230

TW08240

TW08250

TW08260 
$W S U M=0.0$

TW08270

DO $1510 \quad I=1, N$

TW08280

$W S U M=W S U M+R C(L 22 A)$

$Y B A R=Y B A R+R C(L 22 A) * R C(L 11 A)$

TW0 8290

$L 22 A=L 22 A+1$

$1510 \quad$ LIIA $=\mathrm{L} 11 \mathrm{~A}+1$

YBAR=YBAR / WSUM

TW08300

TW08310

TW08320

LIIA=I IRGS (1)

TW08330

$\mathrm{L} 22 \mathrm{~A}=\mathrm{L} 22$

TW0 8340

$Y Y B A R=0$.

TW0 8350

DO $1520 \quad I=1, N$

TW0 8360

YYBAR $=R C(L 22 A) *(R C(L 11 A)-Y B A R) * * 2+Y Y B A R$

TW0 8370

$\mathrm{L} 22 \mathrm{~A}=\mathrm{L} 22 \mathrm{~A}+1$

1520 LIIA $=L 11 A+1$

$R 2=1 .-S S Q^{*}(S U-F M) / Y Y B A R$

IF (R2.LT.0.) $R 2=0.0$

IF (R2.GT.1.0) $R 2=1.0$

$R C(L 55 B+6)=R 2$

$1530 R C(L 55 B+5)=S S Q$

$1540 R C(L 55 B+4)=S S$

$1550 R C(L 55 B+3)=S U-F M$

$1560 R C(L 55 B+2)=F M$

$1570 \quad \mathrm{RC}(\mathrm{L} 55 \mathrm{~B}+1)=\mathrm{SU}$

1580 IND2 $=$ ND $2+1$

IND $3=N D 3+1$

IND $4=N D 19+1$

IP I $C=1$

I $Y=I$ I RGS ( 1 )

YSUM $=0.0$

$\mathrm{L} 22 \mathrm{~A}=\mathrm{L} 22$

DO $1600 \quad I=1, N$

IP $=$ IP IC

$S P=0.0$

DO 1590 I I $=1, M$

$S P=S P+A(I P) * * 2$

$1590 \quad I P=I P+N P M$

$A($ IND2 $)=F S Q R T(S P) * S S$

IP IC $=$ IP IC +1

IND $2=$ IND $2+1$

TW08380

TW08390

TW0 8400

TW08410

TW08420

TW08430

TW08440

TW08450

TW08460

TW08470

TW08480

TW08490

TW08500

TW085 10

TW08520

TW08530

TW08540

TW08550

TW08560

TW08570

TW08580

TW08590

TW08600

TW08610

TW08620

TW08630

TW08640

TW08650

$A($ IND3) $=R C$ ( IY ) $-A$ (IND 4 )

TW08660

IND $3=$ IND $3+1$

TW08670

I ND $4=$ IND $4+1$

TW08680

YSUM $=Y S U M+R C$ (IY) **2*RC (L22A)

TW08690

$L 22 A=L 22 A+1$

$1600 \quad I Y=I Y+1$

$L S T=I$ I RGS $(M X A R G S+3)$

IND9 $=$ ND 9

DO $1610 \quad I=1, M$

IND9 $=$ IND $9+1$

$R C(L S T)=A$ (IND9)

$1610 \quad \mathrm{LST}=\mathrm{LST}+1$

IF (NROW- $(M+1)) \quad 1650,1630,1620$

$1620 R C(L S T+1)=A$ (ND9)

$1630 R C(L S T)=(S U-F M) * S S Q$

TW08700

TW08710

TW08720

TW08730

TW08740

TH08750

TW08760

TW08770

TW08780

TW08790

TW08800

TW08810

$\mathrm{LST}=\mathrm{LST}+2$

IF (M+2.GE.NROW) GO TO 1650

TW08820

LSTA $=L S T+M-1$

IF $(2 * M+2 . G T$. NROW $) \quad L S T A=I I R G S(M X A R G S+3)+N R O W-1$

TW08830

TW08840

IND $8=$ ND 8

TW08850 
D0 $1640 \quad I=L S T, L S T A$

TW08860

IND $8=$ IND $8+1$

TW08870

$1640 \mathrm{RC}$ (I) $=A$ (IND 8)

TW08880

1650 LSTOR=IIRGS (MXARGS +2$)$

TW08890

IP I $C=1$

TW08900

I ND $2=$ ND $2+1$

TW08910

DO $1660 \quad I=1, N$

TW08920

$\mathrm{RC}(\mathrm{LSTOR})=\mathrm{A}$ ( IND2)

TW08930

IND $2=$ IND $2+1$

TW08940

$L S T O R=L S T O R+1$

TW08950

IF (IARGS (5)-IARGS (4)-1) $1670,270,560$

TW08960

RETURN

TW08970

TW08980

TW08990

C

1680 FORMAT (//,31X,34H ANALYSIS OF VARIANCE FOR TWO-WAY, I2,3H X , I2,6TW09000 IH TABLE, //,4X,7H SOURCE, 13X,5H D.F.,10X,14HSUM OF SQUARES, 13X, 12HMTW09010 2EAN SQUARES, 10X,17HF RATIO F PROB. (1)

1690 FORMAT $(4 X, 20 \mathrm{H}$ BETWEEN ROWS

1700 FORMAT $(4 X, 20 H$ BETWEEN COLS

$, 14,50 A 1,6 X$, OPF $11.3, F 9.3)$
$, 14,50 A 1,6 X$, OPF $11.3, F 9.3)$

TW09020

TW09030

1710

FORMAT $(4 X, 20 H$ RESIDUALS

$, 14,50 \mathrm{Al})$

TW09040

1720 FORMAT (4X,2OH TOTAL , I4, 25Al//)

TW09050

TW09060

1730 FORMAT (9X,40HA WEIGHTED LEAST SQUARES ANALYSIS USING , I4,22H NON-TWO9070 IZERO WEIGHTS AND, I4,24H ZERO WEIGHTS IN COLUMN , I4/)

TW09080

1740 FORMAT (39X, 3IHTUKEY'S TEST FOR NON-ADDITIVITY//,4X,20H NON-ADDITITHO9090 IVITY I I , 50A1,6X, OPFII.3,F9.3)

1750 FORMAT (4X,20H BALANCE ,I4,50Al)

TW09100

1760 FORMAT $(1 X)$

TW09110

TW09 120

1770 FORMAT (//5X, 1 IHCOEFFICIENT, 14X, 8HESTIMATE, 17X, 9HSTD. DEV . / $5 X, 10 H T H 09130$ IGRAND MEAN, $50 \mathrm{Al} / 1$

1780 FORMAT $(5 X, 7$ HROW $, 13,50 A 1)$

TW09 140

TW09 150

1790 FORMAT (5X,7HCOLUMN, I3,50A1)

TW09 160

FORMAT (5X, 1OHRESIDUAL, $50 \mathrm{Al})$

TW09170

1810 FORMAT (//10X, I2,3H X, I2,86H TABLE OF RESIDUALS, STANDARDIZED BY TWO 9180 IDIVIDING EACH RESIDUAL BY ITS STANDARD DEVIATION.)

1820 FORMAT $(/, 8 \mathrm{H}$ COLUMN, $15(3 \mathrm{X}, 14,1 \mathrm{X}))$

1830 FORMAT (8H ROW)

1840 FORMAT $(2 X, I 4,2 X, 15(2 X, F 6.2))$

TW09190

TW09200

TW09210

TW09220

END

TW09230 
DATA $N(1), N(2), N(3), N(4), N(5), N(6), N(7), N(8), N(9), N(10), N(11), N(12$ VAR 50

$1), N(13), N(14) / 10705,2604,16038,16767,17496,18225,18954,1377,15001$, VAR 60

C $25 * 01$

\section{LOOKUP NAME IN VARIABLE-NAME TABLE}

\section{NAMES IN TABLE}

NRMAX, COLTOP, $V, W, X, Y, Z$

DO $10 \quad \mathrm{I}=1,7$

IF (NAME (1).EQ.N(I).AND.NAME (2).EQ.N(I+7)) GO TO 20

\section{CONTINUE}

$$
I=0
$$

$20 \quad A R G=I$

RETURN

END
VAR 70

VAR 80

VAR 90

VAR 100

VAR 110

VAR 120

VAR 130

VAR 140

VAR 150

VAR 160

VAR 170

VAR 180

VAR 190

VAR 200

VAR 210
C

SUBROUTINE VECTOR (A,J)

C VERSION 5.00 VECTOR

COMMON /BLOCRC/NRC,RC(12600)

COMMON /BLOCKD/ IARGS(100), KIND(100), ARGTAB (100), NRMAX, NROW, NCOL, NVEC 40 IARGS, VWXYZ (8), NERROR

DIMENSION ARGS $(100)$

EQUIVALENCE (ARGS (1), RC(12501))

VECTORIZE A IN TO COLUMN STARTING AT J

IF (NRMAX.EQ.0) GO TO 20

$K=J+N R M A X-1$

DO $10 \quad I=J, K$

$10 \quad R C(I)=A$

20 RETURN

END
VEC 10

VEC 20

VEC 30

VEC 50

VEC 60

VEC 70

VEC 80

VEC 90

VEC 100

VEC 110

VEC 120

VEC 130

VEC 140

VEC 150

VEC 160 
SUBROUTINE XECUTE

C

C

C

C

C

C

$$
\text { C }
$$

100 CALL RESET

GO TO 9999

C PRINT PRINT A-F

200 CALL PRINTX

GO TO 9999

C PUNCH

300 CALL PUNCH

GO TO 9999

C APRINT APRINT A-F

400 CALL APRINT

GO TO 9999

C READ READ A-F

500 CALL READX

GO TO 9999

C ABRIDGE

600 CALL ABRIDG

GO TO 9999

C MPRINT MPRINT A-F

700 CALL APRINT

C NPRINT NPRINT A-F

GO TO 9999

800 CALL PRINTX

GO TO 9999

C LI=9 AVAILABLE

900 RETURN

C LI=10 AVAILABLE

1000 RETURN

C ADD, SUB, MULT, DIV, RAISE, SUBTRACT, DIVIDE, MULTIPLY

1100 CALL ARITH

GO TO 9999

C SIN, ASIN, SIND, ASIND, SINH, ASINH

C COS, ACOS, COSD, ACOSD, COSH, ACOSH

C TAN, ATAN, TAND, ATAND, TANH, ATANH, NEGEXP

C COT, ACOT, COTD, ACOTD, COTH, ACOTH

C ABS , ABSOLUTE, EXP, EXPONENT , LOG, LOGE , LOGTEN , ANTILOG, SQRT , RA ISE

C INTEGER, FRACTION, SQUARE
XEC 10

XEC 20

XEC 30

XEC 40

XEC 50

XEC 60

XEC 70

XEC 80

XEC 90

XEC 100

XEC 110

XEC 120

XEC 130

XEC 140

XEC 150

XEC 160

XEC 170

XEC 180

XEC 190

XEC 200

XEC 210

XEC 220

XEC 230

XEC 240

XEC 250

XEC 260

XEC 270

XEC 280

XEC 290

XEC 300

XEC 310

XEC 320

XEC 330

XEC 340

XEC 350

XEC 360

XEC 370

XEC 380

XEC 390

XEC 400

XEC 410

XEC 420

XEC 430

XEC 440

XEC 450

XEC 460

XEC 470

XEC 480

XEC 490

XEC 500

XEC 510

XEC 520

XEC 530

XEC 540

XEC 550

XEC 560

XEC $\mathbf{5 7 0}$

XEC 580

XEC 590 
C $\quad \mathrm{L} l=13$

XEC 620

1300 GO TO $(1301,1302,1303,1304,1305,1306,1307,1308,1309,1310$,

XEC 630 $11311,1312,1313,1314), \mathrm{L} 2$

\section{GENERATE}

1301 CALL GENER

GO TO 9999

C SET

1302 CALL SET

GO TO 9999

C FIXED

1303 CALL FIXFLO

GO TO 9999

C FLOATING

1304 GO TO 1303

C PLOT

1305 CALL PLOT

GO TO 9999

C PAGE PLOT

1306 CALL PLOT

\section{GO TO 9999}

C L1=13 L2=7 AVAILABLE

1307 RETURN

C NEW PAGE

1308 CALL PAGE(4)

GO TO 9999

C SPACE

1309 CALL SPACE

GO TO 9999

C CGS

1310 CALL PHYCON(0)

GO TO 9999

C SI

1311 CALL PHYCON(-1)

GO TO 9999

C FLEXIBLE

1312 GO TO 1303

C PRINT NOTE

1313 CALL NOTEPR(3)

GO TO 9999

C ROUND

XEC 640

XEC 650

XEC 660

XEC 670

XEC 680

XEC 690

XEC 700

$X E C 710$

XEC 720

XEC 730

XEC 740

XEC 750

XEC 760

XEC 770

XEC 780

XEC 790

XEC 800

$X E C \quad 810$

XEC 820

XEC 830

XEC 840

XEC 850

XEC 860

XEC 870

XEC 880

XEC 890

XEC 900

XEC 910

XEC 920

XEC 930

XEC 940

XEC 950

XEC 960

XEC 970

XEC 980

XEC 990

XEC 1000

XEC 1010

XEC 1020

XEC 1030

XEC 1040

1400 GO TO $(1401,1402,1403,1404,1405,1406,1407,1408,1409,1410,1411,1412$, XEC 1050
XEC 1060 $11413,1414,1415), \mathrm{L} 2$

XEC 1070

C BEGIN

1401 CALL BEGIN

XEC 1080

GO TO 9999

XEC 1090

C SCAN

XEC 1100

1402 GO TO 1401

XEC 1110

C REPEAT EXECUTE PERFORM

XEC 1120

$1403 \mathrm{~J}=1$

XEC 1130

CALL REPINC (J)

IF (J) $9999,9999,90$

XEC 1140

XEC 1150

C $L 1=14 \quad L 2=4$ AVAILABLE

XEC 1160

1404 RETURN

XEC 1170

C $\quad L 1=14 \quad L 2=5 \quad$ AVAILABLE

XEC 1180 
1405 RETURN

C INCREMENT

XEC 1190

$1406 \mathrm{~J}=3$

XEC 1200

CALL REPINC (J)

XEC 1210

GO TO 9999

XEC 1220

XEC1230

C L1 $=14 \quad$ L2=7 AVAILABLE $\quad$ XEC1240

1407 RETURN

XEC1250

C RESTORE

XEC 1260

$1408 \quad J=3$

XEC1270

CALL REPINC $(J)$

XEC 1280

GO TO 9999

XEC 1290

C IFLT

XEC 1300

1409 CALL IFS

XEC 1310

GO TO 9999

XEC 1320

C IFEQ

XEC 1330

1410 GO TO 1409

XEC1340

C IFGT

XEC 1350

1411 GO TO 1409

XEC1360

C IFGE

XEC1370

1412 GO TO $1409 \quad$ XEC1380

C IFNE $\quad$ XEC1390

1413 GO TO $1409 \quad$ XEC 1400

C IFLE $\quad$ XEC1410

1414 GO TO $1409 \quad$ XEC1420

C COMPARE $\quad$ XEC1430

1415 GO TO $1409 \quad$ XEC1440

C MDEFINE MZERO MERASE MIDENT MDIAGONAL $\quad$ XEC1450

C ADEFINE AZERO AERASE $\quad$ XEC1460

1500 CALL MOP $\quad$ XEC1470

GO TO $9999 \quad$ XEC1480

C MINVERT INVERT SOLVE $\quad$ XEC1490

1600 CALL INVERT $\quad$ XEC1500

GO TO $9999 \quad$ XEC1510

C Ll=17 NO L2 VALUES ARE NEEDED IN SUBROUTINES XEC 1520

1700 GO TO $(1701,1702,1703,1704,1705)$, L2 $\quad$ XEC1530

C MMULT MMULTIPLY $\quad$ XEC 1540

1701 CALL MMULT $\quad$ XEC1550

GO TO $9999 \quad$ XEC1560

C MRAISE

1702 CALL MRAISE

GO TO 9999

C MKRONECKER

1703 CALL MKRON

GO TO 9999

C MTRAIN

1704 CALL MTRIAN

GO TO 9999

C MEIGEN

1705 CALL MEIGEN

GO TO 9999

1800 IF (L2.GT.8) GO TO 1809

C MADD MSUB MTRANS SCALAR AMULT MSUBTRACT

C AADD ASUB ATRANS ARAISE ADIVIDE ASUBTRACT AMULTIPLY

CALL MATRIX

GO TO 9999

C ACOALES AAVERA

XEC 1570

XEC 1580

XEC 1590

XEC1600

XEC1610

XEC1620

XEC1630

XEC1640

XEC1650

XEC1660

XEC1670

XEC1680

XEC1690

XEC1700

XEC1710

XEC1720

XEC1730

XEC1740

XEC 1750

XEC 1760

GO TO 9999

C NORMLAGUERE LAGUERE HERMITE LEGENDRE TCHEBYSHEV UCHEBYSHEV

XEC 1770 

GO TO 9999

C $L 1=21$

XEC 1830

2100 GO TO $(2101,2102,2103,2104,2105,2106,2107,2108,2109,2110,2111$, XEC1840 $12112,2113,2114,2115,2116,2117,2118,2119), L 2$

C ROWSUM ROW SUM

XEC 1850

XEC 1860

2101 CALL PROROW

GO TO 9999

XEC 1870

XEC 1880

C PRODUCT

XEC1890

2102 GO TO 2101

C DEFINE

XEC1900

2103 CALL DEFINE

XEC 1910

GO TO 9999

XEC1920

XEC1930

C $L 1=21 \quad L 2=4$ AVAILABLE

XEC1940

2104 RETURN

XEC 1950

C MAX MAXIMUM

XEC 1960

2105 CALL EXTREM

XEC 1970

GO TO 9999

XEC 1980

C MIN MINIMUM

XEC 1990

2106 GO TO 2105

C $\quad L 1=21 \quad L 2=7 \quad$ AVAILABLE

XEC2000

2107 RETURN

XEC2010

C SQRT

2108 CALL SORDER

GOTO 9999

C ORDER

2109 GO TO 2108

C ERASE

2110 CALL ERASE

GO TO 9999

C EXCHANGE

2111 CALL EXCHNG

XEC2020

XEC2030

XEC2040

XEC2050

XEC2060

XEC2070

XEC2080

XEC2090

XEC2100

XEC2110

XEC2 120

GO TO 9999

C FLIP

2112 CALL FLIP

GO TO 9999

C CHANGE

2113 CALL CHANGE

XEC 2130

XEC 2140

XEC2150

XEC2160

XEC2170

XEC2 180

GO TO 9999

C HIERARCHY

XEC2190

XEC2200

2114 GO TO 2108

XEC2210

C LIST

XEC2220

XEC2230

2115 CALL LIST(0)

XEC2240

GO TO 9999

C NO LIST

C WRITE SUBROUTINE

XEC2250

XEC2260

XEC2270

2116 CALL LIST(1)

XEC2280

GO TO 9999

C NULL

XEC2290

XEC2300

2117 GO TO 9999

C ERROR

XEC2310

XEC2320

2118 CALL FNEC

XEC2330

GO TO 9999

XEC2340

C CERF

XEC2350

2119 GO TO 2118

XEC2360 
C POLYFIT FIT MORTHO

XEC2370

C SPOLYFIT SFIT

XEC 2380

2200 CALL ORTHO

XEC 2390

GO TO 9999

XEC 2400

C $\quad \mathrm{Ll}=23$

XEC2410

2300 GO TO $(2301,2302,2303,2304,2305,2306,2307,2308,2309,2310,2311$ 。

XEC 2420

12312), L2

C CLOSE UP

XEC2430

2301 CALL MISC2

C GO TO 9999

XEC 2440

XEC2450

XEC2 460

2302 GO TO 2301

C SHORTEN

XEC2470

2303 GO TO 2301

XEC2480

C EXPAND

XEC2490

2304 GO TO 2301

XEC2500

C DUPLICATE

$X E C 2510$

2305 GO TO 2301

XEC 2520

C MOVE AMOVE MMOVE

XEC2530

2306 CALL MOVE

GO TO 9999

XEC2540

XEC 2550

XEC2560

C LI $=23 \quad L 2=7$ AVAILABLE

XEC 2570

2307 RETURN

C Ll=23 L2=8 AVAILABLE

2308 RETURN

C L1=23 L2=9 AVAILABLE

2309 RETURN

C PROMOTE

2310 CALL PDMOTE

XEC2580

XEC2590

XEC2600

XEC2610

XEC2620

XEC2630

XEC2640

XEC 2650

GO TO 9999

XEC2660

C DEMOTE

XEC 2670

2311 GO TO 2310

XEC 2680

C DIMENSION DIM

XEC 2690

2312 CALL DIMENS

XEC2700

GO TO 9999

XEC2710

C $\quad \mathrm{Ll}=24$

XEC 2720

2400 GO TO $(2401,2402,2403,2404,2405,2406,2407,2408,2409,2410$,

XEC 2730

XEC2740

$12411,2412,2413,2414,2415)$, L2

C STATIS

2401 CALL STATIS

XEC2750

XEC2760

GO TO 9999

XEC2770

XEC 2780

C SSTATIS

XEC2790

XEC 2800

XEC 2810

XEC2820

XEC2830

XEC2840

XEC2850

XEC 2860

XEC2870

XEC2880

XEC2890

XEC2900

XEC2910

XEC2920

XEC2930

XEC2940

XEC2950 
C NHISTO

XEC2980

2409 CALL HISTGM

XEC2990

GO TO 9999

XEC 3000

C FREQUE

$X E C 3010$

2410 CALL FRDIST

XEC3020

GO TO 9999

XEC3030

C CORREL

$X E C 3040$

2411 CALL CORREL

XEC3050

GO TO 9999

XEC3060

C SCORRE

XEC3070

2412 CALL CORREL

XEC3080

GO TO 9999

XEC 3090

C ONEWAY

XEC 3100

2413 CALL ONEWAY

XEC3110

GO TO 9999

XEC 3120

C SONEWAY

XEC 3130

2414 GO TO 2413

XEC3140

C UNIFORM RANDOM

XEC3150

2415 CALL FNKC

GO TO 9999

C SELECT SEARCH CENSOR MATCH

XEC3160

XEC3170

2500 IF (L2.EQ.4) GO TO 2504

XEC 3180

CALL SELECT

GO TO 9999

XEC3190

XEC3200

XEC 3210

2504 CALL INTERP

XEC3220

GO TO 9999

$X E C 3230$

C MVECDIAG MVECMAT MMATVEC MVECDIAGONAL

XEC3240

2600 CALL EXPCON

XEC3250

GO TO 9999

C MPROPERTIES APROPERTIES SMPROP SAPROP

XEC3260

XEC3270

2700 CALL MPROP

GO TO 9999

XEC3280

XEC 3290

C ITERATE ISETUP ISOLATE

XEC3300

2800 CALL ITERAT

$X E C 3310$

GO TO 9999

XEC3320

XEC3330

XEC3340

C SEPARATE INSERT MAXMIN EXTREMA

XEC 3350

GO TO 9999

$X E C 3360$

C BESSEL SUBROUTINES

XEC 3370

XEC3380

$X E C 3390$

XEC3400

XEC3410

$X E C 3420$

XEC3430

XEC3440

XEC 3450

XEC 3460

$X E C 3470$

XEC 3480

XEC 3490

XEC3500

XEC3510

XEC 3520

XEC 3530

XEC 3540 


\begin{tabular}{|c|c|c|}
\hline C & $\lfloor l=43$ & $X E C 3550$ \\
\hline C & $L l=44$ & $X E C 3560$ \\
\hline 3300 & RETURN & $X E C 3570$ \\
\hline 3400 & RETURN & XEC 3580 \\
\hline 3500 & RETURN & $X E C 3590$ \\
\hline 3600 & RETURN & $X E C 3600$ \\
\hline 3700 & RETURN & XEC 3610 \\
\hline 3800 & RETURN & XEC 3620 \\
\hline 3900 & RETURN & $X E C 3630$ \\
\hline 4000 & RETURN & $X E C 3640$ \\
\hline 4100 & RETURN & $X E C 3650$ \\
\hline 4200 & RETURN & $X E C 3660$ \\
\hline 4300 & RETURN & XEC 3670 \\
\hline 4400 & RETURN & $X E C 3680$ \\
\hline$C * * * *$ & * THE FOLLOWING CARDS ARE NEEDED ONLY FOR TAPE OPERATIONS & XEC3690 \\
\hline C & THE STATEMENTS $4500-5000$ WERE RETURN STATEMENTS & XEC 3700 \\
\hline C & READ TAPE & XEC 3710 \\
\hline 4500 & CALL TAPOP2 & $X E C 3720$ \\
\hline & GO TO 9999 & XEC 3730 \\
\hline C & CREAD TAPE & XEC 3740 \\
\hline 4600 & GO TO 4500 & XEC 3750 \\
\hline C & WRITE TAPE & $\mathrm{XEC} 3760$ \\
\hline 4700 & GO TO 4500 & $\mathrm{XEC} 3770$ \\
\hline C & SET TAPE & XEC 3780 \\
\hline 4800 & GO TO 4500 & XEC3790 \\
\hline C & CSET TAPE & $\mathrm{XEC} 3800$ \\
\hline 4900 & GO TO 4500 & XEC 3810 \\
\hline C & ENDFILE TAPE, REWIND TAPE SKIP TAPE, BACKSPACE TAPE & XEC 3820 \\
\hline 5000 & GO TO 4500 & $\mathrm{XEC} 3830$ \\
\hline $\mathrm{C} * * * * *$ & *********************************************************) & *XEC 3840 \\
\hline C & $M\left(X X^{\prime}\right) \quad M\left(X^{\prime} X\right) \quad M\left(X A X^{\prime}\right) \quad M\left(X^{\prime} A X\right)$ & $\mathrm{XEC} 3850$ \\
\hline 5100 & $\begin{array}{l}\text { CALL MXTX } \\
\text { GO T0 } 9999\end{array}$ & $\begin{array}{l}X E C 3860 \\
X E C 3870\end{array}$ \\
\hline C & $M(A D), M(D A)$ & XEC 3880 \\
\hline 5200 & CALL MDAMAD & XEC 3890 \\
\hline & GO TO 9999 & XEC 3900 \\
\hline C & $M\left(V^{\prime} A\right) \quad M(A V)$ & XEC3910 \\
\hline 5300 & CALL ARYVEC & XEC 3920 \\
\hline & GO TO 9999 & XEC3930 \\
\hline C & $L l=54$ & XEC 3940 \\
\hline 5400 & GO TO $(5401,5402,5403,5404,5405,5406,5407), L 2$ & XEC 3950 \\
\hline C & $L 2=1$ & XEC 3960 \\
\hline 5401 & RETURN & XEC 3970 \\
\hline C & DUMMY A & XEC 3990 \\
\hline 5402 & CALL DUMMYA & $\mathrm{XEC} 4000$ \\
\hline & GO TO 9999 & XEC4010 \\
\hline C & DUMMY B & XEC 4020 \\
\hline 5403 & CALL DUMMYB & XEC 4030 \\
\hline & GO TO 9999 & XEC 4040 \\
\hline C & DUMMY C & XEC 4050 \\
\hline 5404 & CALL DUMMYC & XEC 4060 \\
\hline & GO TO 9999 & XEC4070 \\
\hline C & DUMMY D & XEC 4080 \\
\hline 5405 & CALL DUMMYD & XEC 4090 \\
\hline & GO TO 9999 & XEC4 100 \\
\hline C & DUMMY E & XEC4110 \\
\hline 5406 & CALL DUMMYE & XEC 4120 \\
\hline & GO TO 9999 & $X E C 4130$ \\
\hline C & DUMMY F & XEC 4140 \\
\hline
\end{tabular}


C $\quad L l=55$

X.EC4170

C $\quad L l=56$

XEC4180

XEC4190

C $\quad L l=57$

XEC4200

C $\quad L l=58$

XEC4210

XEC4 220

XEC4230

XEC4240

XEC4250

XEC4260

XEC4270

XEC4280

XEC4290

XEC4300

XEC4310

XEC4320

XEC4330

XEC4340

XEC4350

XEC4360

XEC4370

XEC4380

XEC4390

XEC4400

XEC4410 


\section{SUBROUTINE XFORMT}

C

C

C

10

C

C

C

20

IF (IND.EQ.0) RETURN

COMMON /BLOCKA/ MODE,M,KARD (83), KARG, ARG, ARG2, NEWCD (80), KRDEND

LOOK FOR LETTER A-F FOLLOWED BY NON-ALPHANUMERIC CHARACTER

XFO 40

$X F O \quad 50$

$X F 0 \quad 60$

XFO 70

XFO 75

XFO 80

XFO 90

XFO 100

XFO 110

XFO 120

XFO 130

XFO 140

XFO 150

XFO 160

RETURN

XFO 170

SUBROUTINE XHEAD $\quad$ XHE 10

$\begin{array}{llll}\text { C VERSION } 5.00 & \text { XHEAD } 5 / 15 / 70 & \text { XHE } 20\end{array}$

COMMON /BLOCKA/ MODE, M,KARD (83), KARG, ARG, ARG2, NEWCD (80), KRDEND XHE 30

COMMON /BLOCKD/ IARGS $(100), \mathrm{KIND}(100)$, ARGTAB $(100), \mathrm{NRMAX}, \mathrm{NROW}, \mathrm{NCOL}, \mathrm{NXHE} 40$

IARGS, VWXYZ (8), NERROR

COMMON /ABCDEF/ L (48)

XHE 50

GO TO 20

XHE 60

$M=M+1$

XHE 70

10

IF (KARD (M).GE.10) IF (KARD(M)-46) $10,30,10$

XHE 80

20

CALL AARGS

XHE 85

$I=A R G$

IF (KARG.EQ.O.AND.I.GT.O.AND.I.LE.NCOL) GO TO 60

XHE 90

XHE 100

XHE 110

CALL ERROR (204)

RETURN

$50 \quad M=M+1$

60 IF (KARD (M).NE.36) IF (KARD (M)-46) $50,30,50$

C

C

C

80 CALL PREPAK $(2$, IND , I , IR , IR $)$

RETURN

END

XHE 120

XHE 130

XHE 140

XHE 145

XHE 150

XHE 160

XHE 170

XHE 230

XHE 240

XHE 250 
IF PRINT COMMAND HAS DEC. ARGS FLEXIBLE FORMAT WILL BE USED

FLEXIBLE COMMAND WILL CHANGE SWITCH TO 0

XOM 25

IF PRINT WITH ALL INTEGER ARGS AND SWITCH=0 USE FLEXIBLE FORMAT XOM 27

IF PRINT WITH ALL INTEGER ARGS AND SWITCH=1, USE SPECIFIED FORMAT (FIXED OR FLOATING)

COMMON /BLOCKA/ MODE, M,KARD (83), KARG,ARG,ARG2,NEWCD (80), KRDEND

XOM 28

XOM 29

COMMON /BLOCKB/ NSTMT, NSTMTX, NSTMTH, NCOM, LCOM, IOVFL, COM(2000)

XOM 30

COMMON /BLOCKC / KIO, INUNIT, ISCRAT, KBDOUT, KRDKNT, LLIST

XOM 40

COMMON /BLOCRC/ NRC, RC (12600)

XOM 50

XOM 60

COMMON /BLOCKD/ IARGS(100), KIND(100), ARGTAB (100), NRMAX,NROW, NCOL, NXOM 70

IARGS, VWXYZ (8), NERROR

DIMENSION ARGS $(100)$

XOM 80

EQUIVALENCE (ARGS(1), RC (12501))

COMMON /HEADER/ NOCARD $(80), \operatorname{ITLE}(60,6)$, LNCNT , I PRINT, NPAGE , IPUNCH COMMON / PKSWT / IHCNT, IHTP

COMMON/FMAT / IFMTX (6) , IOSWT , IFMTS (6), LHEAD (96)

COMMON / BLOCKX/INDEX $(6,8)$, LEVEL

COMMON /SPRV/ NERCON, NERR, ISWERR

COMMON / ABCDEF / L (48)

COMMON/PCONST / JPC, P (40), N (40)

C

C

C

C

C

C

10

20

30

C

C

C

40

50

60

IF LG IS NEG, FIRST CARD WAS NOT 'OMNITAB' CARD. IF LG=0, FIRST CARD $=$,OMNITAB, , ELSE SUBSEQUENT 'OMNITAB' CARD FOUND.

IF (LG) $40,20,10$

GO THROUGH 'STOP' SEQUENCE AND RETURN

CALL XSTOP

DO $30 \quad I=1,80$

NOCARD (I) $=\operatorname{NEWCD~(I)~}$

INITIALIZE SYSTEM

DO $50 \quad I=1,60$

DO $50 \mathrm{~J}=1,6$

$\operatorname{ITLE}(\mathrm{I}, \mathrm{J})=\mathrm{L}(45)$

DO $60 \quad I=1,6$

XOM 90

XOM 100

XOM 110

XOM 115

XOM 120

XOM 125

XOM 130

XOM 140

XOM 150

XOM 170

XOM 180

XOM 190

XOM 200

XOM 210

XOM 220

XOM 230

XOM 240

XOM 250

XOM 260

XOM 270

XOM 280

XOM 290

XOM 300

XOM 310

XOM 320

XOM 330

$\operatorname{IFMTX~(I)~}=\operatorname{IFMTS~(I)~}$

IOSWT $=0$

I HCNT $=0$

XOM 340

XOM 350

XOM 360

CALL PREPAK (3, IND, IND, IND, IND)

MODE $=1$

NRMAX $=0$

NRO $W=201$

$\mathrm{NCOL}=62$

KRDEND $=80$

LLIST $=3$

NERROR $=0$

NSTMT $=0$

NSTMTH $=0$

NCOM $=1$

$L C O M=2000$

XOM 370

XOM 400

XOM 410

XOM 420

XOM 430

XOM 440

XOM 450

XOM 460

XOM 470

XOM 480

XOM 490

XOM 500

LEVEL $=0$

XOM 505 
IOVFL $=0$

$\mathrm{NPAGE}=0$

$N R C C=N R C+100$

DO $90 \mathrm{I}=1, N R C C$

$90 \quad R C(I)=0$.

NERR $=0$

NERCON $=100$

I $S$ WERR $=0$

DO $100 \quad \mathrm{I}=1,8$

$100 \quad \operatorname{VWXYZ}(\mathrm{I})=0.0$

$J P C=-1$

CALL NOTEPR (0)

RETURN

END
XOM 510

XOM 520

XOM 530

XOM 540

XOM 550

XOM 560

XOM 570

XOM 580

XOM 584

XOM 586

XOM 588

XOM 600

XOM 605

XOM 610 


\begin{tabular}{|c|c|c|c|}
\hline \multirow{8}{*}{$C$} & SUBROUTINE XPND $(T, K, Y, K N D)$ & XPN & 10 \\
\hline & VERSION $5.00 \quad$ XPND & XPN & 20 \\
\hline & COMMON /BLOCRC/ NRC, RC (12600) & XPN & 30 \\
\hline & COMMON /BLOCKD/ IARGS $(100)$, KIND $(100)$,ARGTAB $(100)$, NRMAX, NROW, NCOL & NXPN & 40 \\
\hline & IARGS, VWXYZ (8), NERROR & XPN & 50 \\
\hline & DIMENSION ARGS $(100)$ & XPN & 60 \\
\hline & EQUIVALENCE (ARGS (1), RC $(12501)$ ) & XPN & 70 \\
\hline & DIMENSION T (2) & XPN & 80 \\
\hline C & & XPN & 90 \\
\hline C & THIS SUBROUTINE TAKES A ' 'STATEMENT'" REFERENCE AS STORED & XPN & 100 \\
\hline C & AND EXPANDS IT INTO THE PROPER ARGUMENT WITH CHECKING. & XPN & 110 \\
\hline C & & XPN & 120 \\
\hline C & $K$ IS RETURNED $O$ IF ARG IN STATEMENT IS ONE WORD LONG & XPN & 130 \\
\hline C & $K$ IS RETURNED I IF ARG IN STATEMENT IS TWO WORDS LONG. & XPN & 140 \\
\hline C & $K$ IS RETURNED -( ERROR NUMBER ) IF ERROR OCCURS. & XPN & 150 \\
\hline C & & XPN & 160 \\
\hline & I $T=-T(1)$ & XPN & 170 \\
\hline & IF (IT.LT.I6) GO T0 40 & XPN & 180 \\
\hline C & & XPN & 190 \\
\hline C & ''ROW, COL'.' ENTRY & XPN & 200 \\
\hline C & & XPN & 210 \\
\hline & I $T=I T-8208$ & XPN & 220 \\
\hline & IF (IT.GT.O.AND.IT.LE.NROW) GO TO 10 & XPN & 230 \\
\hline & $K=-16$ & XPN & 240 \\
\hline & GO TO 20 & XPN & 250 \\
\hline 10 & $\operatorname{IARGS}(100)=\operatorname{ABS}(T(2))-8192$. & XPN & 260 \\
\hline & $\operatorname{KIND}(100)=0$ & XPN & 270 \\
\hline & CALL ADRESS $(100, \mathrm{~J})$ & XPN & 280 \\
\hline & IF (J.NE.0) GO TO 30 & XPN & 290 \\
\hline & $K=-11$ & XPN & 300 \\
\hline 20 & RETURN & XPN & 310 \\
\hline 30 & $J=J+I T$ & XPN & 320 \\
\hline & $K N D=0$ & XPN & 330 \\
\hline & IF $(T(2) . L T .0) K N D=1$. & XPN & 340 \\
\hline & $Y=R C(J-1)$ & XPN & 350 \\
\hline & $K=1$ & XPN & 360 \\
\hline & GO TO 20 & XPN & 370 \\
\hline C & & XPN & 380 \\
\hline C & NRMAX, $V, W, X, Y, Z$, REFERENCE. & XPN & 390 \\
\hline C & & XPN & 400 \\
\hline 40 & $I U=I T / 2$ & XPN & 410 \\
\hline & $K N D=I T-2 * I U$ & XPN & 420 \\
\hline & $K=0$ & XPN & 430 \\
\hline & IF (IU.LE.I) GO TO 50 & XPN & 440 \\
\hline & $Y=V W X Y Z(I U-2)$ & XPN & 450 \\
\hline & GO TO 20 & XPN & 460 \\
\hline 50 & $Y=N R M A X$ & XPN & 470 \\
\hline & GO TO 20 & XPN & 480 \\
\hline & & XPN & 490 \\
\hline
\end{tabular}


SUBROUTINE XSTOP

C VERSION $5.00 \quad$ XSTOP $5 / 15 / 70$

XST 10

COMMON /BLOCKC/ KIO, INUNIT, ISCRAT, KBDOUT, KRDKNT, LLIST $\quad$ XST 30

COMMON /BLOCRC/ NRC,RC(12600) $\quad$ XST 40

COMMON /BLOCKD/ IARGS (100), KIND (100), ARGTAB (100), NRMAX, NROW, NCOL, NXST 50

IARGS, VHXYZ (8), NERROR $\quad$ XST 60

DIMENSION ARGS $(100)$

EQUIVALENCE (ARGS(1), RC (12501))

COMMON /HEADER/NOCARD $(80), \operatorname{ITLE}(60,6), \mathrm{LNCNT}, \mathrm{IPRINT}, \mathrm{NPAGE}, \mathrm{IPUNCH}$

XST 70

XST 80

COMMON /SCRAT / NS,NS2,A(13500)

DIMENSION ITEMP (84)

EQUIVALENCE (ITEMP(1), A(1))

DATA IZ, IP , NO , KOMMA / IHZ, IH+, IHO, IH, I

XST 90

XST 110

XST 120

XST 130

XST 140

XST 150

C THIS ROUTINE REHINDS THE SCRATCH UNIT AND PRINTS IT.

XST 170

REWIND ISCRAT

XST 180

LLIST $=0$

IF (NERROR.EQ.0) LLIST=3

XST 190

XST 200

CALL PAGE (0)

XST 210

WRITE (IPRINT, 90)

DO $40 \mathrm{~J}=1,50$

XST 220

XST 230

READ (ISCRAT, 100) ITEMP

IF (ITEMP (1).EQ.IZ) GO TO 50

XST 240

IF (ITEMP (1).EQ.IP) GO TO 30

XST 250

IF (ITEMP (1).EQ.KOMMA) GO TO 20

XST 260

WRITE (IPRINT, 110) ITEMP

XST 270

GO TO 40

$20 \quad$ LLIST $=3$

IF $(I T E M P(2), E Q$. NO . AND .NERROR .EQ . 0) LLIST=0

XST 280

XST 290

XST 300

XST 310

GO TO 40

30 WRITE (IPRINT, 120) (ITEMP (I), I=2,84)

40 CONTINUE

GO TO 10

50 REWIND ISCRAT

IF (NERROR-1) $80,60,70$

XST 320

XST 330

XST 340

XST 350

XST 360

XST 370

WRITE (IPRINT, 130)

GO TO 80

XST 380

XST 390

$\begin{array}{ll}70 & \text { HRITE (IPRINT, 140) } \\ 80 & \text { NERROR }\end{array}$

XST 400

XST 410

WRITE (IPRINT, 150)

XST 420

WRITE (IPRINT, 160)

XST 430

RETURN

XST 440

C

$\begin{array}{lll}90 & \text { FORMAT } & (/ / 19 X, 39 H \\ 100 & \text { FORMAT } & (84 A 1)\end{array}$

XST 450

XST 460

FORMAT $(20 X, 84 A 1)$

FORMAT $(18 X, 3 \mathrm{Al}, 3 \mathrm{X}, 80 \mathrm{Al})$

FORMAT (///4OX,2OHONLY ONE FATAL ERROR)

XST 470

XST 480

XST 490

XST 500

XST 510

140 FORMAT (///40X, 14,7H ERRORS)

XST 520

FORMAT (1HO/33X,

160 FORMAT (IHI)

XST 690

END

XST 700 


\section{Appendix-OMNIT Flow Chart}

\section{Flow Chart Notes}

(1) The flow chart of the OMNIT subprogram was generated by the FLOGEN program on the UNIVAC 1108 and drawn by the CAL-COMP plotter; see CAL-COMP [1968].

(2) When the flow of the diagram is broken, an arrow points to a box with two symbols. The top number refers to the page and the bottom symbol is the entry point. At each entry arrow, the number inside the box indicates the page from which the flow comes and the symbol outside is the entry position.

(3) The INPUT subprogram reads an OMNITAB instruction and stores the information in the array KARD. Each character is stored in one location starting with the character of the first column in KARD(3). See page 2 of the flow chart.

(4) The subprogram NNAME takes the first six or less characters of the first nonnumeric word and converts them into two numbers by combining the values assigned to each letter. (See NNAME for the values assigned to each letter.) The numbers are stored in NAME(1) and NAME(2). See page 5 of the flow chart.

(5) Pages 5 through 11 of the flow chart check to see if the OMNITAB command is one of the following: OMNITAB, FINISH, FORMAT, NOTE, HEAD, TITLE or STOP.

(6) The subprogram EXPAND converts the values in ARGTAB, described on page 18 of the flow chart, to floating-point numbers and stores them in either the array IARGS or the array ARGS, depending upon whether an argument is an integer or a floatingpoint number. Also, EXPAND sets $\operatorname{KIND}(\mathrm{I})=0$, if an argument is an integer, and sets $\operatorname{KIND}(\mathrm{I})=1$, if an argument is a floating-point number; see pages 19 and 22 of the flow chart.

(7) A table look-up is done by the subprogram LOOKUP using NAME(1), NAME(2) and sometimes NAME(3) and NAME(4). Also, a set of unique numbers are assigned to the variables L1 and L2. If $L 1=0$, no command was found; see page 20 of the flow chart.

(8) If a command is found, the subprogram XECUTE is called to execute the instruction; see page 22 of the flow chart. 
市

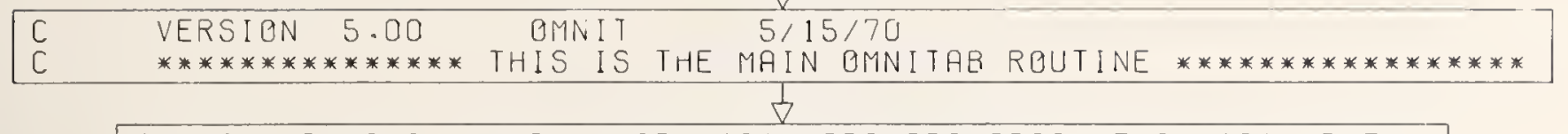

COMMON /BLOCKA/ MODE,M.KARD(83),KARG,ARG,ARG2,NEWCD(80),KRDEND

COMMON /BLOCKE/ NSTMT.NSTMTX.NSTMTH.NCOM.LCOM.IOVFL, COM(2000)

COMMON :BLOCKC/ KIO.INUNIT, ISCRAT, KBDOUT, KRDKNT, LLIST

COMMON /BLOCRC/ NRC.RC(12600)

市

COMMON /BLOCKD/ IARGS(100), KIND(100),ARGTAB(100),NRMAX,NROW,NCOL,N ARGS. VWXYZ( 8). NERROR

DIMENSION ARGS( 100$)$

EQUIVALENCE (ARGS(1),RC(12501) )

COMMON/BLOCKE, NAME ( 4$), L 1 . L 2$. ISRFLG

COMMON/HEADER/NOCARD $(80)$, I TLE $(60.6)$, LNCNT, IPRINT . NPAGE , IPUNCH

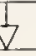

C THE FOLLOWING CARDS ARE NEDDED ONLY FOR TAPE OPERATIQNS

COMMON /TAPE/ NAME4(2).NTPCT.IPUNCP. INUNIP.LITP $\downarrow$

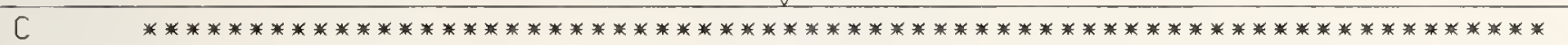

$\frac{\nabla}{\text { DATA IBLANK/1H/.LETSGE/-1/] }}$

$\begin{array}{ll}\text { C } & \text { THIS IS THE MAIN OMNITAB PROGRAM } \\ \text { C } & \text { SUBROUTINES CALLED BY THIS PROGRAM. }\end{array}$

SETUP. INPUT, ERROR. STMT.NNAME, ARRGS.ASTER.SETQ.READQ.STQRE, XECUTE

AERR, XOMNIT, XFORMT , LOOKUP

MOD = 1 INTERPRETIVE MODE

$=2$ DATA MODE (READ SET)

$=3$ STORAGE MODE (BETWEEN BEGIN AND FINISH)

$=4$ [MPLIED STORAGE MODE (STATEMENT NUMBER GIVEN)

$\downarrow$

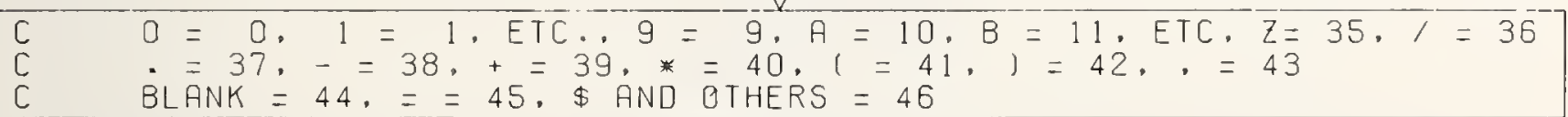

$\checkmark$

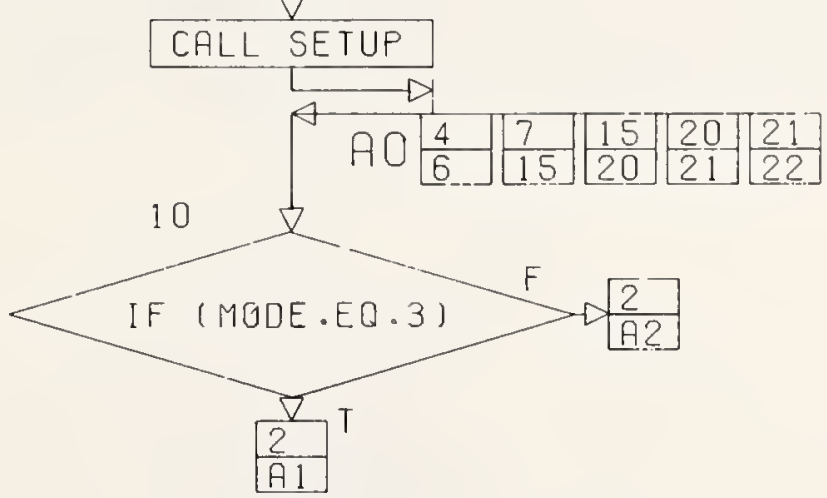

CONT. ON PG 2

PG 1 OF 22 


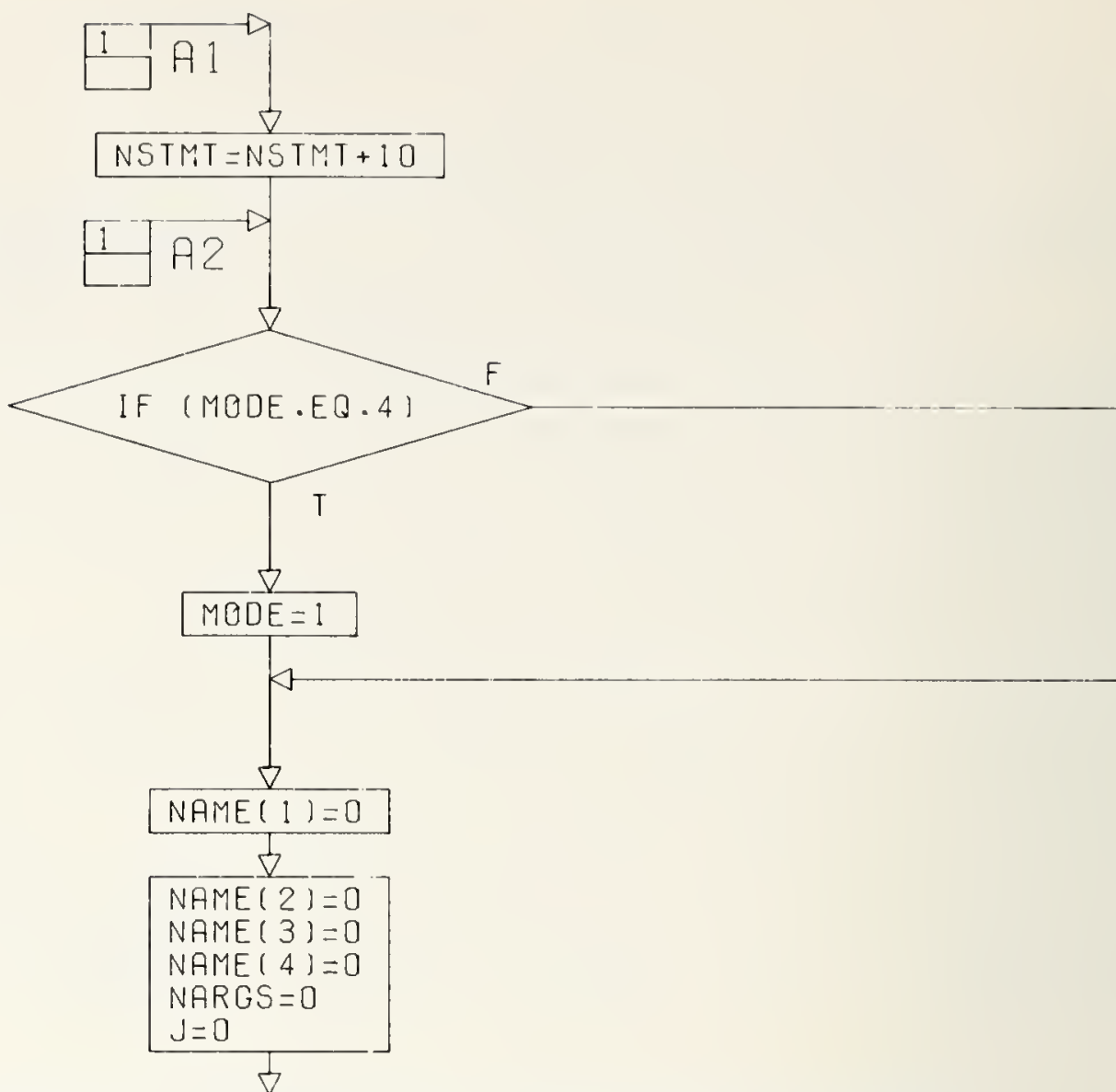

C CHECK FOR ACCUMULATED ERRGRS DURING LAST EXECUTED COMMAND I

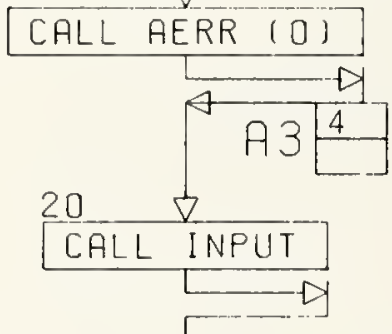

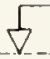

C SCANNING BEGINS WITH THE THIRD CHARACTER. THE FIRST TWG ARE DUMMY C TO KEEP THE PROGRAM OUT OF TRQUBLE. SCANNING TERMINATES WITH A $\$$ C A \$ HAS BEEN PLANTED IN THE (KRDEND+!)-TH POSITIGN.

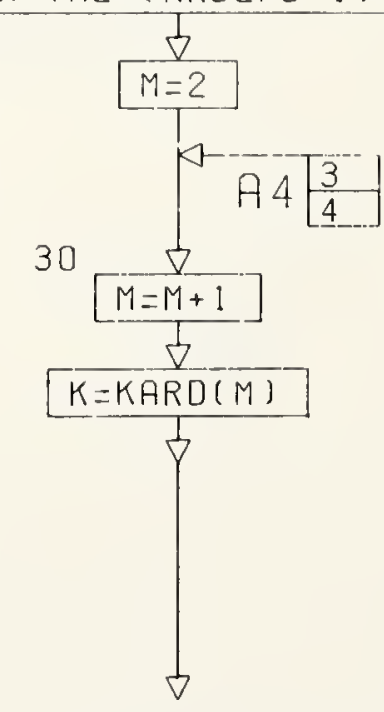

CONT. GN PG 3 

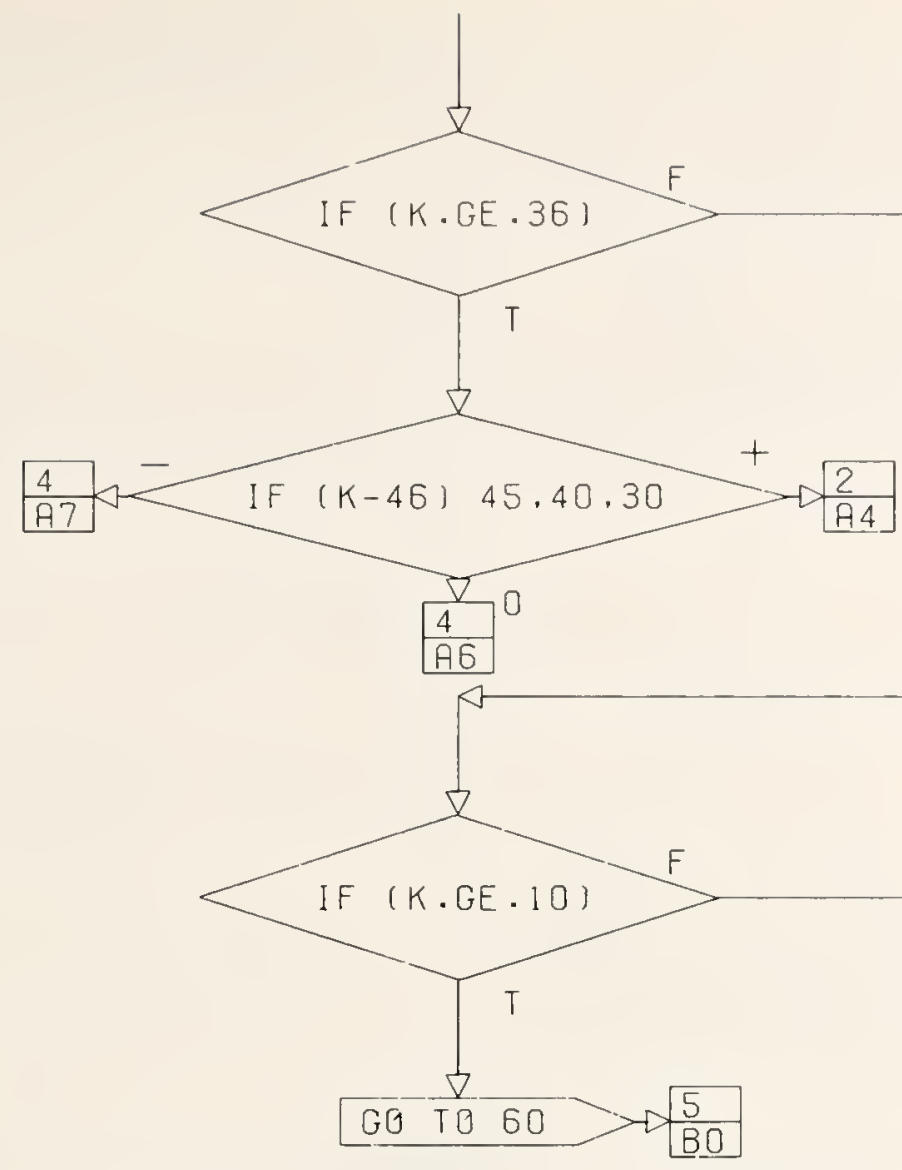

A NUMBER IS THE FIRST ALPHANUMERIC CHARACTER ENCOUNTERED. ERROR IF
C IN MUDE 3

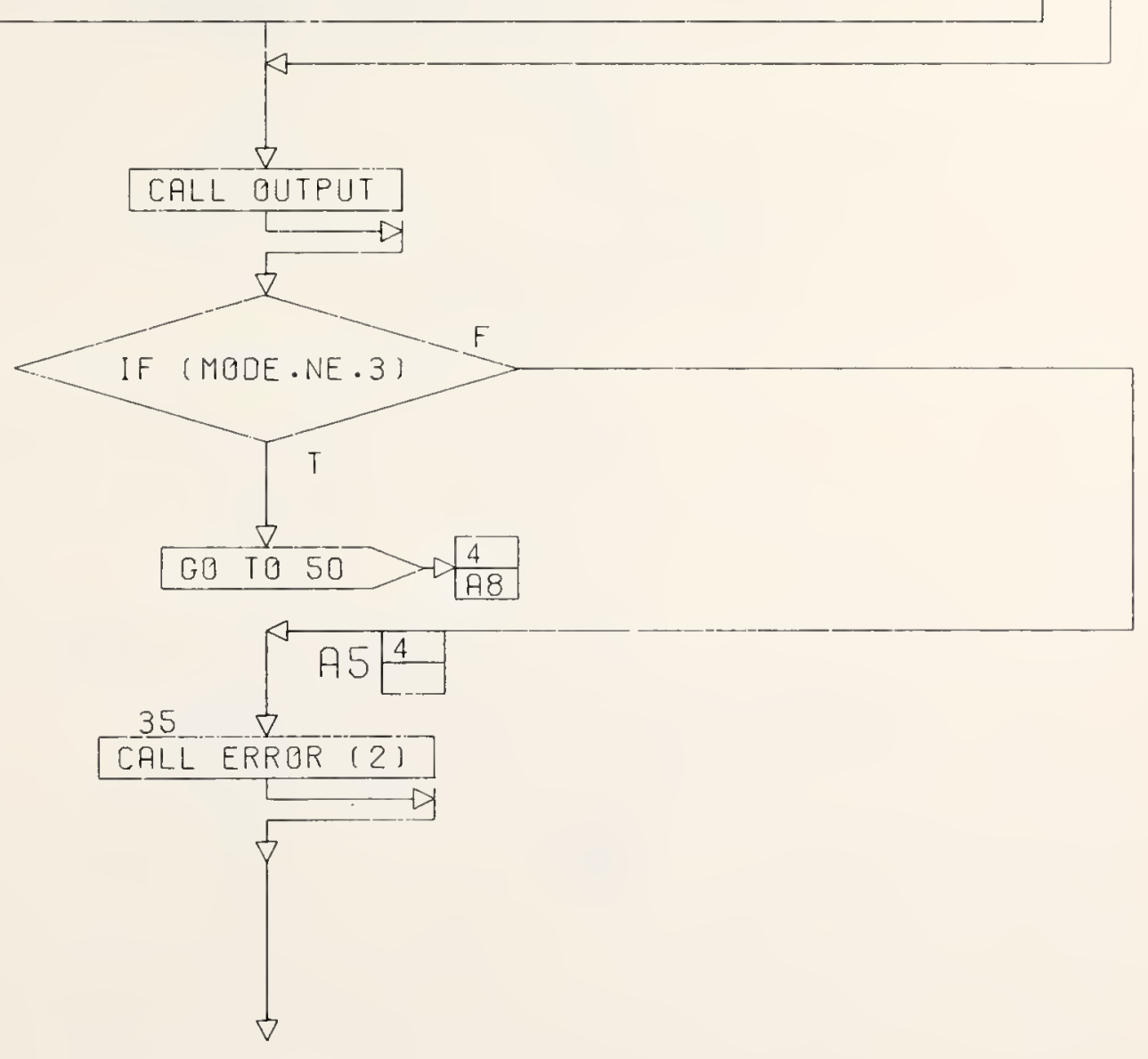

CONT. GN PG 4 


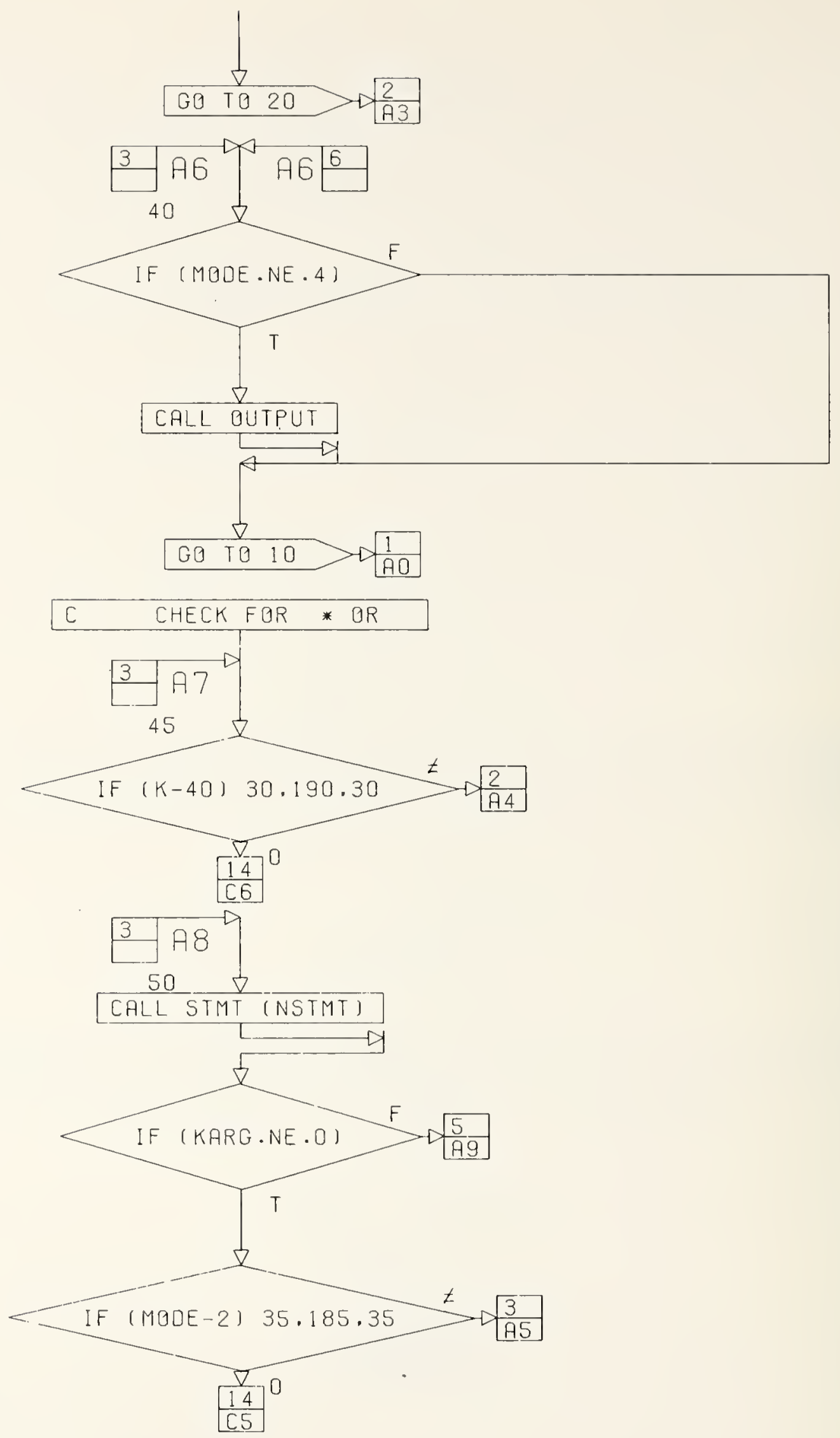

CONT. GN PG 5

PG $4 \quad$ OF 22 
C IF AN ILLEGAL STATEMENT NUMBER WAS FEUND, KARG $=1$ (KARG $=0$ IF
C

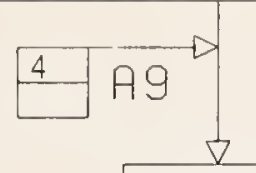

$M O D E=4$

1

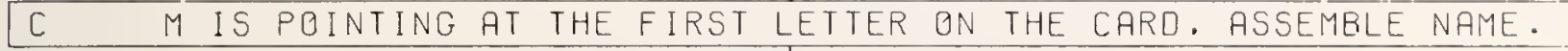

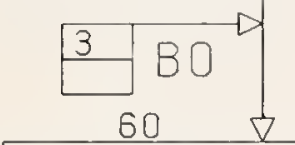

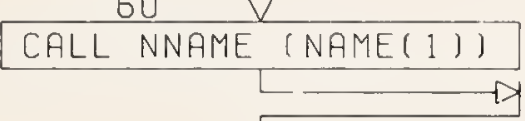

$\nabla$

C CHECK THE FIRST NAME FOR SPECIAL NAMES ...

C GMNITAB, FORMAT. NOTE. FOOTNOTE. HERD. TITLE C GMNITAB

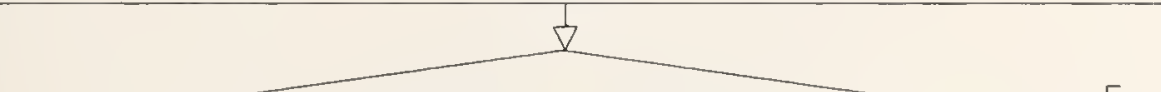

LIF (NAME( 1 ).NE.11300.OR.NAME(2).NE.7102) F

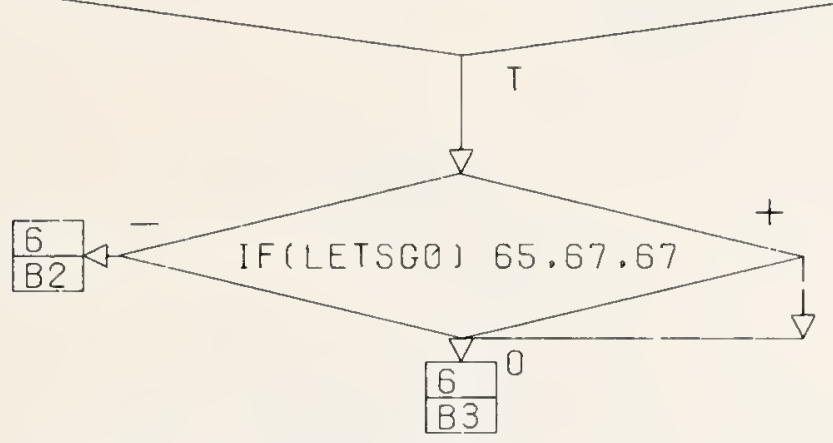

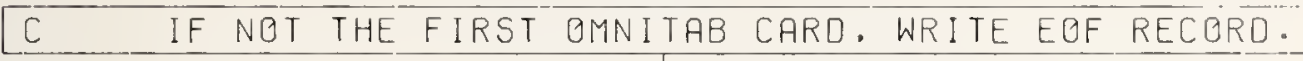

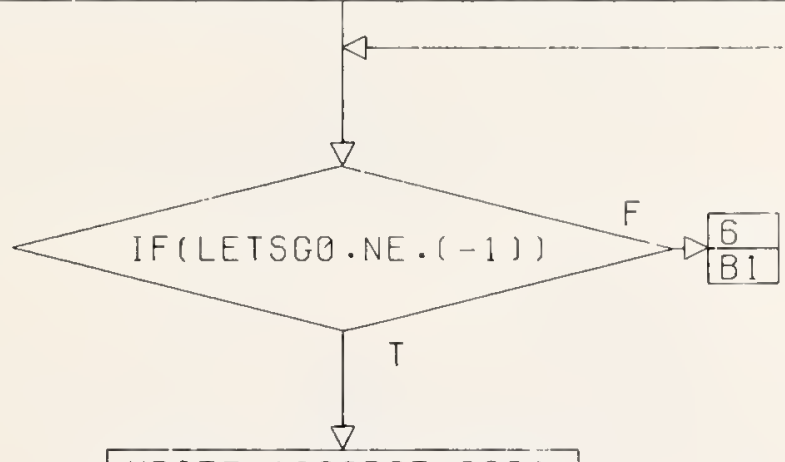

WRITE (ISCRAT.390)

CONT. ON PG

6

PG 5. OF 22 


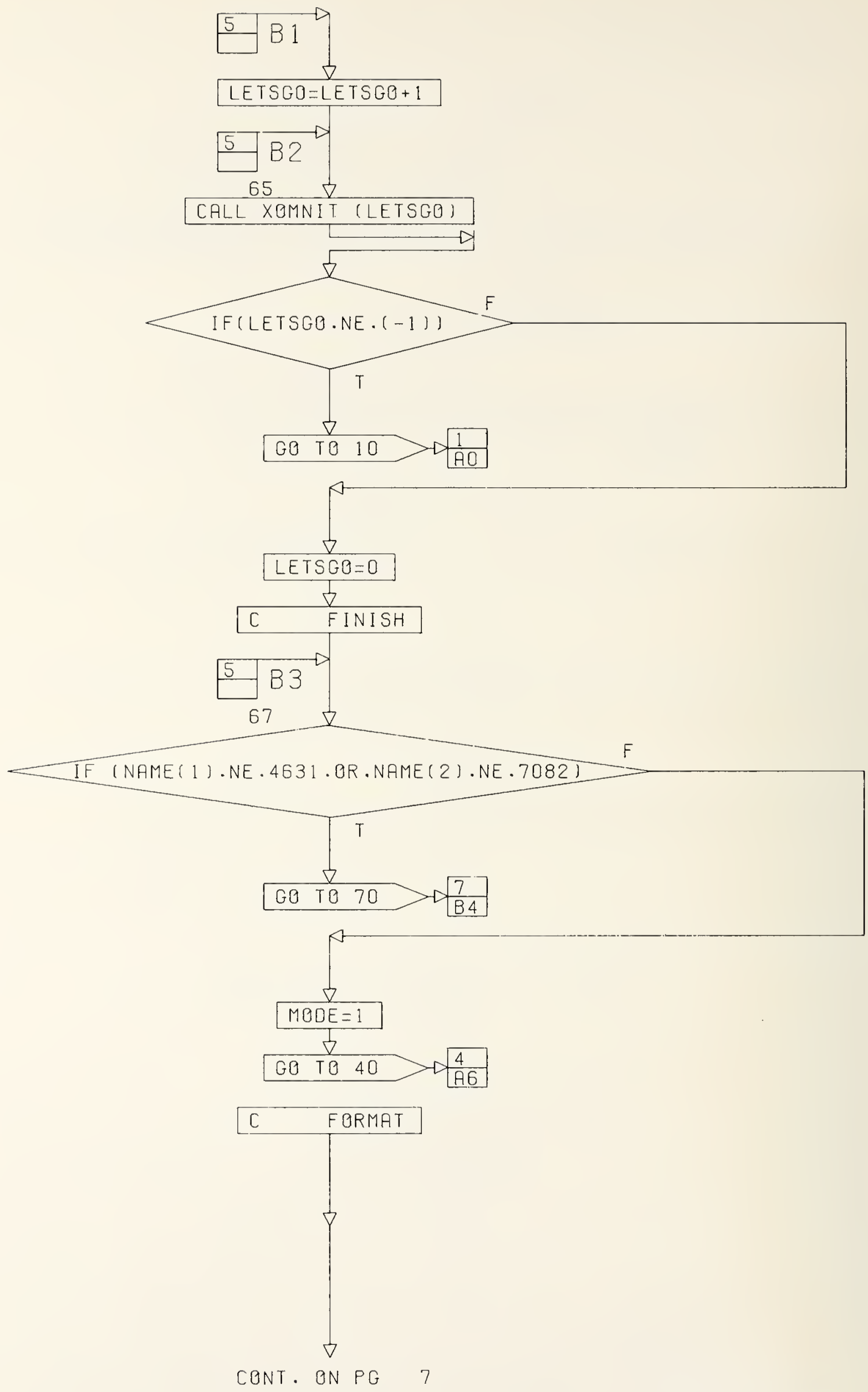

PG 6 OF 22 


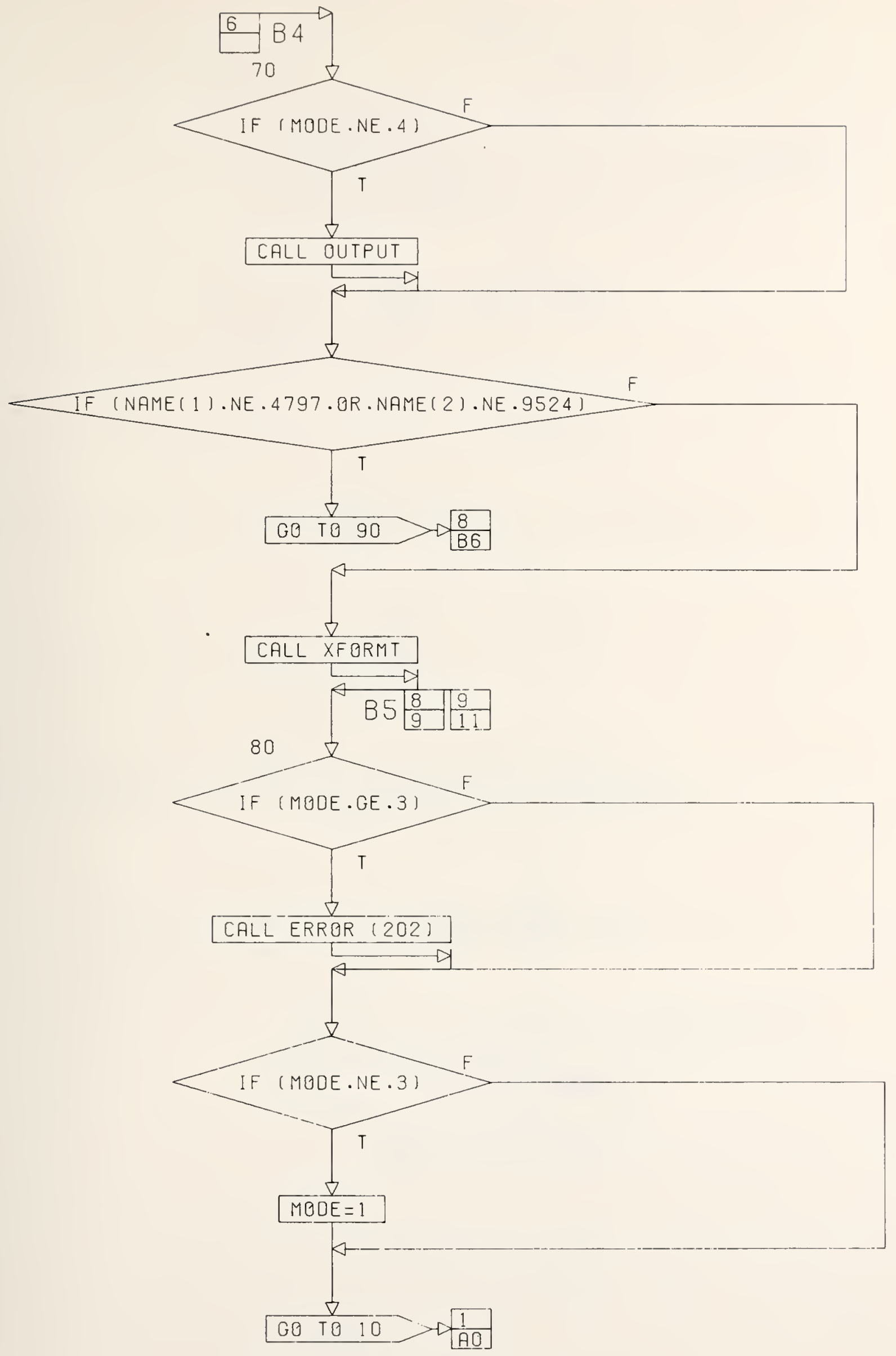

CONT. ONPG 8

$P G \quad$ I OF 22 


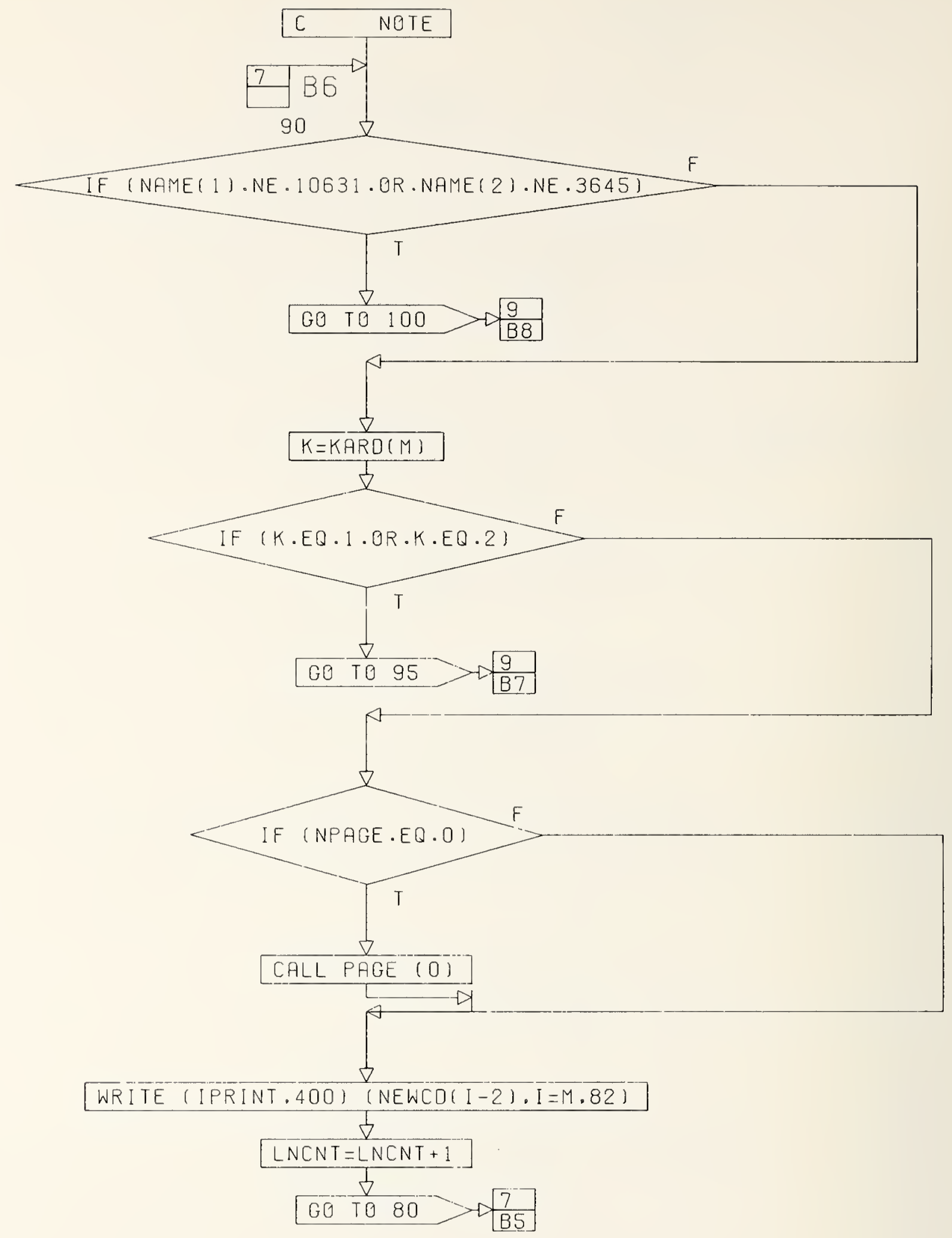

CONT. ON PG 9

$P G \quad 8$ OF 22 


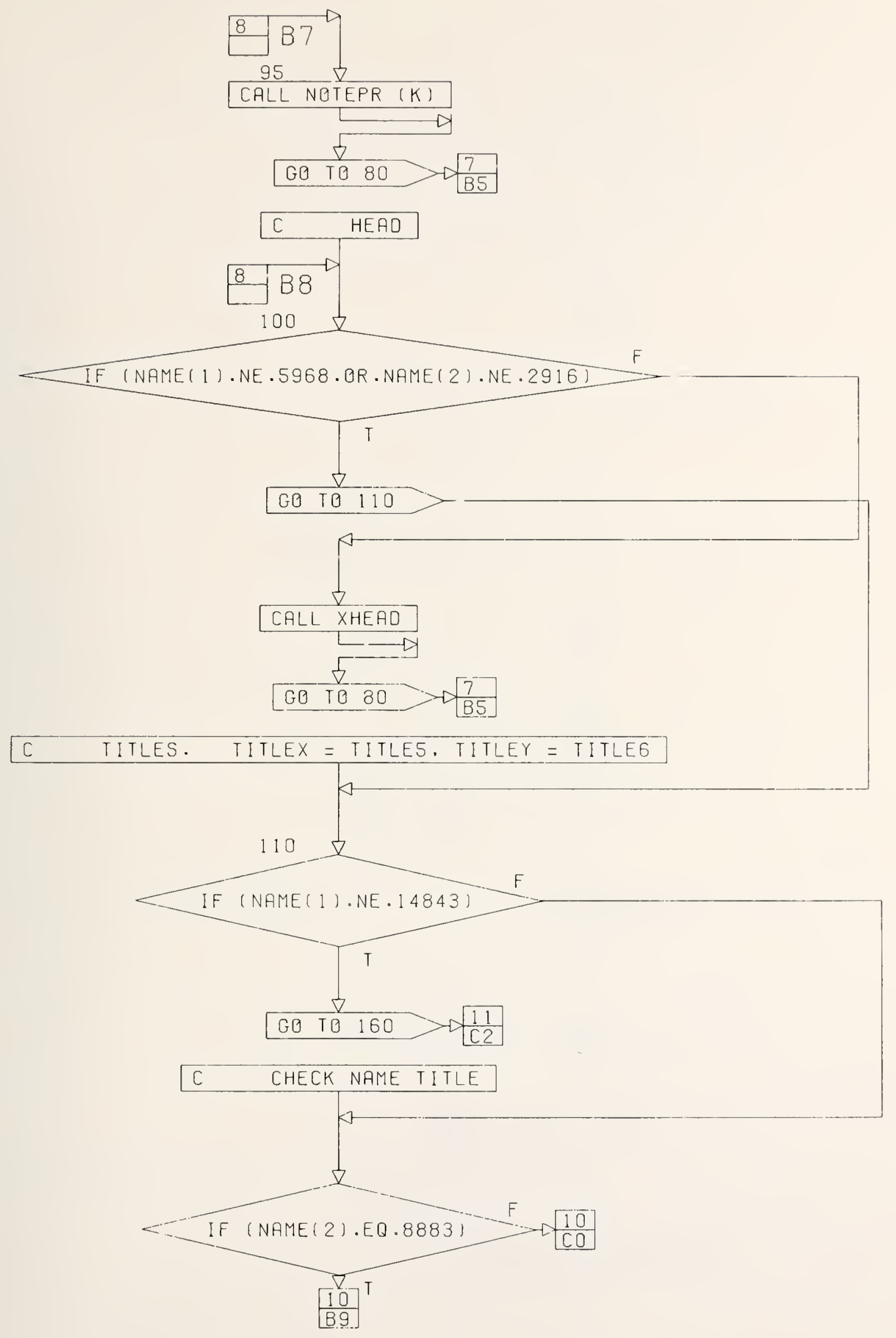

CONT. ON PG 10

PG $9 \quad$ OF 22 


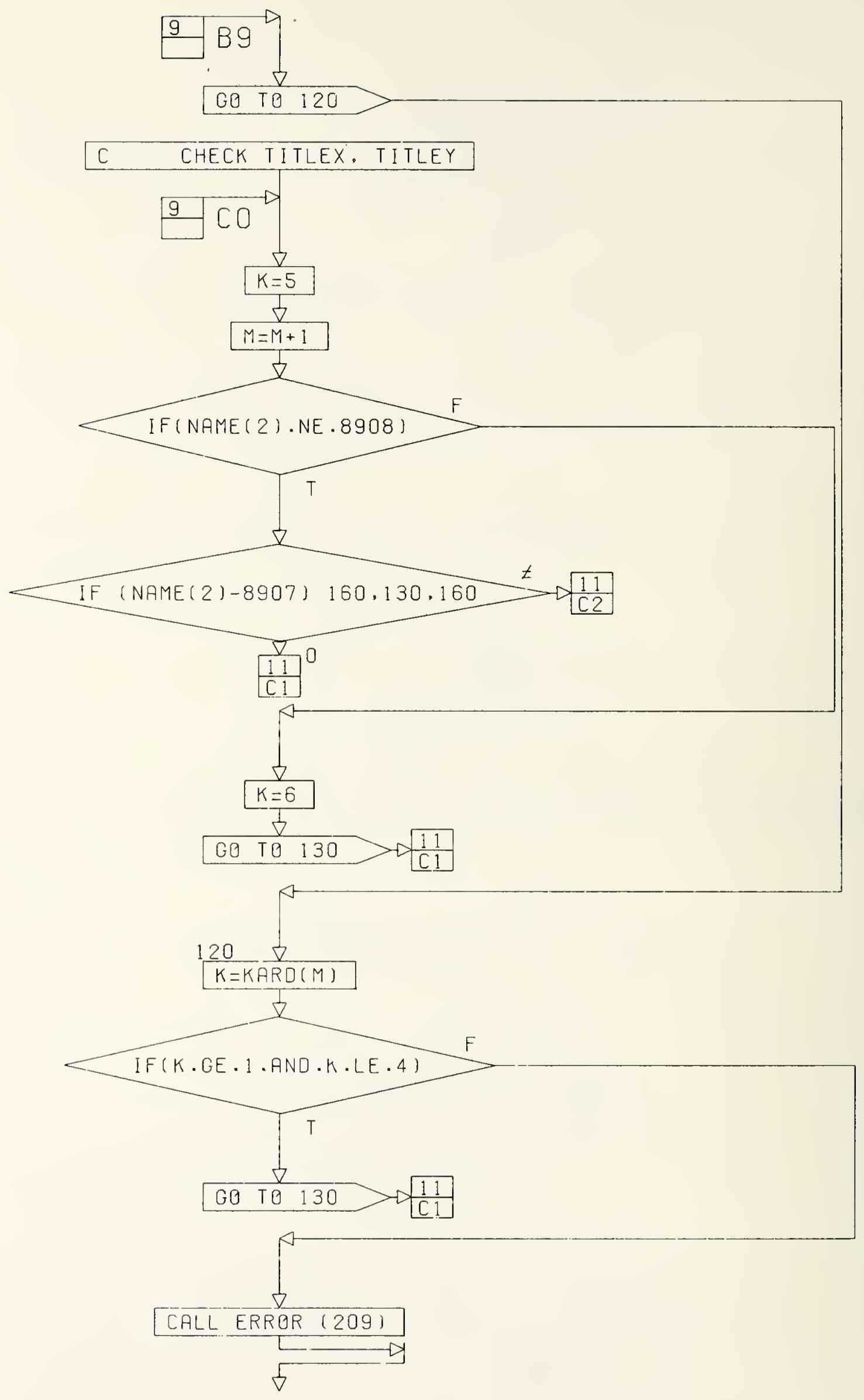

CONT. ON PG 11 


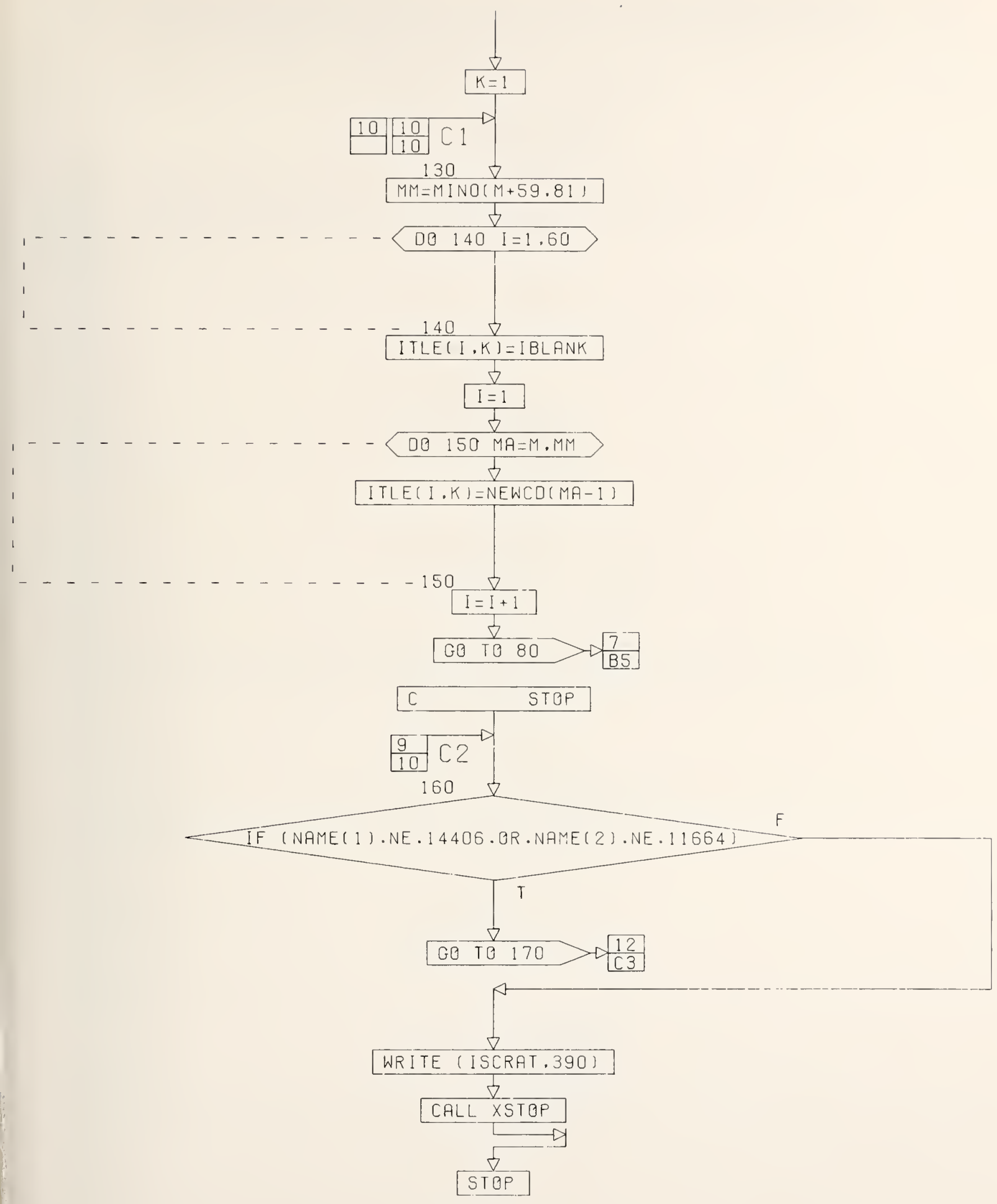

CONT. ON PG 12

PG 11 GF 22 
C M IS POINTING AT THE FIRST NON-LETTER AFTER NAME. LOOK FOR POSSIBLE NAME QUALIFIER OR ARGUMENTS OR END OF CARD.

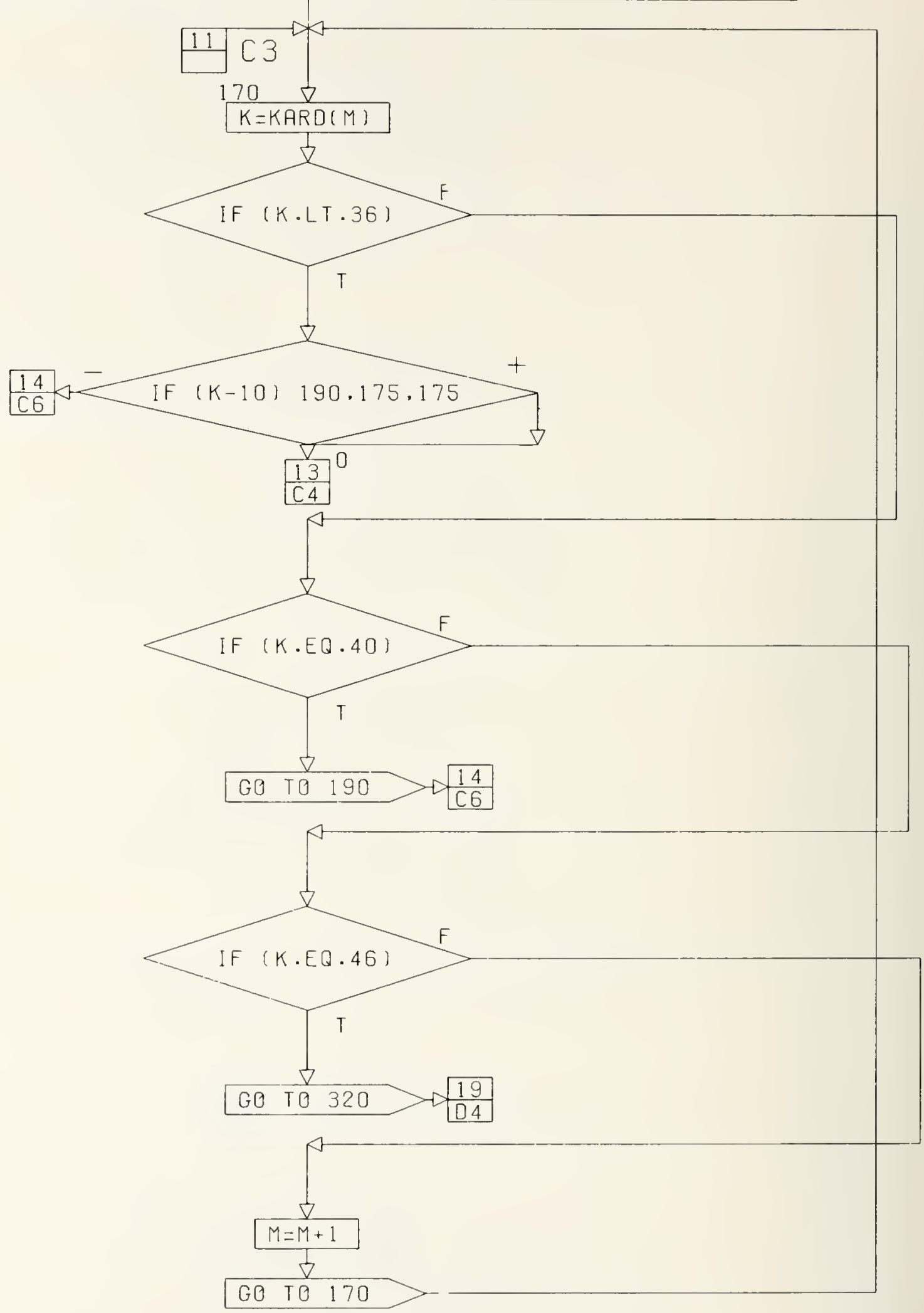

CONT. ON PG 13 
C A LETTER FOUND. ASSEMBLE SECOND NAME (COMMANO QUALIFIERI.
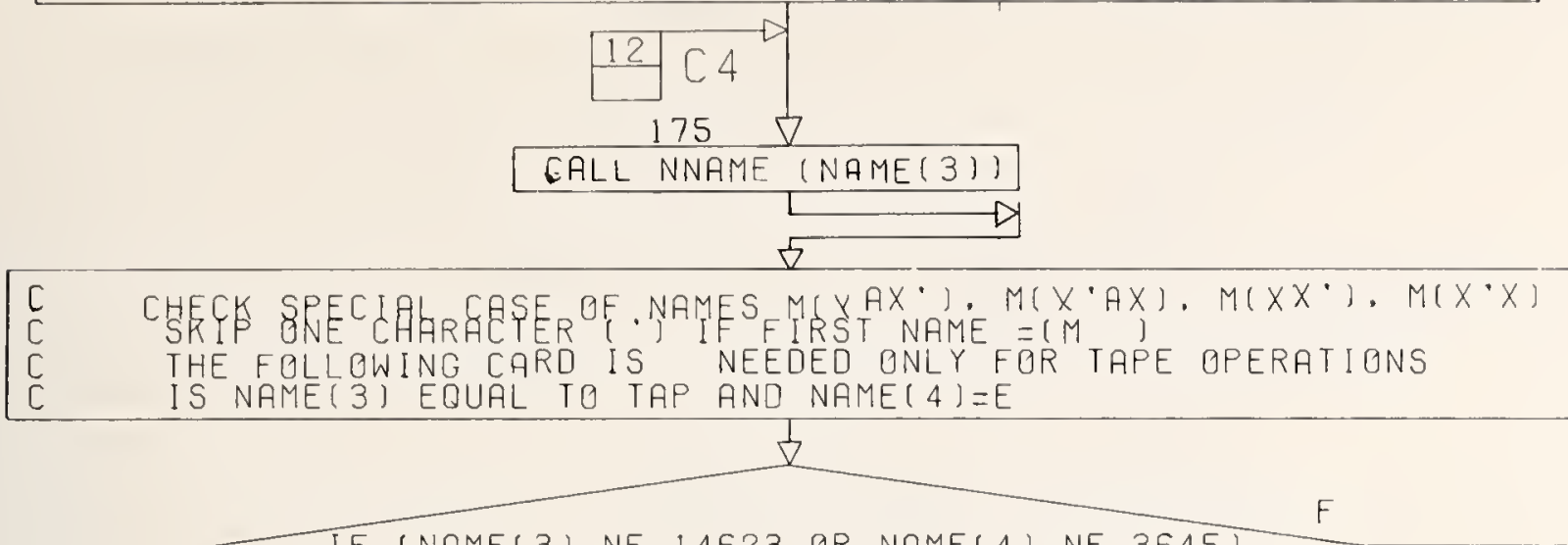

IF (NAME(3).NE.14623.OR.NAME(4).NE.3645)
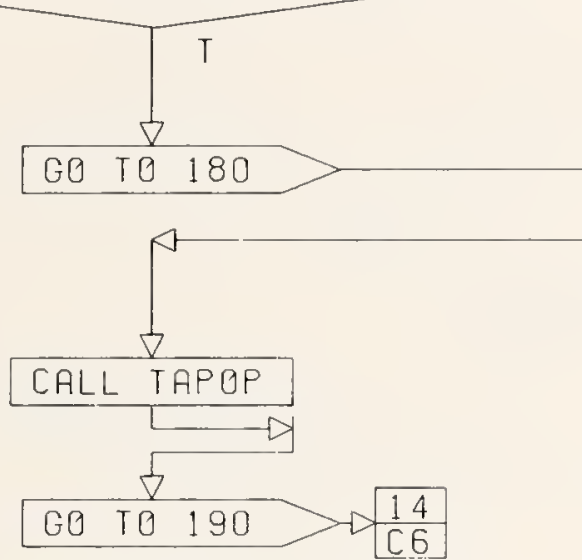

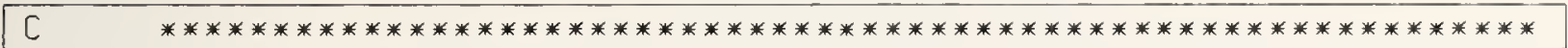

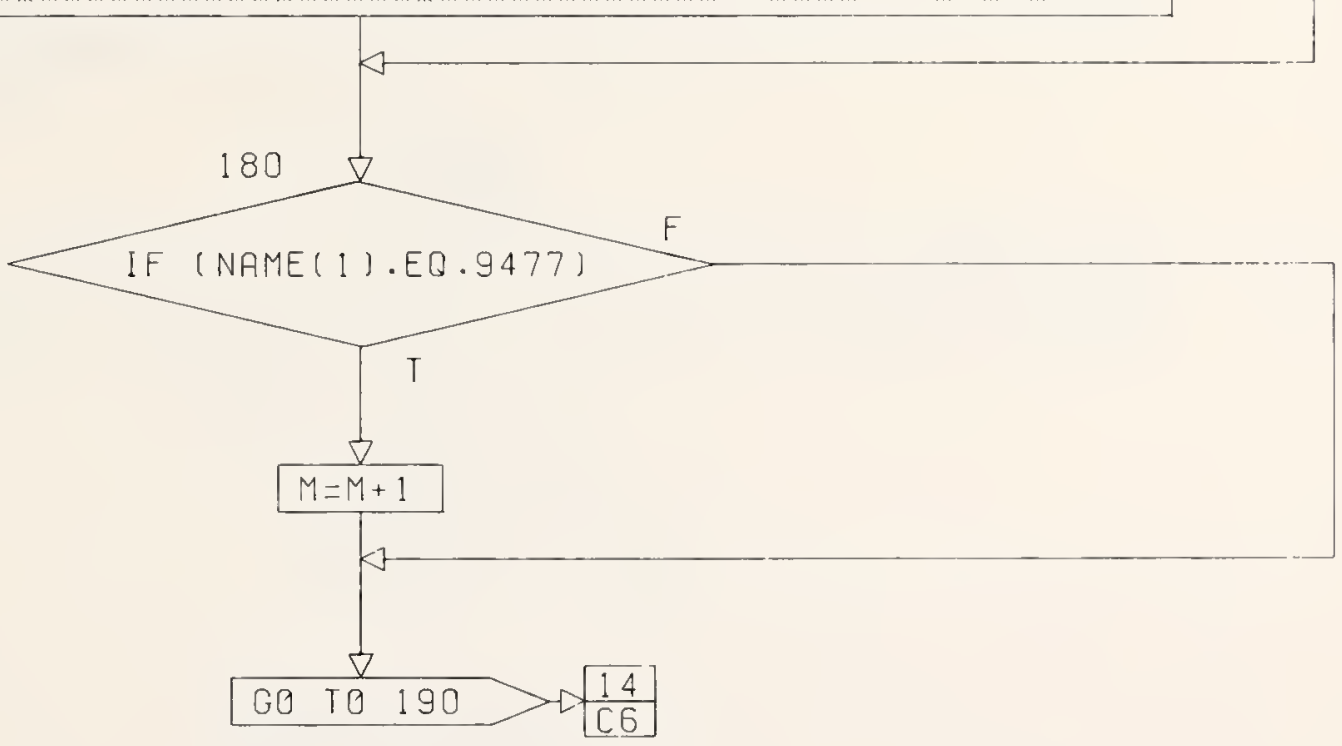

CONT. ON PG 14 


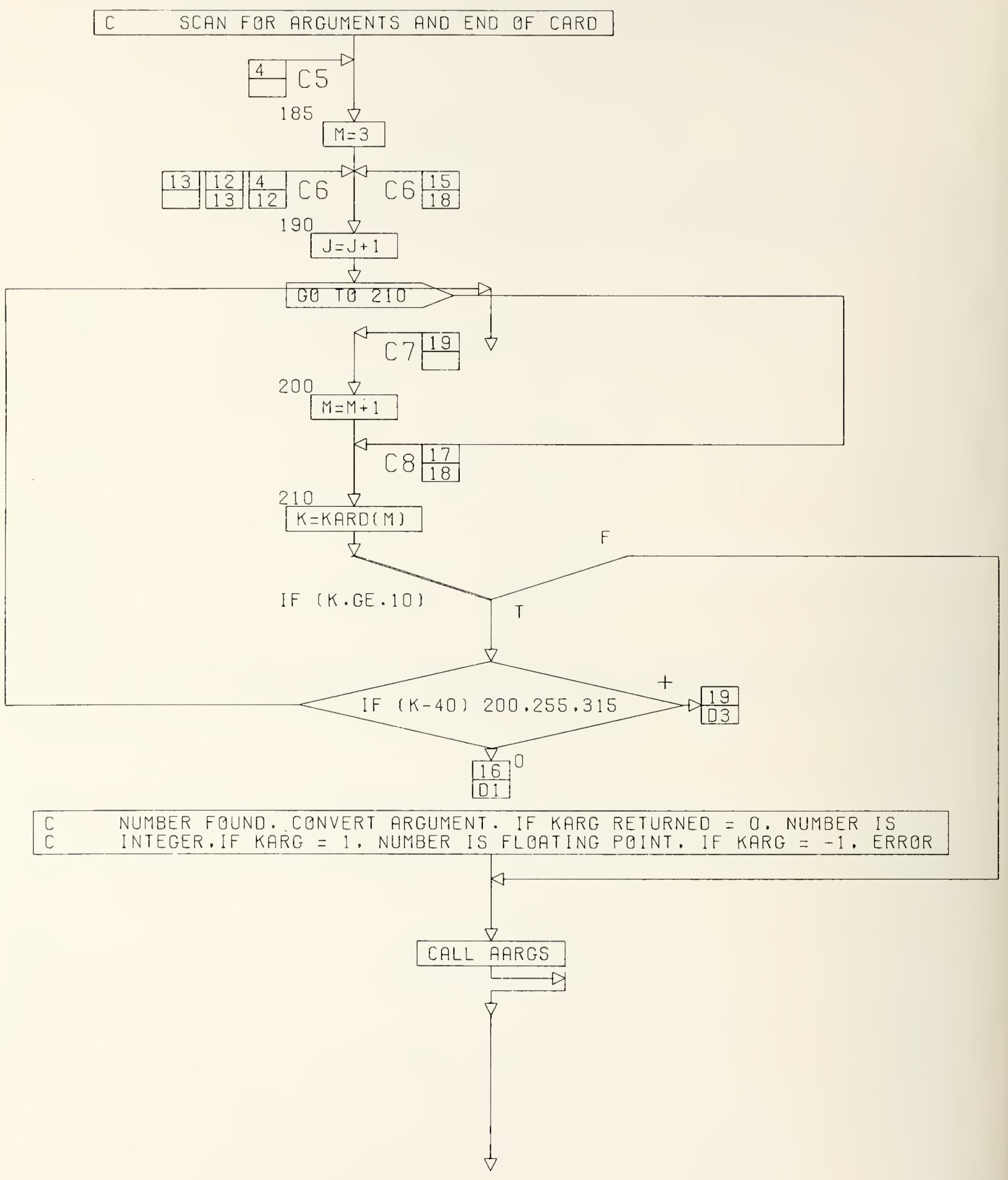

CONT. ON PG 15 

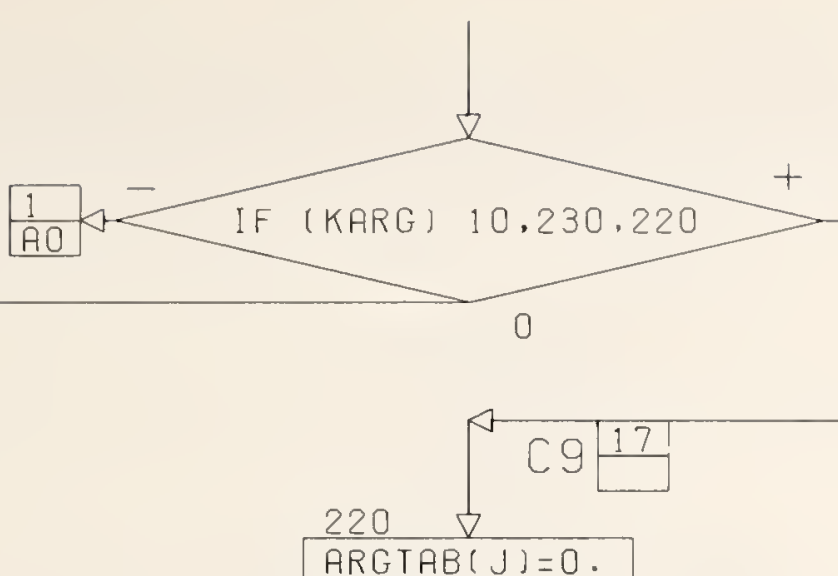

$\operatorname{ARGTAB}(J)=0$.

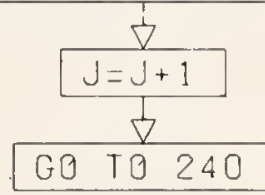

\begin{tabular}{|ll}
\hline C & ARGUMENT IS AN INTEGER. ADD A BIAS OF 8192 THEN CHECK THAT IT IS \\
C & .GT. O
\end{tabular}
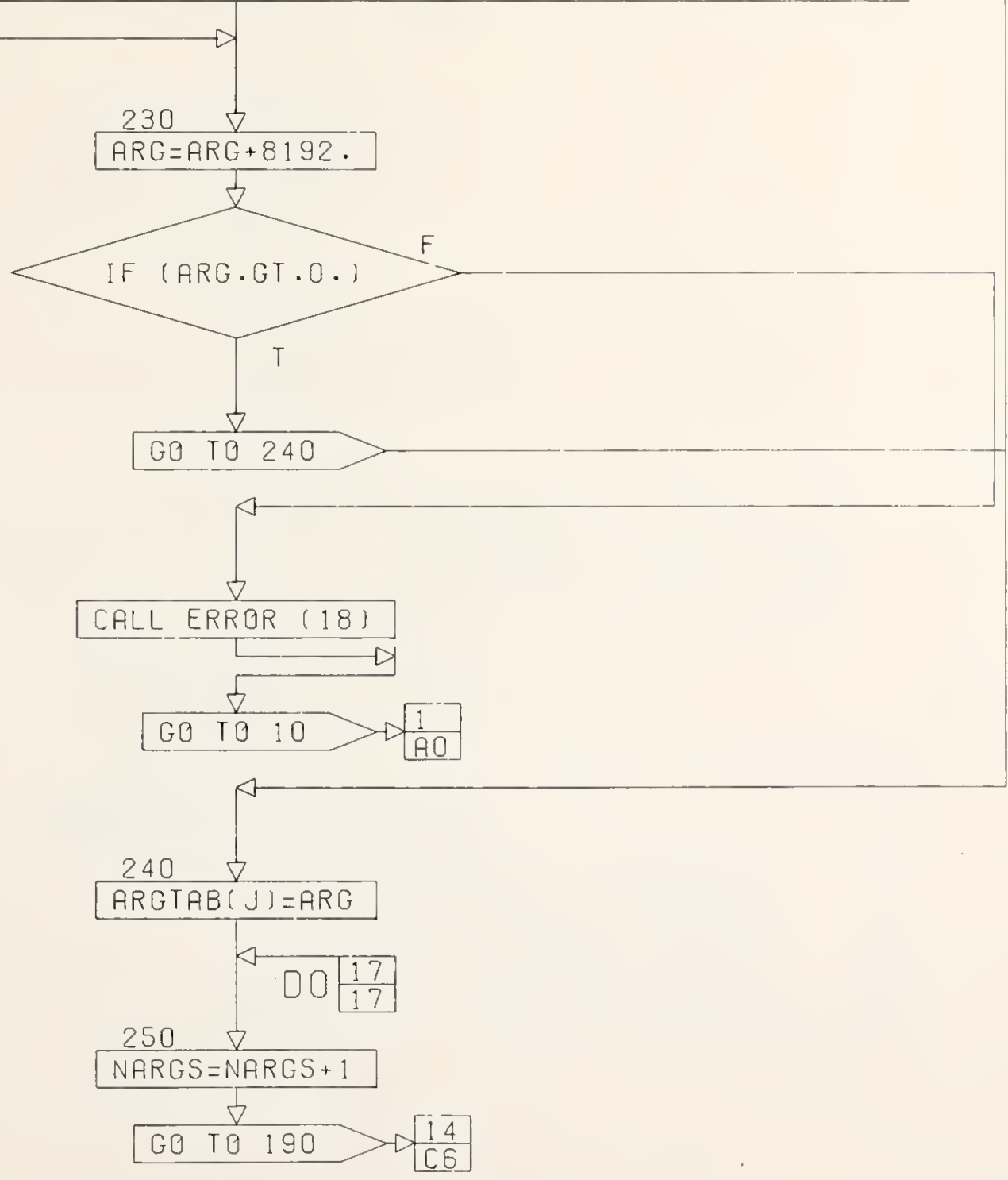

CONT. ON PG 16 
ASTERISK FOUND. CONVERT

IF BRACKETED BY SINGLE ASTERISKS, QUANTITY IS TO BE USED AS A

FLOATING POINT ARGUMENT. IF BRACKETED BY DOUBLE ASTERISKS. OUANTITY IS TO BE TRUNCATED AND USED AS AN INTEGER RRGUMENT.

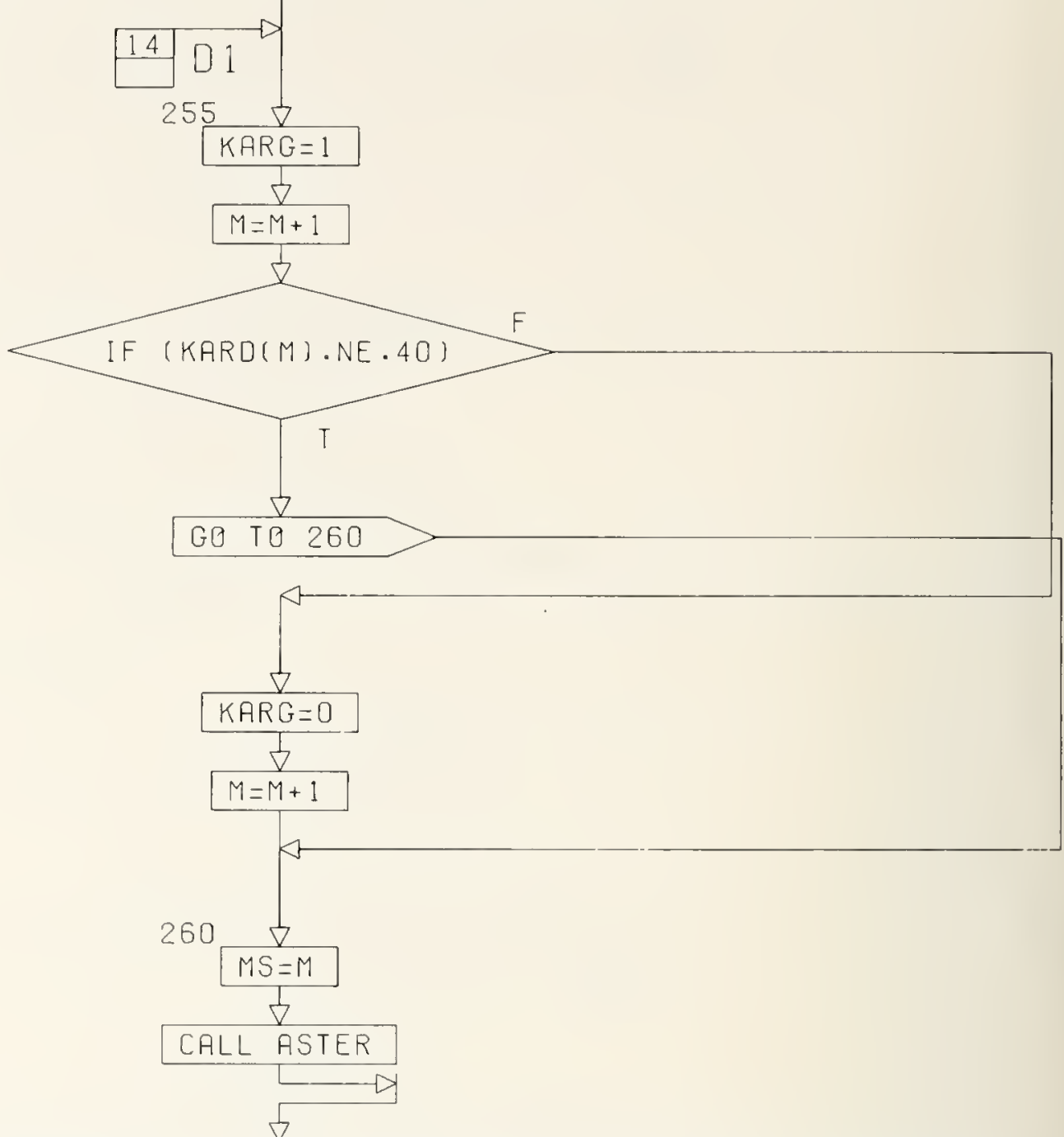

THE TERMINAL ASTERISK(S) HAVE BEEN CHECKEO TO BE THE SAME AS THE INTITAL SET (IF NO ERROR) AND M IS POINTING AT THE FIRST CHARACTER AFTER THE LAST ASTERISK.

KARG RETURNEO AS 1 = ERRQR FOUNO

$$
\begin{aligned}
& 2=\text { FLOATING POINT CONSTANT, Z.B. *PI* } \\
& 3 \text { = INTEGER NAMED VARIABLE, Z.B. * NRMAX** } \\
& 4=F L \text {. PT. NAMEO VARIABLE, Z.B. *NRMAX* } \\
& 5 \text { = INTEGER ROW-COLUMN }
\end{aligned}
$$

PT. ROW-COLUMN.
PT

$6=$ FL. PT. ROW-COLUMN.
$7=$ STRING OF ASTERISKS
$7 \cdot$ B

$Z \cdot B \cdot * 1,2 *$

A STRING OF THREE OR MORE ASTERISKS IMPLIES - THRU-

EXAMPLE..

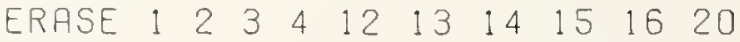

IS EQUIVALENT TO

ERASE 1 $1 * * 4,12 * * * 16,20$

PRINT $1 \begin{array}{lllllllll}20 & 19 & 18 & 17 & 16 & 15 & 14\end{array}$

PRINT 1. $20 * * * 14$

IS EQUIVALENT TO 


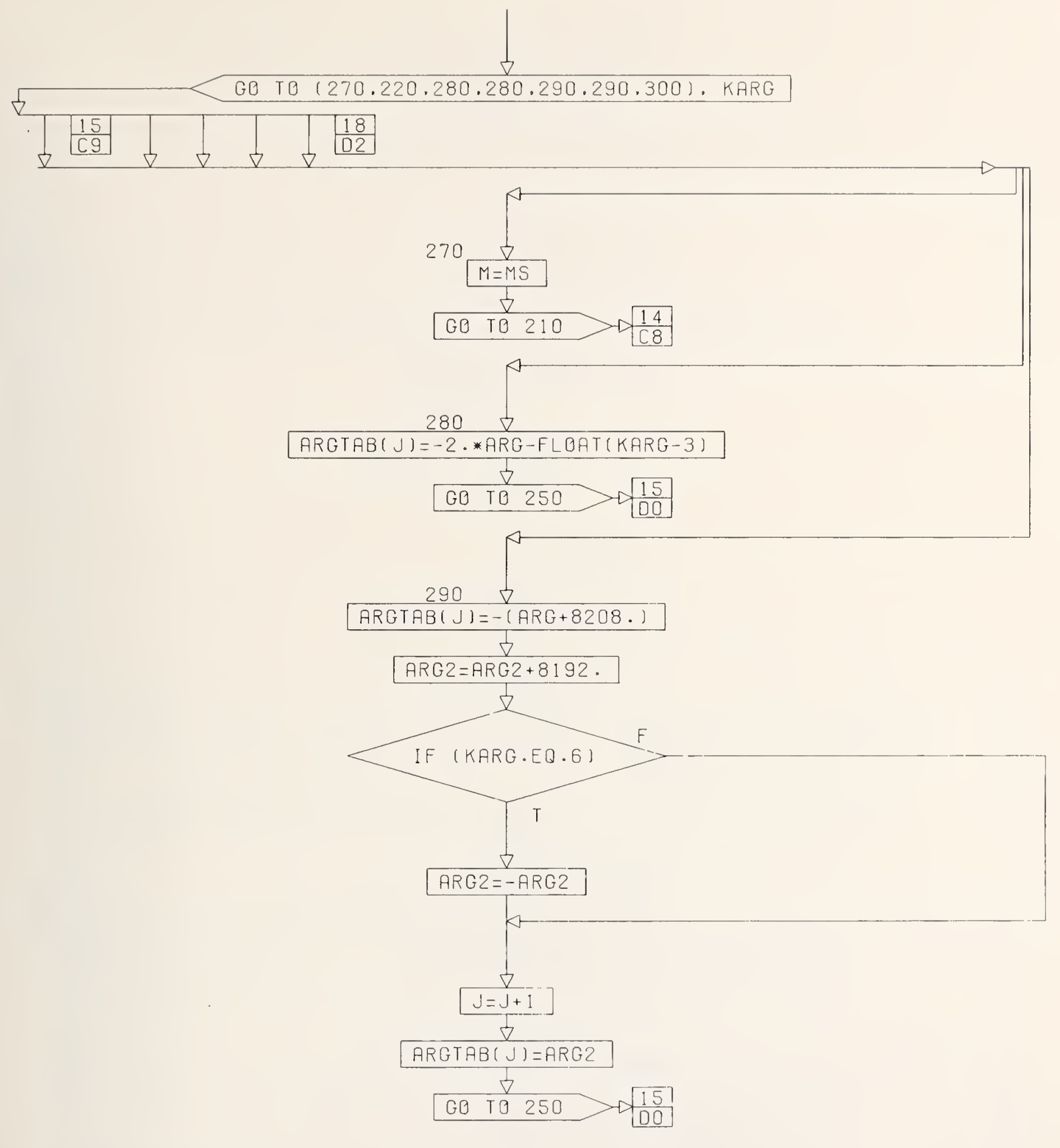

CONT. ON PG 18

PG 17 OF 22

363 

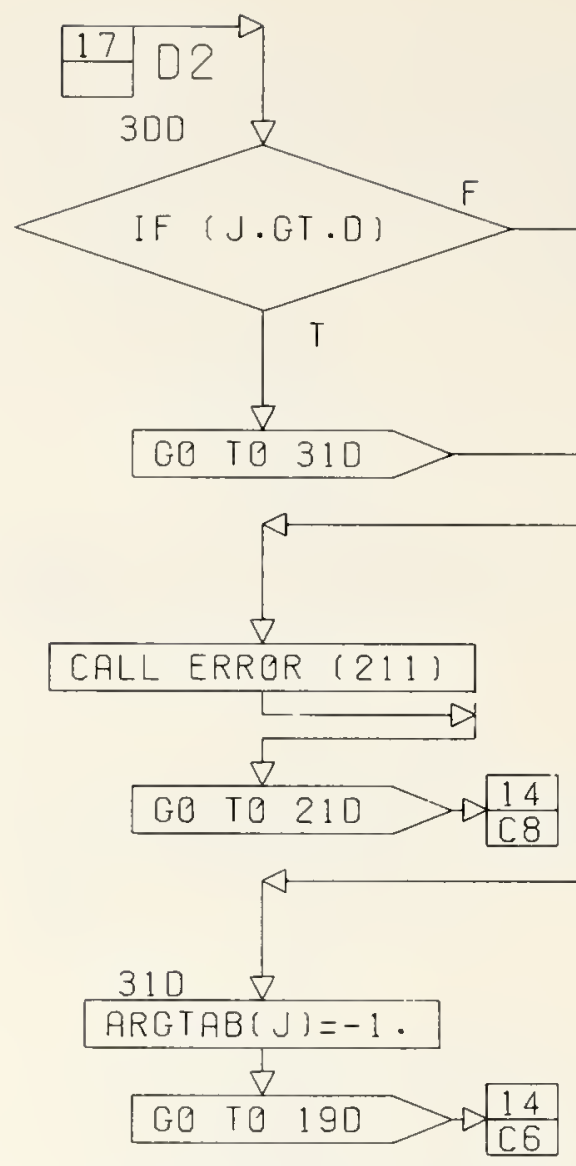

ARGTAB SETUP

IF ENTRY .GT. D. IT IS AN INTEGER CONSTANT ( $Z$.B. COLUMN NUMBER) TO WHICH A BIAS OF 8192 HAS BEEN ADDED. THIS IS TO SAY THAT A NEGATIVE INTEGER ARGUMENT MAY NOT BE EXPLICITLY GIVEN OR MODIFIED TO BE LESS THAT -8191

IF ENTRY .EQ.D. THE NEXT ENTRY IS A FLOATING POINT CONSTANT.

IF ENTRY .LT. D. ARGUMENT IS A VARIABLE. SET SIGN POSITIVE AND.. IF ENTRY .LT. 16. IT IS A NAMED VARIABLE REFERENCE NUMBER

\begin{tabular}{lllllll}
\hline$C$ & 2.3 & NRMAX & 6.7 & $V$ & 10.11 & $X$ \\
$C$ & & & 8.9 & $W$ & 12.13 & $Y$ \\
$C$ & & & & 14.15 & $Z$
\end{tabular}

$V, W, X, Y, Z$. ARE FGR PRGGRAMMING CONVENIENCE GNLY AND DO NUT AFFECT THE OPERATION OF OMNITAB

IF ENTRY IS EVEN. CURRENT VALUE TO BE TRUNCATED AND USED

AS AN INTEGER ARGUMENT.

IF ENTRY IS OOD. THE CURRENT VALUE IS TO BE USED AS ${ }^{\circ} A$

FLOATING POINT ARGUMENT.

IF ENTRY.GT. 16, IT IS A WORKSHEET REFERENCE (ROW.COLUMN) TO WHICH A BIAS OF 8192 . HAS BEEN ADDED.

ENTRY - $8208=$ ROW NUMBER

ABS (NEXT ENTRY) = COLUMN NUMBER TO WHICH A BIAS OF 8192. HAS BEEN ADOED.

IF NEXT ENTRY IS NEGATIVE, WORKSHEET CONTENTS RRE TO BE USED AS A FLORTING POINT CONSTANT. IF +, WORKSHEET VALUE $\nabla$

C TO BE TRUNCATED AND USED AS AN INTEGER ARGUMENT.

$$
\text { CONT. ON PG }
$$




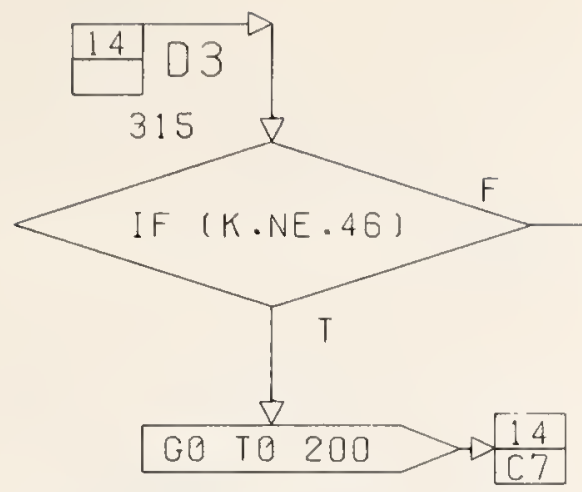

C THE TERMINATION OF CARD FOUND ( \$ ENCOUNTEREDI

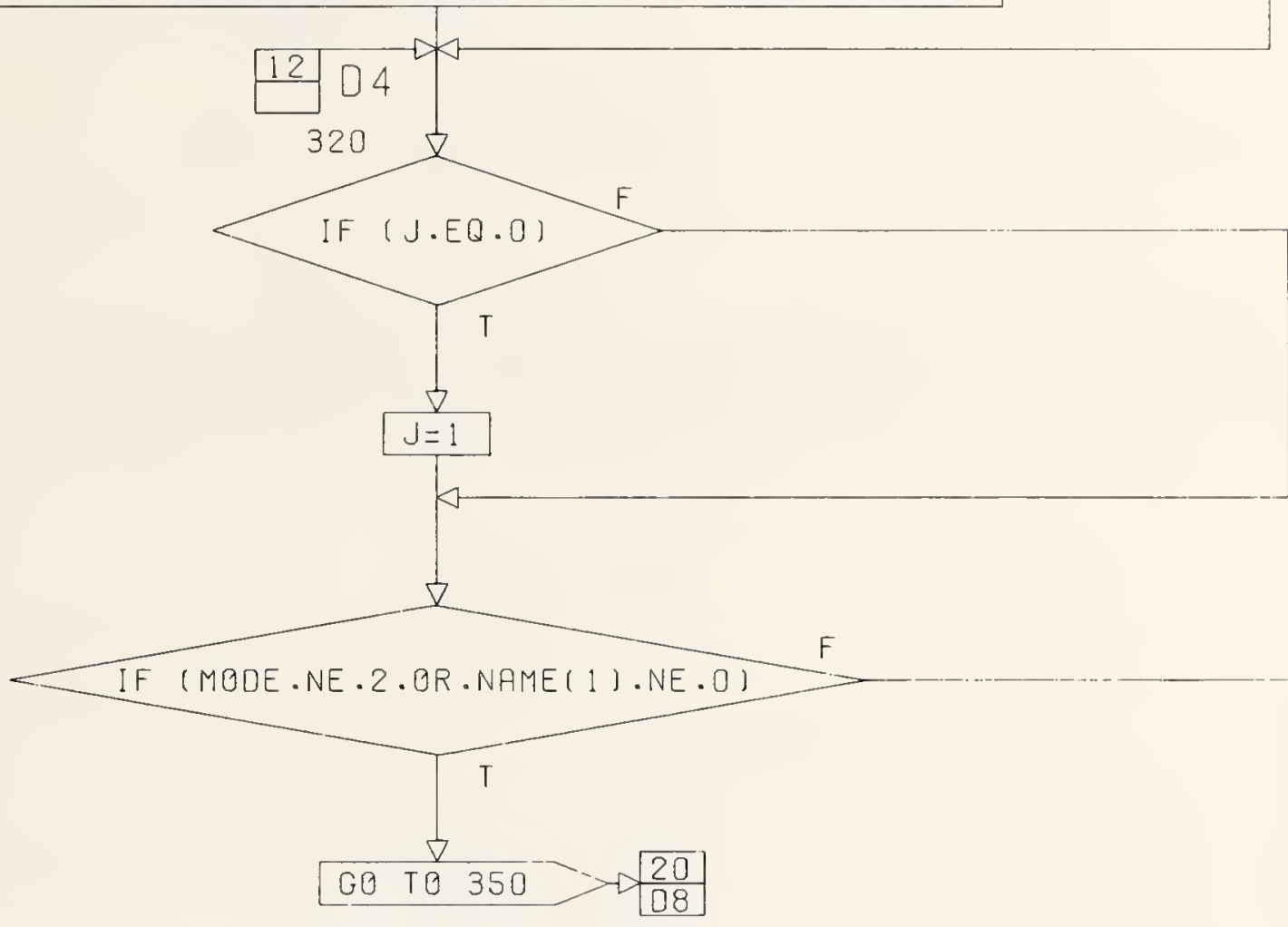

C IN INPUT MODE ANO NO POSSIBLE NAME. RETURN TO SET OR READ ROUTINE

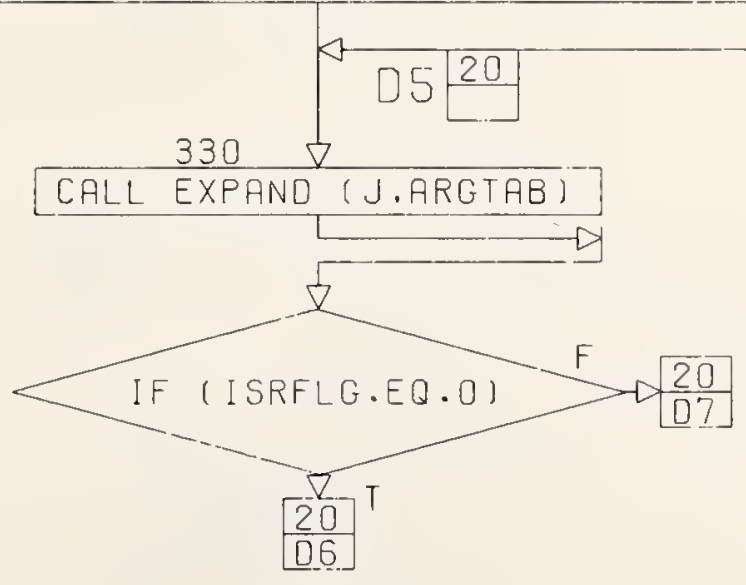

CONT. ON PG 20

PG 19 OF 22 

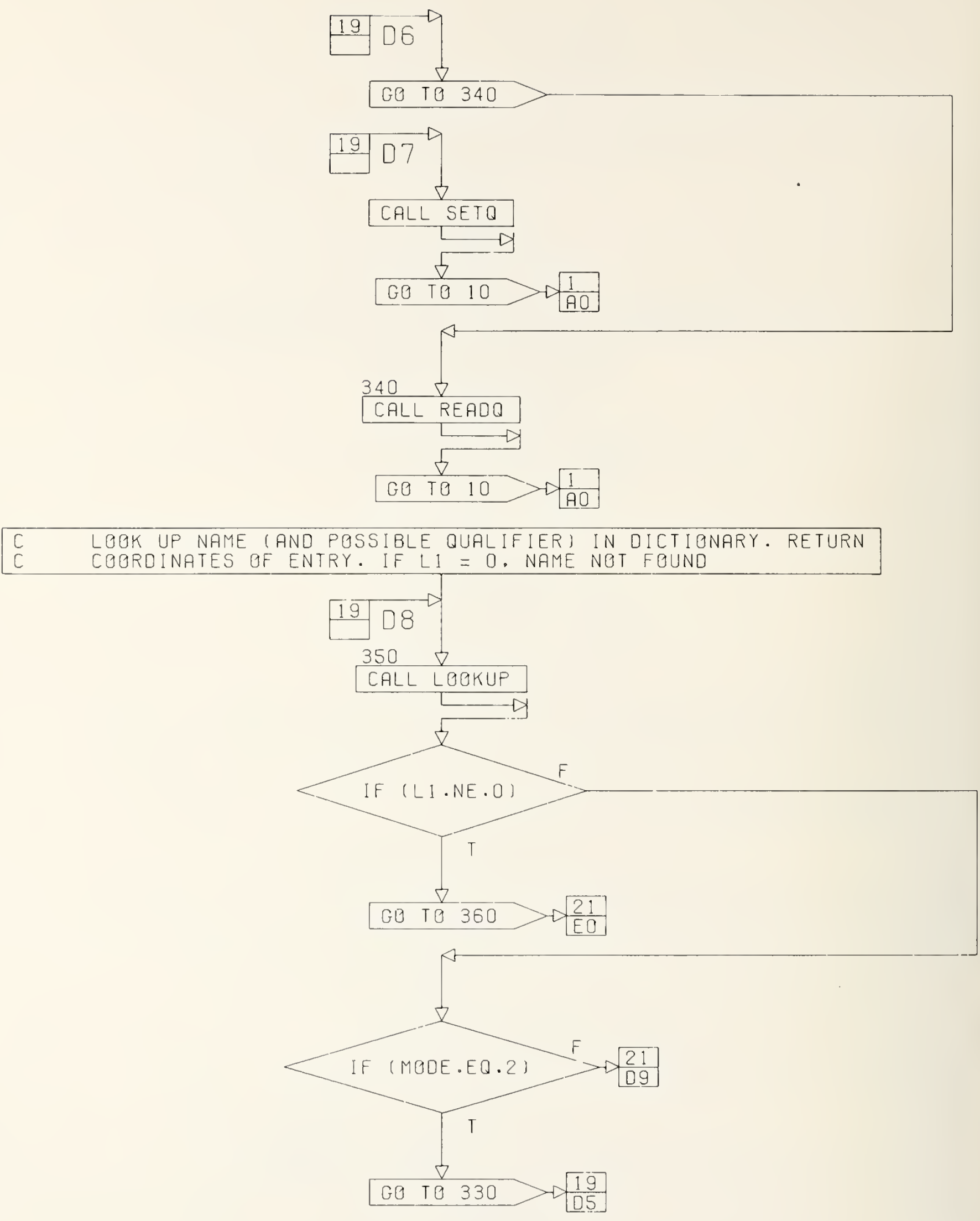

CONT. ONPG 21 


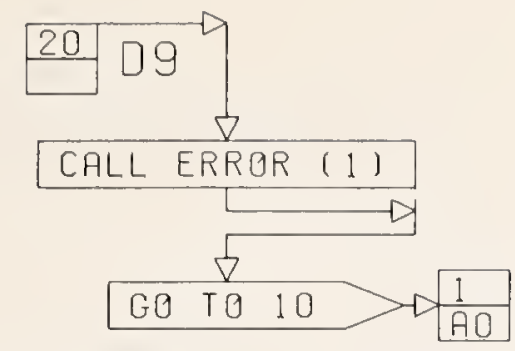
\begin{tabular}{llll} 
C NAME FOUND & & \\
C & THE FOLLOWING CAROS ARE NEODEO ONLY FOR TAPE OPERATIONS \\
C & STATEMENT WAS 220 IF (MOOE.EQ.2) MODE=1 & \\
\hline
\end{tabular}

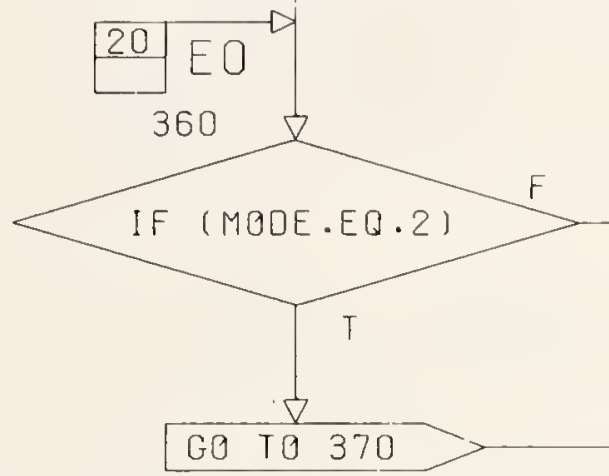

C

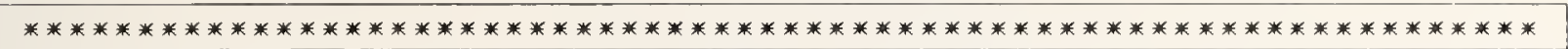
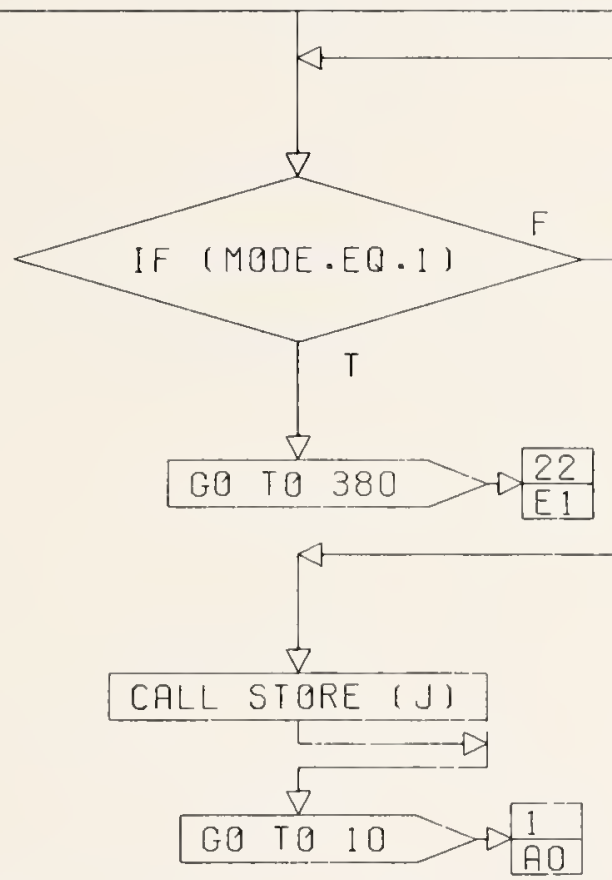

C THE FOLLOWING CARDS ARE NEODED ONLY FOR TAPE OPERATIONS

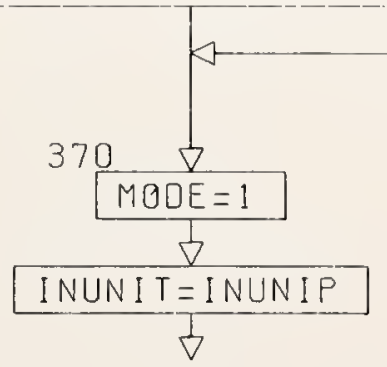

CONT. ON PG 22 
Latest developments in the subject area of this publication, as well as in other areas where the National Bureau of Standards is active, are reported in the NBS Technical News Bulletin. See following page. 


\section{HOW TO KEEP ABREAST OF NBS ACTIVITIES}

Your purchase of this publication indicates an interest in the research, development, technology, or service activities of the National Bureau of Standards.

The best source of current awareness in your specific area, as well as in other NBS programs of possible interest, is the TECHNICAL NEWS BULLETIN, a monthly magazine designed for engineers, chemists, physicists, research and product development managers, librarians, and company executives.

If you do not now receive the TECHNICAL NEWS BULLETIN and would like to subscribe, and/or to review some recent issues, please fill out and return the form below.

Mail to: Office of Technical Information and Publications National Bureau of Standards

Washington, D. C. 20234

Name

Affiliation

Address

City State Zip

Please send complimentary past issues of the Technical News Bulletin.

Please enter my 1-yr subscription. Enclosed is my check or money order for $\$ 3.00$ (additional $\$ 1.00$ for foreign mailing). Check is made payable to: SUPERINTENDENT OF DOCUMENTS. SP 339 


\section{PERIODICALS}

JOURNAL OF RESEARCH reports National Bureau of Standards research and development in physics, mathematics, chemistry, and engineering. Comprehensive scientific papers give complete details of the work, including laboratory data, experimental procedures, and theoretical and mathematical analyses. Illustrated with photographs, drawings, and charts.

Published in three sections, available separately:

\section{Physics and Chemistry}

Papers of interest primarily to scientists working in these fields. This section covers a broad range of physical and chemical research, with major emphasis on standards of physical measurement, fundamental constants, and properties of matter. Issued six times a year. Annual subscription: Domestic, $\$ 9.50$; foreign, $\$ 11.75^{*}$.

\section{Mathematical Sciences}

Studies and compilations designed mainly for the mathematician and theoretical physicist. Topics in mathematical statistics, theory of experiment design, numerical analysis, theoretical physics and chemistry, logical design and programming of computers and computer systems. Short numerical tables. Issued quarterly. Annual subscription: Domestic, $\$ 5.00$; foreign, $\$ 6.25 *$.

\section{- Engineering and Instrumentation}

Reporting results of interest chiefly to the engineer and the applied scientist. This section includes many of the new developments in instrumentation resulting from the Bureau's work in physical measurement, data processing, and development of test methods. It will also cover some of the work in acoustics, applied mechanics, building research, and cryogenic engineering. Issued quarterly. Annual subscription: Domestic, $\$ 5.00$; foreign, $\$ 6.25^{*}$.

\section{TECHNICAL NEWS BULLETIN}

The best single source of information concerning the Bureau's research, developmental, cooperative and publication activities, this monthly publication is designed for the industry-oriented individual whose daily work involves intimate contact with science and technology-for engineers, chemists, physicists, research managers, product-development managers, and company executives. Annual subscription: Domestic, $\$ 3.00$; foreign, $\$ 4.00^{*}$.

* Difference in price is due to extra cost of foreign mailing.
NONPERIODICALS

Applied Mathematics Series. Mathematical tables, manuals, and studies.

Building Science Series. Research results, test methods, and performance criteria of building materials, components, systems, and structures.

Handbooks. Recommended codes of engineering and industrial practice (including safety codes) developed in cooperation with interested industries, professional organizations, and regulatory bodies.

Special Publications. Proceedings of NBS conferences, bibliographies, annual reports, wall charts, pamphlets, etc.

Monographs. Major contributions to the technical literature on various subjects related to the Bureau's scientific and technical activities.

National Standard Reference Data Series. NSRDS provides quantitative data on the physical and chemical properties of materials, compiled from the world's literature and critically evaluated.

Product Standards. Provide requirements for sizes, types, quality and methods for testing various industrial products. These standards are developed cooperatively with interested Government and industry groups and provide the basis for common understanding of product characteristics for both buyers and sellers. Their use is voluntary.

Technical Notes. This series consists of communications and reports (covering both other agency and NBS-sponsored work) of limited or transitory interest.

Federal Information Processing Standards Publications. This series is the official publication within the Federal Government for information on standards adopted and promulgated under the Public Law 89-306, and Bureau of the Budget Circular A-86 entitled, Standardization of Data Elements and Codes in Data Systems. 




